\title{
Framing Wicked Problems Using CoDesign and a Hybrid Design Toolset
}

\author{
Thesis
}

Presented in Partial Fulfillment of the Requirements for the Degree Master of Fine Arts in the Graduate School of The Ohio State University

By

Erika Leigh Braun, B.S.

Graduate Program in Design

The Ohio State University

2016

Thesis Committee:

Elizabeth B.-N. Sanders, Ph.D., Advisor

Alan Price

David Staley, Ph.D.

Elliot Bendoly, Ph.D. 
Copyright by

Erika Leigh Braun

2016 


\begin{abstract}
Design is not simply an outcome, but a process of problem solving. The current transitional process for adults with Autism Spectrum Disorder (ASD) and the challenges faced by their healthcare providers and parents is a wicked problem that needs to be addressed. Wicked problems are not good or bad, but are often made up of multiple stakeholders and an indeterminate end-state. Design problems are often wicked; there is no definitive formula or an existing solution. Trained and practiced in reshaping complexity and ambiguity through Design Thinking to give form to new ideas that do not yet exist, Designers have the capacity to expand their role from simply 'makers' of artifacts to 'makers' of sense, building new tools and integrating new ways of problem solving to assist with framing wicked problems. The objective of this body of research is to investigate a hybrid CoDesign approach (using digital and non-digital Design Thinking tools), and the expanding role of the Designer and stakeholders in tackling wicked problems through an exploratory autism case study.
\end{abstract}

The role of the Designer in addressing wicked problems and the use of technology for collective sensemaking in the Design process were investigated through the development of a digital prototype in parallel with an exploratory Participatory Action Research (PAR) case study centered on issues surrounding transitional care and support for adults with autism and their families. A diverse group of stakeholders was brought together to participate in an iterative CoDesign process aimed at building shared understanding, stimulating new ways of thinking, and reframing the wicked problem to create new resolutions for the Center for Autism Services and Transition 
(C.A.S.T.), a clinic for adults with autism connected to The Ohio State University Wexner Medical Center.

The outcomes of the case study and findings from the development and testing of the digital prototype support the value of sensemaking and reframing, and the significance of diversity, inclusivity and immersion in PAR to engender intangible outcomes as well as design concepts that can be carried into the next stages of Design development. Additionally, the findings support the contribution potential of people on the autism spectrum in CoDesign roles and the significance of a Designer's skillset in facilitating collaborative sessions around a multi-faceted problem and creating the tools and seeing/maker spaces through which a collective team of Designers and CoDesigners can frame problems and innovate. The case study promotes the benefit of a hybrid PAR framework that incorporates face-to-face interaction, physical toolsets for shared/critical making, and technology applications to engender new forms of engagement and to nurture each expert's ability to meaningfully contribute to creating true value in a wicked problem. When Design is no longer perceived as only an aesthetic outcome, but instead as a valuable asset for tackling complexity, Designers will be able to see themselves, and be seen by others, as framers, new types of form givers in the front-end of the Design process - extending their value and reach to tackle more pressing issues in society. 


\section{Dedication}

For those in the autism community dedicated,

not to changing people with autism,

but instead, to changing the world they live in. 


\section{Acknowledgements}

I would like to extend my appreciation to the following people for their guidance and encouragement throughout my graduate studies. First, I would like to thank my graduate committee. To Liz Sanders, I am grateful for your support throughout this thesis, as well as for your continued mentorship and the genuine passion you share for designing with people. To Alan Price, your influence during the development of the tool helped to extend my abilities and scope of possibilities in the area of technology. To David Staley, your enthusiasm and provocation to rethink new, possible futures were instrumental in helping me understand autism and Design's role in the future. And, to Elliot Bendoly, your feedback on information visualization helped me to see sensemaking and data visualization tools as opportunities to continuously iterate and learn.

Thank you to the IDEA Studio for the opportunity to work on the C.A.S.T. project. To the adults on the autism spectrum, parents, and providers and administrators from The Ohio State University Wexner Medical Center, Nisonger, C.A.S.T., and Nationwide Children's Hospital who graciously shared their time and energy as participants, your contributions, engagement, and feedback were invaluable to the implementation and outcomes of this project.

Thank you to my peers in the Department of Design for your support, insights, and feedback. The breadth and depth of this thesis grew as a result of our collective discussions.

Most of all, thank you to my husband Joe Mullins and to my parents. Your continued support and encouragement enable me to tackle new challenges and grow, and for that I am truly grateful. 


\title{
Vita
}

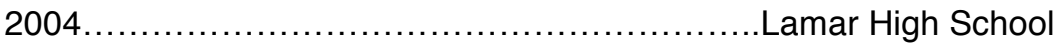

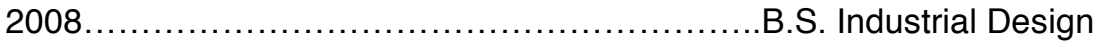 \\ The Ohio State University

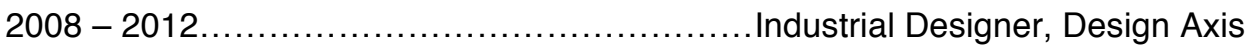 \\ Columbus, Ohio

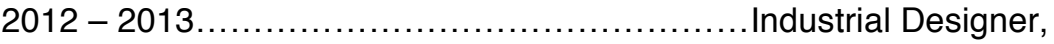 \\ Technology and Commercialization Office, \\ The Ohio State University, Columbus, Ohio \\ 2013 to present..................................Graduate Teaching Associate / \\ Graduate Research Associate, The Ohio \\ State University Department of Design
}

\section{Publications}

Braun, E., Moreland, J., Sanders, E., \& Gill, C. (2014). Designers in Design Thinking. In DS 78:

Proceedings of the E\&PDE 2014 16th International conference on Engineering and Product Design, University of Twente, The Netherlands.

\section{Fields of Study}

Major Field: Design 
Table of Contents

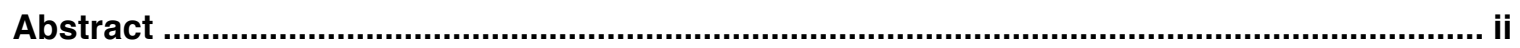

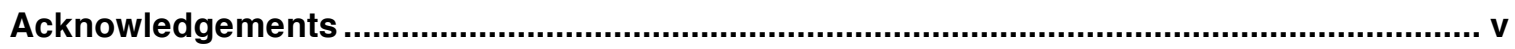

Vita

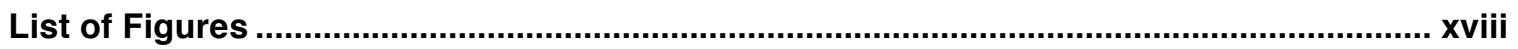

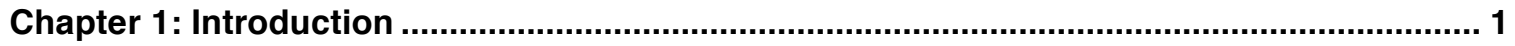

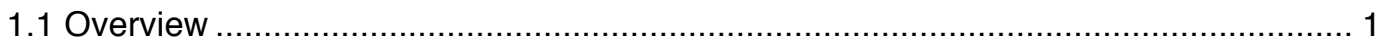

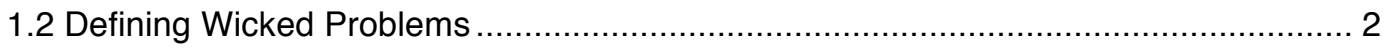

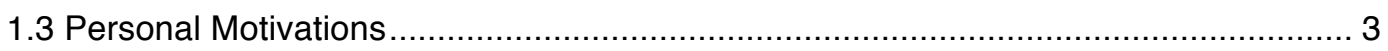

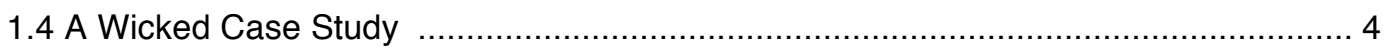

1.4.1 Transitional Care and Support for Adults with ASD ................................ 5

1.4.2 Exploring Digital Capabilities in the Design Process............................... 6

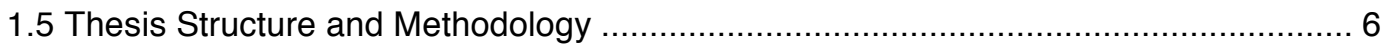

1.6 The Expanding Role of Designers in Wicked Problems ........................................ 8

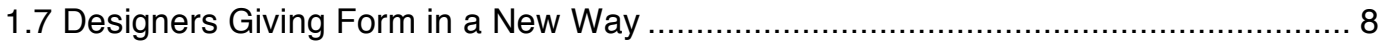

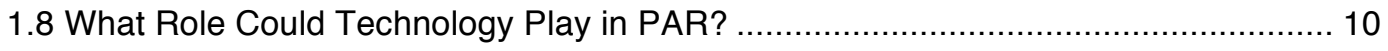

1.9 This Thesis is Exploratory and Provocative in Nature......................................... 11

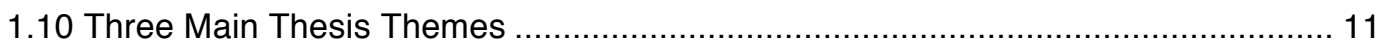

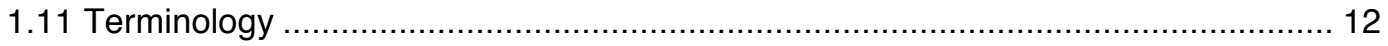

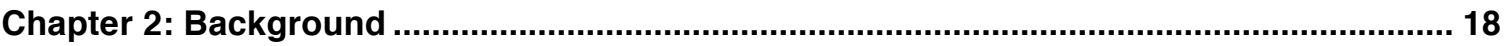

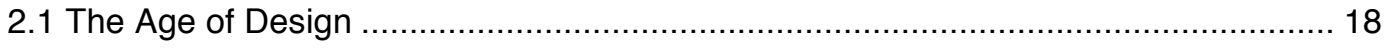




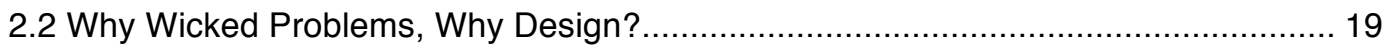

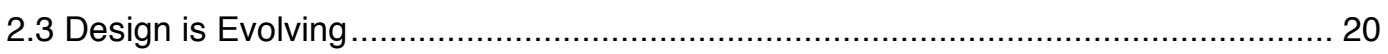

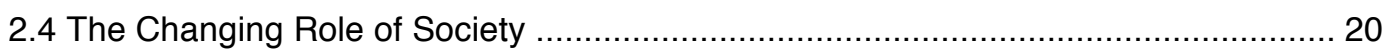

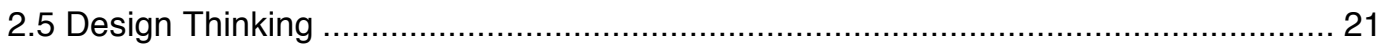

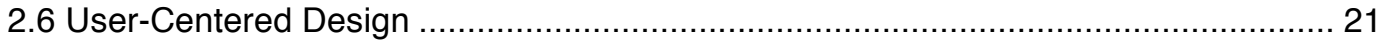

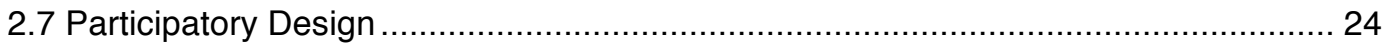

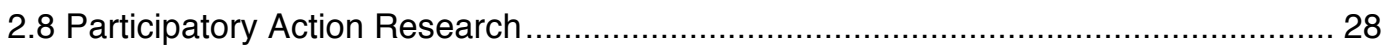

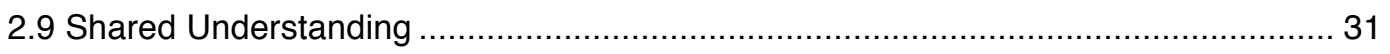

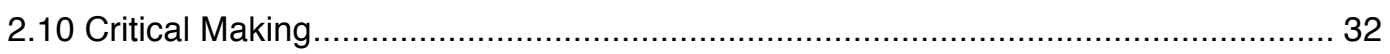

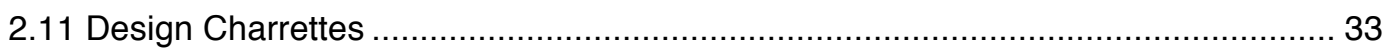

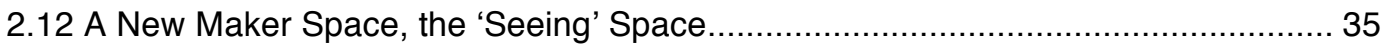

2.13 How We Chose to Look - Sensemaking and Synthesis ....................................... 36

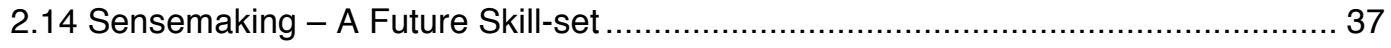

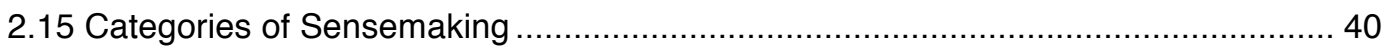

2.16 Sensemaking and Future Perspectives ........................................................ 44

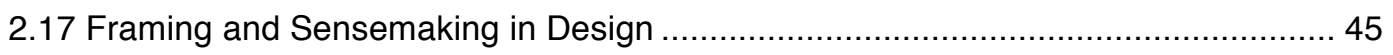

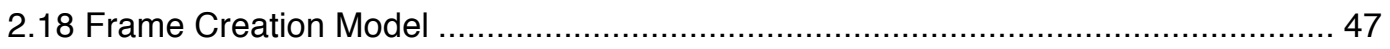

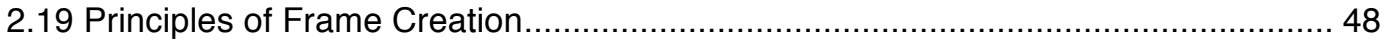

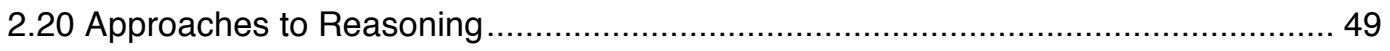

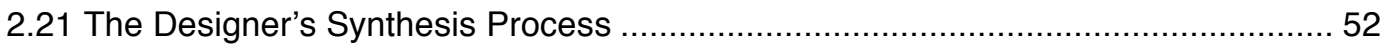

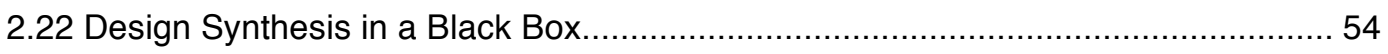

2.23 Designers as Facilitators and Visual Interpreters............................................ 56

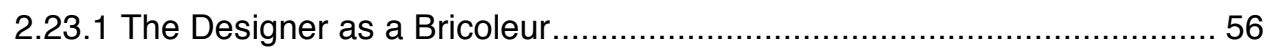

2.23.2 Problem Architect (Giving Form to the Problem) ................................5 57

2.23.3 Curator of Information and the 'Seeing' Space ................................... 58

2.24 Who's Flying the Plane? - The Roles of the Designer and Stakeholder ................61 61 


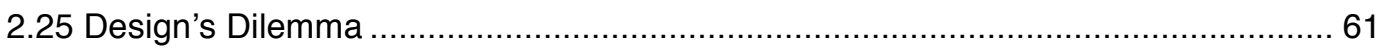

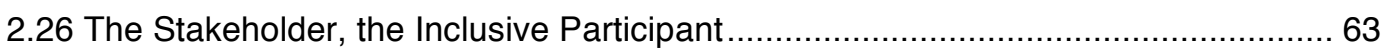

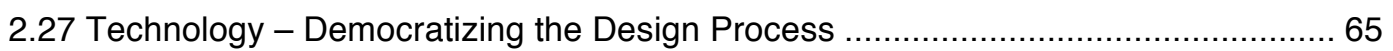

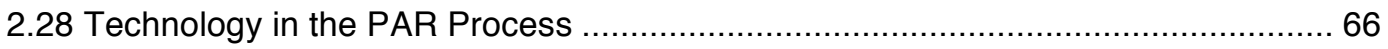

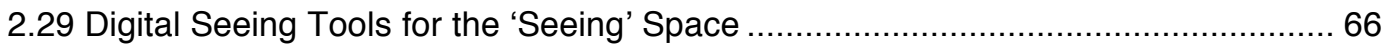

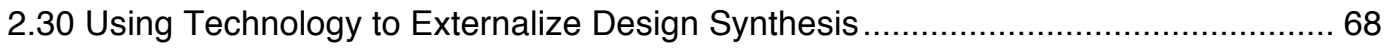

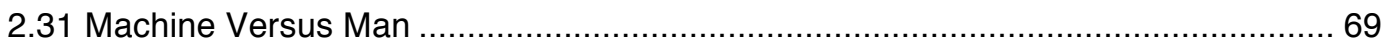

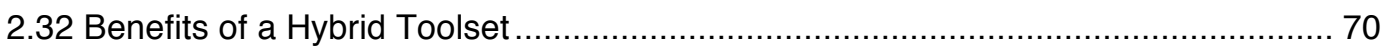

Chapter 3: Group Dynamics and Design Thinking Approaches ...................................... 72

3.1 Learning From Ants. Change from the Ground Up .......................................... 73

3.2 Studies on Group Dynamics in Collaboration..................................................... 74

3.2.1 Production Blocking, Social Inhibition, and Cognitive Uniformity .............. 74

3.2.2 Oral Communication, Democratic Interactions, Analogies and Open

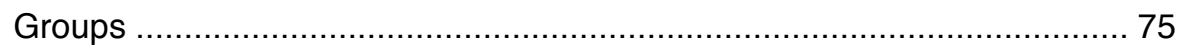

3.3 Collaborative, Cooperative, and Competitive Gameplay.................................... 77

3.4 Lessons Learned from Designing Collaborative Games ...................................... 78

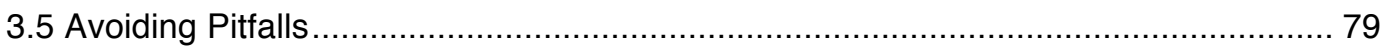

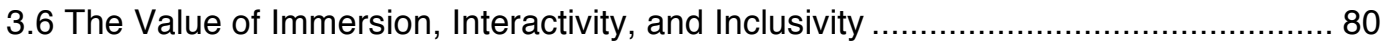

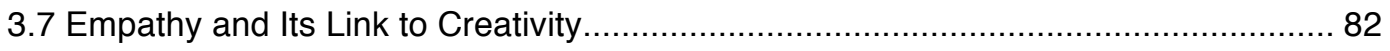

3.8 Finding a Common Language Through Enacting, Telling, and Making ................... 83

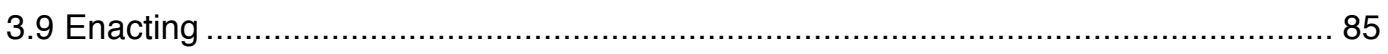

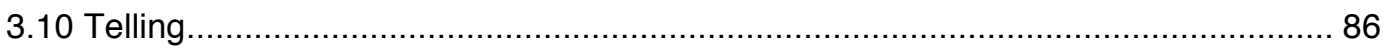

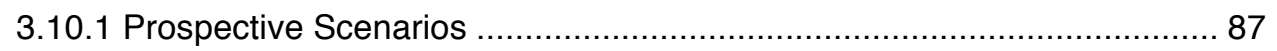

3.10.2 Stories Are Usually Told from a Purposeful Perspective........................ 88

3.10.3 The Influence of Narratives - An Amsterdam Case Study..................... 89

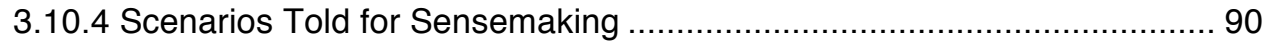


3.11.1 Participatory Prototyping, Probes, and Generative Tools

3.11.1.1 Generative Toolkits ...................................................... 92

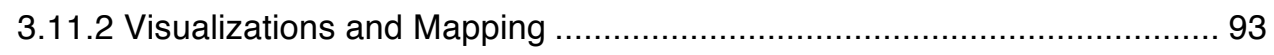

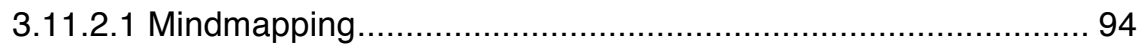

3.11.2.2 Resolution Mapping and Mess Map Diagrams ...................... 95

3.12 Extending Collective Sensemaking Across Time and Space................................ 97

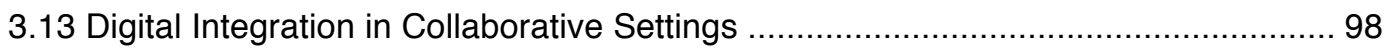

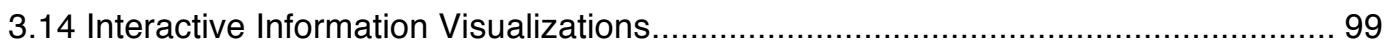

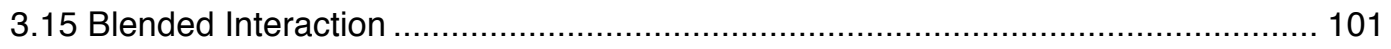

3.16 Personal Versus Shared Digital Devices in Collaborative Spaces........................ 104

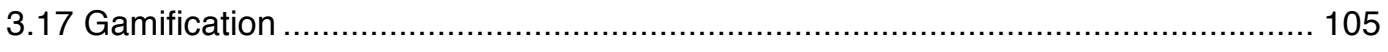

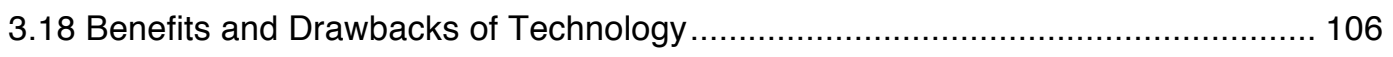

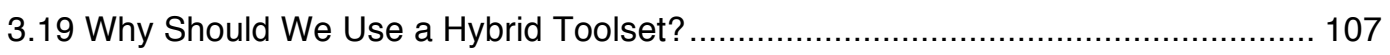

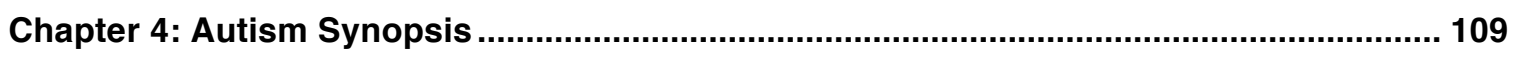

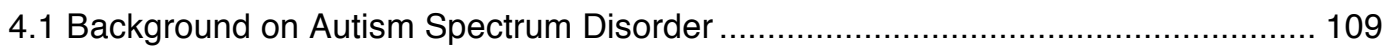

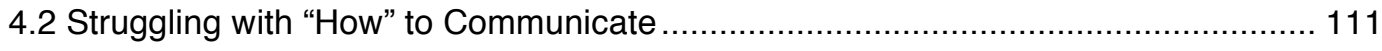

4.3 The Overlooked Potential of the ASD Population ............................................. 112

4.4 Change is Hard - Preparatory Tools for People with ASD ............................... 113

4.5 Visualizing Abstract Concepts Through Associations to Make Sense .................... 114

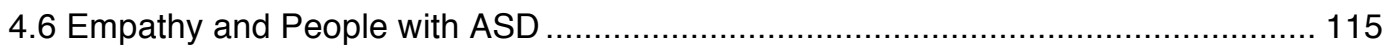

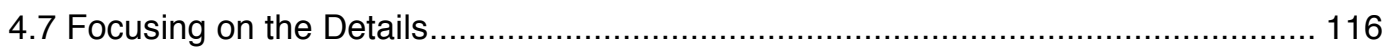

4.8 UK Case Study - Participatory Design with People on the Spectrum..................... 117

4.9 The Value in Bringing People with ASD to the CoDesign Table ............................. 120

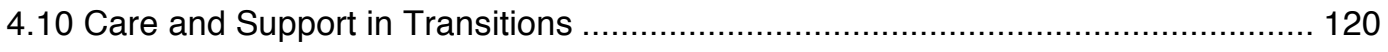


4.11 The Link Between Transitional Support and Future Success

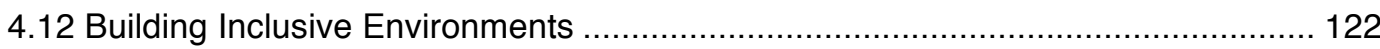

4.13 Transitions are a Wicked Problem ........................................................................ 122

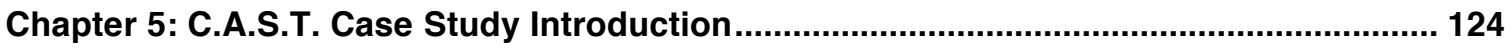

5.1 Stepping Back from Ideation. Framing a Wicked Problem................................... 124

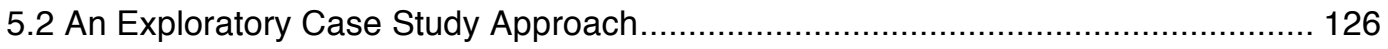

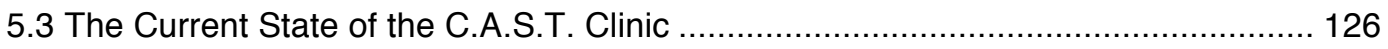

5.4 Involving ASD Participants in the C.A.S.T. Case Study ..................................... 127

5.5 From Participatory Design (PD) to Participatory Action Research (PAR) ............... 128

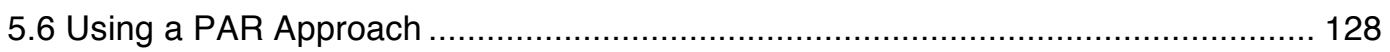

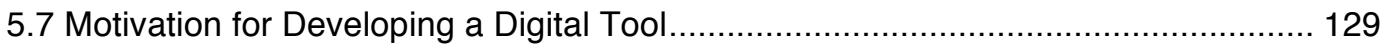

5.8 The Approach for the Hybrid Design Thinking Framework ................................. 130

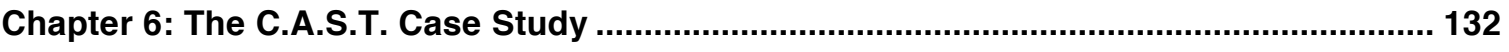

6.1 Understanding Different Perspectives....................................................... 133

6.1.1 Perspective of the Wicked Problem Shared by Adult Participants with ASD 133

6.1.2 Perspective of the Wicked Problem Shared by Parent Participants 134

6.1.3 Perspective of the Wicked Problem Shared by Healthcare

Provider/Administrator Participants 135

6.2 C.A.S.T. Case Study Introduction 136

6.3 The Intent of the Case Study 139

6.4 Overview of the Case Study. 140

6.5 Pre-Phase Materials and Preparation 140

6.5.1 The Journal 141

6.5.2 The Method Cards 143

6.6 Phase 1 145 
6.6.1 Semi-Structured Interviews, Including the Role of Provotypes and Visuals. 145

6.6.2 The Semi-Structured Interviews 147

6.6.3 Focus Group 1 with ASD Participants. 160

6.6.4 Focus Group 2 with Parents and Healthcare Providers/Administrators... 165

6.6.5 Interviews to Prepare Presentations for Workshop 1 ............................ 169

6.6.6 Summarizing Phase 1 179

6.7 The Approach for Phase 2: Workshop 1 180

6.8 Workshop 1: Shared Understanding, Sensemaking, and Defining "What" 184

6.8.1 Workshop 1 Collaborative Environments 185

6.8.2 The Presentations 186

6.8.3 Integrating the New Digital Prototype into Workshop 1 186

6.8.4 The Sensemaking Activity 188

6.9 Phase 3: Workshop 2 Preparation 191

6.10 Workshop 2: Prototyping and Understanding "How" 192

6.10.1 The Collaborative Space for Workshop 2 192

6.10.2 Overview and Initial Discussions/Sharing in Workshop 2 193

6.10.3 Persona Development. 195

6.10.4 Prospective Scenario Journey Mapping......................................... 200

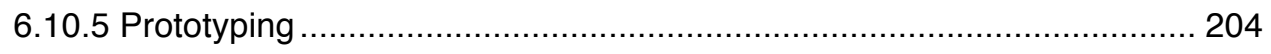

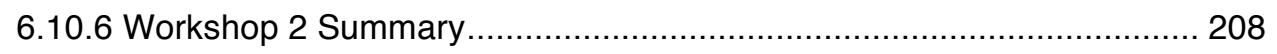

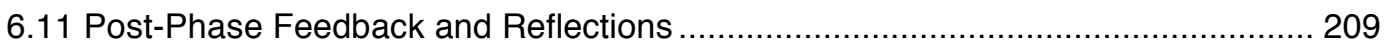

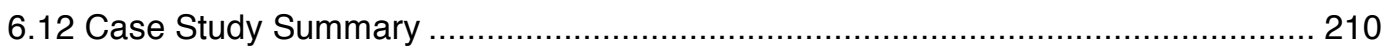

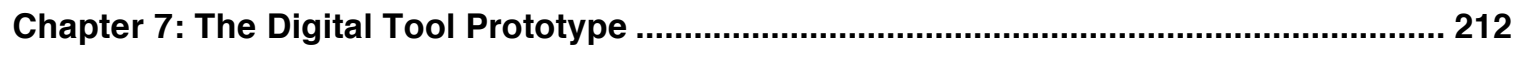

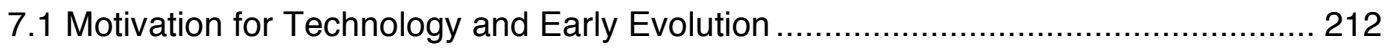

7.2 The Digital Tool Prototype, Designed for Learning and Exploration ...................... 213 
7.3 Introduction to the Digital Tool Prototype

7.4 Phase 1: 2D Visualizations and Paper Prototypes............................................ 216

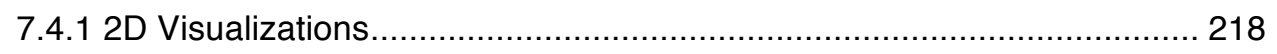

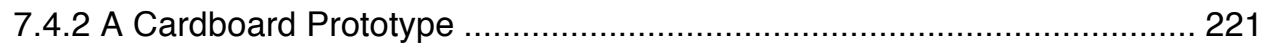

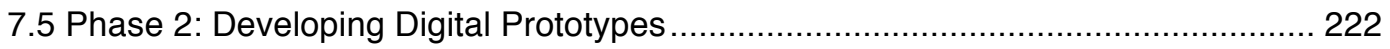

7.5.1 Development of an Initial Digital Prototype ......................................... 223

7.5.2 Development of the Second Iteration ............................................ 227

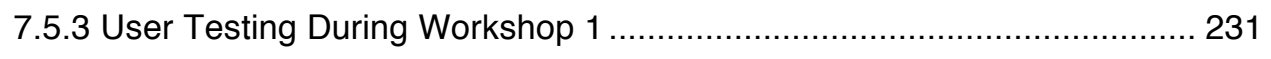

7.5.4 Using the Digital Prototype for Post-Workshop 1 Summary .................. 233

7.6 Phase 3: Final Revisions to the Digital Prototype ................................................ 236

7.6.1 Revised Features of the Final Digital Prototype .................................. 238

7.6.2 Play-testing the Final Digital Prototype ............................................ 242

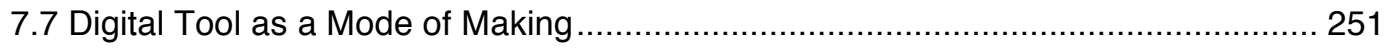

7.8 How This Digital Tool Fits Within the Hybrid Framework .................................... 252

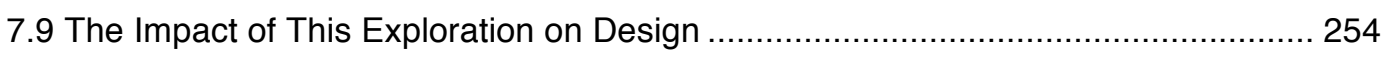

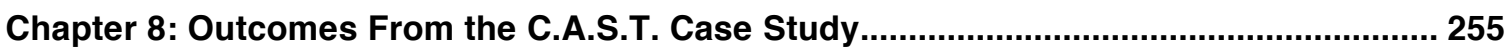

8.1 Pre-Assessment Questionnaire Outcomes ....................................................... 255

8.1.1 Pre-Assessment Outcomes for the Adult Participants with ASD ............ 256

8.1.2 Pre-Assessment Outcomes for the Parent Participants........................ 262

8.1.3 Pre-Assessment Outcomes for the Healthcare Provider/Administrator

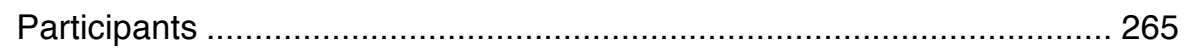

8.2 CoDesign Outcomes from Phase 1 Focus Group Sessions .................................. 269

8.2.1 CoDesign Outcomes from Focus Group 1 with ASD Participants............ 270

8.2.2 CoDesign Outcomes from Focus Group 2 with Parents and Healthcare

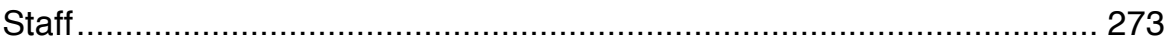

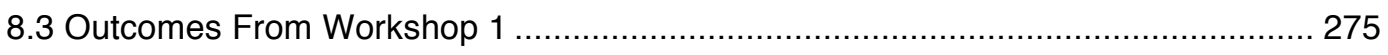




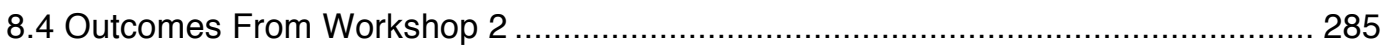

8.5 Post-Assessment Questionnaire Outcomes …................................................. 291

8.5.1 Post-Assessment Outcomes for the Adult Participants with ASD ............ 292

8.5.2 Post-Assessment Outcomes for the Parent Participants ....................... 296

8.5.3 Post-Assessment Outcomes for the Healthcare Provider/Administrator

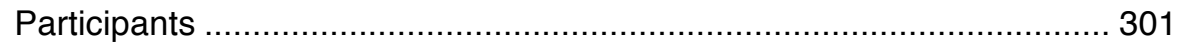

8.5.4 Synthesis of Post-Assessment Questionnaire Outcomes ...................... 305

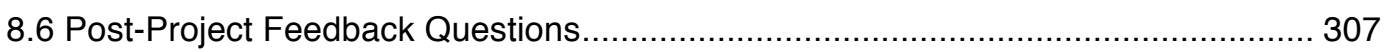

8.7 Discussion Regarding the Participants' Responses........................................... 308

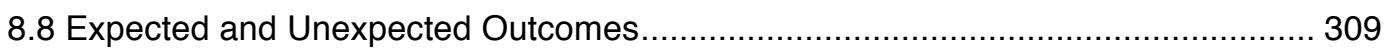

8.9 What Was Learned From the Outcomes of the Case Study ................................. 311

Chapter 9: Reflections Derived From This Case Study …............................................... 318

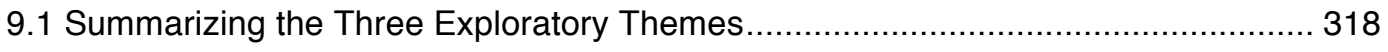

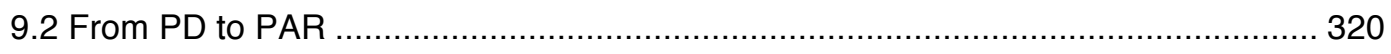

9.3 The Problem Solving Process - Iterative Abductive Reasoning ............................ 322

9.4 The Construction of the Framework for this Case Study ................................... 323

9.5 Reflections on the Methods and Tools Used in Each Phase .................................. 324

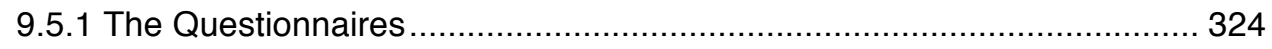

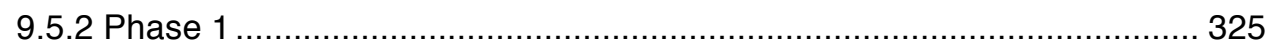

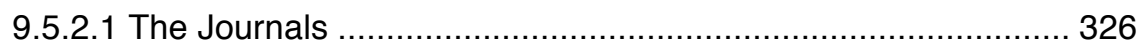

9.5.2.2 The Method Cards ....................................................... 327

9.5.2.3 Interviews and Focus Groups ........................................ 327

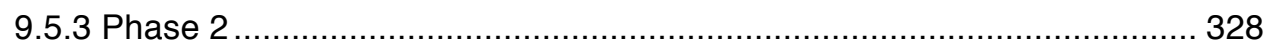

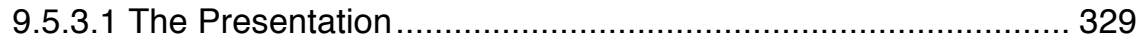

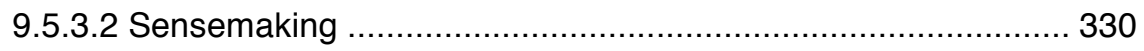

9.5.3.3 Sensemaking with Technology and Sticky Notes................... 331 


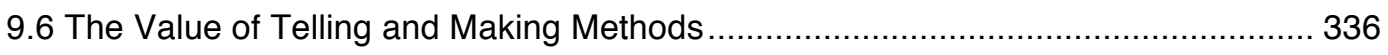

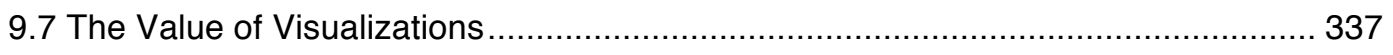

9.8 The Value of Externalizing the Design Process ................................................ 338

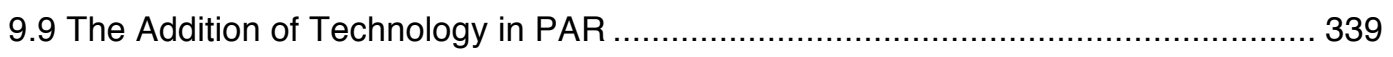

9.10 Designing Seeing/Maker Spaces and Tools for PAR .................................... 340

9.10.1 Designing Seeing/Maker Spaces for PAR, Not Just 'Maker' Spaces ..... 341

9.10.2 Designing Tools Around the Strengths of CoDesigners ....................... 342

9.10.3 Technology as a Tool for Communication....................................... 343

9.10.4 Understanding Tool Design Through Digital Prototype Development.... 343

9.11 Unanticipated Findings 345

9.11.1 More Than One Seeing/Maker Space ......................................... 345

9.11.2 A Digital Tool for Designers and CoDesigners................................ 346

9.11.3 Multiple Tools Used to Externalize the Design Process...................... 346

9.11.4 Long-term Versus Near-term Focus.............................................. 347

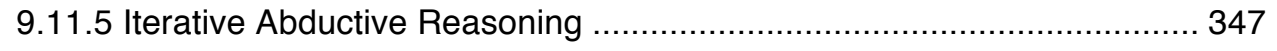

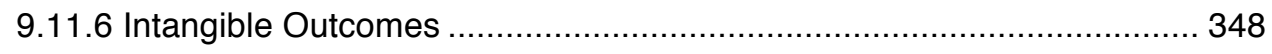

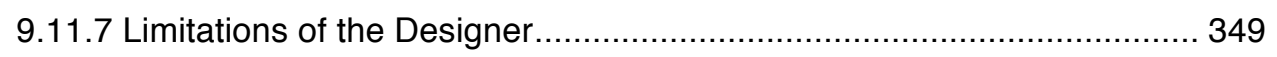

9.11.8 The Value of the ASD Participants.............................................. 350

9.11.9 Designers and People with ASD Think in Similar Ways...................... 350

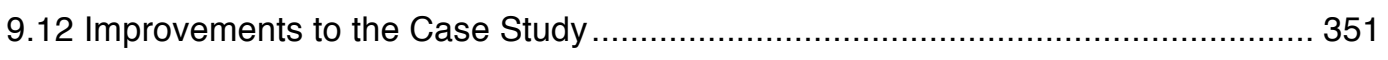

9.12.1 Involving More ASD Participants................................................ 352

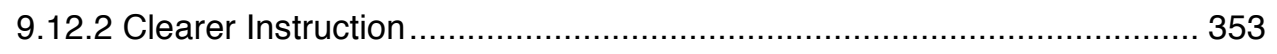

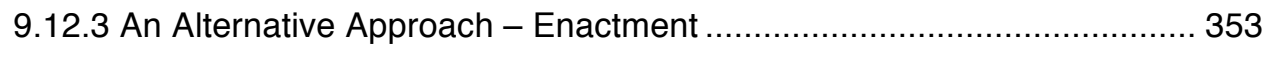


9.13 Influence of This Case Study on the Design Profession...... 354

9.13.1 The Designer's Role in This Case Study......................................... 355

9.13.1.1 Curator, Bricoleur, Problem Architect................................... 356

9.13.2 Extending the Reach of Design.................................................... 358

9.13.3 The Value of Inclusion Through Co-Designing................................ 359

9.13.4 Sharing the Design Thinking Process …........................................... 359

9.13.5 Extending Empathy in the Design Process ..................................... 360

9.14 The Value of Shared Understanding and Sensemaking .................................... 361

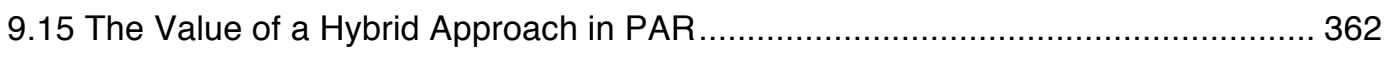

9.15.1 The Hybrid PAR Framework ......................................................... 365

9.16 What Was Learned From This Exploratory Case Study …................................. 369

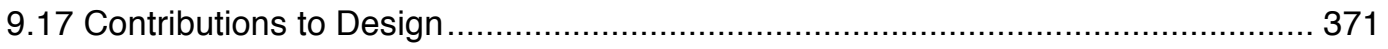

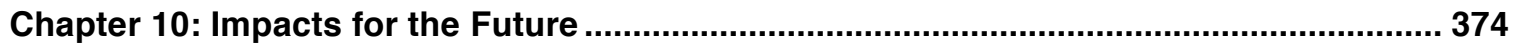

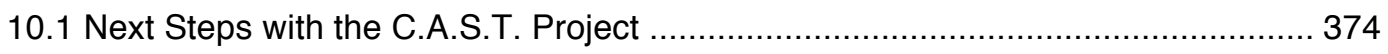

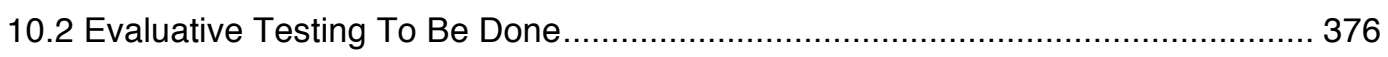

10.3 Where Can Technology Fit in the Front-end of the Design Process? .................. 377

10.4 Future Improvements to a Digital Seeing/Maker Space..................................... 379

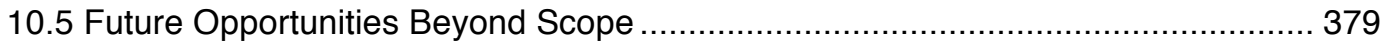

10.6 Implications of this Research on the Field of Design ...................................... 380

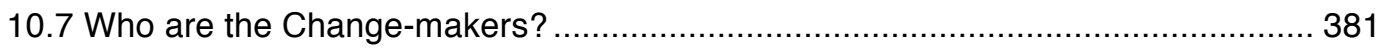

10.8 Expanding Our 'Form Giving' Capabilities ................................................ 382

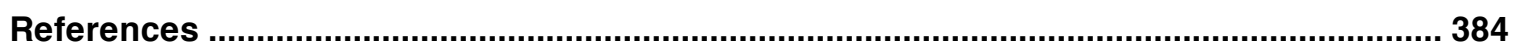

Appendix

Appendix A: Pre-Phase Material and Preparation ................................................. 394

Appendix B: Phase 1 - Interviews and Focus Groups .......................................... 403

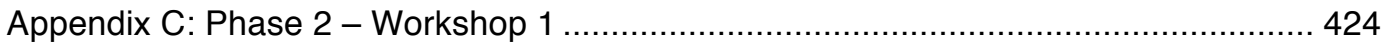




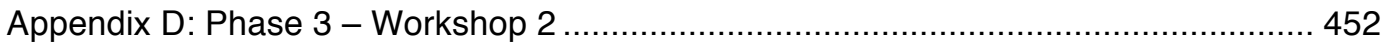

Appendix E: Post-Phase Feedback and Reflections................................................ 463

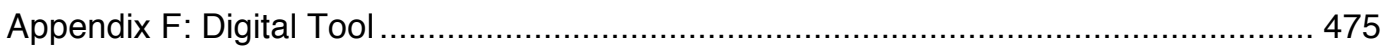




\section{List of Figures}

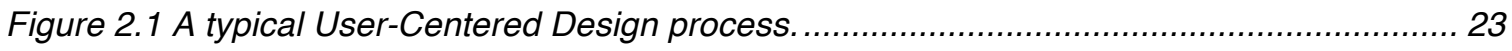

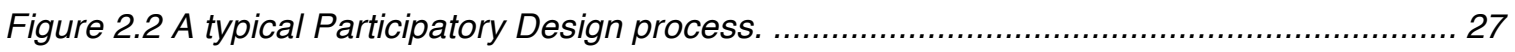

Figure 2.3 A typical Participatory Action Research process. ............................................. 30

Figure 2.4 PAR diagram as an iterative cycle, inspired by Russo (2013)............................... 31

Figure 2.5 Six key drivers that will likely impact our future. (Davies, Fidler, \& Gorbis, 2011)....... 38

Figure 2.6 Ten skill-sets that will likely have the most relevance in the future (Davies, Fidler,

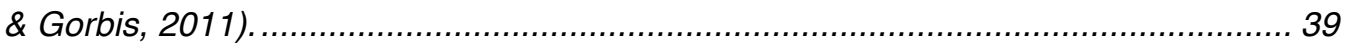

Figure 2.7 Sensemaking fields from a personal (human) level up to a cross-disciplinary

(industry) level (Jones, n.d.).

Figure 2.8 Leaders in various sensemaking fields from a personal (human) level up to a cross disciplinary (industry) level (Jones, n.d.).

Figure 2.9 Frame Creation Model and ten principles of frame creation (from Dorst, 2015,

p. $75 \& 101)$ 48

Figure 2.10 An example of co-occurrence visualizations (Schich \& Coscia, 2011). 59

Figure 3.1 Making, telling, and enacting as complementary, connected activities in co-designing (Brandt, Binder, \& Sanders, 2012).

Figure 3.2 Various generative (Make) toolkit ingredients and how they can be used in making (from Sanders \& Stappers, 2012).. 93

Figure 3.3 An example of a Horn Mess Map (Horn \& Weber, 2007)....................................... 96

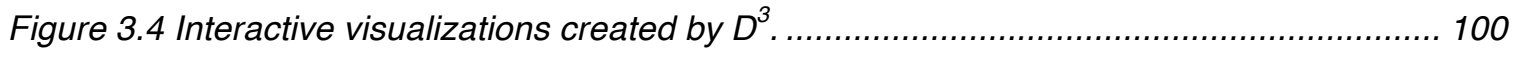

Figure 4.1 Autistic Spectrum Conditions (reinterpreted illustration from What is ASD, n.d.). .... 110 
Figure 4.2 Sensory preference cards (Gaudion et al., 2015).

Figure 5.1 Stepping back in the Design process.

Figure 6.1 The overall front-end PAR approach, from inquiry and framing to prototyping and ideation. 138

Figure 6.2 The first four pages of the Journal, printed on an 11" $x 17$ " piece of paper and then folded into a booklet.

Figure 6.3 Pages five through eight of the Journal.

Figure 6.4 Vision Board on the backside of the Journal.

Figure 6.5 The twelve Method Cards.

Figure 6.6 An overview diagram representing the methods used/referenced in the construction of the three phases. 145

Figure 6.7 Organizational matrix of the players in healthcare connected to ASD. 148

Figure 6.8 Provotyped concepts for the clinic. 149

Figure 6.9 The final iteration of the clinic diagrams.

Figure 6.10 Iterations to the patient/family journey map from childhood to adulthood based on the interview with a parent who had an adolescent on the spectrum..

Figure 6.11 Potential life services/support for person with autism created in an interview with a parent who had an adolescent on the spectrum.

Figure 6.12 The next iteration of the patient journey map based on the interview with ASD

providers from $\mathrm{NCH}$.

Figure $6.13 \mathrm{~A}$ series of visualizations created after the interview with the C.A.S.T. Director that depicts the relationships between the three stakeholder groups: people (patients) with ASD, parents, and providers, as well as society ('community'). 159

Figure 6.14 A birds-eye view of the space used for the focus group with ASD participants...... 161

Figure 6.15 Clinic journey map with problems and ideas that were expressed in the focus group by the ASD participants.. 
Figure 6.16 Visualization of the social interaction negative feedback loop created after the focus group with ASD participants.

Figure 6.17 Photographs from the making activity during the focus group with ASD

participants. 164

Figure $6.18 \mathrm{~A}$ birds-eye view of the space for the focus group with parents and healthcare staff. 167

Figure 6.19 Parent and provider/administrator perspectives from Focus Group 2. 168

Figure 6.20 The life journey map (overlaid with all three stakeholder group priorities)....... 170

Figure 6.21 Samples of the parent presentation co-created in the pre-workshop interview. 171

Figure 6.22 Samples of presentation visualizations created from the information shared during John and Kristin's interview.

Figure 6.23 Samples of the provider/administrator presentation showing education and communication gaps.

Figure 6.24 Priority life journey map shared by the providers.

Figure 6.25 A visualization representing sensory overload, illustrated from secondary research

Figure 6.26 Sanders' (2015) framework for Design Wisdom - bridging together the analytical side and generative side. 180

Figure 6.27 Stakeholder needs/goals (key terms and full descriptions) derived from Phase 1. . 182 Figure 6.28 Needs/goals organized into eight overall categories for use with the digital tool prototype. 183

Figure 6.29 A birds-eye view of the presentation space. 185

Figure 6.30 A birds-eye view of the seeing space created for the sensemaking activity. 186

Figure 6.31 Screen captures of the digital tool prototype. 189

Figure 6.32 Photograph of people interacting with the digital tool. 189

Figure 6.33 Photographs from the whiteboard session and sticky note diagram. 190 
Figure 6.34 Visual synthesis map, re-created with the repaired digital tool. 191

Figure 6.35 Cleaned up visualization of the synthesis map (using Illustrator). 192

Figure 6.36 Two birds-eye views of how the space for Workshop 2 was reconfigured for the discussion/reflection portion (left) versus the collaboration portion (right)..... 193

Figure 6. 37 Persona toolkit - images, situation cards, and headers. 197

Figure 6.38 Owen's current and future personas. 198

Figure 6.39 Sam's current and future personas. 199

Figure 6.40 Prospective scenario toolkit - headers, 2D shapes, and images (similar to the persona kit) 200

Figure 6.41 Owen's Journey Map. 202

Figure 6.42 Sam’s Journey Map. 203

Figure 6.43 Generative tools for prototyping $-2 D$ shapes/materials and 3D objects. 204

Figure 6. 44 Owen's List prototype. 205

Figure 6.45 The props used to communicate Sam's remote Care Team. 206

Figure 6.46 LEGO and resource book props. 207

Figure 7.1 The matrix used to catalog qualitative data from Phase 1 about each stakeholder group....... 217

Figure 7.2 The evolution of the visualization from category radar graphs to a single wheel 219

Figure 7.3 The evolution of rating/rankings of needs/goals within categories to the wheel diagram. 219

Figure 7.4 Illustrating the progression from connections made on the wheel to building the foundation for mindmapping and generative thinking. 220

Figure 7.5 The cardboard prototype of the tool (left) helped to inform the next iteration of the $2 D$ prototype on the right illustrating the transformation of a 'topic' wedge into a 'topic' circle (top right corner) as it is pulled out of the wheel, and the ability to draw connector lines. 
Figure 7.6 The initial digital prototype, including the functions of capturing and presenting data, drawing connector lines, and rotating, scaling, and dragging the wheel of topics. .... 224

Figure 7.7 The initial digital prototype with connector lines, pulled out topics, and scaled (prioritized) topics. 226

Figure 7.8 Comparative images between the wedge wheel and ring of nodes. 229

Figure 7.9 Stakeholder markers placed on topics to depict priority through color and scale. A connector line has also been drawn between two topics to show their relationship. . 230

Figure 7.10 Image of the digital tool (left) with intentionally blue and orange nodes to match the sticky notes (right) that were used to finish the sensemaking activity. 233

Figure 7.11 The initial ring of topic needs/goals and the final output of prioritized, clustered, and connected topics from Workshop 1, using the revised second iteration of the digital prototype

Figure 7.12 The final visual map with the addition of new cluster titles (using Illustrator).

Figure 7.13 A cleaned up version of the new visual narrative (using Adobe Illustrator) showing new topic clusters (left) and connection lines among topics (right)

Figure 7.14 The final prototype with project-specific stakeholder icons (translucent icons on nodes, pictured left) and customizable "idea" nodes (shown above the keyboard) 238

Figure 7.15 Establishing a new cluster from a 'suggested' dotted line to a translucent purple circle. Each new "group" can be given a custom name.

Figure 7.16 Directional arrows and animated line segments show the types of relationships between nodes.

Figure 7.17 The students rotating the ring of nodes, pulling topic nodes out of the initial circle, and clustering them into new groups. 244

Figure 7.18 The students drawing connections between nodes and renaming cluster groups. . 246 Figure 7.19 Stakeholder icons used to assign tasks within the new clusters. 248 
Figure 7.20 The students trying alternate ways to reduce visual clutter - reducing the size of the initial circle and/or moving it to the lower right-hand corner of the screen. ....... 248

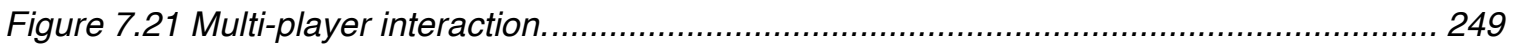

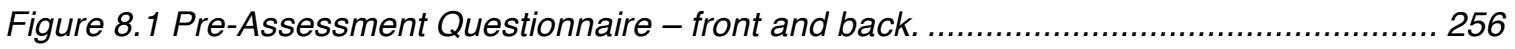

Figure 8.2 Pre-Assessment ASD participant responses for the first six questions. .................. 258

Figure 8.3 Pre-Assessment ASD participant responses regarding their feelings towards collaboration around complex problems. ..................................................... 259

Figure 8.4 Pre-Assessment ASD participant responses to questions about Design Thinking.... 261

Figure 8.5 Pre-Assessment ASD participant responses regarding their feelings towards the design of a new healthcare center for adults with ASD.

Figure 8.6 Pre-Assessment parent responses for the first seven questions, including their current feeling towards collaboration around complex problems.

Figure 8.7 Pre-Assessment parent responses to questions regarding Design Thinking and their current feelings towards a new healthcare center for adults with ASD.

Figure 8.8 Pre-Assessment healthcare provider and administrator responses to the first six questions.

Figure 8.9 Pre-Assessment healthcare provider and administrator responses to the last set of questions.

Figure 8.10 The images above capture: written ideas for the clinic and ways to support people with ASD in the community (top), a drawing of a waiting room with a round intake desk and quiet rooms (lower left), and a model of a waiting room with soft edges and a cat to reduce anxiety (lower right).

Figure $8.11 \mathrm{~A}$ visualization of the new frame. The diagram shows the journey of potentiality for parents and their child on the spectrum, from diagnosis to reaching their maximum potential, as well as providers, outside extenders and assistive technology in supportive roles. 
Figure 8.12 One of the four idea charts, Long-term Planning, compiled after Phase 2.

Figure 8.13 LEGO prop of a 'safe' gaming space for people with ASD and neurotypicals.

Figure 8.14 Building a custom profile that would grow with the child into adulthood - paper

prototype and refined prototype.

Figure 8.15 Finding a future employer that aligns with a unique specialization/fixation - paper

prototype and refined prototype.

Figure 8.16 Finding affordable, independent housing options - paper prototype and refined

prototype.

Figure 8.17 Post-Assessment Questionnaire - front and back.

Figure 8.18 Post-Assessment responses to the first six questions by participants with ASD

Figure 8.19 Post-Assessment responses by participants with ASD regarding their feelings towards collaboration around complex problems. 295

Figure 8.20 Post-Assessment responses by participants with ASD on the final questions. 296

Figure 8.21 Post-Assessment responses by parents on the first four questions.

Figure 8.22 Post-Assessment responses by parents regarding their current feelings of empowerment and expertise.

Figure 8.23 Drawing a comparison between the parents' responses prior to and following the project regarding their feelings towards collaboration around complex problems. .. 299

Figure 8.24 Post-Assessment responses by parents on the final questions. 301

Figure 8.25 Post-Assessment responses to the first set of questions by healthcare providers and administrators. 303

Figure 8.26 Post-Assessment responses to the last set of questions by healthcare providers and administrators. 305

Figure 9.1 A diagram of the PAR process carried out in this case study. 321

Figure 9.2 An Iterative Abductive Reasoning Model. 323

Figure 9.3 Images of the Method Cards and Journal booklet with their plastic folders. 327 
Figure 9.4 The activities embedded in the role of Design facilitator are iterative, feeding into

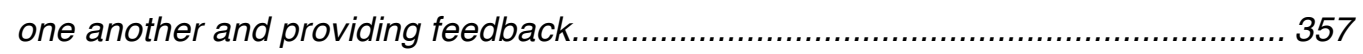

Figure 9.5 A diagram of the PAR process that was carried out in this case study ................... 366

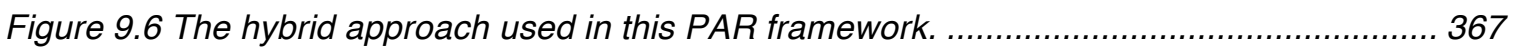

Figure 9.7 Further hybrid opportunities to explore in the PAR framework ........................... 368 


\section{Chapter 1: Introduction}

\subsection{Overview}

"How does action become coordinated in the world of multiple realities?" (Weick, 1995)

The question, posed by Karl Weick, encapsulates the essence of the thesis you are about to read. Framing Wicked Problems Using CoDesign and a Hybrid Design Toolset is an exploration into the evolving roles of the Designer and stakeholders, collaborative dynamics and collective sensemaking, and the integration of digital tools in Participatory Action Research (PAR) for the purposes of provoking new insights, increasing shared understanding, and enacting meaningful change in wicked problems. Three seemingly complex and unrelated topics: the Designer's role in wicked problems, co-designing with the autism community, and the development of a hybrid framework for PAR are brought together through an exploratory case study involving the care and support of transitioning adults with autism, and through the development of a digital prototype for collective sensemaking.

The process and findings of this research are retold from a first person perspective in an effort to connect with the reader on a more personal level, rather than reporting the research and findings from a distant third-person perspective. Design is human-centered; the intent is for this paper to reach the reader (Designer or non-designer) on a human level. The use of "we" will be often be used to refer to Designers, as a call to action to further investigate and challenge our expanding roles and value, as society's problems become increasingly more multi-faceted, systemic, intangible, and wicked. 


\subsection{Defining Wicked Problems}

The term "wicked problem" is complex in itself. In explaining the dimensions of the thesis to Designers and non-designers, it becomes clear that people make various assumptions about "wicked problems." Some relate it to a problem that is "harmful" or "bad"; others view it as pandemonium; most measure it by scale, considering a wicked problem to only be those that are "big" and "widespread." Wicked problems are chaotic, but not always bad, and they are not always associated with scale. Instead, the term "wicked" corresponds to problems, often contributed to by multiple stakeholders with differing needs and perspectives, with an indeterminate end-state (no definitive condition or limit to the problem), and no test of "rightness" when it comes to a solution. Wicked problems are difficult or impossible to solve because of incomplete and often contradictory information, and changing requirements that are difficult to recognize and organize. "Every wicked problem can be considered to be a symptom of another problem" (Rittle \& Webber, 1973, p.165). Consequently, these problems are often wrapped up in ambiguous causal webs. Because there is no idealized solution or end-state, there is no rule to determine the "correct" solution (Rittle \&Webber, 1973, p.165-167). This thesis proposes that wicked problems can benefit from collective sensemaking (an external, action oriented process in which people integrate experiences to seek mutual understanding) and CoDesign (a creative approach allowing non-Design stakeholders to play an inclusive role in the Design process to provide new knowledge and actively participate in developing future concepts with Designers).

Furthermore, wicked problems present opportunities for iterative resolutions to be proposed, developed, tested, and revised based on how they impact the system once they are introduced. System in this body of work refers to the network of connected stakeholders and issues within a multi-faceted problem. Due to their nature, wicked problems benefit from an iterative CoDesign approach called Participatory Action Research (PAR). Unlike Participatory Design (PD), which is often associated with the CoDesign process, PAR is grounded in the notion of repetitive action, 
constant learning and understanding, and iterative creation, rather than generating a singular solution. The word resolution will be intentionally used in place of solution throughout the rest of this paper, because wicked problems can never be truly "solved". Instead, at best, "they are only 're-solved' - over and over again” (Rittle \& Webber, 1973, p.160). Design problems, by nature, are often wicked. There is often no definitive formula for resolving a Design problem because each problem is unique and indeterminate, and the solution does not yet exist. Furthermore there is no limit or finite end-state (solution) to a Design problem - the solutions we engender are more in line with resolutions; there is always room for iteration and improvement (Buchanan, 1992).

\subsection{Personal Motivations}

The motivations that compelled the exploration of this complex topic evolved over the course of many years. Working as a Product Designer for a Design consultancy, I conducted Design Research (using traditional Design Research methods) and developed a wide range of consumer goods and athletic equipment under a User-Centered model. I was creating incremental changes and stylizing products under the assumption that we were "heightening user experience." Whether or not our products were actually contributing to meaningful change, and/or user experiences, was unclear. Jon Kolko calls this "diffused consequence" - not knowing the extent of the influence your designs have on people or society as a whole (Kolko, 2012). Over the course of multiple projects I recognized that the consumer products I was developing seemed to have little value in the larger context of society's problems and I wanted to work on systemic projects that had greater meaning.

In Design Research and brainstorming sessions Designers often use sticky notes to capture and synthesize information/ideas. While useful in some internal brainstorming sessions, I found sticky notes to be limiting, especially in large-scale projects. I wanted to explore alternative ways through technology to collect, organize, and communicate data and ideas to our clients and 
stakeholders, and better illustrate connections between Design Research findings and Design outcomes.

Communicating the value of a Designer as a problem framer and sensemaker, in addition to form giver in the Design process is difficult. I found this ability to be often overlooked by the "pretty sketches' and prototypes I was producing at the end of the Design process. Designers are often regarded more for their technical skills in the later stages of the Design process, with little regard or understanding as to how we arrive at the concepts. We (Designers) need to better communicate our process and the added value we can offer to our users and our clients by helping them to frame problems and Design opportunities during the early strategy phases of a Design project.

I became motivated by three convictions. I wanted to solve more challenging, meaningful problems. I wanted to push the limits of Design Thinking beyond sticky notes and explore the application of technology in the Design Research and brainstorming processes. I wanted to explore better ways to communicate our (Design's) value in sensemaking and problem framing earlier in a project by exploring tools and approaches that could make our synthesis process more transparent and engaging to stakeholders in CoDesign. Motivated by a desire to reevaluate my role as a Designer, better communicate the value of the Designer in the early stages of a wicked problem, and to explore new processes and digital tools for not only tackling problems but also demonstrating our synthesis process, I returned to Design school and partnered with The Ohio State Wexner Medical Center on a wicked project.

\subsection{A Wicked Case Study}

The case study presented in this thesis explores the expanding roles of the Designer and the integration of a hybrid toolset in Participatory Action Research aimed at addressing a wicked 
problem. The wicked problem is connected to the autism community, specifically the problems facing adults with Autism Spectrum Disorder (ASD), and their families, during the transitional phase from childhood to adulthood. This area of ASD embeds all of the qualities of a wicked problem: multiple stakeholders with different needs and goals, an indeterminate end-state, and no "right" solution but an opportunity to resolve unmet needs through iterative thinking and creation. A clinic called C.A.S.T. (Center for Autism Services and Transitions), associated with The Ohio State Wexner Medical Center in Columbus Ohio, had recently been opened. C.A.S.T. is a healthcare clinic specializing in primary care services and transitional care support for teens (transitioning from pediatric care) and adults with ASD and other complex disabilities. However, many of the integral stakeholders with differing prioritized needs and goals had not had an opportunity to voice their views about what the role and value of the clinic should/could be in connection with the ASD community. To tackle this problem, various groups of stakeholders were invited to collectively participate in a series of facilitated CoDesign sessions (comprehensively referred to as the C.A.S.T. Case Study) to discuss, plan, reframe, and generate new resolutions aimed at understanding the true value of the clinic and building a strategy for which to achieve optimal care within the complex web of transitions and ASD.

\subsubsection{Transitional Care and Support for Adults with ASD}

In the C.A.S.T. Case Study three stakeholder groups (adults on the autism spectrum, parents with adolescents on the autism spectrum, and healthcare personnel - providers and administrators) were brought together to examine not only the unmet needs of the clinic, but to also garner greater understanding of the issues and potential opportunities surrounding transitions in ASD and how to create more connected and uninterrupted healthcare support for adults with autism and their families. Transitional care concerns also extend to healthcare providers and administrators (like those from The Ohio State University Wexner Medical Center and Nationwide Children's Hospital in Columbus Ohio) who share a part in the transition from childcare and adult 
care. Stakeholders from all three groups participated in semi-structured interviews, focus group sessions, and CoDesign workshops to discuss the issues, frame a strategy for action, and develop resolution prototypes to improve the current condition. The following chapters examine my role as a Designer and the role of the stakeholders in tackling this wicked problem using a PAR approach and the effects of bringing diverging perspectives together in the same collaborative space to frame a wicked problem, to engender shared understanding and to speculate about future opportunities.

\subsubsection{Exploring Digital Capabilities in the Design Process}

Furthermore, the potential value of introducing a blended interaction tool (a tool that combines individual interaction, physical interaction and social interaction with computer-supported technologies) in future PAR is explored through the development and application of a digital prototype designed in parallel with the autism case study. The digital tool was developed to investigate new ways of engaging CoDesigners and externalizing the Design process. Analysis of the case study (as it relates to sensemaking and the use of a hybrid set of tools) and the process of developing and testing the digital prototype are intended not to prove or disprove the use of the specific digital tool in the PAR process, but to expose new opportunities where technology could be integrated and further investigated in future Design processes.

\subsection{Thesis structure and Methodology}

This section provides a general outline for the content within the chapters of this thesis document.

\section{Chapter Two:}

The research begins with background information and secondary research on wicked problems, Participatory Design, Participatory Action Research, and sensemaking. The 
role of Designers and stakeholders in CoDesign settings are also explored, in addition to the role of interactive technology in future CoDesign and collaborative settings.

\section{Chapter Three}

Chapter Three explores the nuances of collective group dynamics and social practices to better understand which Design Thinking methods (non-digital and digital) could improve communication, sensemaking, shared understanding and empathy, as well as critical/creative thinking among collaborating stakeholders with varying perspectives.

\section{Chapters Four and Five}

Chapters Four and Five provide background information on Autism Spectrum Disorder (ASD) and introduce the transitional process as a wicked problem in autism. An overview of the exploratory case study builds upon the connections made in secondary research and sets the stage for the chapters that follow.

\section{Chapter Six}

Chapter Six recounts each phase of the case study and addresses general findings that were made along the way. The outcomes of each session informed the process used in each subsequent session, as is typical in the PAR process.

\section{Chapter Seven}

Chapter Seven describes the process of developing a digital tool prototype for collective sensemaking, in parallel with the C.A.S.T. Case Study. Findings from user testing, prototype development, and secondary research provide insight for further investigation into and future considerations for the use of technology in PAR and the Design process. 
Chapters Eight, Nine, and Ten

The final chapters reflect on the outcomes of the autism case study and digital prototype (during and after use), and discuss the potential impacts of this research on the future of Design and society.

\subsection{The Expanding Role of Designers in Wicked Problems}

As societal problems become more complex and intangible, the role of the Designer as form giver must also evolve. Design has been expanding beyond the scope of the traditional definition of a Designer as one that gives form to things, to one who can integrate knowledge in new ways and assist with strategic planning and systemic integration (Buchanan, 1992). Jon Kolko is an Interactive and Industrial Designer who wrote a book in 2012 entitled Wicked Problems Worth Solving: A Handbook \& a Call to Action in which he asserts that wicked problems can be 'mitigated through the process of Design, which is an intellectual approach that emphasizes empathy, abductive reasoning, and rapid prototyping" (Kolko, 2012). Trained to reshape complexity through visualization and give form to ideas that do not yet exist, Designers are able to identify multiple issues and perspectives, deliberately shape indeterminate and ambiguous problems through the act of framing, and work through complex problems using an iterative development approach. A Designer possesses skills and expertise that make them suited for not only redesigning tangible artifacts or environments but also tackling and reframing wicked societal problems that expand beyond the traditional scope of Design (Buchanan, 1992).

\subsection{Designers Giving Form in a New Way}

The role of the Designer as primarily a three-dimensional form-giver is evolving into one that can help to shape new perspectives, possibilities, and experiences for people. As the world's problems become more intangible and complex, and people take on more active (over passive) roles in society, the value of the Designer reaches beyond the drawing board to helping to shape 
human experiences and bringing people integral to the problem into the early phases of the Design process. In Participatory Design (PD), non-designers are brought into CoDesign roles with Designers through various Design Thinking activities (Sanders, Brandt, \& Binder, 2010) to help define unmet needs and imagine new possibilities. However, in the context of a wicked problem, with multiple stakeholders, multiple facets, and no single right solution, Participatory Action Research, over Participatory Design, may be a more appropriate approach. Participatory Action Research, or PAR, focuses on iterative research "by, for and with the people who will benefit from it, leading to making practical improvements or transformations" (Howard, Z. \& M. Somerville, 2014). Unlike Participatory Design, the process of framing and reframing the problem, and continually building new knowledge and understanding become the primary focus for PAR over determining specific solutions (Howard \& Somerville, 2014). Therefore, the role of the Designer and stakeholders in tackling wicked problems, using the PAR approach, may have more to do with the process of iterative learning and understanding. The methods built into the PAR approach are not aimed at arriving at a singular ('ideal') outcome, but instead are designed to constantly build onto one another and engage the stakeholders in new ways of knowing, creating, and shared learning, so they feel empowered to continually seek out new understanding and better resolutions.

In CoDesign around wicked problems the role of the Designer can extend beyond the traditional sense of form giver, to one of facilitator and catalyzer of action, by interpreting and helping to frame the complex information (the problem) and the approach for others to see new perspectives, so stakeholders can gain shared understanding and take collective action across many possibilities. The Designer can help to shape ambiguity by creating visualizations, developing a framework of methods, and by configuring collective 'seeing' spaces (Victor, 2014) and tools for sensemaking. Shaping (or framing) the wicked problem and creating shared understanding in this space become as valuable as giving form to new resolutions. Seeded with 
new perspectives and new ways of thinking, the Designer and people who are integral to the problem become the means through which new connections are made and resolutions can emerge.

In shaping the problem and the approach, the Designer may also take on the responsibility of giving form to the new tools and methods that will be utilized in the CoDesign process to elicit new ways of seeing, shared understanding, and generative thinking. In the PAR process, it is important that these methods and the tools are designed for iterative learning and creating. In the next section, the role of technology, as a means through which to extend a CoDesign session (and the communication of the outcomes) beyond a single, real-time experience, is considered as an augmented approach in the PAR process.

\subsection{What Role Could Technology Play in PAR?}

People using technology have created a culture that is active and participatory. Instead of being passive informants or recipients of information, people are actively expressing themselves and creating content (Van Dijck, n.d.). The evolving nature of our society will mean that stakeholders in CoDesign processes will expect to play a more active and integral role overtime. Case studies conducted by other researchers have demonstrated improved communication, discovery, decision-making, creativity, and collective understanding through the use of blended interaction (Jetter et al., 2012 and Blair-Early, 2010). The research presented in this thesis elaborates on the value of a hybrid approach (integrating face-to-face/tangible interactions with digital capabilities) for collective sensemaking and tackling wicked problems. The integration of technology in the PAR process has the capacity to engage active stakeholders in new ways, augment the role of the Designer as form giver to one that can also shape new tools and virtual seeing spaces, and create temporal openness (extending interaction among stakeholders and information across time and space). The Designer in an expanded role, therefore, becomes a problem architect 
through the design of new technology tools and 'seeing' spaces in a hybrid framework for collective sensemaking, personal expression, and social engagement.

\subsection{This Thesis is Exploratory and Provocative in Nature}

For problems that lack definition or clarity, exploratory research is a useful approach. Exploratory research involves user and product testing aimed at gaining an "empathetic knowledge base, particularly when Designers may be working in unfamiliar territory" (Hanington \& Martin, 2012, p.84). Exploratory research cannot validate an idea, but instead can help to inspire speculative thought around new possibilities, and help lay the groundwork for future research and concept development.

The aim of this thesis is not to substantiate a claim or assumption, but instead to identify new opportunities in PAR and provoke new ways of thinking about the roles of the Designer, stakeholders (non-designers), and technology within the realm of wicked problems and collective sensemaking. The findings and outcomes derived from the case study point to the importance of PAR, and the value of the Designer as instigator of new knowledge and facilitator of action. Insights derived from the construction of the digital prototype, developed in parallel with the case study, are not intended to establish whether or not this specific tool is ideal, but is instead meant to inspire future testing and raise awareness about the capabilities of digital technology for collective sensemaking in the PAR process. Results of the approach and methods used in the exploratory case study, along with the digital prototype, point to a new hybrid framework that can be investigated further in future PAR cases connected to wicked problems.

\subsection{Three Main Thesis Themes}

Three overall themes were explored in this case study to inspire the next generation of inquiry around hybrid tools and methods to instigate collective action around wicked problems. The first 
theme concerns user agency and identity within the confines of wicked problems. The evolving roles and value of the Designer, stakeholders, and interactive technology are explored through secondary research, the C.A.S.T. Case Study, and the development of a digital prototype. The second theme deals with investigating collaborative group dynamics and identifying certain social practices (like storytelling, negotiation and sensemaking) that could help to influence/shape shared understanding and empathy, communication, and innovative thinking among collaborating stakeholders. The last theme considers the affordances of non-digital and digital Design Thinking methods and tools in collaborative working environments. The term 'affordance' suggests a relationship between people and the things/experiences/environments with which they interact (Gibson, 1979). Through secondary research and the construction of the digital prototype, the affordances of interactive technology applications are explored to better understand what they could provide to users (Designers and CoDesigners) in the Design process and how they could help the Designer more easily gather, communicate, and organize complex information.

Ultimately, this body of research seeks to uncover a hybrid set of tools and methods (non-digital and digital), which Designers and Design Researchers can use and test in future PAR sessions to facilitate shared understanding, sensemaking and collective action around wicked problems.

\subsection{Terminology}

There are several terms that will be used throughout the body of work that may be helpful to review before reading further. While some of these terms may be commonly used inside and outside of Design circles, their meanings can be somewhat ambiguous. The meaning associated with each term in this list is intended to provide clarity in the context of this study in an effort to make the research accessible to a wide range of readers. 


\section{Affordances}

The term 'affordance' simply refers to what something (an environment, experience, or tool) offers or provides to someone or something (either good or bad) (Gibson, 1979).

\section{Blended Interaction Tool}

An interactive, computer-supported collaboration tool that combines the "power of the digital world with users' pre-existing skills and practices," (i.e., the individual physical or embodied interactions, social interactions, workflows, and physical environment) (Jetter, Geyer, Schwarz, \& Reiterer, 2012).

\section{Bricoleur}

An interpreter who uses a variety of methods, skills, knowledge, instincts and interpretations to synthesize information and gain insight in order to craft an understanding of the world and develop resolutions that are meaningful but temporary (constantly in an iterative state) (Wright, 2005).

\section{CoDesign}

Also referred to as Participatory Design, CoDesign is a creative approach in which people not trained in Design are brought into the Design process to share new knowledge and actively participate in creating future ideas with Designers (Sanders \& Stappers, 2008).

\section{Collective innovation}

A collaborative process, built around shared knowledge, connectivity, and synthesis, through which novel ideas are initiated, prioritized and developed. 


\section{Critical Making}

Intended to bridge the gap between physical creativity and conceptual exploration, this approach emphasizes the process (rather than the outcome) of understanding and productive engagement through the act of making/building (Ratto, 2011).

\section{Curator}

One who acquires, selects, and arranges methods, information, and/or artifacts for display and/or use to guide others towards a specific purpose.

\section{Form giving}

Providing the context or structure for something (e.g., data, narrative, problem, or idea) previously ill-defined in order to help shape and communicate the meaning to others.

\section{Human-Computer Interaction}

A wide field of study that encapsulates the interfacing that takes place between humans and technology, from WIMP interactions (Windows, Icons, Menus, and Pointers) to PostWIMP interactions, integrated into social and physical environments (like multi-touch enabled tabletops, ubiquitous computing environments, and augmented reality) that "exploit and enhance the existing cognitive, physical and social skills of users or groups of users" (Jetter et al. 2012).

\section{Intangible outcomes}

Results that are not tied to specific artifacts or resolutions, but rather to more subjective human qualities such as empowerment and understanding. 


\section{Method}

Loosely described as "a way, technique, or process of or for doing something," a method is "a systematic procedure, technique, or mode of inquiry employed by or proper to a particular discipline or art" (Merriam Webster Online). Methods can be carried out by using tools.

\section{Participatory Design (PD)}

Often referred to as CoDesign, PD is an inclusive approach used in Design practice aimed at designing with, as opposed to for people. In Participatory Design non-designers (i.e., potential users, external stakeholders, and/or people on development teams with non-Design backgrounds) are engaged in various activities throughout the Design process that allow them to share expertise/knowledge with the Design team, express thoughts, and contribute new ideas.

\section{Participatory Action Research (PAR)}

A similar approach to Participatory Design, with the exception that this approach centers around the process of perpetual understanding, continuous knowledge creation, and iterative development throughout the Design process to motivate shared understanding, learning, and action.

\section{Platform}

A single stage (e.g., a physical or digital space) on which multiple people can interact and multiple types of interactions can be simultaneously performed. 


\section{Problem Architect}

A person who gives shape (structure) to a complex problem by designing methods, artifacts, and spaces to scaffold sensemaking by others and to guide them through a plan for tackling the problem.

\section{Prototype}

A "fictional depiction" of an idea or a narrative, something that does not yet exist, but serves as a "rough approximation of the thing we hope to one day build...on the road to something becoming real” (Johnson, B.D., 2011).

\section{Provotypes}

Simply defined as 'provocative' prototypes, provotypes are usually positioned early in the Design process. They are concepts that "embody tensions surrounding an area of interest, in order to support collaborative analysis of that area and to collaboratively explore design possibilities" (Boer \& Donovan, 2012).

\section{'Seeing' Space}

Defined by Victor (2014) as a new type of collaborative environment that facilitates critical making and sensemaking by offering 'seeing' tools (for seeing inside data, across time, and across possibilities) that help people move away from blindly following recipes and towards "deeply understanding what they are doing, inventing new things, discovering new things, and contributing back to the global pool of knowledge" (Victor, 2014).

\section{Sensemaking}

"An action oriented process that people automatically go through in order to integrate experiences into their understanding of the world around them" (Kolko, 2010a). 


\section{Shared Understanding}

Shared understanding means "the stakeholders understand each other's positions well enough to have intelligent dialogue about their different interpretations of the problem, and to exercise collective intelligence about how to solve it" (Conklin, 2009, p.18).

\section{Tangible outcomes}

Tangible outcomes (in contrast to intangible outcomes) are understood to be the Design artifacts (e.g., visualizations, provotypes, and prototypes), as well as the proposed new resolutions (e.g., products, environments, interfaces, and services) that emerge in the Design process.

\section{Tool}

An object or a series of objects (e.g., non-digital artifacts or a digital program) that helps to carry out a method (i.e., a systematic procedure, technique, or mode of inquiry). 


\section{Chapter 2: Background}

An aim in this body of research is to understand the role of Design and a hybrid toolset in tackling wicked problems; therefore it is important to explore the evolving state of society and Design, as they relate to multi-faceted problems with no ideal end-state. Additionally, it is important to understand various Design processes and problem-solving approaches, as well as technology affordances that could be most effective in building a successful Design framework for tackling wicked problems.

\subsection{The Age of Design}

Jeff Conklin, founder of the CogNexus Institute, suggests that society is transforming from the Age of Science to the Age of Design; at its core are wicked problems and the need for opportunity-driven approaches, problem structuring and systematic reasoning, shared understanding, and iterative thinking (Conklin, 2009). In the Age of Science, factual evidence substantiated decision-making, problems were mostly 'tame' and had concrete solutions; the ultimate aim was to solve for the right answer. Therefore, problem-solving processes were mainly linear and decision-makers worked in silos. Today, wicked problems supersede tame problems they are prevalent, dynamic and complex, and require an entirely different approach. Because many involve social complexity (interlocking systemic issues with multiple stakeholders), resolving these problems is "fundamentally a social process" that relies on shared understanding and the ability to "engage stakeholders in a robust and healthy process of making sense of the problem's dimensions" (Conklin, 2009, p.18). Hence, wicked problems require CoDesign 
approaches, such as PAR or PD that promote social learning, shared understanding, and iterative, creative problem solving aimed at reframing the current state of affairs and taking action.

\subsection{Why Wicked Problems, Why Design?}

Design is often misunderstood. "Design is not just about creating beauty, " it is also not all about ideas (Dorst, 2015, p.41). Design is not irrational or mysterious, but instead, in the grander scheme of life, "design is a form of reasoning" (Dorst, 2015, p. 44). For the purposes of this thesis, Design is regarded as an adjective, a process (over a noun or an outcome), wherein shared understanding, intention, and planning are what drive people toward more meaningful resolutions. In Herbert Simon's words, Design is to "devise courses of action aimed at changing existing situations into preferred ones" (Simon, 1969). Ken Friedman and Erik Stolterman introduce Kees Dorst's book Frame Innovation, by explaining that contemporary problems have "increasingly ambiguous boundaries between artifacts, structure, and process, increasingly largescale social, economic, and industrial frames, an increasingly complex environment of needs, requirements, and constraints, and information content that often exceeds the value of the physical substance" (Dorst, 2015, p.ix). These modern issues do not change the definition of Design, but instead challenge our profession in new ways. Therefore we must reestablish the role of the Designer. Today, design projects are more complex - problems are interconnected and cross the boundaries of several organizations, and must therefore meet the expectations of many organizations, stakeholders, producers, and users at every level (Dorst, 2015, p.ix). These problems create the need for transdisciplinary Design teams, a convergence in design practice and Design Research, and a new approach to design that focuses less on developing an end product and more on "analytic and synthetic planning skills." This altered approach is the beginning of "deep design," based on the premise of profound knowledge, systems thinking, and understanding variations (Dorst, 2015, p.ix-xi). 
Through "actions aimed at changing existing situations into preferred ones," (Dorst, 2015, p.vii, viii) designers have "contributed to a wide variety of consequences," and have therefore helped to "shape culture, change behavior, and advance a set of values and priorities" (Kolko, 2012). Design has the potential to shape the way people behave, how they gather information and acquire knowledge, how they communicate across barriers, and how they interact with one another.

\subsection{Design is Evolving}

Adream Blair-Early, an Assistant Professor of Art and Design at the University of WisconsinMilwaukee, explains in her article Beyond borders: Participatory design research and the changing role of design that design is no longer solely about creating individual artifacts or spaces, but in designing systems and communities (Blair-Early, 2010, p.215). The value of designing for experience and systems-based problems is also echoed in a talk, Designers - think big!, given by Tim Brown, CEO and president at IDEO. He asserts that the design profession has a bigger role to play than just making stylish artifacts. Instead, he says we need to take a more systems-thinking approach to design - "with less focus on the object and more focus on Design Thinking as an approach, we may see the result in a bigger impact" (Brown, 2009). These Designers see the role and value of the Designer extending beyond giving shape to a solution and in using their Design Thinking abilities to understand the human experience and design approaches to tackle more complex problems.

\subsection{The Changing Role of Society}

We now live in an active participatory culture (Van Dijck, 2009). Tim Brown in his TED talk, Designers - think big! also hints towards a society that has been shifting from consumption to participation. In turn this means that there is a shift occurring in Design from the passive relationship between consumers and producers that was once dominant in the $20^{\text {th }}$ century to a 
relationship being built on active and more meaningful engagement of everyone (Brown, 2009). Complex design problems that have no right solution rely on knowledge and expertise to be distributed across multiple stakeholders and brought together to create shared understanding, in order to reframe the problem collaboratively (Fischer, 2003). Participatory processes benefit from methods and environments that support active participation and give participants the opportunity to debate and discuss open-ended problems and new resolutions, rather than validate closeended information and solutions.

\subsection{Design Thinking}

Design Thinking has been defined and used by Designers and non-designers in a variety of ways. "Often, in popular literature, many disparate, vaguely creative activities are combined under the label of 'Design Thinking”' (Dorst, 2011, p.531). However, the process of Design Thinking in the design profession is a "quite specific and deliberate way of reasoning" through the use of various methods and tools that help designers capture 'themes' in their research that inform underlying phenomenon (Dorst, 2011 p.528, 531).

Ambiguity is the result of working in a problem space with no definition, because the solution has never existed. At the core of Design Thinking is abductive reasoning and frame creation, which give Designers the freedom and comfort to work in ambiguity, or what Dr. Elizabeth Sanders calls, "the fuzzy front end" (Sanders \& Stappers, 2008 p.6). It is through the activity of synthesizing and making sense of 'themes' that meaningful insights are drawn (Dorst, 2011, p.528).

\subsection{User-Centered Design}

User-Centered Design is targeted at designing for people. Design Thinking methods and tools have been brought into the User-Centered Design process to bridge the language barrier 
between designers and their users and to help draw out the users' thoughts and feelings. "In User-Centered Design, Designers generate solutions placing users mainly in a reactive role" (Fischer, 2003). Sanders and Stappers (2008) further explain that in the "User-Centered process, the user is a passive object of study." The diagram on the next page (Figure 2.1) illustrates a typical User-Centered process from initial exploration and research to concept development.

Figure 2.1 shows that during 'Exploration and Data Collection' Designers/Design Researchers conduct ethnographic and secondary research to understand the problem space and the users. Stakeholders (users) at the core and on the periphery of a problem have unique perspectives (frames) and typically act as informants to the Design team in the User-Centered process. Designers and Researchers use various design research tools (ethnographic research, observation, interviews, focus groups, etc.) to better understand individual users and create a space through which the users can share what they do, think, and feel.

Designers use the Design Thinking methods to empathize with the users and gather various frames of reference. During 'Pattern Finding and Synthesis' the Designers/Design Researchers deconstruct these perspectives (often the needs and goals) of the informants within a 'black box' in search of themes (patterns, connections), as well as outliers (dissimilarities) from which they can draw new meaning and understanding (sensemaking). The term 'black box' is used because the 'Synthesis' and 'Sensemaking' processes are often internalized within the Designer's head or within the design studio (not with the stakeholders). Designers personally reflect and may collaborate with one another (and other industry partners) to synthesize and organize the chaos into frames or design constraints (principles). Within their thinking spaces ('black boxes') Designers will explore different ideas based on their own intuition, the input of other Design team members, and the input of their users. 


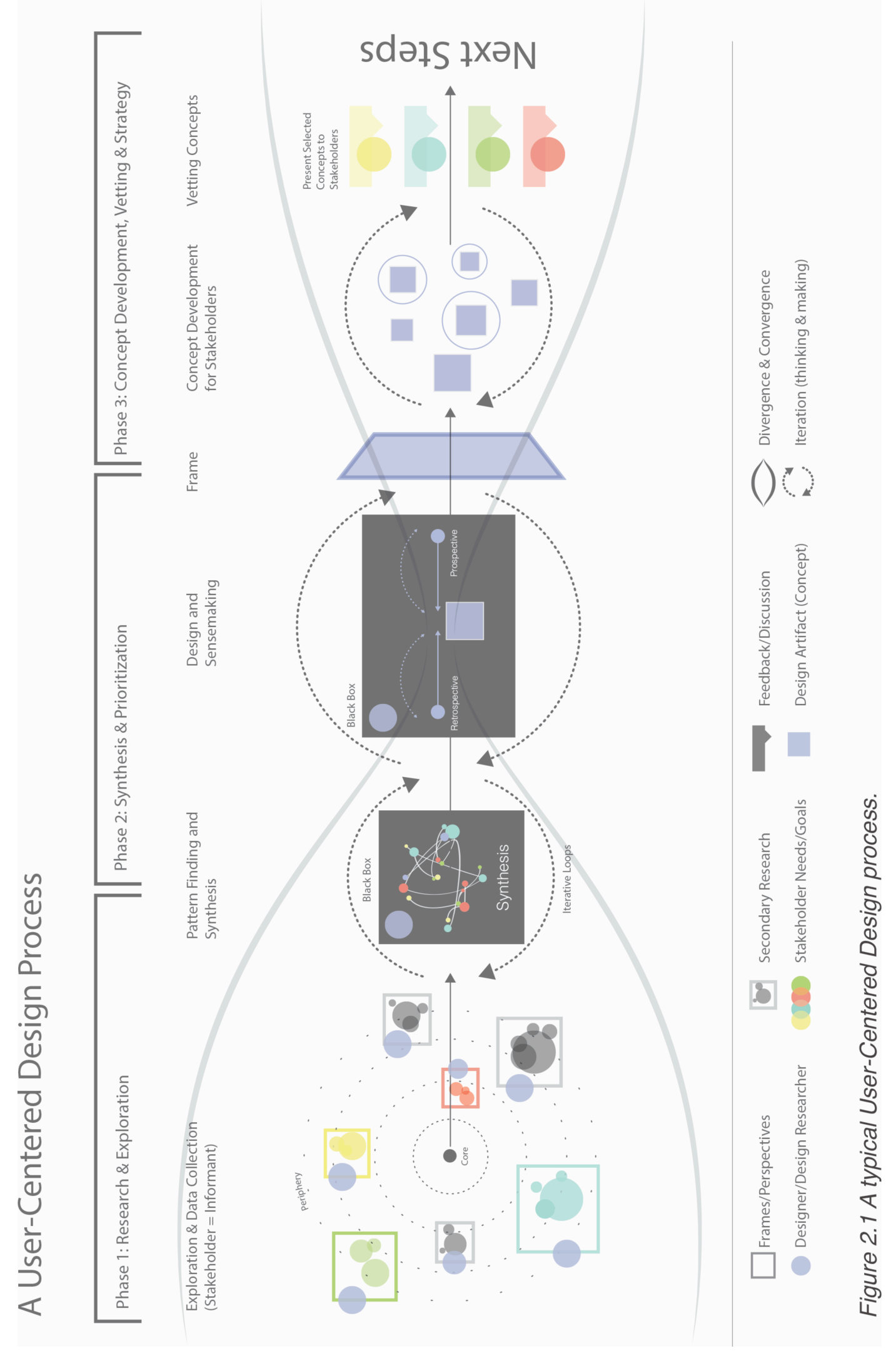


In the final stages of this diagram, the Designers present concepts (artifacts) and decisions, formed in the 'black box,' from their viewpoint or interpretation of the problem and data collected. Users then confirm or repudiate the Designers' assumptions, vetting the Design concepts that have been selected and providing feedback on ways to improve the proposed concepts.

\subsection{Participatory Design}

Wicked problems cannot be resolved from one perspective, but instead require the expertise and knowledge contributions of many integral stakeholders (both at the core and periphery) of the wicked problem system. Wicked problems provide us with an opportunity to evolve and expand beyond designing for people in User-Centered Design processes, to designing with stakeholders in a participatory process. Brandt, Binder and Sanders believe that "taking care of major societal issues often involves several stakeholders with different expertise and responsibilities. Scaling up the complexity of the development tasks calls for Participatory Design practices" (Brandt, Binder \& Sanders, 2012, p.169).

Participatory Design (PD) is an approach used in "design practice that involves different nondesigners [i.e., potential users, other external stakeholders, and/or people on development teams from non-design backgrounds] in various CoDesign activities throughout the design process" (Sanders, Brandt, \& Binder, 2010, p.195). In PD, the roles of 'expert' and 'Designer' are not as clear-cut. The Designer provides stakeholders with tools for ideation so they can express themselves, share their expertise, and generate ideas. The Designer, nevertheless, "still plays a critical role in giving form to the ideas" (Sanders \& Stappers, 2008).

Participatory Design is not a new approach. Its origin can be traced back to two historical instances: in 1971 Design and participation was the overall theme at the second conference of the Design Research Society (Cross 1971), and in the 1980s and 1990s it was associated with 
"the groundbreaking working of American and Scandinavian researchers engaged with systems design and automation" (Brandt, Binder, \& Sanders, 2012 p.147). Participatory Design has been touted as a Design approach that can "contribute to the inclusion and participation of citizens at large in Design and societal planning" (Brandt, Binder, \& Sanders, 2012, p.147). It can be conducted with individuals or with groups of people, but ultimately Brandt et al (2012) believe two types of values guide participation in Participatory Design: democracy and tacit knowledge. Consideration of each person's voice through the use of methods and tools can lead to more legitimate user participation and engagement. The importance of making draws out tacit knowledge and helps guide participants in learning, expressing, and sharing thoughts, feelings, and a sense of belonging through embodied experiences (Brandt, Binder, \& Sanders, 2012, p.147). A typical Participatory Design process, shown on the following page (Figure 2.2), depicts a process that is more collaborative and inclusive than a typical User-Centered Design process. By taking into consideration the viewpoints (thoughts, feelings, ideas) of multiple stakeholders with different backgrounds, and giving them an inclusive role in the process of Design, Participatory Design is, therefore, a more appropriate approach, over User-Centered Design for tackling wicked problems.

Figure 2.2 shows that during 'Research and Exploration' Designers/Design Researchers conduct secondary research, ethnographic research, and use CoDesign methods with stakeholders at the core and periphery of the problem to understand the problem space and the unique perspectives (frames) of the stakeholders. Through ethnographic research and CoDesign, Designers/Design Researchers facilitate methods to better understand what their stakeholders say, do, and make, while bringing their stakeholders into more inclusive Design roles. In PD, stakeholders share their thoughts and feelings, and take part in framing the problem and designing new resolutions with Designers. The 'black box' from User-Centered Design is replaced now with an inclusive Design circle that promotes collective understanding and reframing (using past and future references (i.e. 
expressed needs) to inform new alternatives). Designers give form to a space through which multiple stakeholders can collectively visualize and communicate their perspectives to one another, see data from many views, and prioritize key needs and goals. Through this inclusive process, stakeholders and Designers can learn from one another through telling, making, and enacting methods (Brandt, Binder, \& Sanders, 2012). The methods and tools used in the CoDesign process can also help Designers and stakeholders to identify outliers and patterns within the data, which help to establish 'themes' and frame the problem.

The reframing and synthesis processes are both iterative. As new information is shared, new frames and sensemaking emerge. The synthesis and framing (determining the scope of the problem) processes are facilitated through the Designer/Design Researcher collaborating with stakeholders to organize the 'fuzzy front end' and provide stakeholders with an opportunity to express their own thoughts and ideas, and empathize with alternate perspectives. The act of exploring different alternatives from various perspectives and synthesizing patterns and outliers allows collective teams to reframe the problem in many ways before determining a final approach. Tools and methods for reframing may include: visualizations, journey maps, prototypes, or prospective scenarios. Frames establish the mindset through which the problem will be tackled.

The 'artifacts' or concepts that emerge from the PD approach are the result of co-designing activities. Unlike in User-Centered Design, CoDesigners are also included in the prototyping and fabrication of concepts aimed at meeting the needs within the frame that was established collectively. Through framing and early prototyping, Designers and stakeholders begin to shape ideas, prioritize key concepts, and determine a strategy of next steps. The 'Next steps' will entail taking the concepts further into the Design process. 


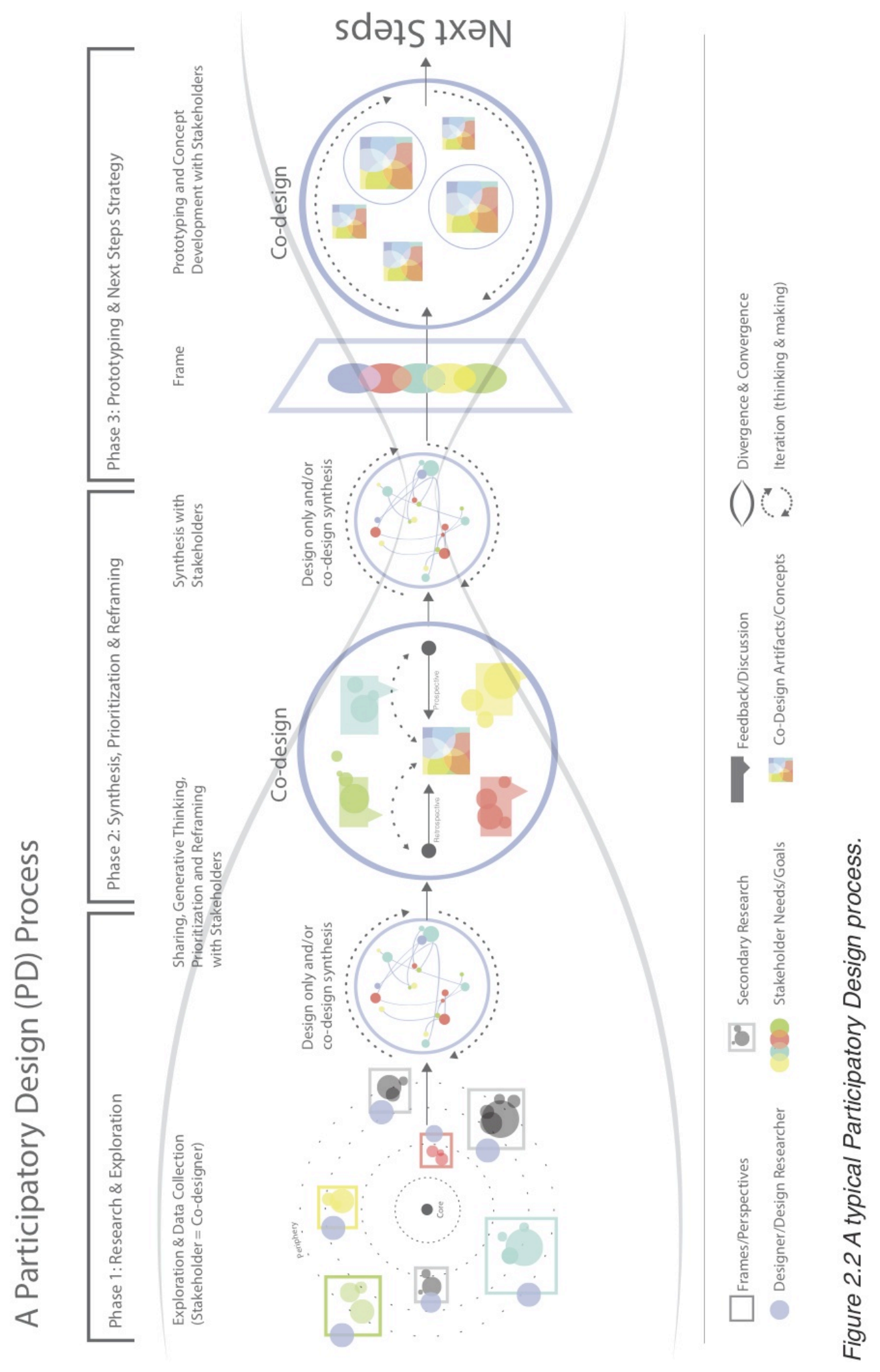




\subsection{Participatory Action Research}

However, due to the multi-faceted, unsolvable nature of wicked problems, Participatory Action Research may be a better approach than PD. Participatory Action Research (PAR) is a CoDesign approach aimed at producing "knowledge and action directly useful to people, and also to empower people through the process of constructing and using their own knowledge" (Shortall 2003, p.225). Unlike Participatory Design, the focus of PAR resides in the process of constant iteration and reflection for the purpose of understanding and knowledge creation, rather than generating an outcome. PAR is a collaborative approach that instigates social networking, problem-solving exploration, shared understanding, and ownership of the problem across transdisciplinary partners. The process is often non-linear, iterative, and aimed at motivating participants to take ownership and act. Typical methods of 'Research and Exploration' in PAR include interviews, observations, and discussion-based workshops, similar to PD, aimed at extracting information and unique perspectives (frames) from stakeholders at the core and periphery of the problem. This initial step is intended to bring stakeholders into an inclusive role in sharing their knowledge and gaining new understanding about the problem space and the needs/goals of other stakeholders.

During the stages of 'Synthesis' and 'Sensemaking,' stakeholders not only contribute to discussions and sharing information, they are also included in identifying patterns, root problems, and drawing connections among the data in order to collective reframe the problem scope around true 'value' and meaningful needs/goals.

The 'Frame' is created collectively. From this collective frame, new resolutions are prototyped and co-designed with the stakeholders. The 'Framing' process, as well as the 'Synthesis' and 'Sensemaking' processes, are iterative, not only within each stage but across the stages in PAR. 
The 'Prototyping' and 'Next steps' phases of the PAR approach are very similar to PD, with the addition of iterative understanding and the stakeholders' maintaining ownership of the problem to carry out the next stage of development. Next steps will entail building a tailored CoDesign team around a specific resolution and taking it deeper into the Design process to ideate further and refine the concepts.

In summary, the emphasis of PAR is designing with stakeholders to engender shared learning, shared knowledge, and collaborative analysis through an "action-oriented and learning-centered approach" that helps collaborative teams frame the problem space and actively address true systematic problems (Howard \& Somerville, 2014). A diagram of a typical PAR process is illustrated on the next page (Figure 2.3). 


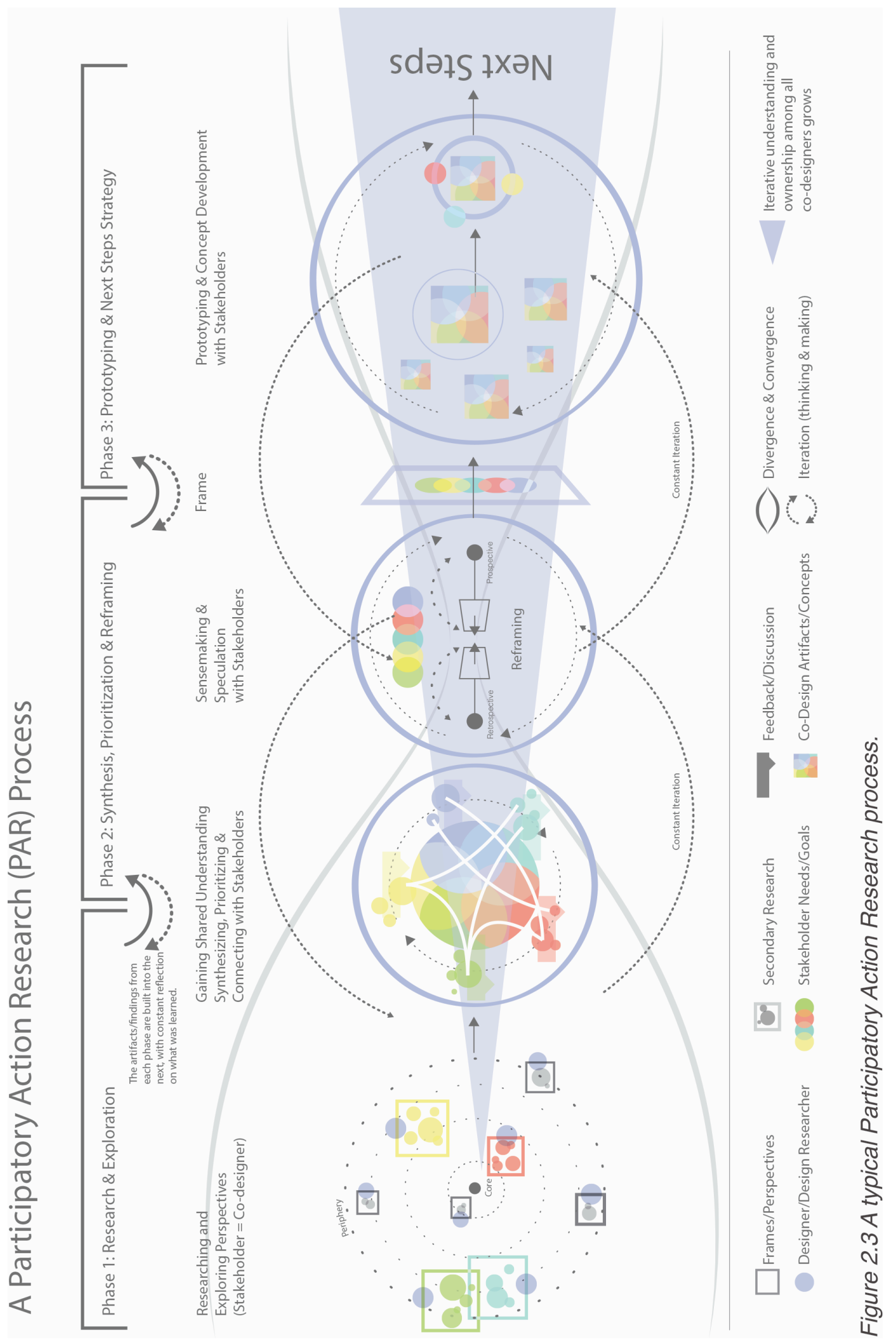



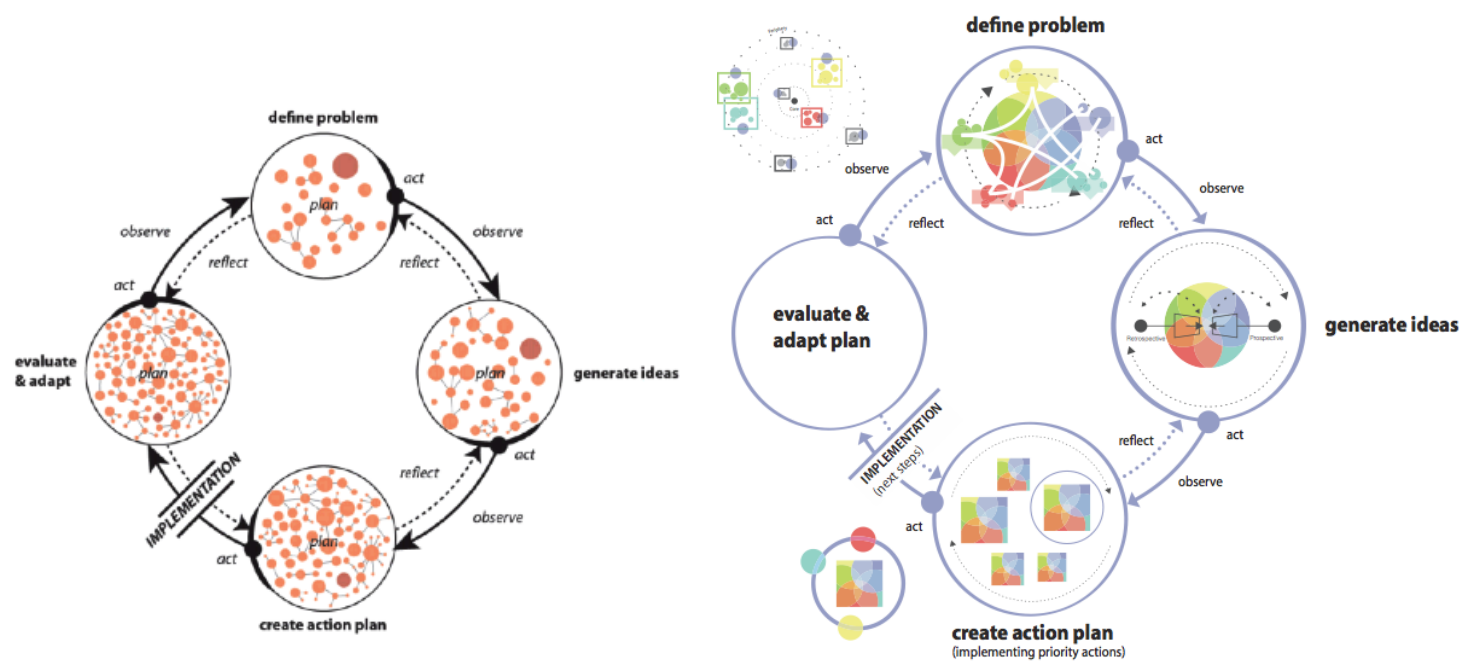

Figure 2.4 PAR diagram as an iterative cycle, inspired by Russo (2013).

Inspired by Michael Russo's (2013) PAR depiction, the diagram above on the right illustrates the iterative steps of PAR in a cyclical relationship (action, observation, and reflection), as explored in the case study. The more linear diagram and cyclical diagram of the PAR process explored in this research focuses on the front-end stages of PAR before implementation.

\subsection{Shared Understanding}

The drive to develop creative and innovative solutions for contemporary problems calls for human-centered, problem-based approaches and cross-disciplinary collaboration (Blair-Early, 2010, p.208). Approaches in Design (like Participatory Design and Participatory Action Research) are becoming increasingly collaborative as more problems become multi-faceted and expand to encapsulate multiple cultures, disciplines, and stakeholders (Blair-Early, 2010, p.214). In PAR, participatory problem solving and collaborative inquiry (directly observing, interacting, and designing with others) materialize when participants directly interact with one another (Blair-Early, 2010, p216). Lee Vygotsky also spoke to the value of collaborative learning. He believed that 
intelligence is often the result of social interactions and experiences. An "essential feature of learning is that it creates a zone of "proximal development," - our internal development process is stimulated when we interact in cooperation with other people (Vygotsky, 1978). Blair-Early explains that collaborative enquiry is one of the most important aspects of PAR. In order for Designers to understand the problem beyond their individual scope, "they need to directly observe, interact and design with other Designers, developers, and end users" (Blair-Early, 2010, p.216). This notion, interacting and designing with others to expand understanding, extends to the CoDesigners (the stakeholders), who are brought in to actively contribute to the sensemaking process around wicked problem and help the Designer expand their scope. If shared understanding is tied to expanding one's scope (to better understand the nuances of a wicked problem from multiple perspectives) and connecting with others, the methods and tools used in Participatory Design research should stimulate cooperative interactions and new ways of learning among Designers and varying stakeholders in a shared space/experience.

\subsection{Critical Making}

Mark Ratto introduces the term "critical making" which he defines as "a mode of materially productive engagement that is intended to bridge the gap between creative physical and conceptual exploration" (Ratto, 2011, p.252). Unlike traditional Design prototyping, critical making "emphasizes the shared acts of making" and "critique and expression" rather than "technical sophistication and function" in a final "evocative object" (Ratto, 2011, p.253). The prototypes produced through critical making are a means to an end, their value is found in the "act of shared construction, joint conversation, and reflection." Ratto goes on to say that he believes this method can be particularly useful for wicked problems (Ratto, 2011, p.253). The artifacts created through critical-making can also be a provotype, or provocative prototype. Provotypes serve a similar purpose as prototypes produced through critical making by exposing tensions in multi-stakeholder 
processes and by supporting collaborative analysis and synthesis, iterative thinking, and exploration into new Design possibilities through making (Boer \& Donovan, 2012).

Brandt et al. (2012) explain, "Through practice we produce the world, both the world of objects and our knowledge about the world. Practice is both action and reflection. But practice is also a social activity; it is produced in cooperation with others" (p.148). Vygotsky's theory of "proximal development' and Ratto's theory of 'critical making' suggest that a key to learning is community and connectivity with others. If, in fact, practice, and shared understanding and learning, are linked to co-producing (co-making) and gaining knowledge through social interaction and shared experiences, then wicked problems may benefit from the design of collective spaces and a Participatory Design approach that support iterative learning and social engagement through making and sharing, and a greater sense of belonging through shared understanding and empathy. The sections below ('Design Charrettes' and 'Seeing Spaces') describe a participatory process and collaborative environments that embody the ideals of critical making and iterative understanding. The research in these sections explores in more depth the role of the players (Designers and non-designers), and the value of diversity, transparency, participatory prototyping, and sensemaking to help people discover the true value they seek in collective innovation.

\subsection{Design Charrettes}

A Design Charrette is a process (or an environment) intended to promote cross-pollination and afford collaborative teams (made up of Designers and non-designers) the ability to explore, share, leverage and build off each other's ideas to create resolutions with true value (Hanington \& Martin, 2012). The physical spaces, often along with low-tech tools for Design Charrettes, are designed to inspire creativity and bring multiple participants together to discuss a wide range of ideas. The ideas presented in Design Charrettes are displayed for everyone to see so they can be discussed and built upon. The Design Charrette process was used in two comparative PAR 
case studies involving the redesign of two spaces (Library Courtyard and Library Facilities) in the Auraria Library in Denver, Colorado (Howard \& Somerville, 2014). Each study consisted of three phases: information sharing, idea generation, and prototyping. Methods used and ideas made and prioritized in each step of the process were built upon in the following phase. While the structure of both Design Charrettes was the same, the type of leadership and participation varied. What the comparative study underscores is the "value of authentic design participation" that comes from designing with rather than designing for people, and creating a space through which participants can learn and gain shared understanding through co-creation and making (Howard \& Somerville, 2014). The success of each of the Design Charrettes in generating authentic design outcomes was measured by the role the Designers played and value of the stakeholders' voices. The Design Charrette used for redesigning the Library Courtyard was perceived as more collaborative and inclusive in its execution than the one used to redesign the Library Facilities because the Courtyard charrette incorporated more open feedback and participatory prototyping. Additionally, the Design team in the Courtyard charrette served as 'interpreters' (instead of directors) of participant ideas, and were directly engaged with the stakeholders in co-developing prototypes. Conversely, the Designers in the Facilities charrette lead the design of many of the concepts, generating ideas and solutions based on gathered assumptions, which ultimately did not align with the priorities of the stakeholders. Inclusivity and participatory prototyping in the Courtyard charrette resulted in stronger relationships and the stakeholders taking ongoing ownership of their space.

The principles of inclusive collaboration, iterative learning through making and prototyping, and the value of information transparency in the Design Charrette process and the environments designed for them can also be realized in 'seeing' spaces. Termed by Bret Victor (2014), these spaces are comprised of tools designed to stimulate new ways of seeing a problem from multiple 
frames of reference and encouraging collective teams to learn from one another to build deeper understanding and identify new discoveries.

\subsection{A New Maker Space, the 'Seeing' Space}

"Create an atmosphere that gives people permission to experiment" (Kelley \& Kelley, 2013, p.197). Spaces designed for collaboration and team cohesion should be designed with intention (Kelley \& Kelley, 2013, p.194). The environment often plays an important role in how people interact with each other and with information. For instance, spontaneous creativity and conversation can arise by creating open workspaces and by incorporating pervasive writing surfaces throughout a space (Kelley \& Kelley, 2013, p.194). What if these spaces could offer more to engage people and help them see new possibilities?

Bret Victor designs tools for people who make software, electronics, music, and animation and the biggest question he has is "how can the creator see and understand what the thing they are building is actually doing?" (Victor, 2014). Traditional forms of craftsmanship take place in 'maker' environments surrounded by tools. In essence, the space, as well as the tools, become extensions of the person's body. Bret Victor highlights three embedded principles found in 'maker' spaces: a shared creative environment, access to powerful and expensive tools (that an individual may not be able to afford), and the motivation to make goods (instead of consume mass produced goods). Taking this concept of the 'maker' space, Bret Victor proposes a new type of collaborative space, one that not only facilitates making, but more importantly helps people to see and understand problems in new ways. He calls this a 'seeing' space. To accomplish these tasks the collaborative space should afford three types of 'seeing': seeing inside (collecting and making data accessible), seeing across time (representing data across time, cause/effect, hierarchy, outliers and patterns), seeing across possibilities (simulated learning, iterations and design alternatives, understanding why or why not). To imagine the 
'seeing' space, Victor says, "picture the NASA control room." This room collects and displays large amounts of data and brings individuals together to see, understand, and control each aspect of that complex system. This way of systems thinking, deep understanding and seeing, Bret Victor believes, needs to be brought to mainstream engineering and making (Victor, 2014). Instead of offering construction tools, Victor proposes that these spaces offer seeing tools that move people away from blindly following recipes and more towards "deeply understanding what they are doing, inventing new things, discovering new things, and contributing back to the global pool of knowledge" (Victor, 2014).

'Seeing' spaces and Design Charrettes are intended to provoke new ways of seeing, while also bringing about shared understanding and new connections (among people and with the information). But, the value of these spaces for bringing about authentic design outcomes and sustainable impacts is found in the role of the Designer (as team member and interpreter, not director), the methods used to ensure that Design is with, not for, people, and the tools designed to stimulate new ways of seeing, learning, and inventing. These interactions ultimately impact how people chose to see new information, learn from one another, make sense collectively, and invent better resolutions.

\subsection{How We Chose to Look - Sensemaking and Synthesis}

"One thing I try to argue is that it's not just about bigger machines to crunch more data, and it's not even about pattern recognition," Mr. Kohn, the painter, said in a phone interview. "[Data visualization is] about frameworks of recognition; how you choose to look, rather than what you're trying to see. Scientists often think of visual images like graphs as the end result of their analysis. I try to get them to think visually from the beginning" (Carey, 2015). 
The quote by Daniel Kohn, a Brooklyn-based painter and conceptual artist collaborating with genetic scientists at the Albert Einstein College of Medicine in New York, describes the value of visual sensemaking as a continual and creative process, not just an end result, through which we frame our understanding of complex information. The notion of perceptual learning (being able to make quick decisions without thinking by mastering subtle differences in clues) and how a person chooses to see contributes to how we make sense of the world (Carey, 2015). Instead of using visual representations and data analysis to tell the end of the story, Kohn believes we should use visualizations and perceptual learning to see in new, different ways (turning the data sideways, upside down, or through another dimension), not just to illustrate what we are "trying" to see. Perceptual learning is active and is often channeled when we are trying to learn new material. It does not require extra effort to think, but instead helps us pay attention to the most meaningful clues (or patterns) and filter out the rest. Scientists and researchers have been using perceptual learning training methods to improve people's instincts in complex fields like medicine and flying airplanes (Carey, 2015). In sensemaking people integrate their understanding and experiences into new meaning to explain (make sense) the world around them (Kolko, 2010a). Our sensemaking abilities may benefit from tools and methods that train us to be more perceptual learners and provide us with flexible visualizations that can be manipulated early in the process to simulate different ways of seeing and provoke new understanding.

\subsection{Sensemaking - A Future Skill-set}

Using gaming platforms and methods like the Delphi technique the Institute for the Future (IFTF) aggregates expert opinions to develop plausible insights about the future through crowdsourcing. In their report, Future Work Skills 2020, Anna Davies, Devin Fidler, and Marina Gorbis (2011) at IFTF identify six key drivers (disruptive shifts) that will likely impact our future. 


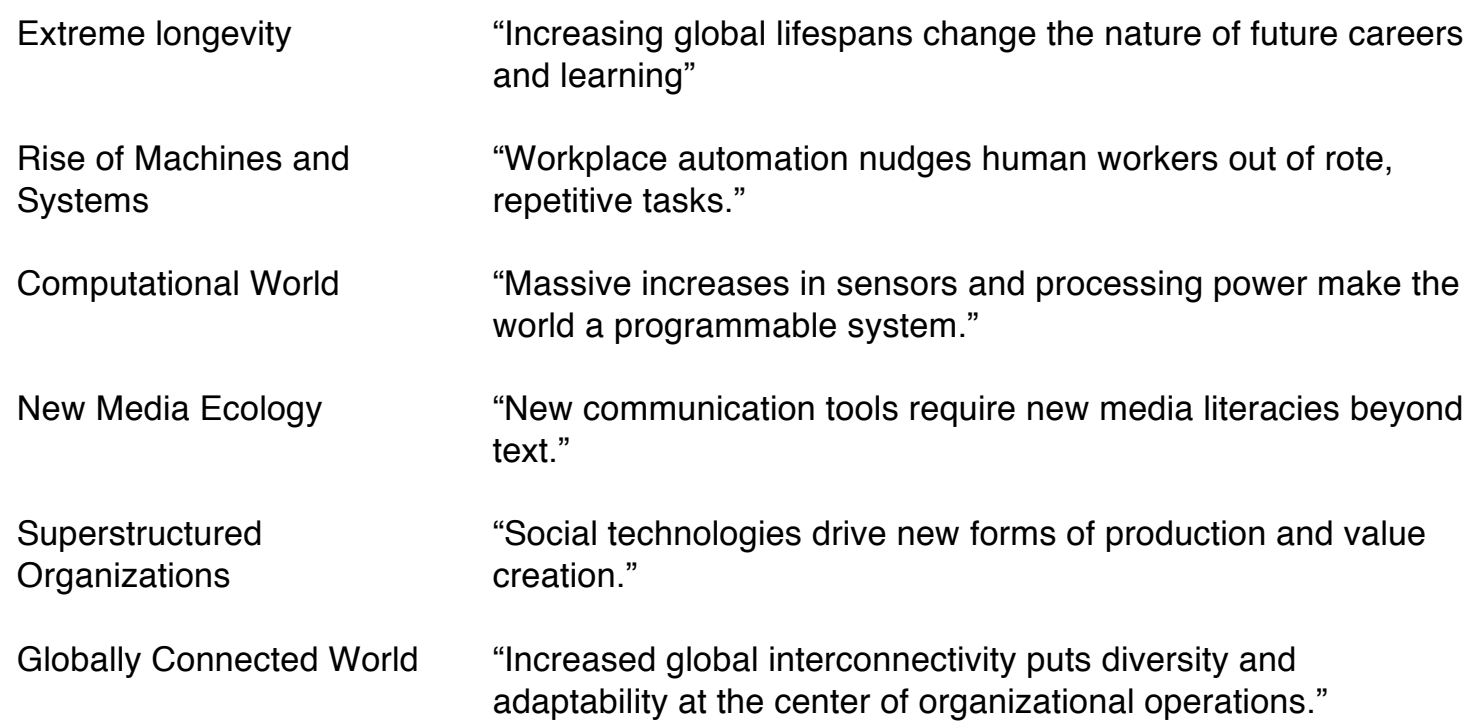

Figure 2.5 Six key drivers that will likely impact our future (Davies, Fidler, \& Gorbis, 2011).

These drivers helped to inform ten skill-sets that IFTF believe will have the most relevance in the future. Below is the list and the definitions IFTF applied to each skill-set. Though each skill-set touches on valuable competencies discussed for tackling wicked problems throughout this body of work, the skill of Sensemaking is highlighted and will be discussed in most depth in the following sections. 


\section{Sensemaking \\ Social Intelligence \\ Novel \& Adaptive Thinking \\ Cross-cultural Competency \\ Computational Thinking}

New Media Literacy

Transdisciplinarity

Design Mindset

Cognitive Load Management

Virtual Collaboration

\section{"Ability to determine the deeper meaning or significance of what is being expressed."}

"Ability to connect to others in a deep and direct way, to sense and stimulate reactions and desired interactions."

"The proficiency at thinking and coming up with solutions and responses beyond that which is rote or rule-based."

"The ability to operate in different cultural settings."

"The ability to translate vast amounts of data into abstract concepts and to understand data-based reasoning."

"The ability to critically assess and develop content that uses media forms, and to leverage these media for persuasive communication."

"Literacy in and the ability to understand concepts across multiple disciplines."

"The ability to represent and develop tasks and work processes for desired outcomes."

"The ability to discriminate and filter information for importance, and to understand how to maximize cognitive functioning using a variety of tools and techniques."

"The ability to work productively, drive engagement, and demonstrate presence as a member of a virtual team."

Figure 2.6 Ten skill-sets that will likely have the most relevance in the future (Davies, Fidler, \& Gorbis, 2011).

Before looking more closely at Sensemaking, it is important to briefly remark on some of the other skill-sets that were mentioned. Many of them appear to be linked to sensemaking. In a wicked problem that relies on multiple experts and iterative thinking processes, it would seem that our ability to determine deeper meaning or significance would be tied to our ability to connect with people and ideas across multiple disciplines in a deep and direct way (Social Intelligence, 
Transdisciplinarity), to think and create resolutions that breach rote thought processes (Novel and Adaptive Thinking), to translate vast amounts of data and work processes into more understandable concepts (Computational Thinking, Design Mindset), and to use a variety of tools and technologies that bridge understanding, filter information, and drive collaborative engagement across time and space (Cognitive Load Management, Virtual Collaboration).

As technology gradually takes over many aspects of our lives, higher-level human faculties like sensemaking will become more and more valuable. Competencies like sensemaking and critical thinking cannot be codified by machines because they rely on the capacity to understand context (Davies et al., 2011, p.8). Our ability to understand context and utilize sensemaking puts humans in a unique position to be adaptive thinkers, create unique insights, and seek out the deeper meaning (significance) of a problem or an idea that is being expressed. Therefore IFTF believes that sensemaking will become a crucial asset in the future for decision-making and innovation (Davies et al., 2011).

\subsection{Categories of Sensemaking}

"Increased complexity requires that more of the design engagement is spent sensemaking" (Jones, n.d.). Peter Jones leads two Design firms: Redesign Research (focusing on Systemic Design) and Dialogic Design International (focusing on Social Design and helping organizations resolve wicked problems). He gave a presentation for Design with Dialog in which he compared the various types of sensemaking and the leaders within each model. Below are two diagrams from his presentation. It is important to look at not only the different levels of sensemaking (Figure 2.7), but also the people in these fields (Figure 2.8). 


\section{Contexts of Sensemaking}

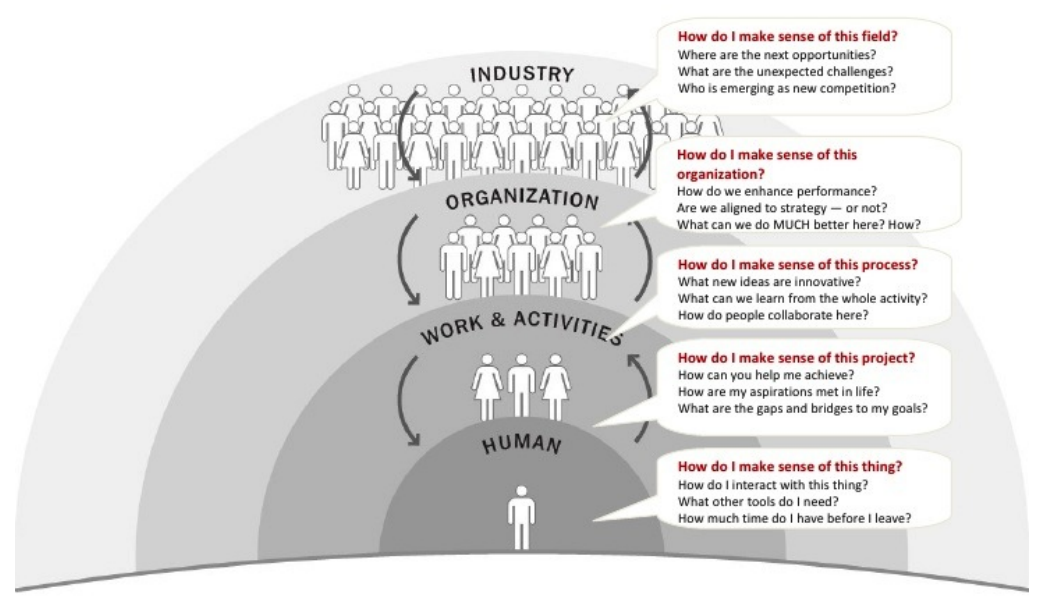

Figure 2.7 Sensemaking fields from a personal (human) level up to a cross-disciplinary (industry) level (Jones, n.d.).

\section{Sense making Models}

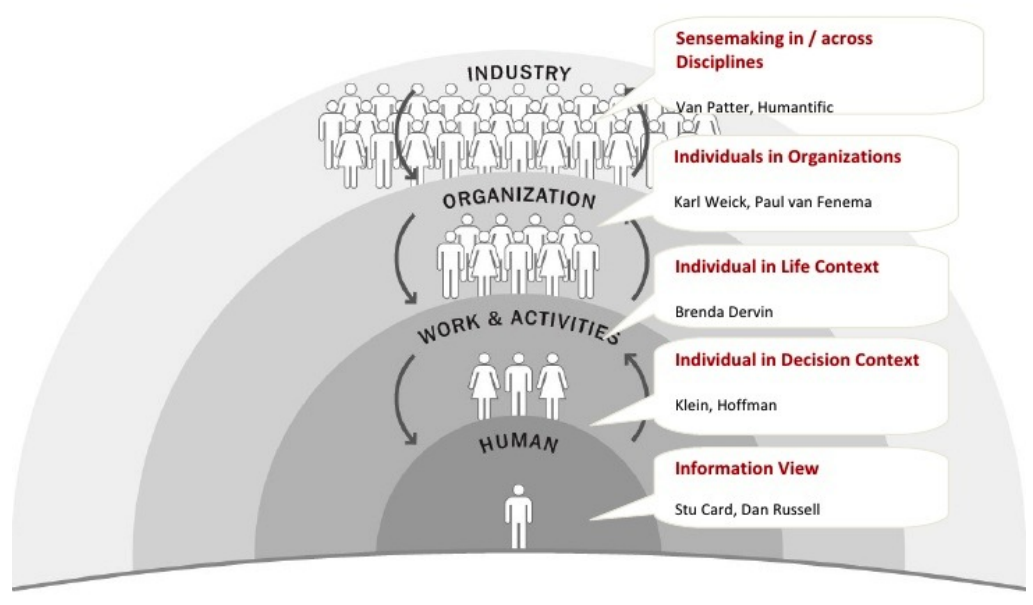

Figure 2.8 Leaders in various sensemaking fields from a personal (human) level up to a crossdisciplinary (industry) level (Jones, n.d.). 
Designers, cognitive psychologists, social psychologists, and communication theorists view sensemaking as an integral part of learning and synthesis (Kolko, 2010a). In his article, Abductive thinking and sensemaking: The drivers of design synthesis, Kolko (2010a), draws a comparison among the views of sensemaking from people in each of those sensemaking fields (represented in Peter Jones' diagrams).

Brenda Dervin is a communication theorist who believes that sensemaking is a subjective process of learning (dependent on personal perspective and point of view) that is contingent on personal experience. In her belief, persistent active learning (through personal experiences) is fundamental to sensemaking and problem solving, and should therefore take place continually and forever (Kolko, 2010a).

Daniel Russell, a researcher at Google, views sensemaking as a personal, highly task specific process through which one forms a representative model (a mental or tangible hypothesis) to "examine, test, and accept or reject specific questions or ideas." Therefore, unlike Dervin, Russell, believes the process of sensemaking is most useful in a finite time period, not something that should remain continuous (Kolko, 2010a).

Cognitive psychologists Robert R. Hoffman, Gary Klein, and Brian M. Moon believe that sensemaking is both a personal and social process, heavily dependent on a person's interpretation and perspective, or point of view (expertise/awareness) of the problem, oneself and/or others based on personal experience and beliefs. Sensemaking, from their understanding, is used to solve complex problems over a long period of time (Kolko, 2010a).

David Snowden is a management scientist studying complex problems prevalent in large corporations. He views sensemaking as a highly collaborative exercise people partake in to make 
sense of complexity in order to act. Like Russell he believes it occurs during a finite period of time. Through his research Snowden has developed a sensemaking framework called Cynefin, which was designed specifically for collective sensemaking and deciding which course to take during the early stages of problem solving. Snowden explains that sensemaking models should be the result of data (i.e., patterns emerging from the data should inform the framework), unlike a categorization model (where the framework proceeds the data) (Kolko, 2010a).

Karl Weick, an organizational behavioral theorist, views sensemaking as a highly collaborative and conversational process. The sensemaking that occurs in a group is biased by the knowledge and expertise of the people in the group who will "talk into existence" a unique set actions based on the subjective frame they establish (Kolko, 2010a). Weick believes that through the interplay of communal activity and shared communication, collective sensemaking can stimulate both long and short term organizational planning and growth.

Karl Weick's work in sensemaking, while on a collective (organizational) level, remains confined to people within a similar organization. GK Van Patter is the Co-Founder of NextDesign Leadership Institute and Co-Founder of Humantific, a Sensemaking and Changemaking consultancy. Van Patter and his company, Humantific, play on an even grander sensemaking scale, incorporating knowledge from multiple disciplines. Van Patter believes "Design Thinking is not one thing but rather changes from scale to scale" (VanPatter, 2015). Strategic Design and using Design Thinking in new ways (what Patter terms 'new practice Design Thinking') goes beyond Design Thinking used in the traditional sense for product, service, and experience design (which is often guided by a predetermined outcome). The activity of CoDesign is at the center of sensemaking. As Designers we are creating the cognitive scaffold that enable collective sensemaking. "To do this requires a much different kind of approach and a different toolbox than those used in traditional Design oriented practices" (VanPatter \& Jones, 2009). In "new practice 
Design Thinking' Humantific uses a diverse set of tools to encourage sensemaking and changemaking, avoiding preconceived assumptions about what the challenges or solution paths may be in a complex problem. New practices for Design Thinking related to sensemaking on an 'industry' level is not transforming traditional Design practice, but instead "creating new paths and new forms of practice" (VanPatter, 2015). This approach to Design Thinking and sensemaking moves Design into strategic roles and allows experts from many fields (points of view) to contribute to collective sensemaking and changemaking.

In each of the descriptions above we get an overview of how sensemaking is being utilized and explored in different areas and in slightly different ways. However, a common thread and a key aspect running through each category of sensemaking is the importance of perspective (point of view) and human perception in the act of making sense of complex information and dynamic systems. Given their multi-faceted and ill-defined nature, wicked problems, Jones (n.d.) believes, benefit most from cross-disciplinary sensemaking. The accumulation of and consideration of multiple perspectives early in the framing and Design process is integral to creating new knowledge and shared understanding to tackle interwoven and complex systemic problems. In bringing together multiple perspectives and experiences to collectively share and make (make sense of a problem and make resolutions), something new can emerge that goes beyond "the everyday practices" of the individual participants (Bandt, Binder \& Sanders, 2012, p.148).

\subsection{Sensemaking and Future Perspectives}

Sensemaking relies on social construction and human perception. Alex Wright defines 'sensemaking' as an on-going, natural tendency in humans to attempt to "craft, understand and accept new conceptualizations prior to action" (Wright, 2005, p.87). An inability to make sense of a complex problem can lead groups (and individuals) down one of two paths: paralysis because of uncertainty and confusion, or misguided (zealous) action as a result of ignoring overall 
complexities and certain 'cues' that do not conform to existing models. As a result, "important opportunities or potential threats" can be overlooked. Instead, Wright (2005) argues that individuals and groups need methods and tools that will help them make sense of ambiguity, prepare them for evolving uncertainty, and help them to develop future perspectives. Prospective sensemaking (covered more in the next chapter) is one approach that can help people imagine future perspectives by constructing "meaningful interpretations of their socially constructed worlds," and becoming "more open to the unexpected." This, in turn, results in transformative thinking and strategizing (Wright, 2005, p.92).

\subsection{Framing and Sensemaking in Design}

"Sensemaking is a constant process of acquisition, reflection, and action," Kolko (2010b) explains, in order that we may frame (or subjectively consider) a situation or idea by integrating various experiences to make sense of the world. Kolko goes on to explain that a "frame is an active perspective that both describes and perceptually changes a given situation" (Kolko, 2010b). Therefore, sensemaking is non-linear, subjective and constantly evolving as new knowledge and new perspectives (frames) are introduced.

'Framing', Kees Dorst (2015) believes, is beneficial for open, complex, networked problems. "Good frames are coherent, and provide a stable basis for further thought" (p.64). As with collective sensemaking, "the frame is also a social entity, as it can help harmonize the thoughts of the various stakeholders within a problem situation."...A good frame...integrates a broad range of issues under consideration and may draw even more issues from outside the original problem arena." Frames should be therefore captivate people's imaginations and inspire new possibilities, while also being robust enough to provide common ground from which participants can discuss the problem and take action around realistic resolutions (Dorst, 2015, p.63 \& 64). 
Sensemaking is a type of synthesis that helps to frame meaning and understanding in order to act. Framing is a universal human ability, but it is particularly central to the Design profession (Dorst, 2015, p.2). Designers like Jon Kolko and Kees Dorst describe the value and connections between sensemaking, synthesis and framing in the Design process. "Designers make explicit the normally implicit processes of sensemaking and framing during design synthesis as they attempt to make meaning out of data through interpretation and modeling" (Kolko, 2010b). The Designer must derive meaning (either through extracting or generating meaning) from the data to frame the problem space. The frame then provides perspective and definition to the problem space, which in turn helps the Designer define the Design principles and direction of the project in order to drive action. Ultimately, frames are the result of 'themes,' which are drawn out by analyzing patterns and outliers, relationships and hierarchy in the data through the process of synthesis. Kees Dorst explains,

"Themes are essentially a sense-making tool, a form of capturing the underlying phenomenon one seeks to understand. They are not clearly positioned in either the problem space or the solution space; their status is unclear until it is determined (retrospectively, after the frame is proposed) where they belong. Distilling themes from a complex situation is described as a process of insightful invention, discovery and disclosure. In design practice we see that 'themes' which could (from a problem solving perspective) be judged peripheral to the central paradox, become the triggers for the creation of new frames that allow the central paradox to be approached in a new and interesting way" (Dorst, 2011).

Themes (extracted from multiple perspectives) can move people away from the situation towards human needs and values. Neither positive nor negative, 'themes' hold the key to new innovation, and provide the foundation from which new frames can be created (Dorst, 2015, p.65). Frame 
creation allows Designers to "solve the unsolvable" and achieve "radical innovation" by identifying opportunities where others see only problems and creating new solutions where others see no answers (Dorst, 2015, p.xv). Frames in the Design process actively change based on new perspectives and information that are received. Sensemaking in the Design process is non-linear and iterative, and is built around active learning, and subjectivity. Based on the intuition of the Designer and the knowledge they gain from research, a similar problem presented to different Designers may be framed differently and will therefore have different outcomes. Ultimately the frame(s) become the window through which flexible Design constraints and the scope of Design are formulated.

In his book Frame Innovation Kees Dorst lays out a process for framing wicked problems. He proposes that Designers participate earlier in the strategy phases of projects and seek out meaningful problems that extend their abilities beyond the scope of "normal design practices." The Young Designers foundation is a program that brings together young Designers and young artists to challenge how Design can contribute to changes for the world and how these Designers can extend their abilities and the scope of their work on social projects. These projects "are a far cry from normal Design practices where one generally starts with a question and designs a solution. In contrast, the YD/project starts with the signaling of an issue in society and seeks interest from a group of initial stakeholders to partner in an open process...or quest... around this issue, shaping the questions that should really be asked" (Dorst, 2015, p.38). Dorst's Frame Creation Model and his ten principles of frame creation are explained in the next two sections.

\subsection{Frame Creation Model}

The Frame Creation Model (Figure 2.9), according to Kees Dorst includes: archaeology, paradox, context, field, themes, frames, futures, transformation, and integration. The first four steps in Dorst's framework (archaeology, paradox, context, and field) lay the foundation of understanding. 
'Themes' encompass analysis and the process of synthesis from which new frames are created. The last four steps (frames, futures, transformation, and integration) explore the implications of future actions (Dorst, 2015, p.75-80, 101, 107-8). In summary, from first identifying the problem to integration, Designers and stakeholders must first define the paradox, context and field to create themes (synthesis) through which new frames, futures and eventually transformation can emerge (Dorst, 2015, p.105).
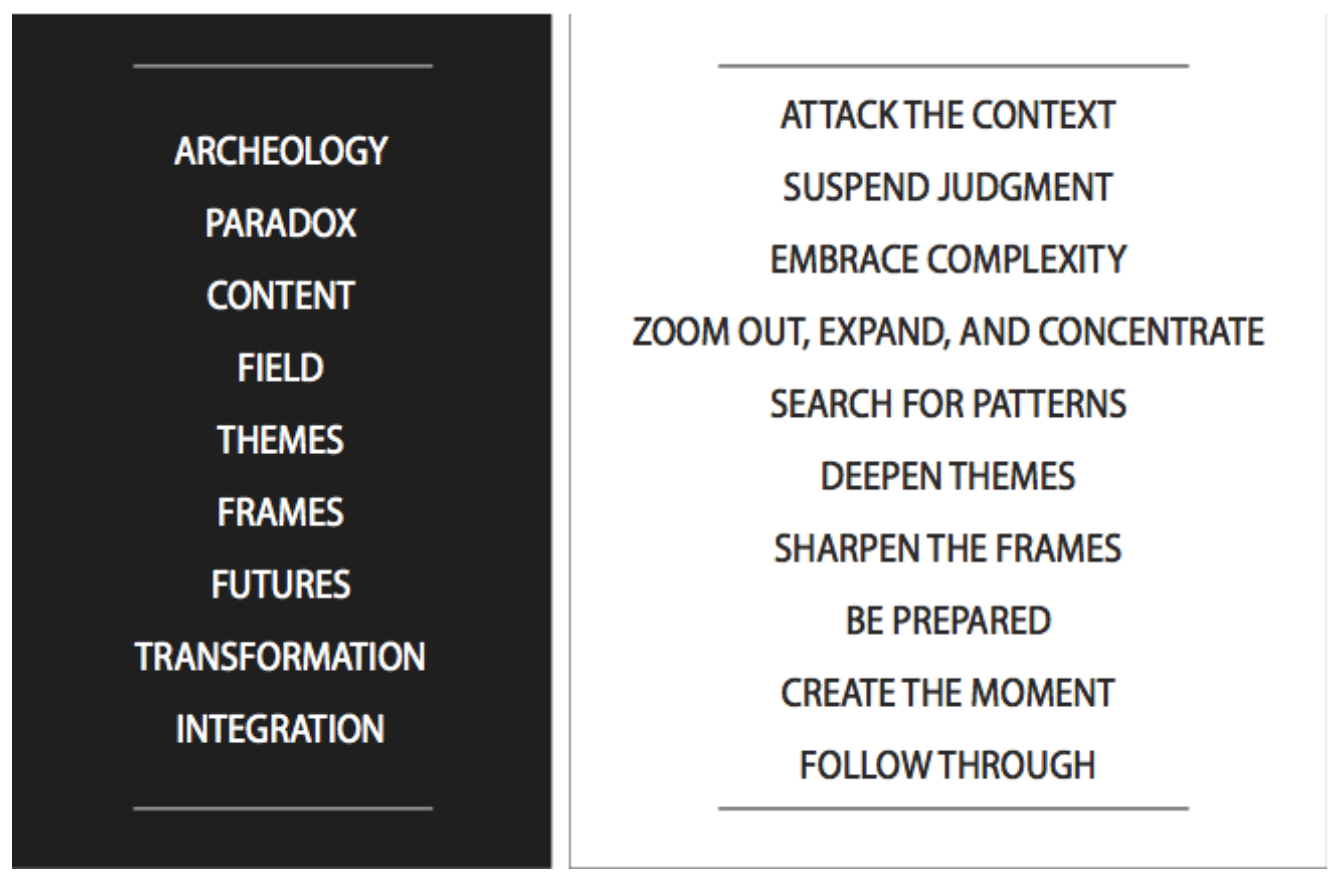

Figure 2.9 Frame Creation Model and ten principles of frame creation (Dorst, 2015, p.75 \&101).

\subsection{Principles of Frame Creation}

Kees Dorst's ten principles of frame creation (Figure 2.9) include: attacking the context, suspending judgment, embracing complexity, zooming out, expanding and concentrating, searching for patterns, deepening themes, sharpening the frames, being prepared, creating the moment, and following through. Each step is crucial when creating frames. We have to let go of our current beliefs in order to make way for new understanding. For example, consider the 
principle of "creating the moment." In this principle information is brought together and a collective innovation team is taken through the frame creation steps by a facilitator. As Designers, our expanding role is in facilitating these moments. The frame creation session is intended to bring diverse groups of people together for different skills expertise and approaches (Dorst, 2015, p.107-8). In the scope of the case studies and the work of the Young Designer foundation presented in Frame Innovation, creative freedom is combined with analysis and a keen sense of relevance to the problem as Designers and integral stakeholders are teamed together to challenge current assumptions and conventional ways of working (Dorst, 2015, p.38).

\subsection{Approaches to Reasoning}

The ability to deal with open, complex problems, create new frames, and challenge existing standards is rooted in how Designers are trained to think. To better understand and appreciate the core thinking process of a Designer, Kees Dorst (2011) illustrates comparisons among three distinct ways of reasoning: deductive, inductive, and abductive.

He begins by diagramming basic reasoning patterns used in problem solving.

WHAT (thing) + HOW (working principle) leads to RESULT (observed)

Then, he moves into the first type of reasoning, deduction.

Deductive Reasoning

WHAT (thing) + HOW (working principle) leads to ??

Deductive strategies rely on finding ways that new approaches/strategies can 'fit' into existing/traditional models (the way things have been done in the past). It is a top-down approach that depends on consistency, tight analysis, and proof. When using deduction we often know the 
'what' and the 'how,' so we can "safely predict the result" (Dorst, 2011, p.523). For instance, given the nature of an object like a piece of fruit and knowing how a juicer works, it is safe to predict that when fruit is placed into a juicer the solid fruit will become a liquid. This type of reasoning is straightforward and anticipatory. It often limits one's ability to expand their scope/thinking and come up with new plausible alternatives. Deductive reasoning informs 'justification', while another form of reasoning, inductive reasoning, informs 'discovery'.

Inductive Reasoning

WHAT (thing) + ?? leads to RESULT (observed)

In inductive reasoning the 'what' and the 'result' are known; what is left to be determined is 'how.' Returning to the earlier example, the nature of the object (the piece of fruit) is known and the end result of extracting juice is desired, however 'how' juice could be extracted from a piece of fruit is completely open for investigation. This frame of logic is how hypotheses are developed. Inductive reasoning generally establishes new strategies and nurtures new opportunities with 'hands-on,' 'trial and error' approaches (Dorst, 2011, p.523). Inductive strategies are externally focused and used to understand the nuances and interrelationships of the environment (or the system), rather than internally focused on how to control it. As a result, inductive strategies are better suited for complexity and interdisciplinary approaches to problem solving than deductive strategies, because inductive reasoning is more organic in nature and "allows for greater flexibility and increased responsiveness" (Wright, 2005, p.93).

While inductive strategies are preferred over deductive reasoning for collaborative problem solving, Wright may be overlooking the potential for abductive thinking practices. For a wicked problem with little definition, multiple facets, and an indeterminate end-goal, it may be best not to 
seek out a specific 'result,' but instead to find opportunities to create true 'value' through abductive reasoning.

Abductive reasoning is unique to Design because Designers are often tasked with developing something that has yet to exist to create value. Hence, when addressing design problems the equation slightly changes (Dorst, 2011, p.523). The outcome a Designer aspires to is more often creating value for others using abduction, rather than seeking a result. Here is how the altered logic equation looks:

WHAT (thing) + HOW (working principle) leads to VALUE (aspired)

Dorst explains that there are two types of abductive reasoning, Abductive -1 and Abductive -2 .

\section{Abductive-1 Reasoning}

?? + HOW leads to VALUE (aspired)

This represents a form of 'closed' problem solving, often used by Designers and engineers to create something new within a set value, using a known working principle. The more complex the problem and the fewer 'knowns', the more open the problem solving process becomes.

\section{Abductive-2 Reasoning}

\section{?? + ?? leads to VALUE (aspired)}

This model of abduction is more in line with wicked problems. The only known element in the equation is the 'value' Designers and others aim to achieve; the 'what' and the 'how' are yet unknown. Take the fruit example. Instead of honing in on the result being 'juice,' perhaps the value we seek is in determining 'what' and 'how' lead to maintaining a healthier diet. This type of reasoning makes the problem solving process more complex and ambiguous than other problem 
solving approaches at the start, but in the end this approach helps Designers expand their initial assumptions and frame unlimited alternatives. While Designers may use all three types of reasoning, abductive reasoning (unlike inductive and deductive reasoning) encourages the development and adoption of new frames by exploring openly the 'what' and the 'how'. The 'how' is understood through the use of thought experiments, iterative thinking approaches, and scenario simulation, wherein a new frame can be created. Establishing the 'how' through this process helps Designers imagining new possibilities that have never existed (Dorst, 2015, p.49 \& 50). Wicked problems may therefore be best tackled using an Abductive-2 reasoning approach.

\subsection{The Designer's Synthesis Process}

The design synthesis process, based on sensemaking and intuition, allows for the possibility of any given problem to be infinite. Design synthesis and sensemaking can occur both implicitly or explicitly through a series of limitless actions that involve: clustering, "pruning, interpreting, and reframing" (Kolko, 2010b). Pruning is a subjective, selective process through which the Designer combs through data and extracts overlapping similarities and rare outliers, based on the meaning (or interpretation) the Designer assigns to the data. Clusters of data, derived from the act of pruning, bring together similar or connected ideas, which in turn help to establish relationships and hierarchy in the data that point to prioritized opportunities for Design. Framing, therefore, is the result of subjective analysis (pruning) and clustered synthesis (Kolko, 2010b). The frame is the bridge between needs (what) and change action (value) (Dorst, 2015, p.130-135). Donald Schön refers to these interpretive perspectives as a normative frames, or appreciative systems. He explains, "it is only within the framework of an appreciative system - with its likings, preferences, values, norms, and meanings - that design experimentation can achieve a kind of objectivity" (Schon, 1984). David and Tom Kelly assert that simple changes in perspectives (frames) can spark new insights, as long as people are willing to let go of their initial assumptions (Kelley \& Kelley, 2013, p.106). Changes in perspective transpire during the process of reframing. 
Reframing occurs often in the Design process as the Designer purposefully shifts their normative lens to look at the problem from new perspectives. The Designer may often juggle multiple frames at once to gain empathy for multiple users and to better understand tradeoffs and relationships within the problem space (Kolko, 2010b). Designers and Design Researchers, practiced in synthesizing multiple points of data and juggling multiple frames (stakeholder perspectives) expose themselves to more experiences and information that filters into their normative lens (Kolko, 2010b). The key to reframing a problem is to get at the core issue and not to just solve for the symptoms (Dorst, 2015, p.39). By continuing to alter their perspectives the Designer gains a broader and deeper understanding of the facets of the system in an effort to see something others have overlooked (infinite possibilities), to be more objective in their synthesis of the problem, and to determine the root need or value they are solving for.

Synthesis is both an act of organization and interpretation in an effort to engender new meaning and understanding in order to reframe a given problem (Kolko, 2010b). Unlike empirical research, which objectively seeks to understand things that already exist, or predictive behavior research that objectively searches for causality to anticipate what people may do, synthesis instead seeks to "understand the facets of things that do not yet exist by bringing them into existence," and "helps to guess what people will do, feel, or think once the thing that does not yet exists, exists" (Kolko, 2010b). Kolko (2010b) believes therefore, that "synthesis is always generative because it always produces more data, information, and knowledge than was present before the actions began." Arguably, synthesis may generate more data and information than what was initially presented, but in terms of wicked problems, sensemaking and synthesis may benefit from a convergence of understanding among participatory stakeholders - bringing all of the perspectives, and elements of the problem together before diverging and seeking out new insights and resolutions. The relationship between convergence of understanding and divergence of new knowledge and ideas may in fact happen simultaneously; as connections are 
made and multiple frames are synthesized, new ideas and new paths may sprout. It would seem in wicked problems that to determine the 'what' and to frame the 'how' in a connected system of stakeholders, shared understanding and empathy should guide synthesis, divergence and idea creation throughout the process. The iterative process of convergence and divergence, framing and reframing in a PAR setting may help the collective arrive at the true value they seek.

\subsection{Design Synthesis in a Black Box}

Design synthesis often occurs organically throughout the ideation phases of the Design process in a black box, (i.e. in the Designer's head). To clients and non-designers our solutions are "magically derived" because "clients don't see the relationship between Design Research and design ideas, and therefore discount the value of Design Research and design synthesis entirely" (Kolko, 2010a). Although sensemaking (a form of synthesis) is typically an automatic and implicit human response to derive meaning from complexity, design synthesis, a learned and intentional process, should be made more explicit and transparent by Designers in the CoDesign process to help CoDesigners and other non-designers appreciate and value our process as being more deliberate and purposeful, than 'magic.'

Sensemaking that occurs between people demands new ways of thinking beyond personal intuition. Today's Design problems are complicated and therefore will need to take place collaboratively, out in the open. Additionally, Design teams are larger and more diverse Designers and stakeholders (non-designers) do not all speak the same language. Kolko espouses that due to rising complexity and the need for collective sensemaking, Designers will need to better understand the "cognitive and social structures that are used to support the various activities involved in synthesis," and formalize the synthesis process (Kolko, 2010b). However, today's wicked Design problems, having no idealized end-state and multiple perspectives, may not require a more formalized understanding of sensemaking, but instead a semi-structured, more 
transparent process that can be made more relatable through the design of 'seeing' spaces, and methods and tools of Design Thinking that afford individuals on a collective team the opportunity to continuously learn and the flexibility to grow from each other through experience.

Dorst believes that frame creation and innovation in wicked problems requires people to see differently, think differently, and do differently (Dorst, 2015, p133-149). To achieve this goal, frame innovation necessitates the engagement of multidisciplinary teams and tools for seeing, thinking, and doing collectively (Dorst, 2015). To create what Kolko (2010b) terms, "a shared 'canvas' for synthesis," Designers have adopted and developed methods and tools that help to externalize their processes of sensemaking and framing. Using dialogue between interaction Designers from frog design as an example, Kolko illustrates three externalized synthesis techniques: reframing from a new perspective (empathizing - putting yourself in someone else's shoes), creative leaps and reframing based on personal sensemaking, and externalization to create shared sense sensemaking (in his example, a whiteboard and shared ideas were pooled together in a "single democratic dialogue and diagram"). In Kolko's example these external sensemaking techniques are positioned in a Design studio. The outcomes of externalizing sensemaking in his case study reflect outcomes that can also occur (given the right tools and methods) in participatory design settings like Participatory Action Research. The notion of a 'shared canvas' for synthesis aligns with Bret Victor's vision of a 'seeing' space. The use of shared spaces and canvases offer ways to bring people into the Design process and stimulate new ways of approaching problems, but in doing so it is important to consider the role of the Designer in these collaborative spaces and these emerging processes.

The sections that follow dive deeper into some of the expanded roles of the Designer alluded to by various researchers and Design professionals like Kolko and Wright, and some that are produced from the compilation of multiple bodies of work. These roles include: facilitator, visual interpreter, bricoleur, problem architect, cartographer, and curator. 


\subsection{Designers as Facilitators and Visual Interpreters}

Given their visualization skills and sensemaking abilities, the role of the Designer in complex problems has extended to "interpreter" (Howard \& Somerville, 2014) and "facilitator" (Kolko, 2012). As a visual interpreter, a Designer's role is to shed light on complex data and make the information usable/meaningful so action can be taken. Using an abductive thinking approach and facilitating Design-led research methods, Designers can help to externalize the sensemaking process by bringing integral stakeholders together in a shared 'seeing' space where information has been gathered and curated. The intent of the space, the visualizations, and facilitation of the methods/information is to build understanding and empathy, and instigate new ways of seeing the problem from different perspectives. In facilitating activities that promote shared understand and speculative thinking, and helping to interpret data in visual forms, different viewpoints can be considered and new possibilities explored. For instance, visual diagrams can help to show relationships among different perspectives or data points, and visual narratives can define the context behind the numbers and help people find common ground. From these examples, the expanded role of a Designer as a visual interpreter and facilitator may encapsulate the activities and responsibilities of a bricoleur, problem architect, and curator, along with those of a moderator.

\subsubsection{The Designer as a Bricoleur}

"Designing in a global context requires Designers that can envision the entire lifecycle and depth of investigation needed to solve a problem rather than develop a product or series of products" (Blair-Early, 2010, p.213). Inexperienced Designers generate proposals for 'what' and 'how' almost randomly, but experienced Designers have "much more deliberate (and efficient) strategies to tackle the complex creative challenge" (Dorst, 2011, p.524). Perhaps the expanding role of the Designer is not transforming all Designers, but instead creating a new path for Designers who think in a more systematic way. Alex Wright speaks to the value of a 'bricoleur' in complex decision-making scenarios. 
"A bricoleur uses skills, knowledge, instinct and interpretation to craft an understanding of the world within which actions are meaningful but temporary...the art and craft of the bricoleur becomes one of synthesizing diverse cues to gain insight" (Wright, 2005, p. 94).

From this description there appears to be a strong parallel between the activities of the bricoleur and the expanding role of the Designer in facilitating synthesis and sensemaking around complex problems. Wright goes on to say that the "need for individuals and groups to enhance their ability to work with ambiguity and equivocality is of utmost importance" (Wright, 2005, p.95). As a bricoleur of new knowledge earlier in the strategic phases of the Design process, the facilitation role of the Designer goes beyond moderating a single session to include interpreting complexity, synthesizing information, and helping to transform understanding.

\subsubsection{Problem Architect (Giving Form to the Problem)}

Designers have the ability to foster discourse and create the tools through which inspiration and reflection can occur (Dorst, 2015, p.69). Brandt, Binder, and Sanders (2012) explain that "participatory tools and techniques can be seen as the scaffolding for the temporary community of practice in the making. They support collaborative enquiry into the intertwinement of the essential questions of 'what to achieve' and 'how to achieve it" (p.418). Designers, in this sense, are the architects of a complex problem, giving form to the wicked problem itself by creating tools and 'seeing' spaces that allow participants to explore new possibilities, priorities and relationships within a multi-faceted problem. Our evolving role as Design facilitators is in giving form or shape to the scaffolding around a problem to provide collective teams with a greater capacity for sensemaking. The scaffold provides the footing through which each participant can play a valuable role and help to construct meaning and resolutions. Through participation everyone determines 'what' needs to be constructed and 'how' to accomplish that goal. After the problem 
has been framed by the Design team (Designers and non-designers), the people connected to the wicked problem can take ownership of the problem and continue working with Designers to build new resolutions.

\subsubsection{Curator of Information and the 'Seeing' Space}

Another emerging responsibility in the expanded role of facilitation is information curator for complex problems in Participatory Design settings (Kolko, 2012). As a curator, the responsibility of the Designer is selecting, arranging, and presenting data in a usable format to guide stakeholders towards shared understanding and sensemaking. The Designer can also help to develop visualizations that help to provide the lay of the land so that collective groups can look at a problem from multiple perspectives and find new meaning in a way they had not been able to from a single frame of reference. The organization of chaos in a wicked problem may benefit from a team's ability to shift between multiple perspectives, instead of being limited to a single point of view. In her article Narratives of Space, Time, and Life, Barbara Tversky (2004) explores how meaning can be constructed by looking at a situation from multiple vantage points. The notion of mapping perspectives and relationships can also be represented by co-occurrence networks. Cooccurrence maps (as shown in Figure 2.10) are constructed using nodes (key categories or elements represented in data) and lines to show visual relationships and hierarchy (Schich \& Coscia, 2011). They can also be referred to as node-link diagrams (Liu et al., 2014). In line with findings from Tversky's research, interactive co-occurrence visualizations could be developed for PAR to help stakeholders construct meaning from both a survey perspective (macro view identifying known 'landmarks' and surveying the intricacies of the systemic problem) and a route perspective (drawing connections among multiple stakeholder frames). 


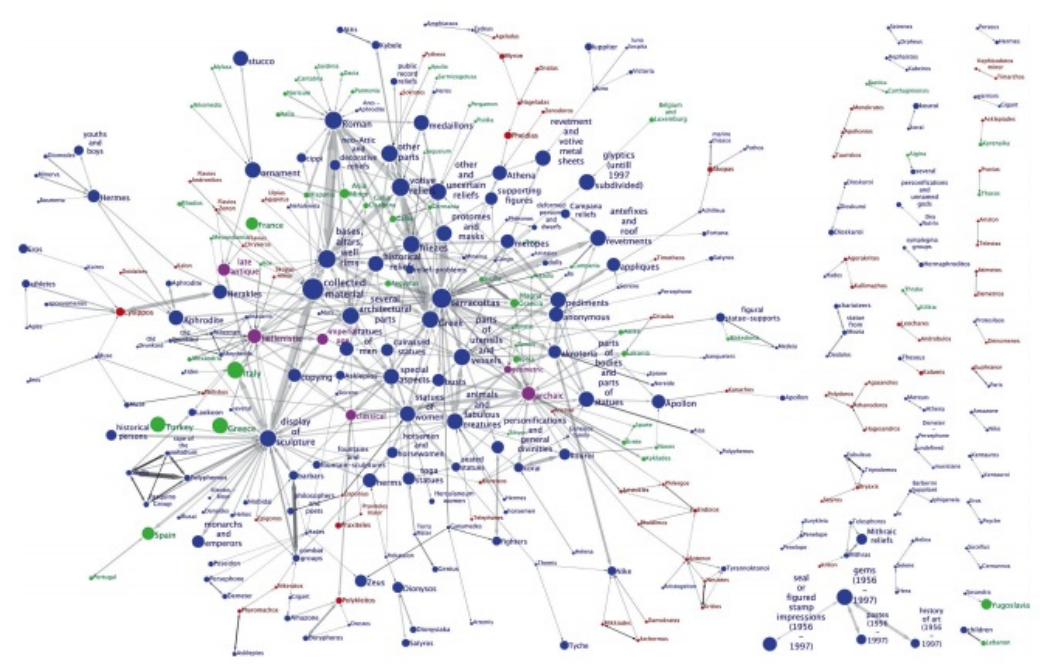

Figure 2.10 An example of co-occurrence visualizations (Schich \& Coscia, 2011).

The activity of curation also extends to bringing people from different backgrounds and perspectives together, arranging the Design Thinking approach, and tailoring spaces for collaboration that provoke new ways of seeing and promote group engagement and unplanned discovery. In his lecture Where Good Ideas Come From, Steven Johnson introduces five notions: liquid networks, slow hunches, blind spots, diversity, and platforms that drive innovation. When aligned with collective innovation, these notions appear to coincide with ways in which Designers could arrange collaborative spaces and PAR methods for wicked problems. Beginning with the analogy of coffee houses in Europe in the 1650s, Johnson explains how crucial the coffee houses were to the enlightenment period. The coffee house is an example of a liquid network where people and ideas could converge organically. The architecture of the space of the early coffee houses afforded people from different backgrounds and fields of interest to intersect and crosspollinate. Liquid network spaces are not designed for eureka moments or lone inventors, but instead are designed to nurture the growth of a network of people and ideas. The 'seeing' spaces proposed by Bret Victor are a type of liquid network, in which large amounts of data and eclectic, multi-disciplinary expertise are gathered together in one space to create understanding and 
generate new ways of 'seeing.' Truly transformative ideas, Johnson explains, are engendered through slow hunches and avoiding blind spots (Johnson, 2010). The same could be said for framing wicked problems. Ideas that have been iterated (built-up) and supported by a community of diverse collaborators broaden the scope of understanding and thinking so that root problems and innovative opportunities are hopefully not overlooked. It is evident that "diversity trumps smartness" (Johnson, 2010) - we are smarter the more diversified we are.

The information, approach, and spaces curated by the Designer for PAR are designed not to attain a single solution, but instead to act as a platform that empowers participants to continue pursuing resolutions and expanding new knowledge and ideas in the future. Innovation happens from a culmination of many people and the iteration of ideas over time (Johnson, 2010). In curating, synthesizing, and giving shape to the problem, the Designer invites a diverse group of experts, gathers multiple frames of information, and orchestrates a framework of tools and methods to stimulate fertility, uninhibited thinking, engagement, and cross-pollination - where ideas and thoughts can freely flow and be built up between people over time.

In each of these three facilitation activities, the Designer is not the lead creator, nor are they solely a moderator, but instead uses his/her abilities to bring order to complexity and stimulate the creativity and critical reasoning of others. At an Innovate conference at The Ohio State University in 2015 Steven Johnson used the metaphor of flying a plane to underscore the value of experiential learning. "Sometimes we have to let people fly the plane to learn themselves." If a critical piece of PAR and CoDesign is empowering participants to 'fly the plane' (actively participate in sensemaking, creative thinking and decision-making) and take ongoing ownership of the wicked problem, do we lose our value as Designers when we bring non-designers into our Design processes or do we enrich the lives of others and the long term outcomes/values we are hoping to achieve? 


\subsection{Who's Flying the Plane? - The Roles of the Designer and Stakeholder}

Van Dijck (2009) assesses user agency in his paper entitled Users like you? Theorizing agency in user-generated content, as it relates to the role of the trained Designer and the participants in CoDesign approaches. As more stakeholders are brought into our Design process (as CoDesigners), many Designers question whether the shared tools and methods used in Participatory Design sessions will negatively affect, or erode, the value or role of a trained Designer. Furthermore, the value of the trained Designer is being questioned as technology continues to improve and things like 3D printing and user-generated web content (material generated on the internet by non-experts) become more accessible to people who previously did not have a voice (Van Dijck, 2009; Sanders \& Stappers, 2008). Consequently, the debate over the value and role of users in user-generated design leaves many Designers concerned with the impact these changes may have on the field of Design in the future (Van Dijck, 2009). Two areas in the realm of wicked problems and Participatory Design practices need further exploration and definition: the value of the Designer and CoDesigners in tackling complex problems, and understanding their roles in the Design process.

\subsection{Design's Dilemma}

Tim Parsons, Associate Professor of Architecture, Interior Arch and Designed Objects (AIADO) at the School of the Art Institute of Chicago, has written a book entitled Thinking: Objects Contemporary Approaches to Product Design and has addressed the 'design dilemma.' He explains,

"There has been much hype about the future of home 3D printing, but it no more makes product designers out of us all than sewing machines made us fashion designers... or desk-top publishing made us graphic designers. If those examples are anything to go by it will actually increase the awareness among amateurs of the skills of the professional." 
In regards to user-generated content and user-generated design solutions, Van Dijck, like Tim Parsons, suggests that CoDesigners have rather limited power to alter the value of the trained Designer (Van Dijck, 2009, p.51). The role of the CoDesigner in PD and PAR is not Designer, nor is it producer, but instead a field expert and a creative collaborator. Participants are usually brought in at the front-end stages of the CoDesign process or at the very end of the Design process in concept validation and customization. The value they add to the Design process comes in bringing clarity and in-depth understanding of the problem and the needs of integral users/stakeholders at different levels. In the realm of complex problems with no clear solution, CoDesigners are brought together to help the Designer with frame creation - determining the root needs and the 'true' value that should be addressed in the networked system of problems.

The same fears associated with the potential depreciation of the Designer in Design Thinking and Participatory Design processes resonates with writers, filmmakers, and other field experts in connection with user-generated Web content. But, Van Dijck (2009) claims that instead of harming or devaluing the role of filmmakers or writers, "user-generated content actually boosts the power of media moguls, enhancing their system of star ratings and upward mobility" (p.53). The role of the populace is more in line with prosumers, rather than producers. Similarly, the attraction to Design through participation and/or self-expression of DIYers, business executives, and other professional fields (e.g., engineering) has actually helped to boost the overall appreciation and regard for the Design profession and its thinking processes (Van Dijck, 2009).

In fact, the value of the trained Designer is being recognized now beyond the drawing board and into boardrooms and on collaborative teams. Therefore, the roles of the Designer and the participatory 'amateur Designers' need to be better defined and understood as society approaches more complex problems. By making Design's thinking and synthesis process more 
transparent and collaborative, we can remove the mystery of what occurs in the 'black box'. The exposure to Design through participatory processes may bring greater understanding and appreciation of what we are capable of producing, but it is also critical that we not lose sight of our own value during this transition from a passive consumer society to a more active and participatory society. The Designer's technical skills and tacit knowledge, gained through experience, will not be replaced by CoDesigners. Although users (stakeholders) are given an opportunity to CoDesign resolutions and modify the system, the responsibility of the final form development remains with the Designer. The role and value of the Designer in PAR is not diminished, but instead expanded and reinforced earlier in the Design process - giving shape to the collective problem-solving space. The contributions of the CoDesigners are not to design the final form, but to ensure that the resolution helps them accomplish their task (Fischer, 2003). The value of the CoDesigner is in helping us define more meaningful opportunities in a world that is too complex to define for ourselves. The inclusion of CoDesigners helps us determine the path towards better design. By creating flexible, customizable systems that allow the users (owners of the problem) to adapt the system to fit their needs and collectively contribute their own vision and objectives, Designers may help to increase engagement and extend ownership of the problem across multiple stakeholder groups connected to the problem. Additionally, underdesigned resolutions (Fischer, 2003) and the collective frame of the problem potentially become more innovative over time as they are allowed to incrementally be refined across multiple experts.

\subsection{The Stakeholder, the Inclusive Participant}

Having more clearly defined the role of the Designer, it is now important that we turn our attention to the participants and their role in PD and PAR. Quick and Feldman (2011) argue that in the domain of public engagement, inclusion in the Participatory Design process is different from simply being an informant. The distinction between being an informant and being an inclusive participant in the Design process is determined by how stakeholders are integrated into the 
Design process. The value of inclusion is demonstrated across four forms of public engagement in four civic planning design case studies (Quick \& Feldman, 2011). In these comparative studies, the researchers found that when stakeholders had inclusive roles in the Design process, the communities themselves became more inclusive - people defined the issues jointly and continued to develop processes for addressing them. High inclusion stimulated open engagement throughout the processes of decision-making and implementation. In contrast, stakeholders in 'informing roles' were limited to soliciting input on specific content and validating decisions at certain stages of the process (Quick \& Feldman, 2011, p.274). These stakeholders were not typically a part of the decision-making process and therefore were more apt to feeling excluded. Low inclusion and participation yielded less buy-in, adversarial tendencies, and disconnection between stakeholders in the case study, because they were given a limited scope of the problem and limited control over implementation. If people do not feel that their participation will produce results they are less motivated to participate and there is a greater likelihood of burnout. Inclusive practices facilitate more 'authentic' input instead of 'pigeonholing' people into 'this or that' choices (Quick \& Feldman, 2011, p.279). Inclusion affords ample opportunities for participants to "redefine the 'what' and 'how' of the problems they are trying to address" (Quick \& Feldman, 2011, p.286). The inclusive role, over the role of informant, promotes more co-designing, co-learning, and coevolutionary tendencies that generate buy-in, stronger community support/engagement, and longterm commitment to a plan of action (Quick \& Feldman, 2011, p.278). Inclusion goes beyond input and validation and instead, "produces new understanding and opportunities for action" (Quick and Feldman, 2011, p.281). It enables more lasting and emergent (less structured and more iterative) engagement. These insights further support the value of an inclusive participatory approach in wicked problems to help stakeholders and Designers determine root problems, build stronger connections and buy-in across issues (and among different stakeholders), and encourage people to act collectively over the course of multiple years. 
Tools and methods designed for PAR should help to promote inclusion and active engagement. Technology, introduced into the PAR process, could potentially facilitate this type of interaction across space and time, which could widen a team's capacity to gain greater knowledge from multiple experts, allow them to simulate and iterate a variety of resolutions over time, and make the Design process, perhaps, even more immersive and engaging.

\subsection{Technology - Democratizing the Design Process}

What is the disruption potential of technology in the Design process? Technology has been shown to facilitate perceptual learning and shared understanding. Jon Landis, Development Executive at Apple, spoke at an Innovate conference at The Ohio State University in 2015. The central theme of his discussion concerned tools for future education. Transformative technologies like the printing press democratized information and changed the human condition. He believes technology apps, in a similar way, can transform how people learn and engage with new information today. An app called Whiteboard is one of those technologies. Students post homework on a shared network. When the teacher corrects mistakes online, failure is no longer perceived as 'bad', but instead regarded as an opportunity for all of the students to learn. Students have an opportunity to redo their homework until they get it right and learn from one another. As a result, their motivation and engagement in the learning space is higher than in the classroom. Landis gave an example of a socially marginalized classroom introvert who became an extrovert on Whiteboard. She used the technology to express her personality and connect with others.

Technology has changed how we learn - information is external, social, and readily accessible across time and space. In that same light, how could technology help to democratize the Design process and allow information to be readily shared across multiple stakeholders connected to a wicked problem, to improve stakeholder motivation/engagement and derive more meaningful 
outcomes? Can technology socialize learning and improve shared understanding through critical making and externalizing the thinking process across many people? It may also help those that may be tentative to speak up in face-to-face situations find a voice, but it should not speak for them. As shown in Kolko's (2010b) examples with the Designers at frog, externalizing sensemaking affords greater participation, inclusion, and social learning. Furthermore, the insights found in Quick and Feldman's (2011) research echo Landis' argument that social learning can stimulate engagement. Our design synthesis process, therefore, may also benefit from being externalized and shared across time and space through digital technology, 'seeing' spaces, and participatory methods in PAR.

\subsection{Technology in the PAR Process}

Today, technology allows content to be amassed and shared by many people (Blair-Early, 2010; Van Dijck, 2009). Digital tools have the potential to be used in PAR to gather, organize and store data, instigate collective prototyping and networking, and make complex information accessible, relevant, and personal. The role of technology in Participatory Design sessions around wicked problems is not to determine the "right" solution for people, but to stimulate 'critical making' and shared understanding among people. Technology is already being used to synthesize messy data and present large amounts of information, but there seems to be an opportunity for technology to also help us map the narrative (provide context for what the data represents), and become a tool for sensemaking.

\subsection{Digital Seeing Tools for the 'Seeing' Space}

The notion of a 'seeing' space was mentioned earlier in a general sense. This section explores the idea of a 'seeing' space, but more specifically through the tools and technology that could be offered in the space. Bret Victor and Fujitsu Laboratories (2015) are a few people working on future workspaces, where collaboration meets technology. In Bret Victor's vision, big data would 
be collected and transmitted to the 'seeing' space. The space, in turn, would display the information on different surfaces (allowing the individual or group to see inside the data). The data can be displayed across time to simulate different occurrences and comparative effects that result from certain situations or decisions. The simulations that have been conducted can be stored in the room/system to be searched for/engaged with at a later time (Victor, 2014). The user should not have to think about bringing information in or understanding how the space works. The system/room presents data in a way that allows people to play with the information freely. Bret Victor envisions that the room and materials (touch-tables, sensors, and interactive walls) would allow users to do test runs, collect data, and compare results to determine the best possibilities, and more importantly understand why their decisions are the best possibility. This type of 'seeing' stretches across possibilities. While sensors and touch walls are expensive, Victor's idea behind the 'seeing' space is akin to a 'maker' space, in which a shared space (available to many people) offers access to expensive tools and evokes the motivation to create something new with others.

The 'seeing' tools and methods implemented in seeing spaces and CoDesign spaces should be designed with intention towards a desired outcome. Bret Victor categorizes tools into three categories: Construction, Conceptual, and Seeing. Construction tools promote tinkering and allow users to "try stuff out," but not understand underlying principles of why something works. Conceptual tools promote tinkering and engineering. They help users understand underlying principles and why certain models work (i.e., Design principles guided by theoretical knowledge). However, if no precedent research or textbook exists to guide theory or knowledge, then our understanding is limited. In order to span all ways of thinking (tinkering, engineering, and scientific thinking) and to move away from recipes, people need seeing tools to prod them towards new territory, creating new kinds of things, and understanding problems deeply enough to build more reliable and robust resolutions (Victor, 2014). 
Fujitsu Laboratories Ltd. (2015) has developed user interface technology that converts a room into a digital space. Their aim is to bring people out of their individual devices to work together on large shared spaces like tables and walls. Their small devices can be linked to the larger screens to enable more fluid sharing between devices. The large format affords information to be displayed and more easily organized in collaborative brainstorming sessions. The issues facing this new technology surround the limited ability for large tablets and whiteboards to link fluidly, making it more difficult to connect multiple display units together to span the entire space of the room during co-creation workshops where multiple users would need/want to take advantage of exchanging information and ideas on a common space. Fujitsu's main focus is on figuring out how to dynamically sync individual devices with large shared devices and validate the effectiveness of this technology in collaborative brainstorming workshops. This collaborative technology space is rich for further exploration in connection with PAR and collective sensemaking.

By designing an interactive digital platform that incorporates a blend of social/physical interactions and collective making, Designers may be able to curate some of the complexity within wicked problems and provoke active inclusion among collaborators. Technology that affords users the flexibility to manipulate different outcomes and to build new, deeper understanding together across time and space could help to create a sense of community and keep sensemaking in the hands of humans (not computers). The digital tool in and of itself could become a virtual seeing-space through which people could interpret new meaning based on collective knowledge by seeing inside, across time, and across possibilities.

\subsection{Using Technology to Externalize Design Synthesis}

The ability to rewind and reframe actions and decisions made in the past, and continue to iterate around new actions and possible resolutions is an added affordance of technology over paper 
tools. Keeping a digital trail of decisions could help teams keep track of what happens in that 'seeing' space over time as new perspectives and information are introduced. The actions taken by the participants: visualizing, framing, and storytelling would be documented in a public space that could be accessed and played back at any time. In addition, these features would further help the Designer and participants in explaining their decision-making process to other stakeholders. A digital tool for sensemaking could help to externalize the synthesis process and, in effect, give credence to future ideas and thought process by tracing the path between identified unmet needs and implementable resolutions. Technology could afford participatory sessions to cross over the limitations of time and space, while immersing stakeholders into collaborative experiences with complex data, and helping to demonstrate the value of synthesis in the Design process.

\subsection{Machine Versus Man}

Referring back to IFTF's list of future drivers, it appears that the relationship among people and their tools will continue to transform with the integration of smart machines and technology. Therefore, we will need to establish the value of humans (Designers and non-designers) to determine what we are uniquely good at, and rethink our work processes. These include skill-sets like sensemaking and social intelligence. Automated machines will replace us in mundane tasks, but in other domains machines and humans will need to work co-dependently. In these roles, machines will be implemented to augment our skills and abilities to understand the context of a problem and adapt our thinking based on new information and empathy (Davies, Fidler, \& Gorbis, 2011, p.3).

In the age of technology, data information, and the Age of Design (Conklin, 2009), there is a strong case for the relevance and necessity of human abilities in the realm of sensemaking. Sensemaking and many of the skill-sets that are projected to be valuable in the future hint at the rise of humans over machines in understanding and making sense of the world around us. 
Although data-mining and analytical tools can help us to identify connections, they are not as effective in contextualizing those findings as humans. Humans, in comparison, can assemble data into narratives that provide context and garner attention. Additionally, unlike machines, humans can also integrate values, morals, ethics and other preferences in decision-making (VanPatter, 2015; Davies, Fidler, \& Gorbis, 2011).

The C.A.S.T. Case Study presented in the following chapters will further assert the value of the 'human contribution' in sensemaking. Though an argument is made for technology and the integration of digital tools in the collective sensemaking process of PAR, the focus is to determine ways in which technology could support human ability and connectivity. Technology can be used as an instrument to promote shared understanding and sensemaking early in the process. As a flexible platform it could be used to trace decisions across time and from multiple locations in order to equip humans with new ways of seeing and organizing data. Technology in the Design process would be intended to enhance collective exploration around multiple possibilities, across multiple disciplines (frames) to help people arrive at deeper understanding and meaningful ideas.

\subsection{Benefits of a Hybrid Toolset}

While technology may help to broaden understanding and increase social engagement in the classroom and in collaborative spaces, it should not be the only way to engage. Landis says we have to balance cyberspace (instant access) with real space (face-to-face) experiences. Technology should not replace the teacher in the school, or replace the Designers and participatory stakeholders in PAR, but instead should augment the value and role of the Designer and the capacity for stakeholders to share and connect; it should enhance their interactions and learning outcomes as CoDesigners in the process of discovering and reframing understanding. Designers have the opportunity to catalyze change and provoke new ways of seeing information connected to a wicked problem through the design of 'seeing' tools/spaces and visualization, and 
a framework of Design Thinking methods (that involve telling, making, and enactment) to facilitate hands-on/face-to-face experiences among Designers and diverse stakeholders.

The skill-set of 'Social Intelligence' draws on our ability to 'feel' (Davies, Fidler, \& Gorbis, 2011). Feeling is as complicated as sensemaking because it is not something that can be computed. Technology cannot sensemake and cannot feel. Empathy and shared understanding allow us to adapt to incoming needs and information and shift our focus (Davies, Fidler, \& Gorbis, 2011). This is why face-to-face human interaction is still vital in collaborative work. While technology may extend our abilities to make sense, it cannot alone replace the human competencies that are necessary to more deeply understand and tackle wicked human problems. There is an opportunity to explore the potential of the integration of the human and the machine in PAR through a hybrid Design Thinking toolset. A hybrid toolset in the PAR process may, in fact, help to externalize the Design Thinking process and engage collaborative teams in new ways of thinking (creatively and critically), while also enhancing our abilities to understand and communicate new information in a shared, collaborative workspace around a wicked problem. 


\section{Chapter 3: Group Dynamics and Design Thinking Approaches}

Blair-Early (2010) asserts that Design can shape the way people behave, how they gather information and acquire knowledge, how they communicate across barriers, and how they interact with one another. As Designers set out to develop tools and methods to bring these properties to life in collaborative settings, it is important for them to understand the nuances of group dynamics and the capacities of different methods and tools for Design Thinking. This section explores the affordances of various methods and tools in Design Thinking aimed at facilitating collaborative learning, creative thinking, and sensemaking. This chapter begins with an investigation into the social aspects of group brainstorming and collaboration through the analysis of prior design research studies and game theory. Next, various non-digital (face-to-face and paper) participatory methods, as well as digital methods and tools are outlined. By examining new tools, current Design Thinking methods, and group dynamics we may gain a better understanding of which approaches may help to insight engagement and deeper understanding, and potentially improve future collaboration efforts around wicked problems. The final portion of this chapter explores the capabilities of technology tools and the fusion of the machine and the human through blended interaction - the combination of physical interaction, social interaction, and technology. The answers to which tools and methods could be best for PAR and wicked problem framing lie in studying the impacts of social group norms, collaboration versus competition, storytelling, and technology on iterative learning, shared understanding, and critical making. 


\subsection{Learning from Ants. Change from the Ground Up.}

Collective intelligence is observed in ant colony systems. Ants within a colony, working at local levels (with limited understanding) impact global behavior by collectively engaging in "improvisational problem-solving" through feedback channels. Improvisational problem solving occurs as ants sense and react to the changing behavior of the ant beside them (Johnson, 2002). A local colony of ants may not be able to make global changes to the system, but by actively seeking patterns and making changes to their micro area they impact the behavior of the greater system through local behavior change. Similar reasoning can be applied to the PAR approach for bringing many people that have different perspectives of the local problem (microscopic view) in on the ground floor to have an influence on the global scope. Additionally, Johnson (2002) explains, the margin of error becomes much smaller when the decision-making process is spread over many individuals and random interactions. Ten ants roaming the terrain for food with predefined orders will not be able to make as intelligent of an assessments of the global state as two thousand ants exploring the space looking for unanticipated/unpredicted encounters. These unanticipated encounters often introduce the ant to new food sources or help them adapt to new environmental conditions (Johnson, 2002). This argument helps to reinforce the need for creating spaces and a PAR processes for wicked problems that support 'unanticipated' encounters with people and information, co-learning and making, and the spread of new understanding.

"Generations of ants come and go, and yet the colony itself matures, grows more stable, more organized," (Johnson, 2002). This phenomenon stems from the fact that as ants learn from one another the global structure strengthens and evolves - new ants learn from global behavior which outlasts finite individual behavior. The same occurrence is noted in how human communities evolve and develop over time. Public sidewalks become the "conduit" that facilitate local improvisational encounters and the flow of information between different people that impacts community behavior (Johnson, 2002). Johnson (2002) suggests that these diverse encounters 
have the potential to expand our perspectives and empathy. It would seem then that meaningful interactions for PAR should be designed into the spaces, approaches, and tools used to facilitate divergent encounters, the flow of information, and to provoke new ways of seeing.

\subsection{Studies on Group Dynamics in Collaboration}

In 1957 Osborn stated that groups, over individuals working alone, could produce "twice as many ideas" using the principles of "brainstorming"' (Osborn, 1957, p.229 as cited in Lamm \& Trommsdorff, 1973). Osborn's principles of brainstorming included: ruling out criticism, welcoming quantity in ideas and the freedom to think openly and freely, and seeking combinations and improvements (Lamm \& Trommsdorff, 1973, p.368). Today, many companies and Design teams use brainstorming principles to arrive at innovative ideas, but is this truly the most effective approach? Lamm and Trommsdorff reviewed past investigations that explored various aspects of group and individual brainstorming activities, and what they discovered is that, more often than not, individual performance, over group performance, is typically superior in quantity and quality of ideas that emerge from brainstorming. The researchers concluded that the facets contributing to the inferiority of groups in brainstorming sessions mostly stem from production blocking, social inhibition, and cognitive uniformity. If wicked problems require the expertise and inclusion of many, then some of the findings from Lamm and Trommsdorff seem troubling. However, by sifting through the details of various brainstorming experiments, we may better define the affordances of certain approaches that could improve the outcomes of collective innovation settings, as well as collaborative engagement, thinking and creativity.

\subsubsection{Production Blocking, Social Inhibition, and Cognitive Uniformity}

Studies suggesting that "individuals working alone produce a greater quantity of ideas than the same number collaborating in a small face-to-face group" (Lamm \& Trommsdorff, 1973, p.380) led the researchers to surmise that this may be due in fact to production blocking. Blocking is a 
rule that follows typical social norms during a brainstorming session; out of courtesy a person is given an opportunity to speak without interruption. As a result, only one person can speak at a time, causing many to refrain from sharing (Lamm \& Trommsdorff, 1973). Secondly, social inhibition, or the fear of negative evaluation, can contribute to the inferiority of groups. Often, the less inhibited (but not necessarily the more capable) group members will lead the discussion, preventing more inhibited (and maybe more capable) participants from speaking up. This pattern is noted in the non-expert vs. expert study done by Collaros and Anderson. When group members felt 'cohesive' and 'equal' to one another, they had "greater ideational proficiencies" (Lamm \& Trommsdorff, 1973, p.378). The third observed pattern, cognitive uniformity (i.e., onetrack thinking) relates to the quantity and quality of group brainstorming ideas. Cognitive uniformity is very similar to the notion of 'groupthink', termed by William H. Whyte, Jr. in 1952. In efforts to maintain harmony and reduce conflict, people in a group may chose to conform to an idea that may or may not be a rational decision. On the other hand, groupthink allowed for cognitive interstimulation (an idea offered by one member resulting in another member suggesting an idea), which "led to a greater variety of ideas," (but, usually within the same category) (Lamm \& Trommsdorff, 1973, p. 382). These three findings suggest that the methods and tools used in collaborative settings should promote more open and organic discussion, democratic sharing practices, and opportunities to see and build onto more than one idea or thought (through iteration), in order to reduce production blocking, social inhibition and cognitive uniformity in collaborative settings.

\subsubsection{Oral Communication, Democratic Interactions, Analogies and Open Groups}

Four other insights emerged during Lamm and Trommsdorf's review of past brainstorming studies. These included the value of: oral communication, democratic interactions, personalanalogies, and open groups. Horowitz and Newman (1964, as cited in Lamm \& Trommsdorff, 1973) "found that subjects produced more responses (ideas) under oral (non-writing) conditions." 
Oral communication tended to prevent awkward and embarrassing periods of silence, and reduced the amount of "screening" that usually "takes place under writing conditions due to a presumably greater sense of commitment by subjects to written than to oral responses" (Lamm \& Trommsdorff, 1973, p.374). Oral brainstorming sessions, as a result, appear to have a more fluid and organic feel than sessions where writing is paramount (Lamm \& Trommsdorff, 1973). Collaros and Anderson (1969, as cited in Lamm \& Trommsdorff, 1973) discovered the impact of democratic interactions in brainstorming. What they found was that the quantity and quality of ideas was lower in groups where people perceive others as being 'experts' in brainstorming. Participants were more willing to share ideas when no one knew the expertise of their group members (Lamm \& Trommsdorff, 1973 p.378). Thirdly, the 'personal-analogy' technique (proposed by Gordon (1961, as cited in Lamm \& Trommsdorff, 1973) was tested by Bouchard (1972, as cited in Lamm \& Trommsdorff, 1973) and compared to ordinary brainstorming. It required that all participants play a "central part of a problem while the group worked on it" (Lamm \& Trommsdorff, 1973, p.375). As a result, the personal-analogy group conditions produced significantly more ideas than those in the normal brainstorming condition, because it allowed the participants to not only personally identify/connect with the topic of discussion, but also to one another. The use of 'personal-analogy,' can be also seen in persona development and scenario creation, which be discussed in later sections. Lastly, Ziller, Behringer, and Goodchilds (1962 as cited in Lamm \& Trommsdorff, 1973) conducted an experiment to test 'open' versus 'closed' groups. Their findings showed that the quantity and quality of ideas in the 'open' groups (those that allowed members to come and go) was superior to the 'closed' groups (Lamm \& Trommsdorff, 1973, p. 379). Allowing a collective group to remain flexible and open may be invaluable to the learning, sharing, and iterative stages of collaboration around wicked problems.

The nuances of group dynamics observed in past brainstorming sessions leads us to question the value of solely writing on sticky notes or writing at all during brainstorming sessions, and 
provokes us to explore further methods for group collaboration and idea generation. Tools that allow people to converse and interact face-to-face appear to offer a more natural engagement and may also prevent social inhibition tendencies. Collaborative tools and methods should also help to establish more 'open' and balanced groups (experts and novices) that afford more democratic engagements, wherein each stakeholder feels that they can interact equally and contribute something unique to the ideation process. Tools and methods that allow the individual team member to connect, not only with the information, but also with other stakeholders and new perspectives may lead to more promising long-term outcomes and interrelationships.

The effects of social norms and tools on collaborative interaction and group dynamics can also be studied in collaborative gameplay and the way collaborative games are designed. Similar to collaborative games, wicked problems often rely on multiple stakeholders working together towards a common goal, rather than working in silos.

\subsection{Collaborative, Cooperative, and Competitive Gameplay}

Jose Zagal, Jochen Rick, and Idris Hsi (2006) assert that collaborative mechanisms have become a prominent part of many computer games today. However, researchers have noticed that even with an increase of collaborative computer games, players still have a tendency to compete or play individually, rather than collaboratively (Zagal, Rick, \& Hsi, 2006, p.24). In Collaborative games: Lessons learned from board games Zagal, Rick, and Hsi (2006) investigate how to create worthwhile, interesting, and attractive collaborative games by studying the intricacies of collaborative gameplay found in board games and role-playing games (RPGs). By delving deeper into game theory and how cooperative and collaborative games are developed, we may discover insights into how we shape PAR sessions to elevate collective sensemaking, creativity, and shared understanding around wicked problems. 
Using game theory, Zagal, Rick, and Hsi (2006) classified games into three categories: competitive, cooperative, and collaborative. In competitive games each person's goal is singular. Cooperative games "lie between competitive and collaborative games" (Zagal, Rick, \& Hsi, 2006 p.26). "Cooperative games do not always guarantee the cooperating players will benefit equally or even benefit at all. Cooperative games include enforceable rules for negotiating or bargaining that allow players to identify a desirable outcome for the parties involved." Collaboration, on the other hand, is defined by, "all participants working together as a team, sharing the payoffs and outcomes." Collaborative players share one goal and the reward/punishment of the decision they make equally (Zagal, Rick, \& Hsi, 2006, p.26). Innovation around complex problems with multiple stakeholders could be defined as cooperative or collaborative, depending on the shared or differing goals of the collective whole. Many of the stakeholders may have different goals and thus may not benefit in the same way from a decision that is made, but if they work in a more collaborative way they may come out better in the end than if they had competed against one another. Coordination between stakeholders to reach a common goal may be advantageous to PAR. When goals are more aligned (shared), there tends to be more engagement and collective motivation. The challenge for collaborative groups (in a game or around complex problems) is in designing interactive approaches that maximize a team's effectiveness.

\subsection{Lessons Learned from Designing Collaborative Games}

From their analysis, Zagal, Rick, and Hsi (2006) derive four over arching lessons Designers should consider when developing future collaborative games (p. 30-32). Some of these principles can be translated into useful Design principles for shaping future PAR approaches and tools.

Collaborative games that call attention to social dilemmas and problems can help nudge players towards more unified action. Collaborative games should encourage co-constructive understanding and sensemaking by allowing people to make and evaluate individual decisions. Thirdly, collaborative games should ensure that the decisions and trade-offs made throughout the 
game are traceable so players can reflect on cause and effect relationships. Increased strategic thinking occurs when players interact with the information frequently. Lastly, when players are given unique responsibilities (that help them recognize the strengths and weaknesses of each player) in collaborative games, selfish decision-making tends to decrease and the group experience is more enjoyable and engaging. Similarly, the tools and methods designed for PAR sessions and collaborative innovation should engage each person and allow them to contribute to active discussion in multiple ways, should provide ways to trace decisions made throughout the process, should encourage co-constructive understanding through making and evaluation, and should be used to tackle meaningful problems.

\subsection{Avoiding Pitfalls}

Two pitfalls that many collaborative groups stumble upon when trying to make sense of a complex problem are: misguided (zealous) action and paralyses (Wright, 2005). Misguided action, caused by ignoring the overall complexity and certain 'cues' that do not conform to existing models, may be avoided by using immersive methods and tools to gain greater awareness. Paralyses (due to uncertainty and confusion) may be avoided by using interpretation tools (like mapping) that allow individuals and groups to interact with new affordances and insights, to draw out meaning and understanding (Glaveanu, 2012).

In addition to describing principles for Design, Zagal, Rick, and Hsi (2006) identify three potential pitfalls to avoid when designing collaborative games. These pitfalls, like those associated with brainstorming and tackling wicked problems, can be taken into consideration when shaping and designing approaches for PAR. In collaborative games, as in problems associated with groupthink and blocking, it is important to ensure that one player does not make the decisions for the team and that sufficient rationale for full collaboration is evident. To avoid this pitfall in Game Design players can be given different roles and abilities based on their strengths and how they 
can best contribute. In turn, this can provoke players to work together by "sharing knowledge and resources [and] exploring the information space as completely as possible to identify the best strategy to use" (Zagal, Rick, \& Hsi, 2006, p.33).

Furthermore, in collaborative games players need to care about the outcomes to stay engaged. Therefore, outcomes that lack a satisfying result need to be avoided. Lastly, a game designed for collaboration needs to offer new interactive experiences each time the challenge is presented, while also providing enough structure so players can have reliable information to formulate strategies, in order to maintain engagement (Zagal, Rick, \& Hsi, 2006, p.34). In extending these findings to the domain of wicked problems, the people integral to the problem should have inclusive roles and feel that they are contributing to meaningful change. Additionally, the tools and methods used in the PAR approach should promote a sense of interactivity, continuous (iterative) learning (that challenges their current assumptions and pushes the limits of their understanding), and ownership of the problem.

\subsection{The Value of Immersion, Interactivity and Inclusivity}

A blend of immersive and interactive approaches can spark innovation and creative thinking (Glaveanu, 2012). The understanding acquired through immersion into a project and the understanding gained by interacting with external influences (experimenting with new ideas, trying new approaches, and exploring different perspectives) can lead to open-mindedness and the ability to not lose sight of the significance of new ideas (Glaveanu, 2012). In What Can be Done with an Egg? Creativity, Material Objects and the Theory of Affordances, Glaveanu (2012) demonstrates that in order to create something new and expand our understanding (find connections to meaning), we have to balance interaction (remaining open-minded to new influences) and immersion (deep reflection and exploration) in our creative processes. 
The purpose of immersion and interactivity is to "integrate and expand" our insights, and to "act upon what is 'possible' in the world in ways that expand our capabilities for action and thought" (Glaveanu, 2012). Immersion into a topic through a single lens can result in small creative steps, however larger leaps and innovative thinking can occur when people pull themselves out of their comfortable immersed state and also interact with new information and approaches. A willingness to transgress previous assumptions can lead to new innovations and new ways of tackling a problem that may have otherwise been overlooked by only remaining immersed in a singular perspective or in a familiar way of thinking (Glaveanu, 2012).

In the same way, the 'seeing' spaces and the approaches (methods and tools) we design for PAR should afford a blend of deep immersion and new interaction encounters (among multiple stakeholders and ways of thinking/seeing) in order to gain new understanding, discover new insights, and build new opportunities. It is important to point out, however, that the value of this process (blending interaction and immersion) has less to do with the final outcome. Its real value has more to do with the process of discovery and new understanding that is derived through critical making and reflection. Whether 'making' is associated with physically crafting a prototype or 'making' sense, that same notion can be applied to how new knowledge and transformative thinking can occur in tackling wicked problems. Through this balanced approach, a collective group may become more open to change and may be more equipped and inclined to question their current assumptions and identify more meaningful resolutions.

Interactions in the PAR creative process also apply to interactions among diverse groups of stakeholders. Inclusivity strives for broad-based engagement and the sharing of 'experiential knowledge.' The quality of engagement within an inclusive group is elevated by the addition of newcomers with new expertise and perspectives. Instead of maintaining a fix or closed set of participants with limited skills and knowledge-sets, creating a flexible team entity allows 
communication and knowledge to flow organically and evolve over time (Quick \& Feldman, 2011, p.274). Removing barriers of understanding and encouraging expansive thought-processes from stakeholders with varying backgrounds helps to promote shared understanding, 'authentic inputs', and ongoing empathy for different stakeholders and diverse perspectives. The involvement of a broad array of experts contributes to collective intelligence, and a constant state of emergence and knowledge growth during the collaborative process (Quick \& Feldman, 2011).

\subsection{Empathy and Its Link to Creativity}

The value of immersion, interaction, and inclusion, in connection to generating deeper understanding and open-mindedness in the creative process, is further supported in Carlozzi, Bull, Eells, \& Hurlburt's research on empathy and creativity. Carlozzi et al. (2005) identified that empathy and open-mindedness are positively related to creativity and concluded that strategies and methods designed to nurture creativity and open-mindedness, could increase the potential of people becoming more empathic. People that are more open-minded are less likely to "distort incoming messages, [and as a result] their perceptions of affective messages communicated by others may be more accurate" (Carlozzi et al., 2005). The value of a Designer's empathy in the creative process towards the users they are designing for is widely recognized (Kouprie \& Visser, 2009). Empathy transcends knowledge and helps us understand why certain experiences are meaningful to people (Kouprie \& Visser, 2009, p.438). Methods used by Designers to connect on an emotional level and reflect on different perspectives include the use of personas and scenarios, which can incorporate a blend of both raw data and supportive patterns (Kouprie \& Visser, 2009). The integration of storytelling can also help people better appreciate someone else's experiences or perspectives (Kouprie \& Visser 2009).

Through the eyes of the Designer, Kouprie and Visser (2009) believe there are four phases of empathy: discovery (entering another person's world), immersion (taking the person's point of 
view), connection (resonating with the person to find meaning), and detachment (stepping back into your role as a Designer to make sense of the other person's world). "Having an emotional response to another's emotional state and being able to reflect on that by perspective taking seems to be the core mechanism of empathy" (Kouprie \& Visser, 2009). During reflection in the detachment stage, once immersion has taken place and new points of view have been assimilated, the Designer can then draw on these insights to create new ideas (Kouprie \& Visser 2009). But, in wicked problems, the role of creator and Designer extends to the stakeholders (CoDesigners) involved. In connection with PAR and wicked problems, we should also consider, then, the value of the stakeholders' empathy as active participants in sensemaking and idea creation. The role of the Designer in PAR, therefore, may extend beyond their own acquisition of empathy, but in also helping others to immerse themselves in new/different perspectives in order to become more open-minded. Creative thinking and shared understanding may benefit more from methods that help to provoke new ways of thinking and expanding the mind not only of the Designer, but also the stakeholders, allowing them to experience the phases of discovery, immersion, connection, and detachment. Empathy, as well as the feeling of being heard and needed, could play a large role in helping stakeholders to seek out new understanding and innovative possibilities, while also motivating them to seek common ground and work together to reach a common goal.

\subsection{Finding a Common Language Through Enacting, Telling, and Making}

From their study of one of the most successful collaborative board games, LORD OF THE RINGS, Zagal, Rick, and Hsi (2006) ascertain that players have a greater capacity to win if they collaborate and actively communicate for the benefit of the group. Designers and non-design stakeholders may not share a common language, so we have to find other means for them to communicate their thoughts, feelings, needs, and ideas, while also keeping them engaged in the process (Sanders, Brandt, \& Binder, 2010). Because Participatory Design approaches usually 
involve a wide range of people, the tools and methods used in the approach are aimed at bridging the communication gap between people who have separate knowledge expertise and perspectives, helping them better articulate their feelings and thoughts. The methods and tools for Participatory Design approaches draw upon three overall categories: making, telling, and enacting (Sanders et al., 2010). The methods and tools within this framework are used in crossdisciplinary sessions to stimulate empathy, open communication, and creative thinking on a personal and a collaborative level with other Designers and non-designers.

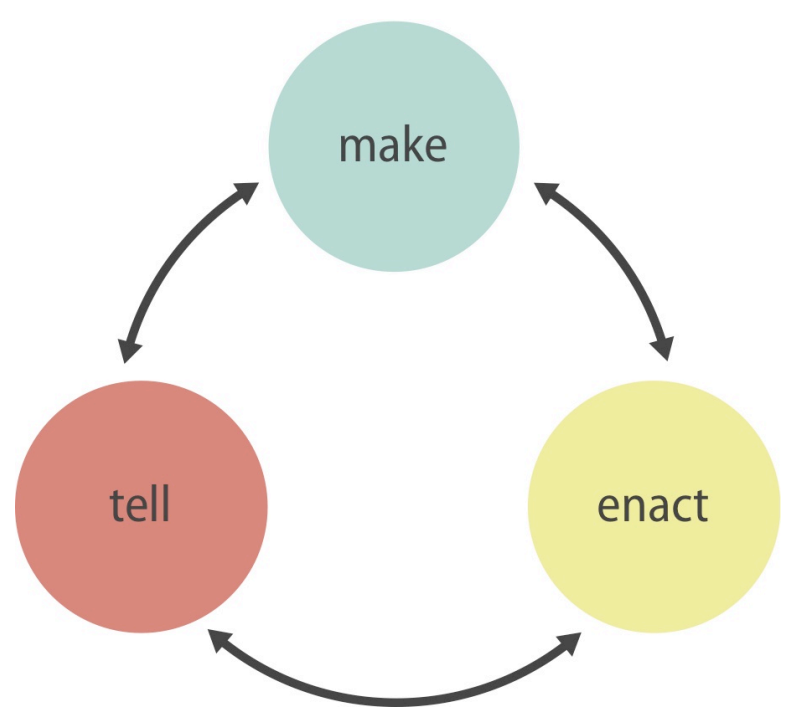

Figure 3.1 Making, telling, and enacting as complementary, connected activities in co-designing (Brandt, Binder, \& Sanders, 2012).

"The making of things, the telling of stories and the enactment of possible futures together provide the basis for forming a temporary community in which the new can be envisioned" (Brandt, Binder, \& Sanders, 2012, p.145). The methods from each category can be used in tandem with one another in the CoDesign process. The choice and order of the methods and tools used are designed intentionally with the participants and the end goal in mind (Brandt, Binder, \& Sanders, 
2012). The following sections will describe, through examples, how enacting, telling and making approaches have been integrated into precedent CoDesign processes.

\subsection{Enacting}

Methods of enactment that have been introduced in Design Thinking sessions include improv and roleplay, which are typically conducted in face-to-face interactions by individuals and/or groups (Sanders, Brandt, \& Binder, 2010). An example of an improv approach used in PD is the Forum Theater technique (Brandt, Binder, \& Sanders, 2012) in which actors perform skits that are directed and altered by an active audience until the audience is satisfied with the outcome. Roleplay and performance are methods of enactment through which people can project themselves and their thoughts, while also immersing themselves into new situations. An example of roleplay can be found in the SeniorInteraction Project (Brandt, Binder, \& Sanders, 2012). Through roleplay, stakeholders acted as both spectators and directors, acting out situations that were then also recorded on the wall. Alternating between actors and audience members in a performance, the participants were pushed to not only view, but also experience and simulate various situations (Brandt, Binder, \& Sanders, 2012, p.164 \& 165).

Deborah Kapchan (1995) studied female street performers in Morocco. While not associated with Participatory Design, these interactive performances demonstrated that empowerment and openmindedness can be derived through enactment when challenging social norms, attitudes, perspectives, and the behaviors of others. By engaging, especially male audience members in the shared act of performing (interaction) and pushing new boundaries, the female performers felt more liberated, while male audience members became more open-minded; the males' involvement in the performances required openness and humility (Kapchan, 1995, p.493, 499).

Lastly, role-playing Games (RPGs) have been shown to exhibit a connection between the value 
of roleplay and a commitment to participation. In RPGs, each player creates a carefully crafted narrative about the persona they represent in the game. Teams then work towards a shared goal using the players they have created. As a result of creating personal narratives and having a shared goal, the players' motivations and commitment to the game increases a great deal more than in a game like Chess, in which a player has little connection to the narrative or the board pieces. RPGs have been shown to create more lasting experiences than these types of games because they build understanding, engagement, and shared commitment among various stakeholders (Zagal, Rick, \& Hsi, 2006, p.34). RPGs' success in collaborative gameplay adds credence not only to enactment, but also to the approach of creating scenarios and personal narratives as methods for immersion and interaction.

\subsection{Telling}

"Narratives...provide a common language through which the ambiguities and equivocalities of everyday life are shared and collectively made sense of" (Wright, 2005, p.92).

Sensemaking and scenario building are natural tendencies humans have when responding to changing situations or reflecting on past experiences to understand outcomes and speculate about the future (Wright, 2005, p.96). "Narratives invite encounters." They help people better understand themselves and invite others into a more meaningful conversation (Wright, 2005, p.92 \& 96). Scenarios are a form of narrative that can be developed by individuals, groups, in face-toface interactions, and through technology and shared across many platforms, through writing, drawing, blogs, wikis, photos, video (Sanders, Brandt, Binder, 2010). Because we all use scenarios to explain past, present and future occurrences, narratives can provide a common language between diverse stakeholders who are seeking to "learn, develop, unlearn, relearn and apply common understandings." In regards to group settings, Wright (2005) describes four notions that Weick (1995-1996) believes must be present to "advance sensemaking capacities": 
improvisation, wisdom, respectful interaction, and communication (Wright, 2005, p.91). Improvisation entails harnessing group/individual intuition and iterative exploration to bring order and creativity to chaos. Wisdom comes from realizing that "absolute knowledge is unattainable" and feeling comfortable in ambiguity. Respectful interaction references the idea of shared understanding and empathy to avoid fragmentation within the collective group. Storytelling allows stakeholders and Designers to communicate in a common language and collectively share constructed elements of reality. Narratives touch on each of these four abilities because they present events in both linear and spatial dimensions, allowing groups and individuals to dig deeper into the nuances and complexities of events and engender a sense of order and meaning.

In an exchange of uncertain and ambiguous information through narratives we can "combine, create, renew and transfer tacit, implicit, explicit and codified processes of knowing from blueprints, ideas, emotional states and fuzzy hunches into problem definitions," with added value (O’Donnell et al., 2000, p.187-200, cited within Wright, 2005). David Snowden will often use narrative and "what if" scenarios to provoke new ways of looking at a problem from a speculative vantage point. Working backwards from a fictional end-state to a factual present-state helps people identify new opportunities and make sense of complexity (Snowden, n.d.). The implementation of scenarios as prospective sensemaking devices is another narrative approach that can enhance inductive thinking abilities when attempting to strategize and understand complex problems.

\subsubsection{Prospective Scenarios}

Prospective scenarios, or future narratives, provide the opportunity for many plausible alternatives within which ideas and assumptions can be 'windtunnelled,' vetted and challenged in a 'risk-free' hypothetical environment. By simulating multiple scenarios, or "alternative constructions of events," (Wright, 2005, p.89) groups can better plan for uncertainties and act on new 
opportunities. However, when sensemaking is retrospective and used to define causal relationships alone, it limits the scope of the problem and narrows the possibility of envisioning new realities. Prospective sensemaking blends the use of imagined futures with retrospective thinking to help individuals and groups challenge their own understanding of the problem, while also envisioning and experimenting with alternative paths and new possibilities. This method, in turn, encourages users to define actionable goals.

Dindler, Brodersen and colleagues suggest that co-created fictional narratives "invoke a sense of suspended disbelief to fuel imagination in participatory prototyping" (Brodersen et al., 2008; Dindler 2010 - cited within Brandt, Binder \& Sanders, 2012). The relationship between fictional narratives and the act of making pulls participants out of their normal ways of thinking to prod them in seeing the future and possible solutions in a new way. The aim then, for Designers and participatory stakeholders dealing with complex issues, may be to utilize prospective sensemaking as a "challenge artifact" to confront existing assumptions and ideas, embrace ambiguity, and avoid using over-simplistic analysis and outdated strategies.

\subsubsection{Stories Are Usually Told from a Purposeful Perspective}

Barbara Tversky (2004) asserts, "the mind constructs narratives from what would otherwise be chaos." Narratives are linear. They help to bring coherence to chaos by organizing events that have taken place in time. However, we live in environments that are non-linear and pervasive (Tversky 2004). "When people tell stories they do so from a particular perspective, for a certain

purpose." "Narrative construction begins with making sense of the world, organizing the space in which we exist, comprehending the events that unfold around us... How they [narratives] are constructed reveals and affects how people think about scenes in space and events in time" (Tversky, 2004 p.389). As noted in her study, participants switched between survey and route perspectives in order to draw out spatial relationships and meaning in the environment. 
Narratives are predominantly told from a subjective point of view for a specific purpose, often to inform or entertain. When used to inform, stories become normalizations - tellers often do not exaggerate but tend to minimize or omit certain details (Tversky, 2004, p.386). From her research (Tversky \& Taylor 1992a; 1996) it would appear that they start by identifying what they know (what is most familiar and concrete and omit what they do not recall) and then using a route (or 'eye-level') perspective to walk through relationships among 'landmarks' (what they know) to extract meaning and communicate key information about the rest of the environment.

\subsubsection{The Influence of Narratives - An Amsterdam Case Study}

Dorst (2015) shares a case study that was conducted in Amsterdam involving the rejuvenation of a neighborhood shared by two types of immigrants from Turkey and Morocco, along with the local Dutch population, who were struggling to live peacefully among one another. Narratives were used as a way of helping the ethnic groups (with differing perspectives and backgrounds) find common ground, shared understanding, and connection. Through a network of shared stories expressed by many, what the neighbors realized was that there were more common values among them than anyone had imagined. This realization created avenues for further contact and understanding among the neighbors, while also helping to defuse tensions and improve the capacity for the neighborhood to deal with any problems that may arise in the future. Several aspects from this case study contributed to the success of rejuvenating the neighborhood. First, the issues were approached on a human level. The researchers looked at situations that directly impacted everyday life. Secondly, the approach was proactive; it did not center on blame. Thirdly by concentrating on human values and commonalities, the community became more connected and stronger around the notion of helping the common good. The method of storytelling and finding common ground created the scaffold for dealing with future problems. What Kees Dorst illustrates in this case study is that open, complex, dynamic, and network problem situations do not have quick direct fixes. Instead, it is crucial to engage integral stakeholders in inclusive roles 
to understand the underlying structures of the specific situation and create new frames (beyond our initial assumptions) so new resolutions and connections can emerge (Dorst, 2015, p.19-21). Experience has shown Dorst "that once core questions can be articulated, possible solutions generally arise very quickly - and these are sure to be nonstandard outcomes, far beyond the reach of where conventional problem-solving would have led " (Dorst, 2012, p.38).

\subsubsection{Scenarios Told for Sensemaking}

Pierre Wack (1985) originally introduced the notion of scenarios (as a strategy for business) to challenge the assumptions and influence the decision-making abilities of Royal Dutch/Shell managers. However, Wright (2005), along with Regner (2003), Woods and Joyce (2002), and Millett (2003) challenge the use of this strategy only with senior managers at the 'organization's center.' In comparing strategizing at the "corporate center" verses strategizing on the periphery with individual stakeholders, Regner (2003) noticed that inductive reasoning (over deductive reasoning) and scenario development was being implemented at the periphery, and as a result the stakeholders' insights were more innovative and transformative. Inductive reasoning created more open dialogue (conversations and negotiations) between stakeholders. Transforming the raw experiences of the stakeholders into narrations helped the managers at the periphery make sense of unfamiliarity. Wright (2005) argues that because scenarios are the result of social constructs, developed not in isolation, but from the influence of experiences and competing/shared understandings among stakeholders, that prospective sensemaking may be more effective at the periphery, with many different stakeholders, than only at the 'center.'

\subsubsection{Design Games to Facilitate Storytelling in Participatory Design}

Sanders, Brandt, and Binder (2010) chart 'games' as a method/tool that can be conducted in Participatory Design with individuals or in groups, and in face-to-face interactions. Brandt, Binder and Sanders (2012) reference a game reported by Johansson (2005) that was designed to 
facilitate storytelling. Based on ethnographic video snippets, participants told imaginary stories that complemented their reflective understanding of current everyday activities. In this way, games and storytelling were combined to illustrate both past and present experiences, while also to express future aspirations. The use of futuring techniques like 'what if' prospective scenarios and games can help stakeholders pinpoint flaws or barriers in the current system, while also giving them freedom to transform their future circumstances. The 'utopian' visions of their future and the requirements for successful transformation from present barriers to future opportunities become "the base for a plan for action" (Brandt, Binder \& Sanders, 2012, p152). The critical component of using futuring techniques, as with other approaches for Design Thinking, is in choosing/designing tools and methods that allow participants to clearly point out their critiques and create their utopian futures in a common language. The incorporation of storytelling and games integrated into methods like futuring and prototyping in a Participatory Design process can support translation and help participants share unique experiences and ideas in a relatable way.

\subsection{Making}

Tim Brown explains that "Design Thinking is learning by making. Instead of thinking of what to build, [we build] in order to think" (Brown, 2009). Brandt, Binder and Sanders (2012) emphasize the importance of making, especially in innovative projects involving multiple stakeholders wanting to generate new concepts for the future, as a mode through which to inspire new ways of understanding, exploring relationships, and building a joint "vision for the future" (Brandt, Binder, \& Sanders, 2012, p.170). The notion of learning through making, as well as telling and enacting is represented in User-Centered Design and in Participatory Design settings. The act of making can be viewed as building a tangible prototype, a visualization, or constructing understanding ('making' sense) to explain or test a concept or idea. All forms of making in turn help us to communicate and understand thoughts, feelings, motivations, needs, and dreams. 


\subsubsection{Participatory Prototyping, Probes, and Generative Tools}

Making methods are often used in the exploratory phases of the Design process as "vehicles for collectively exploring, expressing and testing hypotheses about future ways of living" (Brandt, Binder \& Sanders, 2012, p.155). There are three approaches to making that have evolved in CoDesign: participatory prototyping, probes, and generative tools (Sanders \& Stappers, 2012). Participatory prototyping involves the creation of mock-ups and low fidelity models that represent a future object or scenario and communicates what the future could be through its use (Brandt, Binder \& Sanders, 2012, p.155). Designers use probes to gain inspiration from users and stakeholders by inviting them to reflect on and express their experiences, feelings and attitudes about the probe (Brandt, Binder \& Sanders, 2012, p.155). Generative tools are also used in the front end of the Design process, often in transdisciplinary and Participatory Design projects, to help non-designers express, create, and imagine new futures and ideas (Brandt, Binder \& Sanders, 2012, p.155 \&159).

\subsubsection{Generative Toolkits}

An important part of generative making tools are the toolkits (Sanders \& Stappers, 2012). The toolkits, carefully crafted prior to a session to support the objectives of the research and the abilities of the participants, are a blend of concrete and ambiguous 2D and 3D visual components (from photographs to blocks). They are introduced to participants to "evoke and provoke thoughts and feelings" they may not typically discuss or consider and "usually result in the making of an artifact" (Sanders \& Stappers, 2012). The goal in using generative tools is to help participants craft an infinite set of imaginative possibilities from a finite set of components. Figure 3.2 illustrates various 'ingredients' that can be used in generative 'Make' toolkits to trigger ideas and associations. The descriptions of each ingredient also briefly suggests how they can be use to support certain tasks during the making approach. 


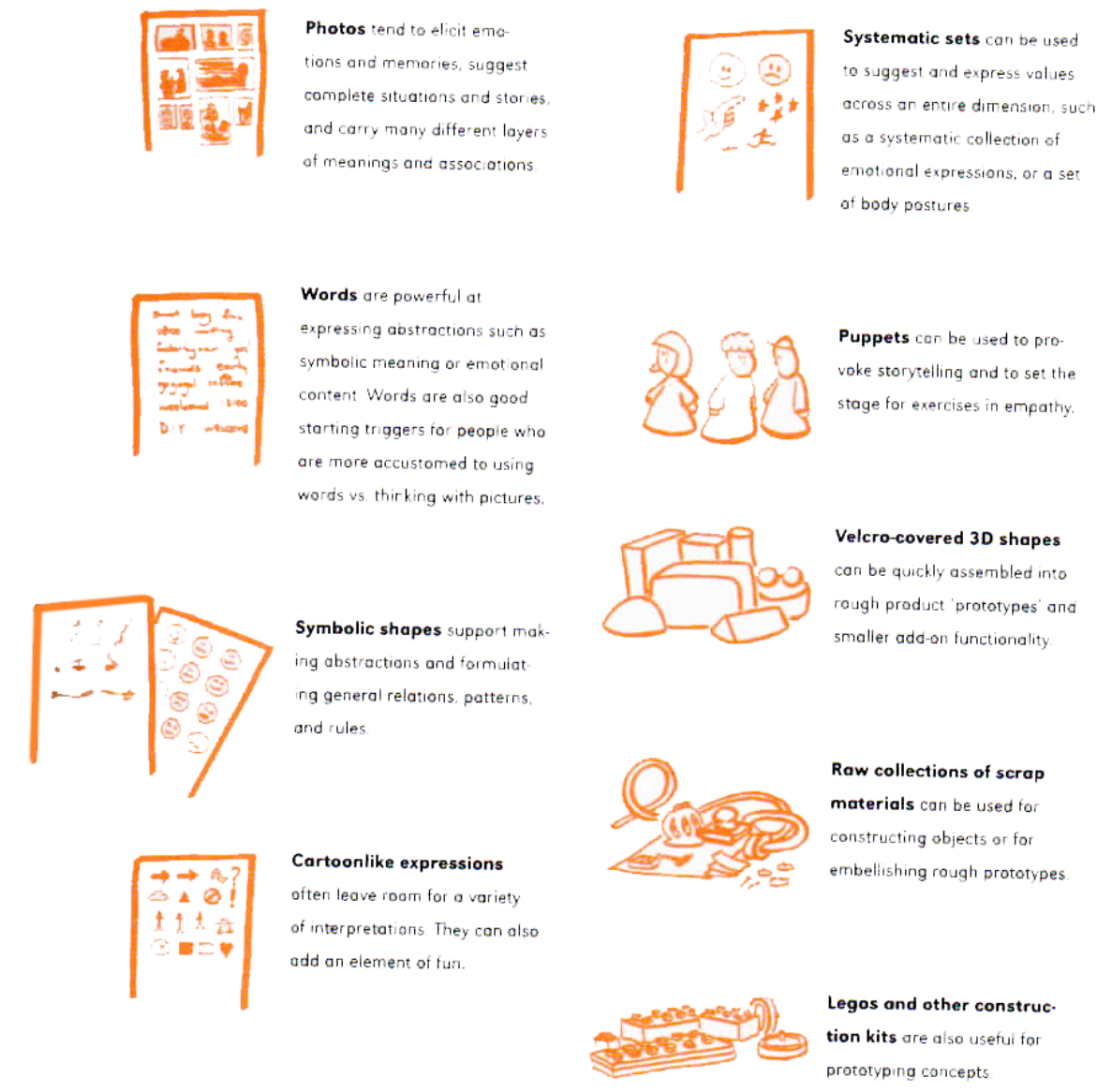

Figure 3.2 Various generative (Make) toolkit ingredients and how they can be used in making (from Sanders \& Stappers, 2012).

\subsubsection{Visualizations and Mapping}

Designers and non-designers use visualizations as a communication vehicle through which to establish a common language or shared understanding around a complex topic (Bendoly, 2016; Horn \& Weber, 2007; Carey, 2015). "Visualization...provides the means by which to explore and discover relationships otherwise hidden from default assumptions" (Bendoly, 2016). The use of data visualizations today is broad, "ranging from finance to sports to politics" (Liu et al., 2014). Active prosumers use visualizations to gain understanding and new knowledge before acting on a decision like voting or buying products (Liu et al., 2014). Additionally, the demands on data 
accumulation and visualizations are becoming more and more interdisciplinary, and therefore rely on activities that support collective sensemaking to breakdown disciplinary biases and ensure more effective decisions are being made (Bendoly, 2016). Data visualizations are often represented in a system of interactive (non-static) idioms (or graphic depictions) designed to convey a story (Bendoly, 2016). However, in the case of a multi-faceted wicked problem the conveyance of a single story may not provide stakeholders with enough information. Often what appears to be left out of data visualizations is the context behind the visual artifact. Bendoly (2016) asserts that data visualizations should be a continuous process of understanding, instead of being viewed simply as an outcome.

Visual materials are often used in the Participatory Design process to "assist the participants in telling about experiences and dreams...building up their own everyday setting or illustrating the flow of activities in a simplified way" (Brandt, Binder, \& Sanders, 2012, p.149). Individuals and groups can use two-dimensional paper components to create visual maps in individual or face-toface interactions (Sanders, Brandt, \& Binder, 2010).

\subsubsection{Mindmapping}

Mindmaps are diagrams usually created around a single central concept. They are used to visually organize and draw associations between information and ideas. Mindmaps may start with givens or previous assumptions, but because of their non-linear nature they elicit divergent thinking tendencies (Kelley \& Kelley, 2013, p.215). The Kelley brothers believe mindmaps promote abductive over linear deductive reasoning. The notion that "we don't know where the idea will take us" embraces the value of indeterminacy and opportunities to see something completely new, which are offered through tools like mindmaps that have no predetermined conclusion (Kelley \& Kelley, 2013, p.215). Given their nature, wicked problems that have many interlocking pieces no single right solution may benefit most from methods and tools that promote 
abductive reasoning, emergent thinking, and iterative Design processes. However, mindmapping alone, because it promotes growth/divergent thinking, may create more ambiguity and added frustration among stakeholders when tackling a problem that has multiple facets and no right solution. Mindmapping without sensemaking and convergence may lead team members further way from shared understanding and identifying root problems. Wicked problems may benefit from the use of visualization tools that support both a convergence and prioritization of collective concerns and stimulate divergent thinking around new possibilities.

\subsubsection{Resolution Mapping and Mess Map Diagrams}

Group decision-making and change implementation around a complex problem should involve various decision-makers (stakeholders) to communicate their perspectives and gain a sense of shared understanding and shared responsibility. However, just bringing them together is not enough. Robert P. Weber explains that, "if you want to change how people think, you first have to show them how they think." Robert P. Weber and Robert E. Horn have developed two approaches, Resolution Scenario Mapping and Mess Map Diagrams, for resolving wicked problems that address issues related to uncertainty and ambiguity, risk and complexity, competing viewpoints, system interactions, and implementation strategies (Horn \& Weber, 2007).

Weber's method, Resolution Scenario Mapping, is similar to prospective sensemaking. It is a 'scenario planning' approach that allows stakeholders to share and evaluate alternative endstates (extreme but plausible futures) of a wicked problem by sequencing/analyzing contingent Events (or milestones) and possible outcomes. "Participants choose their most desirable and attainable outcomes and those milestones or Events [a hypothetical occurrence at a specific point in the future] that lead logically to the desired outcome" (Horn \& Weber, 2007, p.1). Unlike typical 'bottoms up' or 'blank slate' brainstorming exercises (like mindmapping), Resolution Mapping 
provides more structure, through 'simulated hindsight' (working backwards from a goal), while also allowing for creative, organic thought processes (Horn \& Weber, 2007).

Mess Mapping evokes group interaction and visual analytics to help stakeholders understand, organize, share, and evaluate flows and connections among complex sets of data. Mess Maps are visual murals of complex problems that illustrate the interconnections between important chunks of information, in order to help stakeholders acknowledge multiple viewpoints, develop collaborative reasoning, and interact with information at different levels. They allow for both convergent and divergent thinking and afford transparent communication among stakeholders to help them "arrive at a common framework for understanding" (Horn \& Weber, 2007, p.8). The maps (for example, Figure 3.3) are a platform on which data flows can be structured, diverse assumptions can surface, and casual connections can be made (Horn \& Weber, 2007, p.16).

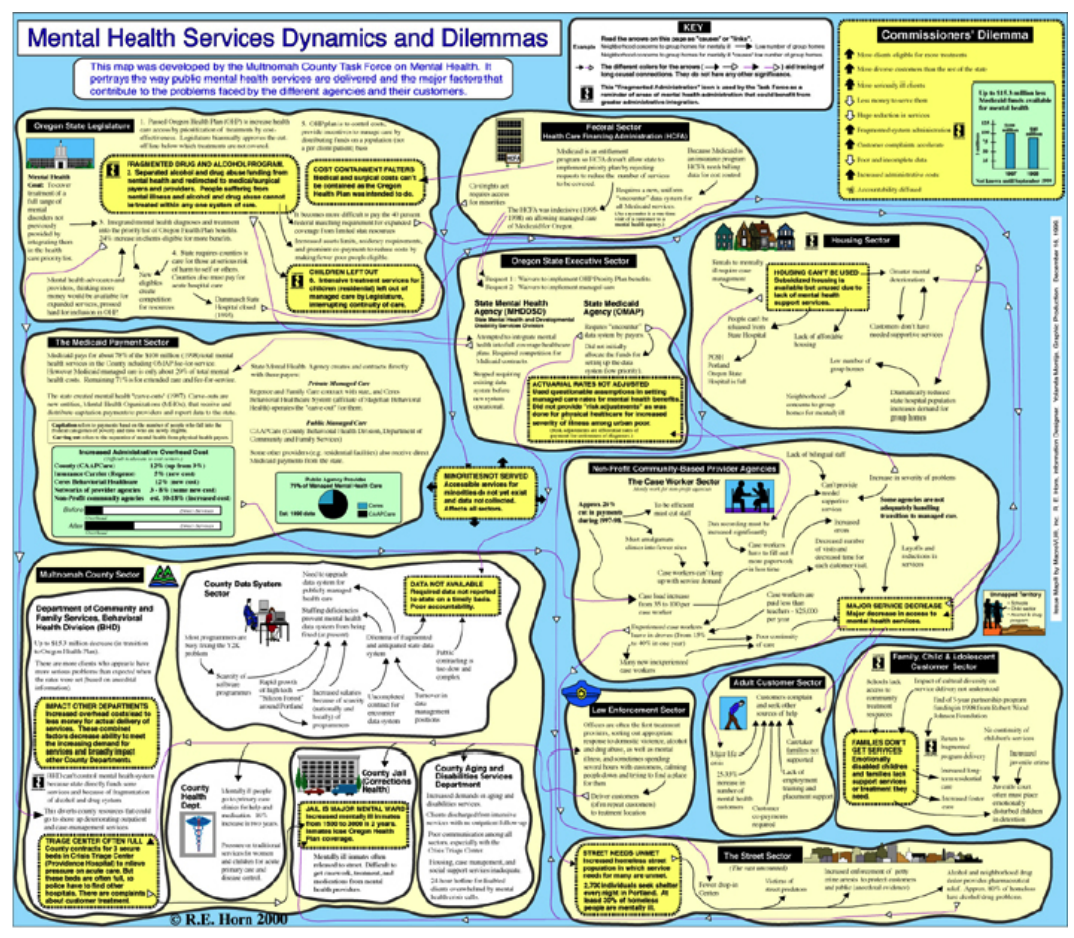

Figure 3.3 An example of a Horn Mess Map (Horn \& Weber, 2007). 
The large 'blobs' in the Mess Map (Figure 3.3) represent each stakeholder (sector) group. Descriptions of the problem as seen from a specific point of view are encapsulated in boxes within the 'blobs'. Cause and effect relationships are represented by the little chunks of text and linked by causal arrows.

Looking specifically at the methods used by Horn and Weber, we recognize that collaborative tools for wicked problems need to be useful and usable by all team members. However, the problem with Horn's visual representations are that while they make sense to those who have helped to construct them along the way in real-time, they are too visually complex for 'newcomers'. Horn claims that the Mess Maps are not designed for "outsiders to find it attractive or informative." Perhaps Horn does not know how to represent the information in an 'attractive or informative' way, or he has not considered the value of extending the sensemaking process in a wicked problem to newcomers and outsiders. This appears to be a missed opportunity. Outsiders should not be left out of the conversation, especially when it comes to wicked problems. Quick and Feldman (2011) spoke to the value and importance of inclusivity (bringing in outsiders and broadening expertise) for more effective collaboration. A tool, whether in visual or narrative form, needs to promote inclusivity and support the emergence of new knowledge and perspectives gained from the inclusion of new team members.

\subsection{Extending Collective Sensemaking Across Time and Space}

The relationship between the design/research team and the participants is an important variable in Participatory Design and Participatory Action Research. A design/research team "may only be able to plan for a one-time event, [but] an ideal situation is a continual relationship that is marked by iterative meetings" (Sanders, Brandt, \& Binder, 2010). It is important that a variety of perspectives be addressed as new people/experts enter the process and ideas continue to mature as design resolutions are moved into new stages. While Weber and Horn's approaches 
allow participants/stakeholders to map and simulate various outcomes, their methods are conducted on rigid platforms (like notecards or single murals) that make it challenging to mold and reshape new resolutions as the group of stakeholders moves through iterative stages. Technology can afford malleable and flexible interactions, and the ability to extend participation across time and space. This flexibility allows for more authentic inputs, ongoing empathy for new perspectives, and continued knowledge growth. Technology offers individuals and groups the ability to connect, share, organize, and construct information/meaning across time and space that paper tools (like sticky notes and Mess Maps) may not be able to accomplish.

A digital tool used in a Participatory Design process could, for instance, offer save features, allowing visual maps to be constructed, deconstructed, and reconstructed as ideas shift and newcomers join. Newcomers could also use the save function as a playback feature to reference the process of creation. The playback feature could further allow those that constructed the map to return to certain aspects and try different simulations. Technology could be used to create flexible narrative maps that could be shared and manipulated by team members in real-time or after collaborative design sessions. Collaborative tools for wicked problems should afford iterative thinking and the inclusion of diverse stakeholders to build and interact with information, assumptions, and insights around complex issues.

\subsection{Digital Integration in Collaborative Settings}

Jeff Conklin (2009) and his colleagues found through their research that social networks, and the use of dialogue mapping and scenario creation (instead of facts alone) help to unite fragmented stakeholders and promote a higher level of shared understanding and commitment in collaborative settings. The use of these methods through collaborative technologies (like projectors) and on multi-touch displays further proved to be effective platforms on which stakeholders could capture input, view various perspectives, determine "what might be," and 
make sense of complex information together to "exercise collective intelligence" (Conklin, 2009, p.18) and uncover a "more coherent sense of meaning" (Conklin, 2009, p.17). In addition to integrating collaborative real-time (non-digital) methods in the Participatory Design process, Conklin calls on Designers to develop socially engaging network tools (beyond blogs and forums which can polarize groups and over-simplify problems) and methods (like scenario development and dialogue mapping) on collaborative technology devices that can be utilized to ignite "rich, multi-stakeholder dialogues... and informed reasoning on a large scale" (Conklin, 2009, p.19).

\subsection{Interactive Information Visualizations}

Information Visualization (or InfoVis) is the "study of transforming data, information, and knowledge into interactive visual representations" (Liu, Cui, Wu, \& Liu, 2014). InfoVis are often used to help people make sense of complex data. Through user interface controls, people can interact with the data to explore and understand multiple perspectives (Liu et al., 2014). Many new visualization frameworks and toolkits have been introduced to help people build interactive information visualizations (Liu et al., 2014). One online interactive visualization resource in particular, Data-Driven Documents $\left(\mathrm{D}^{3}\right)$, inspired the early development of the digital tool for the C.A.S.T. Case Study (Bostock, Ogievetsky, \& Heer, 2011). D ${ }^{3}$ is a web-based JavaScript library that allows people to animate their data using HTML, SVG, and CSS. Below are a series of interactive visualization examples from the $D^{3}$ website. 


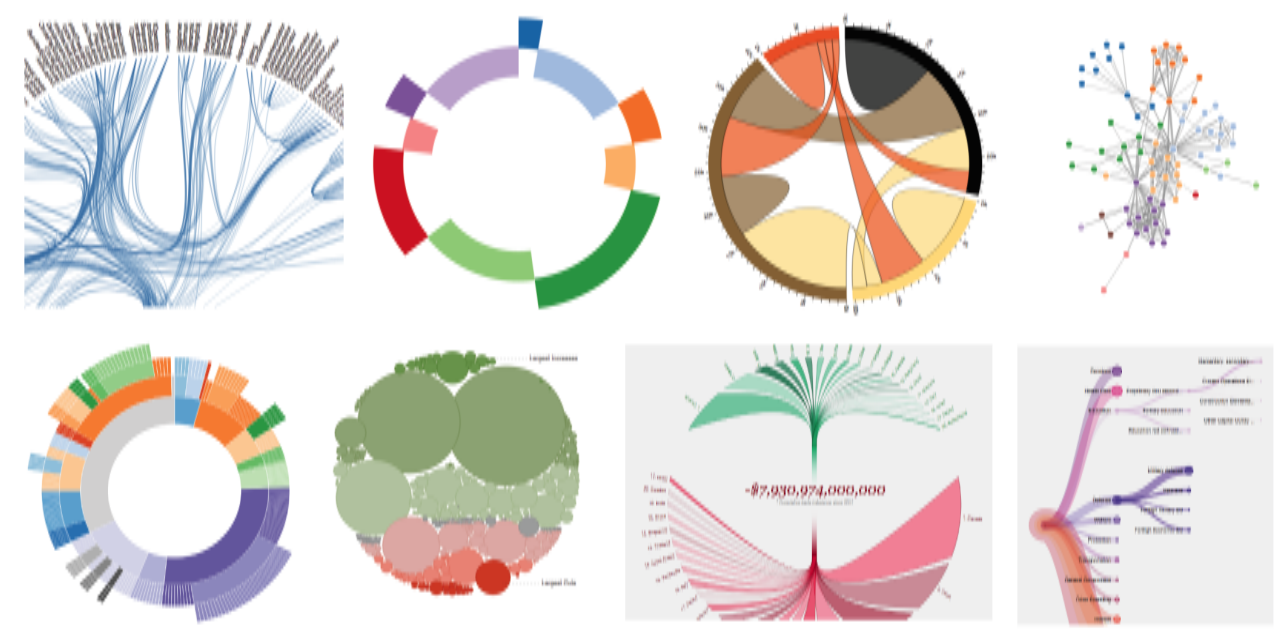

Figure 3.4 Interactive visualizations created by $D^{3}$.

Due to their digital nature, interactive visualizations allow users to play with an infinite number of possibilities. InfoVis tools allow large amounts of data to be transferred, analyzed, converted into categories/clusters and/or filtered (to remove the 'noise') so that specific information can be extracted and mapped (Liu et al., 2014). This affords users the ability to play with various scenarios and filters in order to see new patterns. But, how do we know what we should be looking for? Returning to an earlier quote by Daniel Kohn, the painter, in reference to visualizing data, "It's about frameworks of recognition; how you choose to look, rather than what you're trying to see" (Carey, 2015). Through interactive snapshots with InfoVis tools we are given a summary of the story, but often the end of the story does not provide us with the context or narrative behind the numbers. How do we know the intent of the visual representation, what meaning it is supposed to be conveying to us, and why it is important for us to consider if we do not understand the context of how that slice relates to the whole? Taking the notions of blended interaction and collaborative game-play, as well as the assertion that data visualizations should be a continuous process of understanding, instead of being viewed simply as an outcome, and recognizing that the future of sensemaking is not computers, but humans, there may be an opportunity to alter or extend the capabilities of information visualizations. What added value could information 
visualizations bring if the data points were malleable so that people in a sensemaking session could put the data into their own clusters and draw their own meaning through pattern recognition and connections? What if interactive visualizations were used to facilitate human-to-human interaction in PAR, and could become another vehicle through which CoDesigners could find common ground?

Often real-world data sets can be imprecise and may contain errors (Liu et al., 2014). Given that data visualizations can be subjective or "miss the mark considerably," Bendoly (2016) suggests that there is value in "embracing the prospect of variation" and allowing individuals and interdisciplinary teams to construct and leverage visual representations through the process of sensemaking (Bendoly, 2016). Instead of visualizations only being used as artifacts (or pictorial summations of calculated data points) at the back-end of the process, what happens when we bring interactive visualizations into the front-end of the Design process and use them as a means through which to promote critical making, generate understanding and communion among varying stakeholders, and help collective teams make sense of a wicked problem? Through interaction and immersion with an interactive visualization tool, perhaps the stakeholders also become more empowered and equipped to act on the problem and communicate their understanding to others because they are a part of constructing the narrative, prioritizing what was most important, drawing connections and finding patterns, and getting at the root of the problem from various perspectives, not just passively viewing a visual outcome from one interpretation of data. The next question, then, is how do we best convey and conduct visual interactions and immersive experiences in a collaborative setting?

\subsection{Blended Interaction}

Research in embodied interaction and cognition shows that "interaction with our physical and social environment...[defines] the ways in which we think and reason about the [real] world...[and 
also how we] conceptualize, understand, use, and adopt digital technology and its virtual functionality" (Jetter, et al., 2012, p.2). In their research, Jetter et al. (2012) found that digital tools like Smartboards, Microsoft's Kinect and tangible user interfacing blended the use of natural motor skills and face-to-face communication with "flexible working styles and increased group awareness." Additionally, information visualized through digital interaction helped people recognize patterns and more fluidly interact with large amounts of data (Jetter et al., 2012, p.2).

Jetter et al. (2012) introduce the theory of 'conceptual blending;' the combination of realism and naturalness into digital interaction. They argue for the use of Blended Interaction in the design of interactive collaborative spaces. Blended Interaction combines individual human interaction, social interaction and communication, established workflows, and the physical environment with digital technology in a collaborative setting. Jetter et al. (2012) discovered that the "blended interaction' of digital media interfacing, embodied interaction (physical skills/movements with the human body like using your finger to drag or enlarge objects on a screen), and human cognition in interactive spaces support more holistic understanding, individual interaction, social interaction and communication, and greater workflow in co-located environments. Blended Interaction allows people to learn, communicate, and express thoughts interactively on a shared surface like a multitouch table. Interactive surfaces/tables are often promoted as tools to support efforts around “solving co-located collaborative tasks" (Seifert et al., 2012). Through the element of 'play' and the coordinated interaction between humans using a shared digital device, Blended Interaction tools can promote a shared experience of engagement, more efficient verbal communication, greater group awareness, and collective expression (Jetter et al., 2012). These aspects will be explored further in the sections below and in the case study to determine the relationship between human-to-human communication and the added capabilities of digital technology, as well as the potential to integrate hybrid approaches into future CoDesign processes around wicked problems. 
Reality-based interaction, a framework proposed by Jetter et al. (2012), strives to create computer interactions that emulate socially constructed real-world cognition and gesturing. Jetter et al. identified four aspects within reality-based interactions that could provide the foundation from which to design an ideal collaborative interactive space: promoting individual interaction, facilitating social interaction, supporting workflows, and taking the physical collaborative environment into consideration. Individual interaction requires blended actions that support embodied cognition and interaction, which can be accomplished through "tangible interaction, multi-touch or multimodal interfaces" (Jetter et al., 2012, p.4). Secondly, social interaction and communication in collaborative spaces benefit from digital tools that respect real-world social norms, protocols, and practices. Jetter el al. discovered that blended interaction (combining "abstract computational and visualization concepts" with "tangible around-the-table interaction" on tabletop touch screens) enhanced verbal communication, mutual support, and group awareness. The blended tool also created a fun and effective thinking-process that supported a variety of strategies and collaboration styles, and overall had "a strong impact on user roles, communication and working styles" (Jetter et al., 2012, p.4). Lastly, blended interaction tools (combining both traditional workflows and real-world objects with digital visualizations) were shown to enhance collaboration and established workflows (like sketching and brainstorming in the Design process), which were further supported by integrating "digital tools and ['real-world'] work surfaces" in the physical collaborative environment (Jetter et al., 2012, p.5).

Combining the findings from Jetter et al. and Lamm and Trommsdorff, blended interactions occurring through the use of a shared multi-touch surface could enhance oral communication (over written) and group awareness, which could make communication in collaborative sessions more fluid, organic, and authentic, prompting people to openly express/share ideas and thoughts. By considering the nuances of individual interaction, social interaction, natural workflows, and the physical environment into the design of digital tools for PAR, Designers may create more flexible 
and open platforms on which to garner shared understanding, motivation, and engagement in the framing and sensemaking processes.

\subsection{Personal Versus Shared Digital Devices in Collaborative Spaces}

In consideration of global sharing and some of the facets of group dynamics like production blocking and social inhibition brought up by Lamm and Trommsdorff, some researchers are also exploring the use of personal digital devices in collaborative settings. In addition to the work being done by Fujitsu Laboratories, Julian Seifert and her team of researchers have proposed a concept called MobiSurf, which "establishes a seamless integration of personal mobile devices and an additional shared interactive surface for co-located collaboration" (Seifert et al., 2012). What Seifert et al. found in their research was that given the choice, participants preferred to use both personal and shared interactive devices to gather their thoughts and share them with the others. None of the groups exclusively used one mode of communication/interaction over another, and none of the groups used the device-to-device information sharing feature. All of the information gathered individually was readily shared on the interactive display, and not pushed onto personal devices. The personal devices allowed users to collect their thoughts, while the shared surface allowed for "shared storage and interaction" and prompted open discussion (Seifert et al., 2012). Based on these findings, the fluid interaction between personal (private) devices, along with shared (group) displays demonstrated higher user engagement in the given tasks and gave the participants a greater understanding of the situation. For PAR the blended use of personal and shared devices may elevate shared understanding by allowing individuals to synchronously contribute to more active group discussions (either in the shared space or from a distant location, or at a different time) without having to interrupt another team member. Contributions made anonymously and privately (during the session or prior to a meeting) may also promote more democratic interactions and prevent social inhibition. 
As we saw earlier, collaborative games can stimulate social engagement and shared understanding through shared interactivity and immersion. Aspects of gameplay and gamification, may also help to shed light on new opportunities for interactions/interfaces when designing and developing digital tools for CoDesign to trigger collective engagement and promote new ways to explore and immerse teams in the problem space.

\subsection{Gamification}

Gamification is defined as the process of adding games or game-like elements to something (as a task) so as to encourage participation. - Merriam-Webster Dictionary

Gamification is an emerging area of interest that blends both human cognition and interactivity. Games can be either digital or non-digital. Eric Zimmerman (2013) claims in his manifesto that the $21^{\text {st }}$ century will be defined by games. Media and culture in the $21^{\text {st }}$ Ludic Century (ludic referring to spontaneous and undirected playfulness) are increasingly systemic, modular, customizable, and participatory. Games embody all of these characteristics in a very direct sense. Elements of gameplay, incorporated into digital interactive tools for PAR, might stimulate new forms of engagement among participants, while also providing them with the flexibility to play out different scenarios and create interactive visual representations. When information is put into 'play', game-like experiences replace linear thought processes and may help people find common ground. Technology tools that stimulate interactivity (allowing players to frequently interact with information and one another), and immerse people in the game/problem situation (beyond a single, co-located session) will change the way participants work together in both positive and negative ways (Zagal, Rick, \& Hsi, 2006, p.35). 


\subsection{Benefits and Drawbacks of Technology}

Blair-Early (2010) demonstrates in her research that the use of technology in collaborative sessions affords team members an ability to access, extend, and share ideas and knowledge globally, with an added sense of 'liveliness' (derived from the 'game-aspect' of working online). Technology, in her study, helped students to gain a broader understanding of system relationships. Referencing Ray Land and Sian Bayne (2008), Blair-Early contends that 'printbased' platforms, in contrast " reinforce patterns of authority and individualized authorship," while digital media generate greater "openness, multimodality and collectivity" and "work more from collaborative enquiry and production, inviting contestability." Digital collaboration also, however, requires "consensus and trust" (Land \& Bayne, 2008 cited by Blair-Early, 2010 p.213) which can be a good and bad thing. Positively, it encourages shared understanding and guides teams towards a common goal. Negatively, there can be an "increased risk of deceptive practices" through online communication/interaction (Zagal, Rick, \& Hsi, 2006, p. 35).

Due to their nature, digital tools, over print or paper tools, may allow concepts to remain malleable as collaborative discussions and ideas evolve over multiple stages. Sticky notes, while they offer more flexibility than paper murals can be harder to manipulate (e.g., rescaling or moving multiple sticky notes at a time), store, and play back over time. Save, replay, and reconstruct are added features in digital games that are lacking in non-digital tools. Digital technology can incorporate save features which allow, for instance, computer games to last a lot longer than board games and can "provide continuous in-depth individualized feedback," in order to allow players to try different outcomes or playback certain decisions that were made (Zagal, Rick, \& Hsi, 2006, p. 36). Additionally, digital tools allow for more sophisticated computations to be done quickly and accurately, communication can be more flexible, information can be displayed and organized in different (sometimes more meaningful) ways, and certain data can be analyzed and more dynamically adjusted and tailored to the needs of the user (Davies, Fidler, \& Gorbis, 2011). 
Conversely, while digital information may be easier to save and manipulate, technology can be unreliable. Sticky notes and other paper-based/physical tools can afford flexibility within a realtime session, while also remaining steadfast and familiar. Attention to the level of malleability and global sharing in the tools we design for PAR will become more important as we address more complex problems.

In regards to connectivity, technology can make communication more flexible (across time and space). Alternatively, remote technology can make communication, shared understanding and planning difficult because "many basic cues of identity, personality, and social roles [that are present in face-to-face interactions] are absent in online games" (Zagal, Rick, \& Hsi, 2006, p.35). The same can be said for collaborative teams that are attempting to connect remotely. While technology offers many benefits, it is also important to consider what may be lacking in a process that is only digital. The value of hands-on learning through physical tools and face-to-face interaction and communication, found in the sections on enactment, telling, making, and collaborative Game Design, support the argument for maintaining social interfacing and nondigital approaches that stimulate human-to-human interaction in the PAR process.

\subsection{Why Should We Use a Hybrid Toolset?}

Games and visual materials "invite discussion about problems and opportunities and provide a context that keeps the discussion grounded in everyday experiences" (Brandt, Binder, \& Sanders, 2012, p.149-150). Methods and tools falling under telling, enactment and making afford Designers a great deal of leverage to facilitate transformative thinking and shared understanding, and help to establish new, present strategies aimed at imagined, collective goals within a complex problem. However, some of the real-time approaches and paper-based methods used by Designers and Design Researchers may be limiting and may need to expand to also incorporate technology. As wicked problems continue to supersede tame problems in the 'Age of 
Design' the need for multi-disciplinary teams, opportunity-driven approaches, problem framing, shared understanding, and iterative learning may need to be addressed through a blended toolkit of digital and non-digital approaches that are both interactive and immersive.

There are many tools and methods used in Design Thinking and Participatory Design sessions. The literary review and background research conducted in these early chapters helped to inform the specific non-digital and digital methods and tools selected and developed for use during the case study. The objective of the case study and development of a digital tool prototype for collective sensemaking is to explore the idea of a hybrid approach for Participatory Action Research by incorporating some of the elements and lessons learned from these chapters, in order to develop an effective CoDesign approach for tackling the wicked problem of transitions and adult care in the autism community. 


\section{Chapter 4: Autism Synopsis}

Research on Autism Spectrum Disorder (or ASD) is extensive. For the purposes of this thesis secondary research was conducted to provide a base level, cursory understanding about the disorder before starting the C.A.S.T. project, and to learn about the challenges that come with having ASD from the perspective of people on the autism spectrum.

\subsection{Background on Autism Spectrum Disorder}

As of 2014, the Center for Disease Control and Prevention (CDC) reported that Autism Spectrum Disorder (ASD) affects 1 in 68 children. "This new estimate is roughly 30 percent higher than previous estimates reported in 2012 of 1 in 88 children" (Center for Disease Control, 2014). Research indicates that ASD is almost "five times more common among boys than girls: 1 in 42 boys versus 1 in 189 girls. Levels of intellectual ability vary greatly among children with autism, ranging from severe intellectual challenges to average or above average intellectual ability...and almost half of children identified with ASD have average or above average intellectual ability compared to a third of children a decade ago" (CDC, 2014).

The autism spectrum ranges from "primarily a cognitive disorder" (on one side) to "primarily a sensory processing disorder" (on the other). "At a midpoint along the spectrum, autistic symptoms appear to be caused by equal amounts of cognitive and sensory problems" (Grandin, 2009, p.47 \& 48). While the causes for ASD are uncertain, studies by Thomas Bouchard at the University of Minnesota concluded that roughly half of what a person with ASD becomes is "determined by 
genetics and the other half is determined by environment and upbringing" (Grandin, 2009, p.41). Figure 4.1 illustrates the range of autistic spectrum conditions.

\section{Autistic Spectrum Conditions}

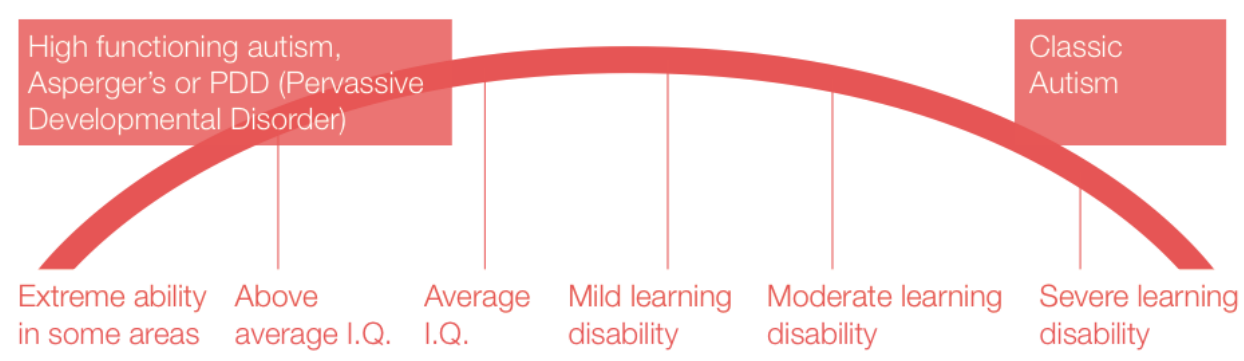

Figure 4.1 Autistic Spectrum Conditions (reinterpreted illustration from What is ASD, n.d.).

The diagnostic categories along the spectrum include "autism, pervasive developmental disorder (PDD), Asperger's syndrome, and disintegrative disorder." "There is much controversy among professionals" about these categories because some believe the categories to be truly separate, while others believe they lie on a spectrum with "no definite distinction between them" (Grandin, 2009 p.35 \& 36). Kanner's Syndrome (named after Leo Kanner) is a form of autism in which a person can learn to talk but struggles with extremely rigid thinking, a poor ability to generalize, and not knowing what to do if something interrupts their routine (Grandin, 2009, p.36). People with Asperger's syndrome are generally higher on the autism spectrum and are capable of more flexible problem solving. People with Kanner's and Asperger's can develop good speech and do well academically, while people with low-functioning ASD often have a harder time understanding spoken words and verbalizing (Grandin, 2009, p.37 \& 38, 42) A lot of these language/cognitive tendencies and impaired social skills have to do with sensory jumbling due to sensory sensitivities. People with mild sensory oversensitivity can function and learn more easily than someone with high oversensitivity who is receiving "jumbled, inaccurate information both visually 
and aurally" (Grandin, 2009, p.42). The adults with ASD who participated throughout the C.A.S.T. case study had relatively strong cognitive and verbal abilities.

\subsection{Struggling with "How" to Communicate}

Many people with Asperger's have complex inner lives (i.e., there is a lot going on in their minds). Their colorful personalities and rich ideas are often hidden behind their challenges with basic communication, which stem from knowing how to communicate with the rest of the world (Generous, 2015). Alix Generous, a brilliant young woman with Asperger's, shared at a TED conference how she has learned to communicate her "inner" dreams. Like many on the spectrum, Generous struggles with language as a form of communication. She is a visual thinker. People with autism tend to be visual (over verbal) thinkers, relying more on "sensory-based thinking" instead of "language-based" thinking (Grandin, 2009, p.30). When a photographer asked Generous to look "flirty" she had no idea what the photographer meant because she could not picture "flirty." Language is exclusive to our species, but Generous admits to feeling a bit more primitive, "like a beta version of Google translate" (Generous, 2015). Temple Grandin is a Professor of Animal Science at Colorado, well admired for her designs in livestock handling, such as a curved chute and race system, her research in areas like cattle treatment and environmental enrichment for pigs (Biography: Temple Grandin, n.d.), and her self-advocacy for people on the autism spectrum. Grandin believes that while thinking in pictures may represent "primitive" modes of perception and understanding, it is not "pathological (Grandin, 2009, p.xvii). Self-advocates like Alix Generous, Temple Grandin, and the ASD participants in the C.A.S.T. Case Study believe that the sensitivities that limit them in some areas of their lives (like socialization), can be seen as gifts or specializations in other areas if they are given a chance to prove themselves. 


\subsection{The Overlooked Potential of the ASD Population}

In her book, Grandin shares her early achievements and why she continued to strive to reach goals. Her drive to overcome small and large challenges (like finishing her undergraduate degree and getting her $\mathrm{PhD}$ ) came from the same energy and passion that sent a "man to the moon'...to prove I could do it" (Grandin, 2009, p.19). As a society we often overlook the potential of people on the autism spectrum, because they lack confidence in social situations and have limited communication abilities. Asperger's and autism, and the traits that go along with it, are often viewed as handicaps or disadvantages. What if these unique attributes were instead viewed as specializations and nurtured?

"It's clear that the genetic traits that can cause severe disabilities can also provide the giftedness and genius that has produced some of the world's greatest art and scientific discoveries" (Grandin, 2009, p.217). Brilliant and creative people like Albert Einstein, Mozart, Andy Warhol, and Lewis Carroll are all believed to have had some form of autism or Asperger's. They all had ideas and abilities, and had they not had the opportunity to share their thoughts and skills (outside of their rich and colorful minds) the landscape of our society would be different. Today, many people on the spectrum are being overlooked or taken advantage of because their ideas and thoughts cannot often be shared due to communication roadblocks and a lack of social skills (Generous, 2015).

For Generous, Asperger's allows her to think innovatively but it does not allow her to speak fluidly or clearly without a lot of practice and support. Our society often favors social ability over intellect. Many companies will not hire a person who lacks basic social skills. Knowing this, Generous decided to apply to Waffle House (believing their expectations and requirements for social skills to be lower than other establishments) - they did not hire her. To give you a brief peak into Generous' background, at the age of 19 she won a research competition for her studies on coral 
reefs, which she then presented at the UN Convention of Biological Diversity. Currently, at the age of 22 , she is preparing to graduate from college and has co-founded a biotech company called Autism Sees. Generous should not have to settle for working at Waffle House because she lacks social skills. She has and can do many great things when given the chance and the right tools to overcome social/communication barriers. An app called Podium, developed by Generous' company, helped her prepare to speak at TED. To get to where she is today, Generous has had to find opportunities, support, and tools along the way to help her achieve her goals. For Generous, an alternative and more promising future would offer autism assistive technologies (like Podium) that could play an integral role in providing people on the spectrum with the tools and resources to succeed (Generous, 2015).

In the following chapters you will hear from two young adults (one female and one male) on the spectrum who actively participated in the case study and voiced similar communication barriers and social observations. Their concerns and ideas for the future mirror Generous' and Grandin's. The female participant is an outspoken self-advocate and college student studying fire and rescue. Her biggest concerns are that people like her, with valuable assets and specializations that could contribute to military logistics and search and rescue missions, are being overlooked because of their perceived disabilities. The male participant shares similar frustrations. He is also an active (and very eloquent) self-advocate and writer, who loves music, yet works as a janitor.

\subsection{Change is Hard - Preparatory Tools for People with ASD}

"People with autism have tremendous difficulty with change" (Grandin, 2009, p.18). To better deal with change, Grandin needed to rehearse or act out the situation first. "Both mental practice and real practice can improve motor skills" Research conducted with athletes and with musicians have shown on brain scans that real practice and imagining playing have the same effect on motor maps (i.e., both contribute to expanding motor skills) (Grandin, 2009, p.23). Apps like 
Podium are tailored to do just that. Many people on the spectrum are capable of doing great things, but they need more time to mentally and emotionally prepare and practice.

Lucid dreaming allows Alix Generous to be at peace, free from judgment and social consequences. However, her goal, similar to Grandin and many other autism self-advocates, is to share her thoughts in the 'real world' and shift people's perspective of people on the spectrum. Generous wants to provide people on the spectrum with technology and supportive resources that allow them to practice certain skills so they can "share their talents with this world and to move this world forward. In addition, we [can] give them the courage to pursue their dreams in the real world, in real-time" (Generous, 2015).

\subsection{Visualizing Abstract Concepts Through Associations to Make Sense}

For some people on the spectrum a visual image or object can be associated with a feeling or experience. An item like "French toast" may represent happiness because of a past feeling of joy remembered while eating it. "The autistic mind works via these visual associations" (Grandin, 2009 p.21). Grandin often "converts abstract ideas into pictures" (Grandin, 2009, p.17). Abstract notions like personal relationships and right and wrong did not make sense to Grandin until she developed visual symbols (like doors and windows) which helped her better understand the concept of give and take. Like windows and glass doors, relationships are fragile and can shatter easily if not approached carefully - carefully opening doors and closing doors (Grandin, 2009, p.18 \& 20). For Grandin, the visual metaphor of being trapped behind glass doors also helped her to explain the way a person with autism feels. There's a feeling of disconnect. Windows allow you to see out but make it hard to communicate with the outside world.

Furthermore, Grandin uses concrete objects and examples like staircases to help her better understand abstract notions like the meaning of life. After watching a construction crew finish up a 
stairway structure that would ultimately become the "entrance to heaven for cattle," (Grandin, 2009, p.230) she became more aware of the preciousness of life. "The Stairway to Heaven," as she called it, gave her something "real" (beyond symbolic meaning) to help her define her life's purpose and convinced her that without the promise of an afterlife, beyond the door at the top of the staircase, people would have no motivation to lead a purposeful life (Grandin, 2009, p.230).

Designers often use visualizations and pattern associations, similar to people with autism, to make sense of complexity and ambiguity. Visuals and physical prototypes are also used by Designers to help bridge communication disconnects in collaborative sessions with nondesigners. The use of visual diagrams and representations, (discussed more in the case study chapter), were integrated into the case study for this reason. Because many people with ASD seem to associate meaning with visual or concrete things, PAR sessions (with ASD participants and neurotypicals) might benefit from more active participation by the incorporation of methods and tools that provide more visual or concrete ways to understand complex information and communicate thoughts, ideas, and feelings across all stakeholder groups.

\subsection{Empathy and People with ASD}

"Some people believe that people with autism do not have emotions. I definitely do have them..." (Grandin, 2009, p.89). People with ASD have emotions (e.g., fear is a dominant one), but their emotions may be simpler than most and they may be expressed in different ways (Grandin, 2009, p.91). Some on the spectrum find it hard to read the emotions of others but are able to empathize. Grandin believes there are different types of empathy. "I have to visually put myself in the other person's place...I have observed that normal people have bad visual empathy." While a person with autism/Asperger's may not be able to understand why someone feels a certain way, they may be able to pick up on the subtle details that many neurotypicals overlook. Grandin says that she gives great driving directions because she imagines herself driving and adds all of the details 
that a person would need in order to reach the destination that a neurotypical may leave out (Grandin, 2009, p.99). Furthermore, in her work as an animal handling process Designer, Grandin puts herself in place of the cattle or the driver and looks for opportunities to make their situation better. Grandin goes on to explain that while "normal people" may have emotional empathy, many lack sensory empathy, which most people on the autism spectrum can empathize with (i.e. they are able to draw on their experiences with sensory pain or chaos) (Grandin, 2009, p.99). Grandin can empathize with cattle on a visual and sensory level. This ability allows her to create some of the most innovative animal handling processes, environments, and machines in the world.

Simon Baron-Cohen says there are two emotional brain types: empathizers and systemizers. Empathizers relate to people through their emotions, while systemizers are more interested in things than people. People on the spectrum tend to lean more towards being systemizers (Grandin, 2009, p.100). One of the parents who participated in the C.A.S.T. case study said that her son, who is non-verbal is very intuitive and perceptive, picks up on other people's emotions and can tell when something is out of place. Due to his heightened oversensitivity he struggles with communicating, and yet he is perhaps more connected than most to the nuances around him, and to the relationship between the people and things in his environment. People on the spectrum may come across as insensitive, when in fact for many it is their oversensitivity that makes it hard to function in a social environment. Given the right tools and space to connect these heightened sensitivities and attention to detail, could people on the spectrum help to see opportunities for Design that many of us overlook?

\subsection{Focusing on the Details}

"My thinking involves putting details together to form concepts. A normal person forms a concept first and tends to ignore the details" (Grandin, 2009, p.201). Barbara Jones is an adult on the spectrum who works in a laboratory identifying cancer cells under a microscope. Her ability to 
recognize patterns (spot abnormal cells instantly) and heightened sense to detail makes her "one of the best technicians in the lab" (Grandin, 2009, p.70). "Nancy Minshew at Carnegie Mellon University in Pittsburgh has found that normal brains tend to ignore the details while people on the autism spectrum tend to focus on the details instead of the larger concepts" (Grandin, 2009, p.26). The value that a person on the spectrum can bring to certain areas of society, through their narrowed, focused attention, is also brought up by two of the most outspoken participants with ASD in the case study chapter.

\subsection{UK Case Study - Participatory Design with People on the Spectrum}

Few studies in PD have been administered with people on the spectrum because of a preconception about their cognitive and social capabilities, and the possibility that some may not be able to communicate verbally (Gaudion, Hall, Myerson, \& Pellicano, 2015). As a result, the subjective thoughts and experiences of people on the spectrum are often not heard or understood and "remain largely unexplored" (Gaudion et al., 2015). Most of the information gathered about autism is relayed in "quantitative terms: as numbers on a bar chart or percentages" or comes from secondary sources. Qualitative insights and personal reflections into how it feels to live with ASD and understanding the feelings/experiences of people with ASD in relation to their environment are not as common (Gaudion et al., 2015). Design research through PD and PAR practices can change that by bringing people on the spectrum into CoDesign roles to express/communicate their point of view and help us better understand the challenges they face.

One case study in particular, conducted in the UK entitled, $A$ designer's approach: how can autistic adults with learning disabilities be involved in the design process?, applied a wide range of sensory Design methods and tools (like sensory preference flashcards and physical 3D props) to mediate non-verbal communication between the Designer and the participants with autism. These methods/tools invited people on the spectrum to express their needs and to co-design 
ideas by engaging with objects (rather than people) and exploring/testing the boundaries of their sensory preferences (rather than completing specific tasks). The researchers, Katie Gaudion, Ashley Hall, Jeremy Myerson and Liz Pellicano, saw value in "breaking down the barriers to participation" with people with social learning disabilities and had them actively participate in codesigning solutions that could improve the "everyday experiences of people living with autism" (Gaudion et al., 2015).

Over the course of three years, Katie Gaudion and her team conducted three Design studies with multiple participants on the spectrum and their supportive staff to explore their interaction and reaction to an outdoor garden (least controllable environment), an everyday object, and redesigning their home (a more controlled environment). The first study invited sixteen autistic adults and their support staff to participate, the second study invited up to thirty-nine autistic adults with their support staff, and the third study involved group activities with sixty support staff. The aim of all three studies was to involve adults with autism (as well as their support staff/family members) in the CoDesign process to help investigate how they "experience their environments" and to "inform the design of spaces, objects and activities that were more meaningful to them" (Gaudion et al., 2015). The research started with first identifying each participant's strengths (i.e., sensory preferences, special interests, and different action capabilities) to determine how best to connect and communicate with them, and adapt the "affordances of each environment" for a more positive experience during the CoDesign sessions (Gaudion et al., 2015). The tasks were to (1) create a new garden, (2) adapt an everyday object (a bubble blowing vacuum cleaner), and (3) add artwork in their home. In each of the three Design studies a variety of Design methods and tools were used including: sensory activities, mirroring interests, making and doing activities participatory observations and shadowing, mapping interests, mapping sensory preference cards (as seen in Figure 4.2), storyboarding, and co-creation workshops. Instead of using questionnaires and interviews to engage with the autistic participants, researchers tried to create 
dialogue and reciprocal relationships with the participants by mirroring their interests and using their interests to engage with them. Many of the methods and tools that were used were hands-on and visual, as well as tailored to the participant's strengths in order to encourage active communication and expression in multiple ways (beyond verbal).

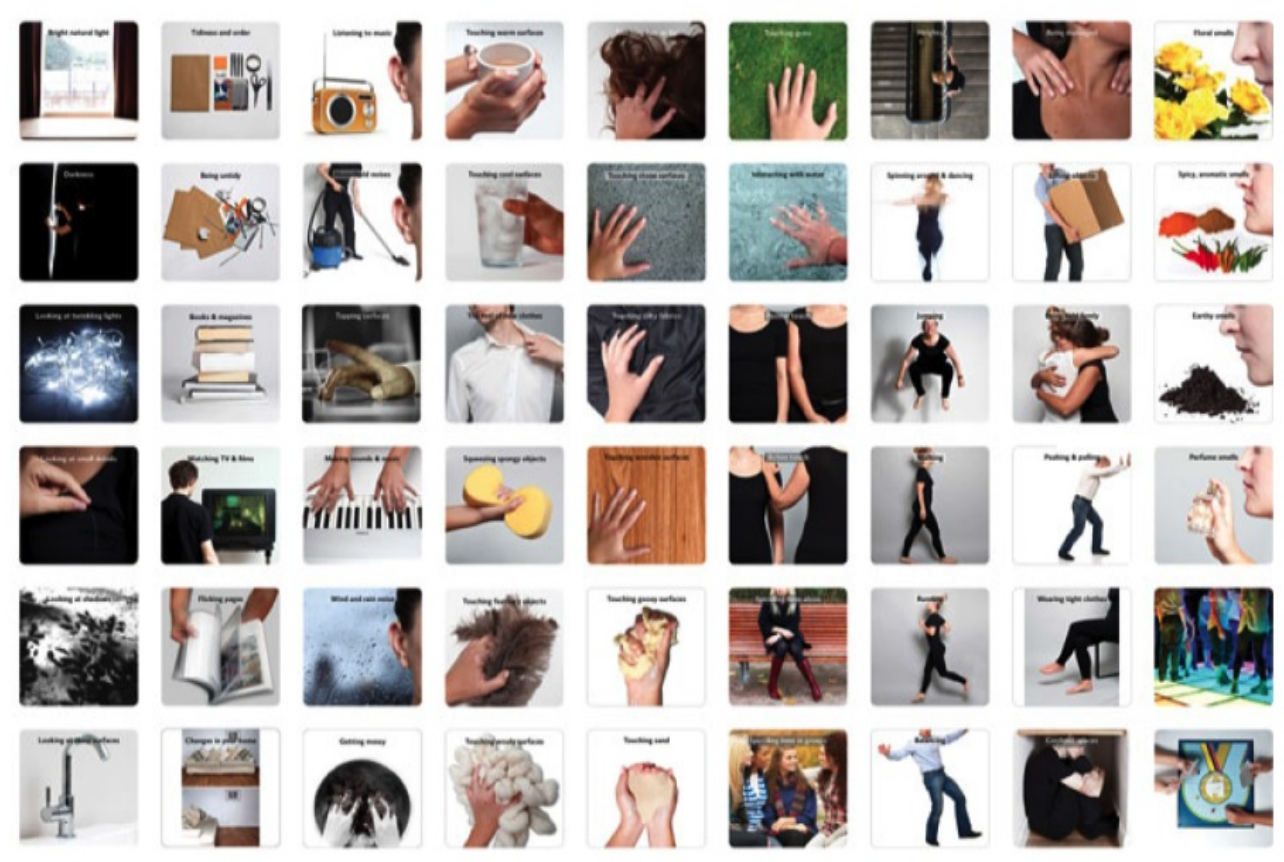

Figure 4.2 Sensory preference cards (Gaudion et al., 2015).

Through this study, the researchers demonstrated that autistic adults with limited speech and learning disabilities "can be involved in the design process." Additionally, "autism-friendly design methods" do not necessarily need to be developed, but instead priority should be "placed on the Designer's empathetic understanding," learning how to engage a participant (who may not be verbal and has different sensory needs) at their level of comfort and understanding when disseminating instructions and leading them through CoDesign sessions. Furthermore, Gaudion et al. (2015) explain that a person's "triad of strengths" can influence the relationship a person with ASD has with their environment. Determining those strengths (along with referencing the 
framework developed in Gaudion et al.'s research) can be a starting point for Designers who want to create a more positive and effective CoDesign experience for people with autism.

\subsection{The Value in Bringing People with ASD to the CoDesign Table}

Wicked problems require the input of many experts and an action-driven approach. Wicked problems connected with ASD would seem to benefit from the expertise and active participation of people with ASD. Taken what was learned in the Gaudion et al. (2015) study and about collaborative games by Zagal, Rick, and Hsi (2006), flexibility and visual/sensory communication designed into future collaborative tools and methods used in PD and PAR may improve shared understanding and feelings of inclusivity among neurotypicals and ASD participants alike through their ability to equally contribute based on their strengths.

\subsection{Care and Support in Transitions}

Most of the Design Research concerning autistic individuals has centered on "children living with autism and only a few projects focus on adults" (Gaudion et al., 2015). The ASD population continues to rise and a majority of a person's life with ASD will occur throughout adulthood, so more opportunities for Design Research need to extend to helping adults on the spectrum. The goal in the C.A.S.T. Case Study (which will be discussed in full depth in the Case Study chapter) is to explore the topic of transitions and the adult care needs for persons with ASD specifically by bringing all integral stakeholders to the table early in the Design process to have an equal chance to voice their thoughts, needs, and ideas and participate in open discussions about the C.A.S.T. clinic and transitional process for ASD adults. This project is intended to hopefully give autistic adults an opportunity to be a part of the discussion around problems that directly affect them and to contribute to co-designing new resolutions. 
Autism Speaks, a leading autism organization dedicated to funding research and increasing awareness of ASD, reports that "approximately 50,000 individuals with ASD turn 18 each year," yet there is a great void in autism research focused on issues facing adults on the autism spectrum (Autism Speaks, n.d.). The care and support for adults on the spectrum and their families is lacking, in comparison to what is offered to children with ASD and their families. Adult care for people with autism is connected to the needs and goals of parents and their children with autism during transitions into adulthood and avoiding what many parents and healthcare providers call the 'Cliff.' Adults on the autism spectrum encounter the 'Cliff' after graduating from high school because there is a lack of supportive services for adults on the spectrum (Autism Speaks, n.d.).

\subsection{The Link Between Transitional Support and Future Success}

Autism Speaks claims that many adolescents on the spectrum have little opportunity to move into higher education or careers, depending on their abilities and their accessibility to resources. Many adults with autism have to rely heavily on their parents, which can put added stress and pressure on the parents' long-term wellbeing. The parents in the C.A.S.T. study add that schools afford people on the spectrum an opportunity to connect socially and stay engaged and challenged mentally. Schools give them purpose and social interaction; with few support services and no plan after high school (whether it is seeking out a career or going into a higher learning program), their purpose, motivations, and enrichment go down. Research by Shattuck et al. (2012) points to support gaps within the transitional process, resulting in young people with ASD having a greater risk of being disengaged from postsecondary education and employment. Dr. Shattuck suggests "the right support services...can increase the chances for an independent and successful adulthood" (Autism Speaks, n.d.). Therefore, services that encourage social learning, social interaction, and learning how to live independently need to be offered to adults on the spectrum to improve their quality of life. 


\subsection{Building Inclusive Environments}

The US National Institutes of Health has identified six needs for adults with autism: vocational training, life skills, mental and physical health support, employment, public transportation, and affordable housing. Elizabeth Deckers, a planner from Kansas State University (with a brother on the autism spectrum) has developed a toolkit to improve accessibility for people with ASD in cities such as Nashville (Ferro, 2014). Her idea centers on connecting public transportation services with green areas, businesses and affordable housing - she is rethinking the network of urban environments and developing " a toolkit to help urban planners design more inclusive communities for adults with autism" (Ferro, 2014). She believes the most important feature for an inclusive city is connectivity. In order to develop an inclusive city, people have to see the city as a whole network of services. The same could be said for the healthcare system and the services offered to people on the autism spectrum and their families. We need to look at clinics, such as C.A.S.T., as part of a system, a connected network of services and care. For instance, if the services at the C.A.S.T. clinic were to be extended to encapsulate a broader patient base how would the offerings be different or re-imagined? Would creating a more inclusive clinic for not only people on the autism spectrum, but also for people with other learning disabilities (and perhaps neurotypicals) allow it to be more sustainable, while also helping to encourage tolerance and understanding in the community? These were some of the initial questions that were being discussed by the providers and healthcare administrators at The Ohio State Wexner Medical Center and C.A.S.T. before the project began.

\subsection{Transitions are a Wicked Problem}

Even this small slice of the greater autism problem, transitioning care and support for families and adults on the autism spectrum, is wicked. It is a problem intertwined in many areas (inside and outside the ASD community), it involves multiple stakeholders with differing goals, needs, and perspectives. There is incomplete and contradictory information about what the current offerings 
are or should be at C.A.S.T. and other care/service facilities, and there is no idealized solution or end-state. Transitional care and support for adults and families is part of a causal web of interconnected issues that extend beyond physical healthcare (into society) that needs to be addressed. 


\section{Chapter 5: C.A.S.T. Cast Study Introduction}

The population of ASD is growing rapidly, as are the challenges and unmet needs facing families and people on the spectrum (especially during the transitional phase from childhood to adulthood). These issues impact not only the greater network of ASD, but society as a whole, so it is important to look for ways to make people with ASD, as well as central and periphery stakeholders, a part of the resolution.

\subsection{Stepping Back from Ideation. Framing a Wicked Problem}

When first discussing wicked problems and the idea of exploring a hybrid toolset and methods for collaborative innovation as a thesis topic with the IDEA (Innovation, Design Thinking and Application) Studio at The Ohio State University Wexner Medical Center (OSUWMC), a unique opportunity was presented. The IDEA Studio was focused on redefining the healthcare user experience through innovation, Design Thinking, and application of integrated solutions to transform healthcare at OSUWMC and the Ohio community. C.A.S.T. (the Center for Autism Services and Transition) in Columbus, Ohio was one of their projects. The clinic is associated with OSUWMC and specializes in providing primary care services and transitional care support for teens (transitioning from pediatric care) and adults with ASD and other complex disabilities. The Design tasks for this project were unique in that the IDEA Studio and the C.A.S.T. clinic were not sure what problem(s) they should be tackling (there was no Design brief). Instead of immediately ideating and generating solutions for a 'better clinic,' or leading a brainstorming session with stakeholders, the IDEA studio wanted to bring Design in for problem framing and 
bringing multiple perspectives (integral stakeholders) together in a shared space to acquire knowledge and understanding, in order to determine how to create true value.

In a typical Design project, the scope would have already been defined and handed to the Designer to carry out. In this project, the focus was on the front-end of the Design process (highlighted by the large white dot in Figure 5.1) and moving the Designer (small white dot in Figure 5.1) into the Define Scope phase at the beginning of the process to explore multiple frames and redefine the problem before ideating. Instead of launching into Design Research and ideation around a provided Design brief, we backed up to converge, build shared understanding, and reframe the scope of the problem to determine the true value we should be seeking to create through Design. Central and peripheral stakeholders (adults with autism, parents with adolescents on the spectrum, adult healthcare providers/administrators from OSUWMC, and pediatric providers/administrators from Nationwide Children's Hospital) were assembled in an exploratory case study to converge around the wicked problem and determine actionable steps towards improving the process, care, and support for adults on the spectrum and their families during and after the transition from childhood to adulthood.

\section{A Typical Design Process}
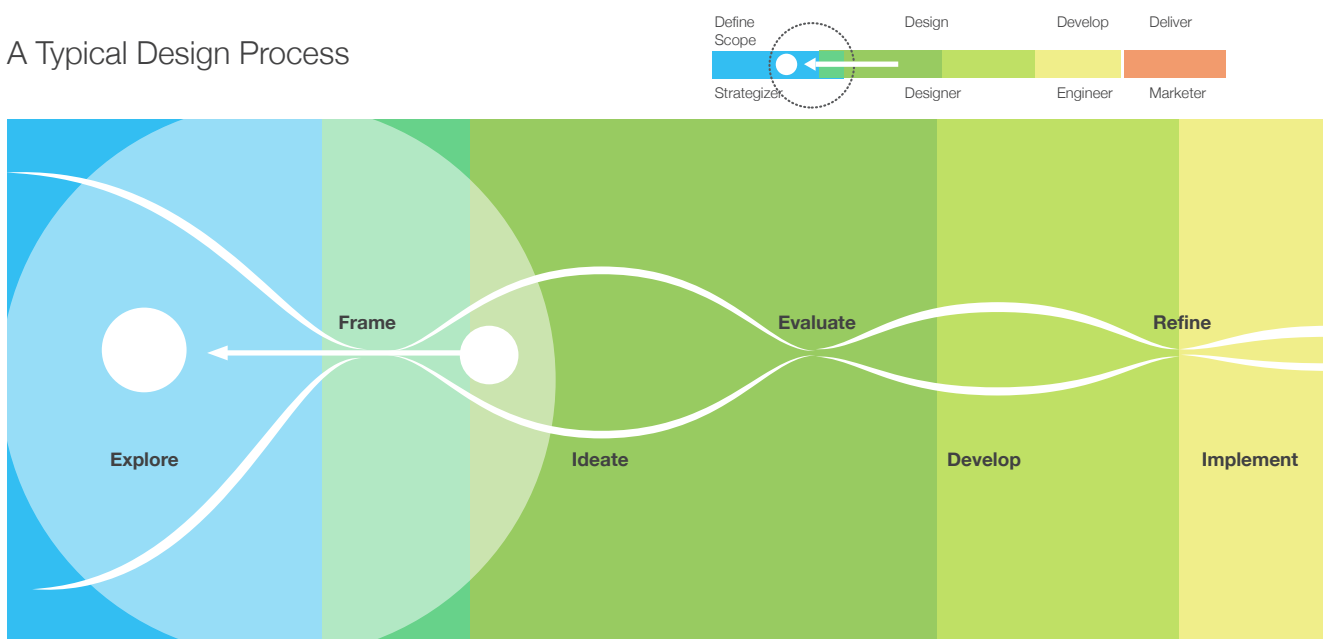

Figure 5.1 Stepping back in the Design process. 


\subsection{An Exploratory Case Study Approach}

The C.A.S.T. Case Study was selected as the research approach for this thesis because it combined four aspects of inquiry worth exploring: (1) the capabilities of the Designer in collective sensemaking and problem framing, (2) co-designing with the autism community, (3) the potential application of a hybrid toolset in a CoDesign approach, and (4) tackling wicked problems using Design Thinking. Furthermore, a case study can present the process and findings of the research in a more relatable and familiar way by using storytelling, a form of communication that people often use to make sense of complex ideas. The objectives of this case study were to explore the expanding role of the Designer, discover opportunities to bring digital capabilities into the frontend sensemaking phase of the Participatory Design research process, and determine various methods, tools, and approaches that could be utilized in future wicked problem cases to enhance the efforts of collective innovation around wicked problems.

\subsection{The Current State of the C.A.S.T. Clinic}

The clinic had recently opened, but many of the stakeholders' needs had not yet been addressed. The questions that needed to be addressed concerned the gaps and missed opportunities in the current clinic and local ASD network, determining how to create true value in the clinic and make it sustainable, how to improve training, connectivity, and awareness across the network for a more patient-centered model, and how to prioritize diverging goals and ideas among the stakeholders. The clinic had a donor and a lot of stakeholders with many different ideas, but no long-term sustainability plan and limited shared understanding/cohesion among the integral stakeholders. Every stakeholder had their own vision for the clinic and views of the problems facing adults on the spectrum and their families, but limited understanding of the needs of other stakeholders connected to the wicked problem. Parents of children (and adults) on the spectrum had met together often but not with people on the spectrum or healthcare staff. The healthcare providers and administrators within OSUWMC and at the Nisonger Center (a center for 
developmental disabilities associated with OSUWMC) met infrequently, while providers and administrators from OSUWMC and Nationwide Children's Hospital rarely met. In fact, some of the psychologists from Nationwide Children's participating in the C.A.S.T. Case Study were not aware that the clinic existed and that there was an adult care option for their adult patients. Yet, the adult and pediatric healthcare providers were all connected to each other in the same, shared system and they all potentially had an integral role in the long-term success/failure of the future clinic. What the clinic project needed was convergence, reframing, and collective sensemaking understanding brought to the stakeholders about each other, the root problems, and unmet potential inside and outside the clinic that could address more meaningful, long-term needs and goals. Many of the stakeholders had taken part in other collaborative sessions, yet they verbally expressed frustrations with these activities. To them, past sessions had entailed a lot of exercises without a clear focus on the problem they should be addressing or tangible next steps.

\subsection{Involving ASD Participants in the C.A.S.T. Case Study}

The participants with ASD in this case study had not participated in previous Design Thinking sessions. Often due to misguided social perceptions about their cognitive and social abilities, people on the spectrum have limited opportunities to self-advocate, especially in the realm of Design and Participatory Design practices that are intended to resolve the issues they face. In the past, parents and providers had spoken on behalf of their children and patients with ASD. But, in order to find true and lasting value for the clinic in this project, the scope and perspectives on the clinic needed to be broadened. People on the spectrum were invited into this case study as equal participants/CoDesigners and were given the opportunity to voice their needs, concerns, goals, and ideas, as well as life experiences from the perspective of someone on the spectrum. This also afforded other stakeholders the opportunity to expand their own understandings and better empathize with/appreciate the unique thoughts, feelings, and beliefs of people on the spectrum by interacting with them and listening to their visions. 


\subsection{From Participatory Design (PD) to Participatory Action Research (PAR)}

The case study began with a Participatory Design approach, bringing integral stakeholders together to gain shared understanding and co-design new resolutions for the clinic. The stakeholders were a part of three overall groups: adults on the spectrum, parents with adolescents with ASD, and healthcare personnel (providers and administrators). However, over the course of the case study, as the wicked problem was reframed, the approach shifted from Participatory Design (PD) to Participatory Action Research (PAR). The realization that needs related to the clinic stemmed from far greater issues beyond the clinic's walls shifted our focus of the wicked problem from developing clinic resolutions to gaining greater understanding of the multiple facets of the wicked problem (transitions in ASD), reframing our scope and approach, and empowering stakeholders to take a more active role not only in identifying and co-designing possible near-term and long-term (disruptive) resolutions in this case study, but continuing to be a part of the evolution, further development, and implementation of these resolutions beyond the scope of this project. It was evident that the goals for the new frame (broader scope) would not get resolved in this case study, but instead our findings and our resolutions/prototypes would help to shape new understanding and build a scaffold for future projects and further development. The process of PAR is aimed less at arriving at a singular resolution, but instead, promotes iterative understanding, continued learning, and on-going action. The nature this wicked problem, like other wicked problems, is that there is no ideal solution because there are so many facets. Therefore, an iterative learning process that provides the capability for knowledge gained through methods and design artifacts to be amplified/iterated and inform the next step, and motivates continued investigation into better resolutions, became the new approach for this case study.

\subsection{Using a PAR Approach}

The C.A.S.T. Case Study was divided into three phases. Phase 1 entailed secondary research, semi-structured interviews/focus groups with individual stakeholders and stakeholder groups. 
Phase 2 was a shared understanding and sensemaking workshop (Workshop 1). Phase 3 was a framing/concept development workshop (Workshop 2). Each session was co-lead with Dr. Elizabeth Sanders. The project began with research to better understand the unmet needs of people associated with the C.A.S.T. clinic. During Phase 1 it became apparent that the transitional care and support for adults with autism and their families was part of a macro-web of interconnected issues that reached far beyond physical healthcare. Discovering early on that the clinic may not be the central concern, but a peripheral concern to issues that were much more pressing led the approach to shift from PD to PAR.

Using a PAR approach and iterative Design Thinking methods, the aim of the case study was to create a collaborative atmosphere of shared understanding and creative thinking, and to facilitate convergence around the wicked problem of transitional care/support and reframe the scope/approach for future action. This strategy required first stepping back to see the problem from multiple frames and collectively redefining the problem around the unmet needs and opportunities that truly needed to be tackled. To do this, a collaborative making/seeing space needed to be designed that brought the varying stakeholders together and promoted open discussion, collective sensemaking, and critical/creative thinking. Additionally, a framework of various tools and methods needed to be designed that would allow the stakeholders to voice unique perspectives, collectively look at multiple angles of the problem, and build understanding, and propose new ways to improve the situation holistically, rather than solve it from a singular frame of reference.

\subsection{Motivation for Developing a Digital Tool}

Returning to the question posed by Weick, "How does action become coordinated in the world of multiple realities?" and considering the unique needs of this project, a digital tool was prototyped in parallel with the case study to explore the possibility of technology as a means through which 
sensemaking could be supported and the visions of multiple stakeholders could be coordinated before acting. Additionally, the digital prototype was designed to explore new capabilities for Designers, new forms of engagement in CoDesign, and the potential value of bringing technology and a hybrid toolset into PAR. It became apparent after Phase 1 that in order to collectively reframe the scope/approach for tackling the wicked problem, the stakeholders would need to be able to visualize multiple frames and collectively synthesize/make sense of the needs and goals that were central to the wicked problem. An interactive prototype was developed to assist the stakeholders and Designers in identifying key nodes within the autism system and the relationships/connections (if any) between those nodes. Analyzing data in Excel and synthesizing needs and ideas into clusters on a wall with sticky notes has been done in collaborative settings to identify concept opportunities, but the possibility of reconceptualizing the Design Thinking space in a hybrid way motivated the exploration of this blended interaction prototype to provoke new ways of seeing and visualizing the scope of the problem.

\subsection{The Approach for the Hybrid Design Thinking Framework}

The data collected in the autism case study were drawn from multiple resources and methods: secondary research, observations, semi-structured interviews, focus groups, visualizations and visual diagrams, physical artifacts from generative research methods, audio and visual materials, and questionnaires. The organization of specific Design Thinking methods and tools, starting with individual expression to collaborative prototyping, were integrated purposefully into each phase to build up empathy, knowledge, and new understanding over the course of the project. The hybrid framework for this CoDesign case study centered on combining the use of making and telling methods, with a digital prototype for sensemaking, to create an iterative learning experience and a scaffold on which to continue sharing and developing the outcomes from the project. 
Semi-structured interviews and focus groups in Phase 1 helped to establish the different perspectives, needs, and goals of each stakeholder/stakeholder group and gave individual stakeholders an opportunity to voice their concerns before collaborating with others in a shared space. The information shared in these sessions established each stakeholder's frame and provided the data that would be used to explore and map priority issues, identify connections between important needs/goals, and build a collective frame in Phase 2. Using this frame to develop personas and prospective scenarios, participants identified opportunities that addressed true unmet needs and began prototyping concepts to help inform next steps and future development. 


\section{Chapter 6: The C.A.S.T. Case Study}

"When we are given an infinite creative space to work within, it can be every bit as paralyzing as the most confining restrictions. A defined space helps us direct our creative energies in a more articulate fashion. We must be more willing to accept at least some small degree of definition of our respective creative spaces before we can effectively learn to define the space by (and for) ourselves." - an ASD participant.

The participant who shared this thought in one of our many discussions is one of the most insightful people you will ever meet. He is a creative writer, active in the arts and autism communities, and was one of the most instrumental participants in the project. For the continuation of this paper he will be referred to as John. John has Asperger's. He also works as a janitor, rides the bus and lives independently. John never explained why he works as a janitor. Whether by personal choice or because he has no choice, John has the passion and potential like so many other individuals on the spectrum to solve more meaningful problems and create better solutions. His thoughts on creativity echo those of many Designers. They also echo the thoughts shared by the Director of the IDEA Studio at The Ohio State University Wexner Medical Center (OSUWMC) when he was explaining his goals for the C.A.S.T. project. Very often it is hard, if not impossible, to truly create something innovative without first establishing a frame or definition of the space through which we seek to engender meaningful resolutions. 


\subsection{Understanding Different Perspectives}

Inquiry, the search for understanding the people and the problem from multiple vantage points, is where this case study began. The perspectives below summarize the viewpoints shared by the three integral stakeholder groups (adults with autism, parents with adolescents on the autism spectrum, and healthcare providers/administrators from OSUWMC and Nationwide Children's Hospital) in Phase 1, prior to meeting collectively in Phase 2 and 3. The statements described in the next three sections are written in their voices, beginning with the adult participants with ASD.

\subsubsection{Perspective of the Wicked Problem Shared by Adult Participants with ASD}

"Over our life journey, the areas that are of most concern to us are early diagnosis and early education planning (getting us the right tools and support sooner rather than later), vocational planning and social group interaction, and securing and maintaining employment and housing for long-term wellbeing. Ultimately, we want to maximize our potential, whether that is achieving interdependence or full independence, and leading rich, meaningful lives."

"Sensory overload is a problem that affects most of us. An over stimulation of sights, sounds, and visual distractions can impede our ability to communicate and socialize, and may also cause us to act out. This is hard for many neurotypicals to understand. What many neurotypicals also do not understand is that we can work on small, concrete problems or large-scale problems. Public perception needs to change and we need to do a better job of self-advocating. We need to be seen as innovators not victims, not only in mainstream society but also within our ASD community. This can start with recognizing and utilizing our specializations and talents. Society segments itself into factions. Instead, we need to find ways to connect and work together by contributing in different (unique) ways through our strengths. Society needs to see ASD as a gift, not a disability, and help us (people on the spectrum) harness (instead of suppress) our unique abilities so we can contribute to innovation and societal progress in a meaningful way." 


\subsubsection{Perspective of the Wicked Problem Shared by Parent Participants}

"One of the biggest problems we face as parents is that there is a weak network of support in the autism community. Many parents are in disagreement about care and treatment options, and therefore remain unconnected. Without solidarity, the mainstream population does not know how to help or respond. We need to generate ideas that bridge stronger connections within our network and with the mainstream population."

"The future outcome of full independence is a fantasy, not a reality to most adults on the spectrum and their families. The reality is that most adults with autism will live with their parents (or 'natural caregivers') for life, but that is not a sustainable model. Parents need care and support, too, if they are expected to be lifetime caregivers. Personalized care and support should be holistic and extend to the whole family. Over the course of our child's life journey our biggest concerns reside in finding information and support programs, the transition process, and whether or not our child will be able to live independently (securing and maintaining independent housing and continued care and financing). We, and our children, deserve to live rich and meaningful lives, but there are few funding options for the majority of families whose children fall between the top and lower ten percent on the spectrum. If we are truly going to tackle these problems with no (or very limited) funding and resources, our 'ideal' future will require disruptive innovation in areas like assistive technologies and affordable independent housing, in order to extend the care, support and longevity of the natural caregiver, so we can better care for ourselves and our adult child. In order to advance the potential of our society, people on the spectrum, and their families, we have to look at both small and large scale resolutions, untapped opportunities and disruptive changes." 


\subsubsection{Perspective of the Wicked Problem Shared by Healthcare Provider/Administrator Participants}

"As autism providers and administrators from OSUWMC and Nationwide Children's Hospital (representing both adult and pediatric healthcare), the biggest challenge we face is trying to apply a system-based approach to solving the tailored needs of patients and their families. Familycentered care coordination requires streamlined channels of communication, education, planning, and finance. The success of a systems-based approach to medicine relies on overcoming two major barriers: education gaps and communication gaps. Education gaps occur between the "interfacer" (adult patient and/or their caregiver) and the system on how to navigate treatment options, financing, and future planning. Education gaps are also found among healthcare personnel inside and outside the ASD network. A lack of training for physicians and specialists on how to care for autism patients limits care resources and makes the care experience unfavorable and difficult for patients and providers. Additionally, communication gaps contribute to misunderstandings between providers and specialists (inside and outside ASD). Communication needs to be more transparent to help providers better understand where they fit in the system so that they know where and how to best care for patients and their families. We need to improve our communication methods (not only amongst ourselves, but also with patients and families) and determine how we are all connected (especially in our local healthcare network)."

"Early diagnosis and determining early education plans are of high priority to us, along with helping people on the spectrum reach their full potential after transitioning into adulthood. Ultimately, we want to maximize family and patient potential so that people on the spectrum can move from dependency towards greater independence. Our aim as healthcare providers and administrators in the ASD system are to improve the quality of life of our patients and their families, increase effective care and personalized medicine within system-based solutions, provide care/support/services to enrich and maximize patient and family potential, and formulate 
sustainable supports and collaborative networks. To meet these goals we need to: provide tools to educate and train physicians and healthcare personnel, provide personalized family care, provide means to enhance communication and understanding, and provide the means to spread resources and responsibilities."

\subsection{C.A.S.T. Case Study Introduction}

The Design process used in this case study took place in the early stages of inquiry and scope definition. This case study began with discovery and problem inquiry to help the Designers and the CoDesign team collectively understand the context of the wicked problem before ideating around new resolutions. Secondary research (shared in the Autism Synopsis chapter) and conventional Design Research methods (i.e., interviews and focus groups) were conducted to become immersed into the world of autism, to better understand Autism Spectrum Disorder (specifically the issues facing families, providers, and transitioning adults on the spectrum), and to better understand the larger system and people connected to C.A.S.T. Figure 6.1 zooms into the front-end of the Design process and shows the orchestrated journey we took from problem inquiry, to framing, and finally to prototyping and ideation. The objective of the project was ultimately sensemaking and gaining collective wisdom (described by Wright (2005) as finding comfort in ambiguity and an understanding that absolute knowledge is not obtainable). The guidelines laid out by Wright (2005) to advance sensemaking (through activities used to harness intuition and iterative exploration to bring creativity and order to chaos) were integrated into phases of the project. A hybrid approach (non-digital and digital) was explored in the process to engage each person and allow them to contribute in multiple ways (i.e., in both individual and group settings), provide traceable paths between perspectives shared, decisions made, and needs/goals expressed throughout the process, and encourage co-constructive understanding through making and evaluation. Open communication and respectful interaction were promoted throughout the project as each stakeholder group had opportunities to independently voice unique 
thoughts and listen to multiple perspectives early on. Just as social interaction, dialogue mapping and scenario creation were shown to unite fragmented stakeholders and promote higher levels of shared understanding and commitment in collaborative settings in Jeff Conklin's research, visual mapping, storytelling and prototyping were integrated into this case study to bring order to the chaos and help the participants collectively find common ground and make lasting connections. The three-phase case study sought to expand understandings and ownership through an iterative process in the hopes of reframing the wicked problem around more meaningful and sustainable opportunities for transitional care and support for people on the spectrum and their families. 

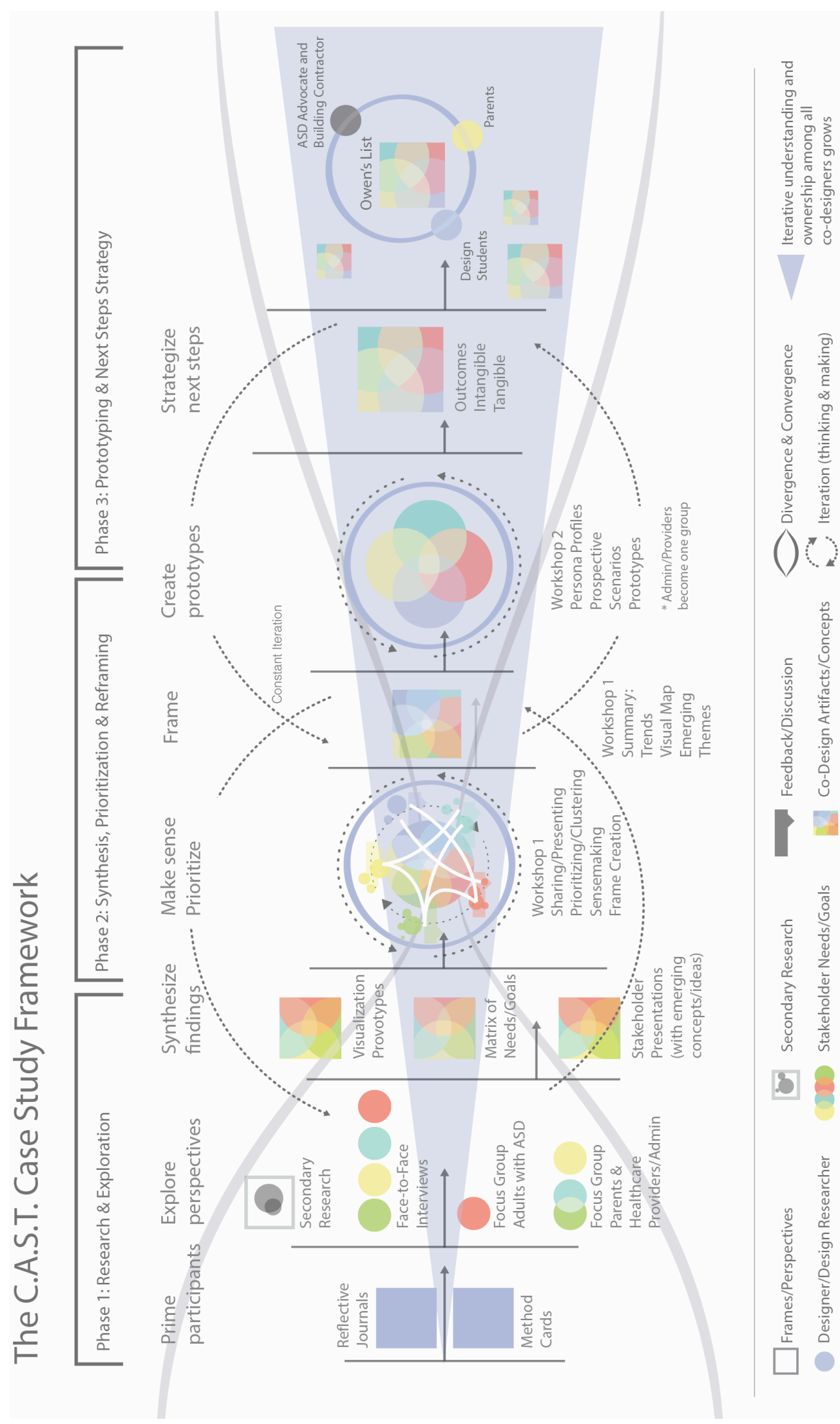

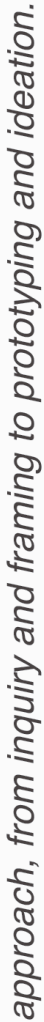
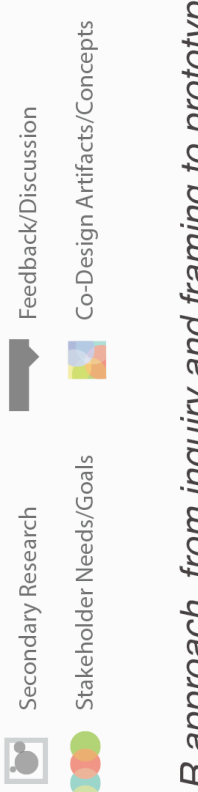

용

$.28 \frac{5}{5}$

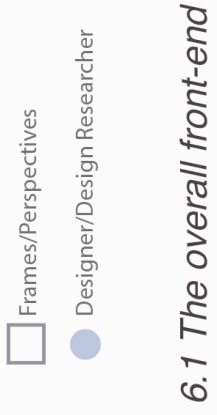




\subsection{The Intent of the Case Study}

The PAR approach used in this case study was intended to engender empathy and more critical and creative thinking among the integral stakeholders in order to bring about more shared understanding, and to draw out new insights that may have been previously overlooked. Following the four phases of empathy laid out by Kouprie and Sleeswijk Visser (2009), participants were given opportunities to discover new/different perspectives, immerse themselves into those perspectives, connect by finding meaning among the perspectives, and step back to make sense of their broadened scope. These means were exercised by:

- Affording stakeholders multiple opportunities to share personal experiences/points of view and to learn from (empathize with) one another

- Allowing the group to connect and make sense of the information by manipulating it collectively (using a blend of interactive non-digital and digital methods and tools)

- Providing a maker/seeing space through which the CoDesigners could speculate about the future, build personas and scenarios, and initiate actionable steps towards both implementable goals and disruptive innovations

Observations were also made throughout the case study about the methods/tools used within and across each phase, in order to identify potential opportunities and gaps in the current Design process, and to speculate about future applications for new tools in PAR. Additionally, the role of the Designer was noted throughout the project. The intent was to demonstrate the value of Design Thinking and the Designer in the front-end phase of a wicked problem to help establish the frame and plan of action for moving forward into the ideation and development phases of the Design process. As a Designer in this project, the role of facilitator and 'form giver' extended beyond giving form to a solution, but in giving form to the wicked problem - helping CoDesigners not only express their thoughts and creativity, but more importantly make sense of the problem. 


\subsection{Overview of the Case Study}

In each section below the process used at each phase of the project will be explained in detail, as well as how each session's outcomes influenced the approach for each subsequent phase in the PAR process. Mention of the digital tool that was prototyped in parallel with the case study will be brought into the accounts of Workshop 1. However a full description of the development of the digital prototype will be explained in the chapter that follows. The phases of the case study are laid out in the subsequent sections as follows:

- Pre-Phase Materials and Preparation: Pre-Assessment Questionnaire, Journal, Method Cards

- Phase 1: Interviews and Focus Groups

- Phase 2: Workshop 1 Presentations and Sensemaking Activity

- Phase 3: Workshop 2 Personas, Scenarios, and Prototyping

- Post-Phase Feedback and Reflections: Post-Assessment Questionnaire, Feedback Questions, Reflection Survey

\subsection{Pre-Phase Materials and Preparation}

The project began with defining the overall framework for the project and determining the project team - the people necessary for addressing and tackling the wicked problem collectively. Preparatory materials (e.g., Method Cards and Journals) were handed out to these stakeholders during the Phase 1 semi-structured interviews and focus groups to immerse them into the project, prime them for each phase, and acquaint them with various tools and methods Designers use to tackle problems. Pre-Assessment Questionnaires were distributed to each participant as a way to benchmark their current understanding/knowledge pertaining to certain aspects of the wicked problem and their feelings/thoughts towards collaboration, the clinic, and what they hoped to gain from this project. The responses to the questionnaires will be shared and analyzed in the Reflections chapter. 


\subsubsection{The Journal}

The eight page Journal (pictured below in Figures 6.2 and 6.3) was divided into sections to be completed individually prior to and following specific phases of the project as a way to guide participants through anticipatory and reflective thinking exercises. The intention of these materials was to give each person an opportunity to consider their role in this project and reflect on their own perspectives, needs, and ideas prior to and after participating in the sessions. All of the Journal questions were printed on the front of an $11 \times 17$ piece of paper, so the back could be used as a large poster board for self-expression (prior to Workshop 2), and so the Journals could be collected and quickly scanned after Workshop 2. The Journal was folded into a booklet for ease of use. Below is an image of the first section of the Journal, to be filled out during Phase 1 (preworkshop sessions) and prior to Workshop 1.

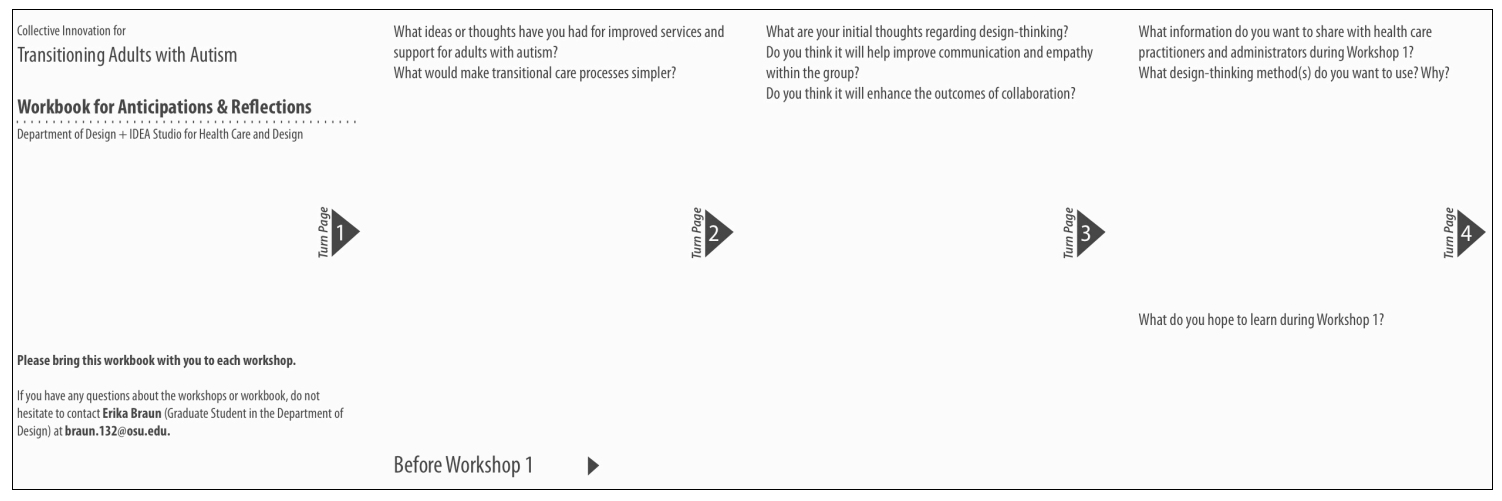

Figure 6.2 The first four pages of the Journal, printed on an 11" $x 17$ " piece of paper and then folded into a booklet.

Journal questions on pages one through four included:

- What ideas or thoughts have you had for improved services and support for adults with autism?

- What would make transitional care processes simpler?

- What are your initial thoughts regarding Design Thinking? 
- Do you think it will help improve communication and empathy within the group?

- Do you think it will enhance the outcomes of collaboration?

- What information do you want to share with health care practitioners and administrators during Workshop 1 ?

- What Design Thinking method(s) do you want to use? Why?

- What do you hope to learn during Workshop 1?

Figure 6.3 below shows the second section of the Journal, to be filled out after Phase 2 (Workshop 1) and prior to Workshop 2.

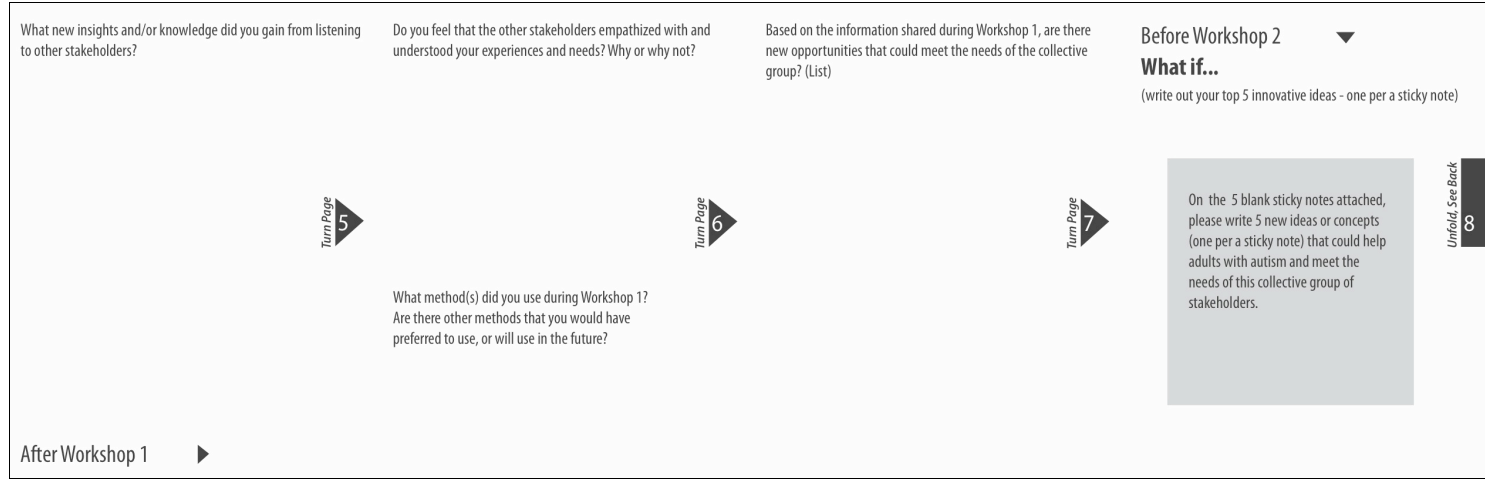

Figure 6.3 Pages five through eight of the Journal.

Journal questions on pages five through eight included:

- What new insights and/or knowledge did you gain from listening to other stakeholders?

- Do you feel that the other stakeholders empathized with and understood your experiences and needs? Why/why not?

- What method(s) did you use during Workshop 1 ?

- Are there other methods that you would have preferred to use, or will use in the future?

- Based on the information shared during Workshop 1, are there new opportunities that could meet the needs of the collective group? (List) 
In addition to filling out the questions, the participants were asked to individually partake in two activities before Workshop 2 to prime them for the final workshop and have them begin to speculate about 'What if...' opportunities. On the eighth page of the Journal participants were given five sticky notes and were asked to write down one idea on each (five total) and bring the filled out notes to Workshop 2. Additionally, on the reverse side of the Journal (Figure 6.4) was a blank page on which the stakeholders could chose to create a Vision Board illustrating their ideal future (hopes and dreams) for adults with autism. The participants were asked to bring both the sticky notes and the Vision Board to Workshop 2. Below is an image of the back of the Journal.

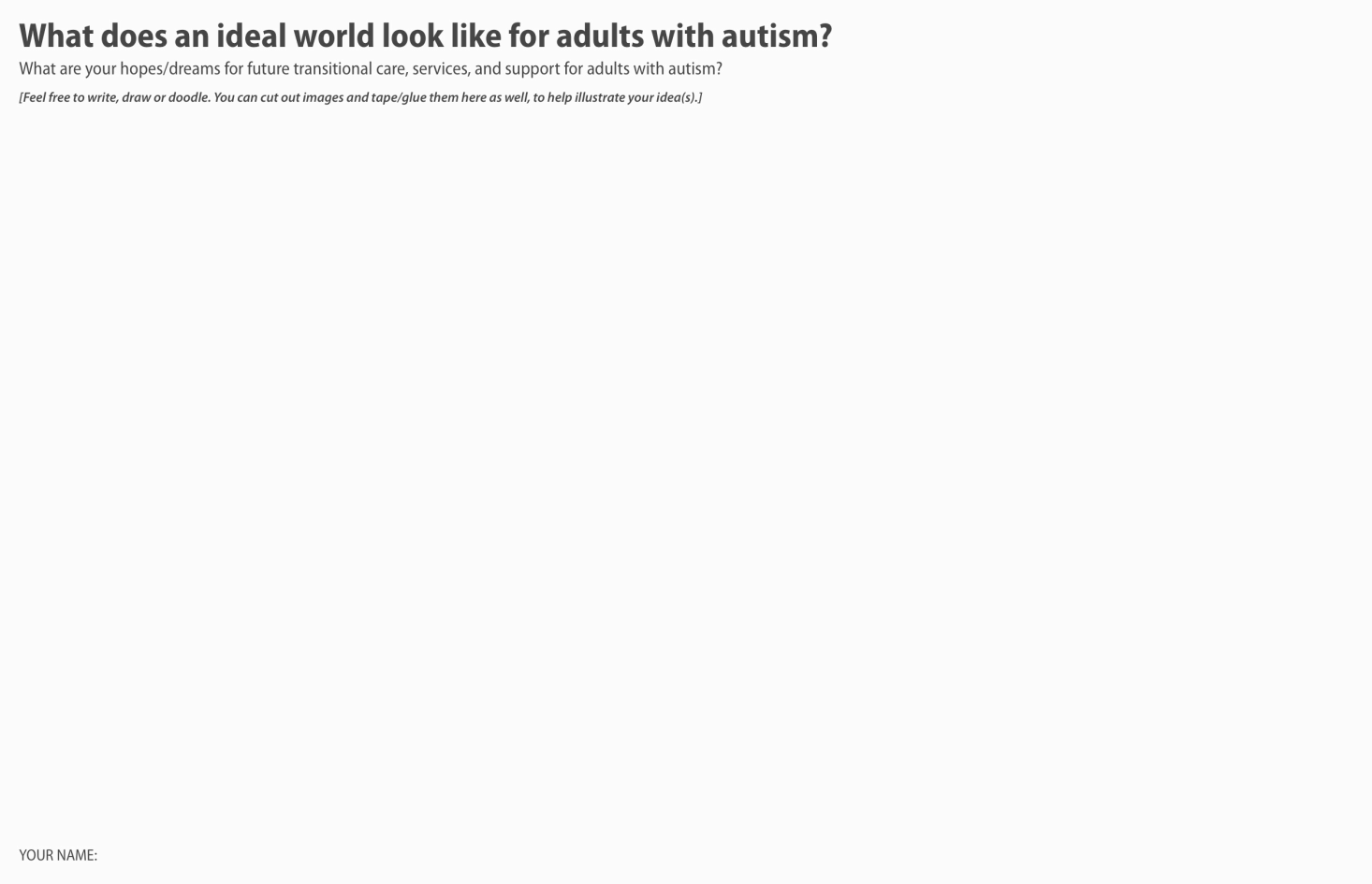

Figure 6.4 Vision Board on the backside of the Journal.

\subsubsection{The Method Cards}

The Method Cards represented an assortment of generative design methods. Eleven method cards were designed for the participants to expose them to a wide range of methods that 
Designers use. Each card defined the method by "how" it is typically used and "why" it is typically used. On the back of each card were lines on which the participants could take notes on each method as they were explained at the beginning of Phase 1 sessions, or while they were used/referenced throughout the project. A twelfth summary card was added to the stack which included a brief summary of how the method cards can be used, along with other references to find/learn about other Design Thinking methods. The eleven methods selected included: Problem Probing, Five Whys, Persona Profiles, Empathy Mapping, Empathy Scenarios, Mapping Needs and Goal, Affinity Mind Mapping, Iterative Prototyping, Trend Analysis, Futuring (Forecasting Scenarios), and Systems Mapping.
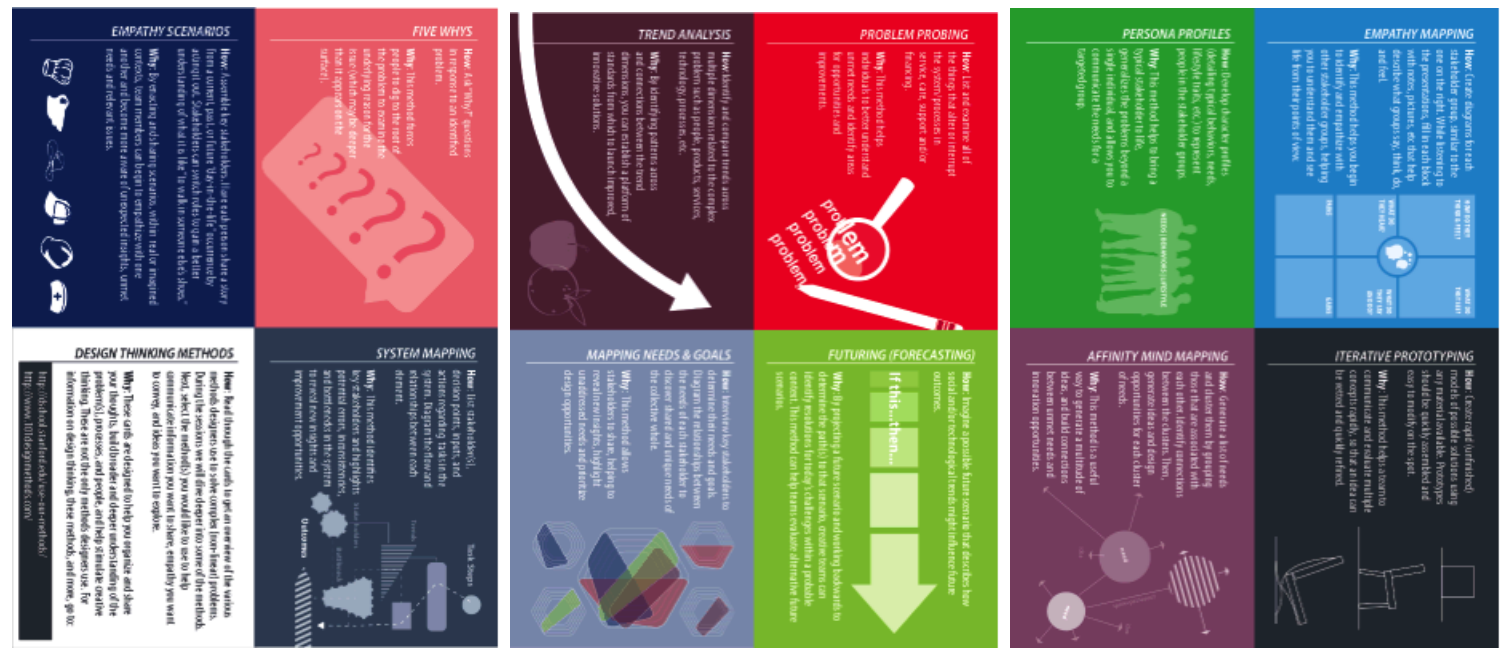

Figure 6.5 The twelve Method Cards.

Some of the methods, not all, were used throughout the project. Figure 6.6 diagrams where in the overall process the selected methods were integrated or used as a reference for the approaches in each phase. The Five Whys, Problem Probing, and Trend Analysis were referenced for the structure of secondary research, interviews, and focus groups to dig deeply and widely into the wicked problem. Empathy Scenarios, Mapping Needs and Goals, and Affinity Mapping guided the design of the approach for Phase 2 to support shared understanding and sensemaking. Phase 3 
incorporated Persona Profiles, Futuring Scenarios, and Prototyping to stimulate generative thinking and Design around new resolutions.

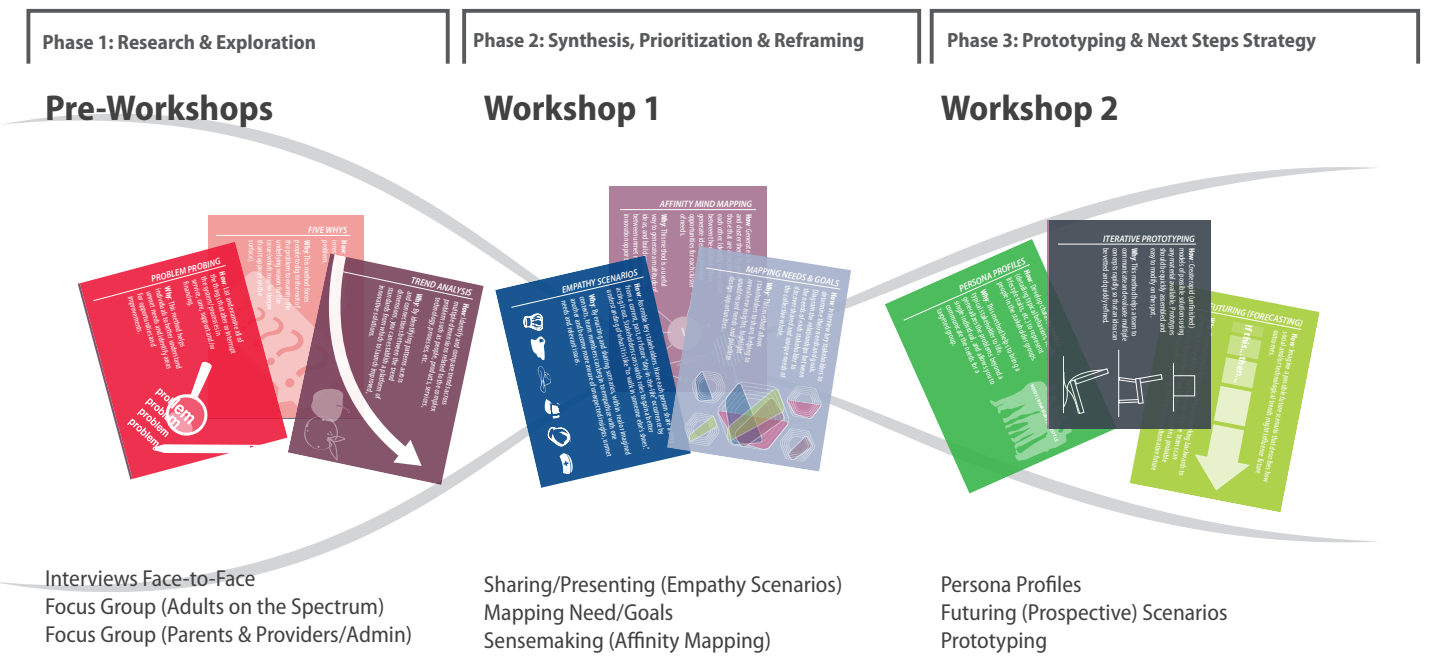

Figure 6.6 An overview diagram representing the methods used/referenced in the construction of the three phases.

\subsection{Phase 1}

Phase 1 meetings included one-on-one semi-structured interviews and two focus group sessions (one with adults on the autism spectrum and the other with parents and healthcare providers/administrators). They were designed to gain a better understanding of the various stakeholder perspectives and the overall scope of the project.

\subsubsection{Semi-Structured Interviews, Including the Role of Provotypes and Visuals}

A series of semi-structured interviews with key stakeholder group representatives were conducted first to gather knowledge about ASD trends and the topic of transitions, as well as stakeholderspecific concerns, unmet needs connected to the clinic and the local autism community, and the various goals each stakeholder hoped we would achieve by the end of this project. The 
descriptions of each interview in the following sections give an overview of the topics discussed and outcomes from the discussions. A more in-depth look at the questions that were posed can be viewed in the Appendix.

Visualizations were developed from the information shared by the stakeholders at each interview. The visualizations included operations charts (e.g., players in the healthcare system), patient journey maps, information graphics, systems maps (showing relationships between the three stakeholder groups), and concepts of what the clinic could/should be. Some of these visualizations were turned into provotypes. The provotypes included concept diagrams of the 'ideal' clinic and a journey map representing the current care and support offerings for ASD patients at various life stages. Laurens Boer and Jared Donovan (2012) explain that provotypes are essentially provocative prototypes. Unlike prototypes, which are typically developed as a reflection on what has been learned, provotypes occur early in the process to stimulate new ideas. Drawing on the skills of the Designer, provotypes blend investigative activity with the design of new possibilities. They are used as a tool of expression and a way to challenge current perceptions (Boer \& Donovan, 2012).

The provotypes, developed iteratively over the course of multiple semi-structured interviews, along with stand-alone visualizations, were used to expose the complexity of the problem, to provoke discussions, and to challenge current perceptions about the C.A.S.T. clinic and transitions during Phase 1. The visualizations and provotypes extended conversations from previous interviews, provoked perspective comparisons, and led to a multi-layered understanding of transitions within ASD. They also helped stakeholders establish where they stood in connection with and/or disconnection to the other stakeholders. The complexity of the problem, as well as the incongruity of the different stakeholders and their strong desires for shared understanding and greater knowledge became more apparent as more stakeholders explained 
unique perspectives based on the questions they were asked and how the provotypes were evolving. The paper-based provotypes were presented in a way that allowed the interviewee the freedom to build onto or introduce alternative perspectives.

\subsubsection{The Semi-structured Interviews}

\section{Project Coordinators at the OSUWMC IDEA Studio}

The first two interviews were conducted with two project coordinators at the OSUWMC IDEA Studio to establish the players and the departments connected to ASD in Columbus that needed to be brought together in the project. It was during these first interviews that the first three provotypes of what the clinic could be were conceived.

In these interviews, a goal for this project that emerged was determining what the focus of C.A.S.T. should be (e.g., transition coordination, medical care or both) and how to make the clinic unique. This question was tied to determining the current state of ASD adults and the future of ASD adult care (i.e., medical, social, financial, vocational, and beyond). Some of the questions that were raised follow. Do occupational therapy/training and vocational care need to serve the full spectrum? Do high/low functioning adults go to the same clinic or should we explore alternative care options that can be more tailored to the individual patient? Some suggestions included care coordination between multiple sites, more family involvement, and better training for healthcare staff to make them and the patient more comfortable.

This patient population often needs more time for examinations, so discovering a tailored and more innovative intake process that was also more effective and efficient for administrators was expressed as being ideal. This was tied to establishing who the clinic should be serving (e.g., full autism spectrum or beyond and at what rate) and how often high/low spectrum patients need to have checkups. Another question yet to be determined was the structure of the facility and the 
roles of the healthcare staff (e.g., providers, administrators, extenders, etc.) Does the clinic need to offer care coordinators and extenders at the clinic, and if not, how can we connect specialists? What services are most important and what services can be coordinated at other sites so the ASD care system is more connected and cohesive? For instance, a navigator at the clinic to work with families and help guide them through transitions seemed to be an valuable asset that could make the clinic's care offerings unique, while also targeting a true need, in addition to medical care. Pictured below (Figure 6.7) are two images: an organizational chart, which was created with sticky notes in one of the two interview sessions to establish the players that are integral to transitional healthcare for people with ASD, and a cleaned up matrix of the same chart that was created after the interview. The images show a wide assortment of departments, related to the clinic, and their interdependencies on one another (represented by small triangles).

Figure 6.8 (top row of images) shows the initial 'visions' for the clinic, created from the insights in the first two interviews. These three clinic concepts were provotypes. They were not intended to be final solutions, but instead became working models in future interview sessions from which to iterate and hone the vision of the clinic. Over the course of multiple interviews the three concepts (shown at the top of Figure 6.8) evolved into the concepts shown on the bottom row of Figure 6.8. Larger images of the provotyped clinic concepts can be found in the Appendix.

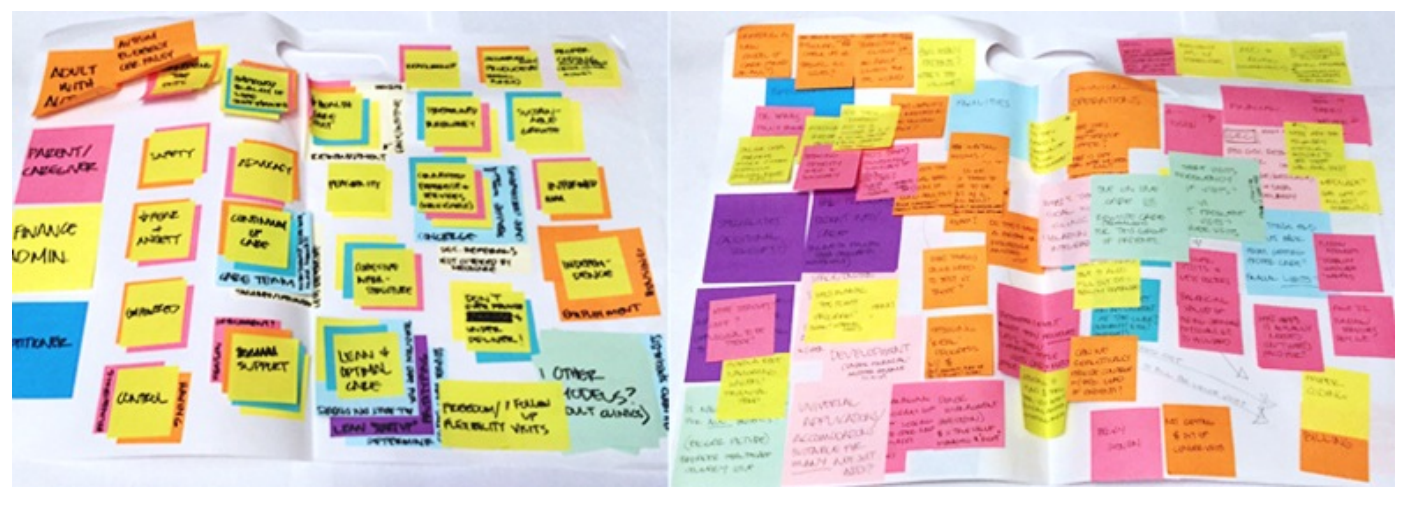

Figure 6.7 Organizational matrix of the players in healthcare connected to ASD. 
Figure 6.7 Continued
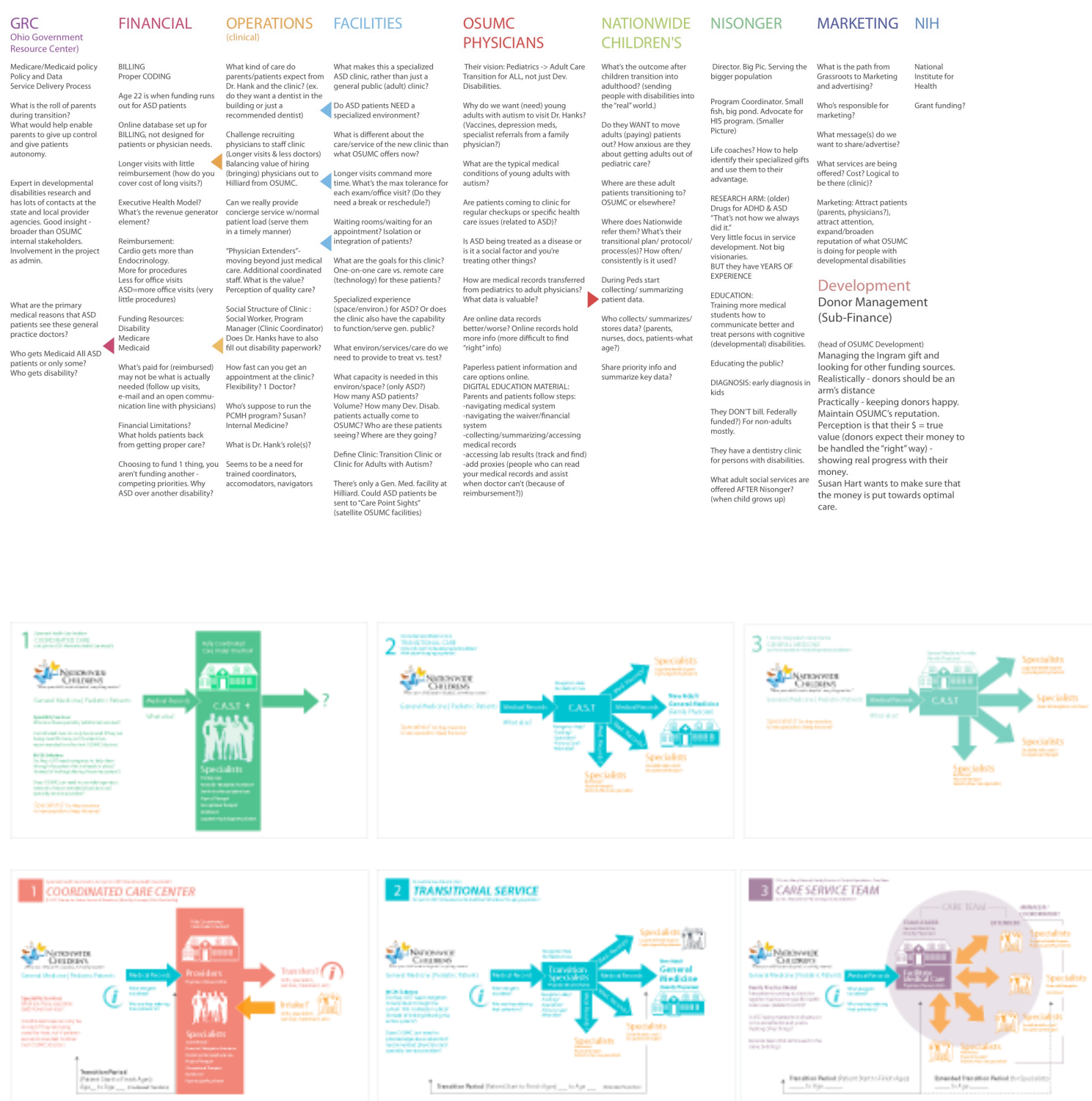

Figure 6.8 Provotyped concepts for the clinic.

\section{High level administrator at OSUWMC}

An interview was conducted with a high level administrator at OSUWMC. She had previously been given little opportunity to voice her concerns about the clinic's fiscal sustainability and the true value they should be creating in the ASD community. Her concerns centered on determining the necessary resources and feasibility of providing for optimal care and core offerings. She 
wanted to better understand the current volume of patients using the clinic, as well as future patient estimations, in order to establish the ideal scale needed to sustain the clinic financially, while also not over-promising and under-delivering care and services. For example, should the clinic provide services only for ASD patients and their families, or should it be broadened to offer care to people with other developmental disabilities, as well? What level of family support is expected?

During the interview she commented on the three clinic provotypes and questions such as whether the clinic should offer concierge or transitional care were explored. She explained that a concierge model is not sustainable because the patients do not stay forever. They move on to the specialists and providers coordinated through the concierge clinic. Through the diagrams of the three clinic provotypes she expressed the 'vision' she most closely identified with and shared her idea for a lean clinic that could create true value and grow incrementally. She also mentioned the value of integrating services and creating a 'Care Team' that would include outside extenders (like nutritionists and social workers) and a care coordinator to help people navigate the system. To make a 'leaner' clinic, the services and care of the main provider at C.A.S.T would need to be extended. More specialists could be less expensive for more tailored services and care. The current medical system is not intuitive or easy to navigate so offering a social worker and/or a navigator was the key to her vision (Figure 6.9).

Given her position, the administrator maintained a systemic view of the wicked problem. Changes made in the clinic, like making physician referrals or adding extenders, created a new set of issues. Referrals are not paid for and currently, while extenders seem necessary for transitional care, they are not covered by many medical insurers. Longer visits with the physician, which may be necessary for this population, modified bill pay. Follow up visits cost more money. Currently coding related to transitions and longer patient visits is lacking and needs to be reformed; the 
coding documentation needs to support what is being done with the patient through the transition phase. If longer visits prevented follow up visits and extenders were necessary, what resources needed to be relocated to provide optimal care? Better coding practices and weighing the cost/benefit for longer/shorter clinic visits were administrator specific problems that she wanted to address during the collective session.

Through the matrix of the healthcare 'players' we discussed various departments and facilities and what value they may add to the optimal care and sustainability of the clinic. The roles of The Nisonger Center, Nationwide Children's Hospital, and the OSUWMC marketing department, for instance, were still undetermined, but deemed valuable to the success of C.A.S.T.

This interview helped to establish barriers within the healthcare system from an administrator's point of view, and provided insight into the day-to-day operations that, while they may meet a patient's need, could disrupt the larger system. By building onto one of the visions of the clinic we strengthened the model for a clinic that could potentially be more sustainable while also providing core offerings. This interview also provided the administrator with greater peace of mind and enthusiasm about the project. She felt more engaged because she had been given an opportunity to voice her concerns and see her thoughts/ideas represented on the paper provotype that would be seen by other stakeholders in future sessions. 


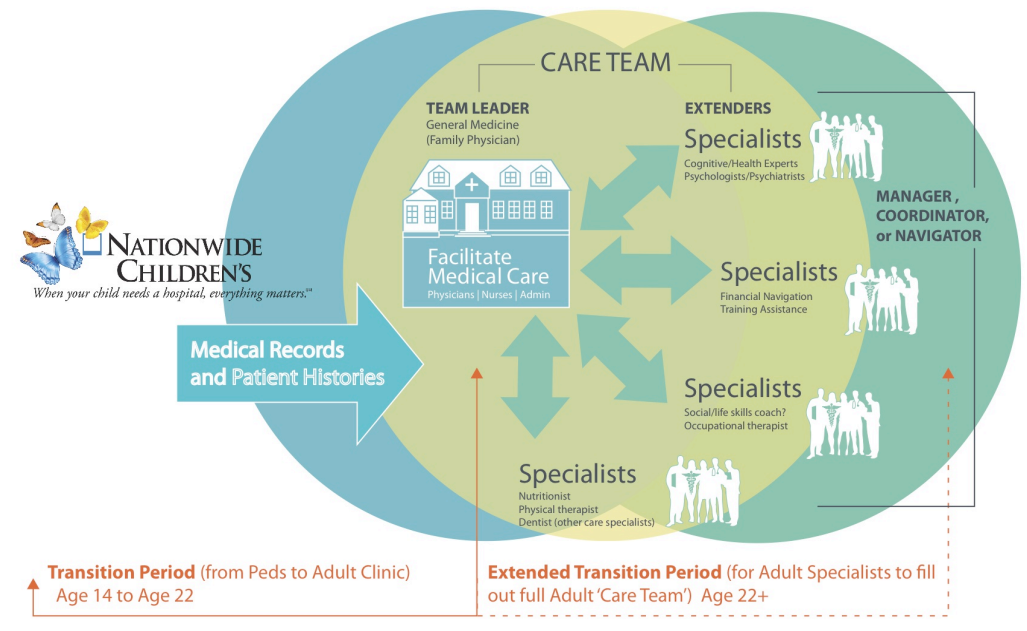

Figure 6.9 The final iteration of the clinic diagrams.

Four major Design opportunities emerged from the first three interviews:

1. Determining core offerings and patient population.

What is the volume of patients and scale and what are the core offerings for optimal value so we do not over-promise and under-deliver?

2. Care coordination and transitional care or medical care.

Should the clinic provide concierge or transitional care services? What are the risks and benefits of either program? How can we extend the main physician and provide quality care, while also reducing costs? 'Care Teams' appear to be a solution for balancing resources, while also creating quality care in a scalable clinic model. 'Care Teams' would include specialists who may be housed, along with physicians, at the C.A.S.T. clinic.

3. Better transitional coding and payment plans.

Coding for transitions, reimbursements, and bill modifiers for patients with autism need to be improved and implemented into the system in a simpler and more intuitive way to better track health histories and account for extended clinic visits so clinic does not have to absorb the cost of the extended time these patients need with their physicians. 
4. Determining roles and core care services.

How broad should services at the clinic extend (e.g., to social, medical, financial, etc.?) How can the Nisonger and OSUWMC marketing teams fit into the current/future model? Family support and their level of involvement at the clinic also need to be ascertained.

\section{Parent with an adolescent on the spectrum}

The fourth interview took place with a parent. An initial journey map was created to represent the support and services a person on the spectrum could need over a lifetime. This map was based on information collected from the project coordinator, Temple Grandin's book Thinking in Pictures, and other secondary sources. Throughout the interview, additions and changes from a parent's perspective were made to the incomplete journey map (Figure 6.10). This provotype helped to guide the discussion around unmet needs, gaps and opportunities in the system that were not currently being addressed for people with ASD and their families at different stages of life. In addition to building onto the journey map, a second map (Figure 6.11) was created that could be used to explain not only the medical services/support that were needed at each life stage, but also to identify the integral network of extenders who needed to be connected at each life stage.

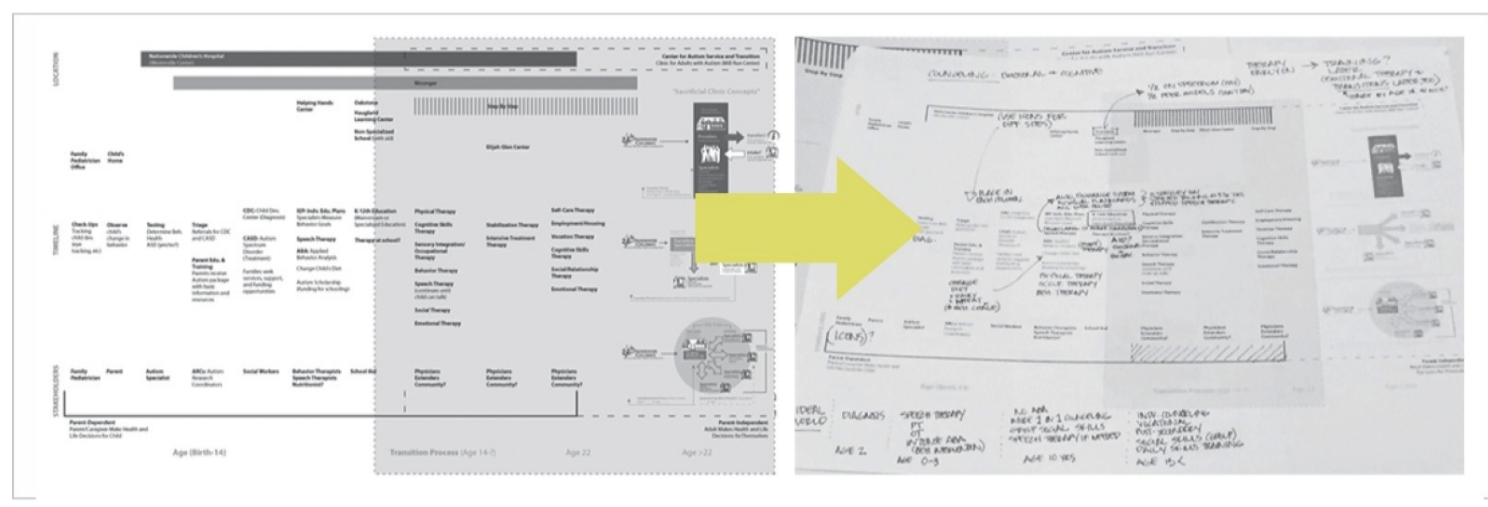

Figure 6.10 Iterations to the patient/family journey map from childhood to adulthood based on the interview with a parent who had an adolescent on the spectrum. 


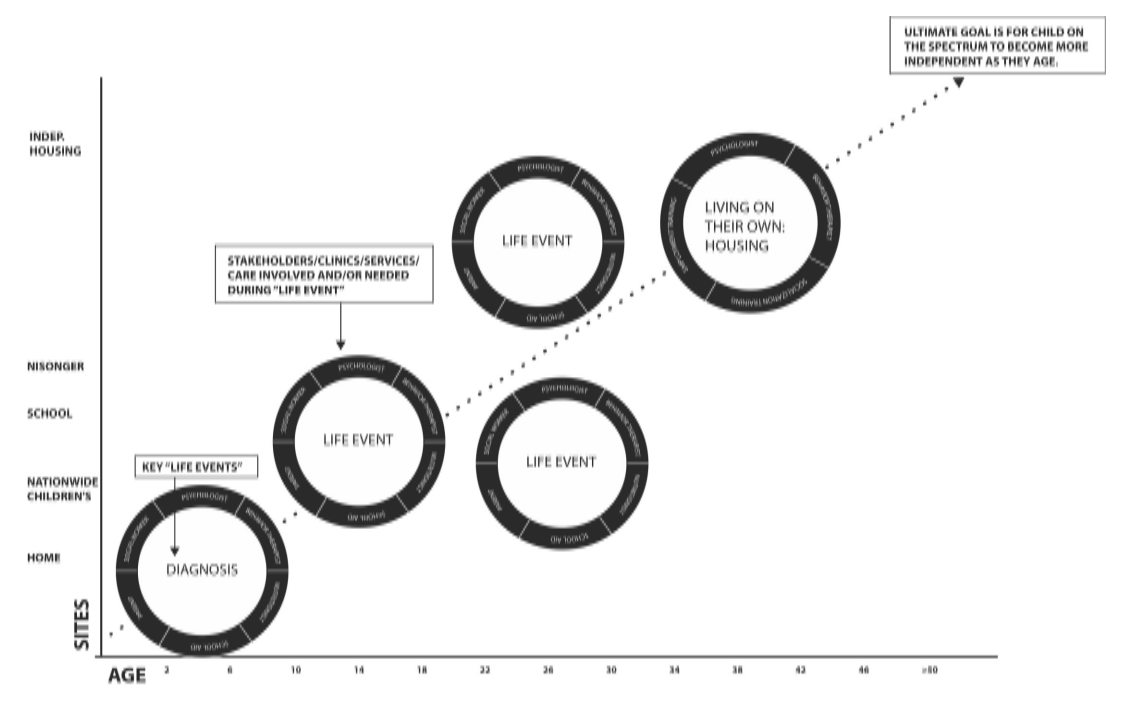

Figure 6.11 Potential life services/support for person with autism created in the interview with a parent who had an adolescent on the spectrum.

\section{ASD child psychologists at Nationwide Children's Hospital (NCH)}

The next interview was administrated with two child psychologists from Nationwide Children's Hospital $(\mathrm{NCH})$. This interview was set up to establish the unmet needs of ASD providers at $\mathrm{NCH}$ during a child's transition from pediatric care to adult care. Currently, $\mathrm{NCH}$ has many adults on the spectrum that they care for because change is hard for people with autism and families struggle with finding new, trusted adult-care providers. On the reverse side, the providers at $\mathrm{NCH}$ do not know where else to send them. In the interview it was clear that these psychologists were not aware that C.A.S.T. existed. The journey map (from the prior parent interview) was used in this session as a provotype (Figure 6.12). Segmented services and support offerings were revised, as we looked at the journey map from a more general perspective. Each child on the spectrum is unique as are the care and services they may need over time. In this meeting we wanted to establish, in more general terms, what the current offerings were and where the gaps in service/support were in the transition process for patient and family care/support from the perspective of the providers. Our discussion and revisions on the map highlighted the fact that 
there was no current system in place for transitioning children from pediatric to adult care. Additionally, the lack of resources (both social and developmental) and funding options for underprivileged families and adults on the spectrum was brought up, which means that many people on the spectrum fall through the cracks, especially during the transitional phase.

In addition to identifying gaps, we began ideating around opportunities that could help to improve the current system like having a more formulaic and cohesive transitional process, coordinated between pediatric care providers and adult care providers. This could start with families laying out actionable milestones with the physicians early on and having 10 and 12 year-olds visit the adult clinic, so change is more expected and gradual, and trust can be built-up (not only for the child, but the parents as well) over time. Children on the spectrum and their parents could benefit from tools that help patients start to speak for themselves. This puts more independence and control in their hands and less pressure on the parents. Furthermore, enrichment opportunities, community support/engagement, and social outreach needs should be available for all people on the spectrum and could be facilitated through the clinic. An idea that was mentioned was forming a "Transitional Group" clinic partnered with $\mathrm{NCH}$, that includes not only primary care providers, but also extenders like social workers and behavior therapists. In addition to providing primary care, the clinic could also be a space for parent meetings. It would have a more holistic care feel. The care given in the clinic should be more proactive and preventative than reactive. Lastly, the conversation lead to the idea that an ideal clinic for adults on the spectrum is one that is designed by them, one that empowers them and gives them more control over the environment. 


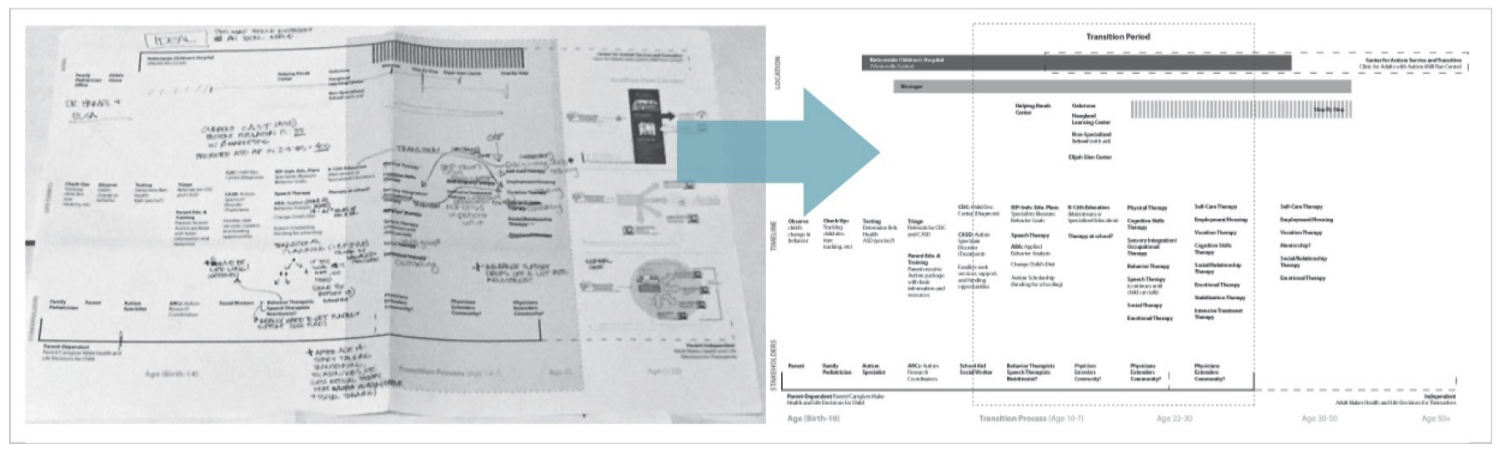

Figure 6.12 The next iteration of the patient journey map based on the interview with ASD providers from Nationwide Children's Hospital.

\section{Director of C.A.S.T.}

The interview with providers from $\mathrm{NCH}$ was followed by an interview with the Director at C.A.S.T. The aim was to get his perspective on transitions from an adult primary care perspective, as well as understand his vision for the clinic using the three visual provotypes (clinic concepts) that had evolved over the course of earlier interviews. His vision aligned with the 'Care Team' vision. An ideal C.A.S.T. clinic would embody a blended 'Care Team' model made up of remotely connected physicians and specialists as well as specialists and extenders (like social workers and a pharmacist) housed within the clinic, so face-to-face collaborative 'Care Team' meetings could happen more spontaneously and organically. An all-in-one clinic is not scalable but makes it easier on the parent by providing one-stop-care and allows for there to be more consistency of care. Alternatively, a completely remote 'Care Team' model (using technology like Telemed) may be more scalable, but information transparency can be difficult to achieve and collaborative meetings can be hard to facilitate.

Few changes were made to the journey map, but the map helped to provoke discussion around outlying issues that impacted whether or not the family/patient could receive the care/support they needed at each stage of life. In continuation to what the $\mathrm{NCH}$ psychologists had mentioned, the 
Director of C.A.S.T. agreed that there needed to be more cohesion between the healthcare centers and a better system in place for slowly transitioning adolescents into adult care. The biggest issue he wanted to tackle in this project is bridging the gap between pediatric care and adult care. In addition, he expressed that communication is a very big problem that needs to be addressed. Things like MyChart can help with communication between physicians, but communication concerns extend beyond physicians, specialists, parents and patients, to communication breakdowns that occur between parents (with differing perspectives on treatments) and how to help parents find financing and resources options once their child becomes an adult.

Funding and resources are often different, not only from state to state, but also from county to county. This makes it hard for the parent to know that a clinic like C.A.S.T. exists or that they can afford to send their child to a specialized clinic. An online resource could help parents and adults find funding and services within their zip code, along with ratings from other parents on physicians that align with their 'treatment' preference. Currently there are very few structured support channels for parents and people on the spectrum. There is a great deal of stress on the parents, not only in care for their child, but also in becoming the expert - having to keep up with navigating the system and understanding different care options. The stress levels of these parents is equivalent to people living in a war zone, which is why many parents form support groups. It became clear in this interview that the 'patient' was not only the child/adult, but also the parents/care-givers. We discussed that achievement for the child and peace of mind for the parents can come from anticipatory guidance. Preparatory tools, such as 'Vision Plans,' that could empower the adolescent/adult to speak for themselves with the physician, while also providing actionable and anticipatory milestones would take stress off the parents and potentially help the child to work toward self-advocacy and independence. A 'Vision Plan' is a navigation tool co-created with parents to chart developmental expectations and goals for the child as they 
progress through different stages of maturation. It serves as a communication/transparency tool to ensure parents and providers are on the same page.

Other new information that came from this interview centered on the lack of special needs training in medical schools. While an adult with ASD will typically see a specialized primary care physician, they will also need to see specialists like a cardiologist, as they get older. Without better training it is difficult for these specialists to take on patients with special needs. It also makes it harder for the primary care physician to recommend a specialist who will be comfortable working with an adult on the autism spectrum. Additionally, there are very few primary care centers for ASD adults in the country. Hence, the lack of training, limited specialized care clinics, and a lack of financial resources forces many adults on the spectrum to go to the emergency room for primary care needs.

Overall the Director of C.A.S.T. believed that the barriers to transitioning resulted from expectation gaps and the dilemma between independence and dependence. Some parents are not ready to let go and give their child independence, while other parents' expectations are too high; some children may never be able to be independent. He believes parents need to widen their view beyond helping their child achieve short-term goals, toward planning for long term goals. Another barrier is lack of funding and support networks (both social and enrichment) for adults on the spectrum. The issue of guardianship has a lot to do with both of these barriers. More severe cases tend to get more support, but those on the edge of independence and minimal guardianship fall through the gap and do not get the support they need. 


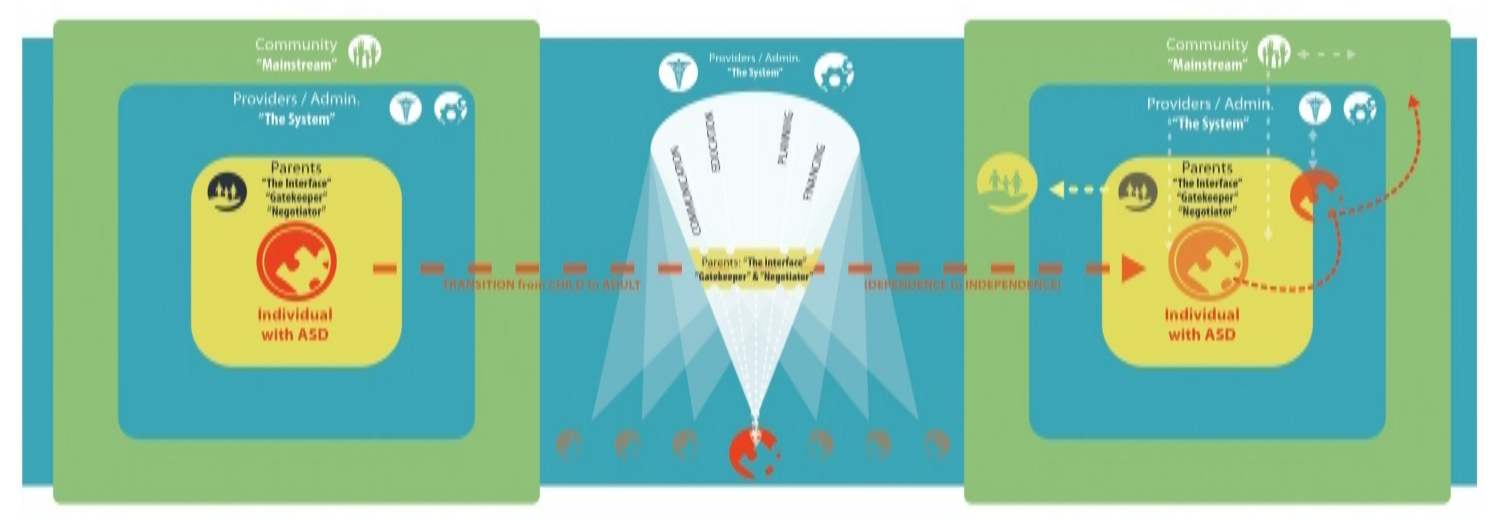

Figure $6.13 \mathrm{~A}$ series of visualizations created after the interview with the Director of C.A.S.T. that depicts the relationships between the three stakeholder groups: people (patients) with ASD, parents, and providers, as well as society ('community').

\section{A Nisonger Programs Coordinator}

The following interview took place with a programs coordinator and a student volunteer at The Nisonger Center for Intellectual and Development Disabilities, part of The Ohio State Wexner Medical Center. This Center is widely respected for their interdisciplinary research, education, outreach programs, and clinical services. This interview was less structured and more conversational. It revolved around better understanding the current program and service offerings in Columbus, Ohio for adults on the spectrum. In our discussion they mentioned that there were limited programs and services for adults with ASD; coaching, independent transportation options, socialization, outreach, and connectivity were areas that needed to be addressed specifically. They emphasized that support and learning cannot stop once a child turns eighteen. Adults with ASD need programs that support social interaction and continued enrichment. This program coordinator runs an eight-week social program called Aspirations for adults on the spectrum. Aspirations is aimed at providing social learning, enrichment, and social interaction opportunities for young adults ranging from the ages of seventeen to twenty-four through group support. Each 
week the members of this group meet at a local Barnes and Noble to have coffee and conversation. At the end of the eight-week program they have a pizza party at Donatos.

After discussing the scope of the C.A.S.T. project with the program coordinator and the student aid, we determined that Donatos, a local pizza eatery, might be the most comfortable venue to do a focus group with his Aspirations members for Phase 1 of the case study. In preparation for the session we made a plan to ensure the ASD participants would be engaged. He suggested that all of the instructions and questions be concrete, to use visuals as often as possible and avoid abstract concepts, to provide space and time for the participants to take breaks or regroup away from the collaborative environment, and to try to limit environmental distractions.

\subsubsection{Focus Group 1 with ASD Participants}

Following the guidelines laid out in the prior interview, a pre-session focus group was conducted with ten adults on the autism spectrum (nine male and one female), and one supportive staff member at Donatos. The aim of this session was to allow each person an opportunity to independently voice their thoughts and ideas, before potentially moving into a collaborative workshop with other stakeholders. Everyone was situated around a large table in a private space at the restaurant. By seating everyone around the same table, we could ensure that the participants were working on the same task. The music was turned off to reduce distractions and the windows allowed for natural light to penetrate the space. 


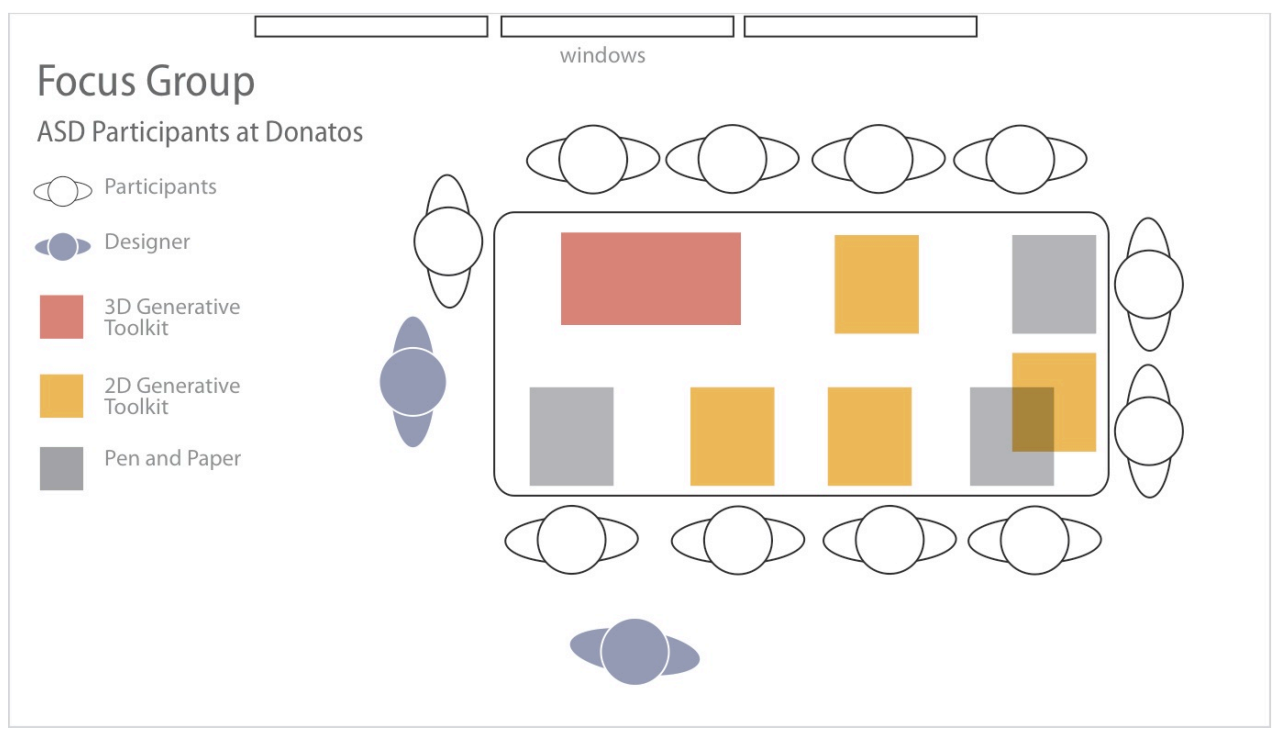

Figure 6.14 A birds-eye view of the space used for the focus group with ASD participants.

The structure of this focus group was divided into two parts. The first part was built around open discussion, creating a safe space to contribute thoughts and ideas about the clinic, or concerns and ideas beyond the clinic, and to share personal experiences. The second stage focused on speculative thinking and helping the participants to begin imagining their ideal clinic experience/environment using 2D and 3D generative design tools. Concrete verbal instructions were given on the agenda/objectives for the session and brief demonstrations were given on how to use the different materials like blocks and cutout shapes (in addition to drawing and writing) for generative Design Thinking to help communicate speculative ideas for a better future clinic.

Participants were asked to consider how the current healthcare clinic makes them feel, how it should make them feel, and if there were any barriers before, during, or after clinic visits that made their experiences unfavorable or difficult. This opened dialog to topics connected to four major themes: the physical environment of the clinic space, patient/doctor relationships and communication, navigation in the clinic and healthcare system, and the merits and unrecognized 
societal value of people on the spectrum. Within each of the themes that were discussed, concerns, needs, goals, and ideas emerged.

During our conversation regarding the typical clinic visit, we asked the participants to share which stages of the 'clinic experience' were the most problematic. Waiting in the lobby and in the exam room ranked second to talking with the physician, which was the most stressful (Figure 6.15).

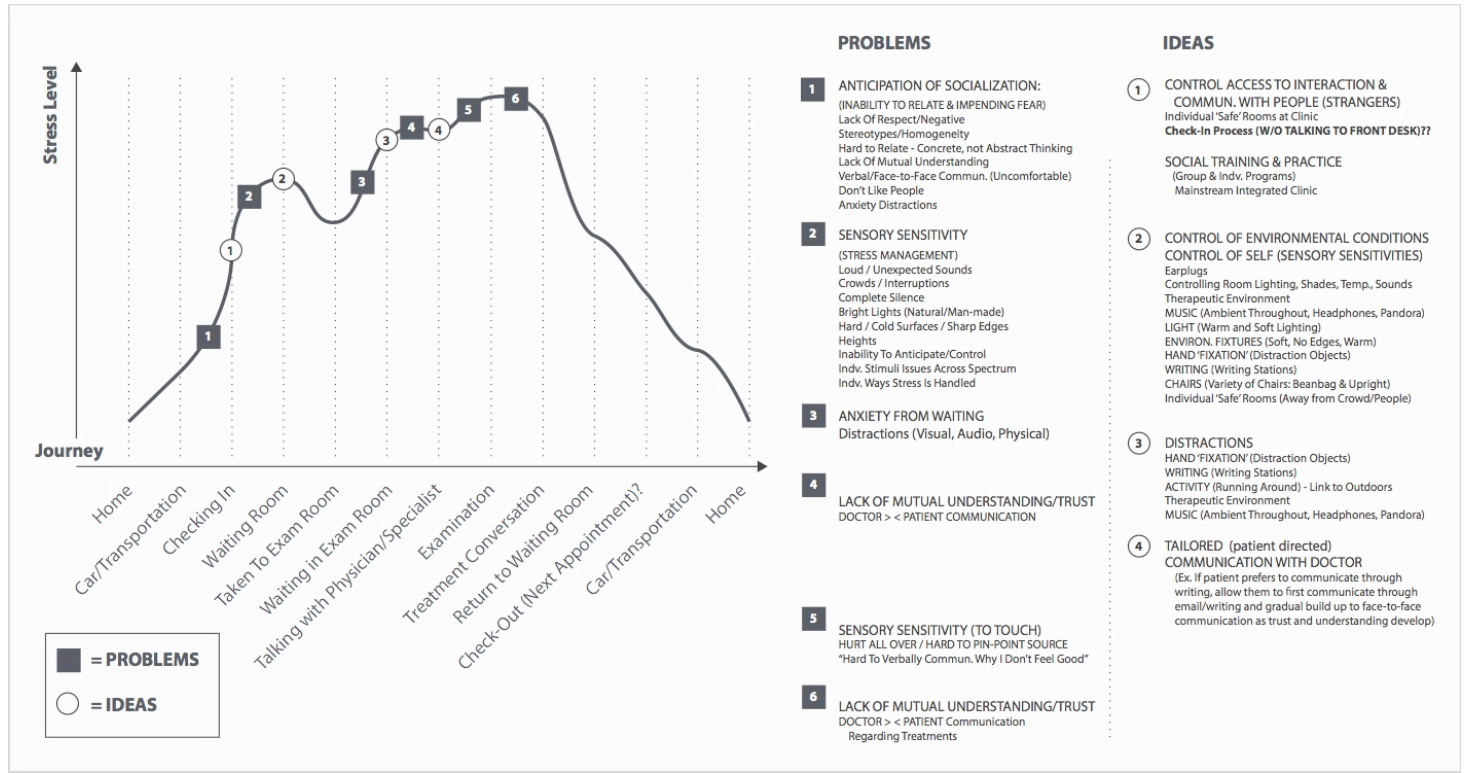

Figure 6.15 Clinic journey map with problems and ideas that were expressed in the focus group by the ASD participants.

Apprehension about going to a clinic visit is a negative feedback loop (Figure 6.16) stemming more from the fear of social interaction than from the actual physical checkup. The anticipation of social interaction with the doctor or other people in the clinic generates anxiety, which causes stress. Elevated stress heightens sensory sensitivities to sounds, lights, and touch, causing overstimulation. Overstimulation (sensory overload) makes it hard to concentrate, therefore making it almost impossible to socially interact. In their anxiety, they have self-fulfilled their fears 
and their emotions have spiraled out of control. Sensory sensitivity and communication seemed intertwined - they both need to be addressed.

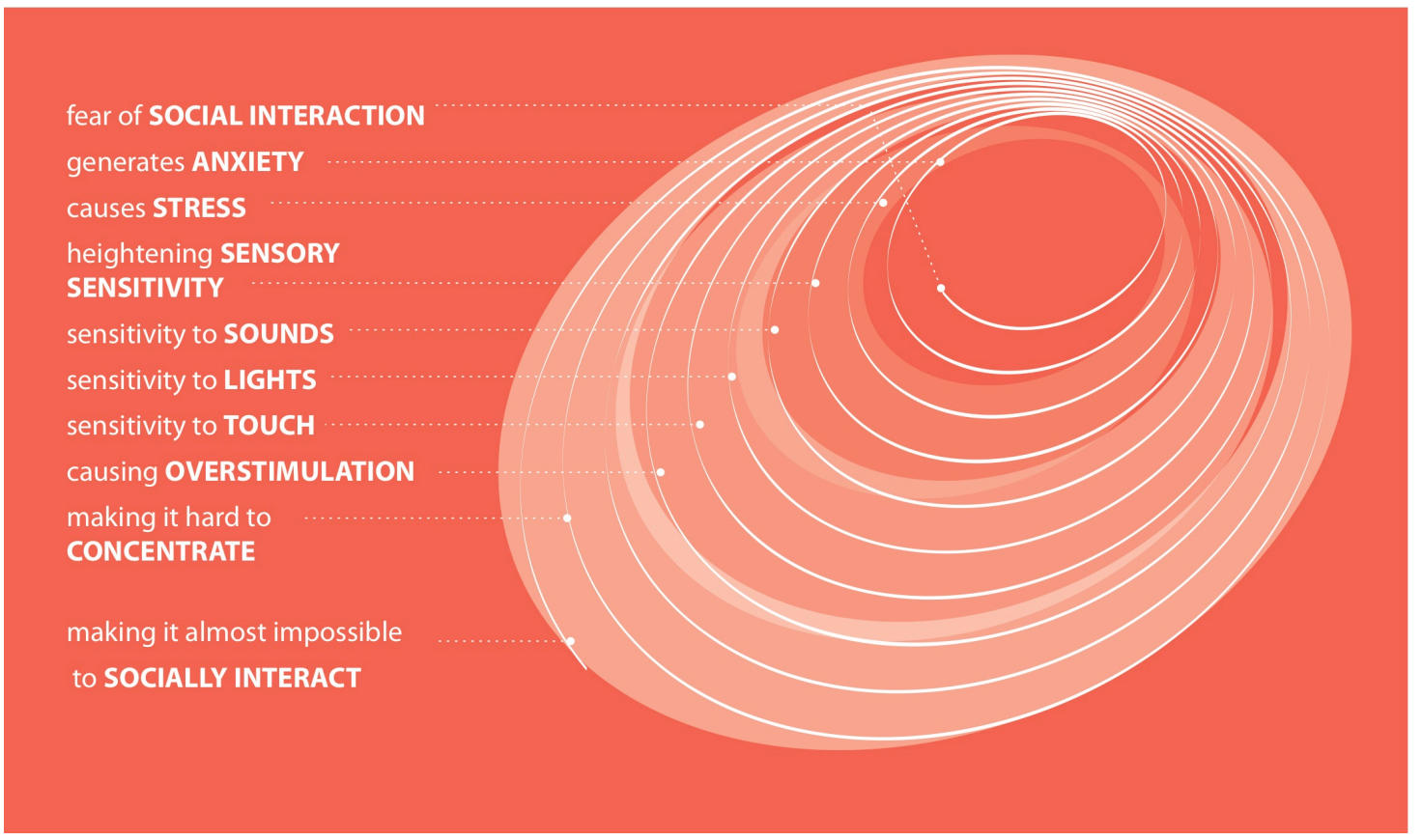

Figure 6.16 Visualization of the social interaction negative feedback loop, created after the focus group with ASD participants.

Sensory sensitivity to loud, unexpected sounds, crowds and interruptions, complete silence, bright lights, hard and cold surfaces, sharp edges and heights, and an inability to anticipate outcomes or control their environment all contributed to stress and anxiety from waiting and distractions in the waiting room that made the clinic experience unfavorable. Additionally, the lack of mutual understanding, communication, and trust with the physician made their experience uncomfortable. It is hard for this population to communicate face-to-face and it is hard for them to read between the lines. They are more comfortable with concrete dialogue that could take place on the phone or through remote technology. 
Ideas for the clinic experience began to emerge, which included tailored (patient directed) communication options with physicians, objects and activities in the waiting room that served as wait-time distractions, comfortable seating, and a therapeutic environment that afforded patients the ability to control their individual experience (earplugs or headphones, a 'safe' room away from crowds, and a connection to the outdoor environment). Acknowledging the ideas that were materializing through discussion, the participants were then instructed to visualize their concepts for the clinic (and beyond) through a making exercise. A limited supply of the generative components/materials (2D cutout shapes, paper/markers, and blocks) were offered to avoid overwhelming the participants, while also providing enough diversity to provoke new ways of thinking. The participants were asked to look over all of the materials that were offered, but did not have to use everything. The generative tools were intended to guide them towards future possibilities, and help them find ways to express the thoughts/ideas in their head in a more visual or concrete way (i.e., beyond verbal communication).
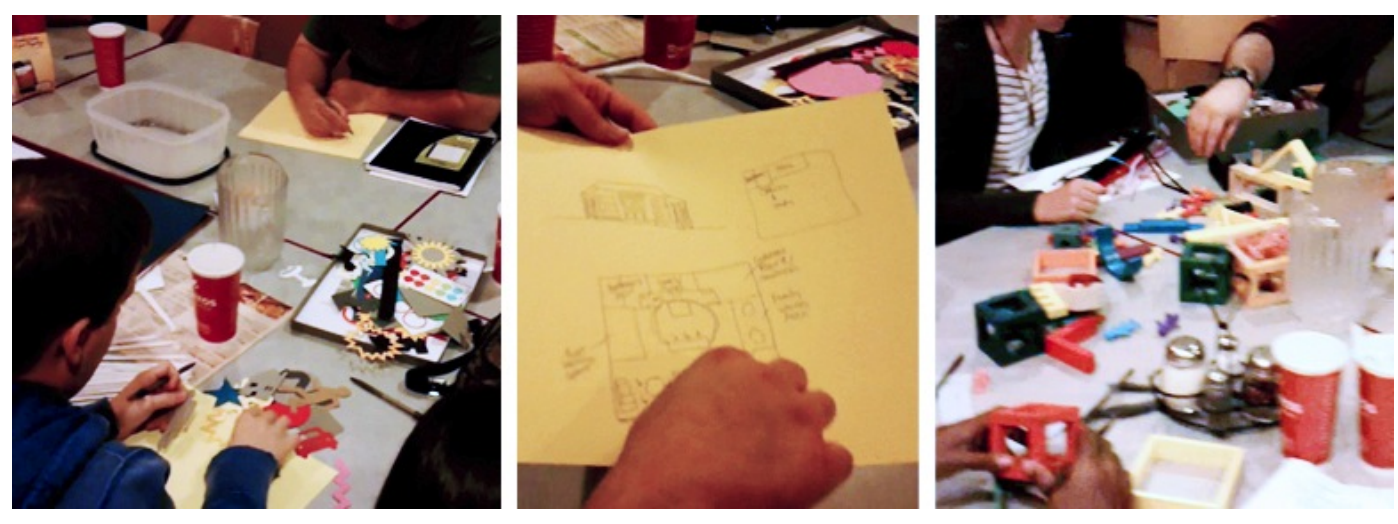

Figure 6.17 Photographs from the making activity during the focus group with ASD participants.

Some drew improved floor plans of the waiting room, one person built a better clinic waiting room using blocks, others listed items for the waiting room, sensory stimuli/sensory reduction ideas, and revised space layouts that could improve the overall experience and comfort of the clinic. Others discussed the need for custom communication channels between patients and doctors, 
and addressed needs and ideas beyond the clinic. One of the participants wrote a specific plan of communication between people on the spectrum and their doctors that began with communicating with the patient on their most comfortable level. Grandin also addressed the comfort many people with autism have with telephone conversations over face-to-face conversations because there is potentially less distracting visual input (Grandin, 2009, p.72). If the patient prefers to communicate through email/writing (like this participant), allow them to do so before and after the exam. Then, with the patience and guidance of the physician, help them to gradually build up to phone conversations, in-person conversations with a partition, and finally face-to-face communication as trust and understanding are developed overtime.

Open discussions and the making activity were video and audio recorded with the participants' permission. After completing their making task, each participant presented the ideas they had created or written down. During this time, other participants added to the ideas and needs that were being expressed. In particular, John (who was quoted at the beginning of this chapter) and another participant (a woman, who will be called Kristin) guided a majority of the conversation. A significant theme that they both wrote and spoke about concerned the need for self-advocacy and materials/service offerings at the clinic to support independent living and tolerance education. They, along with many of the other participants (who were less vocal) saw value in our addressing not only micro clinic needs, but also the bigger issues facing adults on the spectrum in this project. John and Kristin were the only two from this session that chose to participate in all of the following phases of the case study and were integral to the project. The third adult with ASD who participated in the following two phases could not attend this focus group.

\subsubsection{Focus Group 2 with Parents and Healthcare Providers/Administrators}

At this point six overall themes had emerged: connectivity, support, understanding, communication, improving quality of life, and empowerment. Within each of these themes were 
supportive insights and specific needs. These themes and insights were shared with parents and healthcare providers/administrators at the beginning of the second focus group. Three parents, two providers, and two healthcare administrators attended this focus group. The aim of this session was to afford the participants an opportunity to voice their concerns, unmet needs, and goals for the future, as well as help them to begin to discover new perspectives from each other. Unique to this session was an activity of mapping concerns, needs, and goals from their various viewpoints. This method was designed to help the stakeholders also begin to connect with one another and look for new meaning.

Using sticky notes, each group (i.e., parents in one group and providers/administrators in the other group) was tasked with writing down their top ten concerns, top ten needs, and top ten goals. This exercise took longer than expected. The parents remained seated at the main table on one side of the room, while the providers/administrators stood near the wall (with brown paper) where the sticky notes would be presented (shown in Figure 6.18). Audio recorders were placed near the groups to record conversations that took place during the activity. The conversation and body language among the parents seemed more passive and courteous, while the body language and behavior of the standing providers/administrators seemed more active - ideas and comments were shared simultaneously at times. It was during this observation that the value of a collective tool, that encouraged people to stand and interact over one that allowed them to sit and visit, was considered into the design for the digital prototype for Workshop 1. 


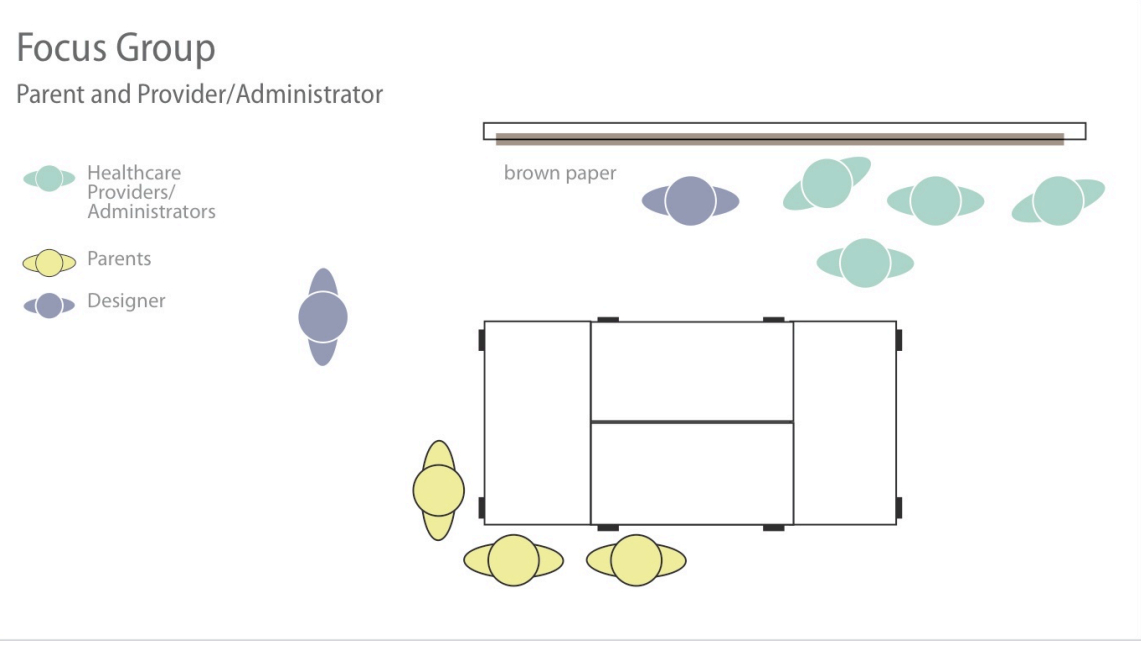

Figure $6.18 \mathrm{~A}$ birds-eye view of the space for the focus group with parents and healthcare staff.

After the writing of the sticky notes, each stakeholder group stood and presented their top concerns, needs, and goals to the other group. The group presentations were audio and video recorded so that what was shared during presentations could be compared and analyzed with what was discussed during the making session. The last part of the session was directed at collectively drawing connections between the expressed concerns, needs, and goals. This final part was not carried out, because time ran out during group presentations. With the time that remained, an organic conversation was facilitated between the groups about the similarities and dissimilarities in how the groups had first approached the topic, in order to begin to draw connections between the overall themes that were emerging.

Starting at opposite vantage points, both groups ultimately agreed on a lot of issues. The similarity in their views at the end was a surprise to everyone. The parents' concerns, needs, and goals began with a micro focus on the small and large challenges their child and their family unit faced during the process of transitioning. Topics ranged from puberty (which can often make an autistic person's sensitivity problems worse (Grandin, 2009, p.48)), dating and making friends, gaining independence, and what to do if the parent could no longer provide care. Daily concerns 
and needs were blended with far, future fears and dreams because the current ASD system was not addressing their greatest need, peace of mind. Providers, alternatively, began with a focus on 'the system'. They expressed big-picture concerns that impacted not only the well-being of their patients but the cohesiveness of the care system. Their concerns ranged from improved training and education for all providers (i.e., ASD specialized and non-ASD specialized physicians) to transparency and cohesiveness between providers (i.e., pediatrics to adult physicians, and ASD physicians to specialized/non-ASD physicians). Eventually both groups came to the conclusion that the issues connecting both the micro and macro viewpoints were optimal care, longevity, peace of mind, self-advocacy, and education. Figure 6.19 captures the priorities that were shared and illustrates that while the stakeholder groups started at opposite vantage points, both groups ultimately agreed on many root (core) issues in the middle.
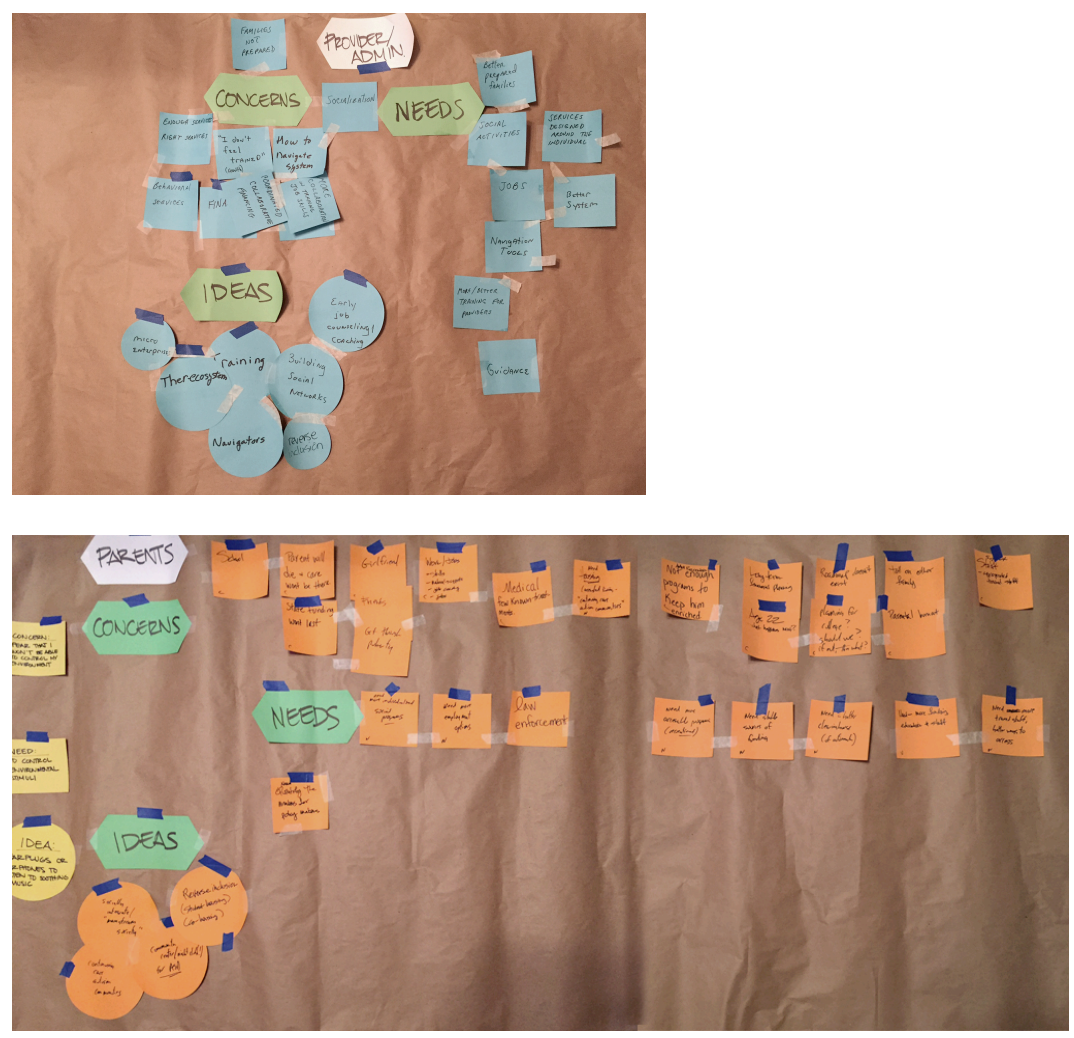

Figure 6.19 Parent and provider/administrator perspectives from Focus Group 2. 
Results of the Top 10 Concerns, Needs, and Goals Exercise

Starting at opposite vantage points, both groups ultimately agreed on a lot of issues in the middle.

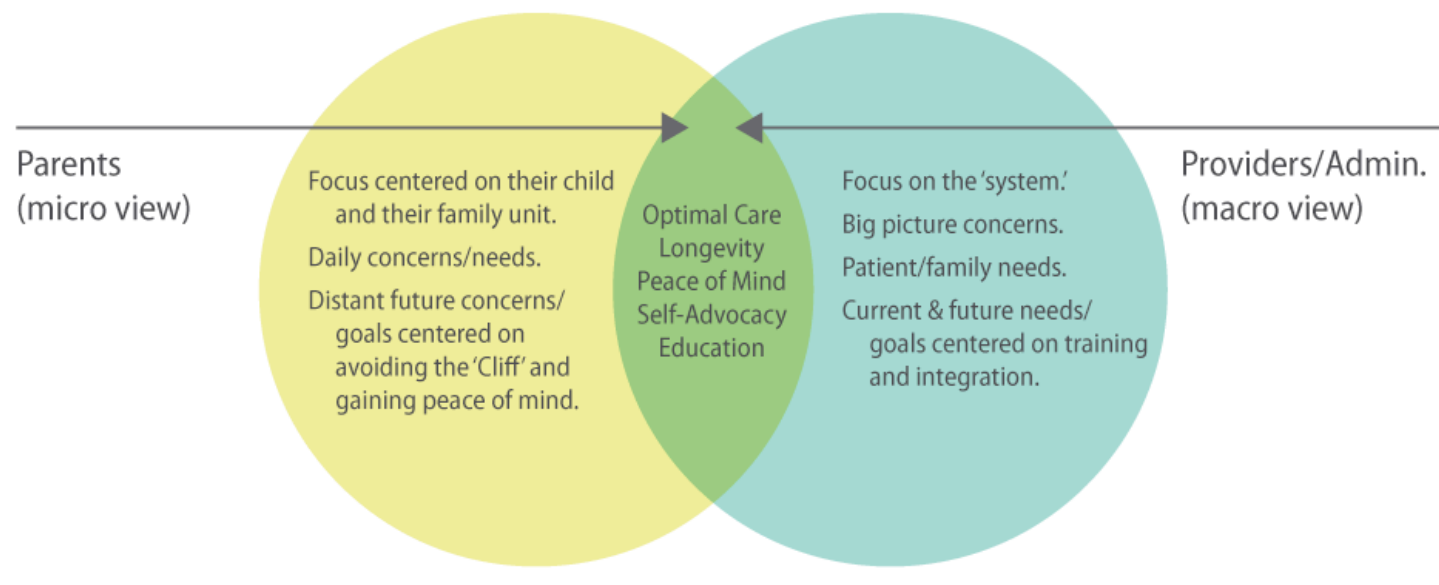

\subsubsection{Interviews to Prepare Presentations for Workshop 1}

The interviews that followed the two focus groups were intended to capture any last thoughts or ideas and to work with stakeholders on designing their presentations for Workshop 1. The presentations for Workshop 1 would be co-designed with the stakeholders interviewed in these sessions and vetted by the other stakeholders who had participated in the focus groups and been interviewed earlier. The intent for the presentations was to allow each group to tell their compelling case to the other stakeholders before the collective sensemaking activity in Workshop 1. A common visualization running through each presentation would be a journey map of key phases or obstacles in a person's life who has ASD. Within this journey, stakeholders would indicate which areas were of the 'highest' or 'lowest' priority to them. Developed with each stakeholder group in the follow up interviews, these journey maps would serve as comparative frames of reference during the presentations. The visualized maps illustrated each stakeholder's priority foci along the life journey. After the final presentation the three maps would be overlaid to show similar and dissimilar priority focus areas. Final presentations for each stakeholder group 
were sent out to members of their respective groups to review and offer additional comments/feedback prior to Workshop 1.

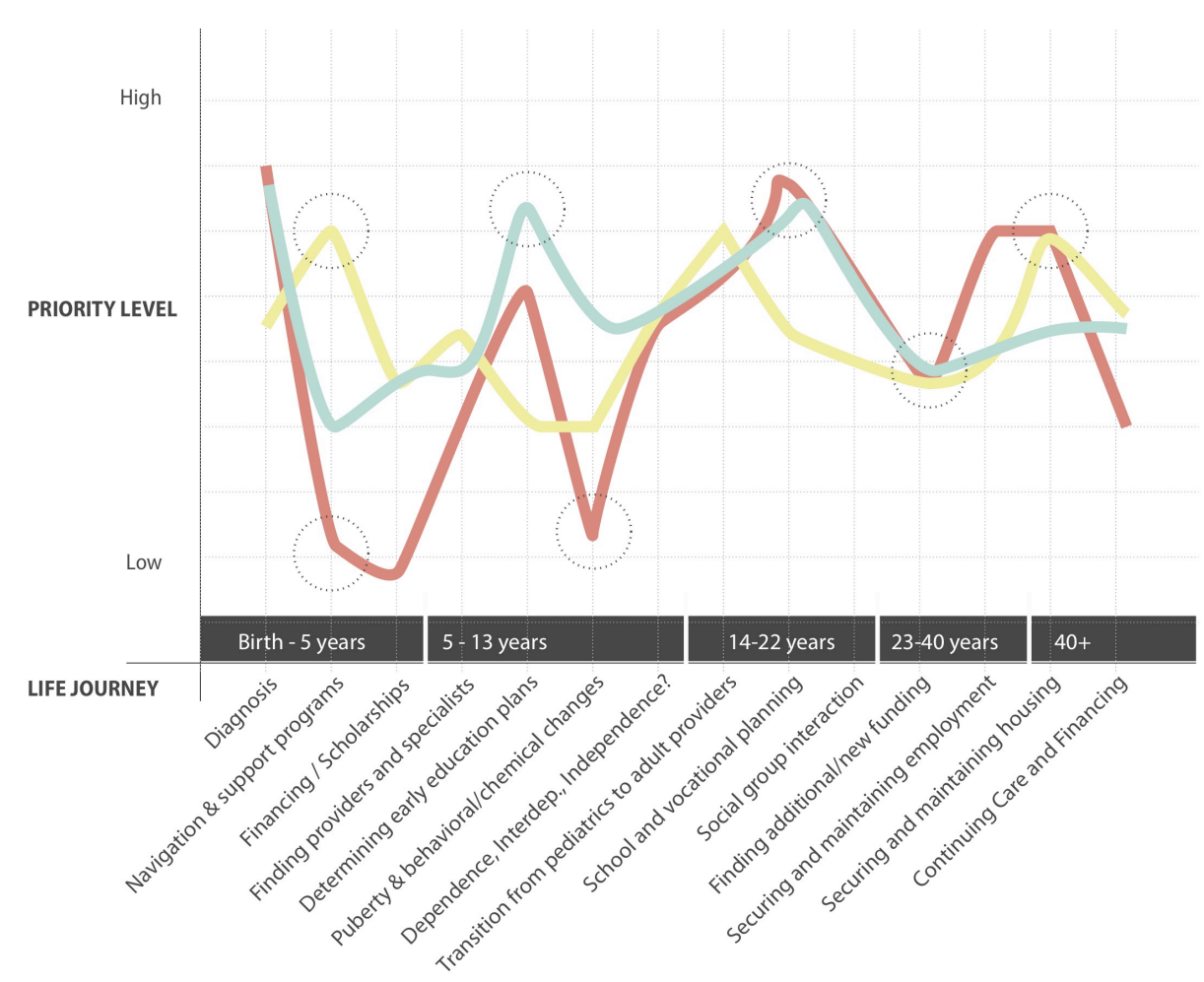

Figure 6.20 The life journey map (overlaid with all three stakeholder group priorities).

\section{Follow-up interview with another parent with an adolescent on the spectrum}

A follow up interview took place with one of the parents from the focus group session to co-create the presentation she would be giving on behalf of the parent stakeholder group in Workshop 1.

The discussion we had and the presentation we would design would be shared with the other parents prior to the workshop. Her vision for the clinic was that it would help to facilitate and care for the holistic (not just physical) needs of the adult on the spectrum and their families. The concerns of parents with children on the autism spectrum extend into many aspects over the course of their child's life, but the biggest needs for innovation fall in finding information and support programs, improving the transitional process, and determining whether or not their child 
will be able to live independently (e.g., securing and maintaining independent housing and continued care and financing). In her mind, the personalized care and support of the patient should also extend to their family. Given these concerns, she felt there was greater opportunity in looking at issues that transcended the clinic and provided an opportunity to collaborate with multiple stakeholders (from various points of view) about transitions. She felt that this would afford this collective group with a unique opportunity to expand the scope and discuss interconnected issues that may be of greater concern in the wicked problem. Ultimately, this interview primarily revolved around not only her daily needs as a parent, but with issues far greater than the clinic, such as the need for assistive technologies, affordable independent housing options, and disruptive innovations to counterbalance the limited amount of resources at their (the parents and adults on the spectrum's) current disposal.
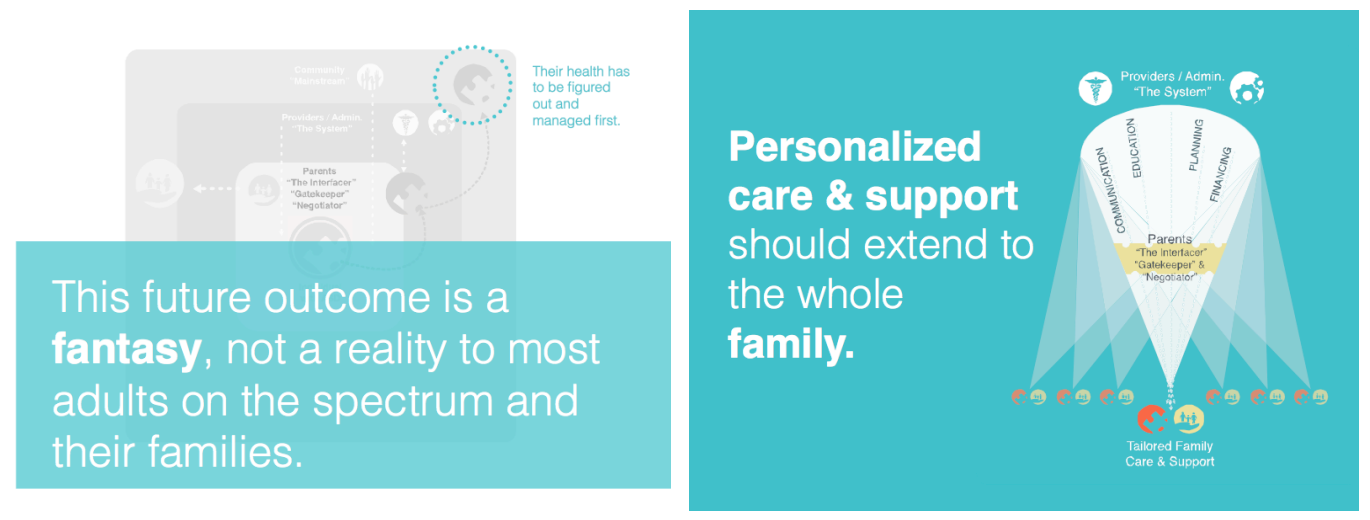

Figure 6.21 Samples of the parent presentation co-created in the pre-workshop interview.

\section{Follow up interviews with two adults on the spectrum - John and Kristin}

An interview was held with John and Kristin after the Donatos focus group to extract more of their thoughts and to help them build the presentation they would be sharing on behalf of their stakeholder group for Workshop 1. In the priority journey map they expressed that early diagnosis and early education planning are key areas that require more attention. Getting the right tools and 
support sooner than later was key to future success. Vocational planning and social group interaction need to continue through transitions and into adulthood. Those are important for being a more confident and well-rounded individual. Lastly, looking for better solutions and opportunities to help adults on the spectrum secure and maintain employment and housing into adulthood were important areas to explore because they had a large impact on the long-term well being of this population.

\section{public perception needs to change}

\section{'victim' $\Rightarrow$ innovator}

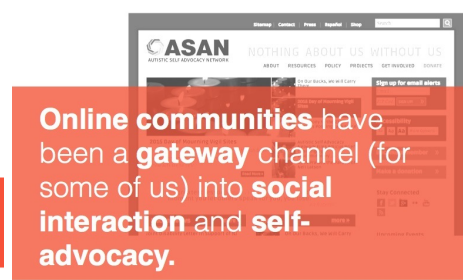

Recognize (our specializations)

Utillze (our specializations)

Our specializations can be harnessed (instead of
suppressed) to contribute to innovation \& progress.

Figure 6.22 Samples of presentation visualizations created from the information shared during John and Kristin's interview.

Most of the discussion centered on recognizing and nurturing the abilities (not disabilities) of people on the spectrum. John and Kristin pointed out that many people with ASD are detail oriented, critical thinkers. "We have heightened sensitivities, and (some would suggest) too much empathy." People on the spectrum constantly speculate about the future and are looking at things from a completely different perspective - they would do well in a futuring career. Everyone faces challenges, yet people with ASD do not seem to be given the same opportunities as neurotypicals to prove themselves - "we have untapped abilities." People with ASD may be very focused and detail oriented, but thinking in broad strokes (from a neurotypical perspective) can cause one to overlook the important details and miss the gaps. Getting distracted easily can lead to less focus and less productivity. Kristin offered that as pattern thinkers "we could contribute to homeland security, military intelligence and data technology fields. Our heightened focus and sensitivity to things like sounds could make us valuable on search and rescue teams." John further stated that 
"our ability to recognize system incongruities, fine details, and gaps could help a company identify skill gaps." They both adamantly stated that people on the autism spectrum can do new things and great things, "we just need to be given time and opportunity to get acclimated." The need for time and practice, and the importance of tapping into the specializations and fixations of people on the spectrum was also brought up by Grandin (2009). If a child or adult on the spectrum likes computers, use computers and games to help them learn. Computers and video games can also help them interact with others and build social confidence.

Merit in society is unbalanced. Society punishes intellect and minimizes 'internal talents', while rewarding social abilities and outward appearance. Often it seems that value is measured by social ability over intellect. "We are here, participating in this C.A.S.T. project, to advocate for ourselves and people on the spectrum and to share our perspective living with ASD." Thousands of other people on the spectrum, like John and Kristin, are self-advocating online. Online communities have been a gateway for some with ASD into social interaction and self-advocacy. However, even within these networks "we do not agree." "How are neurotypicals supposed to respond? We have to have a more united front. "Additionally, shared interests in television shows and movies like Game of Thrones were cited as ways to help to initiate conversation and changes in perception between the neurotypical and ASD communities - "we may be more similar than different. More inclusion and more representation is what the world needs."

\section{Follow up interview with the Director, a nurse, and a pharmacist from C.A.S.T.}

Following the discussion with John and Kristin, an interview was conducted with three providers from C.A.S.T. (who were not able to attend Focus Group 2). Information was collected during this interview to begin shaping the presentation the Director of C.A.S.T. would give in Workshop 1 on behalf of the provider/administrator group. The current ASD system has limited resources to properly care for the growing ASD population. Due to the limited resources and expertise of ASD 
primary care physicians, mainstream extenders and specialists (outside the ASD system) are needed in supportive roles. However, it is often difficult for parents and ASD primary care physicians to find mainstream providers and specialists who will care for an adult with autism. This issue stems from both an education and communication gap, not only between parents and providers, but also between mainstream providers and providers within the ASD system. Factors that contribute to the gaps among providers are related to social stigmas, lack of time, and a lack of comfort. There is an opportunity, therefore, to better educate and train mainstream providers and specialists so they feel more comfortable caring for patients on the spectrum.

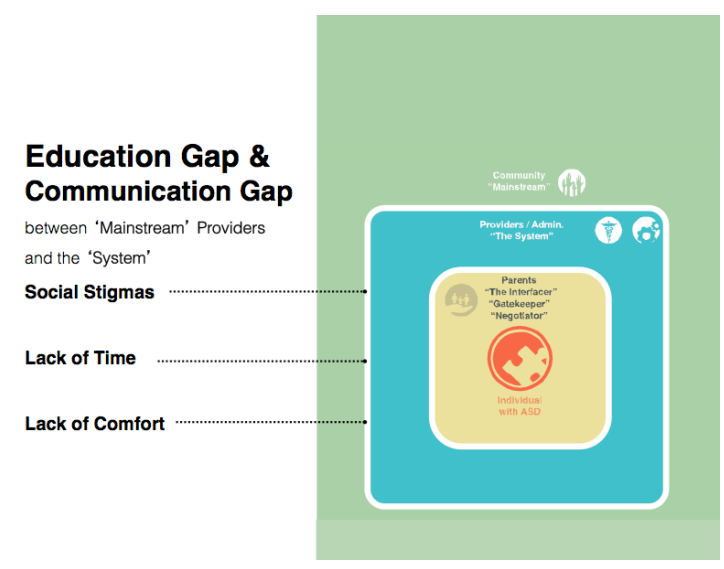

\section{Education Gap}

Parents

(between 'The Interfacer'

and the 'System')

How to Navigate

Adult Healthcare

Treatment Options

Financing Options

and Future Planning

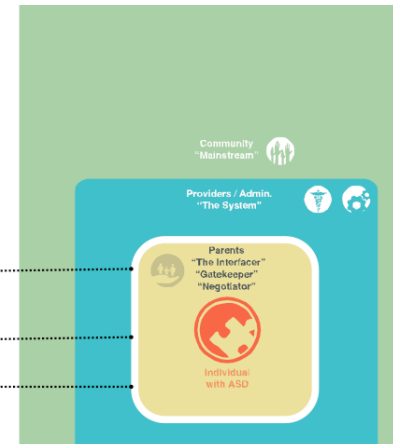

Figure 6.23 Samples of the provider/administrator presentation visualizations showing education and communication gaps.

Providers need to better understand where they fit in the system so that they know where and how to best care for patients and their families. Unlike cancer, autism care is spread out. When someone is diagnosed with cancer a connected cancer 'care team' is formed around their individual needs. When a child or adult is diagnosed with autism, medical information and support resources are scattered across multiple physicians and specialists who remain, for the most part, unconnected. To enhance care coordination the roles of the providers in the ASD system (and outside specialists) need to be defined so their processes are more collaborative and fluid. For 
instance, pharmacists need to be brought in sooner in the transition process to help families and new physicians transfer patient histories and understand treatment needs. A more gradual and guided transition process (introducing the adult clinic/physicians steadily and earlier (e.g., when the child turns 13)) would make the process easier for patients and families. Improved communication and connectivity to the 'Care Team' needs to extend to patients and families. A more connected and streamlined healthcare network (involving the patients/families) would help parents and patients (as well as outside providers) more easily navigate the network.

Shown in the map in Figure 6.24, early diagnosis and determining early education plans were of high priority to providers, along with helping people on the spectrum reach their full potential after transitioning into adulthood. In addition to creating their priority map the providers were also interested in hearing some of the thoughts that had been shared by the ASD participants. The providers were shown visuals that were developed from the Donatos focus group, secondary research, and from the discussion with John and Kristin. They were particularly drawn to the sensory overload diagram and the bee visual in Figure 6.25 (inspired by secondary research shown below). They found the visuals to be eye-opening and informative. 


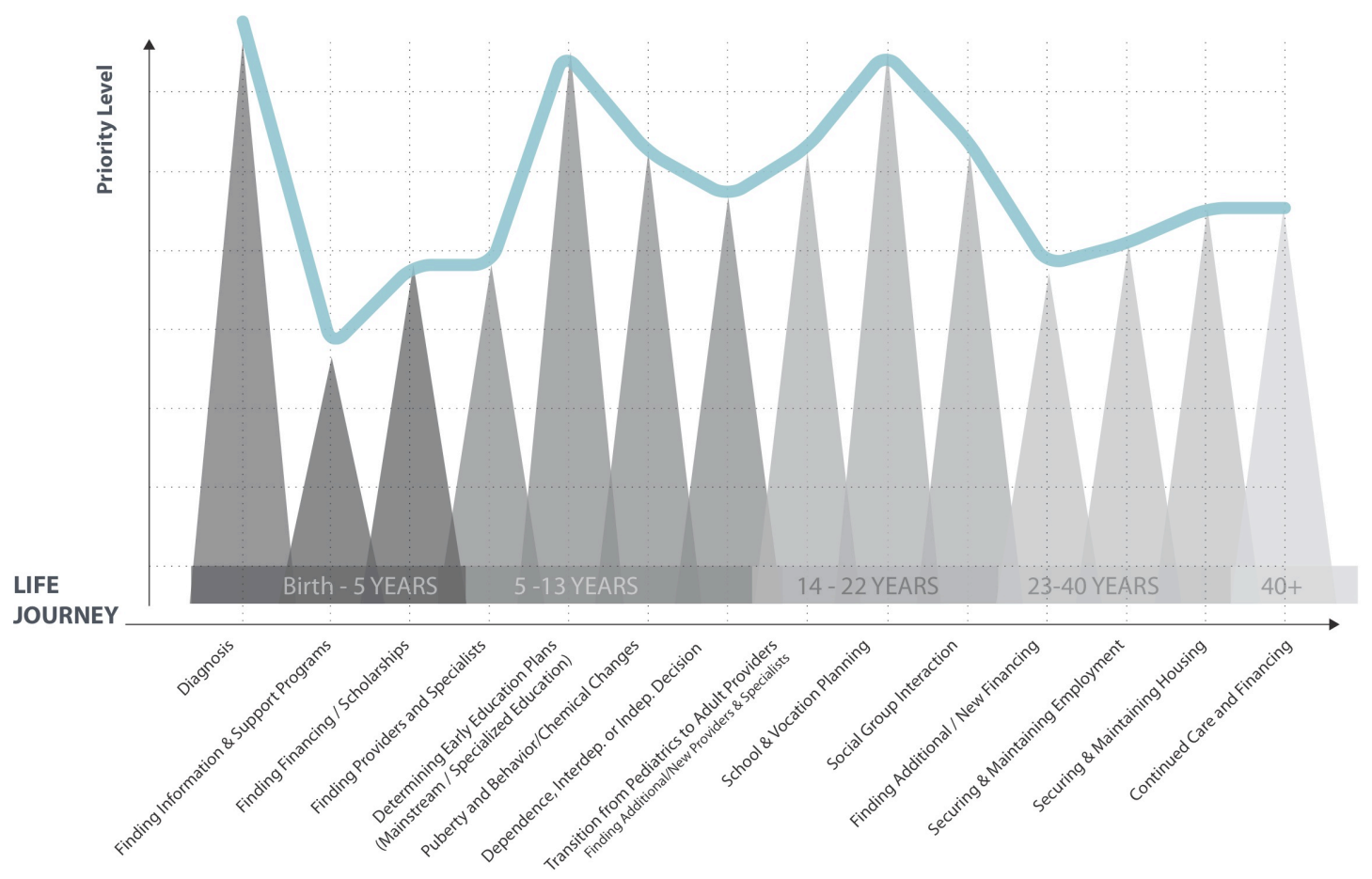

Figure 6.24 Priority life journey map shared by the providers.
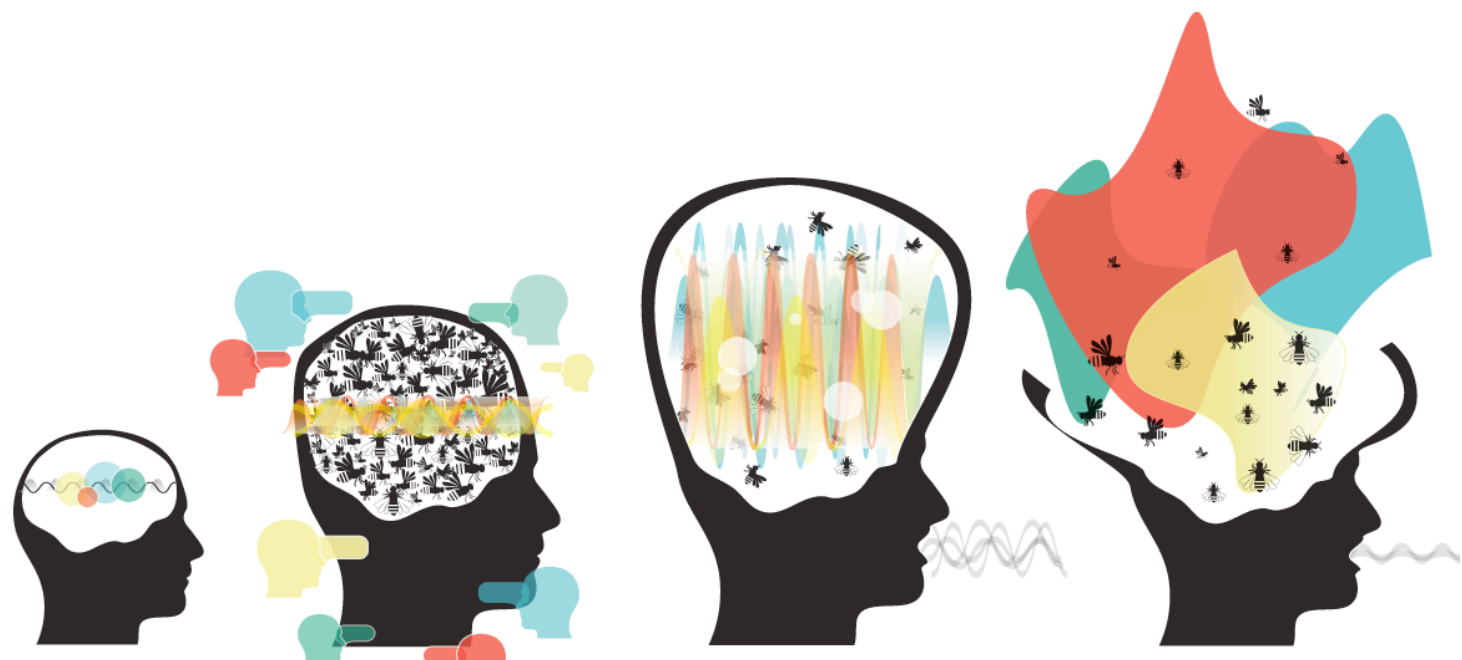

"Sensory overload is the worst feeling in the world!!! I get it when I see too many people walking past me or if I hear too many noises at one time. For example, in a room of people talking, my brain tunes into everyone else's conversation and I struggle to filter the voices out and listen to the carer I am with...When I get sensory overload, it is like I have $\mathbf{1 0 0}$ buzzy bees in my head and my head hurts a lot and feels like it will go "bang" like a balloon. It is the most uncomfortable thing ever!!! I have to try to bang my head on things to try to relieve the pressure in my head to stop the feeling. While I am experiencing sensory overload, I find it very hard to talk or make any sentences, as my speech just won't come out as I want it to and I can't make the words make sense. "Sophie

Figure 6.25 A visualization representing sensory overload, illustrated from secondary research. 


\section{An interview with the Director of Nisonger}

The priority map and the presentation materials that had been developed with the providers at C.A.S.T. were shared with the Director of Nisonger. In addition to the concerns that the C.A.S.T. providers had mentioned, the Director of Nisonger also wanted to discuss the impending 'Cliff', the challenges and barriers faced by most adolescents and families when their child transitions out of high school and into adulthood. Issues related to the "Cliff' revolved around transitional services that need to be offered and various opportunities for adults on the spectrum to maintain friendships and social activities, in addition to helping them figure out the next step, whether that is in a post-secondary program or a job. Children with autism have more services and support options, but as soon as that child becomes 21 , the support drops. Until recently, a lot of the funding has gone towards finding cures instead of helping those who currently have autism, but now more attention is being paid to addressing needs and opportunities for adolescents and adults with autism. People with autism want to connect with others, but many struggle with understanding how to communicate or why they are not good with socialization. Services and programs that support social interaction are just as important as physical care. Nisonger and The Ohio State University offer support and enrichment opportunities for adults to maintain social interaction and get continued training and education including work skills and soft skills, but many of these resources and services are disconnected or hard to find.

Many of the issues related to transitions and the 'Cliff' stem from the fact that there are limited resources. Adults require more specialized care beyond primary care, but many adult healthcare specialists do not have the training or knowledge that is necessary to know how to care for them properly. This puts more pressure on the primary care physician. People with autism have the same life expectancy as neurotypicals, but adults on the spectrum are not offered the same quality of support. They are harder to work with and therefore many specialists are afraid to take them as patients. Given the right tools inside and outside the clinic, a parent may be better able to 
explain, for instance their child's behavior, or help an adult on the spectrum learn how to better communicate their frustrations or fears with a provider instead of misbehaving. Families need reassurance that their child will be taken care of, yet at the same time, parents also need to gain a broader scope of the issues providers face in the transition process. They need to better understand the care the primary physician can provide. Working with physicians to build longterm plans (like 'Vision Plans') could help both the parent and providers.

Lastly, his main concern (related to the clinic) was that it would be tailored to fit needs of a finite group. Many of the suggestions that had been made in this interview and others could benefit not only patients on the spectrum, but also patients with other disabilities. To increase the volume of the clinic and make it sustainable he believed that it should cater to populations beyond autism.

\section{Follow up interview with high-ranking providers at $\mathrm{NCH}$}

The last interview was conducted with two high-ranking providers at $\mathrm{NCH}$. The objective was to review topics that had been discussed with the C.A.S.T. providers and the Director of Nisonger, and to get their feedback on the information and visualization that were being developed for the provider presentation. Providers and administrators from OSUWMC (including Nisonger and C.A.S.T.) and NCH would be present in Workshop 1, so it was important that the case being presented by the Director of C.A.S.T. on behalf of the healthcare provider/administrator group also represented the views from the pediatric side. The providers at $\mathrm{NCH}$ were in agreement with what had been discussed, with the addition of a visual 'patient passport'. The 'passport' would have few words and instead use icons to communicate key information customized to the unique needs/specializations of the patient. It would also offer 'keys to a successful visit'. The passport could be both paper and digital, carried with the patient from one provider to another. 


\subsubsection{Summarizing Phase 1}

The culmination of shared thoughts and ideas from the interviews and focus group sessions revealed that the unmet needs of the clinic were minor in comparison to interlaced issues and opportunities that needed to be addressed in the greater web of transitions and the long-term well being of people on the spectrum and their families. At the root of many of these issues was the realization that there was poor education and communication, and a limited amount of resources. Incremental changes were not going to help us tackle many of these issues. Instead we would have to look for disruptive innovations and opportunities to rethink the needs and goals of the ASD community.

In reviewing the information gathered during Phase 1, it was apparent that the participants had a hard time only voicing needs and goals and working through the levels of understanding (Ackoff, 1989) without also offering ideas or suggesting design opportunities in the process. The activity of bouncing back and forth between needs, sensemaking, and ideas continues into Workshop 1 as well, before we reach the ideation/prototyping phase (Workshop 2). Sanders (2015) describes this behavior as 'Design Wisdom,' (Figure 6.26) where Design Researchers and Designers play in a middle Design space between defining "the world as it is today" (analytical side) and exploring "the world in the future." Sanders (2015) further explains that "when working with wicked problems and/or large systems that are dynamically changing...we need to play on both sides...to address the challenges that Design is being asked to consider today." The expression of ideas, along with sharing needs and making sense in Phase 1 and Phase 2, emerged organically and triggered deeper, richer conversation as CoDesigners worked up from pieces of data to overall insights. The collective team (Designers and CoDesigners) worked on both sides of the Design space simultaneously. For instance, after interviews, as new information was presented in the form of needs and/or goals, visualizations and journey maps were designed and used to provoke further conversation in later interviews and in the presentations in Workshop 1. At each phase, 
ideas that were engendered during the inquiry or sensemaking stages were acknowledged and recorded, along with the expressed unmet needs and goals of the stakeholders, to be used/referenced in later sessions.

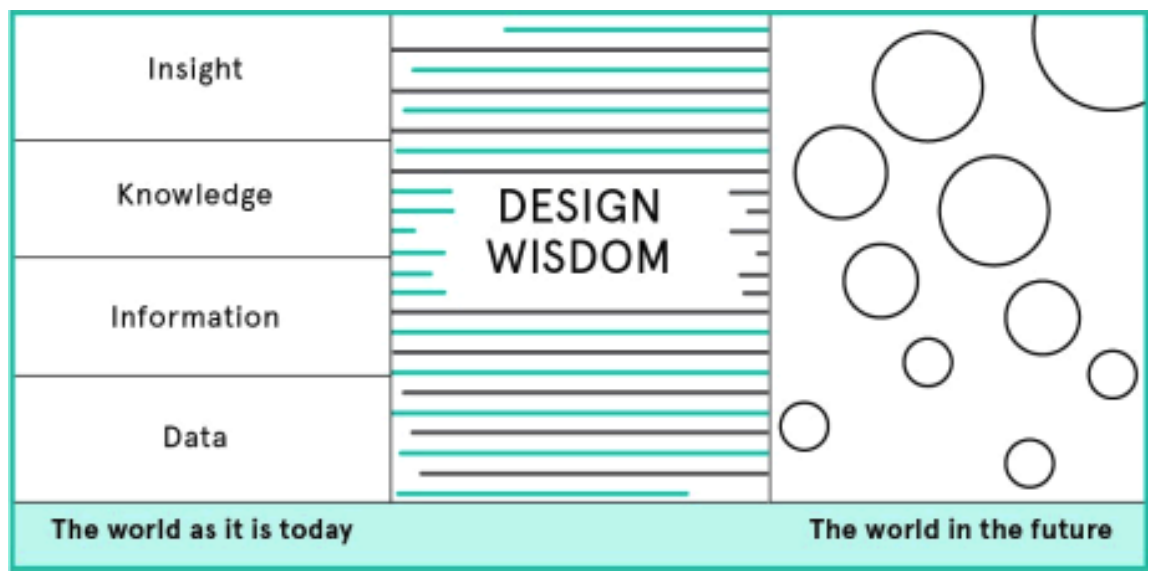

Figure 6.26 Sanders' (2015) framework for Design Wisdom - bridging together the analytical side and generative side.

A majority of the content (needs/goals and ideas) in the stakeholder presentations looked at the wicked problem from a systemic point of view instead of touching on direct issues at the clinic. It became apparent in creating these presentations that to tackle transitions in ASD and identify the true and sustainable value of the clinic, we would be broadening our scope in Workshop 1 and exploring more pressing root problems like communication, education, and peace of mind, as well as long-term goals and self-advocacy needs directed at continuous holistic health.

\subsection{The Approach for Phase 2: Workshop 1}

The visualizations and qualitative data collected over the course of Phase 1 fed into stakeholder presentations that would be given by a representative from each group at the beginning of Workshop 1. The presentations were intended to help each group tell their compelling case to the other stakeholders. The aim of this discovery and empathy exercise was to promote shared 
understanding prior to connecting with the data and seeking meaning among the needs, goals, and ideas related to the wicked problem collaboratively during the sensemaking activity. The presentations were designed to be mostly visual and guide the presenter in not only stating facts but also communicating unique personal narratives and comparative visual maps to help the other stakeholders not only discover, but immerse themselves into different perspectives.

In addition to co-designing visual presentations for each stakeholder group, the insights from all previous research were collected and organized into twenty-four key needs and twenty-four key goals, and compiled into eight categories. Four categories related to needs: Communication, Education, Planning and Finance. The other four categories aligned with goals: Optimal Care/Service/Support, Maximize Potential, Socialization, and Control. This information would be used in the second half of Workshop 1 for sensemaking and visual mapping. The categorized needs/goals were sent to each participant to review prior to Workshop 1. 


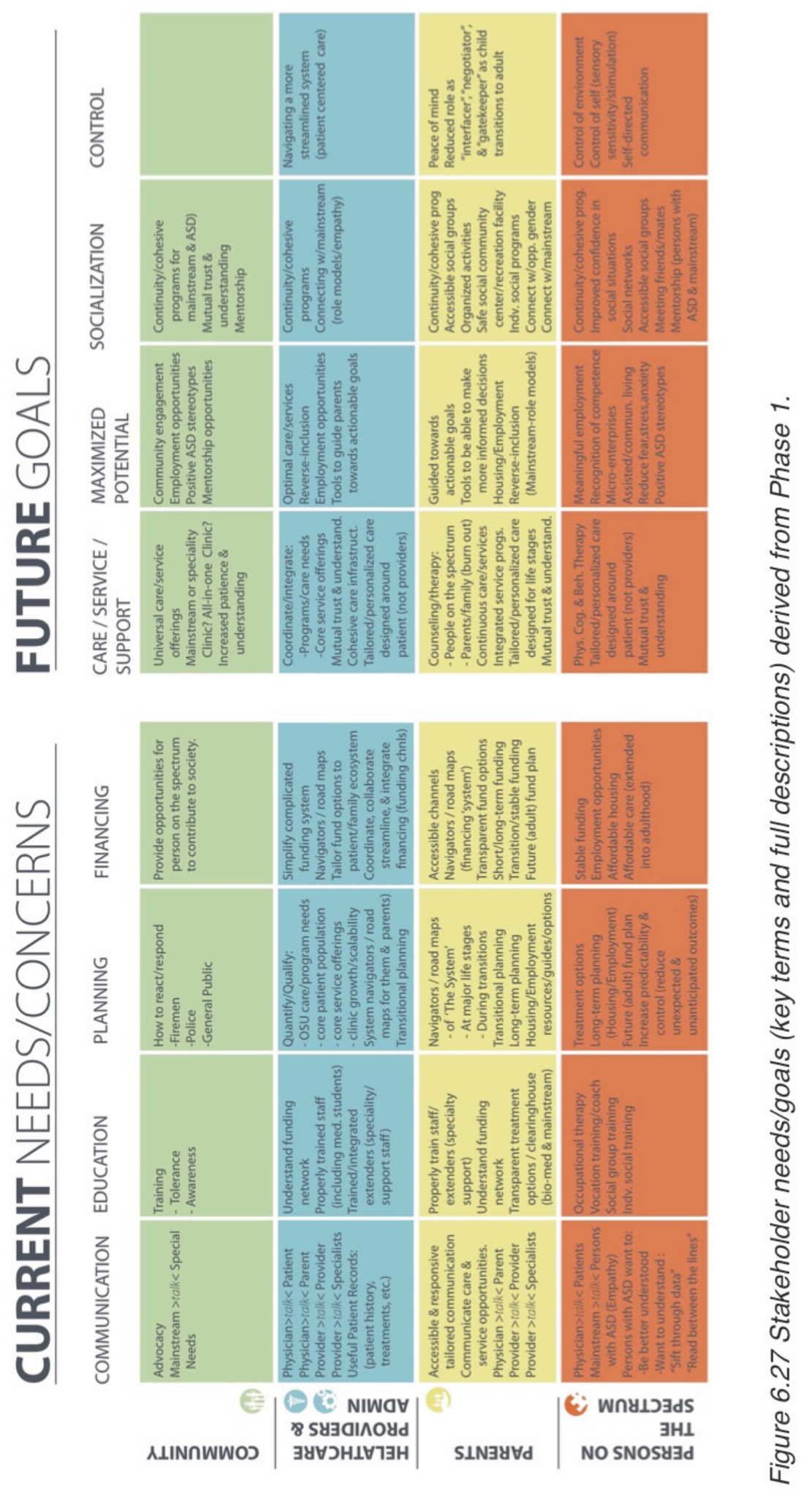




\section{NEEDS}

\section{Communication}

\begin{tabular}{|c|c|}
\hline Sell-Advocacy & $\begin{array}{l}\text { Adults with ASD and their parents need tio be better } \\
\text { understood by the mainstream community. }\end{array}$ \\
\hline Tailored to Families & $\begin{array}{l}\text { Adults with ASD and their caregivers need communication } \\
\text { channels to their healthcare providers that are more tailored } \\
\text { around their needs and ecosystem. }\end{array}$ \\
\hline Accessible \& Responsive & $\begin{array}{l}\text { Adults with ASD and their caregivers need communication } \\
\text { with hoalthcare administrators and providers that is more } \\
\text { accessible \& responsive. }\end{array}$ \\
\hline Provider/Specialists Continuity & $\begin{array}{l}\text { Healthcare providers, specialists, and administrators need } \\
\text { more collaborative, coordinateted, and integrated } \\
\text { communication channels in order to more seamlessly share } \\
\text { information with each other. }\end{array}$ \\
\hline Useful Records & $\begin{array}{l}\text { Healthcare providers need more useful patient records } \\
\text { (history, behavior tendencies, past/current treatments) during } \\
\text { transitions. }\end{array}$ \\
\hline Transition Continuity & $\begin{array}{l}\text { All stakeholders need providers and specialists/extenders to } \\
\text { have more seamless communication channels between one } \\
\text { another, especially during the transition from Pads to Adult } \\
\text { physicians. }\end{array}$ \\
\hline
\end{tabular}

Planning

\begin{tabular}{|c|c|}
\hline Core Programs/Care/Services & $\begin{array}{l}\text { Healthcare administrators need to quantify and qualify core } \\
\text { programs, care options, and service offerings at OSUMC. }\end{array}$ \\
\hline Core Patient Population & $\begin{array}{l}\text { Heatithcare adminisistrators need to better understand the core } \\
\text { patient topulation they should be seving with the clinic. }\end{array}$ \\
\hline Long-Term: Housing/Employment & $\begin{array}{l}\text { Parents and healthcare providers/admin. need to develoo } \\
\text { teilored (and general) road maps for long-term planning: } \\
\text { Housing/Employment/Care }\end{array}$ \\
\hline Short-Term: Treatments & $\begin{array}{l}\text { Parents and healthcare providers/admin. need to develop } \\
\text { tailored (and general) road maps for short term planning: } \\
\text { treatment options, care, and supoort. }\end{array}$ \\
\hline Transitional Planning & $\begin{array}{l}\text { Parents and healthcare providers/admin. need to develoo } \\
\text { tailored (and general) road maps to plan and prepare for } \\
\text { transitions and certain stages of life (like puberty). }\end{array}$ \\
\hline Navigation/Road Maps & $\begin{array}{l}\text { Parents and healthcare providers/admin. need navigation } \\
\text { tools to help guide them through treatment, care, and financial } \\
\text { options within the 'system'. }\end{array}$ \\
\hline
\end{tabular}

\section{GOALS}

\section{Optimal Care/Service/Support}

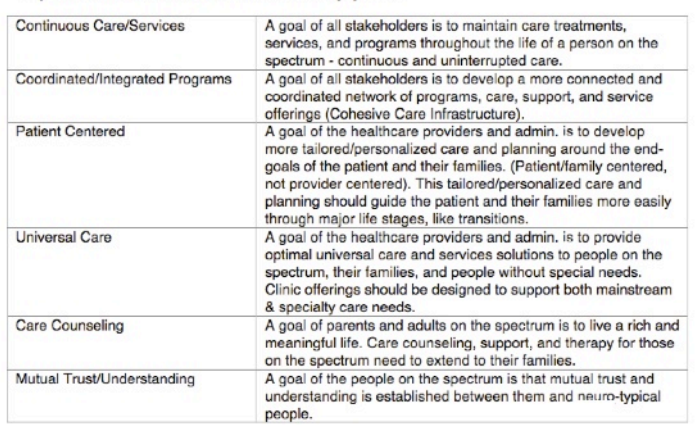

\section{Socialization}

\begin{tabular}{|c|c|}
\hline Integrated Programs & $\begin{array}{l}\text { A goal of parents is to improve the continuity of social programs } \\
\text { and organized activities for people on the spectrum. }\end{array}$ \\
\hline Indv/Group Social Programs & $\begin{array}{l}\text { A goal of parents is for their child on the spectrum to have both } \\
\text { group and individualized social training programs. }\end{array}$ \\
\hline $\begin{array}{l}\text { Social, Physical, Mental } \\
\text { Stimulation }\end{array}$ & $\begin{array}{l}\text { A goal of parents is that their child maintains social, physical, and } \\
\text { mental stimulation. These objectives could be satisfied at a safe } \\
\text { community facilityirecreation center. }\end{array}$ \\
\hline Accessible Social Network & $\begin{array}{l}\text { A goal of parents and people on the spectrum is to make social } \\
\text { groups and networks (online and offline) more accessible and } \\
\text { easier to enter. }\end{array}$ \\
\hline Social Confidence & $\begin{array}{l}\text { A goal of people on the spectrum is to improve their confidence } \\
\text { and reduce their anxiety in social situations. }\end{array}$ \\
\hline Interaction Opportunities & $\begin{array}{l}\text { A goal shared by all of the stakeholders is that people on the } \\
\text { spectrum connect and interact with one another, the opposite } \\
\text { gender, and with the mainstream. Having friends and finding a } \\
\text { partner is important to having moaning and enrichment in life. } \\
\text { Having mainstream role models, mentorshiss, and friendships } \\
\text { could not only improve socialization, but also empathy. }\end{array}$ \\
\hline
\end{tabular}

\section{Education}

\begin{tabular}{|c|c|}
\hline Proper Training & $\begin{array}{l}\text { Parents, providers, and admin. need proper training in } \\
\text { understanding the 'system' and caring for adults on the } \\
\text { spectrum. }\end{array}$ \\
\hline Transparent Treatment Options & $\begin{array}{l}\text { Parents need a plattorm (like a clearinghouse) to become } \\
\text { more informed on providers, programs, and various treatment } \\
\text { options (both hiomed \& mainstream). }\end{array}$ \\
\hline Trained/Integrated Extenders & $\begin{array}{l}\text { The training for providers, admin, extenders, and specialty } \\
\text { support staft needs to be more integrated and begun early in } \\
\text { medical school. }\end{array}$ \\
\hline Social Training & $\begin{array}{l}\text { Adults on the spectrum need to have more opportunities for } \\
\text { social training, in both group and one-on-one settings. }\end{array}$ \\
\hline Occupation \& Vocation Training & $\begin{array}{l}\text { Adults on the spectrum need to continue to have occupational } \\
\& \text { vocational training/ therapy as they grow. Continuous care } \\
\text { is needed as they age. }\end{array}$ \\
\hline Tolerance/Awareness & $\begin{array}{l}\text { The mainstream public needs more tolerance \& awareness } \\
\text { training - how to react \& respond (public/emergency services) }\end{array}$ \\
\hline
\end{tabular}

\section{Finance}

\begin{tabular}{|c|c|}
\hline Streamlined Funding Chann & $\begin{array}{l}\text { Parents and healthcare providers/admin. need more } \\
\text { simplified/streamlined/integrated funding channels. }\end{array}$ \\
\hline Navigating Funding Network & $\begin{array}{l}\text { Parents and healthcare providers/admin. need a better way to } \\
\text { understand and navigate the current funding network. }\end{array}$ \\
\hline ordinated/Connected Network & $\begin{array}{l}\text { Healthcare providers and admin. need a more coordinated and } \\
\text { connected funding network. }\end{array}$ \\
\hline anspare & $\begin{array}{l}\text { Parents need more transparent and accessible funding options, } \\
\text { especially as their child grows into adulthood. }\end{array}$ \\
\hline table $F$ & $\begin{array}{l}\text { Parents and adults on the spectrum need more stable funding } \\
\text { options during the transition phase and into adulthood } \\
\text { (shortlong-torm funding plans). }\end{array}$ \\
\hline Tailored Funding Optic & $\begin{array}{l}\text { More tailored funding options need to be put in place based on } \\
\text { the patientiamily ecosystem. }\end{array}$ \\
\hline
\end{tabular}

\section{Maximize Potential}

\begin{tabular}{|c|c|}
\hline Community Engagement & $\begin{array}{l}\text { A goal of parents and people on the spectrum is to provide more } \\
\text { mechanisms for self-advocacy, empathy, and community } \\
\text { engagement, like reverse-inclusion. }\end{array}$ \\
\hline Meaningful Employment & $\begin{array}{l}\text { A goal of people on the spectrum (and their parents) is that they } \\
\text { have opportunities to seek out and obtain meaningful } \\
\text { employment opportunities that contribute to society's progress. } \\
\text { Peopple on the spectrum want recognition for their unique value } \\
\text { and level of competence in specialized areas. }\end{array}$ \\
\hline Actionable Goals & $\begin{array}{l}\text { A goal of healthcare providers and admin. is to have tools and } \\
\text { processes in place that help to guide parents and adults on the } \\
\text { spectrum towards more actionable and realistic goals. }\end{array}$ \\
\hline Positive Stereotypes & $\begin{array}{l}\text { A goal of all stakeholders is that they need to promole more } \\
\text { positive ASD stereotypes that are not centered on their } \\
\text { limitations, but instead on their potential. }\end{array}$ \\
\hline Informed Decision-Making & $\begin{array}{l}\text { A goal of all stakeholders is to have more mechanisms and } \\
\text { connected resources available that allow them to make more } \\
\text { informed decisions about future needs/care/planning. }\end{array}$ \\
\hline Independent/Assisted Living & $\begin{array}{l}\text { A goal of all of the stakeholders is to find more aftordable housing } \\
\text { solutions for adults on the spectrum. These houses could take on } \\
\text { the form of an assisted living community for both dependent and } \\
\text { interdependent individuals. }\end{array}$ \\
\hline
\end{tabular}

\section{Control}

\begin{tabular}{|c|c|}
\hline Alleviate Dependence & $\begin{array}{l}\text { A goal of healthcare providers and admin. is to try and help those } \\
\text { at the higher end of the spectrum gain more independence during } \\
\text { their transition to adulthood. }\end{array}$ \\
\hline Navigating the Systerm & $\begin{array}{l}\text { A goal of parents, healthcare providers and admin. is to be able } \\
\text { to easily navigate a more streamlined 'system' }\end{array}$ \\
\hline Increase Predictability & $\begin{array}{l}\text { A goal of parents and poeple on the spectrum is to increase } \\
\text { predictability (reduce fear of the unknown'unexpected). }\end{array}$ \\
\hline Peace of Mind & $\begin{array}{l}\text { A gaal of parents and poople on the spectrum is to gain peace of } \\
\text { mind and the feeling that they have more control. }\end{array}$ \\
\hline Environmental Control & $\begin{array}{l}\text { A goal of the people on the spectrum is to be able to better } \\
\text { control their environmental settings and social situations. }\end{array}$ \\
\hline Control of Self & $\begin{array}{l}\text { A goal of the people on the spectrum is to gain a better handle on } \\
\text { themselves and their emotions (sensory sensitivities and over- } \\
\text { stimulation). }\end{array}$ \\
\hline
\end{tabular}

Figure 6.28 Needs/goals organized into eight overall categories for use with the digital tool

\section{prototype.}




\subsection{Workshop 1: Shared Understanding, Sensemaking, and Defining "What"}

Workshop 1 was designed to encourage shared understanding and transformative thinking around the wicked problem. The session was attended by three adults with ASD, six parents, and seven healthcare providers and administrators (from OSUWMC and Nationwide Children's Hospital). The plan for Workshop 1 comprised of: stakeholders sharing unique perspectives and resonating with others, prioritizing the needs and goals from the initial research and discussing those priorities with the other stakeholders on a shared interactive surface. Then, together, the stakeholders would cluster the prioritized needs/goals and draw connections within and across the clustered groups to identify key unmet needs and disruptive Design opportunities. The ultimate goal, by the end of Workshop 1 was to collectively identify major themes, which could then be used to reframe the wicked problem.

Narratives and visual mapping can be used as a common language through which people attempt to make meaning of complex information and connect with others. These two elements were brought into the sensemaking process for Workshop 1. From the unique experiences and perspectives captured in Phase 1, visual presentations were co-created with each of the three stakeholder groups so each group could narrate their thoughts, feelings, and ideas regarding C.A.S.T. and any other topic related to ASD that they felt they needed/wanted to share with the other stakeholders. Individual presentations were a form of storytelling, an opportunity to share personal experiences and perspectives that led to discussions around the conference table (among all stakeholder groups) where new insights and ideas verbally materialized. Personal stories allowed the participants to relate to one another on a more empathetic level and allowed the presenter to not only reflect on past and current situations, but also provide clues to what should be done in the future. 


\subsubsection{Workshop 1 Collaborative Environments}

Workshop 1 was held in the ACCAD (Advanced Computing Center for the Arts and Design) building on The Ohio State University campus. This location was selected for multiple purposes. One consideration was that the ASD participants might be interested in seeing some of the animation work and technology in the space. Additionally, the space housed the multi-touch table and projector, had multiple whitewalls and tall ceilings with plenty of room to move around, and had quiet areas, should people need to step away for a moment. The presentations were held in a conference room, which had a large oval table surrounded by chairs facing a projector screen.

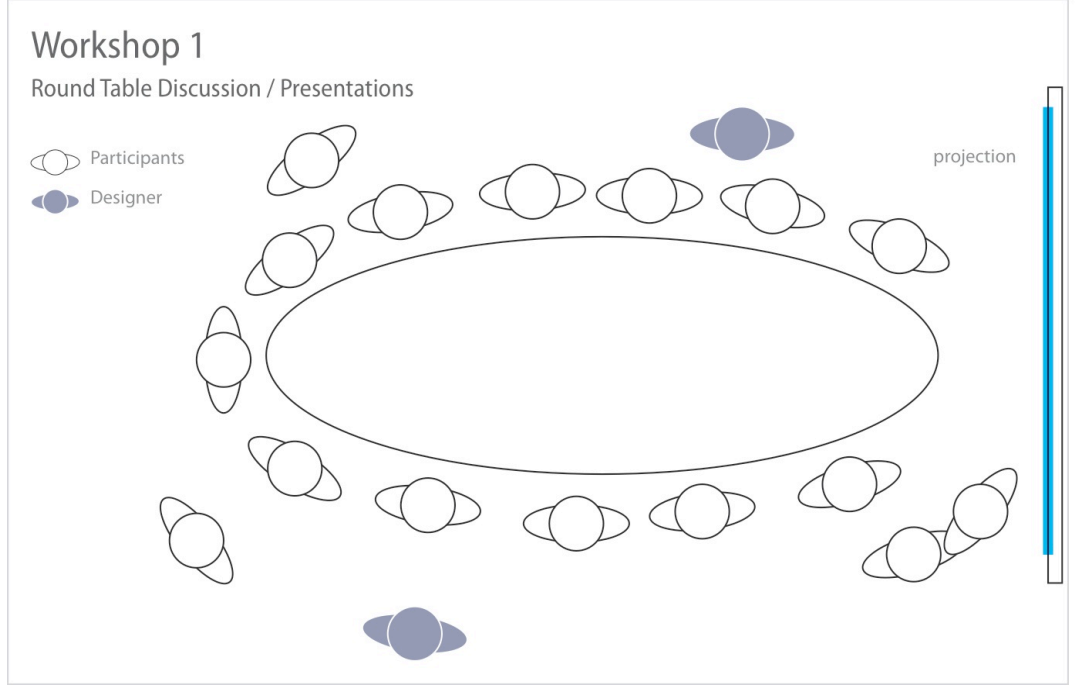

Figure 6.29 A birds-eye view of the presentation space.

The space used for the sensemaking session was semi-open (surrounded on three sides with whiteboard), where people could stand, sit, and walk around freely. The multi-touch table and another projector were also housed in this space. The intention of the whiteboard room with the projector and multi-touch surface was to create a 'seeing' space through which stakeholders could see various perspectives and interact with the data in different ways (digitally or non- 
digitally). While some participants chose to interact with the touch-screen, others could draw on the whiteboard or round paper sticky notes (located on the projection whiteboard wall).

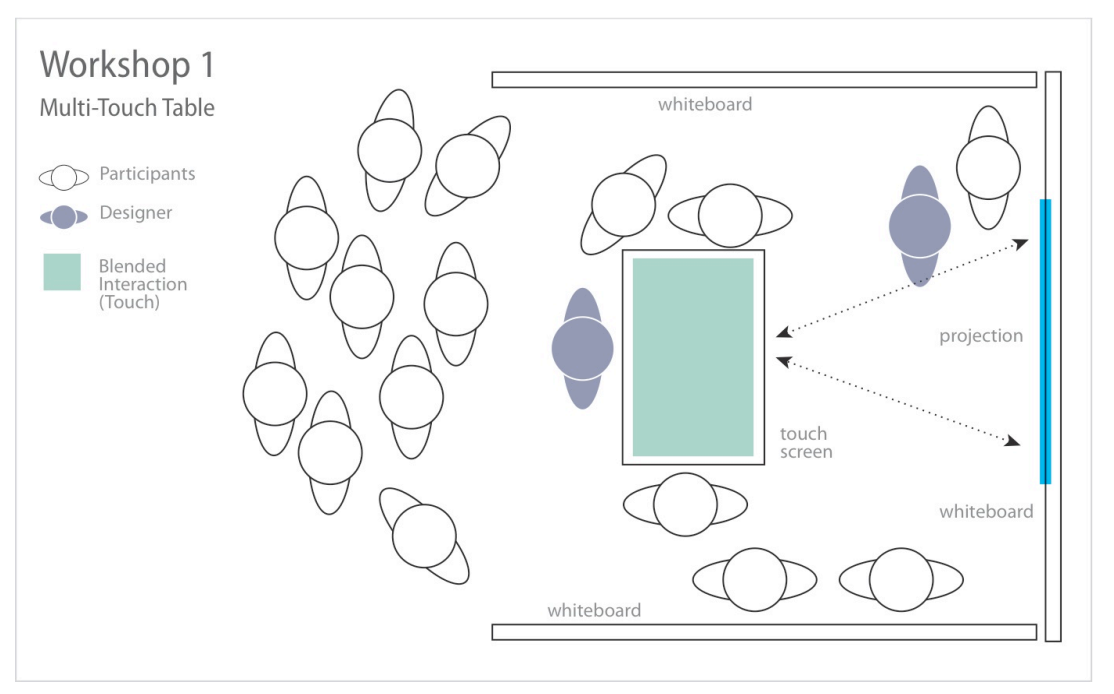

Figure 6.30 A birds-eye view of the seeing space created for the sensemaking activity.

\subsubsection{The Presentations}

The presentations in Workshop 1 were intended to help the stakeholder groups begin to connect (to each other and to new information) through the act of telling and sharing before pulling them together to engage in making sense through the act of collectively creating clusters and mapping a system of prioritized, connected needs and goals. The collaborative discussions and mutual storytelling done during the presentations led to new conversations around greater needs, beyond the clinic, that could help patients and families live more meaningful and productive lives. The telling methods also gave the participants fodder for the collective mapping session that followed.

\subsubsection{Integrating the New Digital Prototype into Workshop 1}

To explore additional capabilities of technology in the PAR process, a multi-touch digital tool was prototyped for the second half of Workshop 1 with Alan Price, a professor at ACCAD. The intent of the digital tool and this stage of the workshop was to help the participants converge around 
multiple perspectives and reframe the wicked problem collaboratively through prioritization and collective mapping. The second half of the workshop (the sensemaking activity) took place in a different type of collaborative space (Figure 6.30) from the one in which the stakeholders presented to one another - moving from a round-table conference room to an open, standing space surrounded by whiteboard walls. Not knowing which tools or platforms would be the most useful for viewing and interacting with the data in a large group, and also not knowing which tools (markers or touch screen) the stakeholders would prefer to use or gravitate toward, the space accommodated multiple means of open and flexible communication. The intent was to create a hybrid "seeing" space through which the stakeholders could interact with each other and the information through digital and non-digital means. A multi-touch table was positioned in the center of the standing space, surrounded by walls that doubled as writing surfaces. The multi-touch table (with projected interactions on the center whiteboard wall), along with paper sticky notes and whiteboard markers, afforded multiple opportunities and various ways for all of the participants to actively engage and converse.

The categorized list of twenty-four needs and goals (Figure 6.28) was converted into interactive circles (nodes) in the digital tool (represented on the multi-touch screen). The design of the digital tool leading up to and following Workshop 1 will be described in more detail in the following chapter. At this point of development, the prototype of the digital tool was in an early phase. It was designed to work on a multi-touch table, connected to a projector that was pointed at a whiteboard wall. The projector displayed what was occurring on the touch-table so those that were at a distance could see the interactions. Drawing from the information on the list, the digital tool converted each need and goal into a circle node that could be moved around the digital space, increased in size based on prioritization, and linked to other nodes. The primary objective of the digital prototype was to provide visual feedback on voiced thoughts and data patterns/relationships in order to help the participants make meaning. The tool had a screen 
capture feature - every fifteen seconds it would take a screen image. After the session was over, all of the screen images could be compiled into a video that would show the train of thought of the participants, from prioritization to clustering to synthesis.

\subsubsection{The Sensemaking Activity}

Following the presentations, stakeholder groups gathered in separate rooms to prioritize their top ten topics (needs and goals combined), from the list of forty-eight. After prioritizing their topics, the stakeholders joined together in the whiteboard space to share and visually exhibit their prioritizations using the touch-screen prototype. At least one representative from each stakeholder group was able to present their prioritized topics using the touch-table that was placed at the center of the space. As each priority stakeholder dot was placed on a topic, the topic grew in size. The thought behind projecting on the whiteboard was so that those who were not directly interacting with the touch-screen could draw connections between circle topics, make notes on the whiteboard wall, or use it as a means to communicate their thoughts through writing/drawing instead of just verbal communication. The whiteboard was not used as expected. Those who were not directly interacting with the screen could see what was happening on the whiteboard and were having discussions with each other, as priorities were being made visible. The group got as far as prioritizing the topics when the digital tool malfunctioned. It was not until later testing, after the workshop session, that we identified a technical glitch in the connector buttons. Clustering prioritized topics and drawing connections between the clusters (using the connector buttons) was to have been the next step of the process. Uncertain of the digital prototype's durability and capacity to cover all of the needs of our collective "seeing" space prior to the start of Workshop 1, forty-eight round sticky notes had been prepared as a backup on the whiteboard wall in the workspace. 

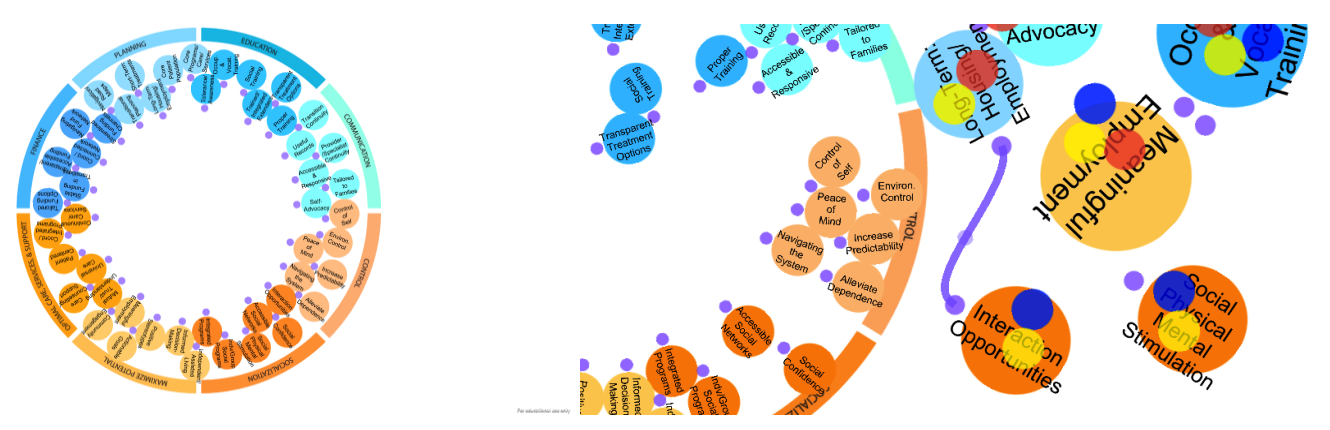

Figure 6.31 Screen captures of the digital tool prototype.

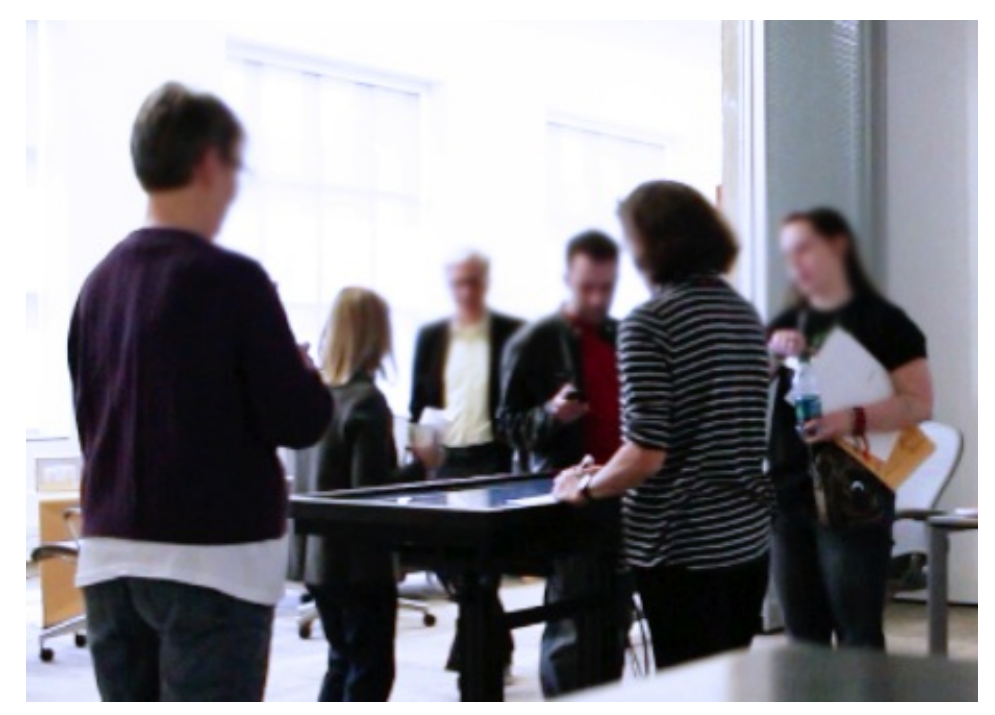

Figure 6.32 Photograph of people interacting with the digital tool.

Once the digital tool malfunctioned, the projector was turned off and the group's focus was shifted to the paper sticky notes on the whiteboard. Using the forty-eight circle sticky notes (that strategically corresponded in color and size to the digital circles) we remapped what had been done on the digital table. Orange sticky notes represented goals, while blue sticky notes represented needs. Larger sized circle sticky notes (with matching color) were used to indicate the needs and goals that had been prioritized by most of the stakeholder groups on the digital screen. The participants who had gathered around the touch-screen stepped back and allowed us to quickly reproduce the pattern of nodes that they had made on the touch screen. The role of the 
participants was then in providing verbal cues and direction. After the prioritized needs/goals ('landmarks') were identified, the collective group rearranged the sticky notes and clustered them into similar categories. Next, the group discussed the relationships among the individual topics and across the clusters, drawing mental connections and reorganizing the hierarchy and visual relationships among the sticky notes. The act of drawing connections was intended to help us begin to move past "what", in order to reframe the problem. The objective was to make meaning between connected and disconnected topics and to identify whether or not more than one goal could be met by accomplishing a shared unmet need.
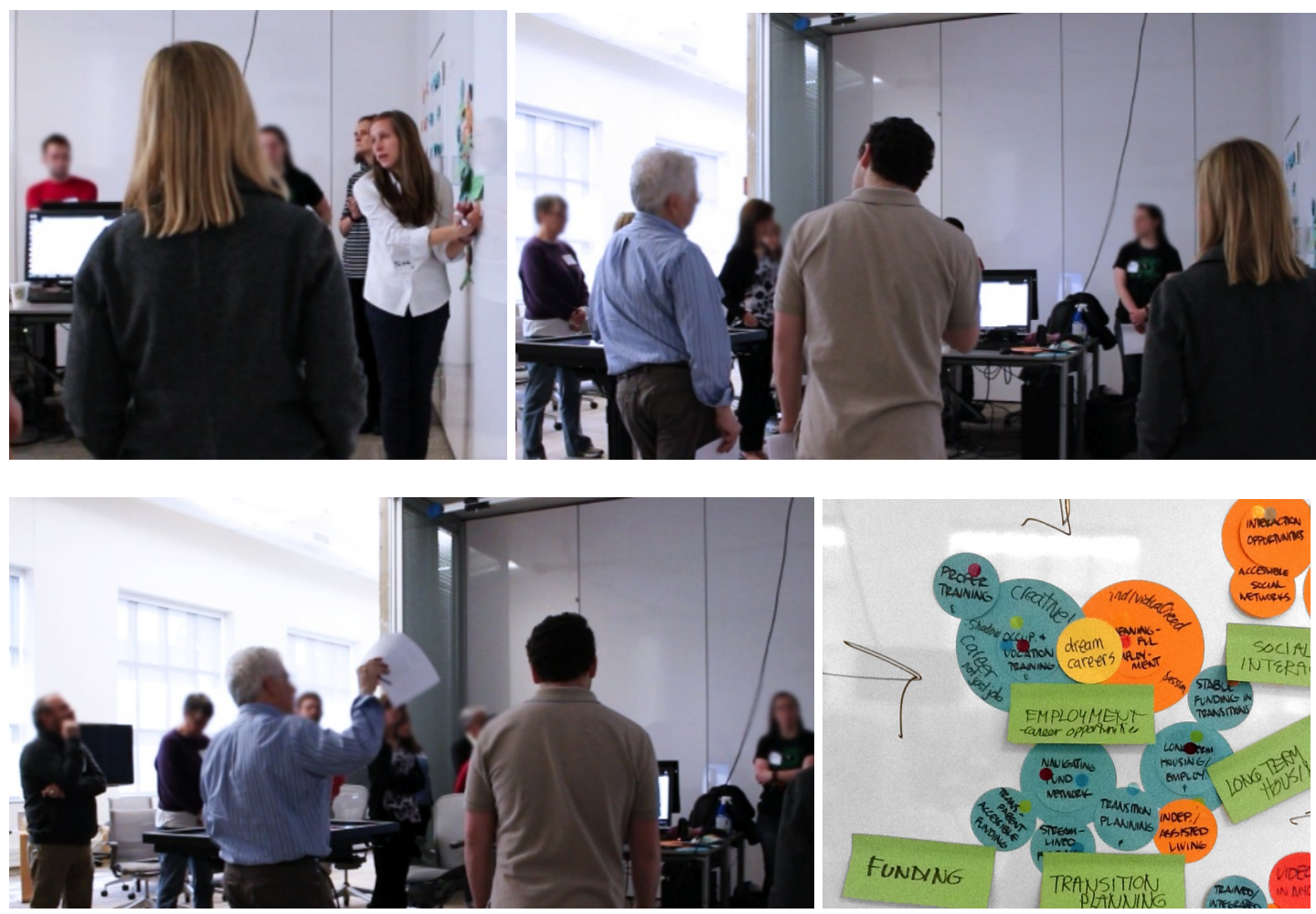

Figure 6.33 Photographs from the whiteboard session and sticky note diagram.

The methods and tools for discovery, immersion, and connectivity in Workshop 1 brought about a new, broader understanding of the wicked problem and its root problems. There was less focus on the 'physical' needs of the clinic, even among people whose primary focus had originally been 
on the clinic. Through storytelling and mutual sharing, collective mapping/sensemaking, and new ways of looking at the clinic's true value/role in the wicked problem of transitions, empathy, new understanding, and critical and creative thinking appeared to grow beyond the everyday understanding and ideas of each individual participant.

\subsection{Phase 3: Workshop 2 Preparation}

Prior to Workshop 2 (Phase 3), a summary of the prioritized needs and goals was compiled along with the ideas that had been generated in Phases 1 and 2 to keep alive the insights and imagined possibilities that had been uncovered and matured up to this point. The ideas (ranging from nearterm to long-term ideas) were grouped into four overall categories, based on the clusters made during the second half of the workshop. By watching video footage from the mapping segment in Workshop 1 and viewing photographs from the final organization of the sticky notes on the whiteboard wall, the digital tool was used to create a video that re-produced the actions (e.g., prioritization, clustering and connections) that had occurred using the digital tool and sticky notes. Prior to Workshop 2 (Phase 3), each participant was given a copy of the video (depicting the train of thought of the participants during sensemaking), along with 'cleaned-up' visualization summaries and matrices of the near and long-term ideas.
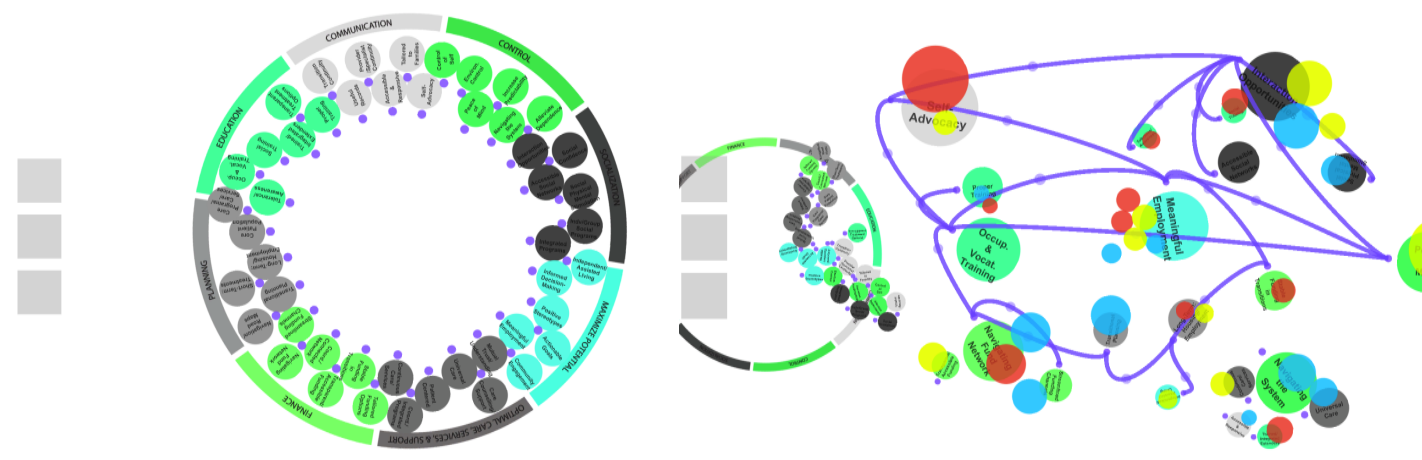

Figure 6.34 Visual synthesis map, recreated with the repaired digital tool. 


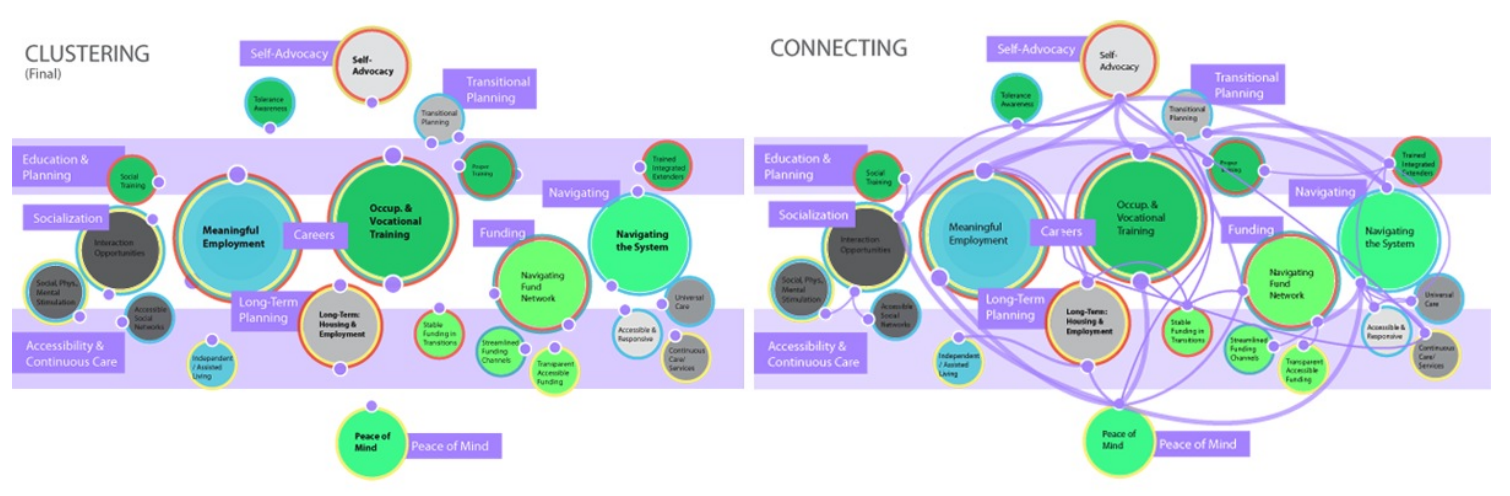

Figure 6.35 Cleaned up visualizations of the synthesis map (using Illustrator).

\subsection{Workshop 2: Prototyping and Understanding "How"}

The creative space and methods used for Workshop 2 were designed around the notion of immersion and connectivity through making while encouraging creative thinking through persona development, prospective scenario building, and prototyping. The aim of this final session was to find a balance between 'what is' and 'what could be' by determining the 'how.' Using themes derived from Workshop 1 the participants created personas and prospective scenarios to establish the frame ('how'). Using intuition and what they gained through immersion, participants then built initial prototypes within the framework of actionable opportunities that could be further developed and integrated into the current or future transitional system of autism. Prototypes were constructed by the participants using generative Design Research methods and tools to help them reflect on what they had learned throughout the research project, and to communicate tangible ideas in a more engaging manner. The method of prototyping allowed participants to connect with one another, reflect, and explore new possibilities simultaneously.

\subsubsection{The Collaborative Space for Workshop 2}

Three healthcare providers/administrators, three adults with ASD, and five parents, all of whom had participated in Workshop 1, attended Workshop 2. The final session was held in the Design building on campus of The Ohio State University. The room was large, let in a lot of natural light, 
and did not have a lot of loud noises. It had plenty of space to walk, stand, and move around freely, similar to the space used for the sensemaking session. Should any of the participants get overwhelmed or need time away from the session, they could easily leave the room and come back. The pin-up boards and tables in the space were on wheels so they could easily be moved; the space could easily be reconfigured based on the needs of the groups. The tables were pushed together at the beginning and the end of the session for group discussion and reflection. After initial instruction and discussion, the tables were pulled apart for group work. The pin-up boards on either side of the room encouraged standing and working together on a shared surface. The central location of the tables with the 2D/3D generative materials made it easy for both groups to access the tools. The space and arrangement of the group tables, along with the pin-up boards and material tables, appeared to keep the ASD participants engaged and facilitated concentrated group work, as the teams could work on opposite sides of the room without distracting the other group.
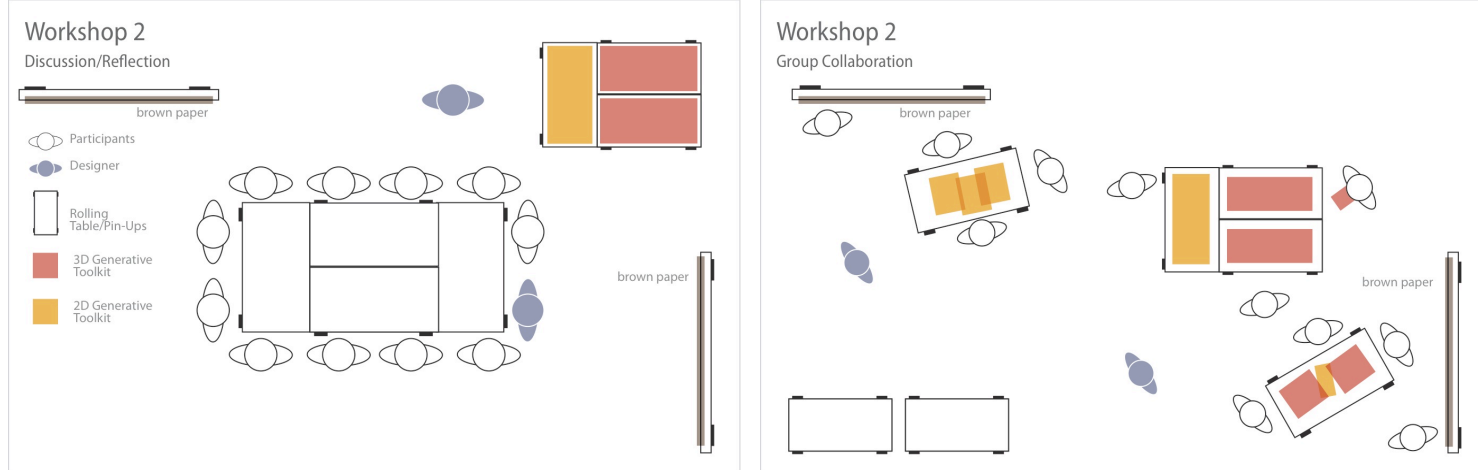

Figure 6.36 Two birds-eye views of how the space for Workshop 2 was reconfigured for the discussion/reflection portion (left) versus the collaboration portion (right).

\subsubsection{Overview and Initial Discussions/Sharing in Workshop 2}

Overall, the structure for Workshop 2 followed a tell/make cycle. This appeared to help participants continue to play in the space of 'Design Wisdom' - bridging sensemaking and 
speculation about future Design opportunities. Sharing and reflection on current experiences occurred at the beginning of the session, followed by the making of current and future personas, creating and telling prospective scenarios (by journey mapping their persona's life in their current state towards an ideal future), and building prototypes that addressed the gaps and opportunities identified along the journey maps.

Prior to collectively developing personas, scenarios and prototypes, the participants were given an opportunity at the beginning of Workshop 2 to communicate their individual thoughts and perspectives on what we had uncovered so far and their ideas for the future by sharing reflections from their Journals. One parent volunteered to share her thoughts and her vision for the future. On the back of her Journal she created a collage of things she wanted for her child and in the center was this quote: "I would not change you for the world. For you, I would change the world." Others chose not to share their Journals, but instead openly discussed the challenges faced by adults on the spectrum who are looking for career opportunities. This dialog led into opportunities about assistive online applications (like Generous' Podium app), to help people who struggle with social skills practice interviewing or practicing skill that could help them reach their full potential. Additionally, options for higher education programs (beyond high school) were discussed between the Director of Nisonger and the parents. The third ASD participant (who we will refer to as Bryan) had attended a higher education program and had done quite well. He and his father had participated in Workshop 1 and were present in Workshop 2. His father and the Director of Nisonger shared information about current education offerings for adults with special needs with the other parents. These programs teach knowledge-based skills, as well as social and everyday skills such as cooking. This conversation culminated in shared personal experiences and created understanding that united the stakeholder groups around gaps and unmet opportunities in the current system to maximize the potential of persons on the spectrum and their families. From the conversations that were being shared individually at the beginning of Workshop 2 it was 
becoming more and more evident that we would not be ideating and prototyping concepts around clinic specific ideas, but instead tackling larger scale ideas that would address long-term goals. The clinic was no longer the main issue. In order to determine how the clinic could create true value in the greater ASD system, our focus had to shift to issues beyond the clinic, first. In this session we needed to explore resolutions that were disruptive and created a more connected system of support and resources that the clinic could/should be a part of in order to reach the collective goal - holistic and sustained wellbeing for people on the spectrum and their families.

\subsubsection{Persona Development}

After our discussion the stakeholders divided themselves into two teams, based on shared interests, to develop personas (current and future), prospective narratives, and initial prototypes. Team 1 consisted mostly of parents and one provider, while Team 2 was made up of all of the ASD participants (John, Kristin, and Bryan), Bryan's father, and two providers. One of the providers had to leave early; Kristin, who came late, took his place on Team 2. Each team was asked to first choose a scope, either near term/clinic specific ideas or long term/disruptive ideas that they most closely identified with and would like to explore further through prospective scenarios and prototyping. Both teams chose to tackle long-term disruptive ideas.

Each team was given a two-dimensional generative toolkit to use to dive deeper into the unmet needs and goals of a specific adult on the spectrum. They created two personas. One persona represented the person on the spectrum today; the second persona represented an aspirational prospect of that same person many years into the future. Each team was asked to consider the lifestyle attributes, fears, and dreams of the present persona and project those attributes, fears and dreams into the future by building a scenario of their future persona. The objective behind using this method in Workshop 2 was to immerse the stakeholders in the lifestyle and challenges faced by a 'typical' adult on the spectrum and their families during transitions. By breaking the 
larger scope of the problem down into one scenario, the participants could better identify concrete opportunities and barriers for success along the transition timeline (present to future) that could help or hinder a person on the spectrum from reaching their maximum potential.

Personas are a method through which fictional characters are developed. Used in the front-end of the Design process, personas can be used to enhance engagement, communicate gathered information from research, and provide people with a deeper understanding of varying perspectives and possible experiences encountered and felt by other people/stakeholders. Personas "utilize our mind's powerful ability to extrapolate from partial knowledge of people to create coherent wholes and project them into new settings and situations" (Grudin \& Pruitt, 2002). Used as an exploratory method early in the Design process, personas can also be used in CoDesign to help to engender empathy for alternative perspectives (to avoid mistaken assumptions) and lead people towards new insights and opportunities related to explicit needs and desires (Grudin \& Pruitt, 2002). In Workshop 2 of the case study the personas were influenced by the shared understanding gained in Workshop 1, and from the personal experiences of the stakeholders. The intent was to use the creation of personas in Workshop 2 as a method through which the participants could dive deeper into the collective prioritized needs and goals uncovered in Workshop 1, in order to find new opportunities to develop prototypes.

The persona toolkits provided to each team contained images, words, headers (About, Lifestyle, Personality, Specialization), and situation cards as guides and inspiration to help them create visual representations of their persona's attributes and lifestyle. The situation cards were intended to help team members consider multiple aspects of the persona's life. The participants were required to look over all of the material but did not have to use everything. They were only required to use all of the headers and at least one face image. One team could not find an image that showed an angry person or a persona having an outburst, so the cutout shapes that were 
given to both teams allowed them to further illustrate their angry persona. Audio recorders were placed at each working station (two stations, one for each team). The participants were asked to communicate out loud as they constructed their personas and made decisions along the way so what was said during the construction and presentation of the personas and narratives could be captured for future analysis. A summary of the results of this activity follow.

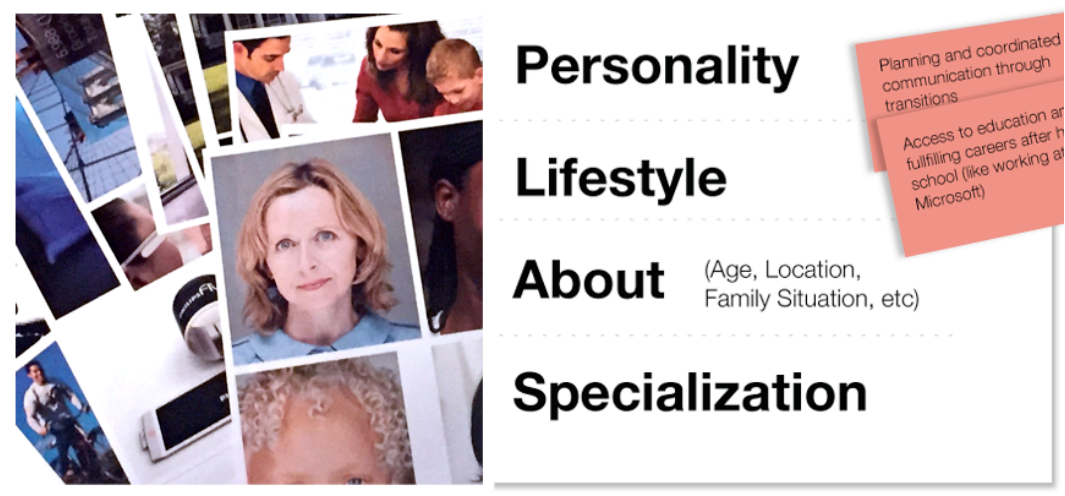

Figure 6.37 Persona toolkit - images, situation cards, and headers

\section{Team 1's Persona - Owen \\ Current persona:}

Owen is a late teenager (17 years of age) with one sibling and two parents, living with his family in a suburban neighborhood of Ohio. He is on the middle of the autism spectrum (he can function and speak, but he is "definitely autistic"). He struggles with explosive, unpredictable behaviors. Owen can be functioning normally, but then there are certain things like anxiety, loud noises and little kids' voices that trigger this explosive behavior. He has zero tolerance for little kids. He enjoys doing things with his hands, as well as getting exercise and being outside. Physical activity is important and encouraged by his parents; it is not self-directed right now. His emerging interests/specializations revolve around electronics, apps, computer games and music. "He has a strong skill in his area of interest." 


\section{Future persona:}

Owen is now 25 years of age. His parents are still around, but they are now in the "background." His independence is emerging and he is making big leaps. He is living away from home and has accomplished a certain level of higher education (whether that is schooling or a trade school). He is living in an apartment in a suburban neighborhood with easy access to public transportation.

He has a tight circle of friends. He wants a girlfriend but is still navigating that area; he still needs skill development in that area. He is still very focused on technology and high-skill areas. He is working and earning money in the field of virtual reality, app development and is able to work independently and work in small teams.
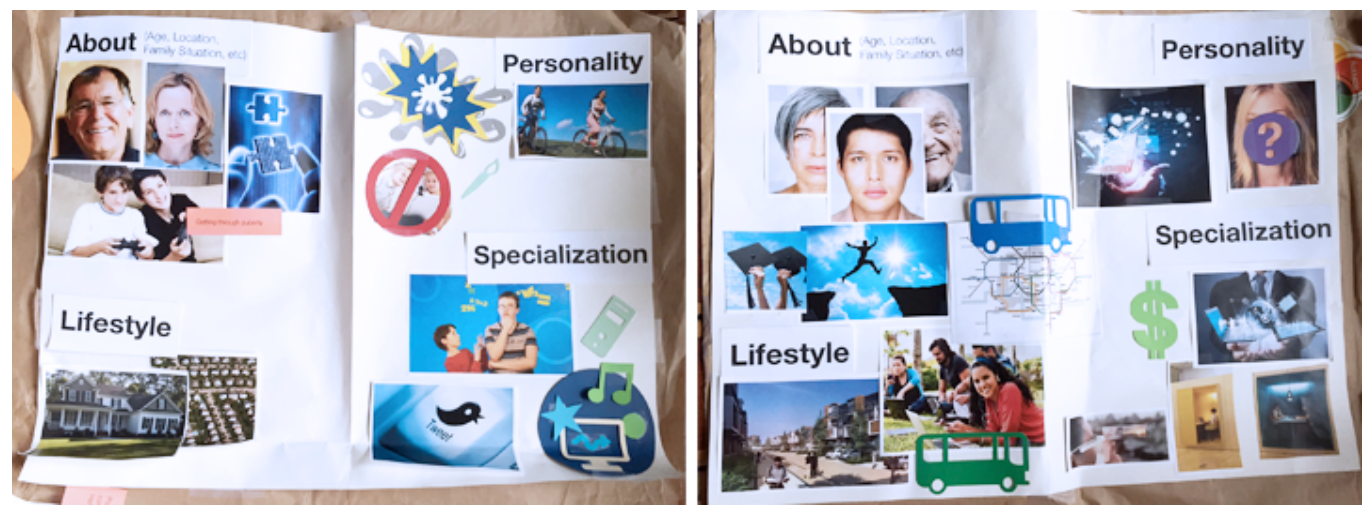

Figure 6.38 Owen's current and future personas.

\section{Team 2's Persona - Sam}

\section{Current persona:}

Sam is a sophomore engineering student living alone in on-campus housing. He is 20 years of age. He is naturally shy and nervous, and enjoys playing computer games. His parents live far away, so at times he feels isolated. Sam does not drive and never exercises. He really does not get out or do much of anything. The only time Sam gets out of the house is going to school and going to work at a local ice cream shop. He does not have any close friends or a doctor - he lacks a social/supportive network around him. 


\section{Future persona:}

Sam has graduated and is working at a computer related job, which is a fulfilling career for him. He lives on his own in independent affordable housing and has a family of his own. He has friendships and is exposed to more physically and mental stimulation and activity. He also has integrated lifetime healthcare.
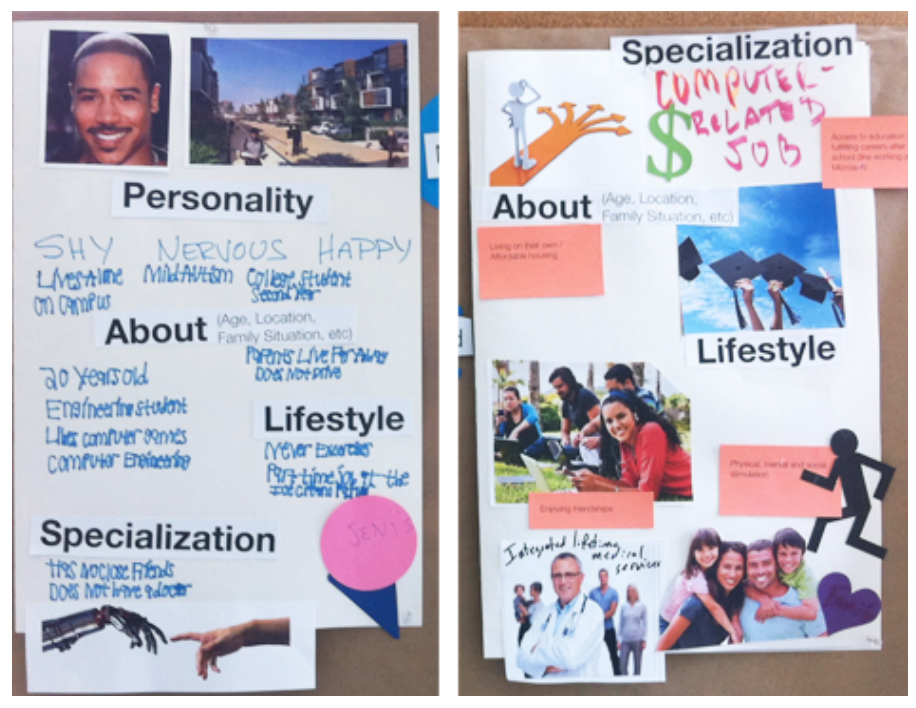

Figure 6.39 Sam's current and future personas.

The current and future attributes of the personas appeared to represent a fusion of both the personal experiences of the CoDesigners and the insights that had been generated in Workshop 1. Both aspirational futures addressed the need for supportive medical and social networks, independent, affordable housing, and meaningful careers that provide mental stimulation and allow the person with ASD to channel their passions in a meaningful way. The persona activity served as a bridge between the individualistic views of the participants and the global, long-term goals/needs of this population. The lifestyle attributes, fears, and dreams expressed through the current and prospective (future) personas became the anchors for building journey maps. 


\subsubsection{Prospective Scenario Journey Mapping}

The two groups presented their personas to each other. This method guided the participants in identifying and jointly communicating the barriers and unmet needs of people, families, and healthcare providers through two different characters. After presenting their current and future (aspirational) personas, the teams returned to their workstations to begin constructing prospective scenario timelines (connecting the present and aspirational future personas) on large pieces of brown paper (on the pin-up boards). During this step, participants could continue using images, words, and the situation cards from the persona generative toolkit. In addition, they were given directional cutout shapes and abstract shapes to construct cause and effect situations along the timeline. They were also required to review new headers, which could be used as guidelines to structure their timelines and to round out their prospective narratives.
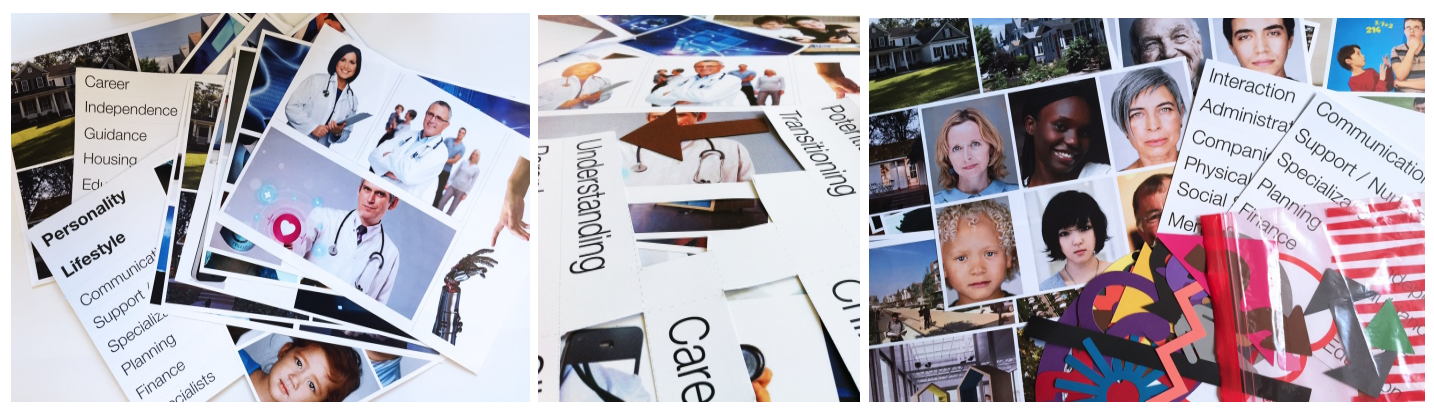

Figure 6.40 Prospective scenario toolkit - headers, 2D shapes, and images (similar to the persona toolkit).

The main objective of this activity was to identify steps along a journey map from the current situation to the future aspiration that could hinder (e.g., unmet needs/gaps) or help (e.g., ideas/opportunities) a person on the spectrum in reaching their full potential. After making their journey maps, the two teams presented to each other. The future personas and prospective journey maps would act as primers for the prototyping to follow. 


\section{Team 1's Prospective Scenario Map}

Owen's Journey

Owen's Journey is shown in Figure 6.41 with Owen's future persona poster. 'Planning' and 'Finance' headers were positioned at the first stage of his journey. 'Education,' 'Career Training,' and 'Mental Stimulation' followed to guide Owen in working towards self-advocacy during high school and before college. 'Interaction,' 'Communication,' 'Support/Nurture,' 'Social Stimulation,' and 'Friendship' were also noted within the journey map. During his journey Owen would have a team of physicians, guidance administrators, and specialists, along with his family, to help nurture and support his holistic wellbeing. For his explosive temper, Team 1 added the idea for technology sensors to monitor and communicate his physiological and behavioral changes. The idea for an Angie's List-like platform for ASD was added to help Owen's parents navigate the system, connect with other parents, and help them begin to locate careers and transitional independent housing options before college. The aspiration for college (or a next level, post-high school education program) was that it would help Owen discover his specialization and nurture his potential. Physical stimulation would continue into adulthood, as well as mental stimulation through training and education built around his specialization. Into his early twenties, Team 1 thought there should be an 'eHarmony' site available for ASD individuals to help them socialize and meet significant others through an online (as opposed to face-to-face) introduction.

Additionally, Owen would continue to receive optimal care and would be living on his own in affordable housing. By the time he turned twenty-five, the age of his future persona, the aspiration was that the opportunities and support presented to him at each stage of his journey would provide him with a more balanced and independent life, while also giving his parents greater peace of mind so they could fade into the background and live a "more normal life." 


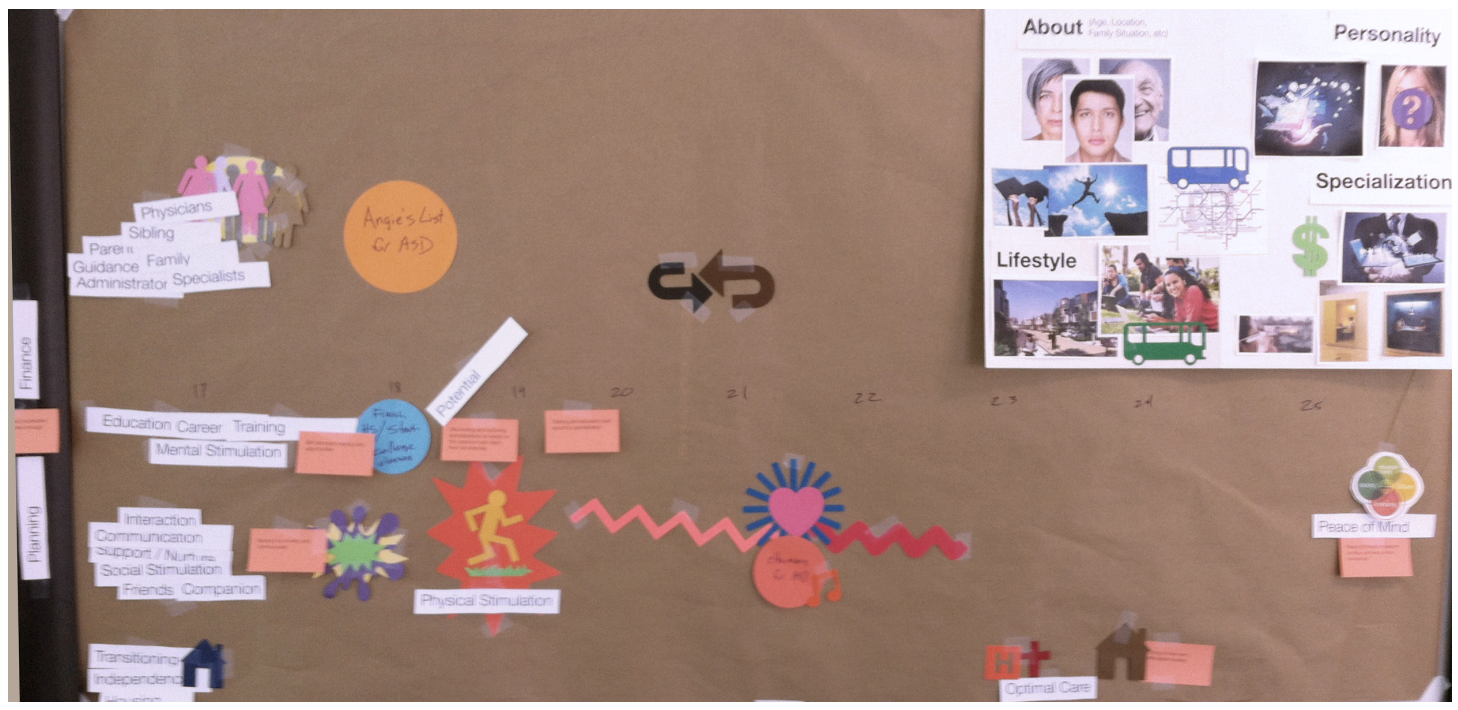

Figure 6.41 Owen's Journey Map.

\section{Team 2's Prospective Scenario Map}

\section{Sam's Journey}

Sam's Journey is shown in Figure 6.42 with Sam's current and future persona posters.

'Motivation' is where Team 2 started Sam's journey. Sam is a shy, under motivated person. In order for him to reach a more promising future he needed to continue receiving optimal care into adulthood from his Care Team (physicians and specialists). Tools and services originating from this Care Team needed to be in place to motivate him, support/nurture his interests, and provide training in areas like communication. Some of these tools/motivators included helping Sam build a schedule and participate in activities that offered social and physical stimulation. Through these activities, the aspiration was that he would make more friends and potentially find a significant other who shared his interests to start his own family. He, like Owen, would live in affordable independent housing with his family and ultimately achieve greater peace of mind. 


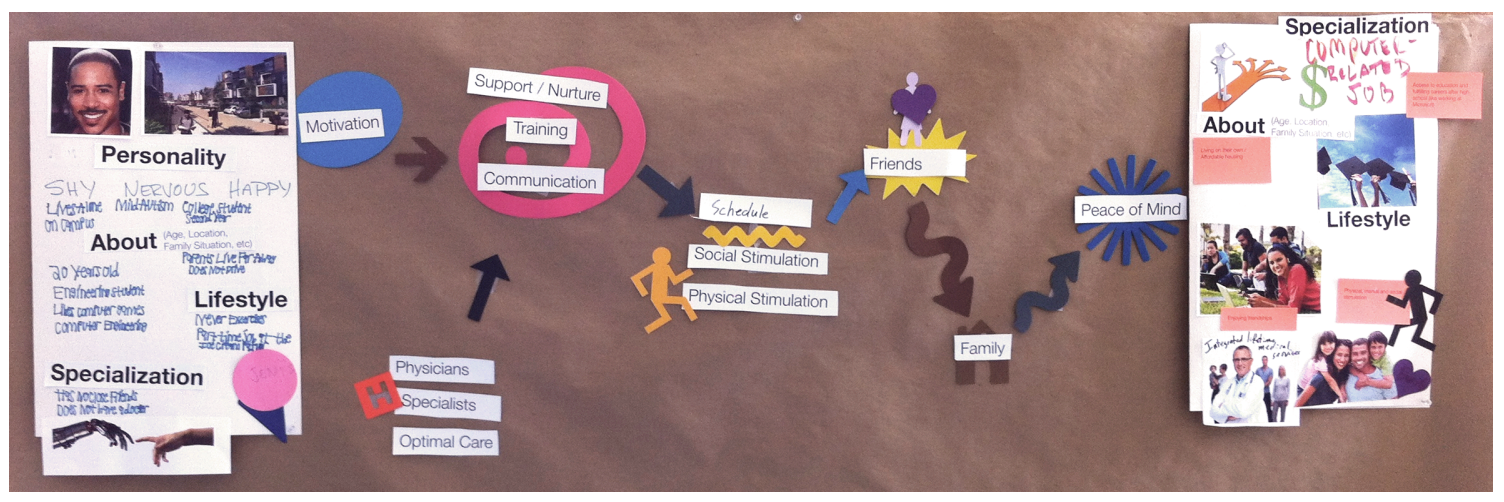

Figure 6.42 Sam's Journey Map.

Similar to the visual need/goal map constructed with circle sticky notes in Workshop 1, the unmet needs and opportunities identified in the journey maps in Workshop 2 culminated in the people attaining peace of mind. For Team 1, peace of mind was achieved by Owen's parents. For Team 2, peace of mind was fulfilled in Sam, who had struggled initially with fitting in and finding his way, through his family and working in a meaningful career that aligned with his passion for computers.

The journey maps and prospective scenarios were intended to act as a springboard from which the participants could start to speculate about the future and imagine disruptive ideas that could be implemented into the current system to fill the gaps and deliver on previously overlooked opportunities. The personas helped the teams create scenarios that transcended current barriers to ideal futures for their personas and their families. In creating and sharing their prospective narratives of their current personas and their progression to their ideal future, teams pointed to specific gaps (unmet needs) in the narratives that needed to be overcome in order to reach their ideal future. Selected opportunities for innovation that were addressed in the journey maps were then translated into more concrete ideas in the final stage of Workshop 2 through prototyping. 


\subsection{Prototyping}

Returning to their workstations, the teams determined which needs/idea(s) they wanted to prototype. Generative tools were set up in the center of the space from which the participants could freely select items that could help them best communicate and represent their ideas/concepts. From what was learned at the focus group with the adults on the spectrum at Donatos, people express creativity, thoughts, and emotions in many different ways, so a wide assortment of two-dimensional and three-dimensional (both abstract and concrete) generative components were provided for the participants to utilize in Workshop 2 (Figure 6.43). The generative materials that were offered included: two-dimensional shapes, large pieces of colored paper and sticky notes, LEGOs, Velcro-covered shapes and an assortment of abstract objects that would stick to the Velcro shapes, hand puppets and dolls, miniature white furniture, and blocks. The focus for this final stage was for the teams to use the generative tools to make sense of the future and further express, create, and imagine new resolutions through the act of making.
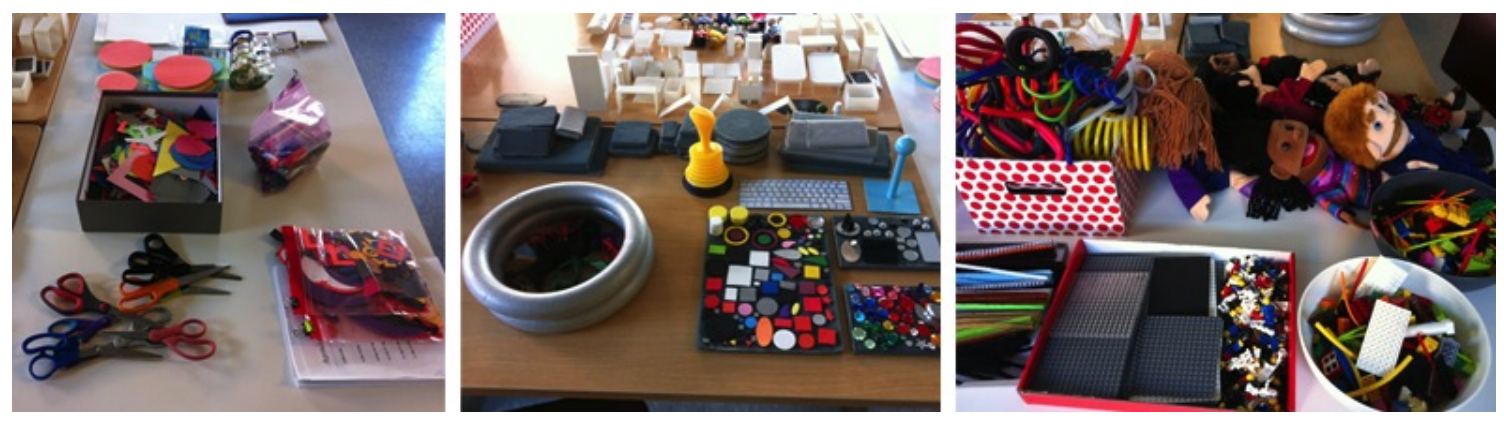

Figure 6.43 Generative tools for prototyping -2D shapes/materials and 3D objects.

Team 1 used mostly two-dimensional materials: 12 "x12" colored paper, markers, and paper shapes. These were used to create a series of wireframes for a new website they called Owen's List. The idea for Owen's List was inspired by the features of Angie's List, which had come up in conversations and evolved over the course of the project from Phase 1 to Phase 3. In Workshop 
2, Team 1 wanted to begin to make the idea more tangible and develop screenshots of different offerings the site could provide to parents and individuals on the autism spectrum.

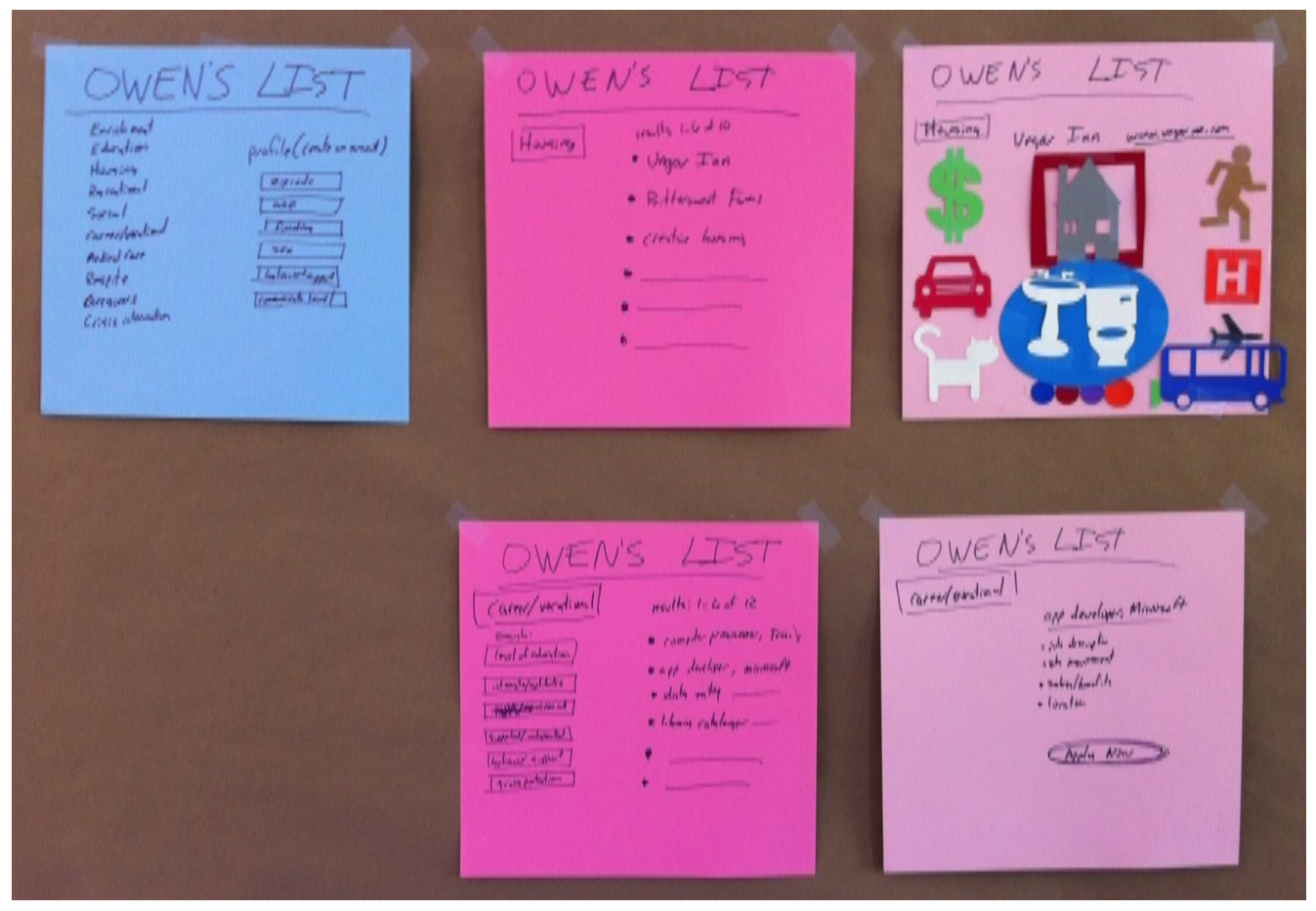

Figure 6.44 Owen's List prototype.

Team 1 followed an expected prototyping path from persona creation to building a concept that resolved a specific unmet need identified in the journey map. Their prototype represented a series of screen shots of Owen's List (Figure 6.44). Walking through each frame, the prototype helped us imagine how the website could function.

Team 2 followed a similar path from persona to prototype, but their outcome and the way they chose to 'prototype' their resolutions was unexpected. Team 2 chose to use both two-dimensional and three-dimensional materials, however their 'prototypes' did not embody a specific concept or idea. Instead the objects they used acted more as 'props' through which they visually narrated connected aspects of their aspirational future. Each 'prop' represented a new opportunity that 
could contribute to a more integrated support system for adults on the spectrum. Bryan, his father, and the Director of Nisonger used the dolls, puppets, and miniature furniture to recreate a 'day in the life' of an adult on the spectrum (like Sam) who was living a more independent life. In their scene, Sam lived independently, had a job, and had a family. He also had an integrated Care Team (support network) that he could contact at any time or day (shown in Figure 6.45). They were connected to each other and to Sam.

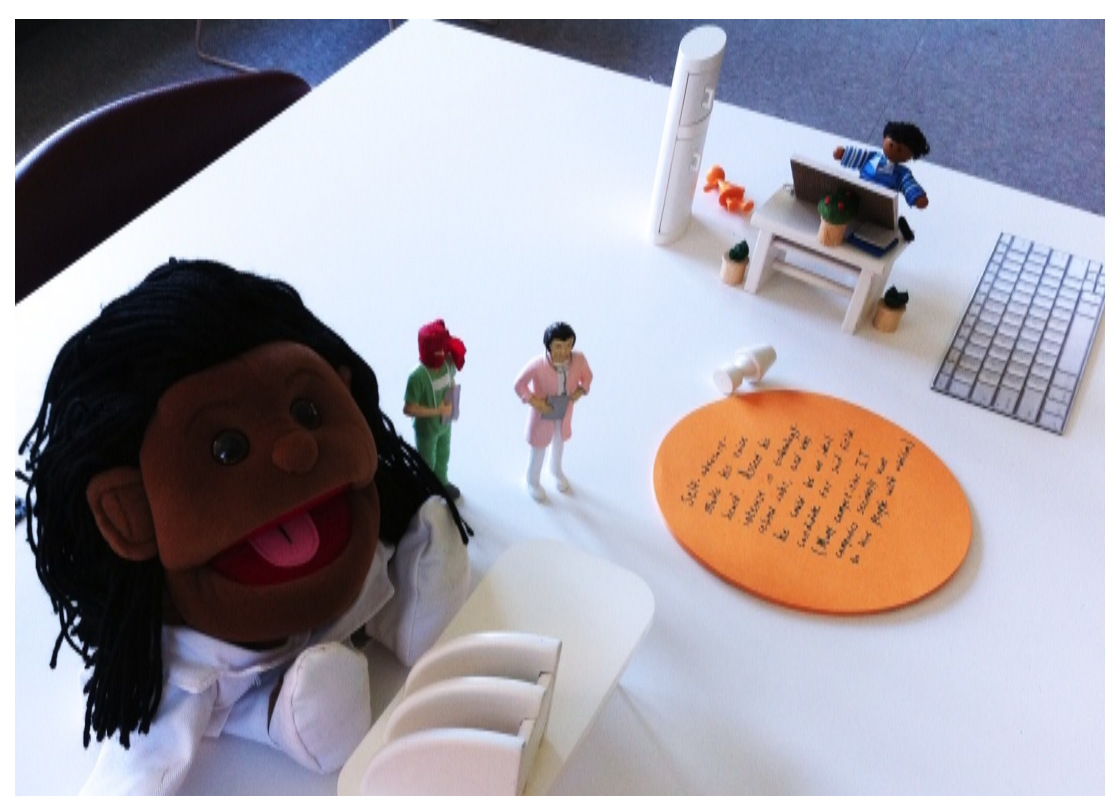

Figure 6.45 The props used to communicate Sam's remote Care Team.

John chose to express his idea somewhat independently. Using LEGOs, John (from Team 2) recreated a setting in which neurotypicals and people on the spectrum were actively engaged in a RPG (role playing game). LEGOs and the game itself were not the concept, but instead represented the need for tolerance, social interaction and mutual respect/understanding. John's representation of socialization fit into his team's integrated system of care and independence. Using paper and markers John also created a 'book of references' for adults on the spectrum. Again, the 'book' acted more as a prop than a prototype. The value in this concept came more 
from the idea that adults on the spectrum needed resources (online or at specific places like the clinic) where they could learn more about themselves, others, and how to become more confident and independent. Some adults on the spectrum like Alix Generous, John, and Kristin are selfmotivated and place themselves in self-advocacy roles. Others, who are shy and nervous like Sam (their persona), lack the tools and resources that could motivate them and build up their confidence to feel empowered to speak for themselves and become more independent. The props created by Team 2, representing social groups, integrated Care Teams, and educational references, were intended to inspire and exhibit specific unmet opportunities interrelated in the system of ASD (from the perspective of the stakeholders). These ideas will need to be explored in more depth through the design development of more specific concepts in the future.
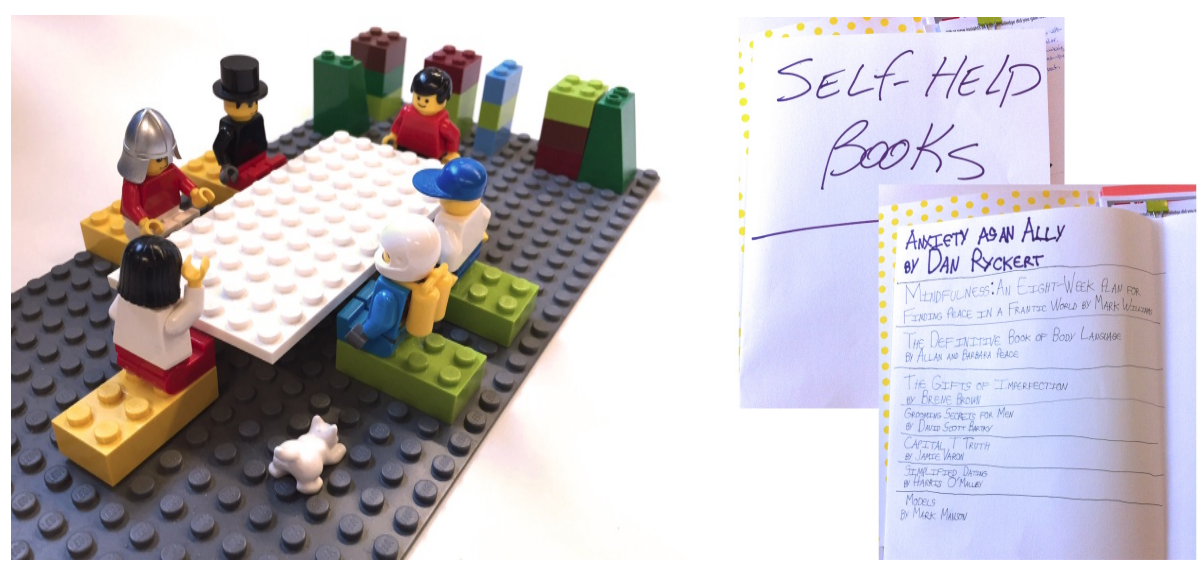

Figure 6.46 LEGO and resource book props.

Kristin's strengths in communicating needs and ideas were exhibited not in making but through the vibrant narratives she wove into the props from her own experiences and the experiences of others on the spectrum. During Workshop 2 she contributed to the 'system' that Bryan and the others on Team 2 had created, but the true value she added to the session came during her team's presentation. In addition to the visual exhibit of a more connected support system and independent life, she reinforced that these things could not be possible if people on the spectrum 
were not better understood and appreciated for what they can offer. Before a person on the spectrum can get a job or socialize with nuerotypicals, society has to look past their limitations and see value in their unique gifts and abilities. This was an unmet need, in her mind, that also needed to be addressed in future Design concepts.

Generative tools were provided throughout all three stages of Workshop 2 to help the participants critically make sense for 'what' needed to be developed or implemented into the ASD system in order to achieve their goals. The term 'critical making' is used here to highlight that making in this session was not aimed at making a perfect single solution, but instead the focus was on learning, connecting, and maturing an idea through the shared act of making. Through immersion in the personas and narratives and through interaction with various materials, the aim was to encourage innovative thinking, speculative thinking, and creativity. Prototypes and narratives were shared at the end to explain what was made and why it was important to the future ASD system (e.g., how it filled the gaps of their prospective futures), and 'how' it could be implemented/developed. Sharing allowed each team's ideas and prospects of the future to be heard; the act of making reinforced engagement, conceptual exploration, learning, and immersion.

\subsubsection{Workshop 2 Summary}

The teams appeared to work well together and completed the exercises without a problem. The act of developing prospective personas, creating scenarios and collectively building prototypes or props (through generative tools) encouraged the participants to outwardly reflect on their own perspectives and establish a connection with their teammates through the act of shared storytelling and making. It also pushed the teams to consider more than one perspective and to establish an empathetic connection with an adult on the spectrum for whom they were developing the prospective scenario. The participants' responses to the attributes, fears and dreams brought the personas to life, which helped to strengthen the emotional bond and urgency between the 
participants and the personas they were developing for the future. This exercise also helped to highlight the potential barriers faced by children and adults on the spectrum and how those barriers could be toppled and redesigned to support a more ideal transitional process, greater peace of mind, and helping people on the spectrum reach their full potential.

The generative tools used to create the prospective scenarios assisted the participants in visually illustrating connections between present and future roadblocks/opportunities, encouraging the participants to reconsider many attributes of the current system and unmet opportunities for a better future. The 2D and 3D tools, used to construct props and prototypes, were aimed at bridging the communication gap between stakeholders and the Designer, to help the stakeholders express their ideas in more concrete terms. General ideas and key features of the props and prototypes can now be taken and further developed and defined by a CoDesign team, made up of Designers and specific stakeholders closely connected to the features of Owen's List, integrating Care Teams, building social groups, and assembling resource platforms.

\subsection{Post-Phase Feedback and Reflections}

Post-Assessment Questionnaires were given to each participant in Workshop 2. This questionnaire was intended to measure and compare the participants' knowledge of and feelings towards the ASD system and connected stakeholders, the C.A.S.T. clinic, and Design Thinking methods used to tackle complex problems collaboratively, after having participated in this project.

Following the project, two feedback questions were sent out to all of the participants (from all phases) to further understand their initial expectations for the project and to gauge if the approach had met their expectations. The questions that were asked:

1. What were your expectations prior to participating in the collaborative sessions/workshops? 
2. What transpired/occurred during/after the sessions (or a specific session) that was different from what you expected?

A lengthier Reflection Survey was also sent out to all of the participants with the summarization of our findings and resolutions from the project. The responses from the two participants who filled it out can be found in the Appendix. The results of the Pre and Post-Assessment Questionnaires, along with the responses from the feedback questions, will be shared in the Outcomes chapter.

\subsection{Case Study Summary}

The continuous cycle of Design-led making and sharing (telling) methods throughout all three phases appeared to establish and support stronger emotional connections between the participants, as well as the people they were creating resolutions for. This was observed during the collective mapping (sensemaking) activity, after shared presentations in Workshop 1, and during the prototyping stage after sharing the personas and scenarios in Workshop 2. The connections between the participants, the information, and the future personas they were designing for deepened as the research progressed through the three phases. Outcomes from each session were brought into the subsequent sessions, where iteration, evolution, and maturation of sensemaking and idea generation was encouraged.

Persona development, prospective scenario creation, and prototyping are relatively versatile methods that can be used in the generative phases of the Design process. These methods were integrated into the last phase of the case study after framing the wicked problem and defining the design space collectively. Exploring the current problem space and considering various stakeholder perspectives within the shared ASD system first in Workshop 1 helped to prime the participants with new knowledge and provide more than one perspective to consider before developing ideas in Workshop 2. The presentations, collective sensemaking activity, and shared 
making methods germinated new understanding, empathy, creative thinking, and new connections between the people and the information. The CoDesign process empowered the stakeholders to contribute to tackling a problem that affected them and strengthened their investment in the ideas and resolutions that were being generated and developed. The PAR approach allowed the participants to take an active role in helping to define and explore the paths future designs could take, giving them ownership of the wicked problem. The sensemaking activity (Workshop 1) and prototyping (Workshop 2) allowed more visible connections to be made between research findings (qualitative data) and decisions made collectively around potential resolutions. Each resolution and prospective scenario constructed in Workshop 2 could be traced back to a prioritized topic and a key issue from an earlier phase. Had we plunged into personas, stories, and prototypes related only to the physical clinic, the path between research and resolution may not have been as clear to the participants. Additionally, more meaningful unmet needs and disruptive long-term opportunities connected to the wicked problem of transitional and adult care for people on the spectrum and their families may have been overlooked.

The findings of the case study, and at each phase of the process, will be discussed in more depth in the Outcomes chapter. By and large, a project which began with a primary focus on finding opportunities to add value to the C.A.S.T. clinic and transition process evolved to encompass a more systematic approach to growing understanding and redefining the value and role of the clinic in the context of larger-scale root problems associated with long-term wellbeing and peace of mind outside the clinic. In the process of converging and understanding multiple needs and goals, we reframed the wicked problem and widened our scope, beyond physical health care, to encompass more meaningful issues that need to be tackled. 


\section{Chapter 7: The Digital Tool Prototype}

This chapter is divided into three sections: motivations and intention for the digital tool prototype created in parallel with the C.A.S.T. case study, the development and testing of the digital prototype across three phases, and what the digital tool could mean for future hybrid participatory frameworks for wicked problems. Keep in mind that the section on development and testing corresponds to the timing of each of the three phases (Pre-workshop sessions, Workshop 1, and Workshop 2) of the case study.

\subsection{Motivation for Technology and Early Evolution}

The reason for building a digital tool prototype for the Design process was to push the limits of the current methods and tools we use in Design Thinking and to explore an alternative way to engage collective teams in thinking and interacting with each other and information differently. The intent for developing the digital prototype in parallel with the case study was to gain a better understanding of user dynamics and workflows in collective innovation settings. Observations made during the sticky note session of Workshop 1, development and testing of the digital prototype over multiple phases, and precedent research were used to inform the functions for a future digital tool for collaborative sensemaking and to explore what benefits technology could afford Designers and stakeholders in the CoDesign process when tackling a wicked problem. The

prototype was designed to provoke collaborative discussion and new ways of seeing a complex problem from multiple angles. The intent for the digital prototype was not to replace, but to support non-digital Design Thinking methods and tools in collaborative settings, and work in 
tandem with face-to-face methods and tools that allow people to share stories and build meaning through a hybrid process.

The initial idea for a collective design digital tool for came from experiencing the limitations of using sticky notes over and over in Design Research and brainstorming sessions. Hundreds of sticky note ideas would be put up on a common wall and clustered into categories. The top five ideas would be selected (usually by the person 'in charge') and the rest would be forgotten, lost in a PowerPoint summary on an administrator's computer or on a rolled up piece of paper in the corner of someone's office. Often, in these brainstorming sessions little consideration was given to converging around root causes, or how the unique needs and ideas of each stakeholder were related, or how these needs impacted the greater problem situation. Drawing from these experiences, the initial objective was to design a digital tool that would facilitate idea generation, collection, and communication. However, the vision for the tool shifted from a tool for brainstorming to one for sensemaking and synthesis during Phase 1 of the case study. The new direction began to take shape as it became apparent in secondary research, discussions with the C.A.S.T. stakeholders, and early prototyping that technology could do more than assist collaborative teams in posting ideas in a shared virtual space. Instead, what was needed for tackling the wicked problem was a digital tool designed to bring people together to converge and synthesize information in the front-end of the Design process, make sense and frame the scope of the problem, and collectively generate meaningful resolutions in a co-located space.

\subsection{The Digital Tool Prototype, Designed for Learning and Exploration}

The intent for designing a digital tool was not to develop a single tool solution or to prove or disprove the use of digital technology in PAR, but instead to explore the process of developing a blended interaction tool, while also studying the social interactions and tasks of potential users, and to identify opportunities where a tool like this could add value to the Design process. The aim 
was to design and develop a semi-functioning prototype that could be introduced during Phase 2 of the case study to help stakeholders make sense of the problem, communicate multiple points of view, discover new connections, and stimulate open discussions around the varying needs and future goals relating to transitional care and support for adults with autism and their families. More specifically, this prototype was developed to test various interactions on a shared digital canvas that could elicit creative and critical thinking, help people pinpoint root problems and make connections, identify shared and distinct needs/goals, and prioritize opportunity areas.

\subsection{Introduction to the Digital Tool Prototype}

Working with Alan Price, a professor at ACCAD (Advanced Computing Center for the Arts and Design) at The Ohio State University, an early prototype of an interactive visualization tool was designed and developed to fit into the hybrid Design Thinking framework established for this case study and future Design projects. In the book Routledge International Handbook of Participatory Design, Brandt, Binder and Sanders (2012) mention the term 'boundary object' (p.148-9). A boundary object is an object that can give meaning to different people even though the people may come from different backgrounds or have different perspectives. Though a boundary object may have different meanings to different users, it also has enough structure to make it recognizable and usable. The aim of the digital tool was that it would serve as a boundary object. It would have enough structure in general use to provide users with simple visuals and interfacing gestures to accomplish a task, but be malleable and flexible enough to allow users to shape and adapt its features to meet their unique needs.

Further findings uncovered in secondary research relating to topics like blended interaction and technology in the Design process, group dynamics and collaborative Game Design, externalizing the design synthesis process, and using interactive data visualizations to build continuous understanding led to new discoveries and new tool features during the iterative phases of 
development. Secondary research revealed the limitations of paper-based tools like Mess Maps to extend participation and trace decisions and sensemaking beyond the present moment (or session), so functions for saving and replaying actions at a later time were explored. Additionally, there appeared to be a lack of digital tools that promoted collective convergence, prioritization, and front-end strategy/framing around multi-faceted problems. Many of the examples of digital tools used in Design Research are used for collecting data and generating/sharing ideas remotely. The aim for this digital prototype was to facilitate communication, new ways of seeing, and group engagement on a shared surface. In addition, it was designed to help externalize the design synthesis process by immersing collective teams in sensemaking. Instead of providing a static summary of computed relationships or a visual summary of the Designer's assumptions drawn from earlier research/encounters, the data would be organized and made available on a multi-touch digital surface were it could be manipulated by the stakeholders together in real-time.

Various interactive interfaces and functions were explored during the development process to prompt users to engage with each other and the data through shared interactions. The goal was for the tool to function in a way that made the Design Thinking and Participatory Design processes more transparent and democratic. Priority needs and ideas from all stakeholder groups would be represented and measured not by rank but by where the collective team deemed they were most important or necessary by visually mapping the interconnected system of stakeholders and their needs/goals. An added feature of the prototype, along with providing transparency and visual feedback in real-time, was tracing thought processes through a string of automatic screen captures and decisions. The interactive digital tool was developed to incorporate different perspectives and be used as an iterative platform from which people could collectively discover a common language through shared engagement and flexible interaction. 
As a tool for sensemaking, the prototype was ultimately designed to explore ways technology could be used to actively engage users, organize and present gathered data, support design synthesis (prioritization and connectivity among data points), and store/communicate decisions for future use in the Design process. Each movement and manipulation made on the multi-touch screen would then be captured and documented in a video that could be replayed after the session. The section below describes the prototyping process and the outcomes derived from each iterative phase of development. The evolutionary changes (inspired by precedent research, observations made during the case study, and trial and error during prototyping) will be described in more depth in each phase of the tool's development below.

\subsection{Phase 1: 2D Visualizations and Paper Prototypes}

In the field of Human-Computer Interaction developers use paper prototypes before developing digital prototypes to visually represent screen interfaces and to quickly walk through various interactions/situations in a series of operations that help users achieve a specific end-state or function (Brandt, Binder, and Sanders, 2012, p.156). Screen interface visualizations were first

developed using Illustrator (an Adobe graphic design software) to help translate the initial idea of the tool from words and thoughts to images that could be communicated and vetted with other Designers. The primary function of the tool, as it evolved from a brainstorming tool to a sensemaking tool, was to present various needs and goals in one shared space so stakeholders could juggle multiple perspectives, prioritize areas of interest, and draw comparisons. The 2D visualization took on many forms, from matrices to graphs, to find visual ways to most effectively communicate similarities and distinctions among the needs and goals of various stakeholder groups. As the early tool was being designed, data from Phase 1 was also being compiled into a matrix, cataloging the struggles, needs, goals, and ideas of each stakeholder group (Figure 7.1). 

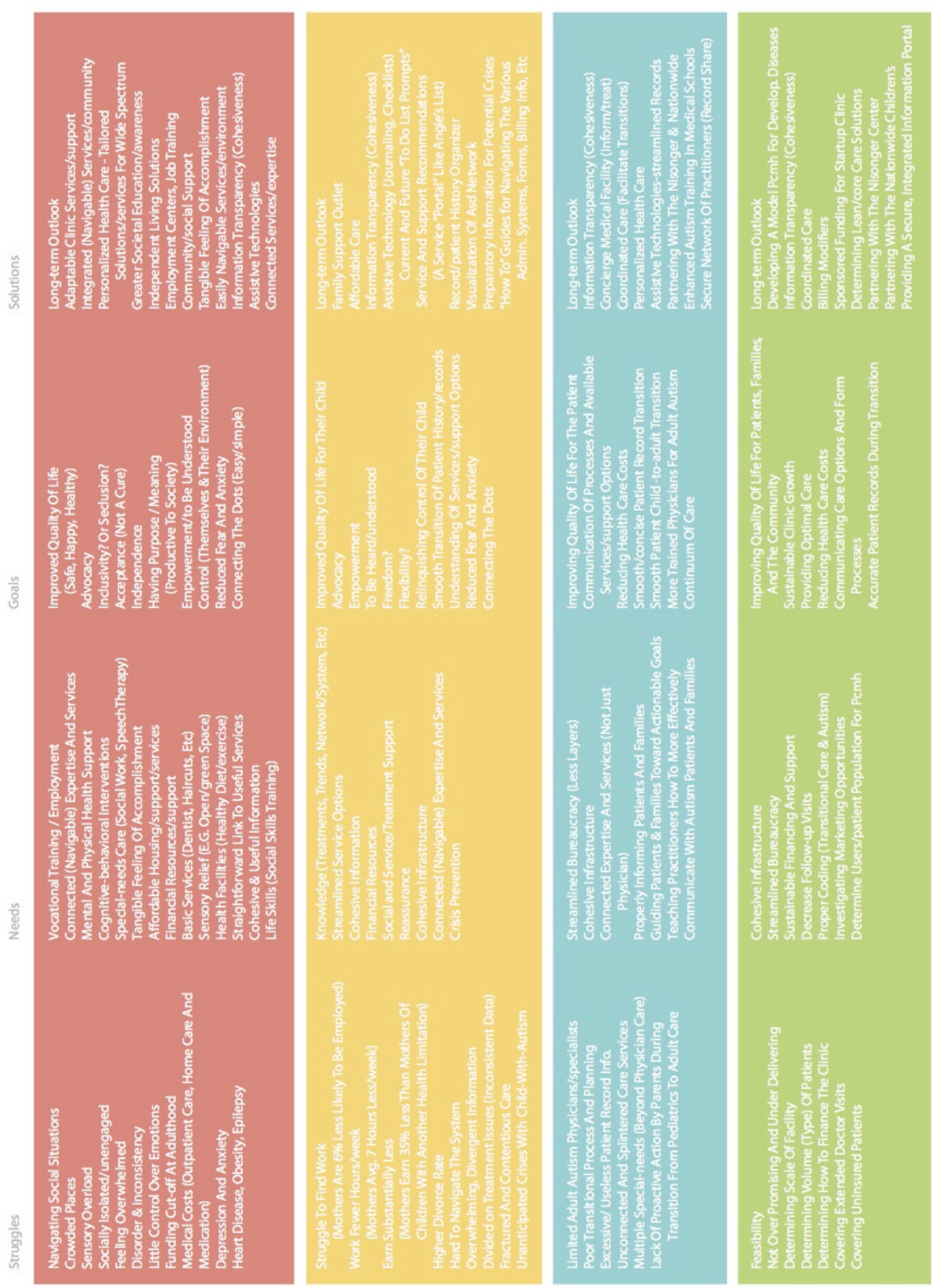

ธุ่

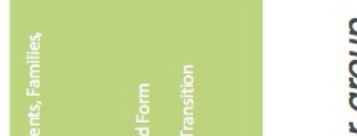

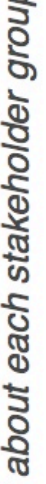

-

ญุ

Q

ह

$\frac{\pi}{8}$

!

ชั

$\frac{8}{\frac{8}{\pi}}$

ชิ

용
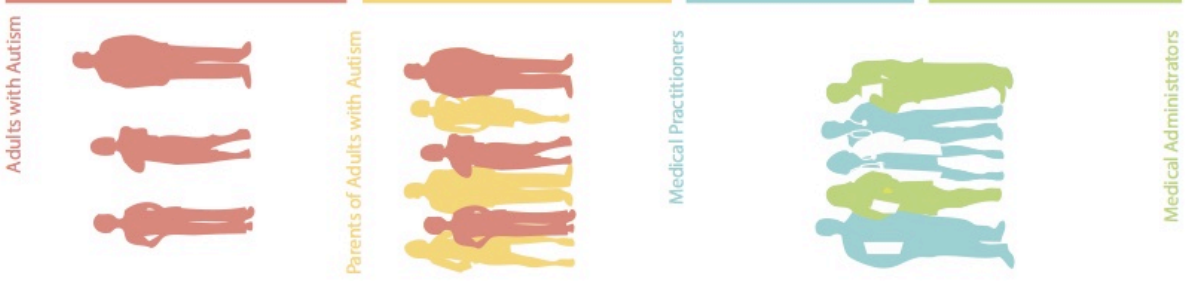

D 
New categories of needs and goals, connected to all of the stakeholder groups, were derived from seeking out patterns and outliers in the data recorded in this matrix from secondary research, interviews, and focus groups. This matrix led to the matrix of Current Needs and Future Goals and eventually the list of forty-eight needs/goals (both shown in the previous chapter).

\subsubsection{D Visualizations}

The needs and goals pruned from the data were arranged in high to low rating charts (far left image in Figure 7.3), as well as radar graphs, to explore various ways people could comparatively depict how important certain needs/goals were. The radar graphs (Figure 7.2) helped to depict similarities and differences between the needs/goals that were most important to the stakeholder groups within each category, however they were visually confusing, especially when trying to look at the wicked problem from a holistic perspective across all categories. Rating charts seemed to limit the ability to express context or background to the decisions being prioritized and, like the radar graphs, these charts also kept the needs and goals within categories separated from one another. In addition to not gaining a holistic view, it would be difficult to draw connections between needs and goals outside categories.

Ultimately, these investigations led to a visualization that took the form of a wheel. This form would facilitate the ability for users to visually see many needs/goals within multiple categories and prioritize those that were most meaningful to them. Stakeholder markers (colored circles assigned to each stakeholder group) could be used to mark priority topics on each wedge of the wheel. The wedge is similar to a 'slice' in a pie chart - each category in the wheel had an equal wedge (depicted in the right image in Figure 7.2). Additionally, the sizes of the stakeholder markers would determine the need/goal's importance to the stakeholder. The wheel would also allow users to draw lines or connections between various topics to communicate relationships within the system across categories (far right images in Figures 7.2 and 7.3). 


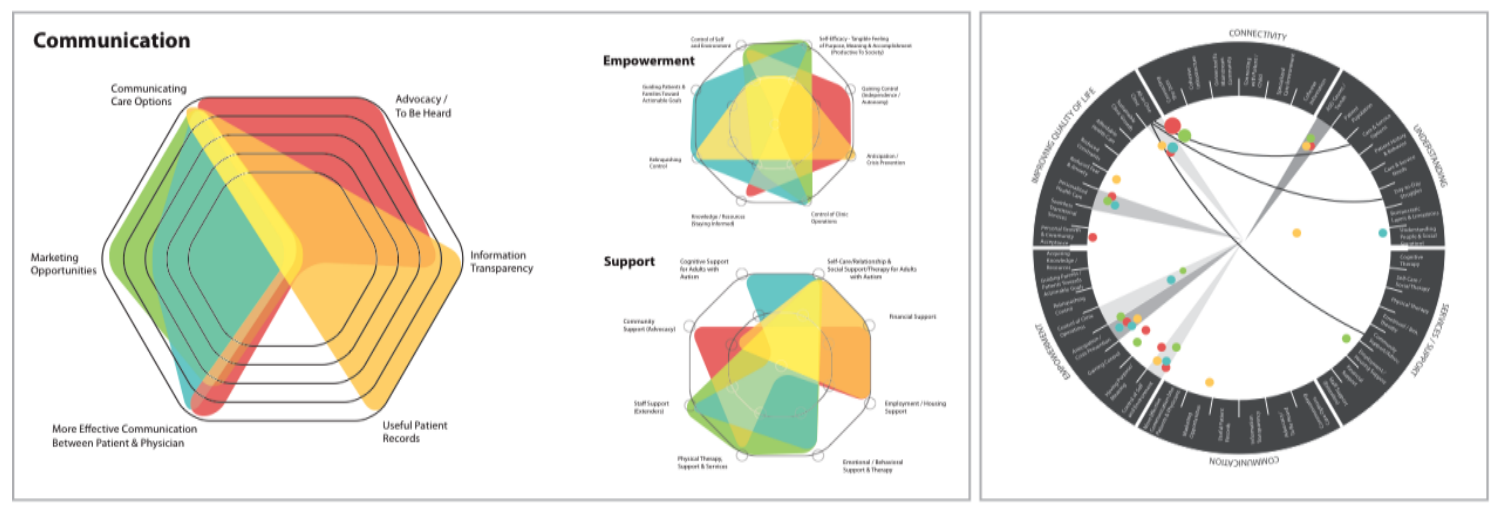

Figure 7.2 The evolution of the visualization from category radar graphs to a single wheel.
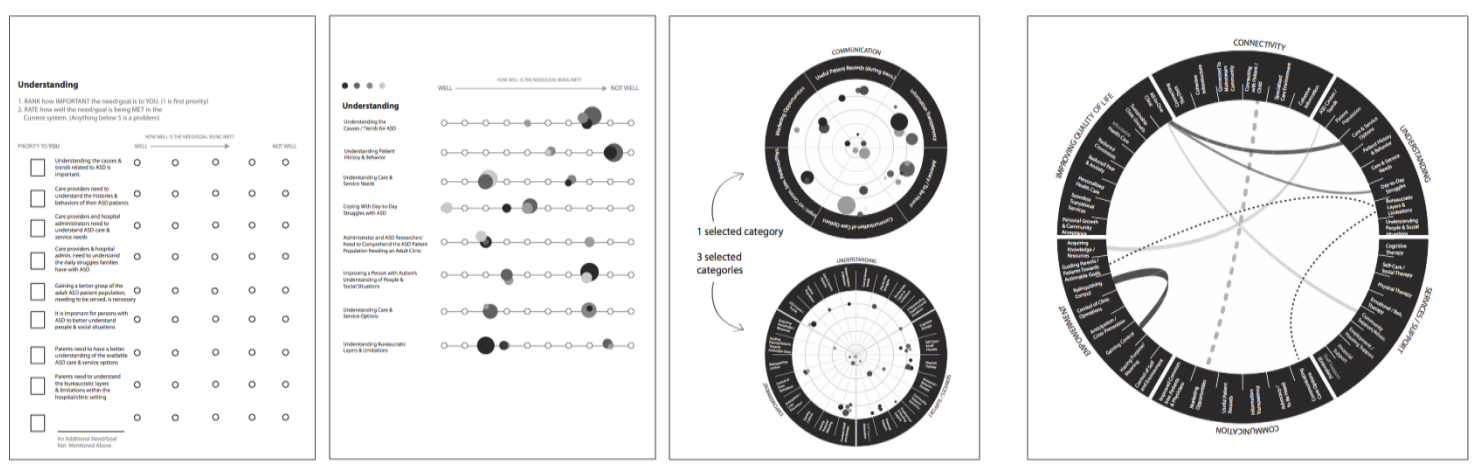

Figure 7.3 The evolution of rating/rankings of needs/goals within categories to the wheel diagram.

The first image in Figure 7.3 list a series of needs within the category of understanding. The boxes on the left were intended to be used for ranking priorities, while the linear dots were to be used for rating relevance to the stakeholder and how well the need was being met in the current system. Testing this prioritization method with various people suggested that ranking and rating topics simultaneously could be confusing. Also, this chart supported individual decision-making tasks and did not allow stakeholders to collectively draw visible comparisons between their decisions. The second image shows a more graphic way of linearly rating and prioritizing needs within the same category. The larger dots represented higher priorities, and where the dots were placed on the rating scale depicted how well the stakeholder group felt the priority was being met. 
This iteration of the ranking/rating charts was designed to help stakeholders compare priorities/relevance to one another and stir conversation when relevance or priorities were visibly in conflict across stakeholder groups. The third image in Figure 7.3 shows the iteration from the rating/ranking charts to a wheel diagram of prioritized topics within one category. The wheel design led to the idea of combining multiple categories within a single form. The large wheel in the fourth image (Figure 7.3) shows all of the categories with drawn connector lines. The ability to switch between macro (all categories) and micro (select category) views, along with the ability to draw lines among the need/goal topics represented on the wheel, were explored further 2D diagrams.

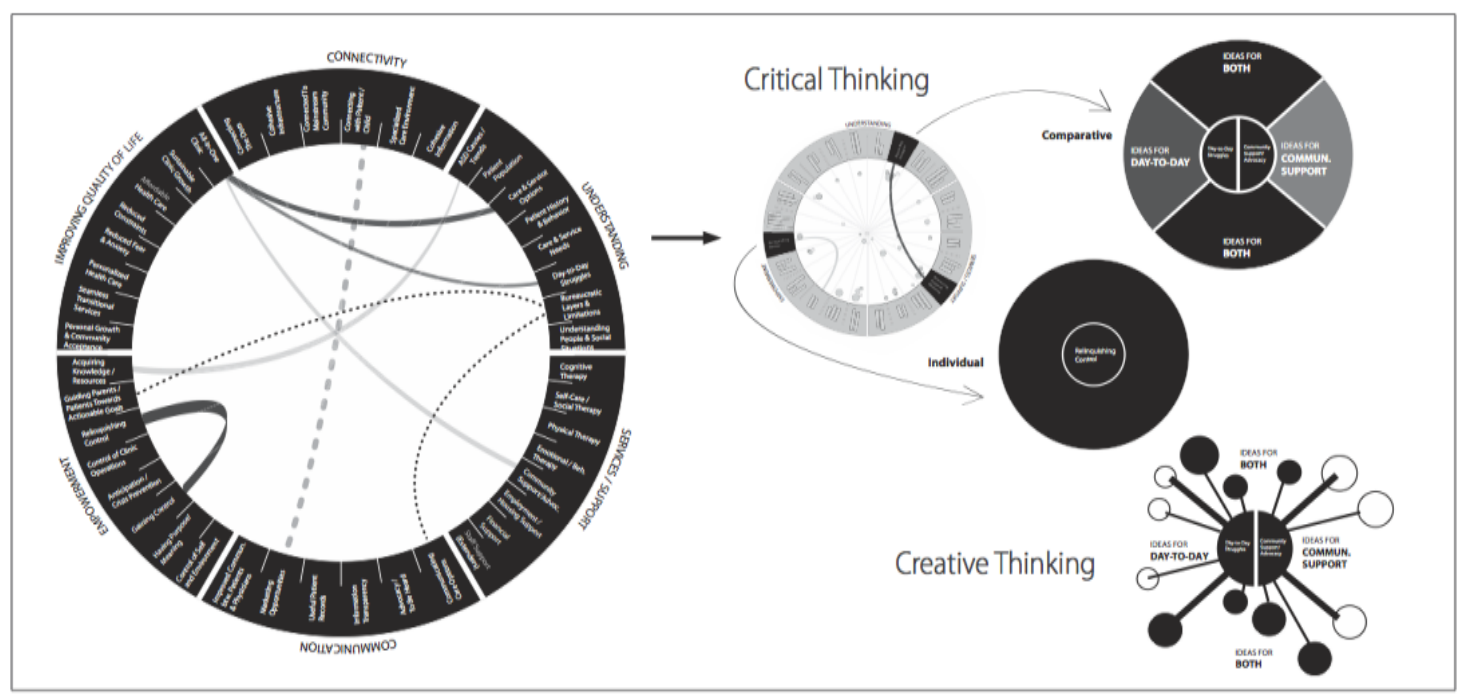

Figure 7.4 Illustrating the progression from connections made on the wheel to building the foundation for mindmapping and generative thinking.

The series of illustrations in Figure 7.4 explored ways to combine critical thinking and sensemaking (prioritizing needs and drawing connections) with creative and generative thinking. Connected topics (needs/goals) could be pulled out of the wheel and used as a foundation for a new mindmap. The mindmap would be grounded in meaning and value (not arbitrary) by the 
prioritization and relationships that had been identified in the wheel. The function of pulling out specific topics to create a new map or create new meaning with the tool led to explorations in how to transform and manipulate the presented arrangement of data. The original presentation (wheel of data) would provide enough of a constraint to begin sensemaking, but would also afford users the flexibility to build context and meaning into the data they were prioritizing/connecting through the shared act of creating new configurations.

\subsubsection{A Cardboard Prototype}

After deciding on this direction, a paper mock-up of the wheel was constructed to investigate the functions of prioritization, connections, and reconfiguring the wedges of the wheel to create new maps. The cardboard model served as an initial physical prototype to transform the $2 \mathrm{D}$ visuals into something tangible and interactive in order to investigate how users might interact with the wheel to prioritize needs/goals, create new clusters, and draw connections. The cardboard wheel was placed on a magnetic whiteboard where it could be spun and each wedge could be lifted out and placed on another area of the board (imitating the notion of pulling out priority 'slices' for brainstorming). The rotation of the wheel would allow data to be read from multiple angles and would allow more than one person to view/interact with the information on the tool from different vantage points. Color-coded stakeholder dots (represented by round sticky notes on the cardboard model) could help to visually distinguish "priority" wedges from non-priority wedges. However, it was evident from the cardboard mock-up that it might be hard to distinguish which priority dots belonged to which wedge. With a set diameter, the width of the wedges was dependent on how many needs and goals were represented in the wheel. Less topics would allow for wider wedge slices, while more data topics would make the wedges narrower. Large dots on narrow wedges may be hard to distinguish readily (refer to the left image in Figure 7.5).

Explorations with the cardboard model additionally showed that a wedge is an awkward shape to drag outside of the wheel to create new maps. Further exploration with $2 \mathrm{D}$ visualizations (far right 
in Figure 7.5) led to the idea of the wedge shapes transforming into circles as they were pulled from the wheel to create the foundation for a new configuration. This is illustrated on the right in Figure 7.5 by the large circle being pulled away from the top right corner of the wheel.
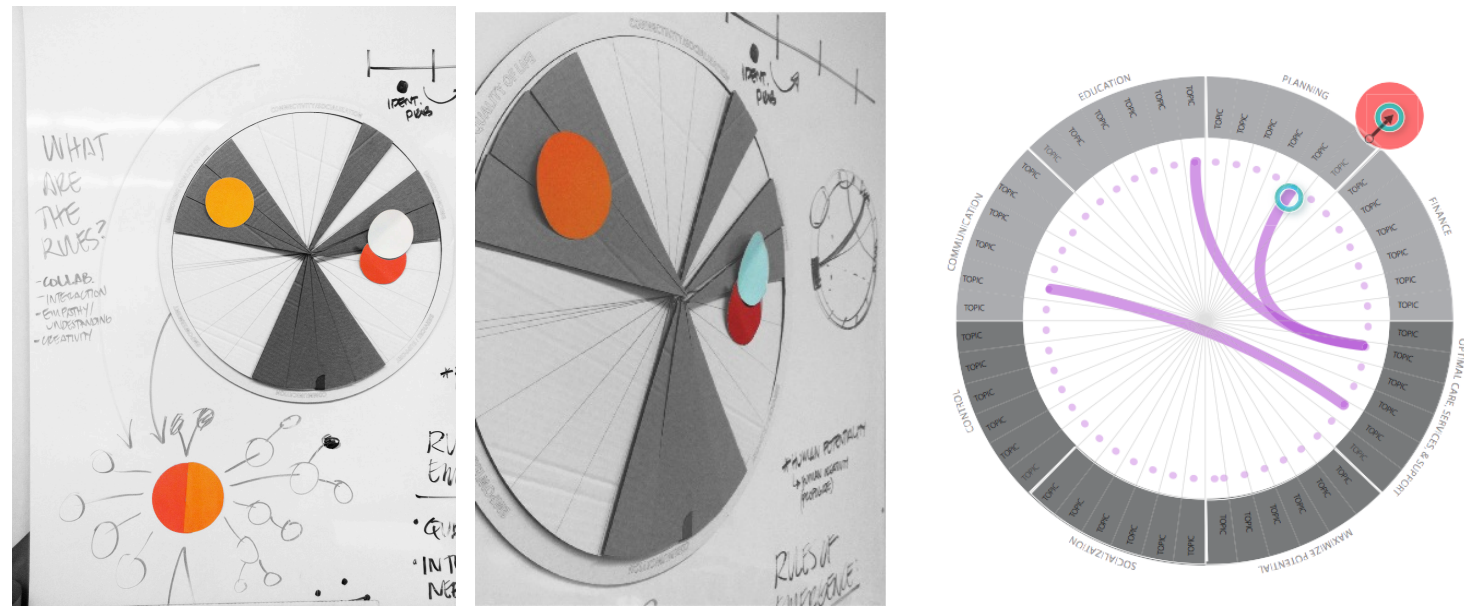

Figure 7.5 The cardboard prototype of the tool (left) helped to inform the next iteration of the $2 D$ prototype on the right, illustrating the transformation of a 'topic' wedge into a 'topic' circle (top right corner) as it is pulled out of the wheel, and the ability to draw connector lines.

\subsection{Phase 2: Developing Digital Prototypes}

The next phase of development was to recreate and test the functions of the paper prototype and 2D visualizations in digital form with touch-sensitive interactions. A more responsive prototype needed to be developed to play out the intended interactions and interfacing between the user and the tool and the series of functions. An initial digital prototype was built to explore the functions of:

- Rotating, scaling, and manipulating the views of the visual map through touch gestures

- Pulling topic wedges out (transforming into circles) to create a new visual map

- Rotating, scaling, and dragging individual need/goal topic circles 
- Capturing, organizing, and communicating various stakeholder perspectives in a single wheel

- Depicting prioritizing needs/goals

- Drawing connections (within and across categories) to represent relationships between data to define similarities \& dissimilarities across multiple perspectives, needs, and goals.

Unity, a game development platform for designing 3D animations and interactive games, was used to create the digital prototype and explore the functions listed above on a multi-touch surface. The Unity program was selected because of its flexibility, game-like interactions, and means through which to establish customized rules of play, instead of being limited to clickthroughs or specific gestures. Unity also offered multi-touch capabilities - using 2D shapes with touch gestures was a relatively new feature in the program that we wanted to explore. In addition to these capabilities, Unity was also selected because it was being taught in two Game Design courses. The courses would be used to not only learn Unity and develop the prototype, but to also test the functions and interfaces of the tool at various stages of development with Design colleagues. Working with Alan Price, who has extensive programming and Unity experience, we began co-developing the digital prototype between Phases 1 and 2 of the case study.

\subsubsection{Development of an Initial Digital Prototype}

The initial digital prototype closely imitated the illustrated 2D visualization in Figure 7.5. Data collected from initial interviews and focus group sessions in Phase 1 were categorized and entered into an Excel matrix, then saved as a CSV file (Comma Separated Values). The Unity program was then able to be coded to read the CSV data and translate the categorized needs and goals into evenly segmented wedges on the interactive wheel. 

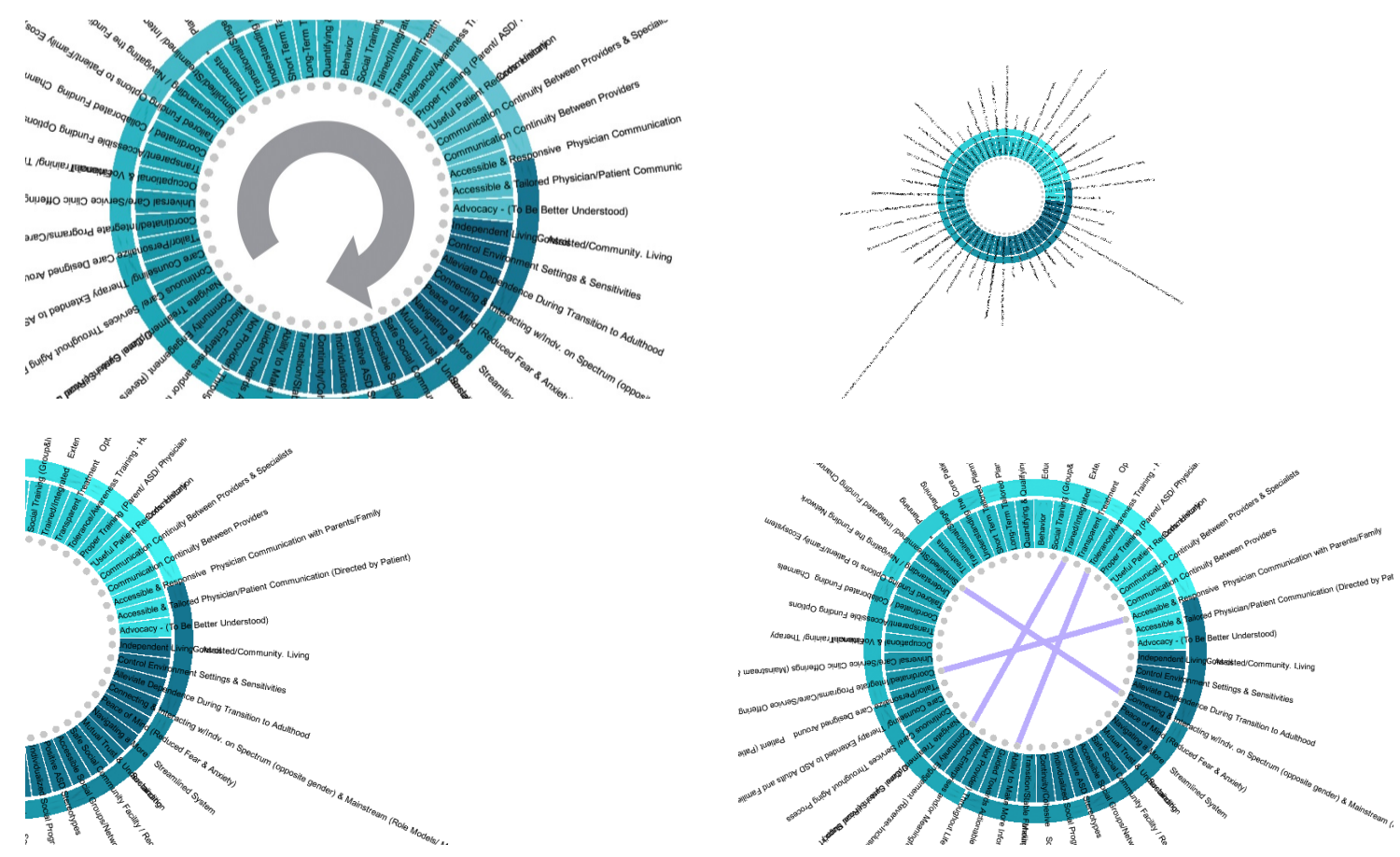

Figure 7.6 The initial digital prototype, including the functions of capturing and presenting data, drawing connector lines, and rotating, scaling, and dragging the wheel of topics.

The main features of the early version of the tool centered on being able to capture and present data from research on an interactive display so that a collective group could juggle multiple topics at once, expand their scope of the problem, and better understand tradeoffs/relationships among the different needs/goals. The prototype also allowed users to scale, rotate, and drag the wheel of topics, visually indicate priority needs/goals (topics) by scaling the circle topics (once removed from the wheel the wedge topics were transformed into circles), and draw connections among topics (unselected wedge topics and selected circle topics) to identify related issues within the interwoven problem.

While being used as a tool for sensemaking to facilitate a convergence of thought and understanding, the digital tool also needed to spark new ways of thinking about the problem and allow for the generation of new ideas and opportunities. This was explored through the function of 
allowing users to pull individual or connected topics out of the wheel to create a new visual configuration. This feature was intended to support the social process of sensemaking (negotiating decisions through data manipulations on a shared surface) and the notion that sensemaking is heavily dependent on the interpretations and perspectives of the people present. The meaning generated from the same set of data may be perceived differently, depending on the stakeholders who are present. Therefore, the digital tool needed to not only present users with information, but also needed to afford those users the opportunity to construct new narratives and meaning by creating a customized visual representation of the topics they found to be most significant.

During pilot testing with other Design students, however, we discovered interruptions and limitations with rearranging the data - pulling wedges outside of and back into the wheel. Additionally, if the needs and goals were to remain connected (or continue to offer the functional capabilities of connecting to other topic circles), connector dots would also need to be attached to the removed wedge (transformed circle) topics and not remain only in the initial wheel. Figure 7.7 shows connector dots added to the removed topics (circles) after making this discovery. The biggest question left unanswered at this stage of development was whether or not the topic wedges, once removed from the wheel and transformed into a circle, could or should be placed back into the wheel.

All in all, the biggest revelation at this stage of the prototype was that the wheel shape was too restrictive. It limited the user's ability to rearrange the topics or to return topics back into the wheel. Two benefits of this wheel, however, were organization and cohesion. The wheel housed all forty-eight topics (needs/goals), it could be easily turned, dragged and scaled to read the content on the wedges, and the connections between the need/goal topics were clearly 
distinguishable in the wheel. Keep in mind that the text at this stage of development had not been refined to fit neatly on the wedge shapes or circles.

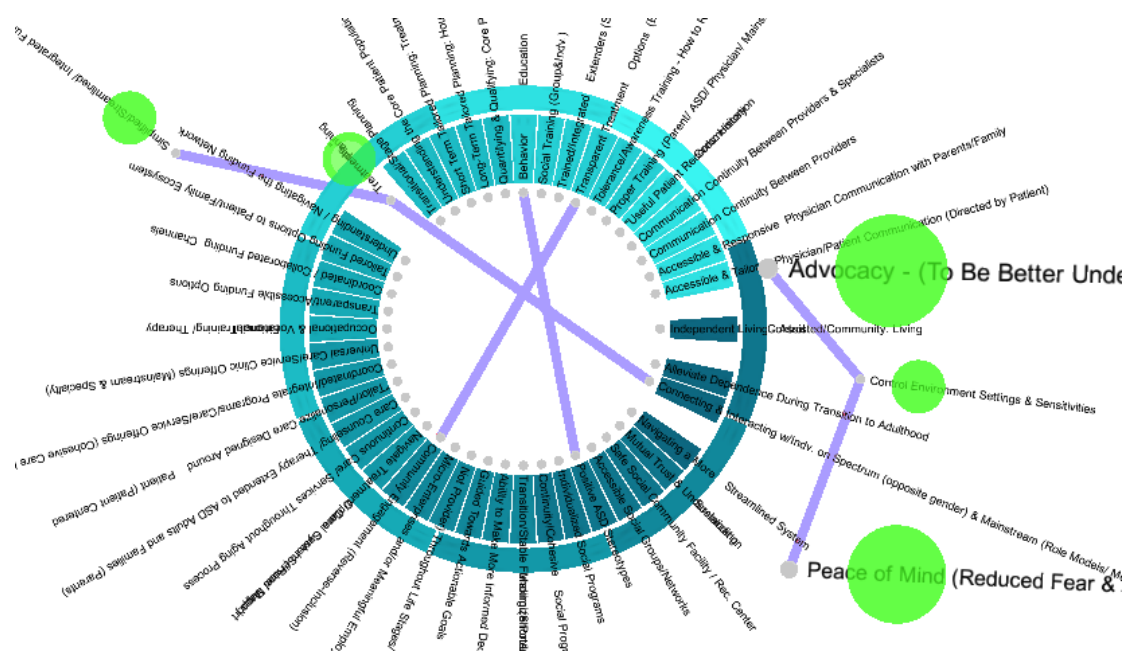

Figure 7.7 The initial digital prototype with connector lines, pulled out topics, and scaled (prioritized) topics.

Jetter et al. (2012) demonstrated that information visualized through digital interaction increased group awareness and contributed to flexible workstyles, while also helping people recognize patterns and more fluidly interact with data. Secondary research into collaborative gameplay and the benefits of blended digital interaction tools expanded the vision for the digital tool in the CoDesign process. Beyond investigating functional features and supporting remote connectivity, technology could create a stimulating environment in real-time for groups and individuals, a safe space for introverts and extroverts, and engage people in collective experiences that embodied both physical and social interactions. The ability for a digital tool to draw people together to seek out patterns and spark new meaning and shared understanding was something that we wanted to explore further in the interfacing and gestures we were building into the digital prototype. Pilot testing demonstrated that the first iteration of the digital prototype was too stiff - it impeded thought progression and workflow. In order to meet the needs of collective sensemaking, the tool 
needed to stimulate collective creativity and provide enough flexibility to allow users to work fluidly and stay curious to seek out patterns and explore new perspectives.

\subsubsection{Development of the Second Iteration}

The next iteration explored more flexible manipulations and game-like interactions in an effort to make the tool more approachable and useful. The first design of the digital tool focused on determining how to input data and prompt both individual and group interactions, but it was too rigid. The second iteration of the digital tool would explore the balance between concrete data and flexible interfacing, and the relationship between individual and social interaction. The fortyeight needs and goals of the stakeholders, curated from Phase 1, would provide the concrete foundation through which the stakeholders could manipulate the organization and representation of the data to provide context and create a new visual narrative. Similar to the way the maps were given context in Tversky's research, prioritized topics would act as landmarks, from which stakeholders could draw routes (or connections). The interactive aspects of the tool on a shared surface would provide flexibility in how the narrative would be told and how it would evolve among the stakeholders present in the sensemaking activity. The connector lines would represent pathways between specific topics and the placement of the topic circles (or nodes) in relation to one another on the screen would determine their hierarchy.

To provide more game-like interactions and support the creation of new narratives, the second iteration of the tool integrated more touch gestures (i.e., scaling, rotating, and dragging), made the components (nodes) more maneuverable and interactive, and allowed for connector lines to be created and deleted. Games can be immersive; they allow people to explore multiple possibilities and to simulate different outcomes. Additionally, they can promote inclusivity when people are working together towards a common goal. The aim in creating more game-like interactions for sensemaking was to promote shared engagement and collective 
creativity/expression, stimulate open communication and negotiation, and provoke users to want to immerse themselves in the data and interact with each other to gain new awareness.

In the second iteration, the research data (identified stakeholder needs and goals) were presented on a large multi-touch device to invite group discussion around decisions that were being exposed on the screen and to promote shared interaction. The maneuverability and scalability of the topics (pulling out key topics and scaling them to show priority), along with the function of the connector lines, were intended to make unique perspectives and root problems more visible to the group so they could make sense of the multi-faceted problem together and reframe the scope (provide context to the data and build a new narrative). Additionally, a screen capture feature was added to record movements every fifteen seconds.

The wheel of connected wedges was converted into a large ring of individual circles (or nodes), each with their own purple connector dot. The nodes could be playfully moved inside or outside the initial ring. This function allowed stakeholders to 'prune' the data and pull selected nodes (individual or connected) apart from the original data set with more ease than the wedges on the wheel. It also afforded users the ability to place nodes back into the original ring without interrupting workflow. While grouped, the ring of data nodes could be moved, spun (to be read from multiple angles), and scaled in synchronicity. Once nodes were pulled out, their manipulation (dragging, scale, and rotation) remained independent from those of the ring. The scalability of the ring provided users with the ability to zoom into topic nodes or make the overall ring smaller. This shrinking feature (along with the ability to migrate all of the nodes in the ring simultaneously) created a more open, blank canvas on which a new visual narrative could be created with the selected nodes (those pulled outside the ring and not scaled down). A 'physics' feature was also applied to the nodes to allow them to bounce off of one another. This element was intentionally added to engender more playful interactions on the screen. The aim of the second iteration was 
to not constrain the data within a rigid shape, but to loosely organize the spreadsheet data into a ring of independent nodes. This loose organization and game-like interactions were done intentionally to promote more playful engagement, stimulate curiosity, and promote manipulation.
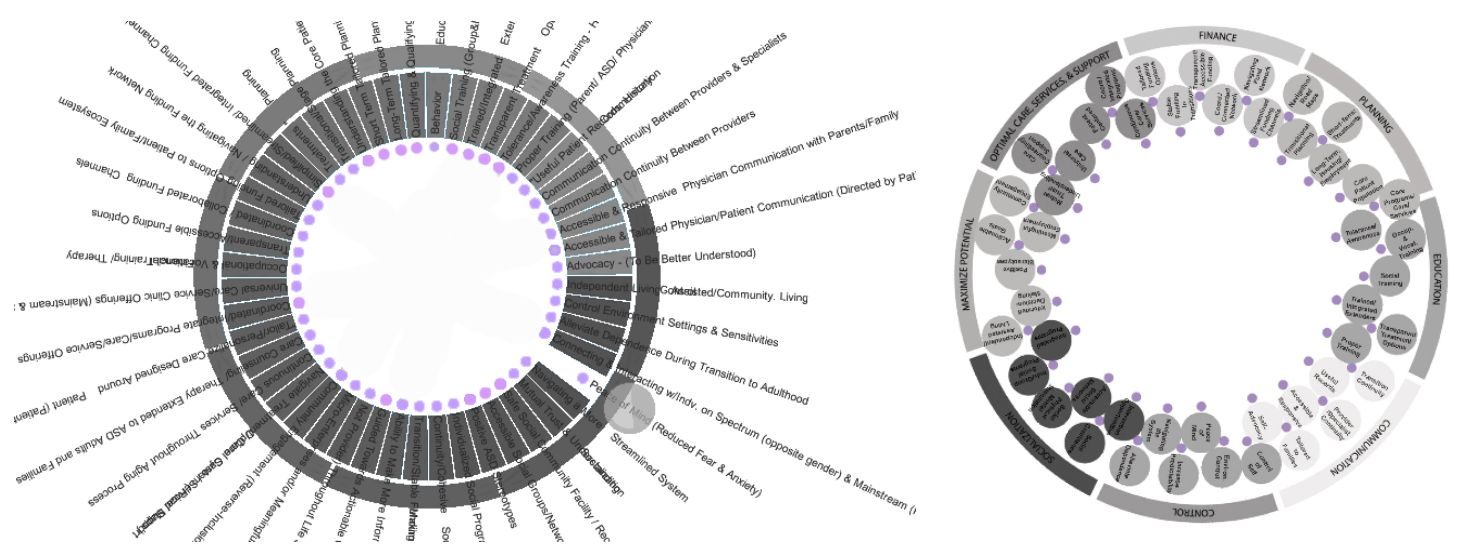

Figure 7.8 Comparative images between the wedge wheel and ring of nodes.

The various touch interactions linked to the nodes allowed users to freely reorganize and reprioritize the data based on their shared understanding and sensemaking. In addition to being able to scale the topics freely through touch gestures (by pinching together or extending two fingers), the topics could also be scaled up incrementally when a 'marker' (colored dot representing a stakeholder group) was placed on them to represent priority. As more 'markers' were placed on the topic, the topic would increase in scale. The marker remained attached to the scaled-up topic while the node was moved around the screen. 


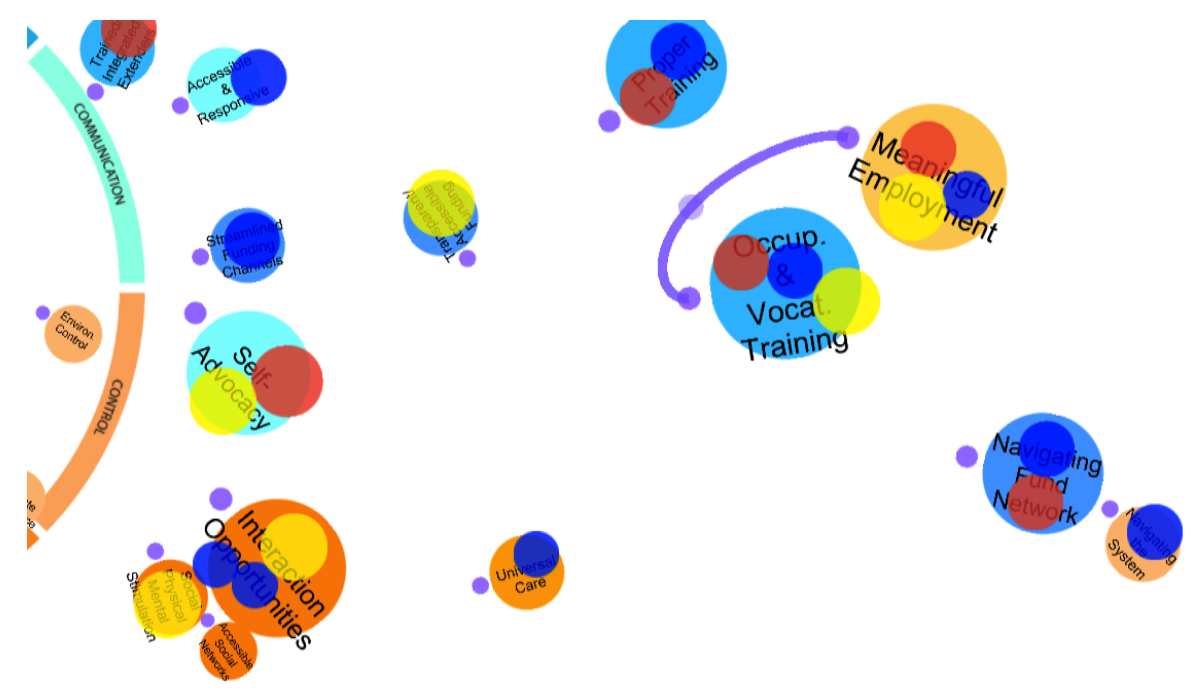

Figure 7.9 Stakeholder markers placed on topics to depict priority through color and scale. A connector line has also been drawn between two topics to show their relationship.

Additionally, the purple connector dots gave users the ability to draw visible connection lines between topics (either within or outside the ring cluster). The connector feature was integrated to help stakeholders construct the narrative and establish linkages between prioritized 'whats' (needs and goals) to determine the 'value' they wanted to create. These functions, conducted on the multi-touch table, were intended to pull the thoughts of the individuals out into the open on a shared surface to promote social interaction and team building around the data points/connections that were most relevant to the group. In doing so, the team could identify common patterns and outliers, gain empathy for one another's perspectives, and obtain a broader understanding of the system relationships/root problems in order to reframe the scope of the wicked problem around meaningful goals.

Key features of the second digital prototype iteration:

- Increased flexibility and playfulness of the ring of topics

- Increased flexibility, playfulness, and maneuverability of individual topics to create new configurations 
- Prioritization - scalability of topics through touch gestures or with colored stakeholder markers

- Drawing connections between data points

- Storing and presenting qualitative data (needs/goals) in one place

- Capturing screen interactions in real-time for future reference/traceability

\subsubsection{User Testing During Workshop 1}

The second iteration of the digital tool was tested during Workshop 1. A multi-touch table and the use of Microsoft Kinect were explored to find the most accessible and meaningful platforms on which to stimulate active engagement, connectivity and new ways to approach the wicked problem through social interaction and a shared making experience. By allowing participants to self-organize around a table and/or wall within a space, the hope was that they would be able to use the tool to actively learn from one another and construct new meaning together. Furthermore, the ownership of the C.A.S.T. problem and the means through which to build better resolutions would be transferred to the diverse team of CoDesigners and would not solely rest on the Designer.

In an effort to find more dynamic and immersive ways to externalize the design synthesis process, we connected the Unity prototype to a Microsoft Kinect to turn a floor-to-ceiling whiteboard in the collaborative space into a multi-touch surface. The thought was that a larger, vertical surface might actively engage more people (since the size of the multi-touch table and the number of touches it allowed were limited to four people). However, the glare of the light on the shiny surface prevented successful touch manipulations. Therefore, we restricted digital touch interactions to the multi-touch table and projected the interactions on the whiteboard wall. The projected table interactions on the whiteboard wall in the space, along with whiteboard markers 
and paper sticky notes (matching the colors of the nodes on the screen), were intended to engage people who were not at the touch-table to see the activity and contribute in different ways.

The dataset used to test the functionality of the second iteration of the tool in Workshop 1 was curated from the needs/goals shared in Phase 1 interviews and focus group sessions. The data had been translated into forty-eight nodes that bounced onto the screen in a ring of eight categories. Nodes colored in various shades of blue represented needs, while nodes colored in various shades of orange represented goals (Figure 7.10). The participants' first tasks were to pull out their top ten topics from the ring and place their designated colored 'markers' on the topics to declare them "a priority need or goal." In doing so, those topics grew in size and the color markers identified which stakeholder group(s) had selected them. This function made their priority needs/goals more evident amongst the rest of the topics and the other stakeholders.

All three adults with autism approached the multi-touch table with a willingness and curiosity to interact with the shapes on the screen, not only to signify their top ten priority needs/goals, but to also play with other functions the tool had. Representatives from the other two stakeholder groups also had a desire to manipulate the topic nodes and draw connection lines, while those who did not have room to stand around the table stood back and watched the projected interactions on the whiteboard wall. However, in the process of dragging topics outside of the original ring and drawing/deleting connector lines the digital prototype crashed twice. There was an unidentified glitch in one of the functions, so we had to revert to using round sticky notes to complete the sensemaking exercise. It was not until later testing, after Workshop 1, that we discovered the small glitch that made the program crash was associated with the function of deleting the connector lines. 


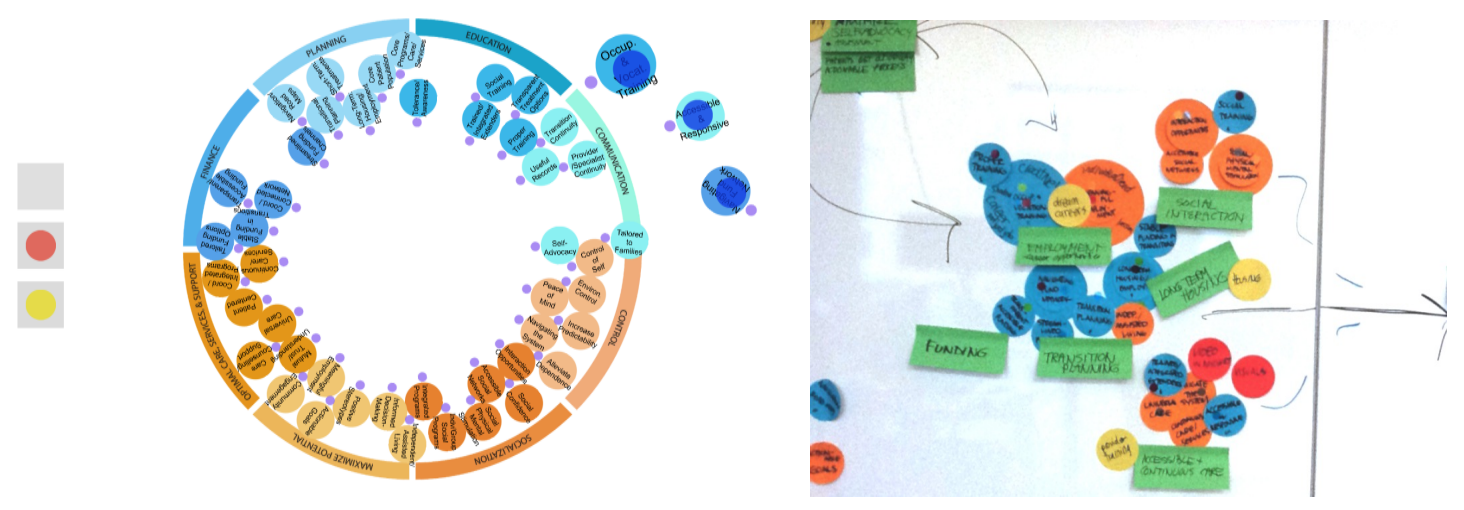

Figure 7.10 Image of the digital tool (left) with intentionally blue and orange nodes to match the sticky notes (right) that were used to finish the sensemaking activity.

\subsubsection{Using the Digital Prototype for Post-Workshop 1 Summary}

An unintended function of the digital tool was that it was used after the workshop to synthesize and visualize insights derived from the organization of sticky notes and from audio/video recordings during the sensemaking activity. Because the prototype failed in use, it was used to visually summarize the sensemaking process and repeat what had been done with the paper sticky notes, so that the stakeholders who could not be a part of the workshop could trace the thinking and decisions made by the group. Before Workshop 2, an animated series of screenshots was sent (in video format) to the participants, along with a final image of the visual narrative they had created with the sticky notes. The video helped to illustrate the decisions that had been made over the course of prioritizing, clustering, and drawing connections/relationships between prioritized needs/goals with the sticky notes. Figure 7.11 shows screenshots from the video, using the revised second iteration of the digital prototype to recreate what had occurred with sticky notes. 

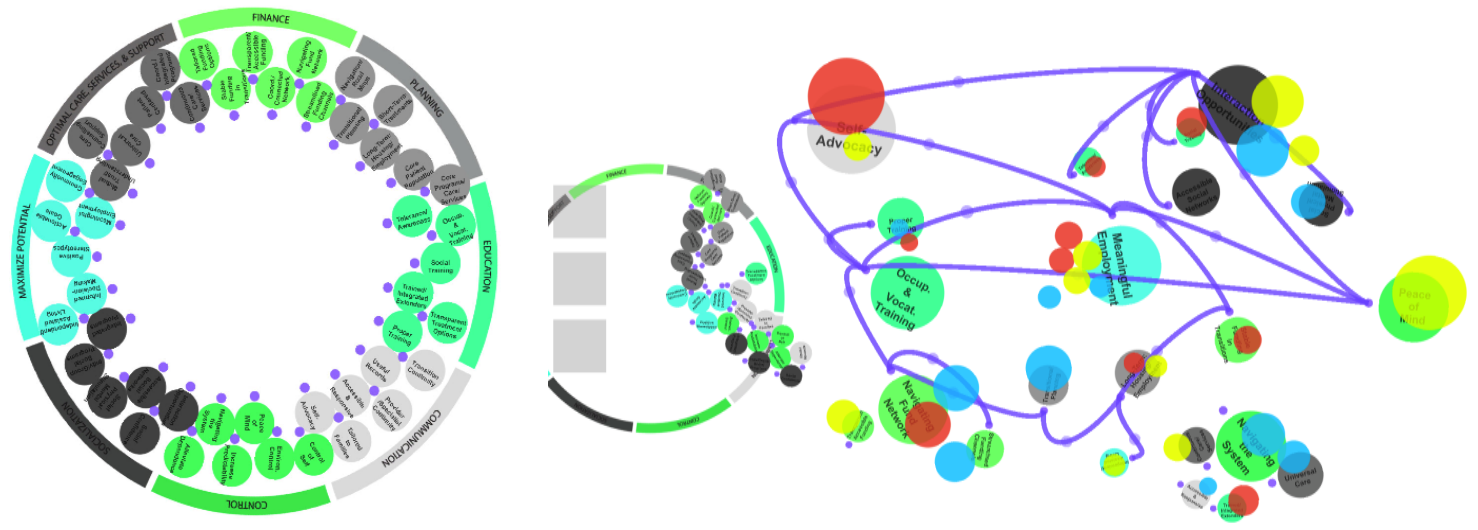

Figure 7.11 The initial ring of topic needs/goals and the final output of prioritized, clustered, and connected topics from Workshop 1, using the revised second iteration of the digital prototype.

The colors of the nodes correspond to the category they are in, rather than needs versus goals. The image on the right depicts which needs/goals were selected from the wheel (and by which stakeholder group by color), how they were clustered and reorganized on the wall (with selfadvocacy (top left) and peace of mind (far right) anchoring all of the new clusters), and the connections that were made among the selected topics to show their relationship to one another.

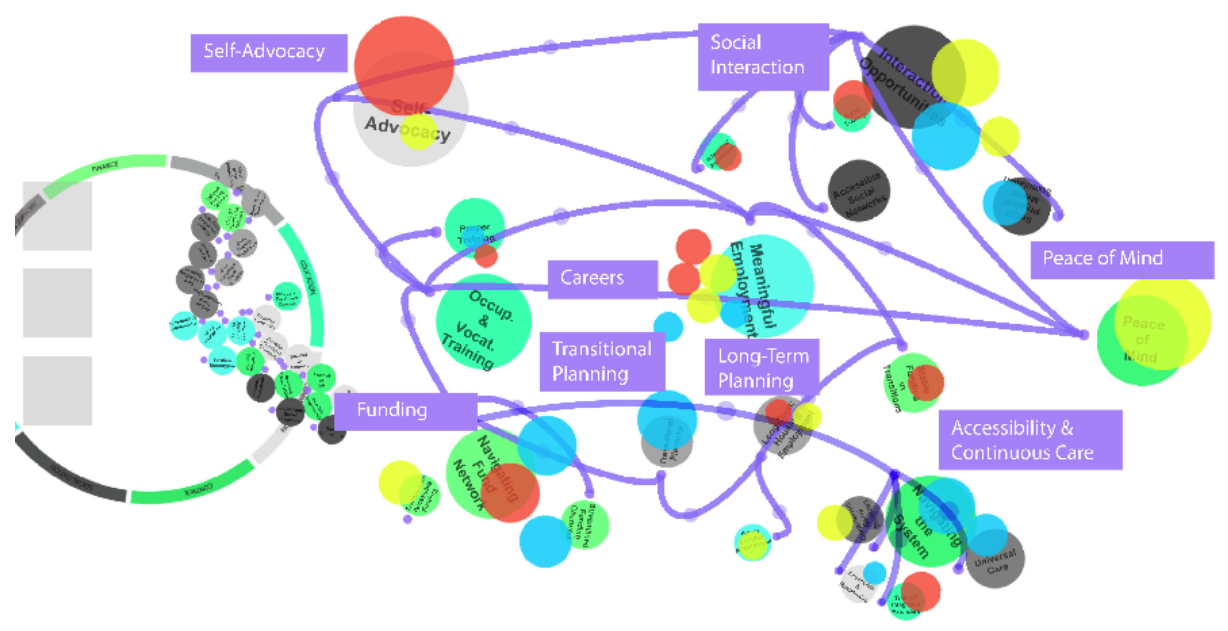

Figure 7.12 The final visual map with the addition of new cluster titles (using Illustrator). 
The addition of the new cluster titles (using Adobe Illustrator) was not a feature in the second iteration of the tool, but was discovered as a necessary feature while clustering the sticky notes in the session and presenting the final visual narrative. A cleaned up version of the new visual narrative (using Adobe Illustrator), was sent out to the stakeholders to communicate/summarize what they had discussed and created with sticky notes (Figure 7.13).
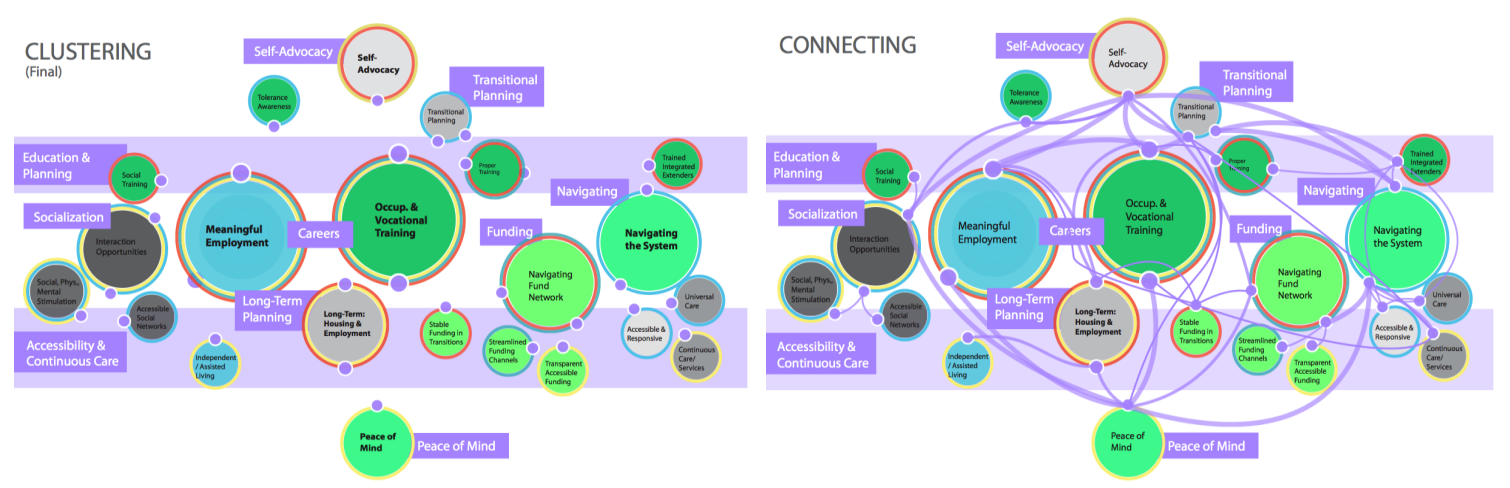

Figure 7.13 A cleaned up version of the new visual narrative (using Adobe Illustrator) showing new topic clusters (left) and connection lines among topics (right).

Visual clutter and chaos is evident in the screen captures of the second iteration of the digital tool in Figure 7.12 Some of this chaos is associated with unrefined functions at this stage of the prototype, some of which were revised in the final stage of the prototype. However, there were also struggles with program glitches connected to the multi-touch capabilities and 2D shapes in Unity. During the process of developing the digital prototype in Unity it became clear that this program was useful as a tool for prototyping the digital concept and multi-touch interfaces to better understand how users might interact with the functions of the concept (that would have otherwise have been hard to replicate in paper-prototypes or a series of 2D visualizations). However, the touch features and 2D shape interactions in Unity are perhaps not refined enough, yet, to use this program to build a final tool. 


\subsection{Phase 3: Final Revisions to the Digital Prototype}

The last phase of development involved taking what had been learned in Workshop 1 and building a final revision of the prototype. In problem-shooting the glitch from Workshop 1 we tried various actions, including bringing in different data spreadsheets. In doing so, we not only discovered that the error had originated from deleting connector lines, but also discovered another complication. The code was not recognizing different category and topic sizes. This realization allowed us to come up with a better solution that would allow various data spreadsheets to be brought in more readily than before. Trial and error, through play-testing, turned problems into opportunities to expand the capabilities of the prototype and make it more useful, and in this case, more versatile.

With the 'connector' glitch repaired and the database input function more flexible, the second iteration of the digital tool was tested with Design students in the Game Design class. Instead of using data from the autism case study, a new dataset was created relating to a topic the students may be more familiar with, the needs/goals of novice bicyclists, casual riders, and expert cyclists. Overall the students found the interactions of the tool playful and engaging, but suggested that certain components and interactions with the prototype seemed impersonal. The colored markers were not representative enough for the people they were intended to 'speak for' on the shared canvas. All in all, their feedback revealed that the tool needed to not only help people make connections with data, it also needed to connect better with the people using it.

Revisions to the tool were also inspired by observations during Workshop 1 while using sticky notes to complete the new visual narrative. After the prioritized needs/goals were recreated on the whiteboard wall (using the orange and blue round sticky notes to match those that had been on the digital screen), the stakeholders then directed the Designers in clustering the selected needs/goals into new categories. After renaming the clusters, the participants were prompted to 
identify connections. During this process they identified cause and effect relationships, as well as determined the overall hierarchy of the topics. For instance, each of the new clusters was in some way linked to self-advocacy and/or peace of mind. Those two ideas became anchors in the new overall frame of the wicked problem, which was focused on designing resolutions aimed at maximizing the potential of people on the autism spectrum and their families. The actions conducted with the paper sticky notes heavily informed the next iteration of the tool, which incorporated a clustering prompt feature, custom naming for new clusters, and directional lines. These revisions were intended to help participants organize their canvas easier, so they could construct a clearer visual narrative and better communicate different casual relationships.

After observing the face-to-face interactions that occurred during the presentations at the beginning of Workshop 1 , and the simultaneous sharing of needs and ideas during interviews and focus group sessions, it became evident that the interface and interactions of the tool needed to support 'Design Wisdom' and fuel more social interaction by not only providing flexible functions, but also providing the means through which users could fluidly switch between convergent and divergent (generative) thinking. It appeared natural in the group's interactions during the presentations that as needs and issues were raised, ideas and resolutions were offered either by the person presenting the problem or by another member of the group. In order to fuel innovative discussions, and foster new understanding and creative thinking, the tool needed to also offer users the ability to contribute new information. As a tool for framing and convergence, along with one that allows for generative thinking, collective teams may be better able to build a strategy from which to enact change (not just understand the problem). This discovery, along with findings from the sticky note session of the sensemaking activity and feedback from the Design students during play-testing, were used as guides to design the final revision of the tool. 


\subsubsection{Revised Features of the Final Digital Prototype}

The final iteration of the digital prototype incorporated more visual feedback, editing capabilities, tailored stakeholder icons (instead of colored markers), and "idea" nodes (with connector dots) to improve user-experience and foster social interaction. The function of adding "idea" nodes was to allow users to not only record potential opportunities or new concepts in real-time, but to also draw connections between those ideas and the prioritized needs/goals. Areas that were refined in the final prototype addressed glitches from the second iteration, as well as the ability to create a clearer hierarchy of topics and a more organized visual narrative of unmet needs and future opportunities/resolutions. Additionally, datasets and stakeholder icons could be quickly interchanged through a web folder.
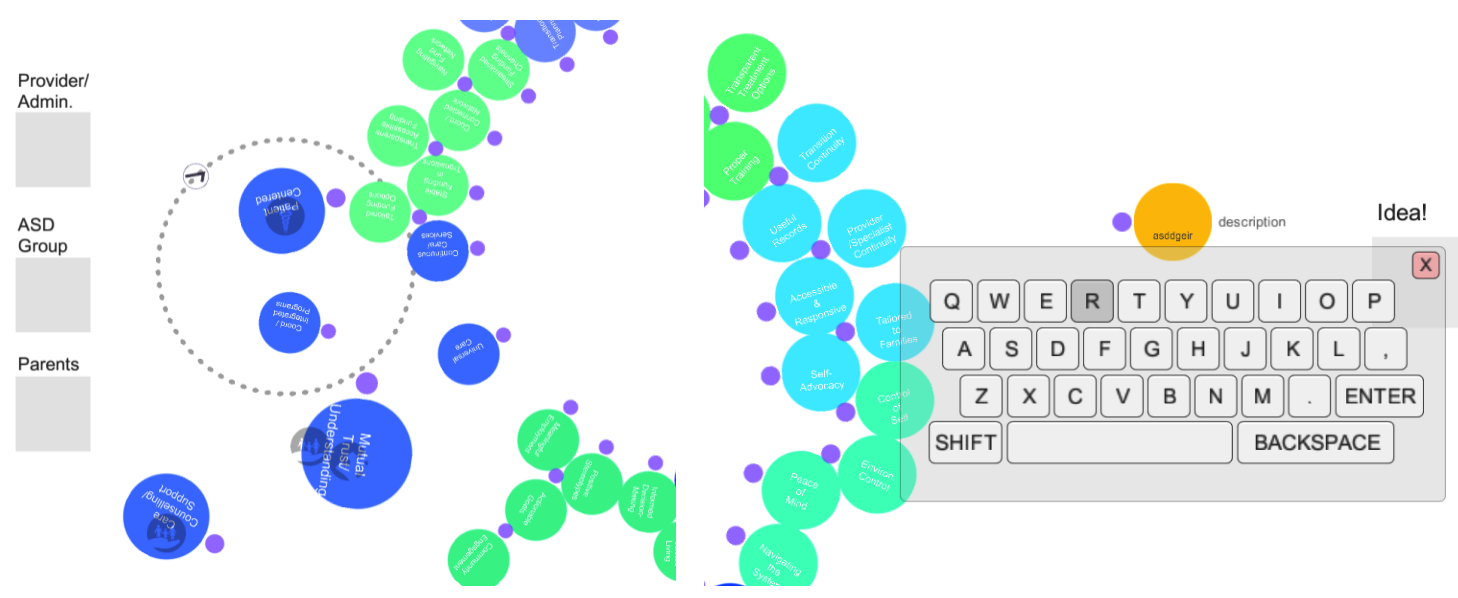

Figure 7.14 The final prototype with project-specific stakeholder icons (translucent icons on nodes, pictured left) and customizable "idea" nodes (shown above the keyboard).

Overall, the final prototype sought to imitate the design synthesis process more deliberately and interactively externalize the process. In order to do this, the functions of the tool incorporated more guidance and visual feedback that more closely mimicked the actions Designers often do in their head or during group synthesis with the sticky notes. The actions guided by the Designers with the sticky notes in Workshop 1, based on the input of the stakeholders, included pruning, 
prioritizing, clustering, and drawing connections with added context (the stakeholders explained 'how' they were connected). The prototype used in Workshop 1 touched on some of these processes, like prioritization and drawing connections, but did not help the stakeholders provide context (the ability to rename clusters or specify the type of lines). It also did not allow the users to easily cluster the nodes or add their own content. Therefore the outcome of the visual map from Workshop 1 was chaotic and messy, and lacked visual context. The playful 'physics' feature, allowing nodes to bounce off of one another, made it difficult to move grouped nodes and to keep the nodes tightly grouped together once they were clustered. Furthermore, after clustering the prioritized need/goal on the whiteboard wall with sticky notes, the stakeholders renamed the clustered groups and rearranged the organization of the clustered groups on the wall to create hierarchy. The acts of clustering, renaming, and determining hierarchy were invaluable in reframing and determining the new scope of the C.A.S.T. project - maximizing potential by targeting unmet needs and achieving goals that resulted in peace of mind and promoted selfadvocacy. Based on these discoveries, the final iteration of the tool increased the familiarity of real-world actions by emphasizing the synthesis workflow observed with sticky notes and incorporated the ability for users to add new information such as new clusters with titles and idea nodes. While still maintaining its playful interactions, the revised functionality was designed to guide CoDesigners in better understanding the process of synthesis in a more interactive and visual way. The goal of the final version of the tool was to balance convergent and divergent thinking, as well as playful flexibility and guidance, so as to engage stakeholders in the sensemaking/creative Design process, while also giving them a better appreciation for the Design Thinking process through immersion. 


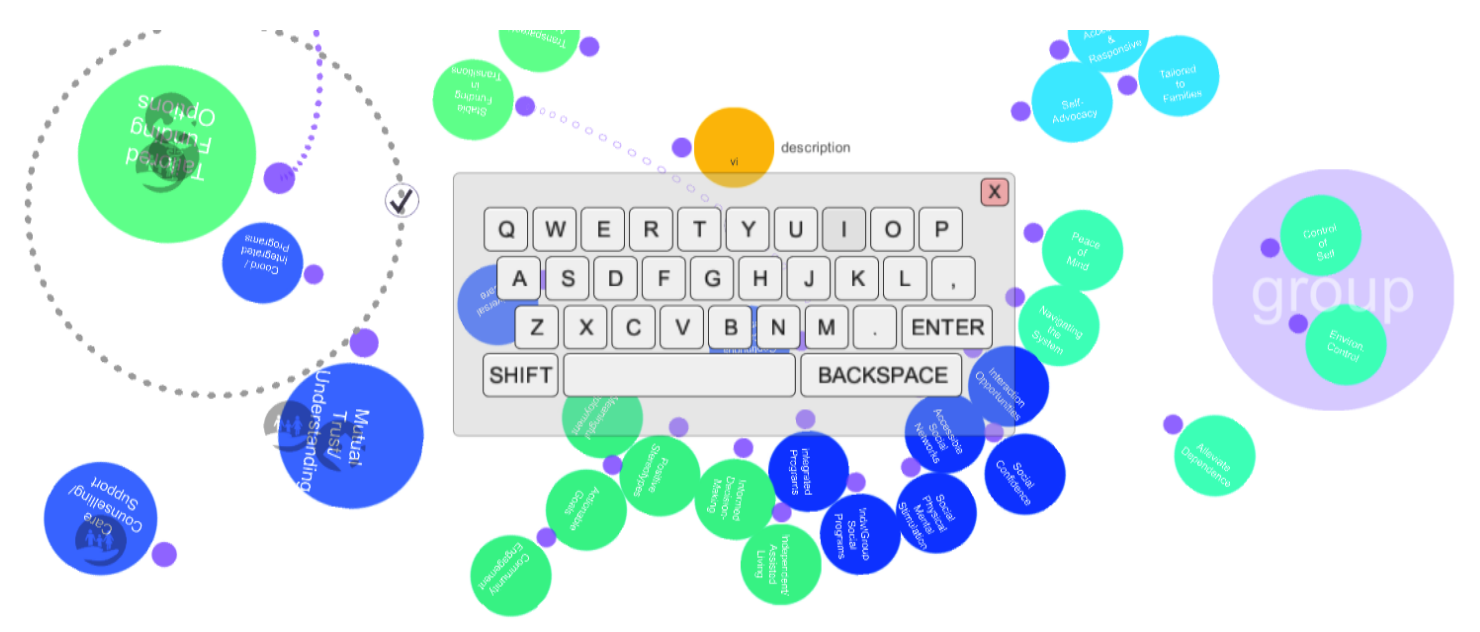

Figure 7.15 Establishing a new cluster from a 'suggested' dotted line to a translucent purple circle. Each new "group" can be given a custom name.

The scalability of the nodes in the final revision was restricted to adding stakeholder icons and no longer using touch gestures to reduce accidental size increases/decreases when scaling the overall ring of nodes, which had been observed in play-testing the second iteration. The 'physics' feature was left on the nodes to stimulate playful interaction, but an automatic feature of a dotted circle around topic nodes was added (as depicted in Figure 7.15 above) to suggest new groups. A dotted circle would appear as nodes moved into close proximity with one another to help participants begin to see potential relationships. Animation was added to the dotted line to increase playfulness and prompt users in deciding whether or not the topics in proximity were related. If the team of stakeholders determined that the grouped topics were indeed a cluster, they could turn the dotted circle into a translucent purple circle, indicating a new cluster had been made. Once the cluster was made, all of the topic nodes within the cluster could be dragged simultaneously. The new cluster could be renamed and dragged to any area on the screen. Directional lines (represented by directional arrows and animated line segments as seen in Figure 7.16) were added to visually show cause and effect hierarchy, in order to better clarify the type of relationship between the nodes. The organization of node clusters, hierarchy, and directional 
arrows was intended to create more linear relationships among the nodes and clusters that could potentially lead to the construction of a clearer visual narrative.

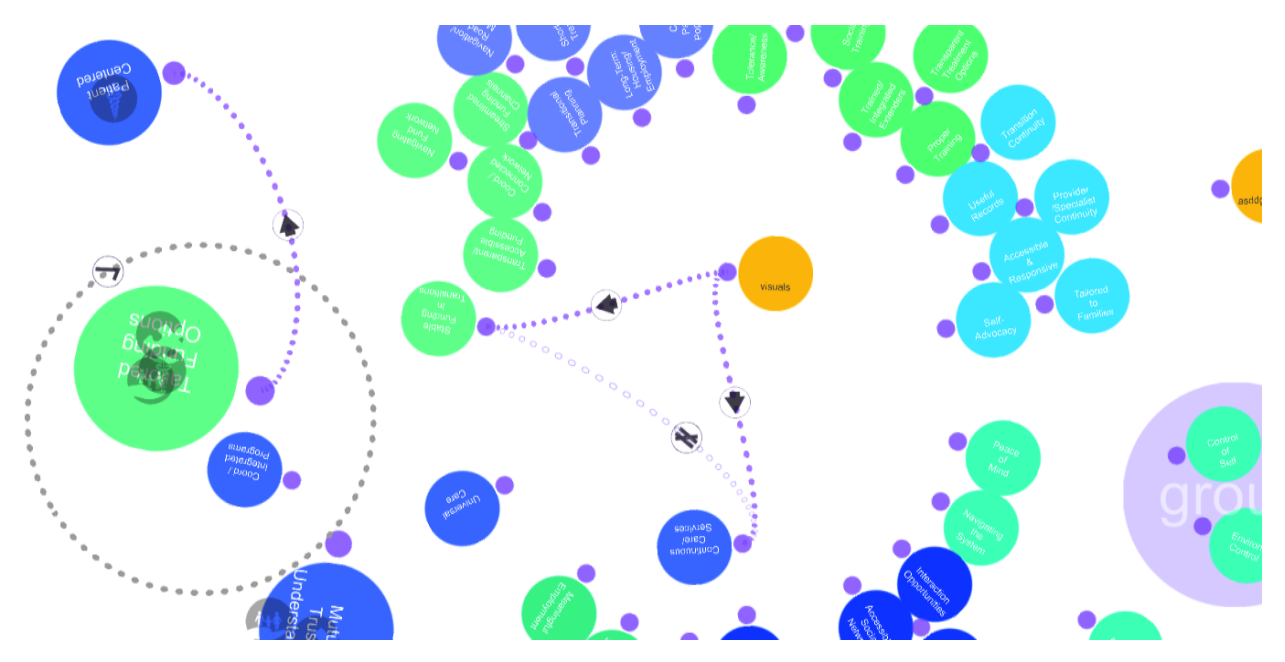

Figure 7.16 Directional arrows and animated line segments show the types of relationships between the nodes.

Key features of the final digital prototype:

- Adaptability - various datasets and stakeholder icons can be brought into the tool

- Increased customization - custom cluster names and descriptions

- Prioritizing (and changing the scale of) need/goal topics with stakeholder icons

- Suggested clustering (dotted circles)

- Creating new clusters/categories (with titles and descriptions)

- The ability to move grouped clusters around the canvas to establish hierarchy

- Supporting the creation of new ideas (with connector capabilities)

- Creating and deleting connector lines

- Defining the types of relationships between nodes with directional arrows/lines

- Capturing, storing and presenting qualitative data (needs/goals) in one place

- Capturing real-time screen interactions for future reference/ traceability in video format 
After revising the final version of the digital tool prototype, two students from the Game Design class volunteered to play-test the new features and functions of the prototype. A new set of data (incorporating various topics related to designing games) was made-up for this play-test. It is important to note that for this play-test that the students would be co-designing a new game. For this task, the wicked problem was Game Design. The use of the tool for 'sensemaking' (using the final prototype) aligned with collectively communicating and understanding the facets of a game, in order to create a visual Design brief for a game they hypothetically wanted to design. The stakeholder icons for the new dataset included: Designer, Engineer, and Writer. The students could pretend to represent one or more of the stakeholder groups as they were building the Design brief for the game, or use the icons however they thought best. The initial ring of categorized data nodes were intended to provide footing and constraints to the students in the beginning, however they were also given the opportunity to contribute new data through the use of the "idea" nodes. Unlike the C.A.S.T. case study, the students had not given input on the topics (data nodes). After giving instructions about the features and functions of the tool, the students were allowed to openly play and ask questions as they encountered functions they were unfamiliar with or unsure how to carry out. The focus of research during this play-test was not to study the outcome of the game they wanted to design, but instead to observe their process and their interactions with the prototype and with each other while engaging with various functions to accomplish the task. Insights from this play-test were derived through casual observations and conversations with the students during this session as they walked through the process.

\subsubsection{Play-testing the Final Digital Prototype}

At the start, the two students began pulling certain points of interest out of the initial wheel of 'nodes,' rotating the wheel, and dragging the selected nodes into new clusters. One of the students pointed out that an advantage of the multi-touch tool was that two people could work together at the same time - while one was creating clusters, the other could spin the wheel to find 
new game elements. Scaling, rotating, and being able to move the ring of nodes to the side were favorable functions. An added feature the students brought up, that was not present on this prototype, was the ability to automatically reorganize the ring of unselected nodes and move the ring to the edge of the canvas. They noticed when moving the ring of nodes to the side that some of the selected nodes would accidentally get bumped. If the ring of unselected bubbles needed to be referenced again, then the same button could be used to "pop" it back out.

They found that pulling individual nodes out of the ring and reconstructing new clusters was useful in deciphering the components of their game. As 'nodes' were added to new clusters, the size of the clusters would increase in size. The 'importance' of the topic clusters could be seen from a distance, which helped the Design students readily determine which task or aspect of the game would require the most effort or design development. Furthermore, the flexibility and ease of the user interface in grouping ideas together and dragging the cluster (with all of its pieces) to other areas of the canvas was a benefit. They commented that when you try to do the same thing with sticky notes you have to move each individual sticky note (grouped or ungrouped) each time new categories or hierarchies are determined. With sticky notes you also do not have a record of how things were organized before they were moved. In this digital tool there is a record. The interactions afforded by the digital tool prototype allow for various clusters and hierarchies to be rearranged quickly and effortlessly. Pulling nodes out and clustering them, automatically scaling clusters with the addition of new nodes, and rearranging hierarchy on the canvas were expressed by the students as favorable features and became the mode of their collaboration. 

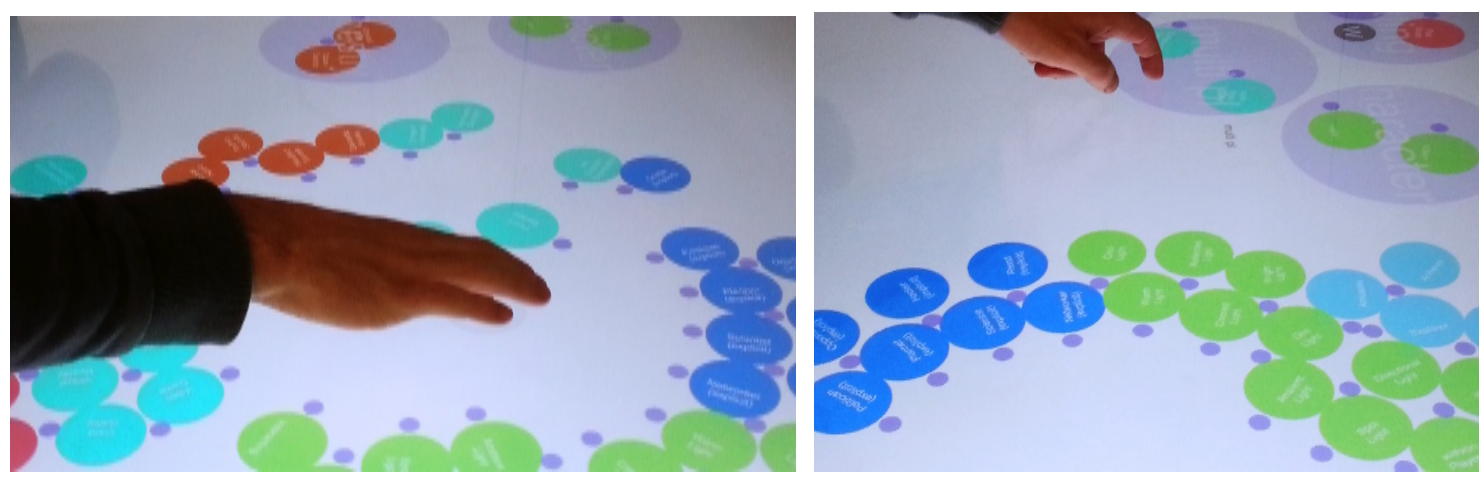

Figure 7.17 The students rotating the ring of nodes, pulling topic nodes out of the initial circle, and clustering them into new groups.

\section{Interaction and communication}

In observing their interactions it was evident that the tool was allowing them to make individual selections, while also provoking them to share verbally what they were doing and why with one another as decisions were being made. These interactions possibly stemmed from the fact that they were tasked with working together to create a collective vision on a shared canvas.

Collaboration consisted mostly of mutual conversation, negotiating priorities, and making selections to elements (like game-type, characters, and setting) for the game they were designing together. Their interactions centered on teamwork and collaboration, rather than the independent expression of ideas. They often spoke out loud just before, or as they were making decisions. As topics were dragged out of the ring the students would communicate their individual visions for the game, and the other person would give their opinion. Through these exchanges the visual Design brief for the game expanded and evolved. The tool facilitated open, casual communication and agreements appeared fluid and natural. Sometimes they would share personal experiences while they were making decisions - their past experiences helped to inform their current decisions and future aspirations. While the students could interact with the components on the screen individually, the students would ask for validation from the other to ensure that as the game evolved, the contributions and decisions were mutual and benefiting the 
common goal. They appeared to find common ground through their interactions on the multitouch screen. "We got the conversation going smoothly. Once we figured out how the tool worked we started building things together intuitively," said one of the students.

\section{Visual and audio recording}

While screen captures were being made throughout their interactions with the data, a second device was recording their conversation. Audio recording helped to capture in-the-moment commentary around the topics being discussed. It was evident during Workshop 1 that much information (including ideas and explanations) had been vocalized but not written down on the sticky notes or captured with the screen shots on the digital tool prototype. In the future, both visual and verbal traceable recordings should be built into the digital tool, so that the context or nature of the decision can be connected to the visual interactions being orchestrated on the screen.

\section{Flexibility, customization, and context}

The students appreciated the flexibility the digital prototype offered, such as the ability to add new ideas/topics (with descriptions), create new clusters (with custom titles and descriptions), and draw four different types of connection lines (with the option to delete the lines). 'Undo' features and customization functions like these allowed the students to change their minds, try different things, and add new context/meaning. The ability to add new ideas was viewed as a valuable feature on the final prototype. As demonstrated in the workshops, and in Sanders' (2015) paper Fabric of Design Wisdom, the ideas and needs for the game were shared almost simultaneously. The students' process blended divergent thinking and clustering - leaping to new ideas while inspired through the convergence of needs and game priorities. They wanted to generate ideas while they were clustering needs/goals. Providing the ability for users to readily add and link new topics/ideas to initial topic 'nodes' was expressed as useful feature by the students. Connector 
lines were only used after the clusters had been made and tasks were being assigned through the placement of stakeholder icons within the clusters. Directional arrows (showing cause/effect relationships, equally balanced relationships, or conflicting relationships) provided additional context behind the decisions that were being made and why. More flexibility features were desired, such as the ability to change the color of individual nodes (once they had been reclustered/categorized) and to change the color of the clusters to add further distinction between the groups. These features would allow users to assign additional/new meaning to the topic nodes and groups, other than the original meaning (color by category) that was assigned at the beginning.
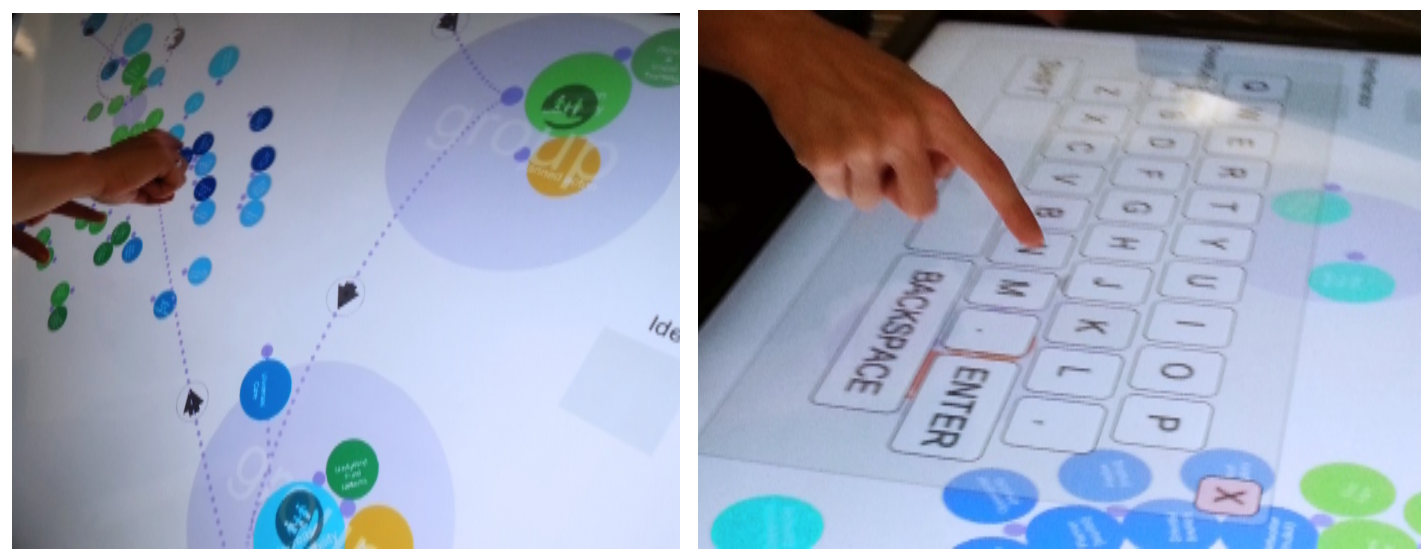

Figure 7.18 The students drawing connections between nodes and renaming cluster groups.

\section{Customizing initial data nodes}

In addition to adding new ideas, one of the students wanted to change a few of the topics on the original nodes. Because the students had not given prior input on the topics, they felt a little restricted in generating a new game concept using the topic nodes they were given initially. Their intuitive gesture was to double tap the original topics to change the text so they could have more say in what the nodes said and represented. The importance of stakeholder contributions early in the research process and prior to interacting using this digital tool for sensemaking was made 
apparent through this observation. The ability to change a need/goal node was a feature that was purposefully left off the prototype in Workshop 1, in order to keep the focus on conversion around the needs and goals that had already been expressed, in order to arrive at a common frame. Depending on the intention of a collaborative session, it may be worth considering the option to allow stakeholders to change the text on the 'nodes', in addition to adding new ideas. Having initial nodes with information was expressed as a useful way to initiate structure around an indeterminate topic, however offering more flexibility would add to the user's ability to customize the tool around their specific needs.

\section{Stakeholder icons}

Towards the end of the play-testing session, the students converged on making more critical decisions about how they would accomplish each task of the game. Surprisingly, the students chose not to use the stakeholder icons at the beginning to assign priority to topics (related to certain stakeholders), but rather used the icons (Designer, Engineer, and Writer) to assign roles and delegated tasks for the next stage of development. Hypothetically, the visual map the students created would be used to not only explain their vision for a new game, but would also be used as a planning tool to build a Design team around the tasks that had been determined for the next phase of Design. For instance, the amount of 'Designer' icons within a cluster (not on a topic) would determine how many Designers would be needed to accomplish a task like building the characters. The digital tool prototype, while designed for sensemaking in Workshop 1 and helping stakeholder groups with different backgrounds and perspectives express unique priorities

and converge around shared needs and goals to develop a new frame, was slightly reinterpreted during this play-test. The flexibility of the functions (like the stakeholder icons) allowed the digital tool to be used in an unanticipated way. However, in both instances (the case study and this playtesting session), the tool served as a framing tool for action. 

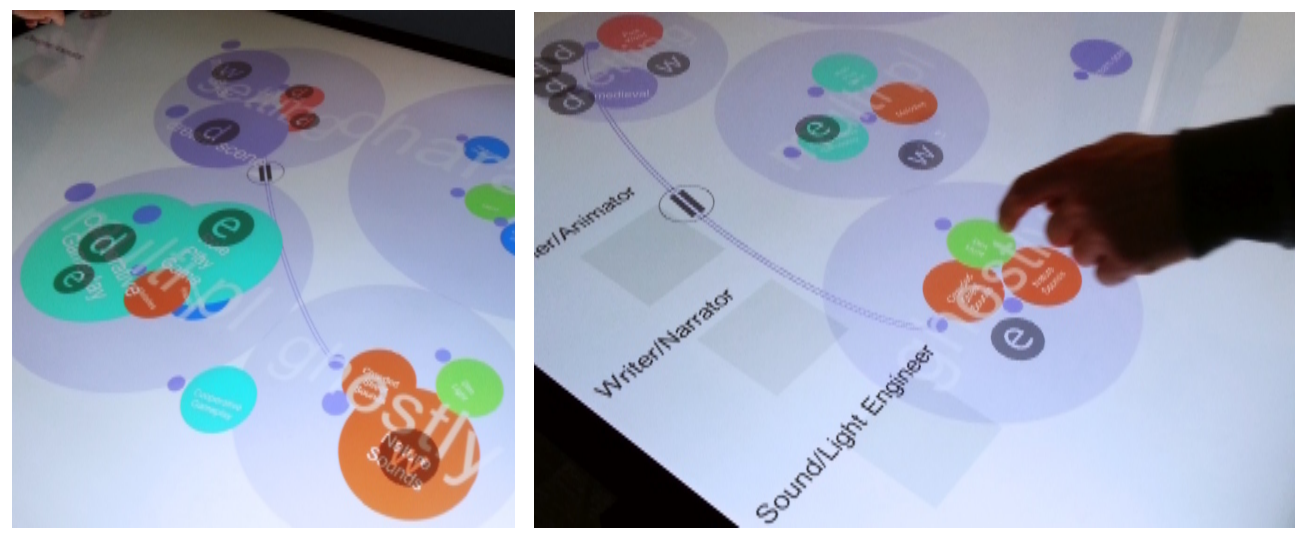

Figure 7.19 Stakeholder icons used to assign tasks within the new clusters.

\section{Organizing visual clutter}

Another insight shared by the students was the need for reorganization. The canvas can get messy as new clusters and connector lines are made overtime. Visual clutter could be reduced in several ways. First, by simply reconfiguring the initial ring of unselected nodes by the push of a button. Another idea proposed was moving the reconfigured circle to the bottom corner or edge of the touch screen to give users more room to create. This 'hide' feature would also prevent unselected nodes from being touched or moved unintentionally.
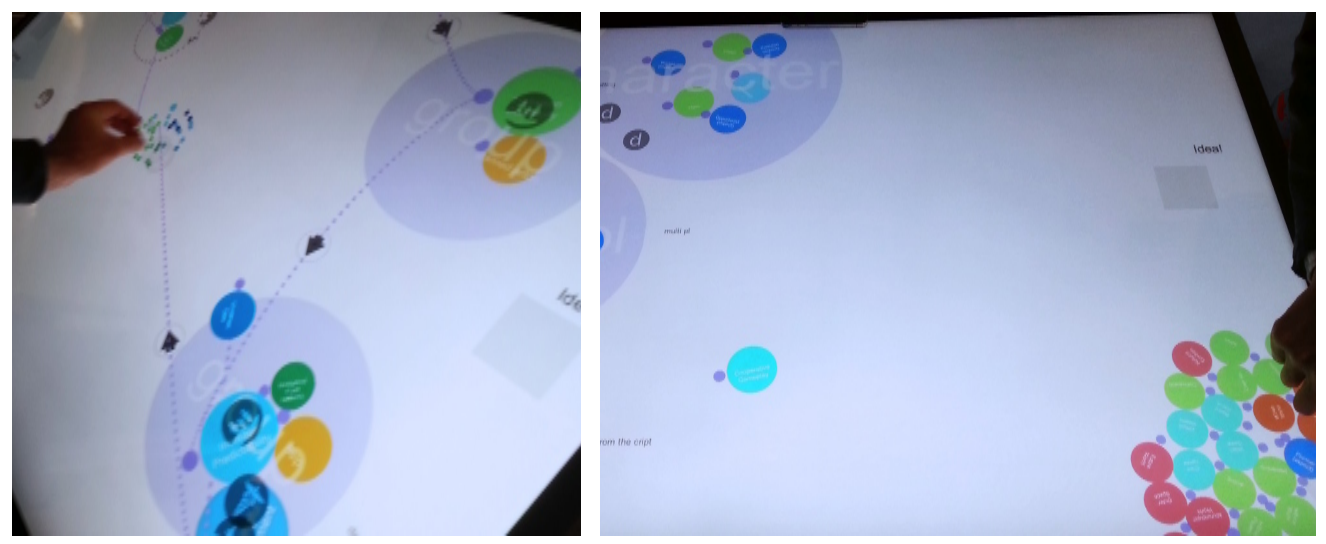

Figure 7.20 The students trying alternate ways to reduce visual clutter - reducing the size of the initial circle and/or moving it to the lower right-hand corner of the screen. 


\section{Playfulness vs. organization - added flexibility}

The students from the Game Design class (including those who had play-tested the second version of the prototype), said that they enjoyed the playfulness and visual aesthetic of the circular nodes, as well as the ability to spin the wheel and read the nodes from all four sides of the table. A comment made by one of the two students after interacting with the final prototype was, "It's fun to play with. There is a playful element that makes me what to engage with it." The students in the Game Design class came from creative departments. Other disciplines may prefer to see the needs and goals in the form of a list or matrix, within their original categories. While less playful, it may be easier to read. Adding a feature that allows stakeholders to read the data in a list or on nodes may provide further flexibility to users with different work styles.
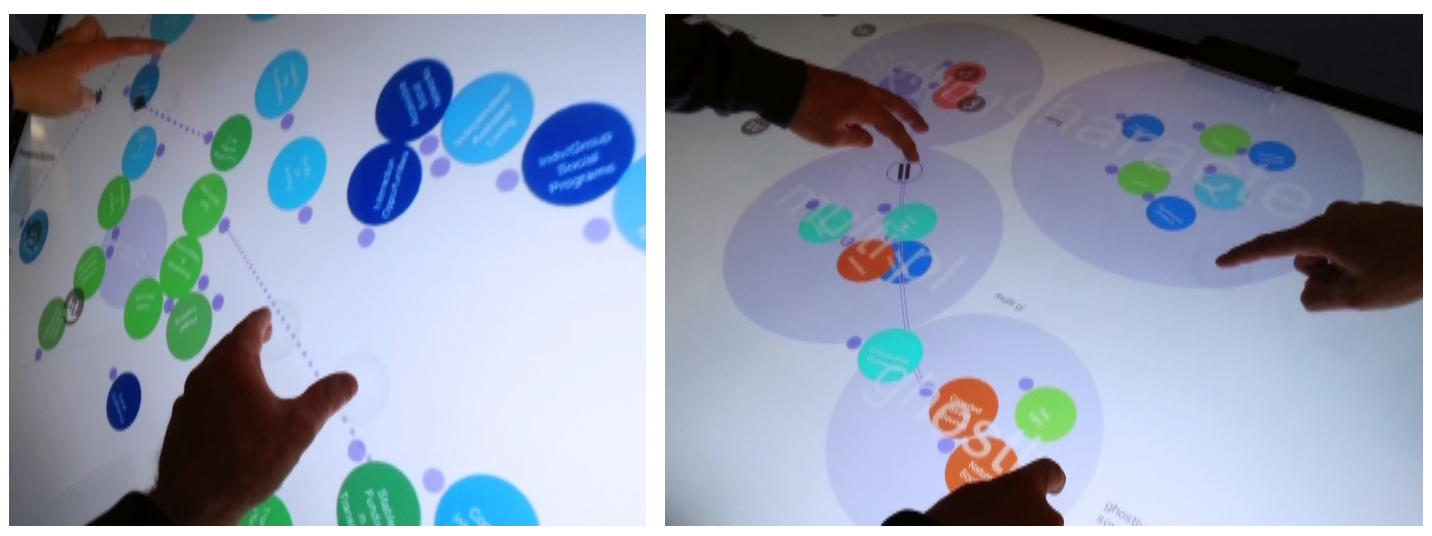

Figure 7.21 Multi-player interaction.

\section{Digital tool prototype versus sticky notes used for brainstorming}

The final discussion with the Design students centered on comparing the use of two tools for generative design: the digital tool they had just used and sticky notes, which they had used in past experiences for brainstorming. The following reflections were shared by the students after interacting with the digital tool prototype. They explained that sticky notes compel people to write things down individually, which can cause brainstorming sessions "to get out of control quickly." 
Hundreds of sticky notes go up from the individuals before discussions are had about how the ideas or needs written on the notes are related. During the play-testing on the shared digital canvas, as new ideas or needs for the game occurred to the students they vetted them with one another and discussed priorities. The students commented that with sticky notes in a brainstorming session people are given individual blank canvases, prompting them to add many thoughts and ideas (listing everything possible), which can place the group in a constant state of divergence and independent thought. The digital tool had initial topics/nodes to consider, which provided some constraints and made the process of developing a new game controlled. The digital interface and initial topics forced the students (in their own words) to "pay attention" and act more "deliberately" as they were generating new ideas and establishing constraints for the game. The nature of having some initial data during their generative design session, and not just a blank canvas, prompted them to consider different aspects of a game carefully and listen to one another while building their new game with intention. One of the students remarked, "When sticky notes are being used [in a brainstorming session], people are not as likely to listen to one another. Ideas can double or triple in size, and in many cases there are duplicates or competing ideas on the same wall. It does not need to be this complicated." The students found value in a creative thinking tool that balanced constraints and structure with flexibility and customization.

The sentiments of these Design students mirror the earlier quote from John (one of the ASD participants) about creativity. "When we are given an infinite creative space to work within, it can be every bit as paralyzing as the most confining restrictions. A defined space helps us direct our creative energies in a more articulate fashion." The balance between structure/intention and flexibility/creativity in the digital tool helped the students find common ground and provided enough definition in the creative space to allow their ideas for a new game to flourish, while being directed towards a collective vision. 
The last sections reflect on the value of prototyping the digital tool (as an exploratory/learning approach), how the digital tool could fit into future hybrid Design processes, and the impact of this exploratory research on the field of Design.

\subsection{Digital Tool as a Mode of Making}

The tool evolved from supporting brainstorming and idea sharing to one that was designed to capture shared input, present various perspectives, and spur generative thinking, while helping stakeholders converge and make sense of a complex problem, so they might exercise collective intelligence and reframe the scope of the wicked problem around more meaningful values.

Brandt, Binder and Sanders (2012) believe that the act of collective making helps to inspire new ways of understanding, recognizing different relationships, and exploring prospective futures together. The act of making, whether it pertains to making sense or building a visual narrative on a shared multi-touch table or on a whiteboard wall with sticky notes, appeared to unite the stakeholders under a shared cause and a shared experience, and provoked them to explore new perspectives and possibilities beyond their individual perspectives.

In a similar way, the act of making and developing the digital tool through multiple iterations with Alan Price inspired new ideas and directions for the tool that had not been considered at first. Additionally, the act of building the digital prototype helped to expose the capabilities and limitations of digital technology, while also inciting new possibilities for the future that may not have otherwise been considered through simply a series of 2D visualizations or a cardboard prototype.

In developing a new interactive tool for the Design process, this research demonstrated there is a lot to be learned and gained by building and testing, even a semi-functioning digital prototype with intended users. The iterative development of the digital prototype, in tandem with opportunities 
and barriers identified during the case study and through development, was in itself a learning vehicle through which to explore new functions and possibilities. Brandt, Binder and Sanders (2011) explain that there are three types of prototypes: prototypes for exploration, prototypes for experimentation, and prototypes for evolution and adaptation ( $p 156)$. For the purposes of this case study, the main intent of the digital prototype was for exploration - investigating new possibilities for integrating technology into the Participatory Design process and clarifying the features and desirability of a digital tool for collective sensemaking, rather than evaluating or validating whether or not this specific tool was the most adequate solution.

The 2D visualizations and cardboard prototype allowed the initial idea to materialize. However, the limitations of the cardboard and static screen images made it difficult to decipher and anticipate certain interactions. Testing (during Workshop 1) and user interfacing with the digital tool at different phases (by classmates) was integrated into the development process to better understand user interaction and tool requirements. A major insight that came from developing the digital tool and testing multiple versions of the prototype in Workshop 1 and with other students was that people tended to voice ideas and propose iterations for the tool through their interactions. These assessments led to the discovery of new insights and revealed limitations and hiccups in the digital tool along the way. Additionally, user testing and trial and error throughout the construction of the digital prototype provided added understanding about the potential capabilities of technology, the process of coding an interactive program, and what the intended users may expect in a digital tool designed for collective sensemaking.

\subsection{How This Digital Tool Fits Within the Hybrid Framework}

In this case study, the second iteration of the digital tool prototype was sandwiched between two non-digital approaches. It was designed to organize and convey the information gathered (through non-digital methods) in Phase 1, and become a plan of action to help guide/direct 
generative thinking and hands-on making/exploration for Workshop 2. Unintentionally, in the case study it was used as a tool by the Designer to summarize and reiterate the decisions and connections that had been made with sticky notes during the sensemaking activity in Workshop 1. The tool allowed the Designer to demonstrate the process of moving from unmet needs to potential goals, show the connections and hierarchy between collected research topics, and map the value the stakeholders hoped to create in the future.

The main idea behind the digital tool was that it would bring all interested stakeholders together to strengthen relationships and spark collective action. The aim was that it would help to build stronger connections and that it would broaden the perspectives of the participants so they could walk away with a new understanding about the problem and/or perspectives of other stakeholders. The digital tool prototype had the capabilities to record each step of the process, each decision that was made, each movement of the nodes, each prioritization (or growth of the nodes), and each connection drawn in order to make the sensemaking process 'open' and allow the decisions that were made in the session to be shared with new people.

As a tool for sensemaking, the value of a future digital tool (similar to this prototype) lies in being adaptable, bridging understanding, framing complex problems, and stimulating innovative thinking. As an interactive digital tool, the final prototype has the capacity to adapt to different problems (e.g., game design or social problems), facilitate social interaction and help stakeholders provide context and meaning to a wicked problem in a face-to-face session, while also allowing them to trace their thought-processes. The ability to extend the sensemaking discussions across time (into multiple sessions) in PAR could provide Designers and CoDesigners with a more fluid process to continue communicating and iterating their frames and their goals. Further exploration and prototyping need to be done with the digital prototype to determine how a saved visual narrative could be reopened to retell what transpired in an earlier 
session, and/or how the narrative could be manipulated with the addition of new expertise/perspectives in order to keep collective learning and iteration nourished, continuous, and malleable. Future improvements and next steps for the digital tool will be touched on in the closing chapters.

\subsection{The Impact of This Exploration on Design}

Collaborating stakeholders and Designers in the future may benefit from the use of a digital tool like the one developed in tandem with this case study. Technology gives us the ability to not only store large amounts of data and convert complex data into visual summaries, but also to present data in a flexible manner. By blending interactive system with the human capacity to tell narratives and create meaning, a digital tool, similar to the prototype developed here, may help CoDesigners and Designers find common ground, better visualize a chaotic problem, and more easily see the relationships between unmet needs and shared goals, in order to discover true 'value' and solve more meaningful problems. If we are to resolve wicked problems, we have to have the right tools to make it easier for collaborative teams to understand one another, explain and negotiate their positions in the context of the larger system, and be able to act on meaningful issues imbedded in a wicked problem.

Wicked problems like autism are a condition of culture, society, and the human condition. As people take on more active roles in the decisions that impact their lives, and our world and our problems become more complex and interlinked, it is crucial that our learning experiences and our perceptions expand beyond our own frame. A hybrid set of tools and methods that bring about collective experiences and shared understanding, while also extending participation beyond a single sensemaking session, may have a greater impact for bringing about the necessary changes for innovation. 


\section{Chapter 8: Outcomes From the C.A.S.T. Case Study}

Many of the ideas and outcomes derived from each phase regarding autism and transitional care were mentioned in the C.A.S.T. Case Study chapter for one of two reasons: they helped to inform the evolving frame of the project, and/or the insights helped to inform the next session and the topics that were investigated further. Due to that fact, there may be some repetition in this chapter as the findings and outcomes of each phase are broken down further. In addition to sharing findings and insights regarding transitional care in the autism community, this chapter will also analyze the outcomes from the Pre-Assessment and Post-Assessment Questionnaires, as well as the responses from the feedback questions and Reflection Survey that were posed to the participants after the project, to uncover what was learned about the PAR process and methods that were used to tackle this wicked problem.

\subsection{Pre-Assessment Questionnaire Outcomes}

A pre-assessment questionnaire was given to each participant during Phase 1 to establish their initial thoughts and feelings towards the current C.A.S.T. clinic, transitional care/support for adults with autism and their families, and the use of Design Thinking approaches in collaborative settings. Their responses are described below and represented in visual diagrams. A summary visual diagram with all the responses from each stakeholder group can also be found in the Appendix. 

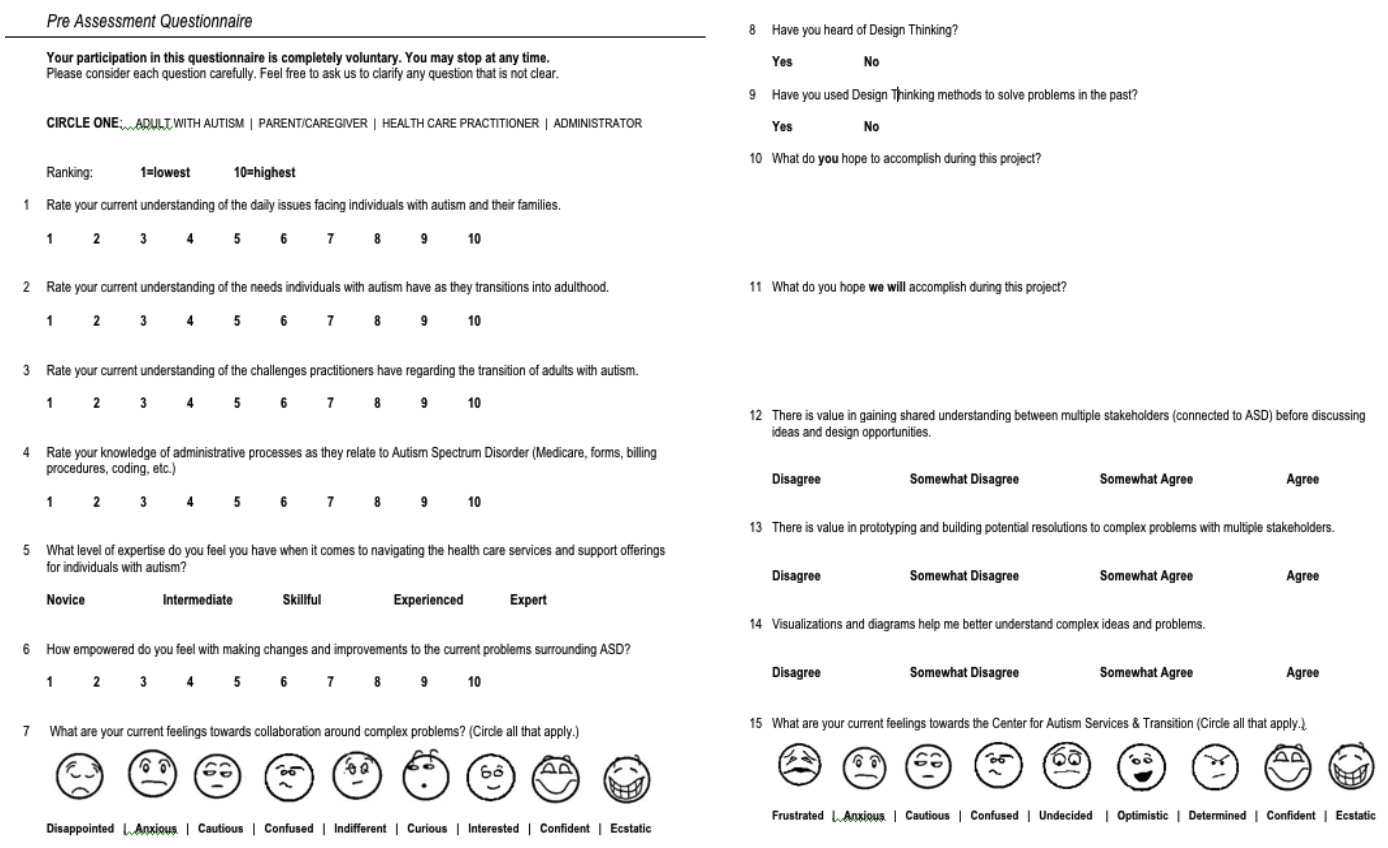

Figure 8.1 Pre-Assessment Questionnaire - front and back.

\subsubsection{Pre-Assessment Outcomes for the Adult Participants with ASD}

Eleven participants filled out the survey; ten were adults on the autism spectrum, one participant was a neurotypical intern in the Aspirations program. Each of their responses is represented by a red dot on the visual summary. The intern's red dot is marked by a (+). Some of the ASD participants did not fill out every question on the survey. The questionnaire was printed on a single sheet, front and back, which may have contributed to why one participant may have overlooked the back page. Another participant stopped after answering the first question on the backside. One person appeared to have circled almost all of the answers on the far right side of the page. His responses are marked by an $\left(^{*}\right)$. It is unclear if this act was purposeful (following a visual pattern) or if his responses actually reflect his true thoughts/feelings.

A majority of the ASD participants rated their current understanding of the daily issues facing individuals with autism and their families as high or above average. Two ASD participants rated 
their understanding just below average (measured as 4 on a scale of 1 to 10,10 being the highest). The intern first rated her understanding as high and then lowered it to just above average (6). Half of the participants rated their understanding of the needs faced by individuals with autism transitioning into adulthood as somewhat high, the intern rated her understanding just above average. The ratings for their current understanding of the challenges practitioners have regarding the transition of adults with autism were mixed (half rated their understanding low, the other half rated their understanding relatively high). The intern rated her understanding at the average mark. The rating for a majority of the participants for their knowledge of administrative processes as they relate to ASD (Medicare, forms, billing procedures, coding, etc.) was very low; the intern rated her understanding just below average. A majority of the ASD participants (including the intern) also felt their level of expertise when navigating the health care services and support offerings were low (ranging mostly from Novice to Intermediate levels). In summary, the participants with ASD knew a lot about issues relating to daily challenges faced by parents and people with autism, but were less knowledgeable about how to navigate the ASD healthcare system, and the issues facing providers and administrators. 


\begin{tabular}{|c|c|c|c|c|c|c|c|c|c|c|c|}
\hline \multirow{2}{*}{$\begin{array}{l}\text { Pre-Questionnaire } \\
\text { Responses }\end{array}$} & \multirow{2}{*}{$\begin{array}{c}\text { Response } \\
1 \\
\text { Low }\end{array}$} & \multicolumn{3}{|c|}{$\longrightarrow$ Multiple Responses } & \multicolumn{2}{|c|}{ ONo Response (NF) } & \multicolumn{3}{|c|}{ ASD Partixipants } & Parent & Providers/Admin \\
\hline & & 2 & 3 & 4 & 5 & 6 & 7 & 8 & 9 & $\begin{array}{c}10 \\
\text { High }\end{array}$ & NR \\
\hline $\begin{array}{l}\text { Rate your current understanding of the } \\
\text { daily issues facing individuals with } \\
\text { autism and their families }\end{array}$ & & & & : & & + & • & : & : & * & \\
\hline $\begin{array}{l}\text { Rate your current understanding of the } \\
\text { needs individuals with autism have as } \\
\text { they transition into adulthood }\end{array}$ & & & $\bullet$ & - & & e+ & : & : & & $e^{*}$ & 0 \\
\hline $\begin{array}{l}\text { Rate your current understanding of the } \\
\text { challenges practitioners have regarding } \\
\text { the transition of adults with autism }\end{array}$ & & - & & - & et & & $:$ & : & & ** & 0 \\
\hline $\begin{array}{l}\text { Rate your knowledge of administrative } \\
\text { processes as they relate to ASD } \\
\text { (Medicare, forms, billing, coding, etc) }\end{array}$ & - & - & $\bullet$ & $0^{+}$ & & & & & & ** & 0 \\
\hline $\begin{array}{l}\text { Rate how empowered you feel making } \\
\text { changes and improvements to the } \\
\text { current problems surrounding ASD }\end{array}$ & & - & - & - & & - & - & - & et & $e^{*}$ & O \\
\hline $\begin{array}{l}\text { What level of expertise do you feel you } \\
\text { have when it comes to navigating the } \\
\text { health care services and support } \\
\text { offerings for individuals with autism? }\end{array}$ & $\begin{array}{l}\text { Novice } \\
-\end{array}$ & $\begin{array}{l}\text { Intern } \\
: \\
: \\
-+\end{array}$ & & Skillful & & perienced & $\underset{*}{\operatorname{Exp}}$ & & $0^{N R}$ & & \\
\hline
\end{tabular}

Figure 8.2 Pre-Assessment ASD participant responses for the first six questions.

There were mixed feelings (ranging from 2 to 10) on how empowered the ASD participants felt making improvements to clinics and services offered to people with ASD. Half felt they had little power to enact change, while the other half (including the intern) felt empowered (above average). The feeling of limited empowerment may have been the result of having limited understanding and knowledge about certain aspects of the transition problem (on the healthcare side) and/or because they feel marginalized. A few of the participants shared that in society and other aspects of their lives they are given few opportunities to voice their opinions/thoughts. This feeling may have resulted in lower ratings on this question. 
Current feelings towards collaboration around complex problems (circle as many as you'd like)

Each now represents a person from a

takeholder group-ie. four rows, four parents)

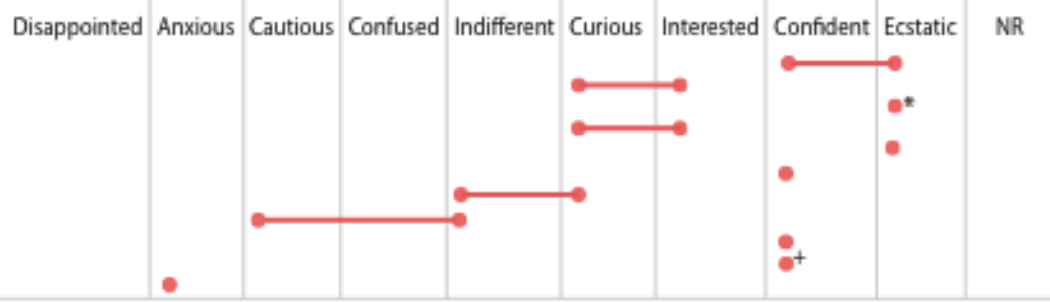

Figure 8.3 Pre-Assessment ASD participant responses regarding their feelings towards collaboration around complex problems.

Their feelings in connection with working collaboratively to solve problems were also scattered from anxious and cautious to curious, interested and confident. A few people even felt ecstatic. As a whole, the majority of the feelings towards collaboration were optimistic (above indifference, which was the medium rating). Overall, their individual goals and the goals they hoped we would achieve collectively centered on themes like: gaining skills for self-advocacy so they could feel more empowered to help others on the spectrum, gaining new understanding and knowledge, and being heard - having an opportunity to share their challenges and unique perspectives with other stakeholders. Here are some of their responses:

What they hoped to accomplish during this project they wrote:

"To help empower others on the autism spectrum in dealing with life's challenges."

"Voice my opinions and thoughts of how ASD-related care should be handled."

"I hope to grasp a better understanding of my autism-related disorder and learn how to prevent it from...[uncompleted]"

"Satisfy my curiosity with this project and offer helpful anecdotes"

"Gain more skills for self-advocacy, and make the dreams, desires, and opinions of myself and others heard."

"To help young adults find true love, marriage, jobs, and families."

"Intern: a better understanding of individuals on the spectrum and how to help them." 
What they hoped we accomplish during this project:

"Get useful information, while establishing a helpful institution for folks on the spectrum."

"Learn first hand concerns."

"I hope the general public will gain higher expectations and views of those thought to be 'disabled."

"Intern: the research you are looking for."

Most of the ASD participants had not heard of Design Thinking or used Design Thinking methods to solve problems in the past. The intern had both heard of and used Design Thinking methods in the past. A majority of the ASD participants, however, "agreed" that there is value in gaining shared understanding between multiple stakeholders before discussing ideas and Design opportunities. The intern somewhat disagreed that there was value in gaining shared understanding first. This was an unexpected response. The ASD participants (who often struggle with social situations) saw more value in shared understanding than the neurotypical intern. Throughout this case study nuances like this, and discussions had with participants on the autism spectrum show how little we may understand people with ASD and how much they have to offer. In regards to the value of prototyping and building potential resolutions to complex problems with multiple stakeholders, and using visualizations and diagrams to help better understand complex ideas and problems, all of the participants agreed (a few somewhat agreed). This was a more expected response, coming from people on the spectrum who are more visual thinkers. Below is a diagram of the responses. Each red dot represents a participant and their response to each question. 
Have you heard of

Design Thinking

Have you used Design

Thinking to solve

problems in the past
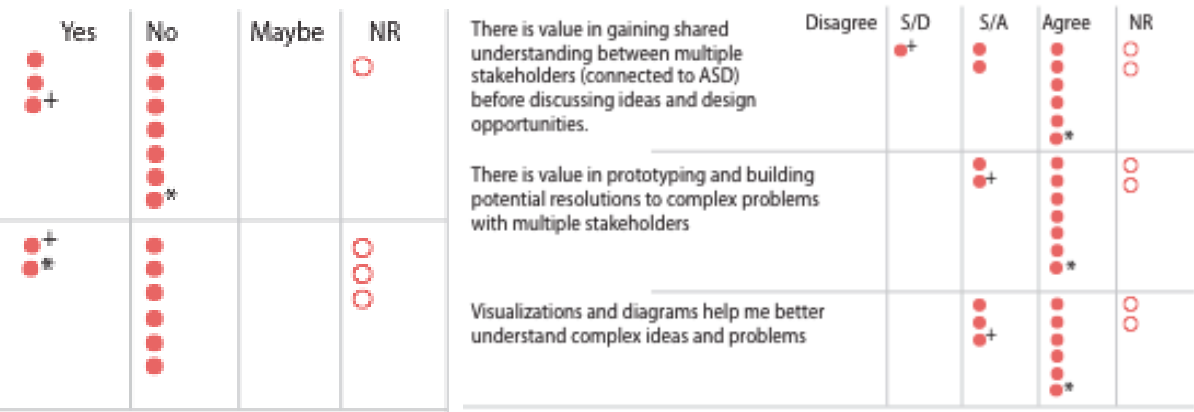

Figure 8.4 Pre-Assessment ASD participant responses to questions about Design Thinking.

Overall their current feelings towards the design of a new health care center (C.A.S.T.) for adults with ASD were mostly positive (above indifference). A majority, including the intern, felt optimistic, confident and ecstatic about the clinic. When we first introduced the project and the objectives for the focus group, before handing out the questionnaires, we mentioned that we would be collaborating around needs and goals related to the clinic to improve care and transitions for people on the spectrum. It is interesting to note that while the clinic was the initial focus of our project, few of their written goals or the goals they hoped we would accomplish collectively aligned specifically with making improvements to the clinic. Looking back at their responses, only two speak to clinic-related goals: "voicing opinions and thoughts of how ASD-related care should be handled" and "establishing a helpful institution." All of the other goals concerned greater issues beyond the clinic.

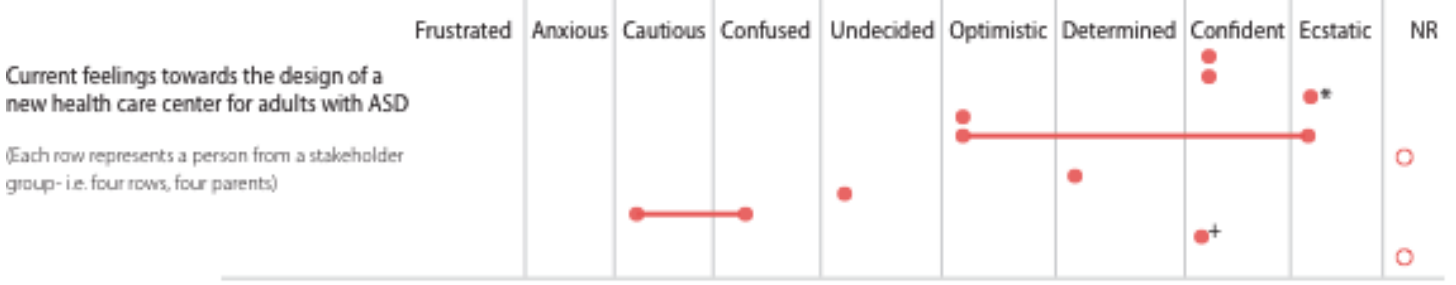

Figure 8.5 Pre-Assessment ASD participant responses regarding their feelings towards the design of a new healthcare center for adults with ASD. 


\subsubsection{Pre-Assessment Outcomes for the Parent Participants}

Four parents took the pre-questionnaire survey. One of the four parents did not fill out the questions on the back, so her responses for the last half of the survey are not recorded. Figure 8.6 shows that the parents self-reported mostly high levels of understanding about daily issues they (families) and adults with autism face, as well as the needs they have related to transitions. Conversely, they indicated moderate to low levels of expertise and understanding about the issues facing providers and administrators, and how to navigate the autism network. They felt moderately empowered to make improvements to the clinic and services offered to people with ASD, and as a whole were interested, but cautious and anxious about working with others to solve complex problems. Similar to the participants on the autism spectrum, the parents' lack of knowledge and lack of understanding regarding the issues faced on the healthcare side of the problem may have contributed to their feeling only moderately empowered. Before this project, the parents and healthcare providers had not been brought together to voice their unique issues or collaborate around new resolutions related to autism. 


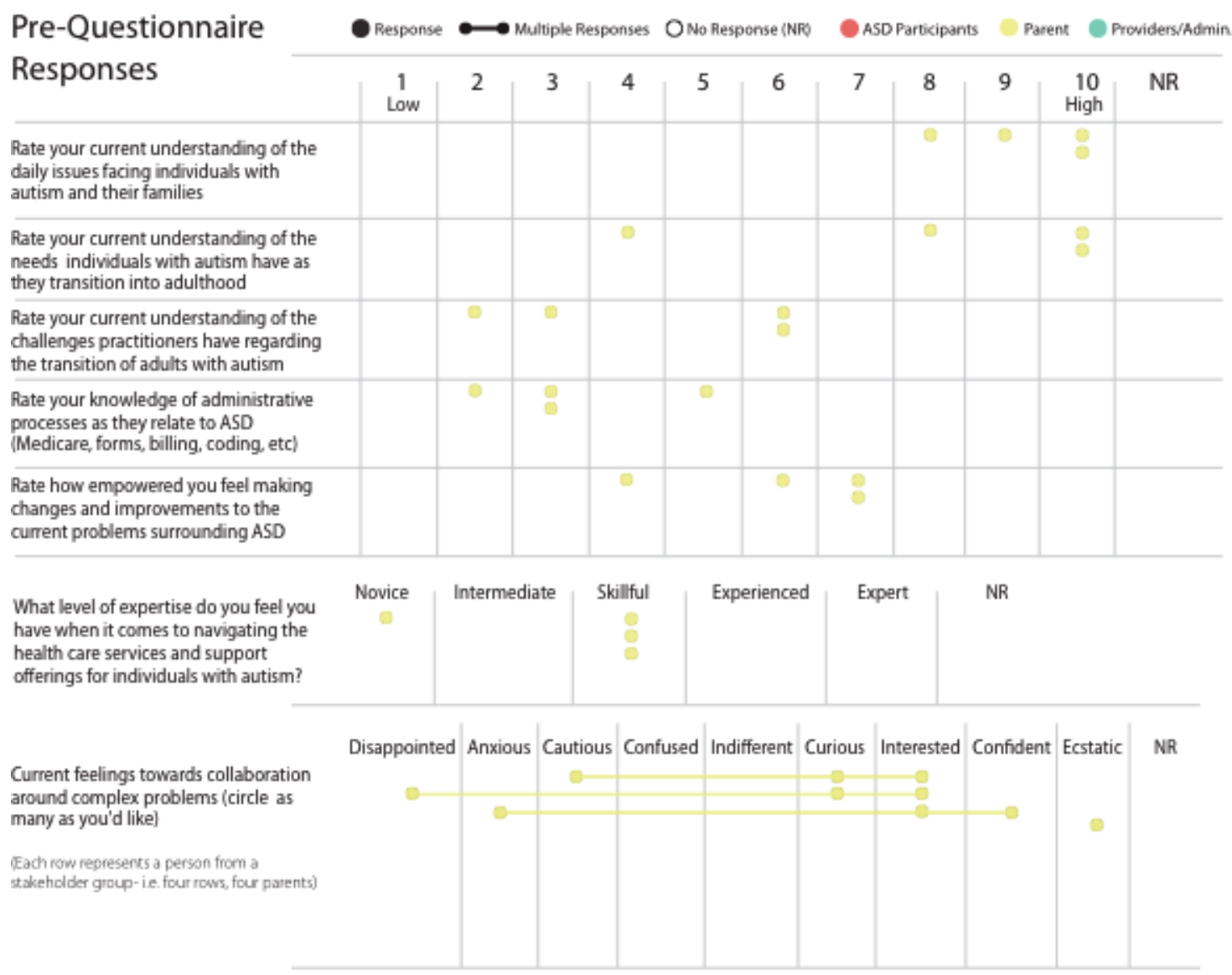

Figure 8.6 Pre-Assessment parent responses for the first seven questions, including their current feelings towards collaboration around complex problems.

As a whole, the individual goals of the parents centered on themes like: gaining greater understanding and knowledge, and contributing in making a difference for people with ASD and their families. They hoped that, collectively, we would be able to share ideas and knowledge and develop meaningful next steps. Few of their goals, like those of the participants with ASD, were aligned specifically with making improvements to the C.A.S.T. clinic. Instead, they were more interested in gaining understanding, feeling more empowered and knowledgeable to act, and having an opportunity to contribute to improving the lives of others in a meaningful way. Below are their responses: 
What you hope to accomplish:

"Greater understanding."

"Contribute as a parent of individuals with autism, make the path easier for those who follow us, learn."

"Gain a greater understanding of OSU autism-related initiatives and goals."

What you hope we accomplish:

"Uncertain at this stage."

"Meaningful steps forward."

"Have clear next steps, share ideas and knowledge."

Only one of the parents reported hearing of, and using, Design Thinking methods to solve problems in the past. However, all agreed that there was value in gaining shared understanding among different stakeholders before discussing ideas, and prototyping and building resolutions to problems. Additionally, they agreed (and somewhat agreed) that using visuals and diagrams helps them better understand complex problems. Their current feelings towards C.A.S.T., similar to the other stakeholders, were very positive and optimistic. In looking just at the current feelings towards C.A.S.T. for all of the stakeholder groups, it seems that most felt optimistic and positive (before the collective sessions had begun). Looking at the goals the stakeholders hoped to achieve individually and collectively it appears that their key aims centered on gaining greater understanding, being a valued contributor to the discussion around transitional care and support, and developing clear actionable steps towards broader goals like connectivity and enhanced wellbeing, over aims related to bettering the C.A.S.T. clinic specifically. 


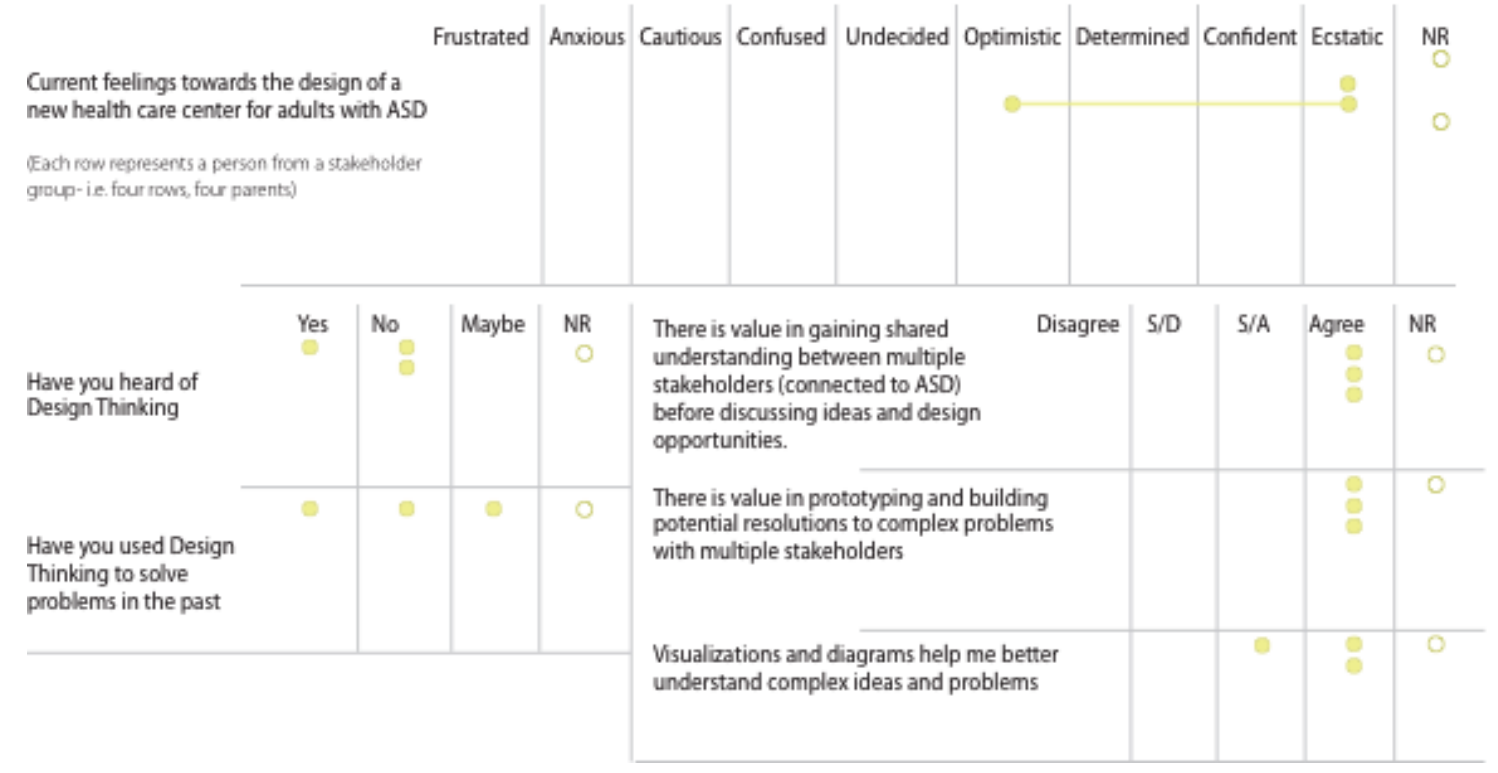

Figure 8.7 Pre-Assessment parent responses to questions regarding Design Thinking and their current feelings towards a new healthcare center for adults with ASD.

\subsubsection{Pre-Assessment Outcomes for the Healthcare Provider/Administrator Participants}

Eight healthcare providers and administrators filled out the Pre-Assessment Questionnaire. Some represented The Ohio State University Wexner Medical Center (OSUWMC), others represented Nationwide Children's Hospital (NCH). Overall, the healthcare providers self-reported higher levels of understanding about the daily issues and transitional needs of adults with autism (and their families) than the administrators (who had mostly average levels of understanding). Providers and administrators had moderate to high levels of understanding about the issues facing providers and administrators in the healthcare system. However, their knowledge of administrative processes were widely distributed from very low to relatively high ratings. Their overall expertise about navigating the autism network ranged from intermediate levels to experienced levels of understanding. As a whole they felt empowered (moderate to moderately high levels of empowerment) to make improvements to the clinic and services offered to people 
with ASD. This may have been the result of having more knowledge than parents and participants on the spectrum about the challenges faced by practitioners and navigating the system.

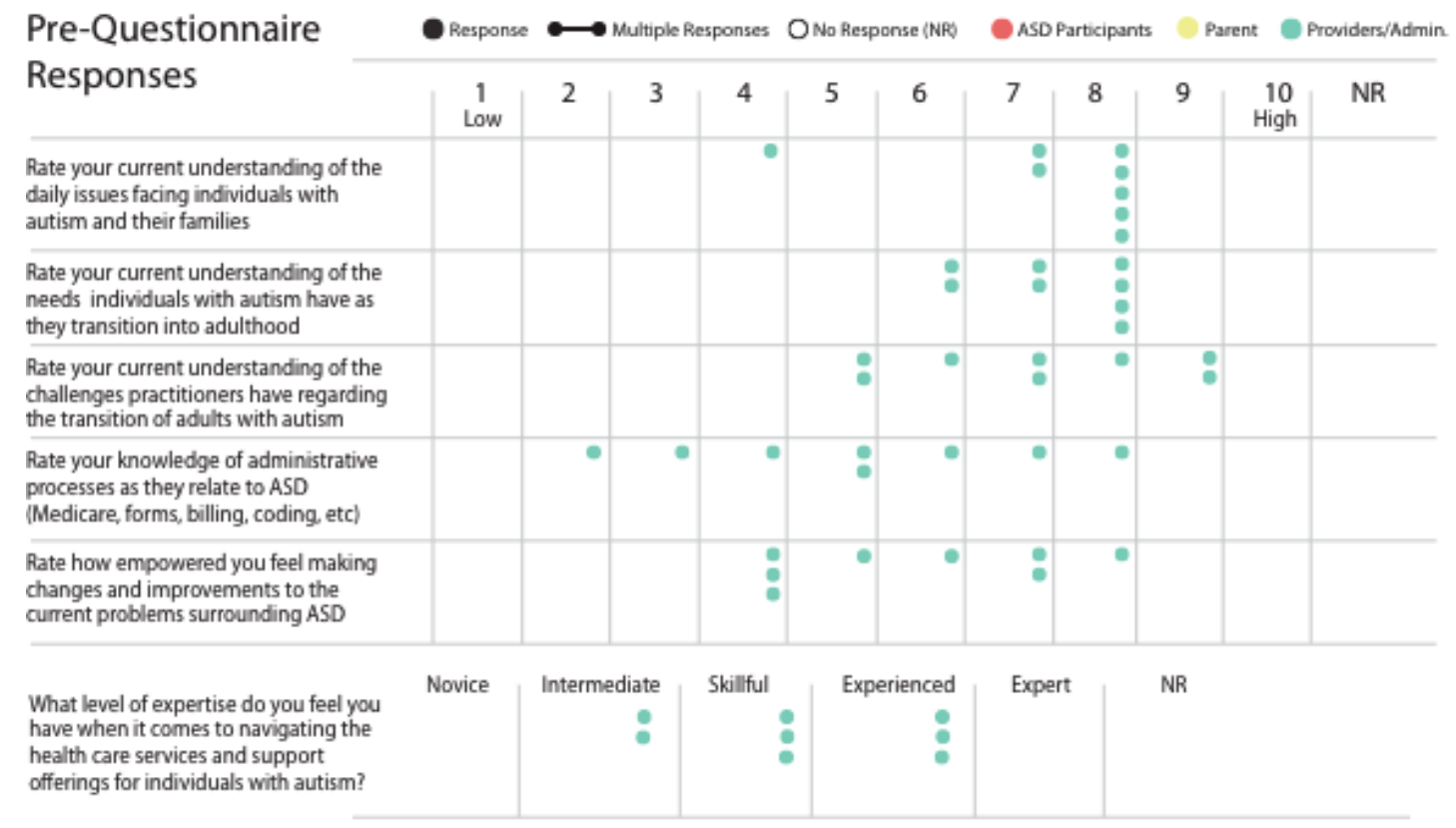

Figure 8.8 Pre-Assessment healthcare provider and administrator responses to the first six questions.

The individual goals of the healthcare providers and administrators centered on themes like: improving opportunities and processes for ASD adults and families. The goals they set for our group were similar to the ASD participants and the parents. They centered on learning from other stakeholders, gaining new understanding and connectivity, and finding ways to improve opportunities and care for people with autism. Collectively, they hoped that we would all become more informed, that we would open the dialogue between stakeholders and identify tangible (actionable) ways to make transitions more fluid and their network more collaborative and cohesive. Here are some of their responses: 
What you hope to accomplish:

"Better understanding of the many issues involved."

"Identify methods of collaboration to improve transitions."

"Ideas for smooth transitioning."

"Find ways to improve the care we provide for people with autism."

"I want to learn how to help patients and families."

"Understanding and being connected to local services \& resources."

"Receiving different perspectives on the experiences of individuals on the spectrum."

"Thinking about how I can improve may own practice."

"Improve opportunities for people with autism."

"Better outcomes and processes for ASD adults and families."

What you hope we accomplish:

"Road map to potential plan for healthcare transitions and adult services for individuals with autism."

"Tangible, feasible solutions to identified problems."

"Help with collaboration among groups."

"Become more informed."

"Open dialogues between stakeholders to learn from each other and appreciate each other's perspectives."

"Improving fluidity/integration of services for early adolescent to adult individuals by linking all stakeholders."

"Improve opportunities for people with autism."

Similar to the other stakeholders, few of their goals aligned specifically with making physical improvements to the C.A.S.T. clinic. A majority of the goals were broader and focused on making 
systems improvements and gaining greater understanding. Overall their current feelings towards C.A.S.T., similar to the other stakeholder groups, were very optimistic. This group of stakeholders as a whole were more positive about working collaboratively than the other two groups. Their feelings towards working with others to solve complex problems, ranged from curious and interested to confident and ecstatic. In studying the comparisons between the feelings held by the parents, it would appear that the providers and administrators may have had some success and/or experience working in collective group settings to solve problems in the past. None of the providers or administrators had used Design Thinking methods to solve problems in the past, but the majority agreed (like the parents and participants on the spectrum) that there was value in gaining shared understanding among different stakeholders, prototyping and building resolutions with together, and using visuals and diagrams to better understand complex problems.

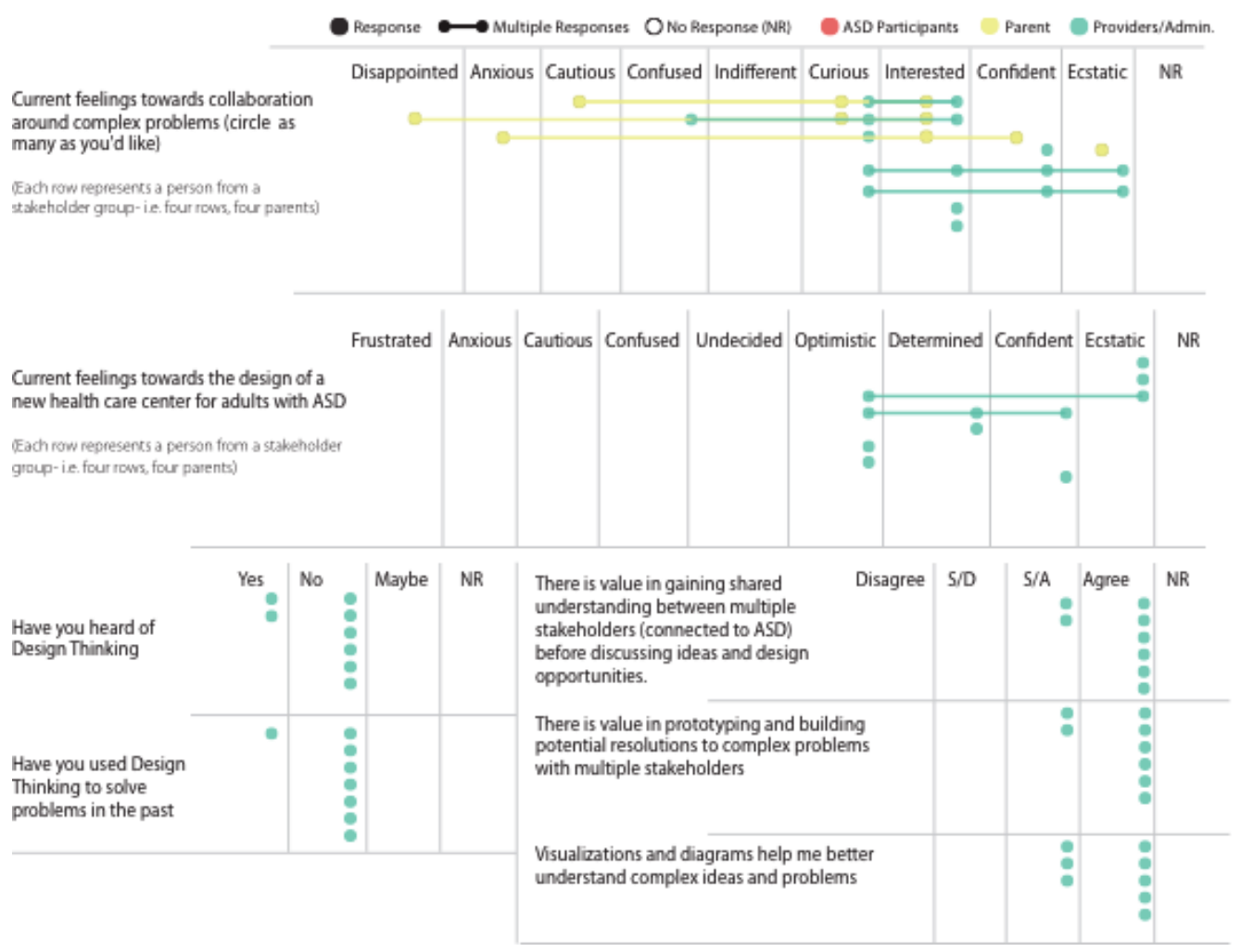

Figure 8.9 Pre-Assessment healthcare provider and administrator responses to the last set of questions. 
A full visual summary with all three of the stakeholder groups' responses combined can be viewed in the Appendix. All in all, the Pre-Assessment Questionnaires demonstrated that there was an imbalance in knowledge and a lack of shared understanding across the interconnected system of transitional care/support in ASD. In studying the goals stated by the participants and the feelings/knowledge they shared coming into the sessions, there appeared to be more interest in gaining knowledge and understanding, and working through complex problems collectively through Design Thinking, visualizations, and prototyping, than there was in fixing the clinic or arriving at a specific answer. The participants understood that this was a complex problem and if we were going to tackle the right issues together, and act with purpose to achieve long-term goals, we had to first understand one another. The results of the questionnaire support the hypothesis that a participatory approach (maybe even more specifically a PAR approach) is ideal for tackling wicked problems - where value is marked, not by arriving at a single solution, but instead by instigating new ways of seeing, and promoting continuous learning, shared understanding, and iterative exploration. The surveys also demonstrate that the non-designers (most of whom had never used Design Thinking) also see value in using visualizations and prototyping as a means through which to make sense of complex problems in a collaborative setting.

\subsection{CoDesign Outcomes from Phase 1 Focus Group Sessions}

As most of the outcomes from interviews were shared in the earlier case study chapter, the Phase 1 outcomes in this chapter will mostly pertain to the findings from the focus group sessions. Many overarching themes and ideas that derived from the interviews and focus group sessions were mentioned in general terms in the case study chapter, as they fed into how Workshops 1 and 2 were organized. Many of the ideas, needs, and goals mentioned in Phase 1 were brought into Workshop 1 to be further explored and defined in the context of collective sensemaking. Following Workshop 1, the ideas from Phase 1 and 2 were captured and 
synthesized into a matrix of short-term and long-term ideas that were shared with the participants prior to action-planning and prototyping in Phase 3 (Workshop 2). As a curator in this project, the role of the Designer was to not only organize the information into usable data for the stakeholder presentations and the sensemaking activity, but to also organize the ideas and prototyped concepts that emerged from each phase to give more direction during Workshop 2 and guidance for future Design phases of the project.

The new frame, created by the end of Workshop 1, centered around maximizing the potential of people on the spectrum, families, and providers/administrators. This new frame emerged as the result of shared understanding, collective mapping, visual provotypes, and an assortment of concepts expressed and generated during Phases 1 and 2. Many of the unmet needs and ideas that had emerged in focus groups and individual interviews during Phase 1 were shared in the Workshop 1 presentations. Through the course of group discussions and collective mapping in Workshop 1 these concepts and the frame of the wicked problem of transitional care in the autism community matured. The sections that follow will discuss in more detail some of the ideas that originated from the focus groups and interviews and were then revised over the course of Phases 2 and 3. Some of the ideas responded directly to the initial scope of needs and goals surrounding the clinic, while other ideas extended beyond the scope of the clinic.

\subsubsection{CoDesign Outcomes from Focus Group 1 with ASD Participants}

In our discussion with adults on the spectrum at Donatos the topic of sensory sensitivity was brought up, as it related to being in different environments. For many in this group, quietness was almost as bad as too many sounds or music that was too loud. White noise or a constant sound was said to be soothing because it was expected and constant. Sounds or occurrences that were unanticipated or were out of their control caused most of their anxiety. In addition, crowds, interruptions, bright lights, sharp surfaces that were cold and hard, and heights were unfavorable 
interior features that generated a lot of anxiety and stress. To manage this stress, many on the spectrum used headphones or hand fixations (playing with objects) to redirect their anxiety. One participant in particular uses writing as a meditative activity to manage stress. Anxiety also comes from waiting and not knowing how long you will have to wait. This fear stems from an experience that is out of their control. When people on the spectrum are overcome with anxiety and overstimulation from sensory sensitivities, they are not able to function and communicate as well. These frustrations may culminate into tantrums and poor behavior. However, communication and comfort can be elevated for both the patients and the healthcare staff at the C.A.S.T. clinic (as well as outside specialized provider offices) if these sensitivities are taken into consideration. In response to these expressed issues, the participants generated a multitude of ideas which included: a waiting room with free earplugs and/or headphones connected to Pandora, a writing station or sensory objects station to keep them distracted, soft repetitive sounds, and quiet rooms (or 'safe rooms', as the participants called them) on the periphery of the waiting room to make the waiting experience in the clinic more calming and controllable. A person on the spectrum could decide to withdraw to a quiet space if the waiting room got crowded or noisy, or decide to stay in the waiting room and use the various stations (sensory, writing, Pandora music) to self-soothe. Stations set up to allow patients to write or interact with sensory objects would keep them distracted while they waited, and could help to calm their elevated sensitivities. Curved corners at the intake desk and in the exam rooms (no sharp edges), and dimly lit spaces would add to a more tranquil environment. Connections to the outdoors and/or places to release energy were ideas mentioned for those that needed space to move around and could not sit for long periods, The ideal clinic space the ASD participants were describing and illustrating in what they were making and sharing verbally sounded more like a spa than a medical clinic. 

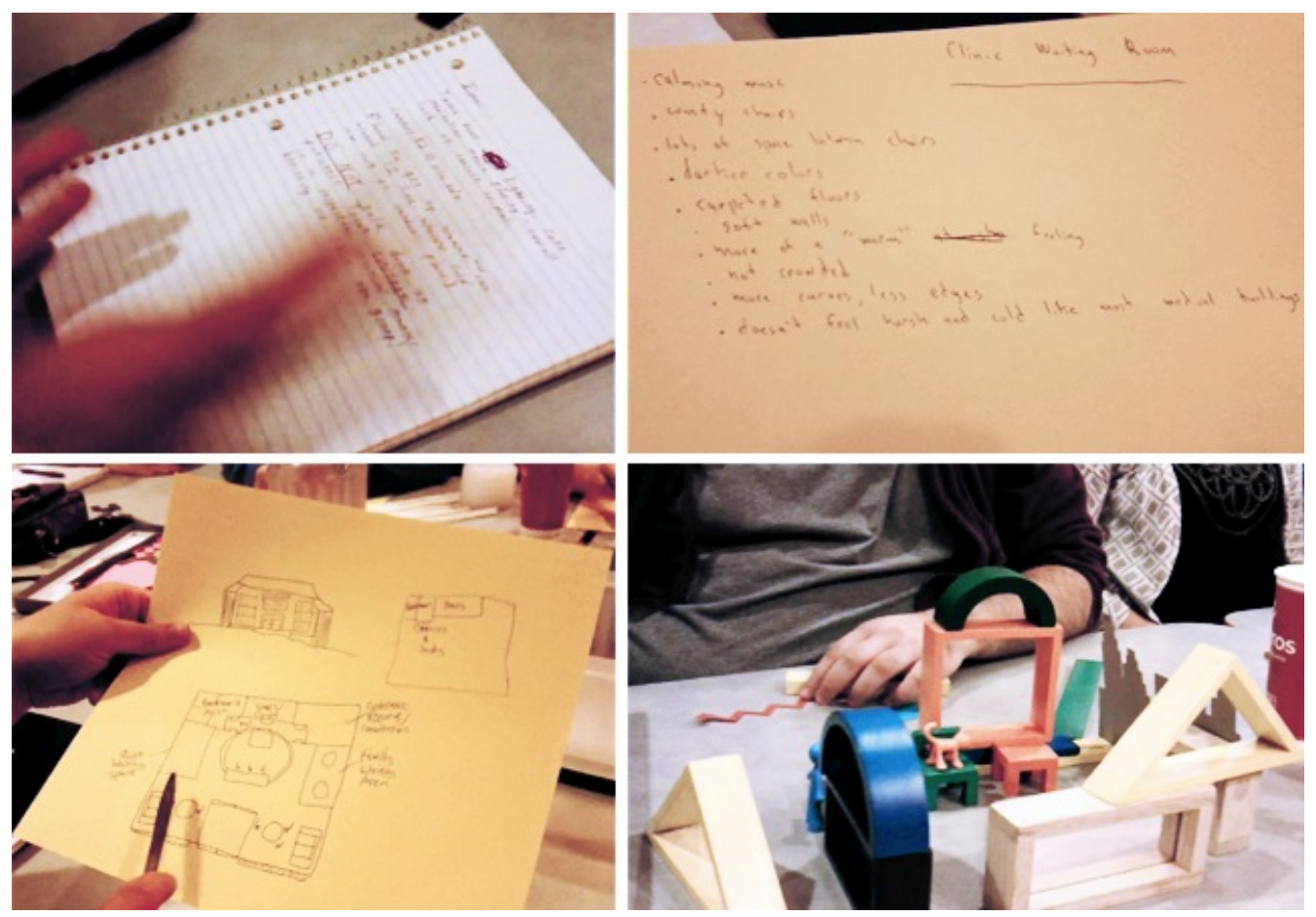

Figure 8.10 The images above capture: written ideas for the clinic and ways to support people with ASD in the community (top), a drawing of a waiting room with a round intake desk and quiet rooms (lower left), and a model of a waiting room with soft edges and a cat to reduce anxiety (lower right).

However, what the ASD participants were proposing was not just a 'spa-like' waiting room environment, but instead one that offered flexibility and gave a person control in a waiting space where we (neurotypicals and ASD people alike) often feel powerless and uncomfortable. Their needs, while perhaps more heightened, seem universal. As you read through some of the other CoDesign concepts generated from the case study, such as those concerning communication between the provider and patient, you may notice other ideas that would not only make a person on the spectrum's experiences easier, but could also resolve a universal need or goal.

"Becoming friends with somebody on the phone is easier than building a face-to-face relationship, because there are fewer social cues to deal with" (Grandin, 2009, p.156). The same concerns 
were expressed by the participants regarding the doctor/patient relationship, and the face-to-face communication channels patients are often forced to use. Many people with ASD are not comfortable with that option and would prefer tailored modes of communication. One of the ASD participants explained that he could eventually achieve comfort with face-to-face communication if the doctor was willing to work up from phone or email conversations, to interactions in clinic rooms with a screen or furniture positioned in a way where the patient did not have to see or face the provider, to then face-to-face interactions. He and others on the spectrum feel they often just need more time to get acclimated to social interactions. Anxiety, associated with a doctor's visit, had little to do (for this group) with getting a physical checkup. Instead, the anxiety of having to communicate and interact with people either in the waiting room or with the doctor heightened sensory overload, therefore making it hard to communicate and socially interact. If communication were tailored to what the individual on the spectrum could handle, it would reduce this anxiety and make for a more pleasant clinic experience. As the person became more and more comfortable with the physician, communication could eventually be elevated to face-to-face interaction.

\subsubsection{CoDesign Outcomes from Focus Group 2 with Parents and Healthcare Staff}

Identifying and exploring the gaps and missed opportunities in the current clinic and ASD network in Columbus occurred at all stages of the case study process. However, the focus group with parents and providers centered on these broader issues, especially. Though starting at opposites ends of the spectrum (parents with a micro-view and providers/administrators with a macro-view) of the system, the participants ended up with a similar frame of core problems that fit more in the middle of the spectrum through open dialog during the prioritization of the top ten concerns, needs, and goals of each stakeholder group.

Successful day-to-day interactions and planning towards a more independent future for their children depended upon the parents' gaining peace of mind from a system that was more 
cohesive and connected on a grander scale. Successful clinic visits and optimum care relied on properly trained staff and outside extenders, as well as streamlined communication and cohesion among providers that were part of a more connected care team model. Aspiration planning and support for independence, along with properly trained outside extenders could help to take the pressure off the parents and ASD providers. ASD providers are not equipped to handle all of the care needs of an adult as they age (i.e., dentist, heart specialists, etc.) and parents deserve to have a meaningful and productive life, as well. The wellbeing of the parents is just as important as their child's. By taking the pressure off parents and giving young adults more tools that empower them to speak up for themselves, we can tackle more than one need and goal.

Similarly, by training outside extenders and medical students and making them more knowledgeable about how to care and cater to the needs of people on the spectrum, ASD providers can offer more tailored and patient-centered support, instead of being spread thin, and patients can receive proper care. The adult care a person with ASD receives should be held to the same standard that neurotypicals expect. ASD providers are typically general practitioners, not specialists. Their resources and expertise are limited; they are not equipped to support all medical issues. Optimized care includes being able to refer patients to specialists/extenders. Training makes these extenders more comfortable, empowered, and prepared to care for the adult ASD population, while also ensuring that clinics like C.A.S.T. are not over-promising and under-delivering. As the ASD population continues to grow, this need becomes more critical.

The goal for greater cohesion extended beyond healthcare and concerned the lack of connectivity among parents in autism network, as well as the lack of connectivity and understanding with the mainstream population. Ideas of how to achieve greater connectivity and tolerance included reverse inclusion programs (i.e., mainstream mentorship) and an online clearinghouse through which parents could openly share treatment information and resources with other parents. The 
clearinghouse idea will continue to be brought up and refined throughout the case study, until it is eventually prototyped in the last phase. The discussion between parents and healthcare providers/administrators culminated in an understanding that there is a limited amount of resources, especially for adults on the autism spectrum and their families. In order to supplement the lack of funding and support, disruptive technologies need to be developed to simplify the lives of people on the spectrum and their natural caregivers. This insight will show up again during the presentations by the parents in Workshop 1.

\subsection{Outcomes From Workshop 1}

At the conclusion of Workshop 1 differing needs and perspectives had been vetted, topics had been collectively prioritized, and the frame of the clinic as the primary focus surrounding transitioning adults on the spectrum had shifted. Findings from Workshop 1 that will be covered in more depth in this section include topics like: nurturing specializations, applications for assistive technologies, improving the clinic experience, gaining a sense of control, navigating a patient's journey, patient-centered universal healthcare, bridging communication gaps, and resources/supportive channels for adults with ASD.

The biggest insight drawn from within our shared seeing-space was that truly disruptive innovation around the needs of patients and their families lay not in reshaping the physical clinic environment, but in designing tools and services that helped parents, providers, and people on the spectrum recognize, assess, nurture, and sustain the specializations and individual potential (over limitations) of people on the spectrum. The realization that the providers from The Ohio State University Wexner Medical Center and Nationwide Children's Hospital made during Workshop 1 was that a truly holistic and sustainable care model hinged on "people succeeding in other areas of their lives." The term 'people' referred to maximizing the potential not only of the patient (adults on the spectrum), but family members and providers (inside and outside the ASD 
healthcare spectrum). Maximizing potential involved building resolutions that provided opportunities for self-advocacy and acquiring peace of mind. For providers it meant knowing that their patients were receiving optimal care and living fruitful lives, for adults with autism it meant creating pathways towards independence and self-advocacy, and for parents it started with having the right navigation tools and finding time to take care of themselves. As the natural caregivers, their health is just as important as their child's. Longevity and achieving maximized potential outside the clinic became our new frame. The new 'value' we were designing for the new frame is visualized in Figure 8.11.

\section{Map of Advocacy \& Potentiality | Recognizing, assessing, nurturing, and sustaining specializations and individual potential}

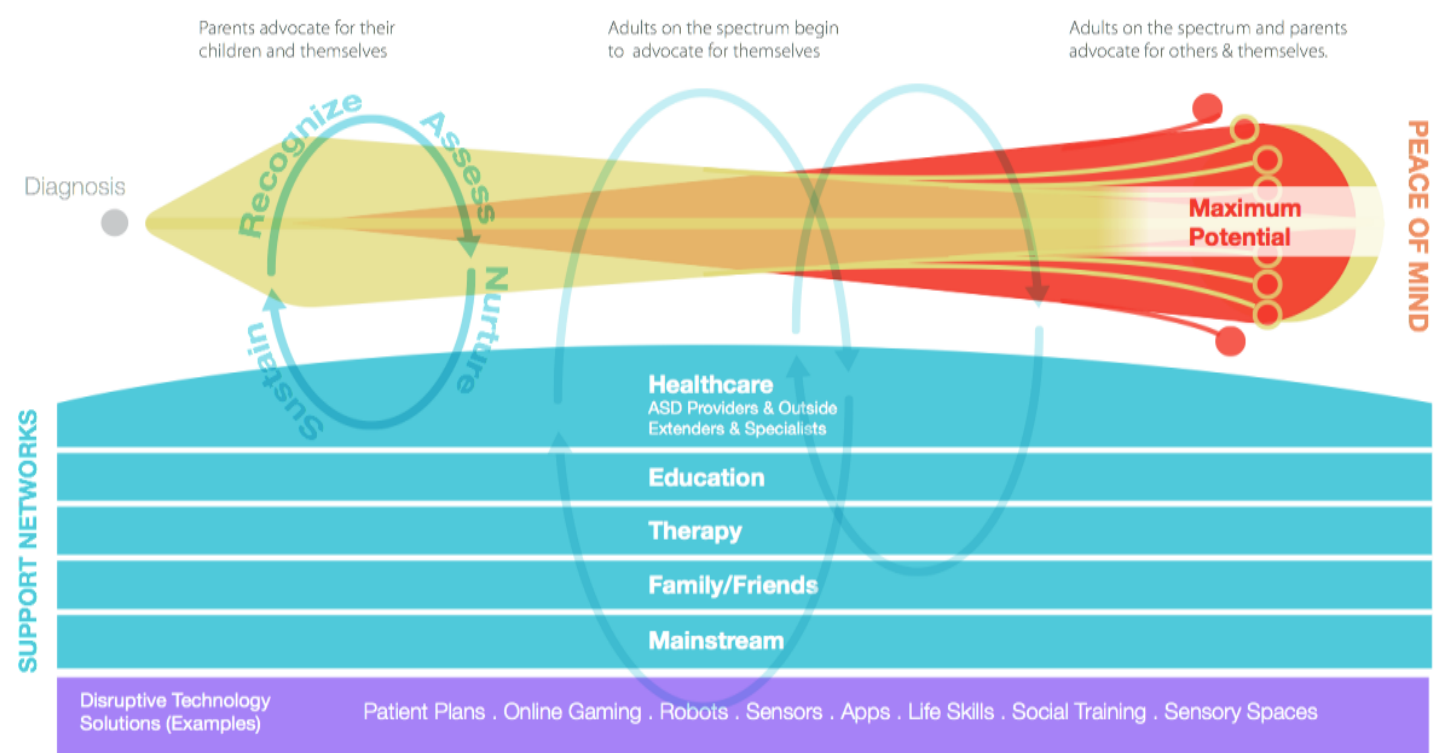

Figure $8.11 \mathrm{~A}$ visualization of the new frame. The diagram shows the journey of potentiality for parents and their child on the spectrum, from diagnosis to reaching their maximum potential, as well as providers, outside extenders, and assistive technology in supportive roles. 


\section{Nurturing Specializations}

Grandin had teachers that used her interests as motivators (Grandin, 2009, p.104). "Leo Kanner stated that the path to success for some people with autism was to channel their fixation into a career" (Grandin, 2009, p.105). Therefore, "teachers [and other people in guiding roles] need to help autistic children develop their talents" (Grandin, 2009, p.105). An idea that came up in our sessions was to give pediatric providers tools to help identify, nourish, and sustain these fixations (i.e., specializations) so that they could be channeled for positive/meaningful careers. The support to nourish these specializations was not just for providers but extended to other areas like schools, mentors, assistive technologies, and online games. "People on the spectrum are often good at one thing and bad at something else" (Grandin, 2009, p.27). A significant insight of our session was in identifying potential resolutions that would help people on the full range of the spectrum (from verbal to non-verbal, visual thinkers to math/music thinkers) identify their unique specializations. Care offerings and supportive services inside and outside the clinic setting should be aimed at helping this population develop and strengthen their gifts/talents in childhood and into adulthood so they lead more productive, meaningful lives, and can give back to society in a significant way.

\section{Applications of Assistive Technology}

Ideas brought up by parents during their presentation to the other stakeholders surrounded assistive technologies and affordable housing resolutions. Ideas around affordable housing were associated with wanting to maximize their child's potential to live more independently, while also increasing the parents' peace of mind when the child was living at home or on their own. Limited support offerings (i.e., caregivers) and funding have made affordable housing and independent living solutions a difficult problem to tackle. Along with exploring building materials (that are affordable and uniquely tailored to sensory sensitivities) and custom floor plans designed for people on the autism spectrum with special needs, parents also wanted to explore disruptive 
ideas, such as integrating assistive technologies into their homes and the homes of people with ASD living independently. Some of the assistive technology ideas that were brought up by the parents (and by some providers in the interview sessions) included: companion robots, the use of Siri and apps that would help an adult with ASD become more independent (i.e., daily/monthly reminders, nutritional information, life management skills, etc.), health tracking sensors and concussion-detection monitoring, biomarkers and information sensors, and big data mining to help parents gather, manage, and stay informed on information and resources.

A common mistake that is made by parents or outsiders is "misunderstanding the motivation of behavior" (Grandin, 2009, p.181). Grandin explains that sometimes bad behavior is the result of an underlying medical problem. One of our parent participants was specifically interested in exploring the area of wearable and ubiquitous sensing technology as way to monitor physical changes in her child. Ubiquitous sensing technology in the home or wearable devices could detect, learn, inform, and intervene in behavior or physical changes in a person with autism, so parents could better to anticipate and/or prevent 'bad' days. This technology could give parents greater peace of mind and control by being able to sense and learn that when their child's blood pressure reaches a certain height they are about to throw a tantrum, for example. Learned physiological patterns could alert parents to proactively intervene and respond with a soothing remedy, or the device could intervene automatically to prevent an outburst. It may also show patterns about time of day or medications that may be causing the physiological changes. Providers added to this idea during the presentation. The same monitoring/ubiquitous sensing devices may also prove useful to providers who want to understand physiological patterns that may be impacting behavior changes, while also helping parents better communicate issues to their child's physician. Providers would receive physiological pattern readouts remotely to help them better prepare for patient visits, and/or reduce the number of clinic visits, while enhancing communication between families, patients, and their providers. 


\section{Visual Cues and Navigation in the Clinic}

The idea of visual time indicators and clinic stage indicators in the clinic evolved from group discussions around the conference room table. Since many on the spectrum are visual thinkers, icons or signs throughout the clinic could indicate at what stage in the process the patient was and help them navigate the environment. Ideas for visual cues in the clinic had been mentioned by individuals in some of the interviews and focus groups. During the presentations these ideas were shared with other stakeholders, who were then able to build onto them. As John and Kristin shared ideas to improve the waiting room experience in their presentation, some of the providers added that while some patients may benefit from a tranquil space or self-soothing distractions, other patients would still have anxiety from not knowing how long they would have to wait. Parents added that a countdown timer or a visual cue in the waiting room could help the patient see how much more time they had left to wait, which might help to reduce their anxiety and give them a better sense of control.

\section{Gaining a Sense of Control}

The importance of a child or adult having a sense of control came up in Temple Grandin's book, Thinking in Pictures, as well as in the case study workshops. Grandin mentions that the sensitivities to loud, harmful sounds like fire alarms for a child with autism can be better tolerated if a child is given control over the volume of the recorded sound. Overtime they can raise the volume to become more comfortable with the noise (Grandin, 2009, p79).

"Some autistic people are very calm when they adhere to familiar routines, but an outburst of temper or aggression can occur if something unexpected happens" (Grandin, 2009, p.199). What was discussed in Workshop 1 was that the clinic did not need to have padded rooms for outbursts, but instead needed to have tools and processes in place that made the experience more anticipatory instead of unexpected. For example, having tools that helped the adult prepare 
for the visit, including what questions to ask or what information to share with the doctor, as well as tools to help the clinic staff prepare for each individual patient, by knowing "what makes for a good visit”.

\section{Navigating a Patient's Journey}

Ideas that originated from the providers' presentation revolved around ways to improve the healthcare system, personalized medicine, and bridging education and communication gaps. Parents need tools that make them more informed and assured while they navigate their child's health care; adults on the spectrum could benefit from tools and skill-sets that give them a better sense of control and empower them to self-advocate. Providing helpful resources for 'outside' (non-ASD) providers, bringing pharmacists into the transition process earlier, supporting a more sensitive transition process, and providing tools like a 'Vision Plan' could help to break down parent and patient barriers, improve navigation through the care system, enhance mutual understanding, and alleviate stressors (for patients, outside providers, and families). The idea behind 'Vision Planning' came from the director of C.A.S.T. who had seen the idea introduced in another clinical setting. 'Vision Planning' involves partnering with families and outside extenders to establish pathways and key achievement goals for their children towards reaching their maximum potential.

This concept does not respond to just one need, but many of the expressed needs of the varying stakeholders. A 'Vision Plan' could help to determine end-goals and a child's potential, help parents navigate the autism network and deal with unrealistic goals sooner rather than later, encourage untapped potential and nurture independence, and provide more personalized care, more accurate and proactive patient histories and road maps for future planning. 'Social stories' and video modeling/simulation were mentioned by provider participants as tools that could be brought into the clinic setting and used to help young adults prepare for clinic visits and reduce 
some of the stress/responsibility on the parents, while also helping the patient increase their social confidence and encourage them to feel more empowered to ask their own questions and communicate directly with their doctor.

To prepare providers (inside and outside the ASD system) for patient visits, a more effective and readily available mechanism needs to be in place so providers can easily view 'key' patient specific information (beyond digital medical records). A 'key to a successful visit' tool that is succinct, meaningful, user-friendly, helpful, and readily available needs to be developed for each patient. Designed like a passport (paper and/or digital-based), a 'Patient Passport,' with simple visual icons, could briefly summarize a patient's sensitivities, both medical and environmental, and be used by physicians, nurses, and clinical staff to craft a more user-friendly experience. Additionally, tied to the 'Patient Passport' could be a medical app, which could offer both extended and abridged health information. Shared between providers and extenders in the 'Care Team,' the app would make information more transparent and keep the 'Care Team' more connected and informed as the child matures. Beyond the clinical environment, the Patient Passport could be used in the community to help others better understand/appreciate public outbursts and individual sensitivities, similar to a medical bracelet.

\section{Patient-Centered Universal Healthcare}

The providers and administrators were interested in understanding how they could better customize care options and clinic visits for people with special needs to avoid having to have padded rooms or increased security measures. The answers to this lay in customizing clinic visits around the abilities and sensitivities of the patient, and creating tools like Vision Plans and Patient Passports. Through the process of learning how to best optimize care for ASD and special needs patients, the administrators and providers also hoped to gain more insight into ways to improve care offerings from a universal healthcare standpoint to ensure they were not over promising and 
under delivering. A universal unmet need, for instance, that was identified in the sessions concerned the breakdown in communication between patients and providers. In interviews and group sessions it became clear that poor communication between patients and their physicians was universal. Anxiety and confusion extended to patients outside ASD. An administrator explained that many patients hated to make appointments over the phone. They expressed a preference to do it through email or online options. Many also did not feel confident, comfortable or prepared to speak to their physician during face-to-face visit. Instead, many patients preferred to converse through email before and/or after the visit. Email allowed them to ask questions as concerns came up and a written response gave them something to reference, instead of having to remember everything the doctor said during a visit.

\section{Communication}

As John and Kristin (ASD participants) raised the need for improved and more tailored forms of patient/provider communication in their presentation to the other stakeholder groups, the idea of MyChart was brought up by the providers. Recognizing that many patients and family members would also like to communicate through email, the providers explained that MyChart, or a similar secure online communication channel, would be more protected than email and would connect directly to the patient's records. Currently, MyChart is being used by many patients at OSUWMC to manage their healthcare. However few adults on the spectrum and their families seem to be using it to its full capability. Using MyChart, patients can ask their doctor questions directly at any time, anywhere. They can also access their medical history, request prescriptions, and manage their appointments online without having to socially interact with a person. This technology exists, it just needs to be made more accessible and its capabilities made more apparent to the ASD community. Extenders within the patient's care team could use MyChart, or a similar shared medical platform, during transitional phases to track current treatments and patient histories and 
stay more connected. It would reduce stress and responsibility on parents who have to repeat and remember past and current medications/treatments for each new provider visit.

In their presentation, parents brought up the disconnect within the ASD system, not just from a patient/provider perspective, but also from a parent-to-parent supportive perspective. The parents needed a single platform through which they could support one another, while also finding highly regarded physicians and specialists for their child. Each child is different and depending on what county or state you are living in, funding and availability to certain resources (e.g., healthcare, housing, or outside support) are often hard to find. In response to this idea, providers added that they would like to create a single online platform. To ensure that proper treatments were being shared among parents, a physician-generated clearinghouse could offer information about 'homeremedy' treatment options (e.g., trying certain diets to help with intestinal issues) to parents to reduce unnecessary follow up visits (that cost the clinic money and take up parent's time). This clearinghouse, or another site similar to an online 'help desk,' could also offer continued support and training to outside extenders who are caring for adults or children on the spectrum.

\section{Resources and Supportive Channels for Adults with ASD}

Another realization from Workshop 1 was that there were a lack of resources and supportive channels to help adults on the spectrum find careers that they were passionate about and that challenged them, not just jobs stacking boxes. "Life and work have to be infused with meaning" (Grandin, 2009, p. 237). The participants determined in the workshop that finding a balance between meaning and passion should start with identifying the specializations of people on the spectrum. Their 'narrow' interests need to be honed and nurtured so they can be directed at helping them reach their maximum potential. "Abilities will grow if the person is encouraged and supported" (Grandin, 2009, p.217). The focus and attention to detail of people on the spectrum can allow them to do many things. Parents and the ASD participants discussed, for instance, the 
link between visual pattern thinking (a trait many people with ASD have) and their ability to code software. This idea is also echoed by Grandin (2009) who explains that "the computer industry is filled with Asperger people...These are the happy people on the spectrum," because they are "with their own people" (p.116). It is clear from this quote, and needs expressed by participants in the case study, that the well-being of a person with ASD may not only benefit from a meaningful career, but also one that may also support their social interaction needs and finding common ground with others.

Opportunities for future careers were also linked to ideas around higher education and creative funding channels. Funding and finding meaningful careers were high priorities among the group. Creative funding channels like micro-enterprises and entrepreneurship were mentioned along with helping people on the spectrum step into emerging technology careers. Ideas were mentioned for redesigning higher education (beyond the current classroom structure) tailored to nurturing individual specializations and connecting students to companies seeking out those specializations. Many people on the spectrum think in patterns and pictures. These talents could be channeled into more meaningful projects and careers working with A.I., assistive apps, developing sensors, or building video games that help people on the spectrum and neurotypicals socialize. The uses of augmented reality and gamification were mentioned in the realm of tools that could better engage this population and teach people on the spectrum life skills, help them practice new tasks, or prepare for social interactions. Self-advocacy mechanisms and platforms, as well as wider caregiver networks that extend to incorporate mentors and extenders beyond physical healthcare could provide people within this population with a better chance to succeed.

Many more ideas emerged and evolved over the course of Phase 1 and Phase 2. Prior to Phase 3 (Workshop 2) these ideas were synthesized and organized into four areas of interest: Longterm Planning, Accessibility and Continuous Care/Support, Improved Navigation, and Social 
Interaction. Near-term and long-term (disruptive) ideas were highlighted within each category. One of the four idea charts, Long-Term Planning, is pictured below. All four can be seen in the Appendix. These idea charts, along with a final visual summary of Phase 2 and the video of our collective mapping (sensemaking) process were sent out to each participant before Workshop 2 .

Ideas: Long-Term Planning

$\begin{array}{ll}\text { NEAR-TERM } & \text { LONG-TERM/DISRUPTIVE } \\ \text {-Tools to help providers \& parents discover \& reinforce } & \text {-Tools to make school counselors more aware } \\ \text { child's specialized 'focus' \& potential } & \text { of post-secondary opportunities for ASD persons } \\ \text {-The C.A.S.T. network extends to life coaches \& } & \text {-Affordable, high efficiency homes (materials \& layouts) } \\ \text { vocation/occupation resources (job placement) } & \text {-Life-skills \& social training + housing'dorms' } \\ \text {-Vision Planning (AASPIRE Toolkit) with IMAGES } & \text {-Innovative funding models (ex. micro-enterprises) } \\ \text {-Pharmacy \& other extenders brought sooner into } & \text {-Careers in technology, futuring, data intelligence, etc } \\ \text { 'sensitive'transition process } & \text {-Post-secondary 'silo'education/training (up to PhD) for } \\ \text {-Life-skills training, modeling \& social stories } & \text { specialized interests } \\ \text {-"Interfacer" tools for young adults on the spectrum } & \text {-Provide means to spread resources and } \\ \text { (alleviate parents' stress \& increase self-advocacy) } & \text { responsibilities: between C.A.S.T. and other healthcare } \\ \text {-On-going training \& support programs for outside } & \text { institutions/specialists, etc. and between parents and } \\ \text { medical specialists (beyond primary care physicians) } & \text { young adults (interfacer roles and responsibilities) } \\ \text {-On-going healthcare and therapy support for } & \text {-Siri \& Apps to help people on the spectrum } \\ \text { parents (at C.A.S.T.) - Family Centered Care } & \text { become more interdependent/independent } \\ \text {-Road maps \& clearinghouse with information for: } & \text {-Maps, tools \& programs to avoid or prepare for "The Cliff" } \\ \text { 'New'ASD parents (maps and support networks) } & \text { after a child on the spectrum graduates from high school } \\ \text { Transitions (road maps and expectations) } & \text {-Programs and housing to support adults on the spectrum } \\ \text { Housing/employment opportunities \& networks } & \text { after their parents pass away. }\end{array}$

Figure 8.12 One of the four idea charts, Long-term Planning, compiled after Phase 2.

\subsection{Outcomes From Workshop 2}

The outcomes of the personas and scenarios, developed in Workshop 2, were described in the C.A.S.T. Case Study chapter. The outcomes of Workshop 2 discussed in this section delve deeper into the ideas and concepts that emerged as a result of collective framing, persona development, and visualizing prospective scenarios. The shared telling and making activities within each phase of the PAR process culminated in the creation of early prototypes addressing the long-term goals and revised frame of our wicked problem. The section below describes the ideas and concepts that were created through this process of framing. 


\section{Social/Gaming Spaces (Digital and/or Face-to-face)}

Grandin mentions that people on the spectrum are drawn to social interactions that revolve around their interests (Grandin, 2009, p.162). Furthermore, these interests, or 'fixations' can help them maintain a social life and make friends (Grandin, 2009, p.105). The link between social interaction, fixations, and shared interests was brought up often during Phases 1 and 2 of our case study. During Workshop 2 John (one of the ASD participants) proposed the idea of creating gaming spaces for social groups, where people with shared interests could easily meet up and engage with one another. John prototyped the idea of a face-to-face "safe" gaming space using LEGOs to demonstrate his future vision of a place where people could self-organize and actively partake in a face-to-face role-playing games or board games with other people on the spectrum, as well as neurotypicals. John's idea came in response to the belief that neurotypicals and people with ASD are more alike than different, and to meet a goal identified in the prospective scenario of Bryan - making friends and having a social life. The idea for a 'safe' gaming space also supported a priority need of the parents and providers to create more social and creative outlets for adults on the spectrum. This idea embodies the essence of the aim for this project. Through shared understanding and mapping, the hope was that the ideas they were constructing in Workshop 2 would meet more than one goal, satisfy more than one stakeholder group priority need, and target a root problem. Neurotypicals and people on the spectrum may have more in common than they think when it comes to entertainment: TV shows, music, books, video games, and movies. Building resolutions that help people with ASD connect socially with neurotypicals and channel their fixations around shared interests can bring about greater tolerance and awareness for this population, while also providing them with an opportunity to express themselves and gain more confidence in social situations. While not directly related, this added social confidence and community tolerance/awareness might in fact be a benefit to other needs indirectly, such as gaining greater independence, keeping a job, and living a healthier, more satisfying life. 


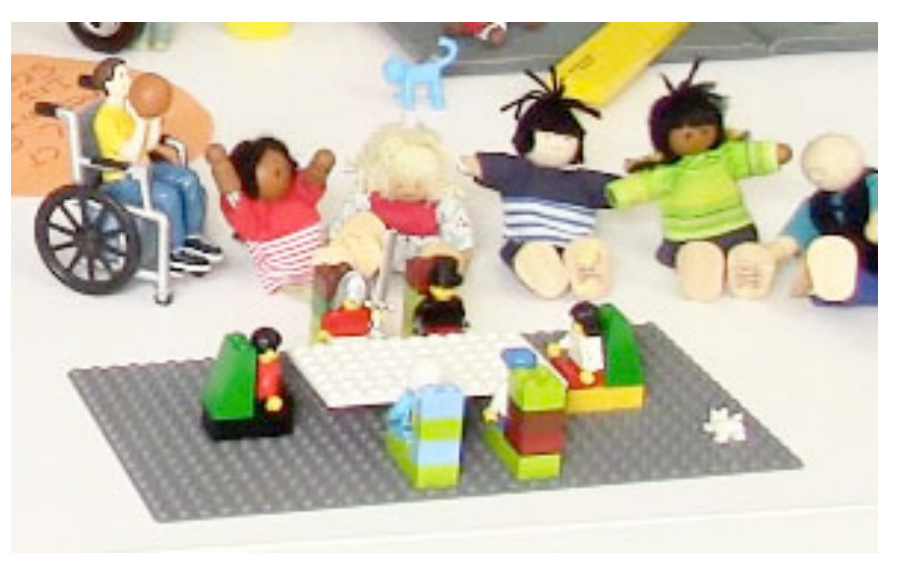

Figure 8.13 LEGO prop of a 'safe' gaming space for people with ASD and neurotypicals.

\section{Owen's List}

Owen's List was the name given to a cohesive online site developed by three parents and a provider from C.A.S.T. This vision for this site was that it would connect people on the spectrum and their families to each other and to resources/services (from medical needs to career opportunities and independent housing options) over the course of a person's lifetime. Similar to Angie's List and Travelocity, service providers could be rated, resources could be found by region, zip code, or search criteria, and search details could increase as you dig deeper. The parents and provider generated this concept using a 2D Make toolkit. The paper shapes in the kit, along with markers, provided the means through which they co-created a series of screen captures on how a person might navigate the site. The website would show the full spectrum of services and a pathway of what to consider at each age/stage of development, what to do next, what services/funding to apply for, how to find housing/employment, etc. After the session, the paper prototypes of the website were documented and refined in more detail for future presentations. 

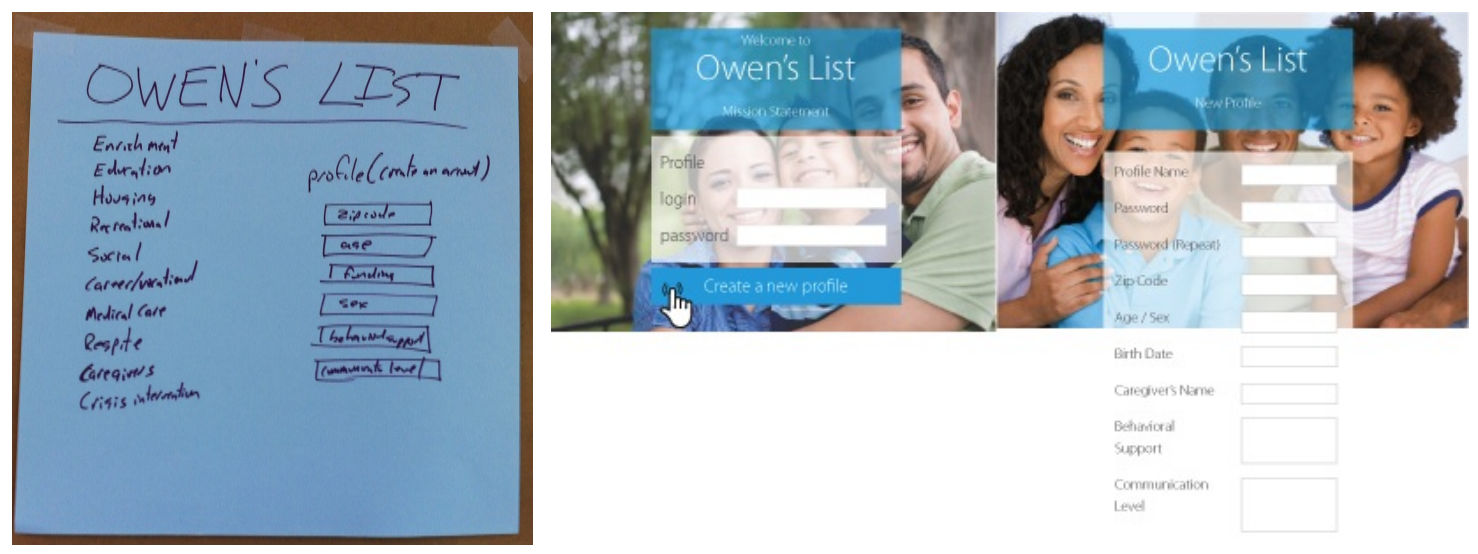

Figure 8.14 Building a custom profile that would grow with the child into adulthood - paper prototype and refined prototype.
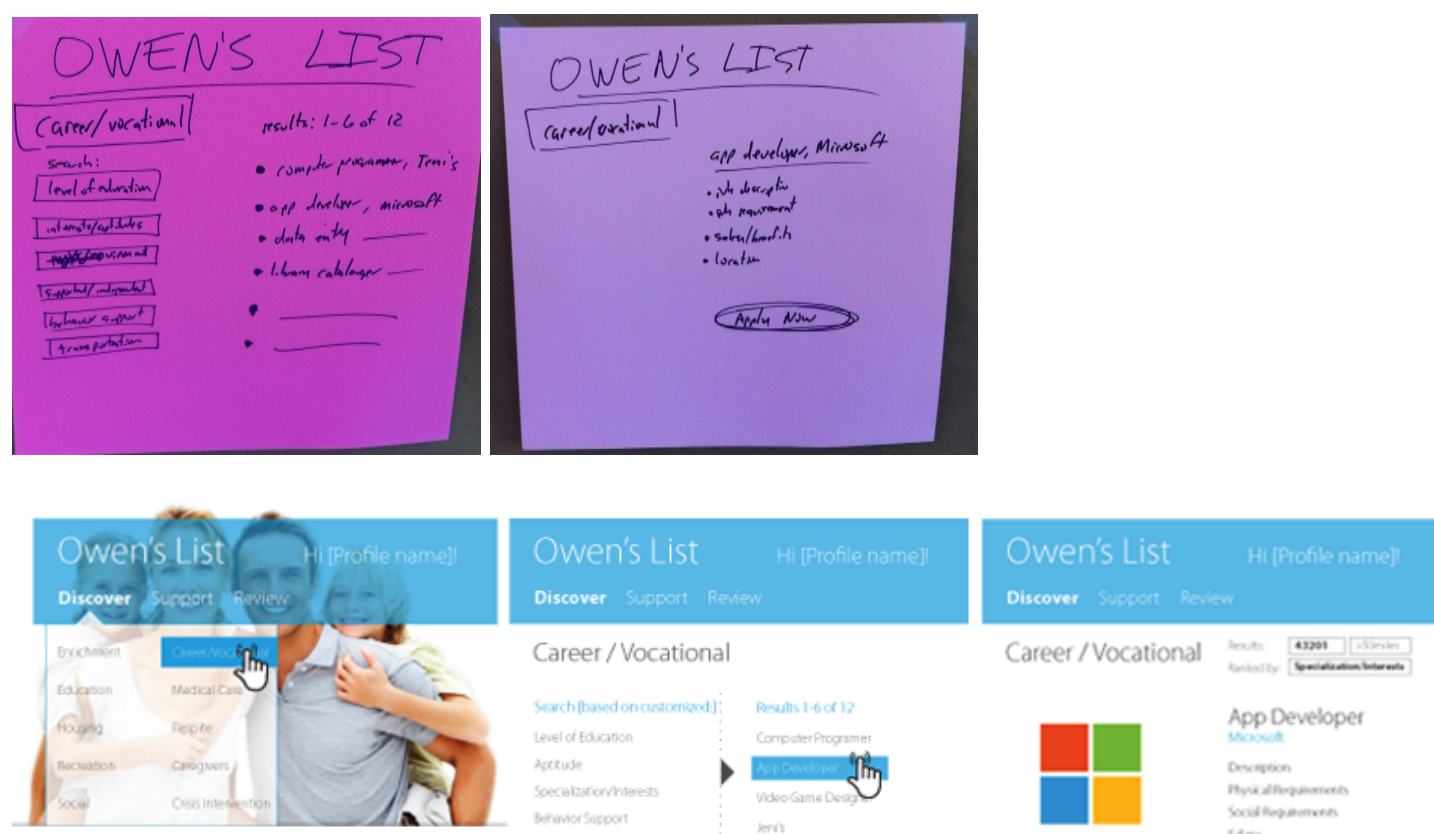

Career/Vocational
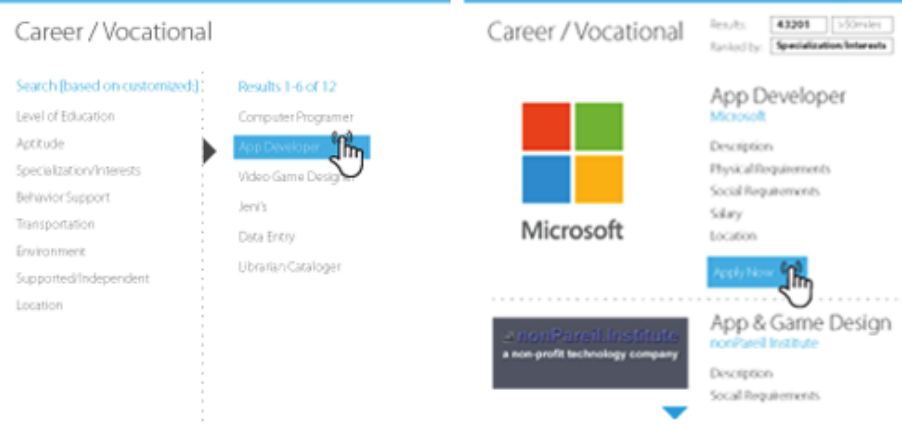

Figure 8.15 Finding a future employer that aligns with a unique specialization/fixation - paper prototype and refined prototype. 


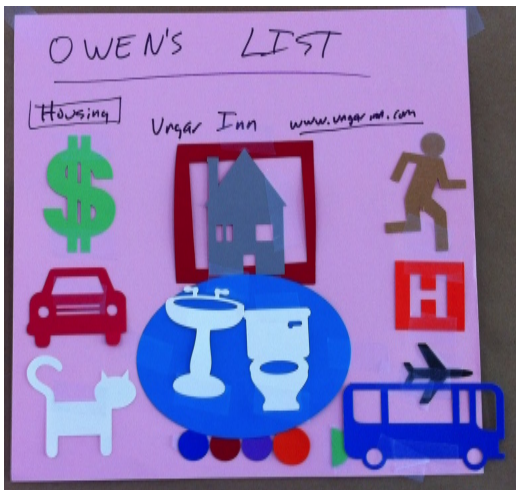

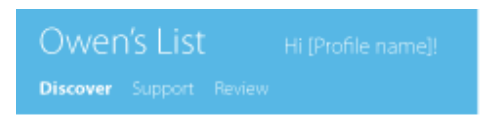

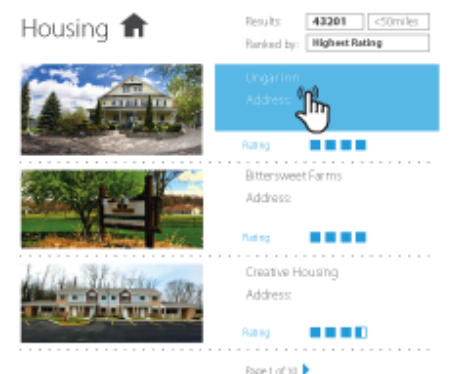

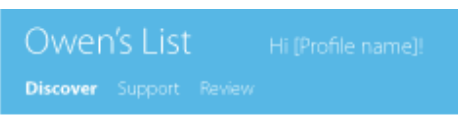

Ungar Inn

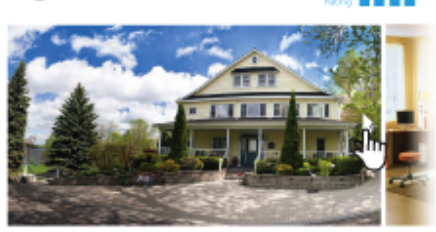

Figure 8.16 Finding affordable, independent housing options - paper prototype and refined prototype.

\section{Resource Directories and Self-Help Resources/Apps/Books}

The idea for digital tools and/or "paper-based" materials offered online and/or at the clinic (and other ASD facilities) was envisioned by John who believed there needed to be an accessible platform that guided people on the spectrum to a wide assortment of tools and resources to help them realize and maximize their potential. This idea was intended to empower people with ASD by giving them more knowledge. John described his idea by building a paper prototype of the 'resource book'. Within the 'resource book' he listed resources that had been suggested to him or had been most useful to him in becoming more independent and empowered to self-advocate. The resources he listed were shared with the Director of Nisonger, with whom he had collaborated in Workshop 2, in an effort to get the ball rolling. In building this prototype and helping parents and providers understand their needs and goals, John and the other participants with ASD met some of their initial goals - "To help empower others on the autism spectrum in dealing with life's challenges, " to "Voice my opinions and thoughts of how ASD-related care should be handled," and to "Gain more skills for self-advocacy, and make the dreams, desires, and opinions of myself and others heard." 


\section{Advocacy and Educational Resources}

An online social platform through which people on the spectrum could self-advocate, while also educating 'outsiders' about what they are capable of accomplishing given the opportunity, was an idea that was brought up during Workshop 2. Though it was not prototyped, it ties together the premises behind both of John's prototypes - social interaction groups and self-help resources. 'Safe' online dialog networks could be a communication gateway for people on the spectrum who struggle with social interaction. This platform, similar to Reddit, would allow people with ASD to self-advocate, while also helping them achieve their goals (such as overcoming obstacles like social interaction) at their own pace. Additionally, an open-dialog platform could promote crosspollination of ideas, shared learning, and emotional support among people with ASD, neurotypicals, and parents.

\section{Integrated Network of Trained Specialists (A Personalized Care-Team)}

The idea for a 'Care Team' was mentioned in Workshop 1 and enhanced from the prospective scenario of Bryan in Workshop 2. 3D objects and miniaturized people were used to demonstrate the facets of the connected 'Care Team' as it pertained to a day-in-the-life occurrence for Bryan and other ASD patients. Using the physical objects, the CoDesigners enacted how communication among the provider, patient, and extenders would function. The 'Care Team' would be able to talk amongst themselves through Skype or MyChart. If Bryan had a question for one or more 'Care Team' members, he could connect with them through a shared online system, without having to make a phone call or go to the clinic. The expectation in the scenario created for Bryan was that the person with ASD should be able to focus their attention on the things that matter most to them, like family, friends, and a meaningful career. The C.A.S.T. clinic and the 'Care Team' should not consume his time or cause him anxiety, but instead be a silent supporter - there when he needs them in a secondary role. This idea responded directly to the new frame of our project. The resolutions tied to medical care and transitional support in this project had little 
to do with the physical clinic and everything to do with maximizing the potential of people on the spectrum and helping them succeed in other areas of their lives. A unified care team would be established for children and adults on the spectrum. The team would be made up of the general practitioner/pediatrician, along with social workers, therapists, program leaders, and other extenders who were pertinent to the child/adult's future success. The ASD 'Care Team' would help to coordinate transitions and could evolve as different support was needed. MyChart or other transparent online resources would help to provide open and flexible integrated communication channels inside and outside the clinic (customized to the patient) so that all members of the careteam members and the patient could stay connected.

\subsection{Post-Assessment Questionnaire Outcomes}

It is important to note that some of the participants who had participated in earlier sessions were not able to attend Workshop 2 and therefore did not complete a Post-Assessment Questionnaire. One parent, who was present in Workshop 2, filled out a Post-Assessment Questionnaire, but had not filled out a Pre-Assessment Questionnaire. All of the questions on the Post-Assessment Questionnaire mirrored those that had been asked on the pre-assessment questionnaire, with the exception of two questions. Instead of asking "have you heard of Design Thinking" or "have you used Design Thinking" on the Post-Assessment Questionnaire, the participants were asked "if they would use Design Thinking for future collaborative projects."

The first set of questions on the survey asked participants to consider their initial understanding/feelings and compare them to their current understanding/feelings. Their responses to these questions, along with similar questions that were asked on the PreAssessment Questionnaire were designed to measure a change or growth in understanding/feelings after the project. The participants' responses are described below and represented in visual diagrams. The participants' prior understanding/feelings for the first set of 
questions are represented by a square; their current understanding/feelings (after the project) are represented by a circle. A black dotted line with an arrow illustrates whether this change was positive or negative. A summary visual diagram with all Post-Assessment Questionnaire responses from each stakeholder group can also be found in the Appendix.

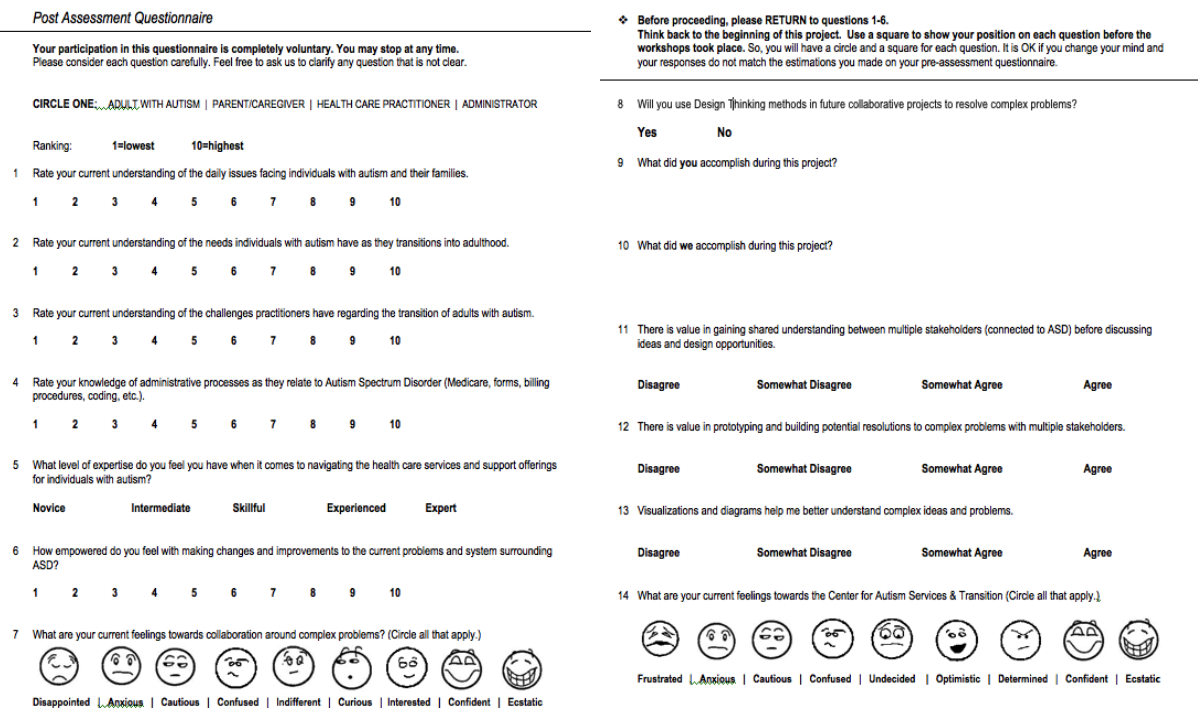

Figure 8.17 Post-Assessment Questionnaire - front and back.

\subsubsection{Post-Assessment Outcomes for the Adult Participants with ASD}

The three ASD participants (two men and one woman), represented in the post questionnaire survey, participated in both workshops. In this Post-Assessment Questionnaire the three ASD participants forgot to mark their prior understanding/feelings with a square, so their prior responses were derived from their pre-assessment questionnaire answers. Overall their understanding of the daily issues facing individuals with autism and their families increased slightly or remained the same. Responses that did not change over time are indicated on the visual summaries by a circle within a square and no directional line. However, the current understanding of the needs of families and people on the spectrum during transitions increased for all three ASD participants. Their indicated change over time in understanding the challenges 
practitioners face were mixed - two reported neutral or greater understanding, while the other participant said their understanding slightly decreased. This may have been for one of two reasons. One, we uncovered a lot of challenges that providers face, but did not address them within the context of the new frame well enough to gain meaning or understanding. The new frame shifted our focus from clinic needs, to unmet needs outside the clinic. This feeds into the second hypothesis that we did not address the administrative and practitioner issues to the same extent as issues faced by adults on the spectrum and their families. However, the three ASD participants reported that their knowledge of administrative processes, while low at the beginning, increased. One participant's knowledge increased a great deal, from 3 to 8 . The level of expertise among the ASD participants in navigating services and care/support offerings changed very little or decreased. This outcome may have resulted from increased exposure to the complexity of the system and the lack of continuity among the players. During the project little attention was paid to how to navigate the system, instead, discussion centered on resolutions to improve communication and navigation. 


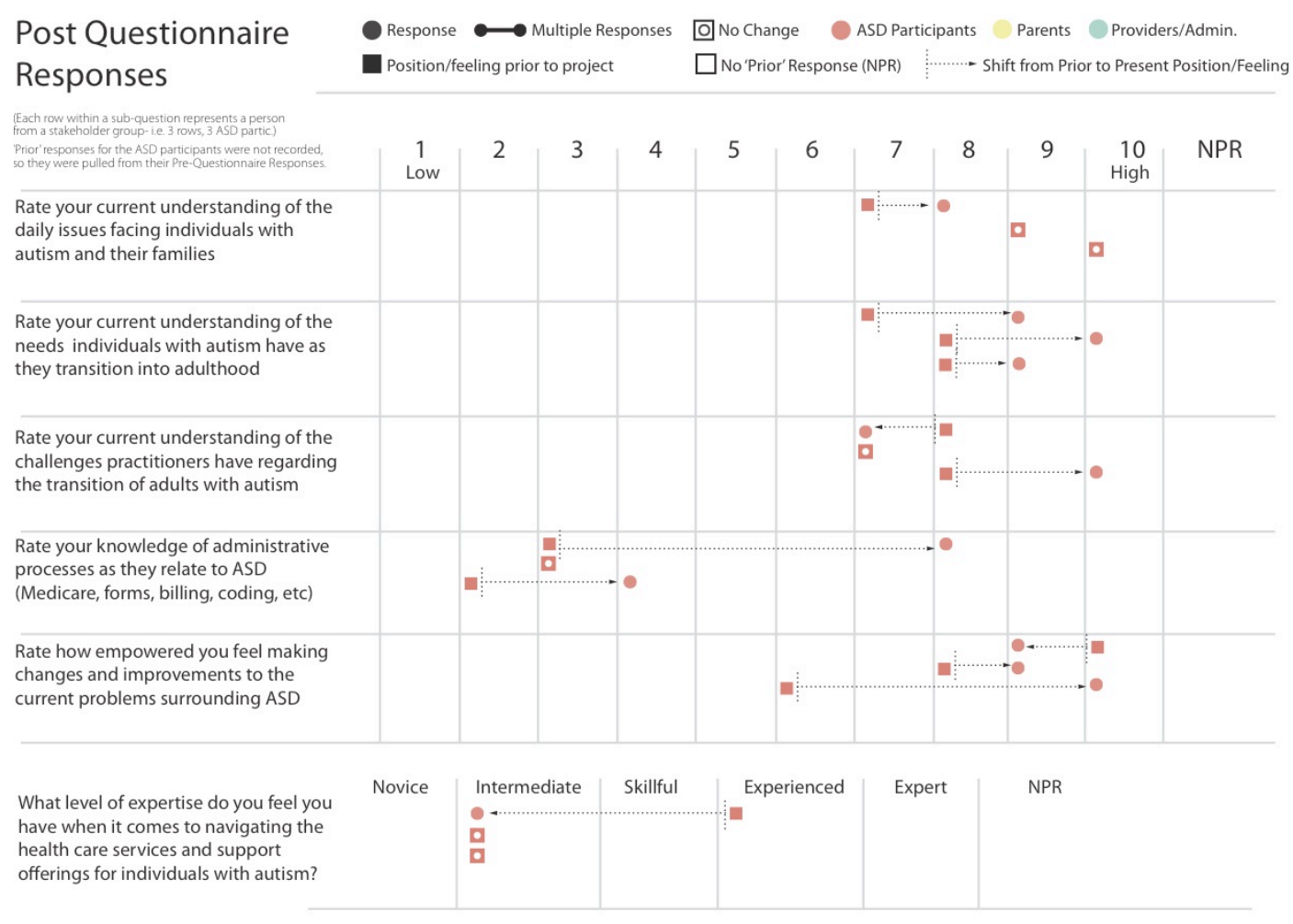

Figure 8.18 Post-Assessment responses to the first six questions by participants with ASD.

After the project two ASD participants felt more empowered to make changes, while the quieter participant felt slightly less empowered. This individual did not have the same level of verbal ability as the other two ASD participants, who had also had more practice in self-advocacy. As a result, those two participants (John and Kristin) became the predominant speakers among the ASD participants throughout Workshops 1 and 2. These factors may have contributed to the quieter ASD participant feeling slightly less empowered to make global changes. However, when asked what he had accomplished during the project, he responded by saying, "I need to accomplish my goal." While he may have felt slightly less empowered to make changes and improvements to the current problems surrounding ASD, he appears to have been motivated and empowered to reach his potential on an individual level. 
Current feelings towards collaboration around complex problems (circle as many as you'd like)

(A) Note: Participant added a written response: "Military's unwaiverable blanket ban on autism including the Air Force which

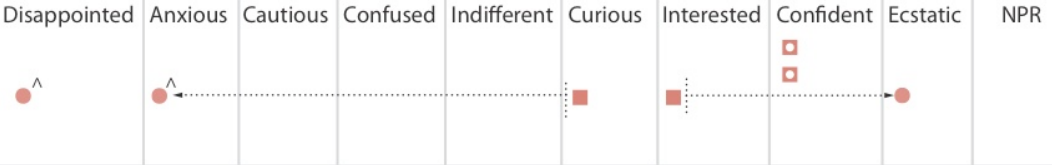

Figure 8.19 Post-Assessment responses by participants with ASD regarding their feelings towards collaboration around complex problems.

All of their feelings towards collaboration remained confident. The most outspoken participant, of the three, remained curious and positive, but also felt anxious and disappointed with collaboration. Following her selected 'feelings' she wrote, "the military's unwaiverable blanket ban on autism?! Including the Air Force which pretty much NEEDS these men and women!? UNACCEPTABLE!" During the sessions this participant was very vocal about the limits society places on individuals with ASD. Her disappointment and anxiety towards collaboration may have stemmed from her frustrations with society's lack of collaboration around these issues.

In reflecting on the things that each of the participants were able to accomplish and what we accomplished, their overall responses were positive and motivated by the belief that through this project they had helped to inform others about their unique perspective, and that collectively we had made strides towards practical resolutions. They seemed appreciative that they had had a chance to self-advocate for other ASD individuals who may not have a voice. Here is what they wrote on their Post-Assessment Questionnaires:

What did you accomplish:

"I need to accomplish my goal." (the quietest ASD participant)

"I informed others about my unique problems and solutions."

"I feel that along with other self-advocates, I initiated in helping individuals to think and begin to realize the strengths of autism over the weaknesses." 
What did we accomplish:

"I need to make sure the [writing unclear] is good." (the quietest ASD participant)

"We made steps towards implementing more practical solutions for people on the autism spectrum."

"I appreciate the consideration that our ideas were taken into."

Their feelings towards C.A.S.T. were confident, determined and optimistic. All three participants said they would use Design Thinking in future collaborative projects and agreed that gaining shared understanding among different stakeholders (before discussing ideas), prototyping potential resolutions with other stakeholders, and using visualizations helped them to better understand the complex problem.

Current feelings towards the Center for Autism Services \& Transition (C.A.S.T.)

Frustrated Anxious Cautious Confused Undecided Optimistic Determined Confident Ecstatic NR

\begin{tabular}{|c|c|c|c|c|c|c|c|c|c|}
\hline \multirow{3}{*}{$\begin{array}{l}\text { Will you use Design } \\
\text { Thinking methods in } \\
\text { future collaborative } \\
\text { projects? }\end{array}$} & Ye & No & Maybe & NR & $\begin{array}{l}\text { There is value in gaining shared Disagree } \\
\text { understanding between multiple } \\
\text { stakeholders (connected to ASD) before } \\
\text { discussing ideas and design opportunities. }\end{array}$ & $S / D$ & $S / A$ & Agree & NR \\
\hline & & & & & $\begin{array}{l}\text { There is value in prototyping and building } \\
\text { potential resolutions to complex problems } \\
\text { with multiple stakeholders }\end{array}$ & & $\bullet$ & : & \\
\hline & & & & & $\begin{array}{l}\text { Visualizations and diagrams help me better } \\
\text { understand complex ideas and problems }\end{array}$ & & & 0 & \\
\hline
\end{tabular}

Figure 8.20 Post-Assessment responses by participants with ASD on the final questions.

\subsubsection{Post-Assessment Outcomes for the Parent Participants}

Two of the five parents did not indicate their initial understanding/feelings prior to the workshops. Prior responses for these parents were marked by an open box under the column NPR (No Prior Response). In general, the parents' understanding related to the daily issues facing individuals with autism and their families were relatively high to begin with. After the project this 
understanding increased for two parents and remained consistent for the other. Their understanding of the needs of individuals with autism during transitions increased or remained the same. The largest growth in understanding came from the parent who initially indicated a low level of understanding. Parents who recorded both past and present knowledge also reported a positive growth in their understanding of the challenges faced by practitioners regarding transitions. Their knowledge of administrative processes either stayed the same or slightly increased. Unlike their understanding of the needs of families and adults on the autism spectrum, the parents' knowledge of practitioner needs and administrative processes was relatively low initially, but their knowledge after the project either remained the same or slightly increased. While none of the changes between past and current understanding/knowledge were negative, there appeared to be the most growth in understanding the needs of individuals with autism and the needs of practitioners during the transition process. These two topics were given the most attention during the project.

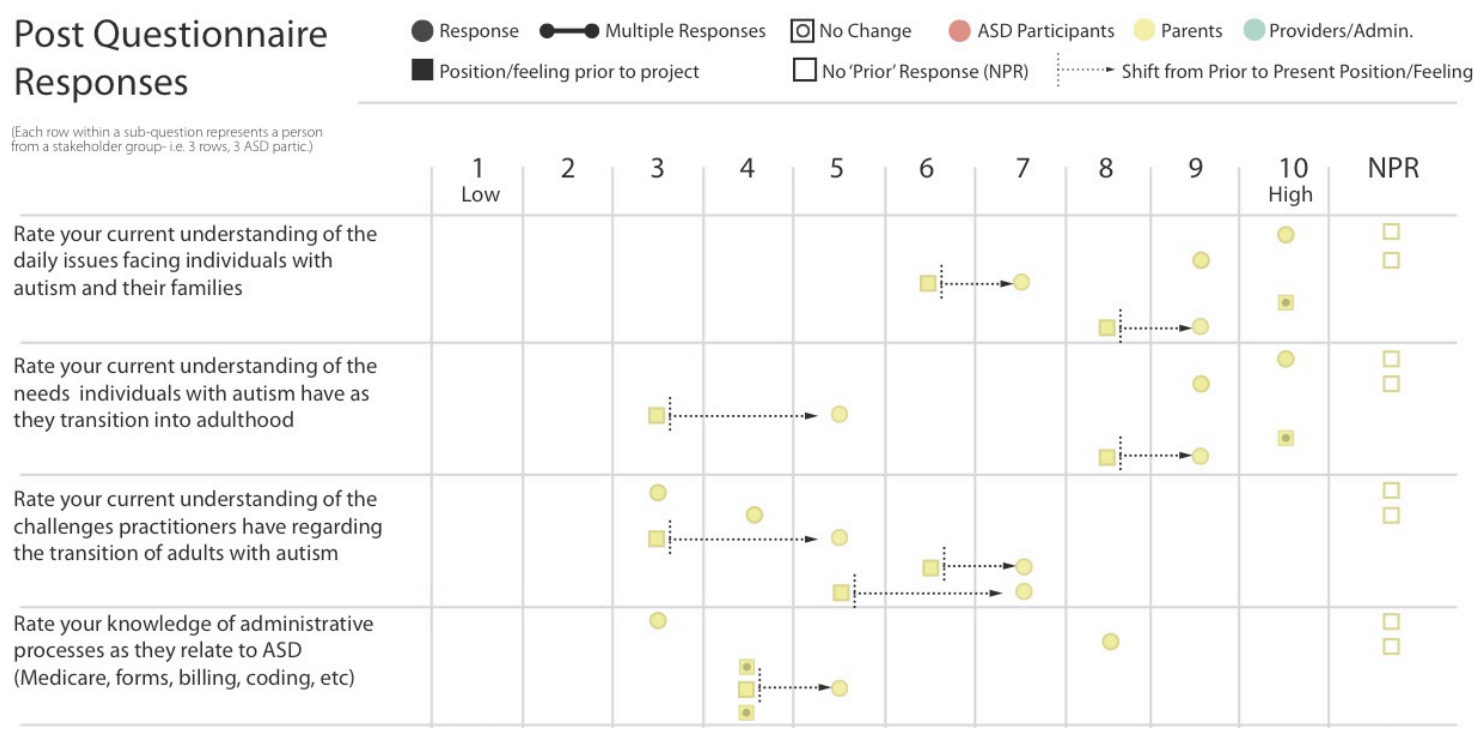

Figure 8.21 Post-Assessment responses by parents on the first four questions. 
The parents indicated consistency in their level of expertise for navigating ASD care and support offerings. It is likely that there is little to no change in this category because the scope of the project centered on finding opportunities and resolutions to improve navigation and communication, as opposed to explaining how a person might navigate the current faulty/disjointed system.

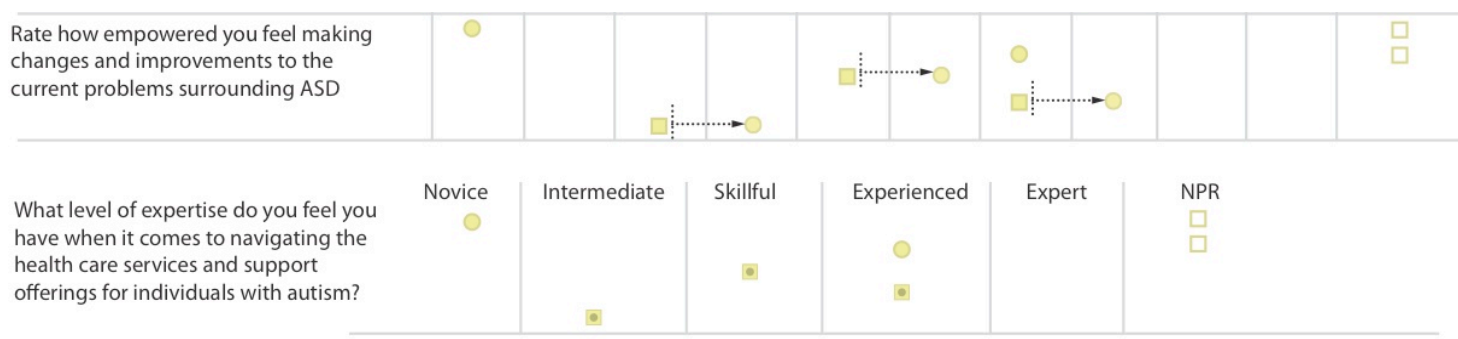

Figure 8.22 Post-Assessment responses by parents regarding their current feelings of empowerment and expertise.

Overall, three parents reported an increased feeling of empowerment after the project. One indicated a low feeling of empowerment, while the other felt a higher level of empowerment for making changes and improvements to the current problems surrounding ASD. It is not clear why one participant had a low feeling of empowerment after the project. Their feeling towards collaboration around complex problems was indicated by the word "ecstatic." Perhaps they feel less empowered on an individual level. 
Current feelings towards collaboration around complex problems (circle as many as you'd like)

Each row represents a persan from a stakeholder group-i.e. four rows, four parents

Disappointed Anxious Cautious Confused Indifferent Curious Interested Confident Ecstatic

NR

Post-Assessment Questionnaire Responses

Current feelings towards collaboration around complex problems (circle as many as you'd like]

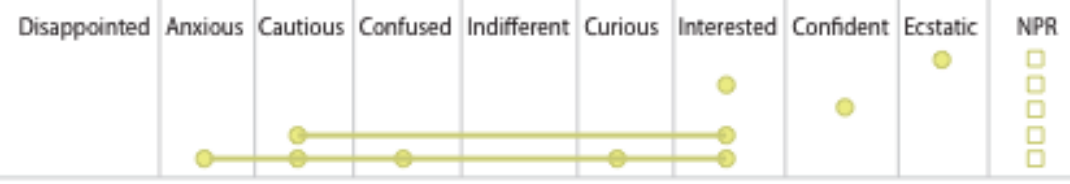

Figure 8.23 Drawing a comparison between the parents' responses prior to and following the project regarding their feelings towards collaboration around complex problems.

The general feelings towards collaborating around complex problems remained similar to the feelings indicated by the parents on their pre-assessment questionnaire. While their feelings remained mostly optimistic and confident, there were two parents who were cautious. Those that indicated feeling anxious and cautious, however, were also interested and curious in the idea of collaboration around complex problems.

In reflecting on what they accomplished personally and what we accomplished together, the parents expressed expanded awareness and networking, as well as the ability to actively generate ideas and propose "real, possible" resolutions to help individuals on the spectrum and their families. For one parent, this session also helped her strengthen the assistive technology ideas she had been working on (outside of this project), which concerned her the most. Here are the parents' responses: 
What did you accomplish:

"Proposed solutions that would help individuals on the spectrum and their families."

"Vet and brainstormed with other parents."

"Expanded my awareness."

"Refined my ideas about supportive technology for ASD individuals and families."

"Good interplay with professionals and people on spectrum."

What did we accomplish:

"Generated ideas and networking."

"Provided a good environment for discussion."

"Several excellent prototypes."

"Shared/increased understanding among stakeholder groups and some real, possible solutions."

"Definition of issues of adults on the spectrum."

Their feelings towards C.A.S.T. were very optimistic and they all agreed that they would use Design Thinking methods in the future. Most of the parents agreed that there was value in gaining shared understanding before discussing ideas and prototyping resolutions with multiple stakeholders. Two parents 'agreed' and three 'somewhat agreed' that visualizations and diagrams helped them better understand complex problems. The spread between 'agree' and 'somewhat agree' points to the notion that while visualizations and diagrams may be useful in some cases to understand complexity, they are perhaps not the only, or most effective way to understand complexity. There are other methods like prototyping, creating personas and prospective scenarios, and engaging in face-to-face discussions that were utilized in this case study that helped people gain new awareness and understanding. Therefore, there is value in conducting a multi-method Design Thinking approach when tackling wicked problems. 
Fustrated Anxious Cautious Confused Undecided Optimistic Determined Confident Ecstatic NR

Current feelings towards the Center

for Autism Services \& Transition

(C.A.S.T.)

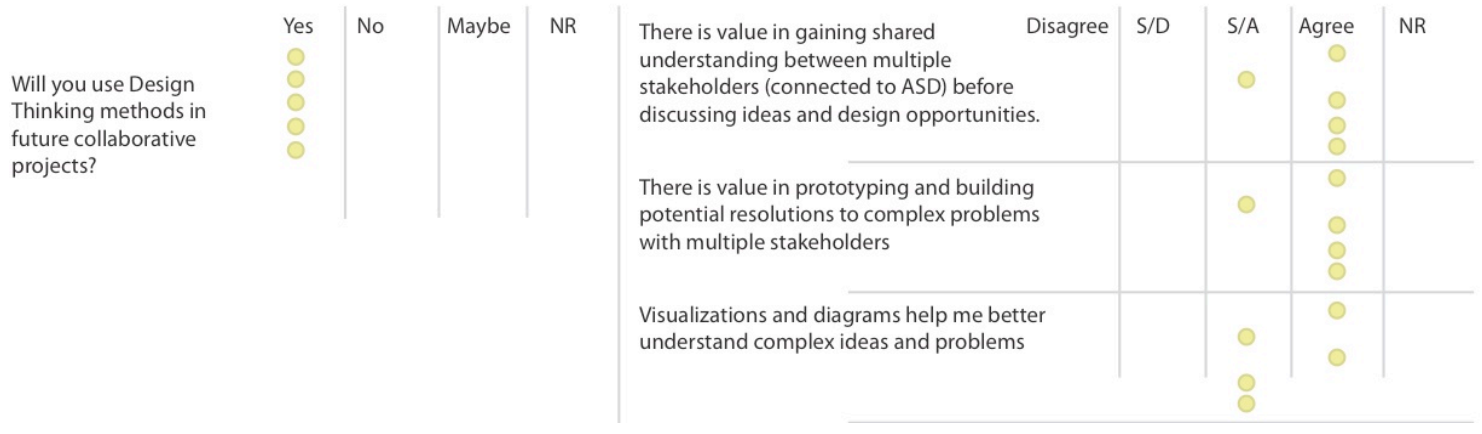

Figure 8.24 Post-Assessment responses by parents on the final questions.

\subsubsection{Post-Assessment Outcomes for the Healthcare Provider/Administrator Participants}

Many of the administrators and providers that had attended the focus group session and

Workshop 1 were not able to attend Workshop 2. The feedback from the third stakeholder group

(providers and administrators) in the post-questionnaire represent the views of a pharmacist at

C.A.S.T., the Director of C.A.S.T., and the Director of the Nisonger Center (who considers himself to be both a provider and administrator). One of the provider stakeholders did not indicate their initial understanding/feelings.

As illustrated in the visual diagram below, the understanding of the providers and administrators for the daily issues facing individuals with autism and their families, as well as their needs in transitions, was high for two participants and grew a great deal from the initial understanding of the third. Their awareness of the challenges faced by providers during transitions were also high. For two, this understanding increased over the course of the project. While their knowledge of administrative processes remained lower in comparison to the other categories, two of the participants' knowledge slightly increased or remained the same after the project. Additionally, their expertise in navigating the ASD healthcare system and the support offerings for individuals 
with autism ranged from 'intermediate' to 'experienced' after the project. One participant remained at a 'skillful' level, while another grew from 'skillful' to 'experienced.' The reason for this increase in expertise (by a provider/administrator from OSUWMC or Nisonger) along with the increase in administrative processes and provider needs across this group of stakeholders represented on their post survey can only be hypothesized. These increases may have come from the involvement of other providers from Nationwide Children's Hospital and/or from speaking with parents. Providers in the sessions often claimed that the parents were the 'real experts.' They read a lot and seek out information so they often have more knowledge about facets of the ASD system and support offerings that take place outside the medical realm. Additionally, during the presentations in Workshop 1 there were mutual discussions about the varying operations in pediatrics and adult medicine (as they relate to ASD) and how the providers from the different hospitals could better align their processes and goals. These discussions may have given this group added understanding of not only care, service, and support offerings outside OSUWMC, but also what the parents have found outside the medical field. 


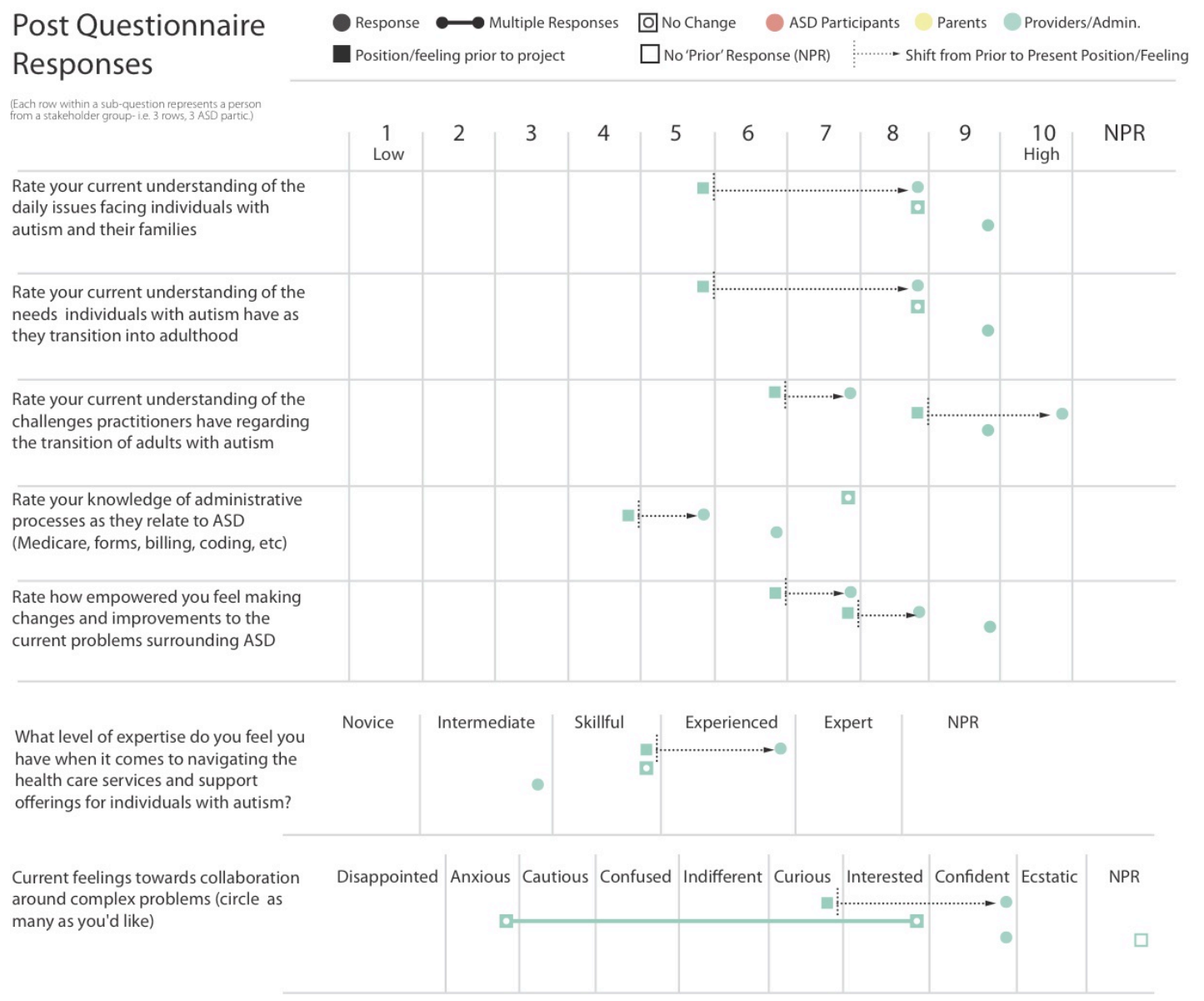

Figure 8.25 Post-Assessment responses to the first set of questions by healthcare providers and administrators.

Their feelings towards collaboration varied, but were mostly positive. One participant indicated that their feelings had grown from 'curious' to 'confident.' Another reported a mixture of emotions, from 'anxious' to 'interested.' In reflecting on their personal accomplishments and our collective accomplishments, this stakeholder group believed the sessions allowed for cross-fertilization and greater understanding around the transitional needs (beyond medical) of individuals on the spectrum and their families. They were also made more aware of the needs and roles of different people helping people with ASD. Below are some of their responses: 
What did you accomplish:

“Increased understanding of individuals' and families' perspectives for transition/needs."

"I came to realize that the medical needs of my patients are often only a small part of their needs."

"Better understanding of parents and autistic children's/adult's needs."

What did we accomplish:

"Increased cross-fertilization between stakeholder groups"

"A better understanding of the overall needs and roles of different people who work with people with ASD"

"Improved my knowledge."

Their feelings towards C.A.S.T. were optimistic. In general, they all agreed that they would use Design Thinking for future collaboration projects and thought that there was value in shared understanding before discussing ideas, prototyping with multiple stakeholders, and using visualizations to improve understanding and construct meaning. 


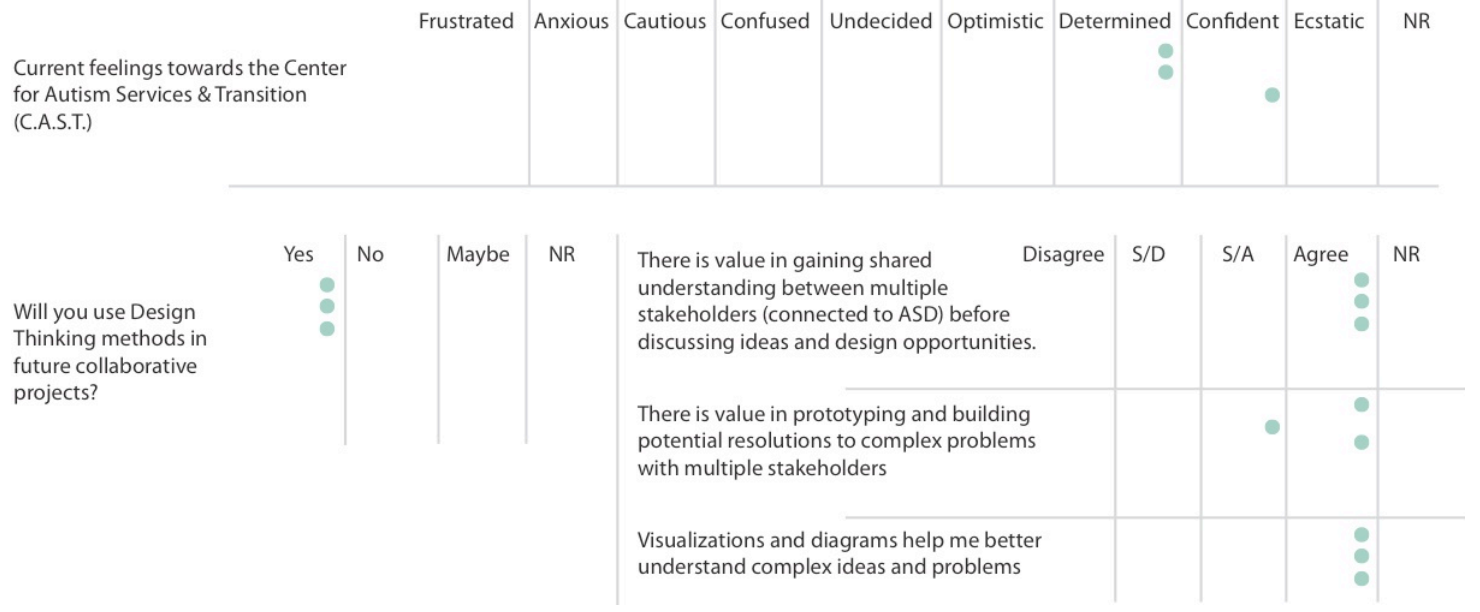

Figure 8.26 Post-Assessment responses to the last set of questions by healthcare providers and administrators.

\subsubsection{Synthesis of Post-Assessment Questionnaire Outcomes}

While the scope of the project initially centered on issues related to C.A.S.T. and transitional care/support connected with the healthcare system, it became more apparent that the unmet needs connected to transitional care stemmed from issues related to peace of mind and longterm well-being. Most of the goals (or root problems) tied to the clinic were interconnected to issues like navigation, communication, overcoming personal obstacles (like anxiety and social interaction), and achieving life goals. What the providers and administrators realized over the course of this project were that they could not offer optimal care if the needs and goals of their patients (and their families) were not being met outside the clinic.

It is important to note that the type of individuals involved during the process of sensemaking in Workshop 1 directly impacted the reframing of this project. Had we had different stakeholders, the frame may have been different. Parents and people on the spectrum, combined, slightly out numbered the administrators and providers who were present, especially during Workshop 2. Therefore, the concerns of the parents and people on the spectrum were more evident. 
Furthermore, having adults on the spectrum present in these sessions was completely new for the parents and providers/administrators who had participated in previous discussions on the topic of autism. The contributions of the ASD participants added a great deal of value to the new understanding of all of the participants and to the eventual frame of this wicked problem. Even though only three were able to participate in Workshops 1 and 2, their thoughts and feelings were unquestionably taken into consideration. Many of the neurotypical participants found the presentation of the ASD participants to be most informative and eye-opening. Therefore, most of the newly acquired knowledge, reported in the post-questionnaires, came from better understanding different stakeholder roles, especially the unmet needs and goals of individuals on the spectrum and their families. Administrators were the least represented group, so it would seem that the limited understanding of administrative processes stems from the fact that less focus was given to their needs and more focus was directed at the adults on the spectrum and the parents.

The post survey confirmed that the participants valued collaboration and Design Thinking processes when tackling complex problems. Additionally, visualizations and prototyping were perceived to be of value to the CoDesigners (with no Design background) in improving their understanding and helping to construct meaning and put forth relevant ideas relating to the wicked problem of transitions in the autism community. These methods should continue to be integrated and tested in future PAR sessions for wicked problems.

The questions in the Pre/Post-Assessment questionnaires were designed initially around needs more directly connected to transitions and the C.A.S.T. clinic. As various perspectives were shared and different priorities emerged, understanding administrative processes and how to navigate the current healthcare services and support were not as central as finding ways to maximize potential outside the clinic and developing new ways to improve the system and overall 
navigation. Ideas were generated to address the unmet needs associated with limited understanding and finding new opportunities to communicate/connect with each other and with information. Navigation was tied to first creating more open communication channels and strengthening the relationships between care/service providers. If parents were not aware of the clinic's existence, for example, focusing our attention in this project on sensory lighting in the waiting room would not have been useful. The aim of this project was to first identify root/connected problems, before selecting directions randomly and spending our time building resolutions that may or may not have been valuable.

\subsection{Post-Project Feedback Questions}

Four participants replied to the two feedback questions that were posed to the participants, following Workshop 2. Below are the questions that were asked and their responses.

1. What were your expectations prior to participating in the collaborative sessions/workshops?

2. What transpired/occurred during/after the sessions (or a specific session) that was different from what you expected?

\section{Responses:}

"I had no preconceived notions prior to attending the workshops. I was merely interested in being a part of a project, which included primary users, parents/family and professionals. I was very encouraged by the unanimity in the groups I was a part of regarding the needs of people on the autistic spectrum after their education is completed. As the numbers of people with autism skyrocket, and the population continues to age, it is imperative that society learns how to deal with and assist this population in as it attempts to navigate life's challenges. This workshop is a good start, but, in my opinion, this topic will become more and more relevant in the future. Clearly, more outreach and availability of post educational programs to help the autistic 
population deal with life are necessary to make sure these folks do not fall through society's cracks."

"I had no expectations. I actually had never participated in this type of workshop before. I enjoyed learning about this process and especially the use of technology to find communalities between the different stakeholder groups. I was pleasantly surprised to see the involvement and participation of the parents and self-advocates. The self-advocates brought a lot to the table as did the parents - especially in bringing the perspectives of individuals with ASD with more significant cognitive and communication deficits."

"I had assumed we would be doing a lot of "designing of space" interventions. Instead, the conversation was much more wide-ranging than I expected. The making/designing of space was part of a much larger discussion."

"I was hoping to get input and opinions from different shareholders regarding what is needed for development of CAST. I quickly realized that CAST was not the biggest need, but just a small part of a bigger, broader need. This occurred in the session where we grouped things together with the circles on the board."

\subsection{Discussion Regarding the Participants' Responses}

The responses of the participants reflected that the process of stepping back and gaining shared understanding before jumping straight into idea generation was unexpected. The value of first converging around similar/dissimilar needs and goals before diverging and generating a multitude of ideas was a concept that was intentionally explored in this PAR process. After reviewing the Post-Assessment Questionnaire responses and the CoDesign outcomes, it appears that this process has merit. Through this case study the participants uncovered root problems and 
pressing issues that were connected to multiple needs and goals across stakeholder groups. As a result, many of the ideas that were conceived responded to more than one stakeholder need/goal. Many of the participants expressed after the sessions that they found value in hearing from one another and uncovering new knowledge. While all of the resolutions and ideas that were expressed or prototyped will need further development, the participants left feeling that they had not only uncovered meaningful resolutions, but had also gained intangible benefits. Had the focus only been on generating tangible ideas/outcomes, and not connecting around a shared sense of purpose, the participants may have left feeling that they had accomplished very little by way of building a 'solution.' Instead, they indicated not only that we had developed "several excellent prototypes" and "some real, possible solutions," but that they also felt motivated to take future action, that they had increased knowledge and understanding, and that they had found new ways to connect with the problem and with each other. These intangible outcomes are invaluable to the continuing PAR process, as more iterations and involvement will be necessary to carry out and test the resolutions.

\subsection{Expected and Unexpected Outcomes}

The initial expectation was that the PAR process would result in actionable decisions and 'tangible' CoDesign concepts, pertaining mostly to the physical environment of the clinic and improving network connections between pediatric physicians and adult providers. What was unexpected from this case study were the intangible outcomes that emerged as a result of the designed framework of methods/tools and the shared experience of the various stakeholders in multiple seeing/maker spaces. In conversations shared with some of the stakeholders during and after the project, they described feelings of increased engagement, empathy, knowledge, connectivity, and creative thinking. The feeling of being heard and gaining new understanding appeared to have had a greater impact on the participants than the resolutions that were generated. One of the parent participants, who is very active in the autism community, said that 
the sessions brought about "engagement, awareness, encouragement, and a sense of community." For her, there was a realization that "there is a broader community than we thought and that we can work together to solve this complex problem." For someone who is already extremely connected in the ASD community to find greater connection and encouragement from these sessions was a valuable outcome. In addition, she realized that "new ways of thinking arose. New connections were made and these connections can lead to future collaborations." The methods, tools, and seeing/maker spaces, though designed to bring about synthesis and creative thinking, also resulted in strengthening the connection between the stakeholders in this wicked problem. For a more lasting impact (especially when problems are wicked), longevity and connectivity become critical to future planning and action because there is no single solution, but instead resolutions that need to be implemented, tested, and iterated. For this reason the Design team needs to stay connected and flexible (able to grow) as new knowledge is gained. We did not reach a final 'tangible' outcome, but instead generated a meaningful plan of action, with a series of related resolutions that need further development. Along with these 'tangible' and 'intangible' outcomes, we also generated visualizations (the sensemaking map of connected/prioritized needs and goals, visual provotypes of the clinic, presentation diagrams, personas, and prospective scenario diagrams) that illustrate a traceable connection between the new resolutions and the initial needs and goals brought up by the stakeholders in Phase 1. For Designers it is often hard to show how ideas and resolutions were derived and why they are important. As Kolko (2012) described, the resolutions we design often appear "magical" to non-designers. Through the process that was taken and the tools/methods that were used, we are able to demonstrate to other stakeholders why the final resolutions are important and how they are tied to priority/root problems that need attention. The value of being able to communicate our Design Thinking process and findings to other stakeholders in the ASD community will become more apparent in the last chapter when next steps for the project are shared. 
After participating in all of the sessions, John (one of the participants with ASD) expressed why he had chosen to stay involved at each phase of the project. He simply replied, "you cannot design something for us without us." The participatory process allowed the various stakeholders to have a voice and to be heard, to step into a CoDesign role and play a critical part in making decisions and coming up with resolutions that directly relate to them. If we are designing for a specific need, all people connected to that need should be heard. Their involvement is critical in helping Designers making sense of the problem, especially a wicked problem. Shared understanding and the use of Participatory Design tools and methods were shown to motivate participants (on an individual level, as well as on a collective level) and bring about greater empathy and awareness for the issues faced by other stakeholders connected to the shared wicked problem. The process and the methods/tools used also promoted a sense of community and collective action around meaningful resolutions, because the participants were immersed in an experience that prompted them to learn from one another, share different views, and engage together in thinking, telling and making.

\subsection{What Was Learned From the Outcomes of the Case Study}

The following sections below provide a summary of what was learned from the outcomes of this case study. The conclusions listed below relate to: the C.A.S.T. clinic, healthcare training, independence and peace of mind, visual information, navigation, and preparedness, building collaborative networks, integrating resources and connections, socialization, advocacy, potentiality, and lastly empowerment and a sense of control. Some of these conclusions reflect not only findings related to the topic of our wicked problem, but also relate to discoveries made about the PAR process through this case study. 


\section{The C.A.S.T. Clinic}

Care and support for families and adults on the spectrum should extend beyond their physical needs and the walls of the clinic. A customized, holistic wellness approach is essential for helping people live successful lives. Patients and families would benefit from a connected and transparent 'Care Team'. The clinic would benefit from having extenders on staff, offering services beyond primary care, establishing a stronger connection with pediatric care and specialized care providers, and allowing patients and families to help design/tailor the clinic experience and their goals for the future.

\section{Healthcare Training}

Providers (inside and outside the ASD care clinics) need to be properly trained so they feel more empowered and comfortable caring for adults on the spectrum, while also widening the referral/care network for people on the spectrum and relieving some of the pressure on ASD primary care providers who cannot offer specialized care. Training and educational resources will optimize care and widen service/support offerings to adult care specialists and extenders (beyond primary ASD care providers). By empowering more extenders and outside specialists to take on ASD patients, C.A.S.T. providers do not over-promise and under-deliver and ensure that people on the spectrum receive the best care.

\section{Independence \& Peace of Mind}

Resources and funding options are scarce for adults on the spectrum and their families. As the ASD population continues to rise, this issue becomes more and more critical and harder to fix. Therefore, we have to find more inventive ways to tackle transitional issues and help people on the spectrum and their families reach their full potential. Disruptive technologies will need to be created to support and sustain greater independence for adults with ASD, while also relieve pressure on the care givers/parents and giving them peace of mind. 


\section{Visual Information, Navigation and Preparedness}

People (especially those on the spectrum) value visual communication, navigation, and the feeling of preparedness. Whether it is short-term navigation in the clinic or long-term navigation in the care continuum and throughout each transitional life stage of a child on the spectrum, visual cues/guidance, progressive 'Vision Plans' and visual ‘Patient Passports' can provide peace of mind and a sense of preparedness/control to patients, providers, and parents to plan next steps and achieve actionable goals (small or large).

Visual information also impacts the PAR process. In this case study, visual diagrams and prototypes were useful tools for communication, shared understanding, and building community engagement. The Director of C.A.S.T. asked to use some of the visuals that had been created in Phase 1 (to represent the thoughts/feelings expressed by John and Kristin) in another presentation he was giving at a medical conference because he felt they more accurately portrayed the struggles people on the spectrum experience than his words could alone. Visualizations and prototypes can act as navigation tools within the Design process to trace ideas/resolutions to identified needs and opportunities found in the research. Additionally, the visual provotypes created in Phase 1, along with the Journals, Method Cards, and summary visuals/video from Workshop 1, were useful in preparing participants for the following sessions.

\section{Collaborative Network of Trained Professionals}

Transitional care is currently a challenge because of unconnected care teams and channels. Medical records and healthcare teams need to be more congruent and transparent. The ASD Care-Team model needs to be more cohesive and connected, especially between pediatric care and adult care, to make the process of transitions more fluid and gradual. Additionally, improved collaboration networks, communication tools, and training among all healthcare staff will increase peace of mind and tolerance, and improve clinical visits. 
Wicked problems require input from a wide range of experts. Engaging a collaborative network of experts from each stakeholder group in the PAR process was invaluable for framing the project and tackling the wicked problem. Varying stakeholders allowed input and accommodation from many perspectives related to the complex problem.

\section{Integrated Resources and Connection}

Parents need a trusted, integrated online resource that: connects and better unites them; helps them share/gather information; guides them through transitional phases and the care/finance continuum; and helps them find customized care options for their child nearby. Additionally, adults on the spectrum need a resource that empowers them to be self-advocates and become more independent, to find trusted resources and people to connect with, and guide them in reaching their maximum potential.

In terms of the PAR process, integrated methods and tools that extend participation beyond realtime sessions allow participants (including newcomers) to more easily trace decision-making processes and expand collaboration potential after the session has ended. The value in developing tools and visualizations that help to extend understanding and participation through the PAR process to new experts becomes critical to motivating participants and giving them ownership of the problem as resolutions continue to be iterated and new knowledge is shared throughout multiple Design stages.

\section{Socialization}

People with ASD benefit greatly from social interaction - more integrated and flexible channels for socialization among people with ASD and neurotypicals are needed. Through socialization, growth and maturation, for both people on the spectrum and neurotypicals, can occur. When given the opportunity and the training tools to improve their social skills, many people on the 
spectrum can learn to speak up for themselves and interact with others. Socialization satisfies many prioritized needs: the need for people on the spectrum to become self-advocates and become less dependent on others, while also helping society become more aware and more tolerant of our similarities and differences.

With regard to the PAR process, social interaction among stakeholders with varying points of view was invaluable to the sensemaking process and the generative design process around the wicked problem not only from a 'tangible' outcome position, but also from an 'intangible' outcomes standpoint.

\section{Advocacy}

Channels and platforms need to be developed to help people on the spectrum self-advocate, and to also help parents develop a sense of community and cohesion. Currently, the outward 'message' of parents and people on the spectrum is disjointed and not based in solidarity - their disconnect weakens the support and understanding they can receive from 'outsiders'.

People on the spectrum have a lot to say and want to be heard. The inclusion of people on the spectrum in this case study was invaluable to the direction the project took and to the outcomes. The PAR process needs to be democratic and inclusive. Young or old, verbal or non-verbal, abled or disabled, everyone has something to say and share. Their needs, visions, and ideas for the future need to be heard and taken into consideration. The approaches used in PAR processes, especially for tackling wicked problems, should provide participants with options in how to make contributions and decisions to problems that impact their lives. 


\section{Potentiality}

We need to create systems, services, and tools that help all of us recognize, assess, nurture, and help to sustain the specializations and individual potential (not limitations) of people on the spectrum. Disruptive technologies and services will be needed to not only give parents and caregivers greater peace of mind and assistance to help them reach their potential, but they will also be needed to guide people with ASD towards becoming more independent, and help them to recognize and advance their specializations so they can reach their maximum potential.

\section{Empowerment and a Sense of Control}

The feeling of control over physical and social environments, as well as the feeling of having control over their future and long-term goals, was a desired goal of the participants with ASD. Empowerment was a root goal connected to more evident problems associated with waiting, navigating, and/or fears of social engagement. Along with empowerment, potentiality and peace of mind became the new frames of our wicked problem. Through PAR, the stakeholders and Design team are now better equipped to understand and apply this frame of thinking to the resolution of new problems that arise inside or outside the clinic. For instance, does a patient's outburst in the waiting room have to do with bright lights, or with a loss of control and peace of mind tied to the apprehensions of socialization and not knowing how long they have to wait?

Parents and people on the spectrum who participated in this session shared that they felt empowered throughout the project because they were CoDesigners. Giving stakeholders an inclusive (equal and valuable) role in determining the scope of the problem and co-designing the initial ideas not only enhances the value of the resolutions, but also yields intangible outcomes (i.e., motivation, empowerment, and connectivity) that are invaluable to the iterative PAR process as possible resolutions are instituted and explored further in the next phase of the project. 
The next steps for this project will be discussed further in the final chapter - Impacts for the Future. A few findings about the PAR process, in addition to findings about transitional care in the autism community were touched on above in the outcome summary. The next chapter will go into more depth and reflect on what was learned from this PAR approach as a whole, and from the hybrid approach that was used specifically in the process, and how those insights may serve as a provocation for further development and research. 


\section{Chapter 9: Reflections Derived From This Case Study}

This chapter is a reflection on the PAR process that was used to tackle the wicked problem of transitions in the autism community, unanticipated outcomes, improvements to the case study, the digital prototype and the hybrid approach for collective sensemaking and collaboration, and the expanding role of the Designer. Reflections into the process, as well as the tangible and intangible long-term outcomes generated from the case study, support the value of using CoDesign methods to elicit shared understanding, sensemaking, iterative exploration, and critical making to tackle wicked problems. Reflections on the discoveries made through the development and testing of the digital tool prototype in parallel with the case study suggest that there may be value in using a hybrid approach and conducting further exploration into technology applications for future PAR processes. Lastly, by the end of this reflection we will look at the implications of these research outcomes on the process of Participatory Action Research and the field of Design.

\subsection{Summarizing the Three Exploratory Themes}

Three themes were explored through a case study surrounding the wicked problem of transitional care and support for adult with ASD and their families. The three themes are described in general terms here, but will be reflected on in more depth throughout this chapter. The first theme centers on understanding the value/role of the Designer, the stakeholders, and technology in tackling wicked problems. The value of 'active' participants in CoDesign roles and the value of Designers as a framer in front-end stages of a wicked problem were explored through the case study. Additionally, the value/role of technology in the Design Thinking process was investigated during the project and in the development of a digital tool prototype for collective sensemaking. Through 
the construction of the digital prototype and the implementation of the PAR process in the case study, the expanded role of the Designer was demonstrated, as one that could give form to the problem (not just building solutions) through the development of seeing/maker spaces and sensemaking tools that engage people in new ways of thinking, seeing, and interacting.

The second theme concerned collaborative group dynamics and methods to improve collective sensemaking around a wicked problem. Various Design Thinking methods were explored, along with a digital tool prototype, in an effort to identify key elements in the PAR framework that could enhance engagement and helped to build seeing/maker spaces that influence connectivity, empathy, information transparency, and creative thinking among Designers and stakeholders.

The last theme focused on understanding the affordances of non-digital and digital tools for the PAR process in tackling wicked problems. The potential for a hybrid toolset in future PAR sessions was explored through the development and introduction of a digital tool prototype. Digital tools have the potential to engage people in new ways of communicating, interacting, creating, and negotiate decisions collectively. Additionally, technology applications can be used by Designers in the Design process to gather, communicate, organize, curate, and store qualitative research data. Lastly, an interactive tool can also be used to immerse non-designers in the design synthesis process, allow people to simulate multiple situations, and extend understanding and decision-making abilities across time and space. Nevertheless, this case study revealed that though technology may offer some benefits and engage people in different ways than paper-based methods and face-to-face discussions, digital tools alone should not take the place of non-digital methods and tools in the CoDesign process. 
The value in using a hybrid approach in PAR, along with a reflection on the non-digital and digital methods and tools used in this case study, will be analyzed in more detail in the sections that follow a reflection on the PAR process as a whole.

\subsection{From PD to PAR}

The multi-faceted problem of transitions and adult care/support faced by adults with autism, their parents, and healthcare providers/administrators, is wicked. There is no single right solution, but instead a need to iteratively research and tackle the problem from many angles with the people

who will benefit most from transformative improvements to the current system through CoDesign. This indeterminate problem presented us with an opportunity to not seek one answer, but instead to actively seek new understanding, to reframe the scope of the problem, and to re-imagine a better future for adults with autism and their families through the design of new resolutions. The value of recognizing indeterminacy can bring people away from only seeing a problem as a means to an end, and instead an opportunity to gain new understanding and see something completely new. The value of bringing stakeholders into CoDesign roles is that the normative lens of the Designer and each individual stakeholder is broadened to allow new insights and connections to emerge, along with inciting motivation, empowerment, and buy-in from the people the problem effects most.

This case study began with a Participatory Design (PD) approach aimed at bringing integral stakeholders together to co-design new resolutions for the clinic. The participatory approach, initially intended to guide stakeholders in sharing needs and co-designing tangible resolutions, resulted in intangible outcomes and an approach more akin to Participatory Action Research (PAR). The approach that was carried out is depicted in Figure 9.1. Through the active participation of various stakeholder groups, our focus shifted toward knowledge creation, iterative problem solving, and action planning built up over the course of the three phases. While many 
ideas were generated, the process of iterative learning, reframing the scope/approach of the wicked problem to create true value, and empowering the CoDesigners became more valuable than the tangible CoDesign outcomes alone. The goals the participants laid out for themselves and for our collective team in the pre-assessment questionnaire centered on gaining more knowledge in order to act and improve the lives of others. Therefore, the framework of methods and tools was redirected to generate shared learning, shared knowledge and greater understanding among the diverse group of stakeholders, so that we could actively target their true needs within this wicked problem. The outcome of the process is new understanding, near-term and long-term ideas, and co-designed visualizations and concepts, which can be carried into the next stage of development.

\section{The C.A.S.T. Case Study Framework}

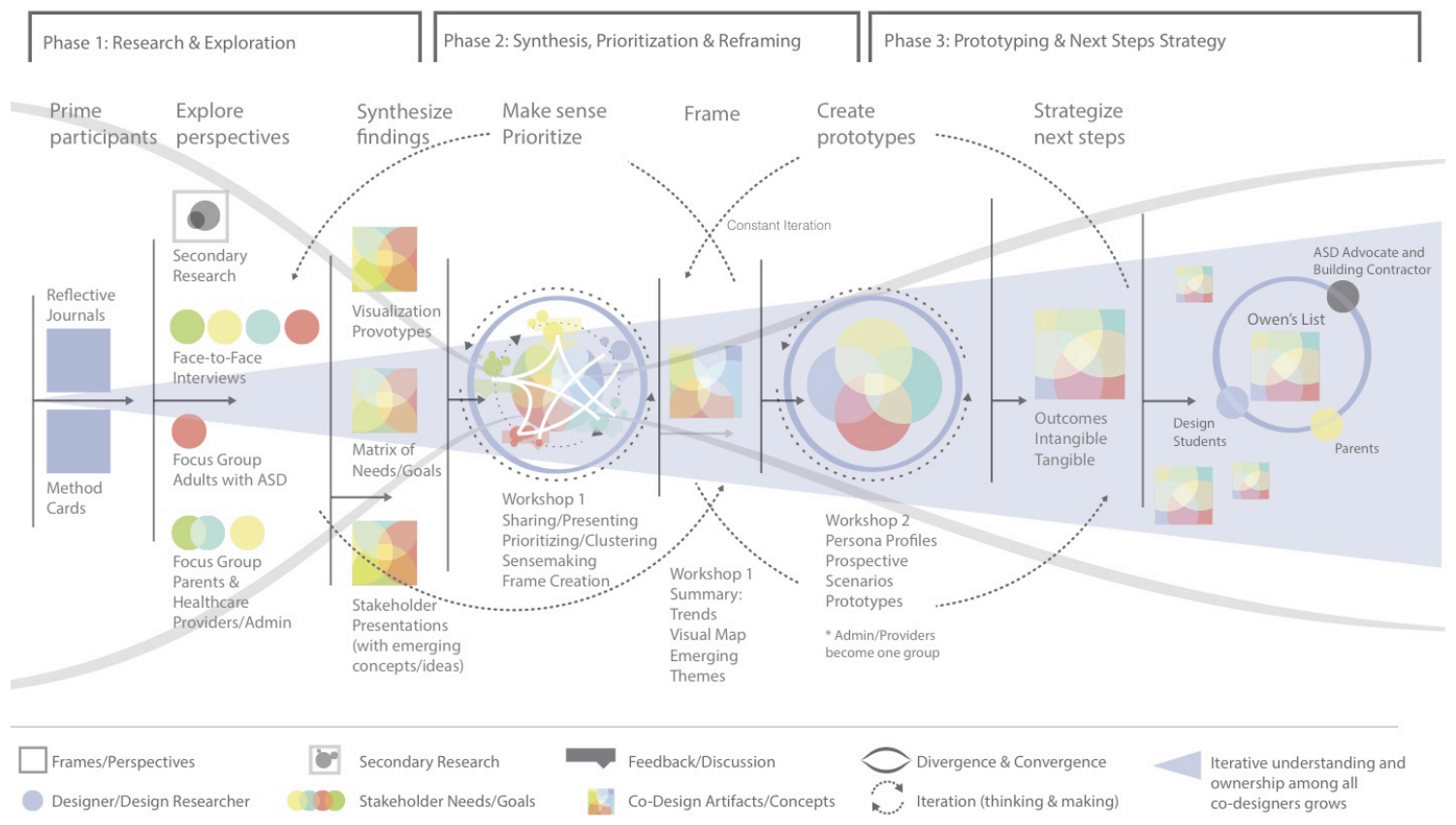

Figure 9.1 A diagram of the PAR process carried out in this case study. 


\subsection{The Problem Solving Process - Iterative Abductive Reasoning}

Design problems are often addressed through abductive reasoning, and unlike deductive and inductive reasoning, the outcome we seek is not in determining a result, but instead in creating value. In the equation proposed by Kees Dorst (2011) below, this form of reasoning allows Designers to remain open in redefining the 'what' and the 'how', in order to discover new possibilities.

?? WHAT (thing) + ?? HOW (working principle) leads to VALUE (aspired)

Given the nature of our wicked problem, CoDesigners were led through abductive reasoning exercises to widely explore multiple perspectives and to imagine new possibilities, in order to collectively frame the problem. The 'what' was generated through individual/group discussion and making exercises during Phases 1 and 2, while the connections between 'what' and 'how' were established during the sensemaking session in Workshop 1 and during persona/prospective scenario development in Workshop 2. These activities deepened our understanding, widened our appreciation, and led to the development of a new frame that encapsulated needs and goals extending beyond the clinic walls. New possibilities and the 'value' we sought to create evolved as 'what' needs and goals were reprioritized during sensemaking and 'how' ideas were generated throughout the sessions. Ultimately, our abductive reasoning process was iterative (Figure 9.2). The initial 'value' we sought was not solidified in the equation once the framing process began. As new knowledge was shared and the 'what' and 'how' were redefined through the PAR process, our original 'value' was replaced by a new 'value' we were seeking to create. 


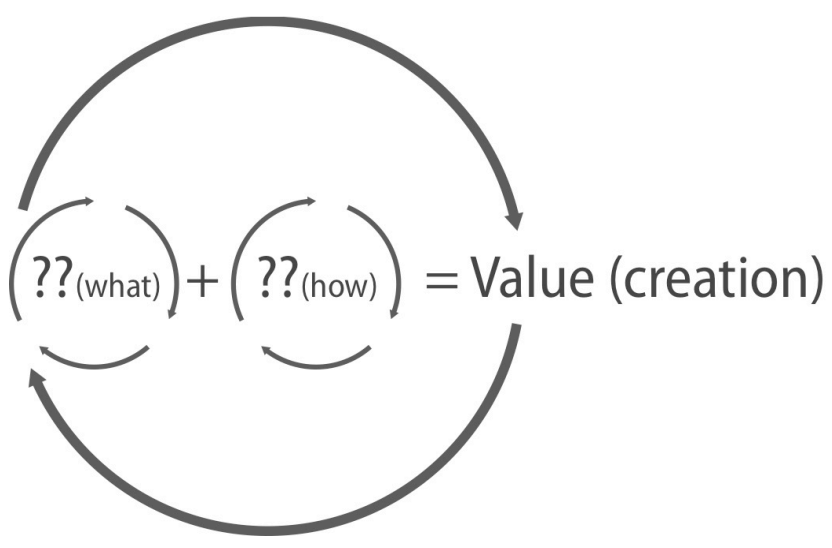

Figure 9.2 An Iterative Abductive Reasoning Model.

The initial 'value' we started with related directly to providing optimal care in the C.A.S.T. clinic and making it sustainable. The new 'value' we sought to create (after Workshop 1) had more to do with ensuring the well-being of people on the spectrum and their families in all aspects of their life and helping them to achieve their full potential. 'Value' shifted from a focus on the needs of the healthcare institution, to servicing the long-term needs and goals of the people in the system.

\subsection{The Construction of the Framework for this Case Study}

The framework and hybrid toolset proposed in this thesis aimed to support the creation of meaningful collaborative experiences by immersing the stakeholders into the wicked problem and bringing them into inclusive CoDesign roles. Using a blend of non-digital and digital methods and tools, the process was designed to assist the Designers and stakeholders in pinpointing root problems, identifying common and distinct needs/goals, understanding macro and micro aspects of the complex issue, and defining the true 'value', in order to generate next steps and more purposeful resolutions. The physical spaces and hybrid approach that were designed into the three phases of the case study were influenced by secondary research findings. The intent was to reduce cognitive uniformity (groupthink), production blocking, and social inhibition, while promoting new forms of engagement and 'seeing', more transparent communication, democratic 
interactions, and stronger personal connections among participants and the information being shared. Each method and phase of the case study were purposefully designed to build onto the next to deepen the team's understanding and creativity, while continuously promoting a sense of connectivity, understanding, involvement, and social interaction.

\subsection{Reflections on the Methods and Tools Used in Each Phase}

New understanding among the stakeholders in the context of the larger system emerged in seeing/maker spaces that encouraged the different stakeholders to connect and cross-pollinate their knowledge through a variety of making and telling methods. These encounters and interactions through methods and tools at a micro level brought about an emergence of new understanding and new concepts related to the wicked problem that could have a large impact on the ASD community. Most of the methods and tools used in the phases of the case study were new to the participants. They were used to support various creative strengths and communication preferences, as well as to challenge the participants to engage in new ways of thinking and interacting with each other and the information. The contributions of CoDesigners in sensemaking, problem solving, and concept creation helped to enhance and support our design efforts to uncover true 'value' opportunities. By leading stakeholders through new methods of problem solving and providing new forms of communication and collaboration, beyond surveys and questionnaires, the 'experts' found new ways to express their thoughts and ideas and provided useful and meaningful information to take into the next phase of development.

\subsubsection{The Questionnaires}

The Pre and Post-Assessment Questionnaires were useful in measuring the participants' changes in knowledge/understanding about the wicked problem, as well as their feelings about Design Thinking, a healthcare center for adults with ASD, and collaboration around complex problems from the beginning of the project to the end of the project. The questionnaires also 
helped to show which goals the participants were aiming to achieve at the beginning and which goals were met through the project. However, some of the questionnaires were not completely filled out. In the future, to ensure both sides of a questionnaire are filled out, surveys should not be printed on the fronts and backs of a page. Additionally, unique instructions (i.e. draw a square around your original ratings) should be made more apparent, so that critical information is not overlooked or lost on the surveys.

Two feedback questions and a Reflection Survey were sent out after the project to gauge whether the project had met the participants' expectations, and for the participants to review the methods/tools that had been used in relationship to the outcomes we had achieved. More responses on the feedback questions and survey would have helped to determine how effective each method/tool was in helping them understand the problem and think creatively, and would have provided more insight into possible revisions for the framework. The survey, in particular, was long and may have deterred some participants from taking time to fill it out. A better approach may have been to have a round table discussion about the specific methods/tools and overall framework with the participants in person during the reflection period at the end of Workshop 2. Alternatively, had the survey been developed through an online format with 'clickable' features, instead of written responses, the survey may have been simpler to use, more efficient to fill out, and may therefore have generated more responses.

\subsubsection{Phase 1}

The Method Cards and Journals, along with stakeholder interviews and focus groups, were introduced early in the process (in Phase 1) to give the participants an opportunity to prepare themselves and reflect on their own thoughts/ideas prior to collective sensemaking and generative design in Workshops 1 and 2. These methods and tools appeared to not only help the 
stakeholders learn more about Design Thinking and prepare for the sessions, but also provided more knowledge about the players and the different facets/perspectives of the wicked problem.

\subsubsection{The Journals}

The Journals were printed on 11"x17" cardstock paper and folded into a booklet for ease of use to guide participants through preparation and reflection on an individual level throughout the project. Only one participant agreed to hand-in her Journal and present her 'ideal' future (on the back page of her Journal) at the beginning of Workshop 2. The other participants refrained from sharing their Journals, so their Journals were not collected at the end of Workshop 2. Therefore, it is not clear how many people used the Journals and whether or not the Journals were useful tools of preparation and reflection during the case study. The participant who did present her thoughts found the Journal useful because it supported a familiar approach she uses to resolve problems. She explained in her Reflection Survey that, "putting thoughts into words is my go-to problem solving method and helps me organize my thoughts. "Knowing this, it may be useful in the future to offer more than one tool/resource to prompt participants in organizing their thoughts and expressing themselves. For instance, a Journal app could be used to allow participants to contribute thoughts/ideas through text or photos more readily. Journal apps on a phone connected to the person at all times may remind them to contribute and to seek out what others are saying/thinking on a shared network. Responses could remain private, or be shared in a private group. What was lost with the paper Journal was that the other participants were not able to share their reflections as easily. It also required that the participants pause to sit down and write responses to questions with pen and paper. This can be a useful approach to pull people into a reflective state, however, it may also deter people from taking time to partake in the activity. In a world that is increasingly digital this may not have been the most comfortable or natural approach for recording ideas/thoughts. Giving participants an opportunity to share their reflections and ideas throughout each phase with a paper Journal or a digital Journal may contribute to more 
iterative understanding and creative thinking, along with continuous shared understanding between sessions.

\subsubsection{Method Cards}

The Method Cards appeared to act as educational tools, helping to increase the participants' awareness of the methods Designers use to resolve problems, while also preparing them for some of the methods we would be using. The cards also served as provocative tools, prompting participants to discover and practice new problem solving approaches. The two participants that responded to the Reflection Survey revealed that the Method Cards contributed a great deal to their overall understanding of the topic and to their creative thinking during the case study.

"Having the reference cards helped me look at the various autism problems/situations in new and different ways." The cards and Journal were printed on cardstock and packaged neatly together in a plastic folder so they could be used as reference tools for future use. The plastic folders with the Journals and Method cards were brought by many participants to each session.
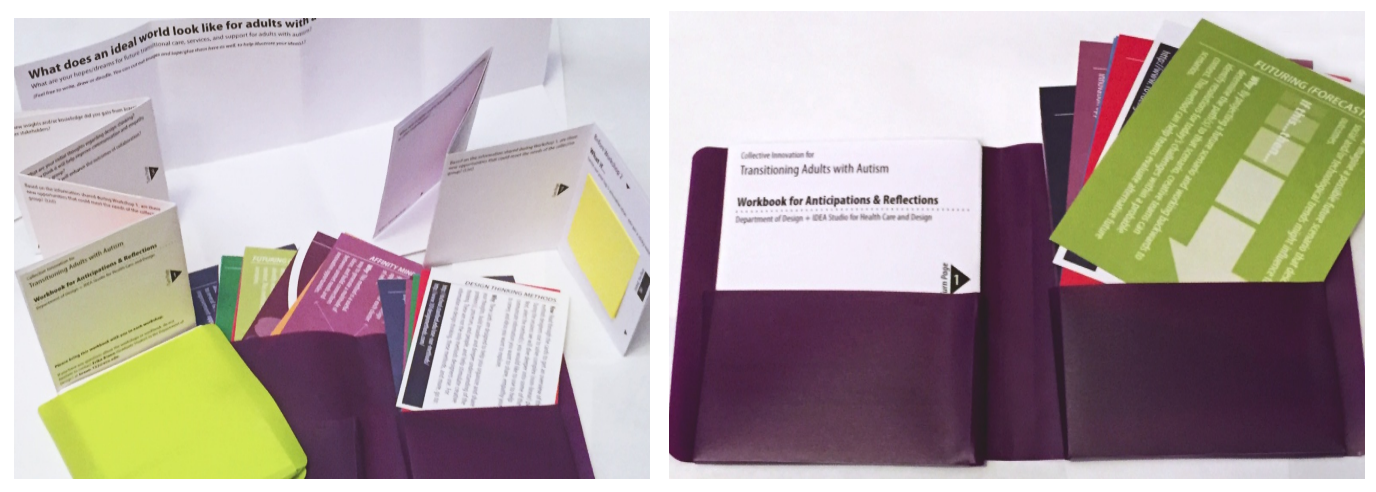

Figure 9.3 Images of the Method Cards and Journal booklet with their plastic folders.

\subsubsection{Interviews and Focus Groups}

Face-to-face interviews and provocative visualizations (that could be readily amended or changed during our discussions) were useful in stimulating conversations, helping individuals express their 
thoughts in a 'safe' space, and provided them with the satisfaction that their needs/goal would be represented in the collaborative sessions. Small focus groups demonstrated the value of bringing similar and dissimilar participants together (face-to-face) to not only express their unique frames, but to also broaden their normative lenses. One of the outcomes that had the most impact in the focus group with parents and healthcare provider/administrator was the realization that their needs and goals were more aligned than dissimilar. The use of sticky notes to express and present concerns, needs, and goals on a shared surface, along with open discussion among the different stakeholder groups, led to this new insight. Hands-on tools and open discussions were also valuable in the focus group session with participants on the autism spectrum. The Make toolkits and open conversation stimulated suppressed thoughts and sparked new ideas, which ultimately contributed to the growth of new possibilities beyond clinic problems. The interviews and small focus groups also allowed the Designer to get to know the various players, empathize with their perspectives, understand the wicked problem from multiple angles and prepare to facilitate the workshops by building stakeholder presentations and curating information for the sensemaking activity.

\subsubsection{Phase 2}

Phase 2 was intended to build shared understanding and convergence around the wicked problem prior to building concepts. Workshop 1 was instrumental in bringing together stakeholders who had not had the opportunity to collaborate around this wicked problem. The seeing/maker spaces and the approaches (methods/tools) that were used gave each stakeholder group an opportunity to voice/represent their thoughts and feel heard. It also gave them an opportunity to listen/see new perspectives and thoughts they had not considered. The visualizations in the presentations and on-the-wall mapping exercise also helped to bridge communication gaps between the stakeholders, promoted shared understanding, and prompted the team to work together towards a common goal. 


\subsubsection{The Presentations}

The presentations at the beginning of Workshop 1 were designed to promote spoken communication, stimulate new understanding and empathy among the stakeholders, reduce social inhibition, and invite more democratic interactions. The presentations were held in a shared space, around a large round table, and given by representatives from each of the three stakeholder groups (parents, people on the spectrum, and healthcare physicians/administrators). This exercise physically brought all of the stakeholders together to listen and share various positions on the topic, inviting them into more meaningful, open conversations. By allowing each stakeholder group to have an equal voice early on, the participants were given an opportunity to contribute to the collective discussion using their expertise (strengths on a certain perspective/topic) openly and without fear of negative evaluation, while also gaining new knowledge in the process. Participants spoke highly about the presentation approach in Workshop 1, revealing that it was one of the most valuable methods.

"This was very powerful." - Healthcare Provider

"I was very encouraged by he unanimity in the groups." - Parent

Additionally, cognitive intersimulation (a favorable aspect of groupthink identified in Lamm and Trommsdorff findings) emerged during the Workshop 1 presentations, as it had in the focus groups. The unique experiences (told through personal narratives) and visual diagrams in the presentations appeared to provide a common language for the participants. Needs, goals, and ideas shared during each presentation also allowed the CoDesigners to connect with the topic and to each other. Each group had an opportunity to express how the wicked problem affected them, while allowing open feedback and communication by other stakeholder groups to feed off of the need/idea or ask for further clarification. The personal narratives, in particular, had a profound 
impact on relatablility and shared understanding, as stakeholders felt comfortable augmenting certain issues or ideas brought up by the presenting stakeholder. Often, stakeholders would expand on the unmet need or the idea that had been proposed. This helped to spur many of those ideas and unaddressed needs further during the sensemaking activity in the second half of Workshop 1 and in some of the issues/resolutions that were explored in Workshop 2.

\subsubsection{Sensemaking}

The CoDesigners in this case study were immersed in a sensemaking activity that incorporated blended interaction. The second half of Workshop 1 was tailored to helping the stakeholders make sense of priority needs/goals, establishing relationships among those needs/goals, and reframing the wicked problem around the more pressing unmet needs and opportunities related to care and support in transitions and adulthood for people with autism. The multi-touch digital prototype, as well as the whiteboards and sticky notes in the seeing/maker space provided the means through which the CoDesigners and Design Researchers could actively engage with one another, make sense of the multi-faceted problem, and together reframe the wicked problem.

The needs and goals (shared and collected prior to Workshop 1) had been transformed into individual topic nodes on the digital screen and pre-written onto the sticky notes. The data on the touch screen and sticky notes was manipulated, reorganized, and prioritized by multiple participants simultaneously. The only 'written' aspect were the addition of new cluster titles and lines drawn with whiteboard markers on the whiteboard wall to explain connections and relationships between the topics and clusters. The aim with the digital tool and pre-written sticky notes was to create a more level playing field, promote more fluid engagement and democratic interactions during the sensemaking session. These tools encourage the CoDesigners to look beyond their priorities and work together on a shared canvas towards a common goal. The interactive multi-touch screen allowed multiple users to synchronously communicate and 
manipulate information, and make decisions verbally and/or visually through changes to the organization of needs and goals on the shared surface. Additionally, those interactions and decisions were projected on the large whiteboard for others to see at a distance, while also being captured and stored through a series of screen shots that could be compiled into a video for later review.

\subsubsection{Sensemaking with Technology and Sticky Notes}

This case study showed that there are benefits to using both sticky notes and digital tools for sensemaking. Sticky notes, often used in brainstorming sessions, fall between paper tools (like MessMaps) and Digital Tools (like the one created in this case study). While they afford the flexibility of sharing and rearranging multiple ideas and issues in a colorful and interactive way, they lack certain features like storage, scalability and traceability that technology can offer. The information/decisions shared on paper (even sticky notes) are often relegated to the activity that occurred in the singular time and space of the session, and therefore have to be photographed and recorded for future reference. Sticky notes used in brainstorming can promote periods of heads-down silence while writing ideas until people are asked to vocalize their ideas. Sticky notes in the case study were pre-written with information that had already been shared in order to avoid individual ideas and heads-down silence. The same data on a shared digital surface can be projected and enlarged, so more people have the opportunity to interface with the data and the choices that are being determined.

Technology has the ability to make collaborative expression more accessible and change the way people engage with each other and information. The digital prototype allowed more than one person to work on the same canvas, stimulated curiosity, contribute to more intentional and shared decision-making, and allowed others to view the information from a distance (with the projector and through a video recapture). During the play-test of the final digital prototype with the 
students, the shared, large digital canvas appeared to perpetuate active discussion, transparent communication, and debate, along with creative thinking as new ideas and needs emerged.

However, the failure of the digital tool during Workshop 1 was a good indicator of the limitations and unreliability of technology. In the future more play-testing and time needs to be factored into preparations for digital tools to ensure bugs are repaired. Nevertheless, while the failure in the program demonstrated the unreliability of digital technology, it also allowed new findings and functions to be uncovered - failure became opportunity. New ways of more readily bringing in various data sets and interchanging stakeholder icons were developed as a result of fixing a glitch that had been overlooked. Additionally, the use of paper sticky notes helped to demonstrate the true actions a team of CoDesigners would use to frame a problem, uncovering the needs for additional functions, such as automatic (or suggested) clustering, moving grouped items together and assigning groups new names. Had the participants been limited to the current functions on the digital prototype, these discoveries may not have been made. In the future, sticky notes may be a useful tool in pilot testing new, collaborative digital tools in the early stages of development.

The digital prototype sparked engagement and interests in some of the stakeholders to interact with the information. This same enthusiasm was not as apparent when we used the paper-based tool. The participants that were interested in the digital prototype (including all three adults on the spectrum) appeared willing to dive in and play with the digital interface and the data. One of the participants on the spectrum and a provider were able to prioritize their individual topics simultaneously on the digital tool, while another provider stood around the touch-table asking about the functions and capabilities of the prototype. One participant reported after the workshop that they had "never participated in this type of workshop before. I enjoyed learning about this process and especially the use of technology to find communalities between the different stakeholder groups." 
The digital tool seemed to engage participants in a different way than the sticky notes. During the sticky notes session, interactions were mostly handled by the Design Researchers while the stakeholders provided verbal guidance on the locations of sticky notes, new cluster titles, and connections among the topics. For the participants who had interacted with the digital screen, there appeared to be a behavior shift from active to passive participation when we migrated to the sticky notes. This may have been for multiple reasons, for instance, time limitations (as it was the last stage of the workshop and time was expiring), the scale of the space in relation to the number of participants may have prevented them and the other participants from feeling comfortable coming close to the wall of sticky notes, or familiarity with sticky notes may have reduced their interests while unfamiliarity and curiosity with the touch screen may have peaked their interest to interact. It is important to note that not all participants inquisitively approached the multi-touch table, some held back. This may have been for one of two reasons: one, that the notion of technology and playing with a new tool was intimidating, or two, that there was not enough room around the table for each person to participate equally on the table.

The insights from this phase of the case study reveal that there is value in ensuring that both digital and non-digital tools are made available to CoDesigners, and supports the idea that there may yet be potential for technology in the PAR process. These reflections further suggest that the size of the group and the diversity of work styles become important points to contemplate when designing tools and seeing/maker spaces for collaborative teams in the future. The value of diversifying the type of inputs and tools for contribution was seen in using both digital and nondigital tools. Sixteen stakeholders attended Workshop 1. While this can be a large number for collaborative sessions, there was value in having that many CoDesigners. In planning for Workshop 1, it was important to have enough representatives from each group (especially providers and administrators, not only from OSUWMC, but also from $\mathrm{NCH}$ ) to ensure that we were covering various stages of transitions, hearing a variety of perspectives, and expanding our 
lenses so we could identify the true needs of the system and root issues during convergence and sensemaking. However, during the sensemaking activity, many people stood back from the activities occurring on the touch-screen/projector and the whiteboard. Finding alternative ways to keep larger groups actively engaged will be key in future collective sensemaking sessions.

\subsubsection{Phase 3}

Workshop 2 had a total of eleven participants representing each of the three stakeholder groups. This worked well for dividing them into two groups for the generative exercises. The methods used in Workshop 2 (persona creation, prospective scenario development, and low fidelity prototyping) helped to put the wicked problem into a human context and prompted participants to speculate about the future. Teams made up of parents, people on the autism spectrum, and physicians immersed themselves into the personas and prospective narratives they were creating. They interacted with one another and shared knowledge from earlier phases, as well as personal experiences to more deeply, and broadly, explore current problems and future possibilities. Ultimately, in creating personas, journey maps, and prototypes together through a shared experience, the participants appeared motivated and empowered to carry out the concepts they had prototyped.

\subsubsection{Developing Personas and Scenarios}

The personas and scenarios they created pushed the participants deeper into the new frame we had established in Workshop 1. A toolkit with paper shapes, images, headers, and markers allowed the stakeholders to quickly create their personas and convey their narrative of unmet needs and potential opportunities on the posters and along a timeline, stretching from now to a more favorable distant future. These activities prompted them to use new tools to look at the complex problem, from both a personal level and a macro level. Creating personas and scenarios in this session encouraged the stakeholders to negotiate priorities and determine 'how', and work 
together to stretch their imaginations and ideas for the future. One participant explained on the Reflection Survey that journey mapping was an "especially helpful technique as it required us to think through how the persona person would accomplish their goals." These two exercises appeared to support secondary research findings around the value of critical making and its ability to bridge the gap between physical creativity and conceptual exploration, and promote shared understanding and productive engagement.

\subsubsection{Prototyping}

Low fidelity prototyping was introduced at the end of Workshop 2. Paper, 2D shapes, markers, and a random assortment of 3D objects (such as LEGOs, dolls, and blocks) were offered to the stakeholders so they could begin to fabricate some of the ideas that had emerged in their prospective journey maps. The physical prototypes were intended to help stakeholders begin making their ideas more concrete, while also strengthening personal connections and empowering them to be a part of the resolution. It is important to note that for some, the idea of prototyping was confusing and intimidating because they had not had experience constructing physical representations of their ideas. Feeling that they lacked creativity made them reticent to build, at first. There was a wide variety of prototyping materials to choose from, however, the diversity of the generative toolset may have helped to inspire those that were not sure how to begin. One of the participants with autism went straight to the LEGOs, while the parents felt paper would be the best tool to use to work through the screen functions/navigation of Owen's List. A healthcare provider and parent who were most outspoken about their perceived lack of creativity, soon found inspiration in the miniaturized people and a doctor puppet. While too many options may be paralyzing, a variety of different tools for different strengths may spark wider imagination and engagement, and contribute to new ways of exploring the resolution. 


\subsection{The Value of Telling and Making Methods}

The methods that appeared to contribute most to the participants' understanding of the topic and creative thinking were those that gave each individual an opportunity to state their case, listen to new perspectives, and generate new understanding and ideas through shared making experiences. These included mapping similarities and differences between the parents/providers in their focus group. A parent shared in their Reflection Survey that "parents often feel they're on an island." Mapping during the focus group showed her how "similarly the different stakeholder groups rated needs and concerns." Furthermore, the methods that empowered individual stakeholders to voice their perspectives provided the means through which new insights and new understanding could be achieved, in order to discover and create meaningful 'value'. The same participant commented that "hearing the other individuals' and groups' perspectives was essential to reaching shared understanding and prioritization."

Methods and tools used within this PAR approach that stimulated open conversation, shared making and telling contributed to a more democratic collaborative atmosphere and increased empathy and understanding, while also empowering people to speak that may have otherwise been overlooked. When asked if the participants felt they were heard and whether the group valued your opinions/thoughts/ideas, a parent responded on the Reflection Survey, "Yes. Due to near-constant advocacy for our ASD kids/adults, parents often feel like everyone else (providers, schools, social workers) are on the other team (e.g., in most IEP meetings there is one parent and at least 8 administrators on the other side of the table). In these workshops, it felt more like every person's input was valued and welcomed." Furthermore, a healthcare provider responded in the feedback questions that they were "pleasantly surprised to see the involvement and participation of the parents and self-advocates. The self-advocates brought a lot to the table as did the parents - especially in bringing the perspective of individuals with ASD with more significant cognitive and communication deficits." 
In addition to methods that facilitated open discussion, methods that were visual or hands-on appeared to help participants see relationships and make connections among themselves and the qualitative data. Visualizations, along with building prototypes with other stakeholders were highly regarded and valued in the Post-Assessment Questionnaire. Creating visual maps (with sticky notes), personas, scenarios, provotypes, and prototypes all fell into this category. For one of the participants who responded to the Reflection Survey, prototyping with other stakeholders was the "most meaningful part of the entire process." The same participant also shared after the workshops that she wanted to use personas in the future to better communicate unmet needs and disruptive ideas regarding this population. Not only did interaction with a new method (personas) in the project help her gain more insight into the issue of transitions, it also helped to promote the value of this method in the Design process.

\subsection{The Value of Visualizations}

"If you want to change how people think, you first have to show them how they think" (Horn \& Weber, 2007).

The value of pictures in helping people with ASD understand and communicate information was shared by our ASD participants and parents during the presentations. Furthermore, visualizations used to tackle complex problems were valued by most of the participants in their responses to the pre and Post-Assessment Questionnaires. Visualizations were used in this case study as provocative tools, tools for sensemaking, and communication vehicles through which the CoDesigners could find common ground and shared understanding. Wicked problems are complex. In this case study, visualizations helped stakeholders shape uncertainty and disparity. Through the use of provocative visuals, interactive tools, and mapping, participants had the ability to actively look at the problem from multiple perspectives and then gain control over the wicked 
problem through manipulation, prioritization, and reorganization of the needs and goals in the sensemaking process.

\subsection{The Value of Externalizing the Design Process}

One of the aims of this PAR case study was to integrate methods and tools into the process to externalize the synthesis process so CoDesigners could gain a new appreciation and understanding for the Design process. Kolko (2010b) provides three techniques for externalizing the synthesis process: reframing from a new perspective through empathy methods, making creative leaps and reframing based on personal sensemaking methods, and creating a shared sense of sensemaking through democratic dialog and diagramming tools. In this case study, reframing from new perspectives was garnered through the sharing of unique perspectives during the visual/verbal presentations, as well as through the development of personas. Creative leaps were made during the presentations (from personal experience to collective understanding), during the visual sensemaking exercise (framing), and during prospective scenario building. Lastly, unique perspectives and ideas were externalized on various shared surfaces throughout the case study, on brown paper with sticky notes during the focus group with parents and providers/administrators, on the multi-touch digital tool, on the whiteboard with sticky notes, and on large pieces of brown paper while collectively building personas and prospective scenarios. By externalizing the design synthesis and creative thinking process, stakeholders were immersed in a new way of problem solving, which invited them to engage with each other and the wicked problem in new ways. Through the process they gained an appreciation for Design Thinking and our unique thinking processes, reporting in their post questionnaires that they valued the approach and would like to use it again in future collaboration to tackle complex problems. The visualizations and the act of externalizing the design synthesis process (during the sensemaking activity) also had the added benefit of visibly linking research findings and problem framing to meaningful resolutions and outcomes. Through the added benefit of technology, interactions and 
decisions conducted in the workshop could be shared and examined by the stakeholders after the session. These design artifacts can be used in the future to convince new stakeholders about the value of the resolutions (and how they were derived), in order to catalyze action around this wicked problem in the future.

\subsection{The Addition of Technology in PAR}

As mentioned earlier, complexity sorted and mapped using paper tools, alone, can be limiting. A digital tool is not limited to real time experiences but experiences that can be shared, reversed and redesigned (iterated) across time and space. Technology provides us with the ability to collect and store a lot of data, in addition to providing the flexibility to interact with data in multiple ways. The ease and flexibility of a digital tool allows users to play in the future by simulating various outcomes and experiences. The aim in developing a digital tool prototype in parallel with the wicked problem case study was to identify opportunities where technology could enhance the collective Design process and the engagement of the participants, and expand the capabilities of the PAR process.

Precedent research demonstrated that blended interaction (combining human interaction with digital technology) supported more holistic understanding, individual interaction, social interaction and communication, and greater workflow in a co-located, collaborative environment. This research, along with experience in the Design field, informed the initial features/functions of the digital prototype, while new insights in the case study and the development of the prototype led to a more meaningful prototype. Blended technology integrated into the PAR process helps stakeholders and Designers visually identify and interact with data across multiple perspectives and collectively draw connections and relationships between topics on a large public surface. Technology also provides a platform through which they can gather, organize, simulate, and store large quantities of data (and real-time interactions). 
It was evident in early interviews that the digital tool prototype needed to help stakeholders converge first to better understand various needs/goals within the wicked problem and how their needs/goals related to one another's in the same network of transitions, rather than simply providing an online platform to share a multitude of ideas. In discussions with the stakeholders there was an apparent lack of shared understanding, a disjointed hierarchy of needs/goals, and a lack of consensus around the value they were seeking to create. The vision for the digital tool evolved into one that could help CoDesign teams uncover relationships and key innovation opportunities through a shared external synthesis process. Additionally, CoDesigners needed the ability to reorganize and prioritize messy data and simultaneously share and record ideas as new connections and unmet needs were addressed. Interactions with the sticky notes in the workshop demonstrated the value of being able to perform these functions. As a result of the case study and development of the tool, the final digital prototype evolved to incorporate features that would capture and store information and interactions, immerse people into the process of synthesis, and help them to create and communicate a new frame.

\subsection{Designing Seeing/Maker Spaces and Tools for PAR}

The sections below reflect on the findings related to the design of 'seeing' spaces and Design Thinking tools, not to push CoDesigners in a specific direction or give them tools only for 'making', but instead to help them express what they know, think, and feel, and provoke them to look deeper and 'see' the problem differently. The spaces and tools developed for this case study were designed with intention to encourage collaboration, open communication and shared making, diverse and innovative thinking, and most of all, deeper understanding. The design and selection of seeing/maker spaces can contribute to the effectiveness of the PAR session. The collaborative environment and the tools offered in the space plays a role in either facilitating engagement or distraction, and preventing or enhancing workflow. In this case study, environments that were familiar to the stakeholders, had low sensory distractions (i.e., sounds 
and lighting), and facilitated 'round-table' discussions, group activity and space to move around, on-the-wall surfaces for collective mapping, and opportunities to build ideas together had a positive impact on collaboration. It is important to take the design of the seeing/maker space, as well as the design of the methods and tools, into consideration when designing a PAR session for participants.

\subsubsection{Designing Seeing/Maker Spaces for PAR, Not Just 'Maker' Spaces}

Collaborative spaces benefit from a hybrid set of tools and methods that stimulate new ways of seeing and learning, making, and sharing information. Instead of offering tools only for constructing solutions, seeing/maker spaces should provide tools for prototyping, synthesis and sensemaking so teams can gain a deeper understanding of the problem and seek out new ways of seeing data and constructing alternative possibilities. The unique benefit for using a multi-touch table for sensemaking and collaboration is that it affords an assortment of individuals the ability to manipulate information and 'make' something new. The design of the digital prototype centered on exploring new ways to bring people together to independently express thought, while also prompting them to collectively see multiple points of view and share new ideas. Physical tools like sticky notes and generative toolkits were also integrated into the PAR process to provide CoDesigners with the ability to learn through critical and shared making. These hands-on approaches and the digital tool were intended to help the CoDesigners think differently and compel them to work together towards common goals. They were designed to enrich their understanding and their creativity, while also encouraging them to see inside, see across time, and see across possibilities, leading them to new discoveries, connections, and motivation. Future collaborative spaces should incorporate the offerings of a 'maker' space, while also providing opportunities for people to more deeply understand a problem collectively. 


\subsubsection{Designing Tools Around the Strengths of CoDesigners}

The tools in the seeing/maker spaces should support peoples' ability to effectively co-design, effectively express what they think/feel, and contribute to the Design process in a meaningful way. Depending on the specialized needs of an individual, group, or method, the Designer may need to customize/adjust the tools used in a seeing/maker space to maximize the potential of the CoDesigners. For instance, people on the autism spectrum with sensory sensitivities and limited cognitive and verbal abilities, may need to use more concrete, tactile tools (Grandin, 2009), or have instructions explained in more literal terminology so they can understand the task and be able to focus without distraction so they can participate in a valuable way.

The spectrum of people with autism is wide - the key is not in designing "autism friendly" methods and tools (Gaudion et al., 2015), but in understanding the strengths/abilities of your participants and offering a spectrum of tools that stimulate their creativity and allow them to comfortably express themselves and their ideas. LEGOs, for instance, were used by one of the ASD participants in Workshop 2 and inspired him to recreate and communicate his ideal future, as it related to social interaction and tolerance. For others, markers and paper were enough to spur them to draw new floor plans or create a paper prop (i.e., a resource guide). In the focus group session with people on the spectrum, generative toolkits with literal images related to healthcare and the clinic, in addition to abstract shapes and blocks, may have helped those that were struggling to communicate more effectively to express their needs and ideas through visual associations. People on the spectrum have an interest in (and are captivated by) technology and gaming. In our session it was no different - all three ASD participants in Workshop 2 approached the multi-touch table fearlessly and wanted to interact with the nodes on the screen. The spectrum of tool offerings can be boundless, but as with all other aspects of Design, the tools that are used or designed by the Designer in their extended role for seeing/maker spaces need to be selected and developed with intention. 


\subsubsection{Technology as a Tool for Communication}

Technology affords multiple ways to contribute. A digital tool could allow participants who are not comfortable speaking out loud to communicate and contribute ideas on a shared surface 'in private.' Additionally, a multi-touch table allows many simultaneous touches, affording CoDesigners the opportunity to work together or individually on a shared surface. One of the participants, who was out spoken in the focus group with other ASD people, chose not to attend the other workshop sessions. He struggles with verbal/face-to-face communication and has to 'prepare' himself for these interactions. After the focus group session, he wrote sent a long list of unmet needs he thought we should address in the following sessions (mostly related to patient/provider relationships), as well as possible resolutions. He felt more comfortable communicating behind a screen than in face-to-face interactions. His needs and ideas were communicated through the presentation given by John and Kristin who presented in Workshop 1. His added contributions (whether virtual or in-person on a touch screen) in other sessions would have added a great deal of value to the group's shared understanding. Perhaps, an added benefit or use for a digital tool in CoDesign is to act as a facilitator or interpreter, to help bridge the communication gap between a person's hesitation to speak face-to-face and their comfort/ability to type and share thoughts through a screen. Interactive visualizations, co-designed with people on the spectrum, may provide another platform through which they can self-advocate and help others "see"/understand their views. Future exploration could be conducted with digital technology in PAR sessions with people on the spectrum to explore this idea.

\subsubsection{Understanding Tool Design Through Digital Prototype Development}

The digital tool has been referred to as a prototype throughout the thesis because it is a physical embodiment of an idea and the means through which to learn and test new possibilities. Its value to this research is in provocation and in beginning to understand how technology can be used to enhance collective sensemaking and other facets of the Design process. Unity was the program 
selected for developing the digital prototype for multiple reasons, but most of all because it allowed the Designer to create custom rules and a more authentic prototype (beyond a clickable step-through). It allowed the users to explore and play with the tool in unexpected ways. Unity allowed the stakeholders to move data freely around the screen and reshape the relationships between the data nodes to construct new meaning. This is a critical feature a digital sensemaking tool should have, and perhaps in a similar way it is critical feature for CoDesign tools (digital or non-digital) in general. Tools in seeing/maker spaces should not direct or guide users towards a direction, but balance structure with flexibility, and task instructions with the freedom to explore alternatives, allowing people's creativity and sensemaking to shape the outcome.

The head of the IDEA Studio at OSUWMC responded to an early vision of a digital tool for Design Thinking in saying that he believed a tool for facilitating collaboration should be like a car. "I don't have to understand it to fully use it (or play with it)." This statement echoes Bret Victor's vision for a 'seeing' space, in which the users do not have to think about bringing information in, but instead the information is presented to them in a way that allows them to interact and play freely. On a much smaller scale than Bret Victor's 'seeing' room, the early digital prototype introduced in Workshop 1 was intended to act as a digital 'seeing' space to help facilitate: discovery, definition, design, and delivery of shared/new information in the PAR process. The final digital prototype has the potential to provide initial constraints (in the form of nodes with prior collected data) and rules of use (how a person interfaces with the different functions), while at the same time offering a flexible platform of play and discovery. The nature of the tool leaves room for interpretation, allowing users to transform the functions of the tool and the arrangement of the data to meet tailored needs and generate new meaning. 


\subsection{Unanticipated Findings}

In the Reflection Survey, participants were asked if there was something they had expected that had not occurred in the sessions. The participant that replied said, "no, actually more occurred than I expected." The following sections reflect on that same question in relation to tackling the wicked problem of transitions in the autism community, the approaches and the process that were used, the people that were involved, and the outcomes. In these areas there were expectations that shifted or changed and there were findings that emerged during the case study and development of the digital prototype that were unanticipated.

\subsubsection{More Than One Seeing/Maker Space}

The original intent was to create one 'seeing' space for collective sensemaking through the design of the digital prototype and the surrounding collaborative space of whiteboard walls and sticky notes in Workshop 1. Instead, multiple seeing/maker spaces were created throughout the phases of the project. The environments, methods and tools used for each phase were designed to engage the participants in various making and telling methods that encouraged them to see inside, see across time, and see across possibilities. Seeing/maker spaces were created on paper through visual provotypes, as well as in the methods and spaces used for the focus groups and the workshops (i.e. sticky note mapping, prototyping, and prospective narrative mapping). The provotypes allowed stakeholders to not only make visual representations of ideas, but to also see others' ideas and build on to them. The sticky note maps and presentations encouraged the stakeholders to see inside the problem and across possibilities. The act of creating personas, journey maps, and prototypes encouraged stakeholders to 'make' collaboratively, while 'seeing' inside the problem, across time, and across possibilities. 


\subsubsection{A Digital Tool for Designers and CoDesigners}

Another unanticipated discovery was that a digital tool for PAR could not only be useful for collective sensemaking with Design teams and non-designers, but could also assist Designers in synthesizing and curating the decisions and ideas that had been expressed and discovered in research. This opportunity presented itself while using the digital tool to not only organize and share forty-eight collected needs and goals, but in recreating the actions that had taken place with sticky notes in the sensemkaing activity. A digital tool may allow Designers to externalize the CoDesign process by helping them visualize and synthesize decision pathways and insights. The digital tool in this case study was used to organize messy data from Phase 1 in preparation for sensemaking in Workshop 1. It also helped to communicate and retrace the decisions that were made and the findings that were uncovered in Workshop 1 to show our train of thought - from priority needs to opportunities for meaningful change.

\subsubsection{Multiple Tools Used to Externalize the Design Process}

An aim in developing the digital prototype was to see if, through immersion in design synthesis, CoDesigners might gain a better appreciation for the value of the Design process as something more intentional than "magic." In the Post-Assessment Questionnaire, all of the participants said "yes" that they would use Design Thinking methods in future collaboration projects. But, the digital prototype broke in Workshop 1. So what methods and/or tools helped to communicate the value of our Design approach? While the sticky notes took the place of the digital tool and helped to externalize the synthesis process on a large whiteboard wall, other methods and tools also helped to externalize and demonstrate the value of the Design process with the non-designers. In reflecting on the outcomes of the case study and reading responses to the feedback questions and Reflection Surveys it would seem that, in addition to mapping with sticky notes, the Method Cards, visualizations, persona creation, prospective scenario development, and prototyping contributed a great deal to the participants understanding and appreciation for our abductive 
thinking approach. Through immersion and interaction in these methods, and with unfamiliar tools (i.e., generative toolkits), non-designers were exposed to new ways to tackle the problem and found value in Design Thinking as a way to build understanding and design new opportunities in the future.

\subsubsection{Long-term Versus Near-term Focus}

Similar to the participant who expressed in the feedback questions that he believed "we would be doing a lot of 'design of space' interventions," the original objective for this project was that the CoDesigners would be prototyping and proposing resolutions to improve the clinic experience. However, a shift occurred and our focus changed; long-term goals and disruptive innovation ideas became of greater importance than near-term clinic needs/ideas. The unanticipated shift from clinic needs to helping adult patients and family members achieve wellbeing outside the clinic was a realization that was made during the presentations. Ironically, this insight was first voiced by the stakeholder group (providers) who had originally been the driving force behind the project (i.e., optimizing care/support offerings in the clinic). While both near-term and long-term ideas were collected and presented to the stakeholders prior to Workshop 2, all of the participants in the workshop chose to prototype big ideas and tackle unmet needs that had long-reaching, disruptive impacts, rather than those that were associated with small, incremental changes. Over the course of the project the 'value' we had initially been seeking to create evolved and changed, which leads into the next unanticipated finding.

\subsubsection{Iterative Abductive Reasoning}

Though C.A.S.T. is where we started, it is not where we ended up, because transitional care/support in ASD is a wicked problem. In a typical Design project, with a given scope and Design brief, the 'value' component of the Abductive Reasoning equation is the only thing that is a 'given.' However, because of the nature of the wicked problem in this case study and its 
connection to a greater networked system of people and issues, we discovered that in order to create true 'value' for the C.A.S.T. clinic, our approach needed to become iterative and our initial 'value' needed to evolve. In our Abductive Reasoning equation the 'value' component was an indeterminate figure. As new knowledge was shared and new understanding was acquired throughout the iterative process, a new frame and a new value was created.

\subsubsection{Intangible Outcomes}

In tackling problems using Design Thinking methods, the assumption was that prototypes and Design artifacts (i.e., persona posters, maps, and visualizations) would be generated, along with shared understanding and empathy. However, unexpectedly, this PAR process also resulted in the perception of social wellbeing, empowerment, motivation, and lasting connections between the stakeholder groups - intangible outcomes. After conducting the PAR sessions participants reported higher levels of understanding on problems specifically related to the struggles of adults with autism and their parents, which in turn led some to want to share what we had uncovered, as well as continue pursuing some of the resolutions that were proposed and prototyped. Rory Sutherland explains in his TED talk, (Life Lessons from an Ad Man) that intangible things (perceived value) are almost more important than tangible value (things). Intangible perceptions about an object or an experience can, at times, have a large impact on our behavior. Adding value in a wicked problem and designing a better human experience relies not only on creating new concepts (tangible outcomes), but in also creating a meaningful human experience in a PAR processes that is designed to expand understanding and encourage collective action. Sutherland says we need to "help people appreciate what is unfamiliar," and more importantly help them "gain a greater appreciation... on those things which are already existing," rather than just building new things. The parents and the providers had more in common (shared similar goals) than they had imagined as we learned in their focus group in Phase 1. Without methods and tools that 
allowed them to 'see' and build shared understanding, we may have begun designing "new things" for the clinic that were not addressing true problems.

Sutherland goes on to say that social networking can help us gain appreciation for the things which already exist, because they help people share news, even around trivial activities. The focus groups, presentations, and sticky note mapping in the case study had a similar effect - they provided a platform on which news could be spread and the team could collectively determine where focus should be placed, related to the wicked problem. Therefore, the value of the PAR process is not only in arriving at resolutions, but in helping people to 'see' the problem and each other in unfamiliar ways by bringing varied perspectives together and allowing them to play more integral roles in sensemaking, framing, and co-designing resolutions for a problem that affects them. The intangible (perceived) outcomes, such as social wellbeing and motivation are invaluable to a wicked problem that will benefit from continual iteration during the implementation and testing of new resolutions.

\subsubsection{Limitations of the Designer}

An initial assumption was that an extended role for the Designer in PAR would encompass building the scaffold for the wicked problem and being a cartographer, mapping the lay of the land related to the wicked problem. However, the case study suggests that this role may not be the sole responsibility of the Designer. While the Designer synthesized and curated qualitative research into 'maps' (i.e. visual provotypes and the digital tool) and provided the initial lay of the land, the information was presented in a malleable format. The 'maps' continued to be iterated and redesigned throughout the process. The PAR experience provided the means through which the CoDesigners could play a more valuable role in constructing meaning and helping the Designer to frame the problem. Landmarks (points of interest) were initially charted from different perspectives acquired in Phase 1 to stimulate conversation and provoke new/different ways to 
look at the problem in Phase 2, but no roads or hierarchy were drawn to guide stakeholders towards a destination. The 'maps' were given true meaning by the CoDesigners who reorganized the placement of landmarks, projected significance onto them through discussion, and built roads (connections) between their hierarchy of needs/goals to establish a new frame.

\subsubsection{The Value of the ASD Participants}

While constructing the framework and recruiting participants for the case study there was some uncertainty as to whether or not people with autism would be able to take part in the project. Interfacing with Nisonger and their Aspirations coordinators afforded the opportunity to engage with multiple adults on the autism spectrum and bring them into CoDesign roles. While only three adults on the spectrum continued through to the end of Phase 3, they each played an invaluable role in the PAR process. Their active participation and contributions through telling and making methods provided new insights and essential information. Before this session, providers and parents had not had an opportunity to listen to adults with ASD speak up for themselves and for

others on the spectrum. The unique perspectives they brought, as individuals with ASD, provided the collective with a new frame of reference and a better appreciation for what people with autism can contribute and want to achieve. This understanding led to the shift in our frame - helping people on the autism spectrum was less about caring for their 'disability' and instead supporting and nurturing their 'abilities' and 'specializations.' In doing so, we (as a society) can support a population that is often overlooked and give them the tools to help make our society better.

\subsubsection{Designers and People with ASD Think in Similar Ways}

"The really social people did not invent the first stone spear. It was probably invented by an Aspie who chipped away at rocks while the other people socialized around the campfire. Without autism traits we might still be living in caves" (Grandin, 2009, p.122). 
Another unanticipated finding that was uncovered in this case study were some of the similarities between people with ASD and Designers. Many people with ASD use pictures and patterns to make sense of information. "An autistic man who composes music...makes 'sound pictures'...a computer programmer...sees the general pattern of the program tree" (Grandin, 2009, p.16). Grandin's insight into the capabilities and thought processes of people on the autism spectrum mirrors what was observed in the sessions with the ASD participants, as well as what John and Kristin shared in their presentation. The ASD participants explained that creating new things, finding patterns and gaps that are often overlooked, and identifying new opportunities is an underutilized strength that many 'Aspies' have because they are highly sensitive to their surroundings and remain focused. Designers and Design Researchers are trained to think about problems and observe their surroundings in a similar way - seeking out opportunities that are often overlooked by people who are not detail oriented. Grandin (2009) explains that her "thinking pattern always starts with specifics and works towards generalization in an associational and not sequential way" (Grandin, 2009, p.16). Her thinking process, similar to the Design process is nonlinear. Through associations in research Designers identify patterns that help to inform frames of understanding. Through this understanding they can then begin to diverge and consider new ideas. In this case study the CoDesigners were led through a similar process. The Design field and the processes of PD and PAR may benefit a great deal from involving more people on the spectrum in not only co-designing resolutions for the ASD population, but in tackling other complex, societal problems.

\subsection{Improvements to the Case Study}

In reflecting on the overall process, the methods and tools used during the case study, and the outcomes of the project (intangible and tangible), there are changes that can be made, in addition to the improvements that have already stated, to enhance the effectiveness of future PAR sessions and future CoDesign sessions with ASD participants. 


\subsubsection{Involving More ASD Participants}

One of the questions on the Reflection Survey asked the participants to share things they would change about the session or the overall process. The response by the participant that answered the question was, "maybe having more ASD adults participate. There was such consistency from the parents' perspective (despite the range of severely-affected to more-independent ASD children) that I don't think having additional parents would have changed the outcome." Many of the other neurotypical stakeholders also spoke of the value the ASD participants, especially, brought to this project. Prior to this project, many of the neurotypical participants had not heard the perspective of this population. This project demonstrated the value of empowering those with a perceived 'weaker' voice to speak up for themselves and others. However, maintaining the number of participants we had over the course of multiple sessions became more difficult. Those that were at Donatos were tentative in their participation and did not feel comfortable moving forward in subsequent sessions. Along with improving the way explanations were given and activities were tailored to the participants in the focus group (to make them more comfortable with sharing), more effort could have been placed in getting in contact with their parents and staying in close connection with the Aspiration leaders. Initially, the parents of the ASD participants in the focus group were intentionally left out to encourage the adults with ASD to speak for themselves. However, the parents of two of the ASD participants wanted to be involved. The parents did not preclude their child from speaking or contributing. Instead, the parents were valuable in ensuring that their child stayed involved in the project through to the end. Many of the adults on the autism spectrum are dependent on their parents. John and Kristin were exceptions - they were more independent. Therefore, they were more inclined to respond to emails and get to the sessions on their own. The third ASD participant relied a little more on his parents. In the future, the parents of adults on the spectrum should be brought into the recruitment process, under the guidelines that their child not be spoken for, but instead speak for themselves and contribute independently. 


\subsubsection{Clearer Instruction}

The questionnaire responses and conversations with the participants in this project suggest that people (especially people on the spectrum) respond well to visual communication. Many of the ASD participants who were only involved in Phase 1 were uncomfortable speaking and/or did not understand some of our instructions. In future PAR sessions, especially with this population, direction and guidance may be clearer and better received by ASD participants if the instructions and the Design methods/tools also incorporate a demonstration or concrete example. The use of more concrete visual symbols and pictures on information sheets (questionnaires and instructions) may improve the effectiveness of these tools with people on the spectrum, and perhaps with neurotypical participants, as well.

\subsubsection{An Alternative Approach - Enactment}

A benefit of a digital tool is that it can help simulate multiple data situations and outcomes. The ability to play with various situations and compare cause/effect relationships invites people to iteratively tackle problems. This same idea can be explored through enactment and performance. Having participants enact various situations or ideas through bodily experiences and face-to-face interactions, along with creating visual provotypes, prototypes, and narratives, could provide another level of understanding in a wicked problem.

Enactment was a method that was not explicitly explored in this case study but it was used spontaneously by Team 2 in Workshop 2. Precedent research shows that methods and tools for enactment and roleplay can bring about transformative understanding, empathy, and meaningful connections (Kapchan, 1995; Brandt, Binder \& Sanders, 2012). Shared experiences through performance and roleplay encourage people to explore unfamiliar roles and situations, help to structure individual and group identities, and situate people in a shared space and time. If we were to take the notions of immersion and enactment and incorporate them into future PAR 
sessions for wicked problems we may find deeper empathy and meaning for different perspectives, beyond what we could obtain from only hearing someone else's story.

Enactment does not always have to be full scale (or full bodied), but can also be done in smaller scale with small props (Brandt, Binder \& Sanders, 2012, p.168). In Workshop 2, Team 2 used the 3D materials from the generative toolkit as props. LEGOs and dolls were used to explain a holistic future narrative of an adult on the spectrum with a job and a family who wanted to socially interact with others (adults on the spectrum and neurotypicals), and needed to stay connected to a support/care team to help him reach his full potential. Bringing full-scale enactment into the prototyping phases, among stakeholders with different perspective, may also help CoDesigners envision and test new possibilities, and increased their empathy for the unique situations (related to transitions in ASD) in which each group found themselves. Playing out unmet needs, situations, and new resolutions may also have helped to expose unmet needs or opportunities, and allowed stakeholders to more expressively portray and play-out current (or aspirational) situations. By involving various stakeholders in enactment, telling, and making methods in future PAR for wicked problems they may be able to further simulate the implications of the priority needs and new resolutions they are proposing for the system.

\subsection{Influence of This Case Study on the Design Profession}

At the end of her paper, Blair-Early (2010) poses a question pertaining to the design of future collaborative classrooms. For the purposes of this thesis the same question can be extended to future collaborative workspaces, "how can the use of collaboration, Participatory Design research and multidisciplinary teams encourage creative innovation and critical thinking....and...create more sustainable designed solutions?" (Blair-Early, 2010, p.217). 
The answer to the question lies in the evolving role and value of the Designer in shaping the PAR experience by giving form to seeing/maker spaces and tools that allow multiple perspectives to be shared and explored, and helping to giving form visually and interactively to complex data through which the collaborative team can navigate chaos and make sense of the problem space. The value of the new Designer in an expanded role is not only as a 'maker' but also as a 'framer' in the Design process. The new Designer plays a critical role as a facilitator in front-end strategy, which incorporates the activities of curation, interpretation, and helping to build the frame. In this role the Designer designs a sensemaking experience that empowers multidisciplinary teams to cross-pollinate and collectively take action.

\subsubsection{The Designer's Role in This Case Study}

The Designer's role in the PAR process is valuable. Guiding CoDesigners through Design Thinking methods requires a skilled understanding of the Design process and comfort in ambiguity to facilitate Design Thinking sessions around messy problems, and the ability to challenge stakeholders to see a problem through different lenses using Design-based methods and 'seeing' tools. The Designer is tasked with bringing multiple disciplines to the same seeing/maker spaces and providing participatory experiences that nurture collaborative engagement, as well as critical and creative thinking. The responsibilities carried out by the Designer in the C.A.S.T. project included:

\section{Preliminary Responsibilities}

- Designing a three-phase CoDesign framework

- Recruiting participants from all stakeholder groups

- Selecting ideal collaborative spaces for sessions

\section{Pre-Session Responsibilities}

- Gathering qualitative data from multiple perspectives (primary and secondary research) 
- Curating the messy data into useful and usable information/visualizations

- Selecting methods and developing tools for creativity and sensemaking

- Reserving and preparing the seeing/maker spaces

\section{Session Responsibilities}

- Guiding stakeholders through Design Thinking methods

- Facilitating discussions and provoking new ways of thinking/looking

- Capturing/recording information shared and presented

\section{Post-Session Responsibilities}

- Analyzing the data/information that was shared

- Synthesizing the data and summarizing discussion points/decisions

- Presenting the results in a useful, usable, and desirable manner

- Generating a follow up plan of action

In reviewing the list of responsibilities within the role of Design 'facilitator' in this PAR case study it is evident that in addition to moderation the Designer also acts as a curator, bricoleur, and problem architect.

\subsubsection{Curator, Bricoleur, Problem Architect}

Figure 9.4 describes three additional responsibilities of a Design facilitator in CoDesign, in addition to moderating sessions, as well as the relationships among those activities. In facilitating a PAR session around wicked problems, these activities are iteratively linked, often feeding into and/or building onto one another, while also providing feedback. 


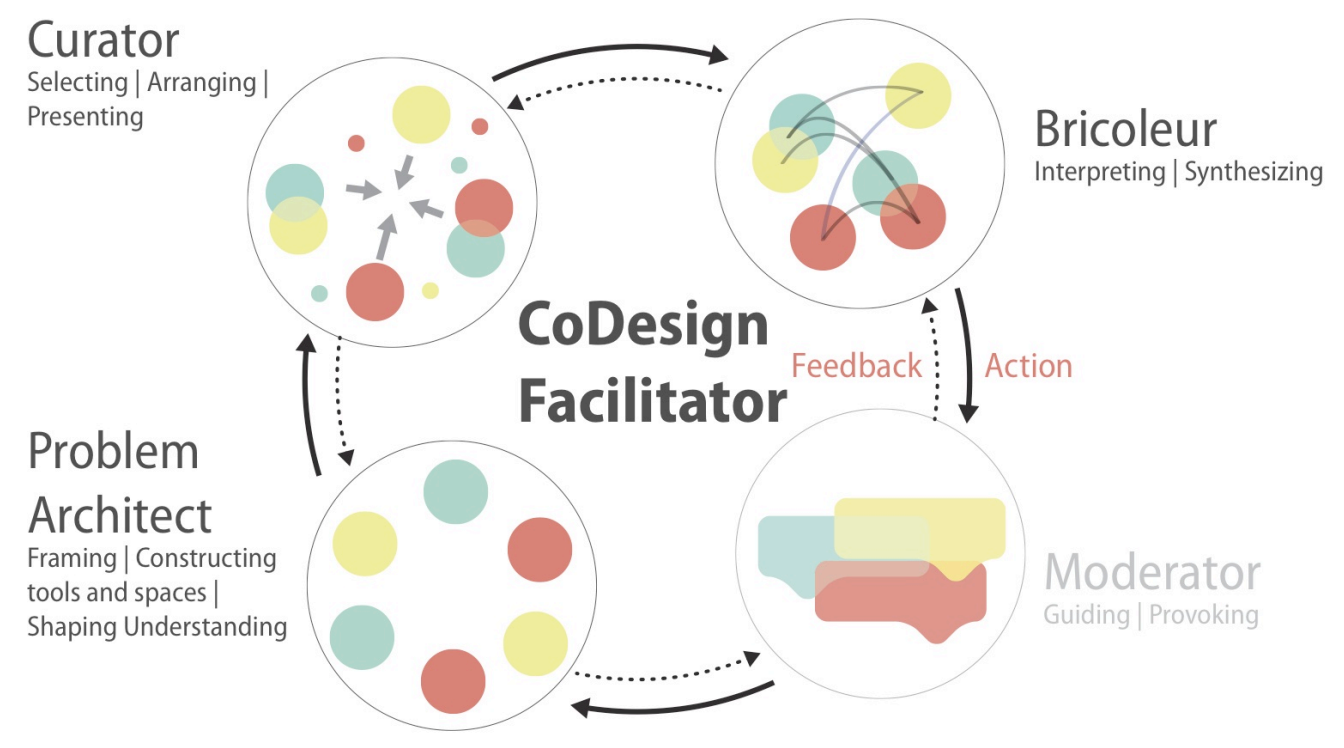

Figure 9.4 The activities embedded in the role of Design facilitator are iterative, feeding into one another and providing feedback.

In the PAR case study, curation and moderation involved bringing people together and exploring and gathering information, various perspectives, and new insights from Phase 1 and Phase 2 which could be used to provoke new ways of 'seeing' and provide fodder for 'making' and 'seeing' in the following sessions. As bricoleur, the information that had been gathered in the prior phases was interpreted and synthesized by the Designer. As problem architect, this synthesis was then transformed into useful and usable data (i.e., provotypes, visualizations, and presentations) that encouraged the stakeholders to reexamine the wicked problem and allowed them to reframe the scope of the problem. The curated forty-eight needs and goals (synthesized from Phase 1), for instance, on the wall with sticky notes, allowed the CoDesigners and the Designers to construct new understanding and make sense of the wicked problem in order to collectively redefine the true 'value' that needed to be created. 


\subsubsection{Extending the Reach of Design}

"Design can help create new innovations beyond incremental improvements, but we need to take a more expansive view of Design and less the domain of a "professional priesthood" (Brown, 2009).

Tim Brown boldly states that for systems-based problems we have to get the Design process out of the hands of only Designers - "Design is too important to be left to Designers." What Brown is saying is that Designers do not have all of the answers. To tackle wicked problems, we need to design from within, and bring those closest to the problem into the fold of problem framing and resolution building. The Design process should not be hidden within a black box or out of the reach of those who are not trained, but instead externalized to not only empower integral stakeholders to see their problem in a new light, but to provide the Designer with more useful and meaningful opportunities from which to design better resolutions.

We should not begin by resolving wicked problems from a pre-assumed set of ideas without first exploring alternative perspectives and synthesizing our understanding about the multi-faceted system/problem and all of its parts. To discover new ways of seeing and reframing the problem, we have to determine the true 'value' we should be creating. To do this, we have to make sense collectively. By allowing integral stakeholders to play an inclusive role as CoDesigners, motivation is sparked and multiple perspectives are actively engaged in new ways of thinking to collectively frame a problem that will not have a singular solution.

Designers can have a more meaningful and lasting impact on society by helping to tackle wicked problems. But, to achieve this goal, our role needs to evolve beyond traditional (tangible) formgiving at the periphery and move into more central strategic roles at the front. We have the 
capacity to help frame a wicked problem by giving shape to collective seeing/maker spaces that provoke shared understanding, discovery, innovative thinking, and new ways of seeing.

\subsubsection{The Value of Inclusion Through Co-Designing}

The case study showed that the inclusion of CoDesigners can bring new understanding and more meaningful knowledge to the Designer about the multi-faceted problem from within. Additionally, their immersion in Design Thinking methods and interactions with other stakeholders helps to engender longer-lasting connections and can motivate some to take ownership. The sense of ownership and motivation provide hope that new resolutions and more productive action will be carried out after the Designers and the scaffolding are removed. CoDesigners immersed into the Design process for this case study were encouraged to think differently, they were made a part of the solution, and were empowered to be agents of change, rather than simply being informants. A parent said in her Reflection Survey that by providing the Method Cards she was able to "look at the various autism problems/situations in new and different ways." She also mentioned that she would like to continue working on the caregiving technology, housing, and Owen's List projects "so others can benefit from what we have learned and the ideas generated," and present our research to Autism Speaks and the national autism organizations. It is encouraging to see that some of the stakeholders were motivated to further explore and share our findings after the case study. The future implications of this research and our aims to progress the findings and resolutions from this case study will be explained in the final chapter.

\subsubsection{Sharing the Design Thinking Process}

Design Thinking approaches pull people out of typical ways of problem solving. There is value in externalizing the design synthesis process and sharing our Design methods, allowing stakeholders to step into CoDesign roles at the beginning of a project. Some Designers fear that by bringing CoDesigners into our processes we minimize the value of a trained Designer. In 
contrast, reflecting on the approach and outcomes of this case study, CoDesign helped the stakeholders have a new regard for Design and our processes for problem framing and synthesis. By bringing non-designers into the 'black box' of design synthesis Designers will help themselves in the long run. We can ensure that the problems we are tackling have true meaning to the people we are designing with (instead of for), we create buy-in and motivation among the stakeholders who will be undertaking and implementing our resolutions, and we also make it easier to explain and demonstrate our process - how we arrived at resolutions through a process of intention, not magic.

In the case study the stakeholders found an appreciation for the Design Thinking methods as a means through which to communicate and connect with other stakeholders and the problem in a new way. Immersing the stakeholders in methods like persona and prospective scenario creation, prototyping, and sensemaking guided them through collaborative making sessions which helped them better understand each other and the problem beyond their own lenses. The interactive making sessions also encouraged them to dive deeper into the unmet needs that had been overlooked and to propose new possibilities. One CoDesigner expressed that the persona exercise helped her a great deal in expressing unmet needs through the story of a boy that had similar qualities of her son, as well as other adolescents on the spectrum. She felt personas were a more compelling way to explain why certain needs needed to be addressed and to help imagine and speculate about the future.

\subsubsection{Extending Empathy in the Design Process}

Methods for empathy and shared understanding were initiated prior to generative thinking activities (like prototyping) to open the minds of the stakeholders and expose them to new ways of seeing the problem. In the case study, collective action (i.e. mapping and prototyping) was effective when our understanding was shared and we were able to look at the problem from 
multiple frames of reference. The element of 'empathy' was not brought up in Bret Victor's vision of a 'seeing' space. The notions of being able to 'see' and draw out understanding were mentioned, but in order for understanding around a complex, multi-faceted problem to be gained, empathy should also be present. The seeing/maker space needs to not only be designed to stimulate participants' creativity and allow them to openly express their thoughts and feelings, but should also foster open-mindedness and connectivity among team members. The presentations at the beginning of Workshop 1 and the personas developed in Workshop 2 helped our team better understand 'why' certain needs/goals were important and how they related to the whole.

\subsection{The Value of Shared Understanding and Sensemaking}

“Hearing the other individuals' and groups' perspectives was essential to reaching shared understanding and prioritization." - Parent

The desired outcome of this project was not aimed at seeking a 'right' answer, but instead bringing diverging perspectives together in one Design space and engaging them "in a robust and healthy process of making sense of the problem's dimensions," (Conklin, 2009, p.18) to collectively arrive at more meaningful systems resolutions and shared understanding. Sensemaking is a subjective framing process, based on the intuitions and shared perspectives of the people present in the sensemaking process. Collective sensemaking, for the purposes of tackling a wicked problem, should be external, action-oriented, and inclusive. By considering multiple perspectives of the stakeholders connected to the wicked problem, and supporting iterative and integrated learning experiences in a collective space (especially early in the Design process), an interdisciplinary team can derive new meaning and a more robust understanding of the system and the paradoxical themes that make up a wicked problem through which to establish Design opportunities and propose innovative resolutions. 
The realization of how to create true value in the wicked problem and for the new clinic came not from offering independent ideas for physical improvements to the clinic environment or systematic changes to the healthcare system, but in looking at the human factors together and realizing that to provide optimal care, the clinic needed to address the holistic well-being of the patient and their families. A high ranking administrator at OSUWMC, whose priority concern at the beginning had encompassed finding resolutions to finance and sustain the clinic over time, had been in meetings about the new C.A.S.T. clinic, prior to this project, but had not felt that the real issues were being voiced or addressed. After Workshop 1 she expressed that the presentations had been invaluable. "It was very helpful to attend and hear the issues of the patients, parents and providers... I think I can be most helpful in trying to move those priorities forward." The issues and connections that emerged from the structure of Workshop 1 provided her with greater understanding (beyond her initial scope). Through new understanding she felt more empowered and motivated to take action. A provider at C.A.S.T. "quickly realized that C.A.S.T. was not the biggest need, but just a small part of the bigger, broader need." For him, this realization came in Workshop 1 during the sensemaking activity when "we grouped things together with circles on the board." These outcomes illustrate the connection between shared understanding and sensemaking, and support the value of gaining shared understanding and empathy to activate creative thinking and motivate participants throughout the PAR process.

\subsection{The Value of a Hybrid Approach in PAR}

While many of the methods and tools used by Designers in CoDesign sessions are paper-based or non-digital in nature, this body of research suggests that there is value in integrating technology into the Design process. A hybrid toolset that supports making and telling methods, through digital and non-digital means, has the potential to enhance future Participatory Action Research around wicked problems. While a digital tool (such as the prototype built in this case study) has the capacity to engage Design teams and diverse collaborators in new ways, it cannot 
replace the value of face-to-face interactions and hands-on making. For instance, in this case study the participants found value in exploring hypothetical futures through persona and scenario creation, and building ideas and new understanding together through on-the-wall mapping and prototyping. Digital tools can offer certain advantages, such as capturing and storing large amount of data, elevating group understanding through visualization and interaction, and extending the sensemaking process across multiple players and multiple sessions, but it can not replace the engagement and motivation of a diverse team built up through face-to-face interactions and critical making in a shared space.

In this case study, technology did not replace non-digital tools or face-to-face methods, but acted in a supportive role, providing the means through which to curate, store, and synthesize research, as well as communicate information that had been shared. Presentations created digitally, in addition to the digital prototype, were designed to promote transparency among the stakeholders, to help facilitate and support the human perspectives they were telling and the sense they were making. Additionally, technology was used to communicate the process of sensemaking and the findings from our sessions, as well as to organize various perspectives and turn them into useful data points for the CoDesign process. The blend of paper-based methods, face-to-face interactions, and visual synthesis tools led to new connections and new plans for action, which sought to transform the current situation for adults on the spectrum and their families in the future, beyond the everyday understanding of each individual participant.

Paper-based visual diagrams are static and do not explain progression well. They often just convey the end of the story. Technology can help Designers create an immersive and interactive CoDesign experience across time and provide the flexibility for an infinite amount of exploration and simulation. Beyond tangible tools, the digital tool prototype had the added features of playfulness, scalability, responsiveness, and traceability. A blended interaction tool also has the 
capacity to unite face-to-face interaction and hands-on making, while supporting social interaction as users build ideas and negotiate on a shared canvas. A blended interaction tool in the PAR process has the potential to not only provide recording capabilities (as decisions are being made), but may also bring participants together (in the present time or at a future time) to explore multiple possibilities through a more dynamic game-like experience. While more research and experimental prototyping will need to be conducted to test this observation, the findings suggest that technology has the added benefit of making collective innovation around wicked problems more accessible and effective over the course of multiple sessions.

On the other hand, face-to-face interactions and hands-on making (using paper-based tools) sparked connectivity, mutual sharing, and organic conversation among a diverse group of stakeholders. Narratives, visualizations, and paper-based tools were used as communication vehicles through which the CoDesigners could find common ground and provoke new ways of seeing. Visual provotypes printed on paper could be easily changed or enhanced by simply being drawn on. Understanding and communication around the true needs and opportunities were garnered through the making of persona posters, prospective scenarios, and prototypes using paper-based tools. While digital (interactive) provotypes, storyboarding, and prototyping may have the capacity to afford CoDesigners the ability to build and evaluate multiple scenarios/outcomes for their persona, digital tools may also add confusion (a learning curve for understanding a new, unfamiliar program) and the potential for unreliability (as observed with the digital prototype in Workshop 1). Familiarity with paper tools and physical objects allowed the focus of the stakeholders in the sessions to be placed on making and openly sharing - rather than having to learn a program or be distracted by unresponsive functions. Making with paperbased/physical objects provided the means through which they could easily construct ideas/maps, learn from one another, and communicate new concepts to reach a common goal. 


\subsubsection{The Hybrid PAR Framework}

Figure 9.5 shows the C.A.S.T. Case Study Framework again as a frame of reference to help explain a more abstract representation of the hybrid approach of methods and tools explored in this PAR process in Figure 9.6. The framework is divided into seven stages that correspond to the process that was carried out in the case study: Priming Participants, Exploring Perspectives, Synthesizing Findings, Making Sense and Prioritizing, Framing, Creating Prototypes, and Strategizing Next Steps. Within each stage are the seeing/maker spaces that were created for each phase of the project. Each seeing/maker space is represented by a colored circle to indicate whether or not technology was used in that space. Following Figure 9.6 is a revised look (Figure 9.7) at how future PAR processes could become more hybrid with the addition of alternative nondigital tools and methods (i.e., enactment) and technology (i.e. digital storyboarding). Some of the modifications/additions that have been mentioned in this chapter (for both digital and non-digital methods/tools) are listed in Figure 9.7 below the diagram. More exploration and experimentation will be needed to determine whether these additions would improve seeing/maker spaces and the PAR process as a whole. 


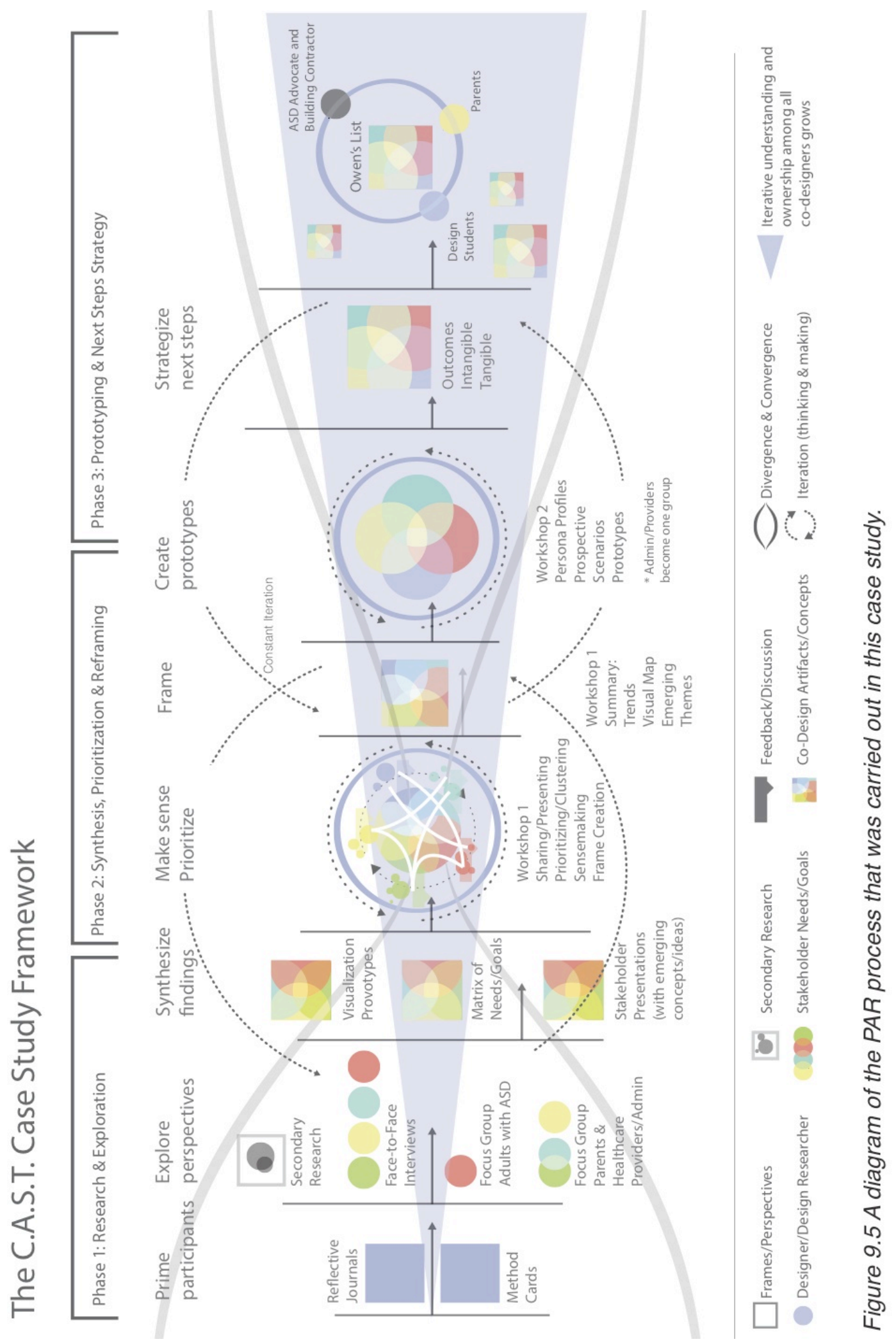




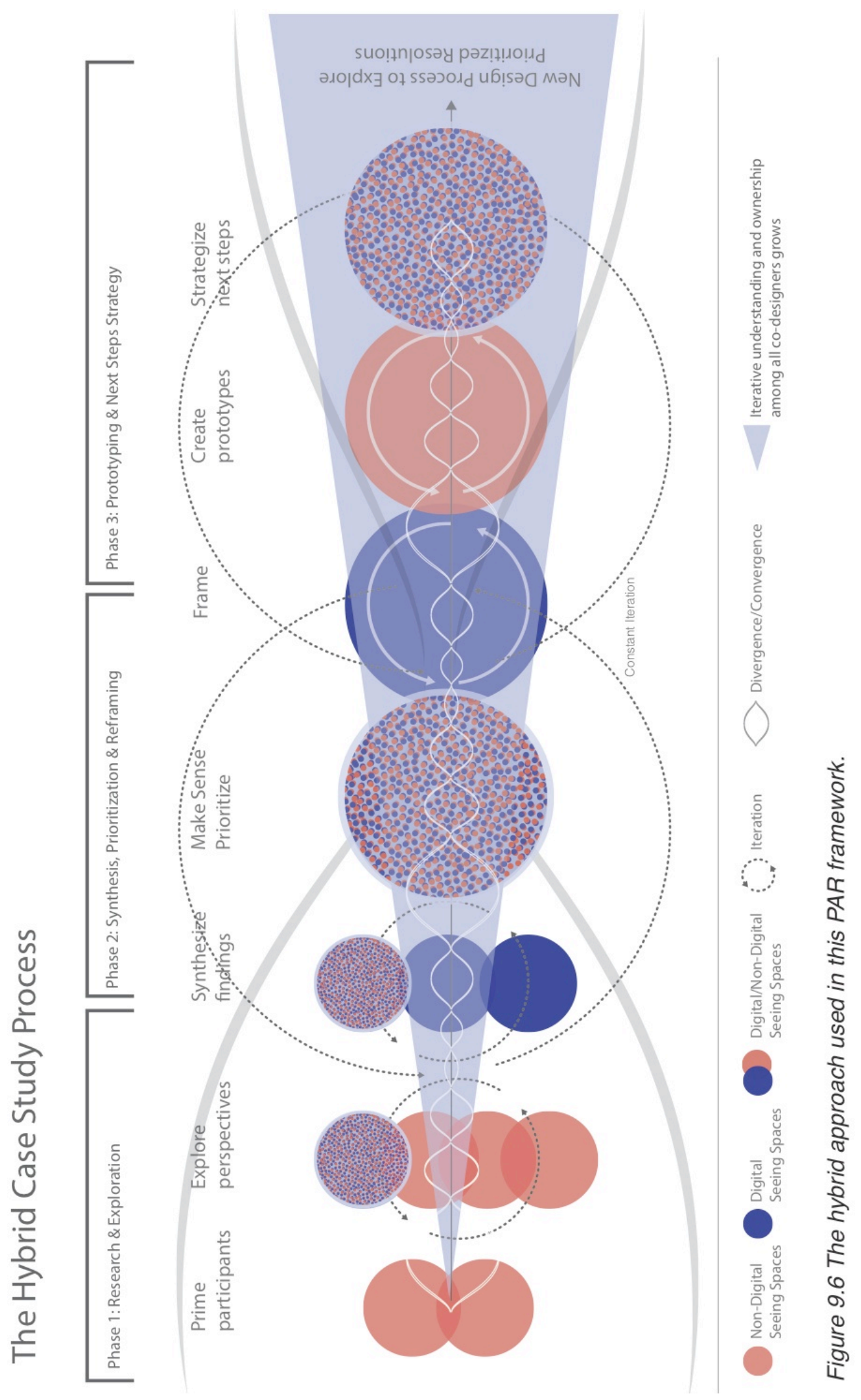




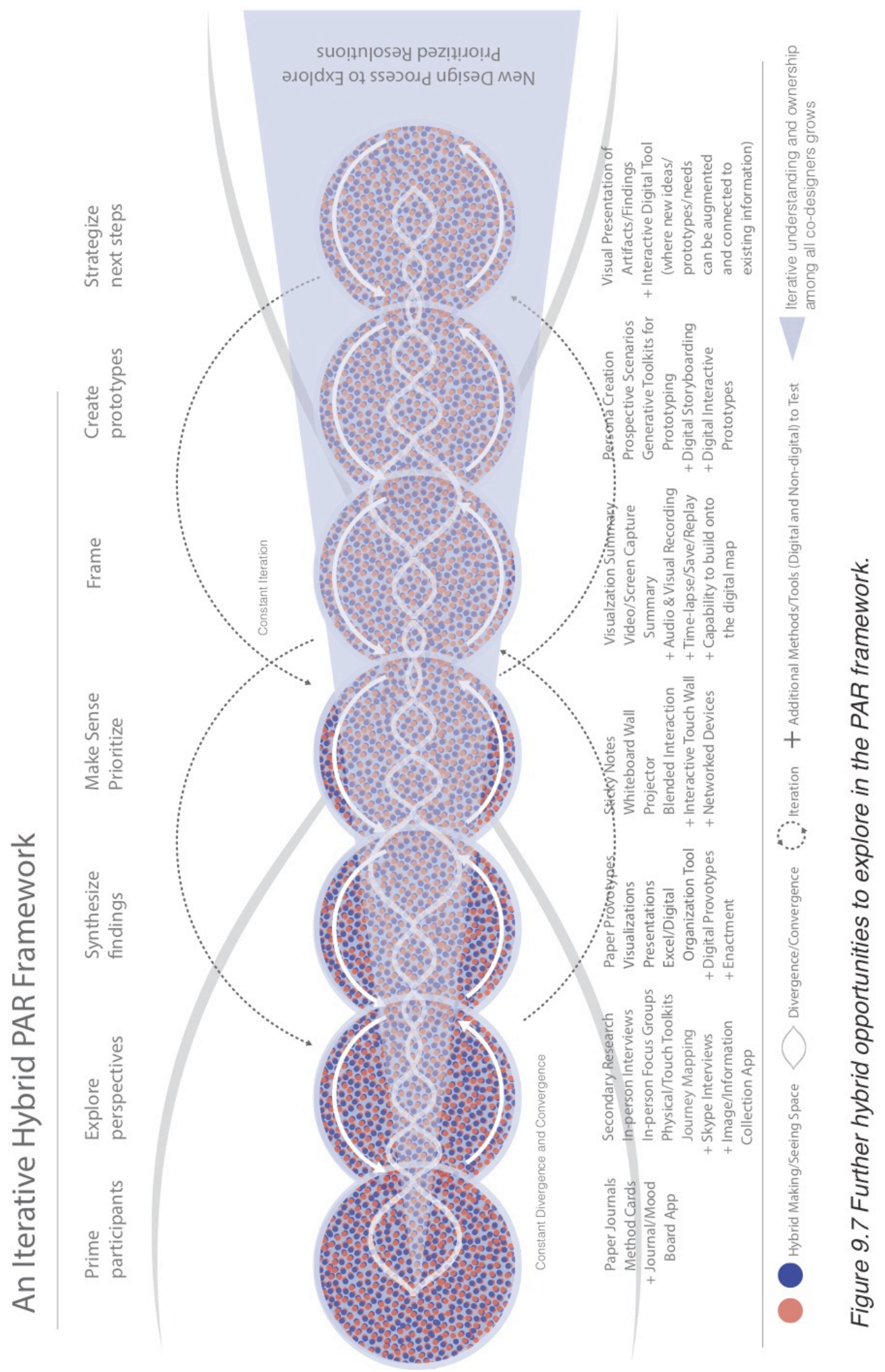




\subsection{What Was Learned From This Exploratory Case Study}

\section{The current transitional process for adults with autism is a wicked problem.}

The problem has multiple facets both inside and outside the healthcare system. The autism population is expanding, while the resources, support and care offerings for adults with autism and their families are severely lacking. The findings and outcomes derived from this case study bring to light opportunities where Design can have a positive influence on this social problem.

\section{Wicked problems benefit from the iterative abductive reasoning and inclusivity.}

This project showed the value of an iterative CoDesign approach (i.e., PAR) to tackle wicked problems. For an iterative process to continue, however, there has to be motivation and buy-in from the people who will be carrying out the change. This case study supports the idea that inclusivity, and immersion through CoDesign methods can help to build connections and motivation. Furthermore, co-designing with various stakeholders allows input from many perspectives. "Diversity trumps smartness" (Johnson, 2010). Therefore, it is critical to provide methods and tools through which shared understanding of the system and its parts can be achieved, and new, meaningful possibilities can be imagined by looking at the wicked problem through multiple lenses.

\section{Everyone should have a voice.}

Young and old, typical and a-typical, verbal and non-verbal, all people need to be heard. Those on the spectrum have a lot to offer society. Their insights had a large impact on the frame and concepts that were developed, as well as the growth of shared understanding about the wicked problem. Given the space and the right tools/methods through which to share their needs, knowledge, and ideas, those with autism can do great things and society will be better for it. 


\section{Sharing and sensemaking led to reframing and collective innovation.}

Stakeholders widened their scope and dug deeper into understanding multiple facets of the transition problem. They discovered that there was little to gain by simply changing aspects of the clinic (the original frame) and more to lose if the root problems (i.e., maximizing long-term potential, and nurturing independent growth in other areas of the lives of those with autism) were not addressed. Recognition of these root problems shaped a new frame. Through collective reframing, CoDesigners felt compelled to tackle long-term resolutions that could have a greater impact, rather than near-term opportunities that would have resulted in incremental changes.

\section{CoDesign outcomes extend beyond 'tangible' resolutions.}

The value gained by involving stakeholders in the beginning phase of the Design process extends beyond helping Designers arrive at tangible Design opportunities, such as innovative products and services, but also provides a sense of engagement, inclusivity, and empathy. The case study suggests that these 'intangible' resolutions are invaluable to PAR and may help to motivate participants to extend their involvement in a project.

\section{Designers can do more than generate ideas.}

The value and reach of a Designer's skills can extend beyond that of idea creator, stylist, and traditional form giver. A Designer's role and value can also encompass an ability to frame and help others see problems in a new way at the front-end of the Design process. Perhaps not all Designers can or want to step into this extended role. Those that do have the potential to give form not only to outcomes, but also to building understanding and framing the complexity of a multi-faceted problem from the beginning. 


\section{There is value in a hybrid Design Thinking approach and seeing/maker spaces.}

The case study and the process of developing a digital tool presented opportunities for technology to be integrated into the PAR process to engage stakeholders in new ways of interacting, seeing, and making, and to also help the Designer externalize the Design process. In addition to immersing non-designers in the synthesis process, interaction with new methods (like personas) helped to promote the value of Design Thinking methods as purposeful problem solving approaches. Blended technology has the potential to encourage new forms of social engagement and making in a collective Design setting. Seeing/maker spaces that offer both digital and non-digital tools are needed to help people look inside a problem, across time, and across possibilities, to broaden their thinking capacities and deepen their understanding in order to help them discover innovative opportunities through shared making and iterative exploration.

\subsection{Contributions to Design}

\section{CoDesign through a PAR process was applied to a wicked problem that is timely.}

The value in applying CoDesign is, by now, well established (Halskov, 2012). In this case study, CoDesign was applied in the early front-end of the Design process to tackle the wicked problem of transitions and adult care/support in the autism community. This issue is timely and the use of CoDesign to tackle and frame wicked problems is an emerging landscape in Design.

\section{People with perceived disabilities were brought into the CoDesign process to help tackle wicked problems.}

While this is not the first time people with autism have been brought into CoDesign roles (Gaudion et al., 2015), it is an emerging approach that is of high value in society today. The outcomes from the project highlight the untapped potential of adults on the spectrum to confront wicked problems and develop resolutions that not only directly affect them, but others on the autism spectrum as well, and society as a whole. The participation of this population, given their 
unique abilities and interests in the future, could provide them with a greater sense of empowerment/self-advocacy and provide the world with potentially more innovative resolutions.

\section{A hybrid toolset was integrated into the PAR process.}

The introduction of technology into the PAR process was explored in this case study. In addition, play-testing of this technology highlighted added advantages, as well as potential pitfalls. The use of the blended interaction digital tool prototype to facilitate social interaction and collective sensemaking, as well as organize and summarize session discussions and outcomes, sheds light on how technology could be integrated into future PAR processes to enhance shared understanding, critical making, and collaborative engagement.

\section{Expansion of the value of the Designer beyond traditional form giver and facilitator.}

The Designer's role in facilitation extended to curator, bricoleur, and problem architect. In these extended activities, Designers develop spaces and tools to facilitate seeing and making among collaborators, and stimulate their sensemaking abilities. This includes gathering multiple perspectives and creating flexible methods and tools through which a diverse team can collectively express thoughts, feelings and ideas as well as negotiate decisions, reframe the problem, speculate about the future, and build new resolutions together.

\section{Design of new materials - seeing/maker spaces and tools for sensemaking.}

This case study supports the notion that people can be an extension of the Designer. Their ability to contribute to Design in a meaningful way relies on the Designer's attitude, as well as the design of the spaces and tools that encourage people to think differently, empathize with multiple perspectives, and interact to promote learning, creativity, and understanding. In this case study a new tool was developed, along with seeing/maker spaces that not only facilitated creativity and expression of thought and feeling, but also helped the team understand each other, see the 
problem in new ways, and dive deeper to construct a meaningful frame and ideas that could create true value in the wicked problem. 


\section{Chapter 10: Impact for the Future}

"As the numbers of people with autism skyrocket, and the population continues to age, it is imperative that society learns how to deal with and assist this population as they attempt to navigate life's challenges." - Parent

This chapter considers the future impact of the research in this case study on the topic of transitions in ASD, on the stakeholders involved, the field of Design, and society as a whole. Design is not an object or an outcome, but a process of problem solving. CoDesigners are brought into our Design process, not to replace the Designer, but to extend our ability to tackle the true problem. When Design is no longer perceived as only an aesthetic and user-friendly outcome, but instead as a valuable asset for tackling ambiguity and complexity, Designers will be able to see themselves, and be seen by others, as framers and new types of form givers in the front-end of the Design process - extending our value and our reach to tackle more pressing issues in society.

\subsection{Next Steps with the C.A.S.T. Project}

The CoDesign approach encouraged the stakeholders to look at the wicked problem in new ways and discover innovative opportunities. Each concept that emerged from our sessions has the potential to address a true need in the autism community. In the Reflection Survey, a parent expressed that "the outcomes of this project were a significant example of the collaboration required to address the complex, multi-faceted issues facing individuals with autism and their families. Many of the ideas generated can be furthered developed and implemented. The longer- 
range ideas (i.e., Owen's List, housing, disruptive caregiving technologies, virtual and physical communities) must happen, as there are no existing solutions for the long-term care and support of the ASD adult population." The artifacts from the case study are also living documents of our process and findings; they provide a transparent pathway illustrating how decisions were made and which stakeholders had an impact on those decisions. These will be useful guides and references for team members and others in the autism community who did not participate in the case study. After the project ended, a few of the stakeholders wanted to carry some of the concepts into the next stage of development and to share our findings and our process with a larger audience - "This work must be presented to the national autism organizations for further advancement!" Through PAR, we not only developed innovative concepts to improve the lives of people on the spectrum and their families, but also generated enough new understanding and feelings of ownership among the stakeholders such that they wanted to continue being part of the resolution.

Both near and far term concepts will require various 'experts' and resources to be further developed. To bring these concepts into the next phase of Design, new multi-disciplinary project teams will need to be built up. This includes end-users as well as experts brought in to further develop the concept into a more tangible space, product or service. The concept will then be moved into the ideation phase and continue to be co-designed with the multi-disciplinary team through the development of more refined prototypes and iterative testing before a resolution is finalized and introduced into the ASD system.

The C.A.S.T. clinic has hired a programs coordinator to do more outreach and to address the greater needs of patients and families beyond the care that happens in the clinic. The artifacts and findings from our case study have been presented to her. She was overwhelmed by what had 
been uncovered and created in the workshops, and would like to see many of these ideas explored in the next phase of the Design process.

Students in a Participatory Design research class at The Ohio State University worked with one of our parents from the case study to further develop Owen's List and tackle three important needs that came out of the sessions: navigating and understanding the ASD system, long-term housing and career solutions, and self-advocacy. The students were given an overview of the process of the case study and the findings, in addition to images of the paper prototype of Owen's List that had been developed in Workshop 2. The students' objective was to take what had been started and delve deeper into the research-related needs and how they could be addressed through an interactive prototype to help parents and adults on the autism spectrum. To better understand the needs associated with long-term housing and navigating ASD, new stakeholders (including more parents and a housing contractor) were brought into the CoDesign process with the Design students. Collectively the team developed a more refined visual prototype of Owen's list that can be presented to other stakeholders and people with the ability to see it to the next level of refinement and development.

\subsection{Evaluative Testing To Be Done}

The complex and interwoven nature of wicked problems with an indeterminate end-state, multiple stakeholders, and no 'right' solution present an exciting and challenging opportunity to further test and explore the capabilities of technology and how it can be integrated into the Design process to widen participation, support Design Thinking methods, and elevate social interactions in future PAR sessions. Evaluative testing will need to be conducted with the digital tool prototype and the methods proposed in the hybrid framework for future CoDesign sessions, in order to determine the effectiveness of the hybrid toolset across multiple topics and stakeholder groupings.

Additionally, a study could be conducted to compare the hybrid toolset versus PAR sessions that 
utilize only a digital toolset versus only a non-digital toolset to better understand the impact of the three approaches. Whether or not the digital tool prototyped in this case study is an ideal solution for PAR was not determined - the prototype served as an exploration into alternative technological approaches that could enhance aspects of the Design process. More testing needs to be conducted with this prototype, along with broader exploration into alternative visualization interfaces and interactive tools to investigate the further use of technology in collaborative settings.

\subsection{Where Can Technology Fit in the Front-End of the Design Process?}

Technology is integrated into many aspects of our lives; does it have a place in the Design process? If so, how can it be seamlessly assimilated to make Design and CoDesign processes more effective? The research suggests that interaction through digitally shared platforms, inspired by collaborative games and design synthesis processes, can help to provide a shared common language, allow stakeholders the opportunity to co-produce and make sense of complexity, and sustain temporal openness and commitment necessary for resolving wicked problems.

In addition to provoking new forms of engagement, technology also offers save, undo, and timelapse features, allowing people to customize information, rapidly simulate several possibilities, and construct, deconstruct, and reshape decisions before implementing an idea. Global and colocated communication and connectivity can also be made more accessible and fluid through digital media, in contrast to real-time or paper-based methods. Blended interaction has been shown to promote greater group awareness, verbal communication, and collective expression in secondary research. This was also supported in the final round of play-testing. Yet, the use of technology and blended interaction are underutilized in the Design process. Our first instinct is to fall back on customary methods and tools, because of their reliability and familiarity. However, technology offers added capabilities to the Design process. Digital tools, similar to the prototype 
designed with this case study, that are integrated into various stages of the Design process can record thinking trails and real-time activities within seeing/maker spaces, as well as create a liquid network through which people with various backgrounds can collaborate around a common problem. Digital capabilities can be brought in during the early framing stages of the Design process, as they were in this study, to gather, store, and organize research data, guide CoDesigners through the design synthesis process, and help Designers communicate postsession findings/insights.

Individuals may use technology in the Design process to stay more connected to information, and/or as a means through which to self-express, reflect, or communicate thoughts, needs, and ideas prior to, during, or after real-time sessions. Interdisciplinary teams may use it to compare multiple viewpoints, interact with different stakeholders, and establish common ground on which to deliberate and ideate around unmet needs and future possibilities. Designers and Design teams can use technology programs and blended interaction techniques to curate and synthesize large amounts of data, and generate new possibilities, while interacting face-to-face on a shared surface.

While the prototype was developed for sensemaking and converging around a collective objective, interactions among the participants demonstrated that convergence and divergence often happened simultaneously. Additionally, play-testing with the students showed the prototype's capabilities to not only help with sensemaking, but to also stimulate divergent thinking. The flexibility in the tool's actions and the ability to bring in different data sets allows users the ability to customize the tool and its functional components (i.e., idea nodes, clusters, connections, stakeholder icons) to meet a tailored need. Basic functions (established rules designed in the program code), along with the 'given' data nodes at the start, provide the constraints or foundation from which creative teams can then freely construct new meaning. 


\subsection{Future Improvements to a Digital Seeing/Maker Space}

Future prototypes of the digital sensemaking tool should further investigate ways to see inside the problem from different levels (both macroscopic and microscopic views) and from various frames of reference. Zoom features embedded in the digital tool could allow teams/individuals to select and work on specific areas (for a closer look) or to zoom out and work on the whole system. The ability to see across time and possibilities could be improved by not only taking screen shots, but building in save/pause/undo capabilities and a time-lapse feature so various alternatives could be juxtaposed over the course of many sessions. These decisions and explorations could then be saved and referred to later in the 'seeing' space by others at a different time.

Additional future functions of the digital sensemaking tool could also include:

- Organizing visual clutter by reassembling the initial ring of nodes and pulling it to the corner

- Audio and visual recording to capture not only what was created, but also what was said

- Sound and visual feedback/interaction, along with animated features, could be integrated into the interface to add 'life' to the digital canvas

- Networking - shared interaction between a shared touch device and individual touch devices

\subsection{Future Opportunities Beyond Scope}

Findings uncovered through this research expose other opportunities to explore further. The value of a collaborative seeing/maker space was exposed in the findings from the research. The idea for a single hybrid, interactive war-room with digital capabilities, whiteboards, touch interaction, physical making tools, and face-to-face interfacing should continue to be explored. This space could support iterative making, and present information from multiple reference points so new knowledge and mutual understanding could continue to evolve and new opportunities could 
continue to be shaped around wicked problems. More of these spaces should be developed for collaboration, especially around wicked problems that would benefit from the input of diverse teams of experts and long-term iteration. Additionally, continued exploration into visual, digital, and physical tools for seeing/maker spaces, designed for people with autism and neurotypicals, should be investigated in further PD and PAR sessions to discover more effective ways for all people to find common ground, cross-pollinate thoughts/ideas, and create innovative concepts in a shared space.

Furthermore, digital information sharing between stakeholders prior to, during, and post collaborative sessions is an area that needs further exploration. Extending access to the information and the collective team (including the Designers) outside a single session may help to strength the bond between them and help to coordinate next steps. Community between stakeholders and Designers can be extended over time and space by allowing team members to upload information in a journal/mood board app from personal devices linked to the interactive digital software. This information would feed into the sensemaking tool along with the additional data the Designer gathers during pre-session interviews prior to the collective thinking session. Additionally, a journal/mood board app would double as an immersive activity to encourage individual critical and creative thinking outside the collaborative session. Because many of us are constantly on personal technology devices, perhaps the preparation/reflection tool in PAR needs to meet the users where they are - digitally.

\subsection{Implications of this Research on the Field of Design}

The case study presented in this thesis addressed the front-end of the Design process and explored various methods and tools used in Participatory Action Research. Focusing on the frontend of the Design process afforded the opportunity to explore Design Thinking approaches, as well as the role of the Designer and the value of sensemaking in the early phases of a wicked 
problem. Designers who choose to play within such an expanded role must evolve from simply form-givers to sensemakers and be able to coordinate the interactions and goals of a diverse team of experts. The findings from the PAR approach in this case study demonstrate that innovative thinking around a wicked problem benefits greatly from the active inclusion of Designers and other stakeholders of the complex problem. Information transparency, crosspollination, and inclusion among Designers and CoDesigners are important components in framing and reframing a wicked problem.

Through their abductive thinking processes and design of tools and seeing/maker spaces, Designers can help to provoke new ways of seeing, learning through critical making, and inspire new insights from others, while also bringing unique mindsets and perspectives together. The tools we (Designers) develop to externalize this collective process and our attitude (collaborator instead of director) in facilitating Design Thinking methods in CoDesign can be valuable in elevating the regard of the Designer and helping non-designers better appreciate and understand our non-linear approach. We can break down the black box and dispel the myth that our work is 'magic', and instead demonstrate that Design is intentional and deliberate by immersing nondesigners in the CoDesign process and allowing them to help us identify patterns, establish a frame, and illustrate connections between unmet needs and new design opportunities.

\subsection{Who are the Change-makers?}

“When we can fully engage people's minds, hearts, and bodies in imagining and expressing future situations of use, we can be assured that they have an opportunity to influence future ways of living, learning and being" (Brandt, Binder \& Sanders, 2012, p.176).

This case study demonstrates the value of the PAR process (as one that stimulates iterative learning and ownership) and suggests that while the Designer may act as a provocateur and 
influencer, people (not Designers alone) become the agents for change through the informed design of a system and a hybrid framework for collective sensemaking. The IDEA Studio at OSUWMC closed half way through the project. The reason for this was unrelated to the ASD project, but because it was the driver and the body of influence/authority on the project there was some concern that our outcomes would be lost and futile. Instead, a parent further developed Owen's List with a team of Design students, and a high-ranking administrator at OSUWMC facilitated a discussion with the new outreach coordinator at C.A.S.T. to present the work/outcomes of our project and determine next steps of action. The Designer's role in the next phases of the project will be to communicate our findings and resolutions, and to further develop and realize the concepts that were prototyped. However, without buy-in and support from the people that were included in the PAR sessions, those ideas may not have been further realized or implemented. New connections made through the PAR process and among the stakeholders accounted for an interest to continue what we had started. While the Designer may act as facilitator and provocateur of new knowledge, with a hope for change, it takes people integral to the problem to make real change possible. The Designer can only help to build the scaffold. If they have done their job through PAR, the people in most need of change are armed with new knowledge, connections and empowerment to implement the changes - they become the change-makers. In their expanded role, Designers are serving change, not just serving the marketplace.

\subsection{Expanding Our 'Form Giving' Capabilities}

The role and value of the Designer on a collaborative team is not only the sum of the Design artifacts and tangible outcomes at the back-end of the process, but more importantly their comfort in ambiguity, creative and critical thinking abilities, visualization skill-sets, empathy, abductive reasoning, non-linear processes, and systematic thinking approaches that equip them to step into new roles and contribute to the front-end stage of a wicked problem. The role of the Designer is 
expanding beyond being a 'maker' of artifacts to one who can help to, curate information, interpret ambiguity, and give form to a wicked problem by designing sensemaking tools and seeing/maker spaces that provoke people to see a problem differently and collectively discover new opportunities.

Our job as Designers is to provoke new ways of seeing and working with CoDesigners by building scaffolds (or living frameworks) on which new resolutions can be iteratively explored to collectively make sense of wicked problems. Designers need people integral to the problem to actively take part in knowledge sharing, sensemaking, and co-designing of the resolutions that directly impact their lives. The ideas mentioned and prototyped by the non-designers are invaluable if we (Designers) are going to develop implementable resolutions that have true value. The role of the Designer as 'maker' is still vital to the Design process. The abilities of the Designer beyond a 'maker' of solutions to a 'maker' of sense and the abilities of non-designers to be active contributors and change-makers extends our capabilities and opens the door for greater possibilities. The aim should be to balance and nurture each expert's ability (Designer and nondesigner) to contribute in a meaningful way to society's most complex problems through the frameworks we develop, the tools we create, and the seeing/maker spaces we design. 


\section{References}

Ackoff, R. L. (1989). From data to wisdom. Journal of Applied Systems Analysis, 16(1), 3-9.

Autism Spectrum. What is ASD? Visual diagram. (n.d.). Retrieved February 22, 2016 from http://www.autisticspectrum.co.uk/what-is-asd/

Autism Speaks. Mounting Evidence of Critical Need for Adult Transition Support. (n.d.). Retrieved February 22, 2016 from https://www.autismspeaks.org/science/science-news/top-tenlists/2012/mounting-evidence-critical-need-adult-transition-support

Bandura, A. (1977). Social Learning Theory. Englewood Cliffs, N.J: Prentice Hall.

Biography: Temple Grandin, Ph.D. (n.d.). Retrieved February 22, 2016 from http://www.grandin.com/temple.html

Blair-Early, A. (2010). Beyond borders: Participatory design research and the changing role of design. Visible Language, 44(2), 207-218.

Boer, L., \& Donovan, J. (2012, June). Provotypes for participatory innovation. In Proceedings of the Designing Interactive Systems Conference. ACM, 388-397.

Bostock, M., Ogievetsky, V., \& Heer, J. (2011). D3 data-driven documents. IEEE Transactions on Visualization and Computer Graphics, 17(12), 2301-2309.

Brandt, E., Binder, T., \& Sanders, E. B.-N. (2012). Tools and techniques: Ways to engage telling, making and enacting. In Routledge International Handbook of Participatory Design. Routledge, 145-181. 
Brown, A. D., Stacey, P., \& Nandhakumar, J. (2008). Making sense of sensemaking narratives. Human Relations, 61(8), 1035-1062.

Brown, T. (2009). TEDTalks: Designers - think big!. Retrieved February 22, 2016 from https://www.ted.com/talks/tim_brown_urges_designers_to_think_big?language=en

Buchanan, R. (1992). Wicked problems in design thinking. Design Issues,8(2), 5-21.

Carey, B. (2015). Learning to See Data. Retrieved from

http://www.nytimes.com/2015/03/29/sunday-review/learning-to-see-data.html

Carlozzi, A. F., Bull, K. S., Eells, G. T., \& Hurlburt, J. D. (1995). Empathy as related to creativity, dogmatism, and expressiveness. In The Journal of Psychology, 129(4), 365-373.

Center for Disease Control. (2014) CDC estimates 1 in 68 children has been identified with autism spectrum disorder. Retrieved February 22, 2016, from

http://www.cdc.gov/media/releases/2014/p0327-autism-spectrum-disorder.html

Conklin, J. (2009, Winter). Building Shared Understanding of Wicked Problems [Interview by K. Christensen]. Rotman, 17-20.

Cooper, Alan. (2008) The origin of personas. Cooper Journal. Retrieved from http;//www.cooper.com/journal/2008/5/the_origin_of_personas

Davies, A., Fidler, D., \& Gorbis, M. (2011). Future Work Skills 2020. Institute for the Future for University of Phoenix Research Institute, 540.

Digital Tools for Design Research (n.d.). Retrieved February 22, 2016, from http://www.ideo.com/images/uploads/work/pdf/Labs_digital_tools_0914.pdf

Dorst, K. (2011). The core of 'design thinking' and its application. Design Studies, 32(6), 521-532.

Dorst, K. (2015). Frame Innovation: Create New Thinking by Design. MIT Press. 
Ferro, S. (2014). Urban Design Can Change The Lives Of People With Autism. Retrieved February 22, 2016, from http://www.fastcodesign.com/3030109/urban-design-can-change-thelives-of-people-with-autism

Fischer, G. (2003). Meta-design: Beyond user-centered and participatory design. Proceedings of HCl International. 88-92.

Fujitsu Laboratories Ltd. / Fujitsu Design Limited / Fujitsu Social Science Laboratory Limited. (2015) Fujitsu Develops User Interface Technology that Converts Entire Rooms into Digital Spaces [Press release]. Retrieved February 22, 2016, from http://www.fujitsu.com/global/about/resources/news/press-releases/2015/0727-01.html

Gamification (n.d.). In Merriam Webster Online, Retrieved February 22, 2016, from http://www.merriam-webster.com/dictionary/gamification

Gaudion, K., Hall, A., Myerson, J., \& Pellicano, L. (2015). A designer's approach: How can autistic adults with learning disabilities be involved in the design process?. CoDesign, 11(1), 49-69.

Generous, A. (2015). TEDTalks: How I learned to communicate my inner life with Asperger's. Retrieved February 22, 2016 from http://www.ted.com/talks/alix_generous_how_i_learned_to_communicate_my_inner_life_with_as perger_s?language=en

Gibson, J. J. (1979). The Ecological Approach to Visual Perception. Boston: Houghton Mifflin.

Glaveanu, V. P. (2012). What can be done with an egg? Creativity, material objects, and the theory of affordances. The Journal of Creative Behavior, 46(3), 192-208.

Grandin, T. (2009). Thinking in Pictures. Bloomsbury Publishing.

Grudin, J., \& Pruitt, J. (2002, January). Personas, participatory design and product development: An infrastructure for engagement. In Proceedings of the Participatory Design Conference, 144152. 
Halskov, K. (2012). PDC 2012: Embracing New Territories of Participation: Proceedings of the 12th Participatory Design Conference: August 12-16, 2012, Roskilde University, Denmark. New York, NY: ACM.

Hanington, B., \& Martin, B. (2012). Universal Methods of Design: 100 Ways to Research Complex Problems, Develop Innovative Ideas, and Design Effective Solutions. Rockport Publishers.

Horn, R. E., \& Weber, P. R. (2007). New tools for resolving wicked problems. MacroVu Inc and Strategy Kinetics. Retrieved from: http://www. strategykinetics.

com/files/New_Tools_For_Resolving_Wicked_Problems.pdf.

Horowitz, M. W., \& Newman, J. B. (1964). Spoken and written expression: An experimental analysis. The Journal of Abnormal and Social Psychology, 68(6), 640.

Howard, Z., \& Somerville, M. M. (2014). A comparative study of two design charrettes: implications for codesign and participatory action research. CoDesign, 10(1), 46-62.

Jetter, H., Geyer, F., Schwartz, T., Reiterer, H. (2012). Blended interaction - Toward a framework for the design of interactive spaces. Workshop Designing Collaborative Interaction Spaces (DCIS), (12).

Johnson, B. D. (2011). Science fiction prototyping: Designing the future with science fiction. Synthesis Lectures on Computer Science, 3(1), 1-190.

Johnson, S. (2002). Emergence: The Connected Lives of Ants, Brains, Cities, and Software. Simon and Schuster.

Johnson, S. (2010). TEDTalks: Where Good Ideas Come From. Retrieved from http://www.ted.com/talks/steven_johnson_where_good_ideas_come_from?language=en

Jones, P. (n.d.). Collective Sensemaking. Lecture presented at Designing with Dialogue. Retrieved from http://www.slideshare.net/peterjones/dialogue-collective-sensemaking

Kapchan, D. (1995). Performance. The Journal of American Folklore, 108(430), 479-508. 
Kelley, T., \& Kelley, D. (2013). Creative Confidence: Unleashing the Creative Potential Within Us All. Crown Business.

Kolko, J. (2010a). Abductive thinking and sensemaking: The drivers of design synthesis. Design Issues, 26(1), 15-28.

Kolko, J. (2010b). Sensemaking and framing: A theoretical reflection on perspective in design synthesis. Design Research Society Conference.

Kolko, J. (2012). Wicked Problems: Problems Worth Solving : A Handbook and Call to Action.

Kouprie, M., \& Visser, F. S. (2009). A framework for empathy in design: Stepping into and out of the user's life. Journal of Engineering Design, 20(5), 437-448.

Lamm, H., \& Trommsdorff, G. (1973). Groups versus individual performance on tasks requiring ideational proficiency (brainstorming): A review. European Journal of Social Psychology. 3(4), 361-388.

Laperche, B., Munier, F., \& Hamdouch, A. (2008). The collective innovation process and the need for dynamic coordination: General presentation. Journal of Innovation Economics \& Management, 2(2), 3-13.

Liu, S., Cui, W., Wu, Y., \& Liu, M. (2014). A survey on information visualization: recent advances and challenges. The Visual Computer, 30(12), 1373-1393.

Macdonald, A. (2012). Wicked problems: Design approaches in complex health-care scenarios. Innovation, 31(4), 39-42.

Making Sense of Strategic Design Practice 2015. (n.d.). Retrieved February 22, 2016, from http://issuu.com/humantific/docs/humantific_makingsenseofstrategicde/1?e=1209717/31130792

Method. (n.d.). In Merriam Webster Online, Retrieved February 22, 2016, from http://www.merriam-webster.com/dictionary/method 
Millett, S. M. (2003). The future of scenarios: Challenges and opportunities. Strategy \& Leadership, 31(2), 16-24.

Mulder, S., Yaar, Z., \& Broschinsky, D. (2007). Book Review-//The User is Always Right: A Practical Guide to Creating and Using Personas for the Web. Interactions-New York, 14(4), 5252.

O'Donnell, D., O'Regan, P., \& Coates, B. (2000). Intellectual capital: A Habermasian introduction. Journal of Intellectual Capital, 1(2), 187-200.

Osborn, A. F. (1957) Applied Imagination. New York, Scribner's (revised edition).

Parsons, P., Sedig, K., Didandeh, A., \& Khosravi, A. (2015). Interactivity in visual analytics: Use of conceptual frameworks to support human-centered design of a decision-support tool. In System Sciences (HICSS), 2015 48th Hawaii International Conference on (pp. 1138-1147). IEEE.

Parsons, T. (2009, October) The Myths of User-Centered Design. Retrieved from http://www.designcurial.com/news/the-myths-of-user-centred-design

Quick, K., \& Feldman, M. (2011) Distinguishing participation and inclusion. Journal of Planning Education and Research, 272-290.

Ratto, M. (2011). Critical making: Conceptual and material studies in technology and social life. The Information Society, 27(4), 252-260.

Regnér, P. (2003). Strategy creation in the periphery: Inductive versus deductive strategy making. Journal of Management Studies, 40(1), 57-82.

Rittel, H. W., \& Webber, M. M. (1973). Dilemmas in a general theory of planning. Policy Sciences, 4(2), 155-169.

Russo, M. (2013). Redefining process and progress for the Puget Sound Partnership: Employing Participatory Action Research to move the Agenda forward. White paper. Retrieved from https://actionresearch.mit.edu/sites/default/files/documents/Russo_PAR2013final_1.pdf 
Sanders, E. B.-N., Brandt, E. \& Binder, T. (2010, November). A framework for organizing the tools and techniques of participatory design. In Proceedings of the $11^{\text {th }}$ Biennial Participatory Design Conference. (pp. 195-198)..

Sanders, E. B.-N., \& Stappers, P. J. (2008). Co-creation and the new landscapes of design. Codesign, 4(1), 5-18.

Sanders, E. B.-N., \& Stappers, P. J. (2012). Convivial Toolbox: Generative Research for the Front End of Design, BIS, Amsterdam.

Sanders, E. B.-N. (2015) The fabric of design wisdom. Current Design Research Journal, (6).

Satoh, I. (2006). Location-based services in ubiquitous computing environments. International Journal on Digital Libraries, 6(3), 280-291.

Schich, M., \& Coscia, M. (2011). Exploring co-occurrence on a meso and global level using network analysis and rule mining. Minign and Learning with Graphs, KDD Workshop.

Schön, D. (1984). Problems, frames and perspectives on designing. Design Studies, 5 (3), $132-$ 136.

Seifert, J., Simeone, A., Schmidt, D., Holleis, P., Reinartz, C., Wagner, M., ... \& Rukzio, E. (2012, November). MobiSurf: improving co-located collaboration through integrating mobile devices and interactive surfaces. In Proceedings of the 2012 ACM International Conference on Interactive Tabletops and Surfaces (pp. 51-60). ACM.

Shattuck, P. T., Narendorf, S. C., Cooper, B., Sterzing, P. R., Wagner, M., \& Taylor, J. L. (2012). Postsecondary education and employment among youth with an Autism Spectrum Disorder. Pediatrics, 129(6), 1042-1049. http://doi.org/10.1542/peds.2011-2864

Shortall, S. (2003). Participatory Action Research. In The A-Z of Social Research: a dictionary of key social science research concepts., R.L. Miller and J.D. Brewer (Eds), 225-226. London: Sage.

Simon, H. A. (1969). The Science of Design: Creating the Artificial. In The Sciences of the Artificial. Cambridge, MA: MIT Presss. 3rd ed. 1996, 111-138. 
https://courses.washington.edu/thesisd/documents/Kun_Herbert\%20Simon_Sciences_of_the_Arti ficial.pdf

Snowden, D. (n.d.). SenseMaker® - Cognitive Edge. Retrieved March 01, 2016, from http://cognitive-edge.com/sensemaker/

Sutherland, R (2009). TEDTalks: Life lessons from an ad man. Retrieved from https://www.ted.com/talks/rory_sutherland_life_lessons_from_an_ad_man?language=en

Talking up SenseMaking. (n.d.). Retrieved February 22, 2016, from http://www.humantific.com/tag/sense-making/

Tversky, B. (2004). Narratives of space, time, and life. Mind and Language, 19(4), 380-392.

Van Dijck, J. (2009). Users like you? Theorizing agency in user-generated content. Media, Culture, and Society, 31(1), 41.

VanPatter, G. (2015). Making Sense of Strategic Design 2015 [Interview by A. Barroso]. Retrieved from http://www.humantific.com/making-sense-of-strategic-design-2015/

VanPatter, G. K., \& Jones, P. (2009). Design 1.0, 2.0, 3.0, 4.0-The Rise of Visual SenseMaking. NextD Journal. White paper. Retrieved from http://www.humantific.com/the-rise-ofvisual-sensemaking/

Victor, Bret. (2014) Seeing Spaces. Beast of Burden. Video presentation at the EG conference. Accessed October 30, 2015. http://worrydream.com/.

Visual Sensemaking Booklet. (n.d.). Retrieved February 22, 2016, from http://issuu.com/humantific/docs/visualsensemaking_booklet

Vygotsky, L.S. (1978) Mind in Society: The Development of Higher Psychological Processes. Cambridge, MA Harvard University Press. 71-91. 
Wack, P. (1985). Scenarios: Uncharted waters ahead; how Royal Dutch-Shell developed a planning technique that teaches managers to think about an uncertain future. Harvard Business Review, 63.

Weick, K. E., Sutcliffe, K. M., \& Obstfeld, D. (2005). Organizing and the process of sensemaking. Organization Science, 16(4), 409-421.

Woods, A., \& Joyce, P. (2002). The arrival of postmodernist strategic theory: Its academic manifestation and the development of a new modernist perspective. Strategic Change, 11(2), 7180.

Wright, A. (2005). The role of scenarios as prospective sensemaking devices. Management Decision, 43(1), 86-101.

Zagal, J. P., Rick, J., \& Hsi, I. (2006). Collaborative games: Lessons learned from board games. Simulation \& Gaming, 37(1), 24-40.

Zimmerman, E. (2013) Manifesto for a Ludic Century. Retrieved from http://ericzimmerman.com/files/texts/Manifesto_for_a_Ludic_Century.pdf

Zinsmaier, M., Brandes, U., Deussen, O., \& Strobelt, H. (2012). Interactive level-of-detail rendering of large graphs. IEEE Transactions on Visualization and Computer Graphics, 18(12), 2486-2495. 


\section{Appendix}


Appendix A: Pre-Phase Materials and Preparation 
Your participation in this questionnaire is completely voluntary. You may stop at any time.

Please consider each question carefully. Feel free to ask us to clarify any question that is not clear.

CIRCLE ONE: ADULTWWITH AUTISM | PARENT/CAREGIVER | HEALTH CARE PRACTITIONER | ADMINISTRATOR

Ranking: 1=lowest $\quad 10=$ highest

1 Rate your current understanding of the daily issues facing individuals with autism and their families.

$\begin{array}{llllllllll}1 & 2 & 3 & 4 & 5 & 6 & 7 & 8 & 9 & 10\end{array}$

2 Rate your current understanding of the needs individuals with autism have as they transitions into adulthood.

$\begin{array}{llllllllll}1 & 2 & 3 & 4 & 5 & 6 & 7 & 8 & 9 & 10\end{array}$

3 Rate your current understanding of the challenges practitioners have regarding the transition of adults with autism.

$\begin{array}{llllllllll}1 & 2 & 3 & 4 & 5 & 6 & 7 & 8 & 9 & 10\end{array}$

4 Rate your knowledge of administrative processes as they relate to Autism Spectrum Disorder (Medicare, forms, billing procedures, coding, etc.)

$\begin{array}{llllllllll}1 & 2 & 3 & 4 & 5 & 6 & 7 & 8 & 9 & 10\end{array}$

5 What level of expertise do you feel you have when it comes to navigating the health care services and support offerings for individuals with autism?
Novice
Intermediate
Skillful
Experienced
Expert

6 How empowered do you feel with making changes and improvements to the current problems surrounding ASD?

$\begin{array}{llllllllll}1 & 2 & 3 & 4 & 5 & 6 & 7 & 8 & 9 & 10\end{array}$

7 What are your current feelings towards collaboration around complex problems? (Circle all that apply.)

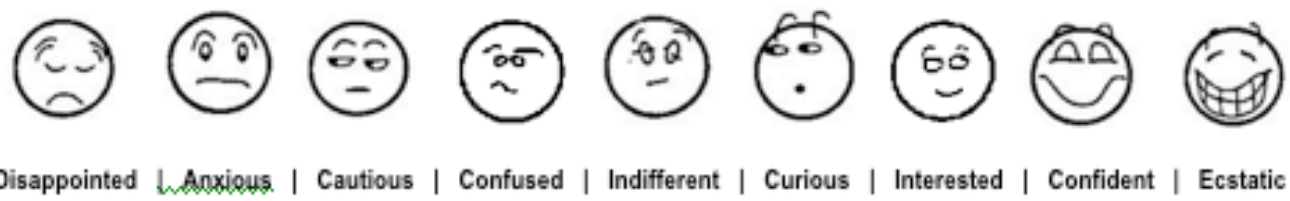


8 Have you heard of Design Thinking?

Yes No

9 Have you used Design Thinking methods to solve problems in the past?

Yes

No

10 What do you hope to accomplish during this project?

11 What do you hope we will accomplish during this project?

12 There is value in gaining shared understanding between multiple stakeholders (connected to ASD) before discussing ideas and design opportunities.

$\begin{array}{llll}\text { Disagree } & \text { Somewhat Disagree } & \text { Somewhat Agree } & \text { Agree }\end{array}$

13 There is value in prototyping and building potential resolutions to complex problems with multiple stakeholders.

Disagree $\quad$ Somewhat Disagree $\quad$ Somewhat Agree $\quad$ Agree

14 Visualizations and diagrams help me better understand complex ideas and problems.

Disagree $\quad$ Somewhat Disagree $\quad$ Somewhat Agree $\quad$ Agree

15 What are your current feelings towards the Center for Autism Services \& Transition (Circle all that apply.).

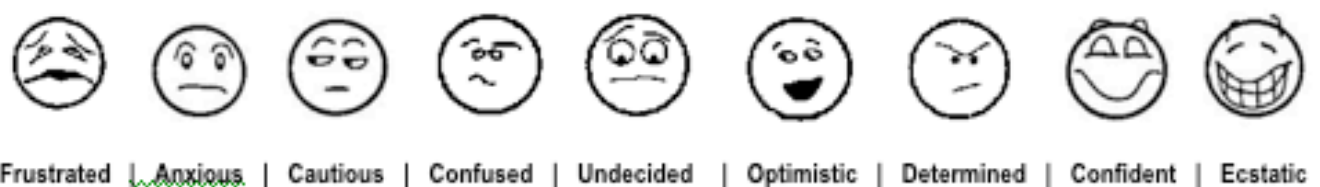




\section{Pre-Assessment Questionnaire Response Summary}

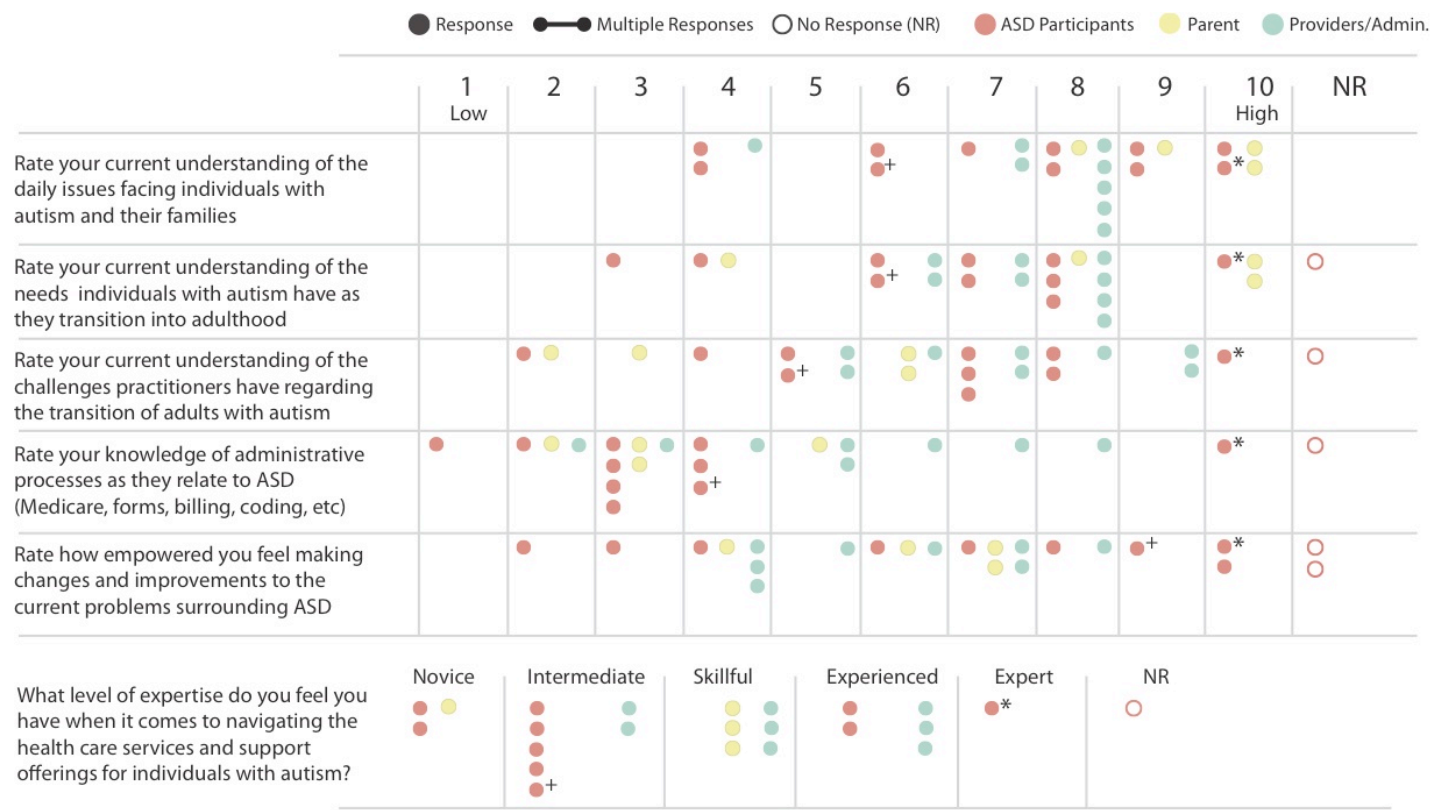

Current feelings towards collaboration around complex problems (circle as many as you'd like)

(Each row represents a person from a stakeholder group- i.e. four rows, four parents)

Disappointed Anxious Cautious Confused Indifferent Curious Interested Confident Ecstatic NR

Current feelings towards the design of a new health care center for adults with ASD

(Each row represents a person from a stakeholder group- i.e. four rows, four parents)

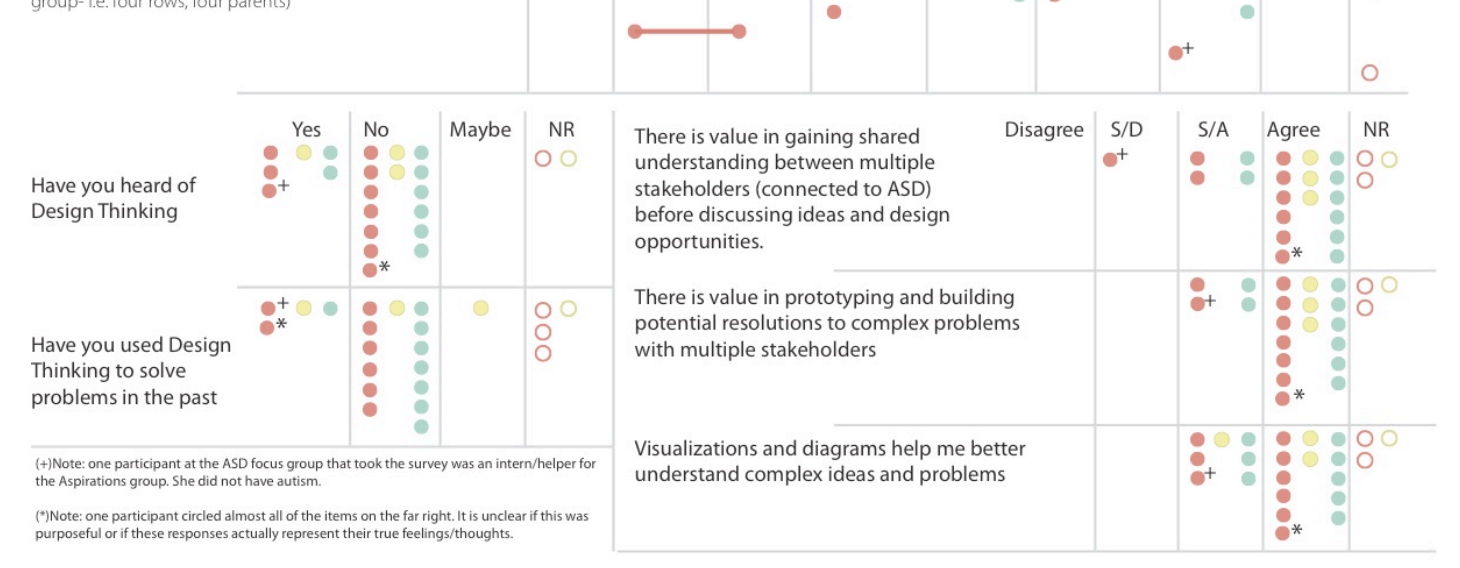

\begin{tabular}{c|l|l|l|l|l|l|l|l|} 
Frustrated & Anxious Cautious & Confused & Undecided & Optimistic & Determined Confident Ecstatic NR
\end{tabular} 


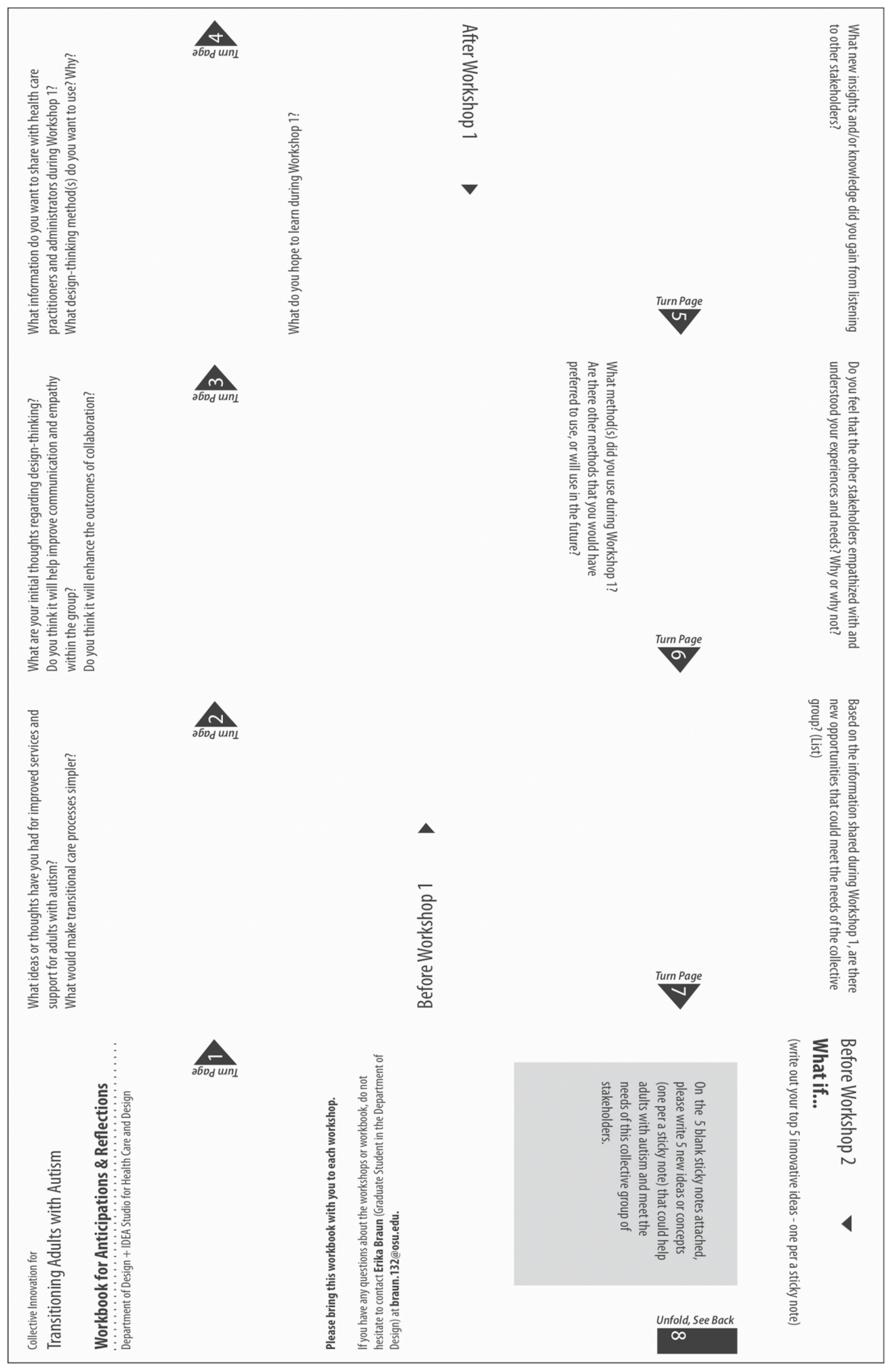




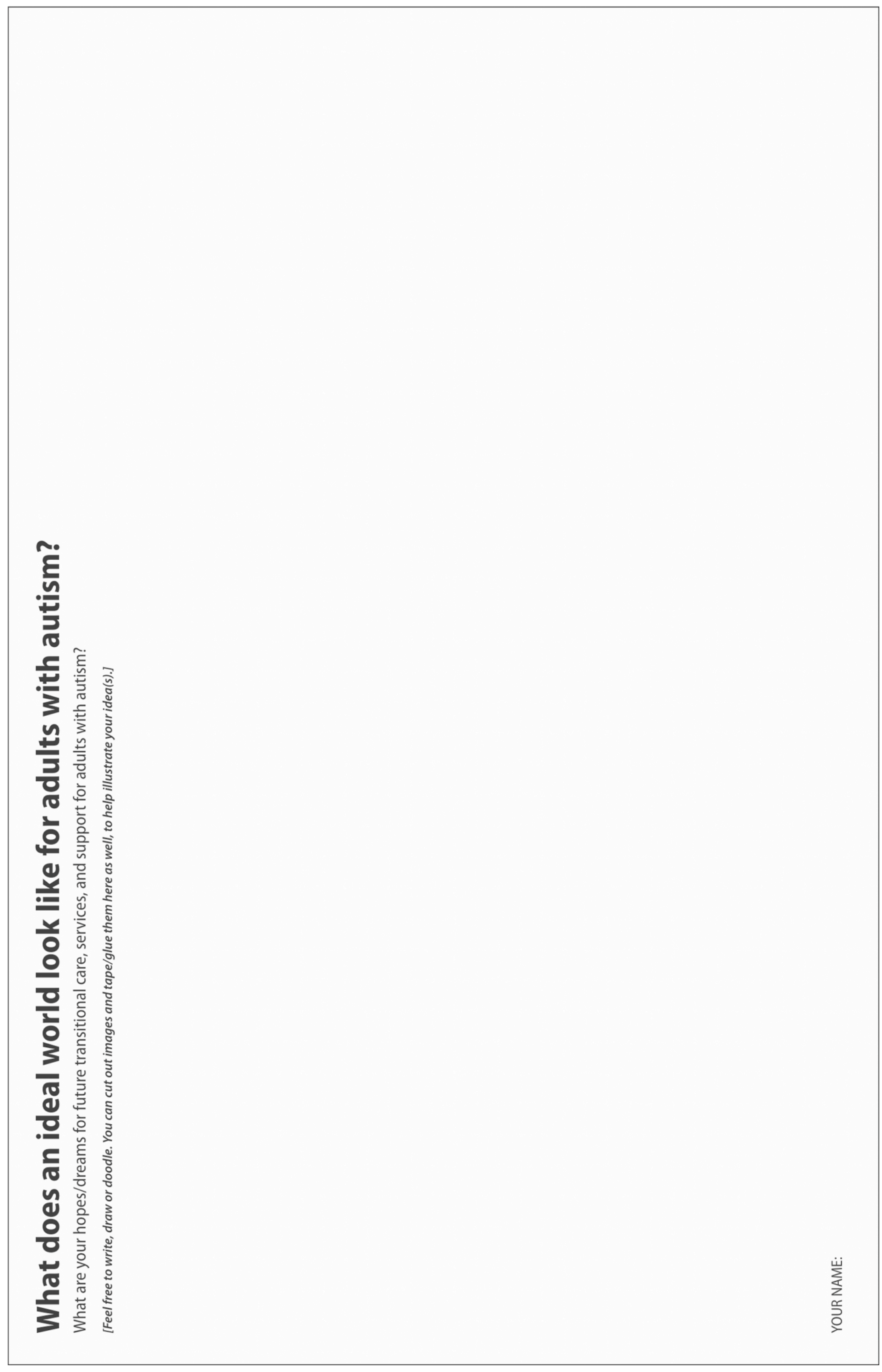


How: Read through the cards to get an overview of the various methods designers use to solve complex (non-linear) problems. During the sessions we will dive deeper into some of the methods. Next, select the method(s) you would like to use to help communicate information you want to share, empathy you want to convey, and ideas you want to explore.

Why: These cards are designed to help you organize and share your thoughts, build broader and deeper understanding of the problem(s), processes, and people, and help stimulate creative thinking. These are not the only methods designers use. For information on design thinking, these methods, and more, go to:

http://dschool.stanford.edu/use-our-methods/ http://www.101designmethods.com/
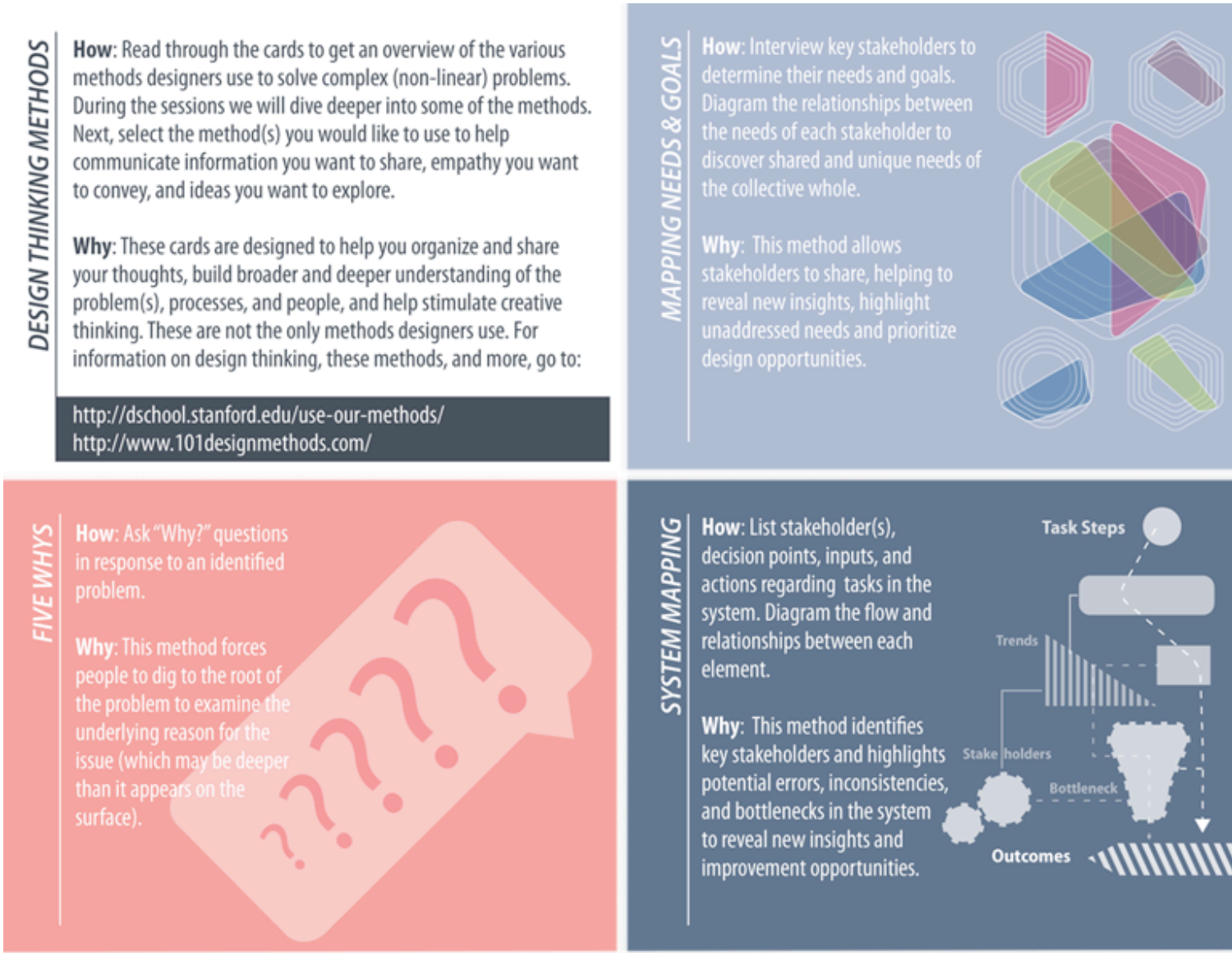

How: Assemble key stakeholders. Have each person share a story from a current, past, or future "day-in-the-life" occurrence by acting it out. Stakeholders can switch roles to gain a better understanding of what it is like "to walk in someone else's shoes."

Why: By enacting and sharing scenarios, within real or imagined contexts, team members can begin to empathize with one another and become more aware of unexpected insights, unmet needs and relevant issues.
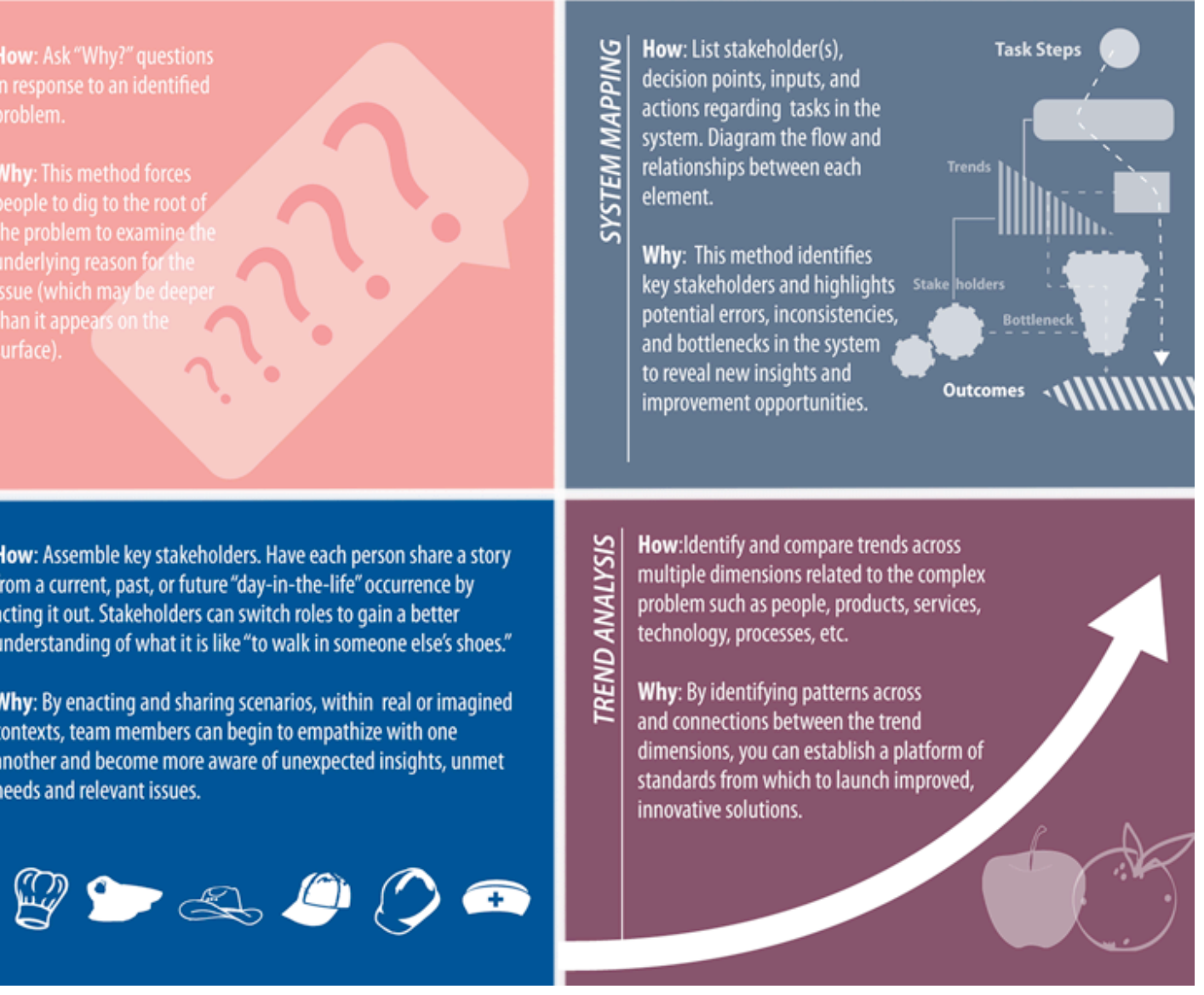

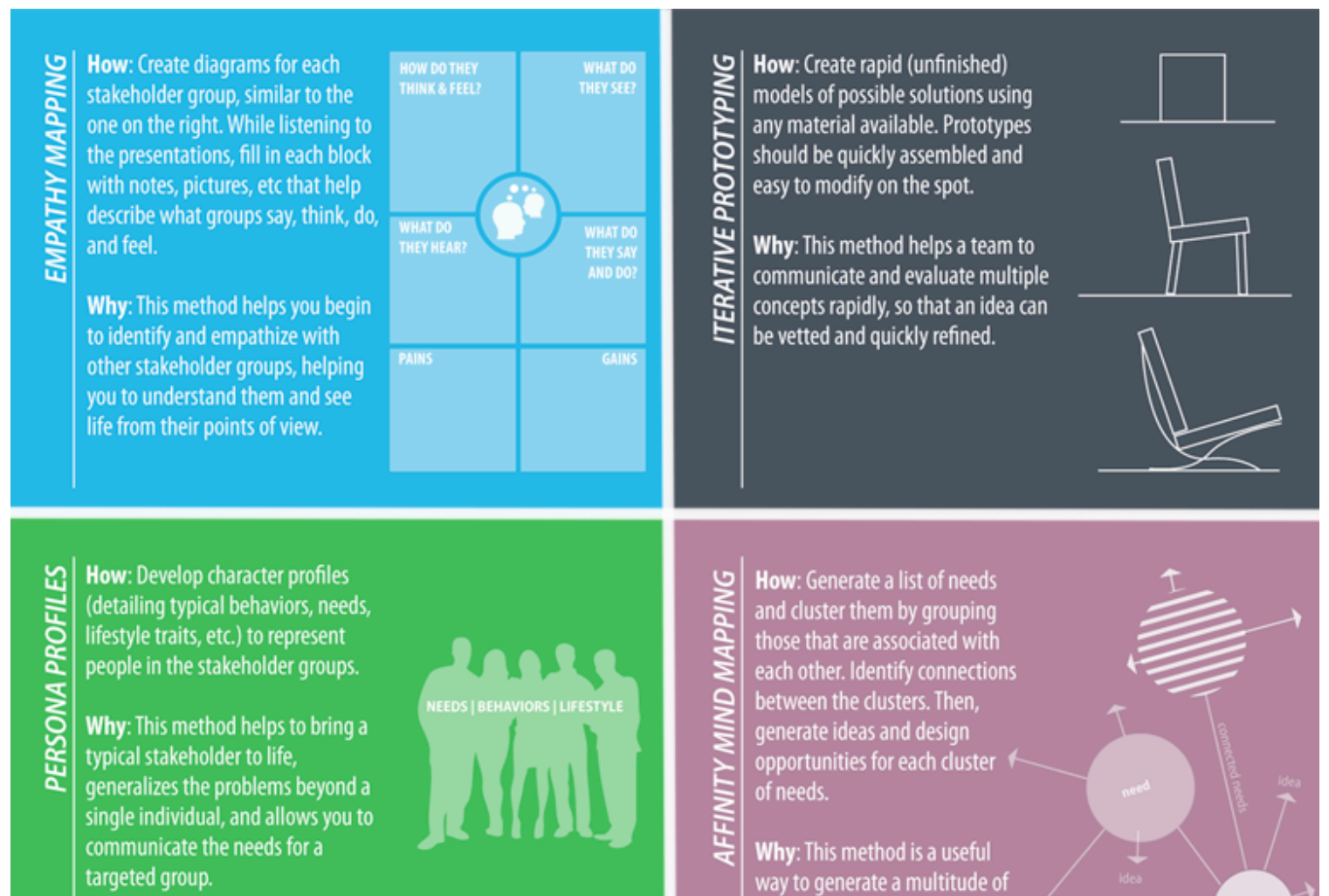
targeted group.
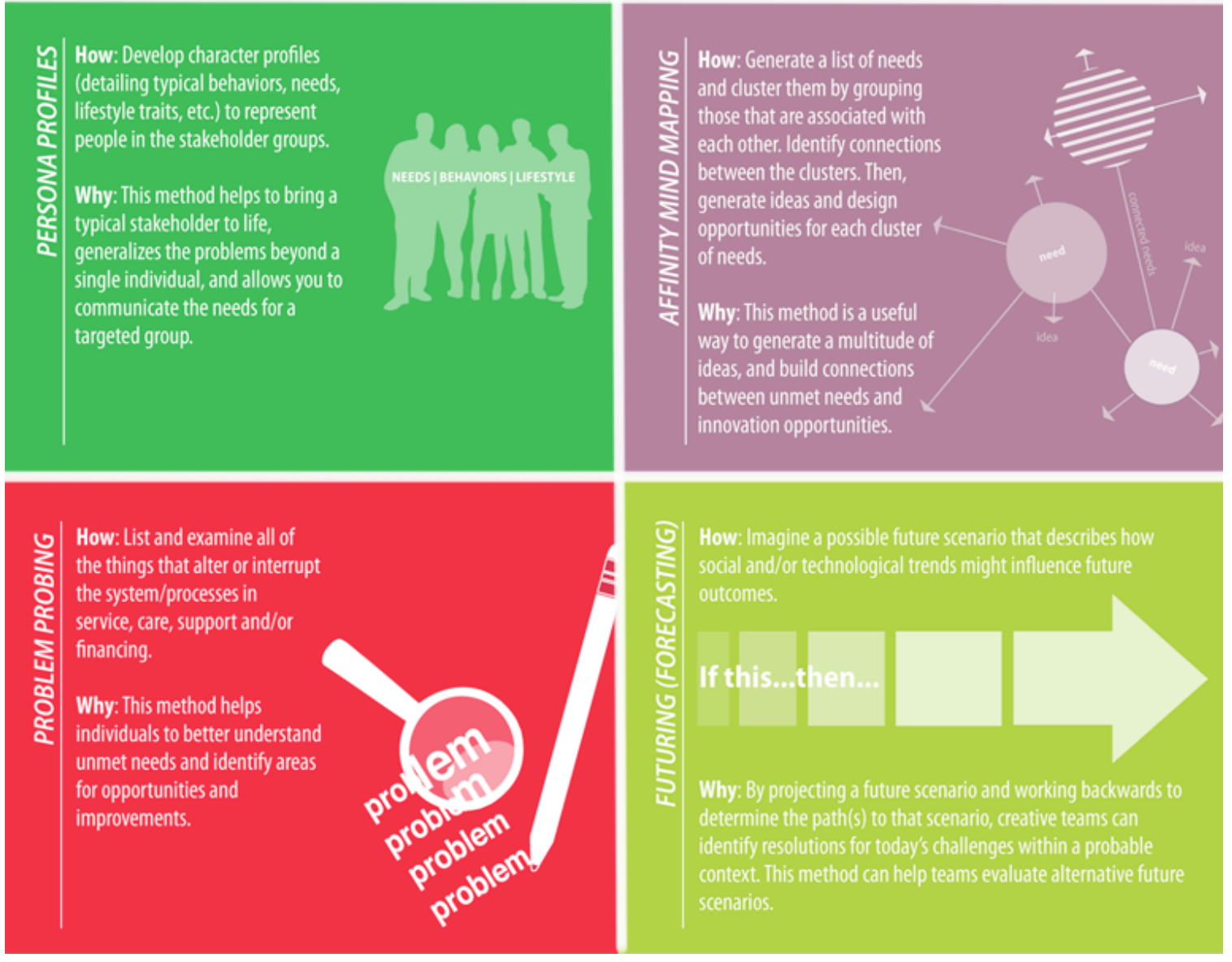


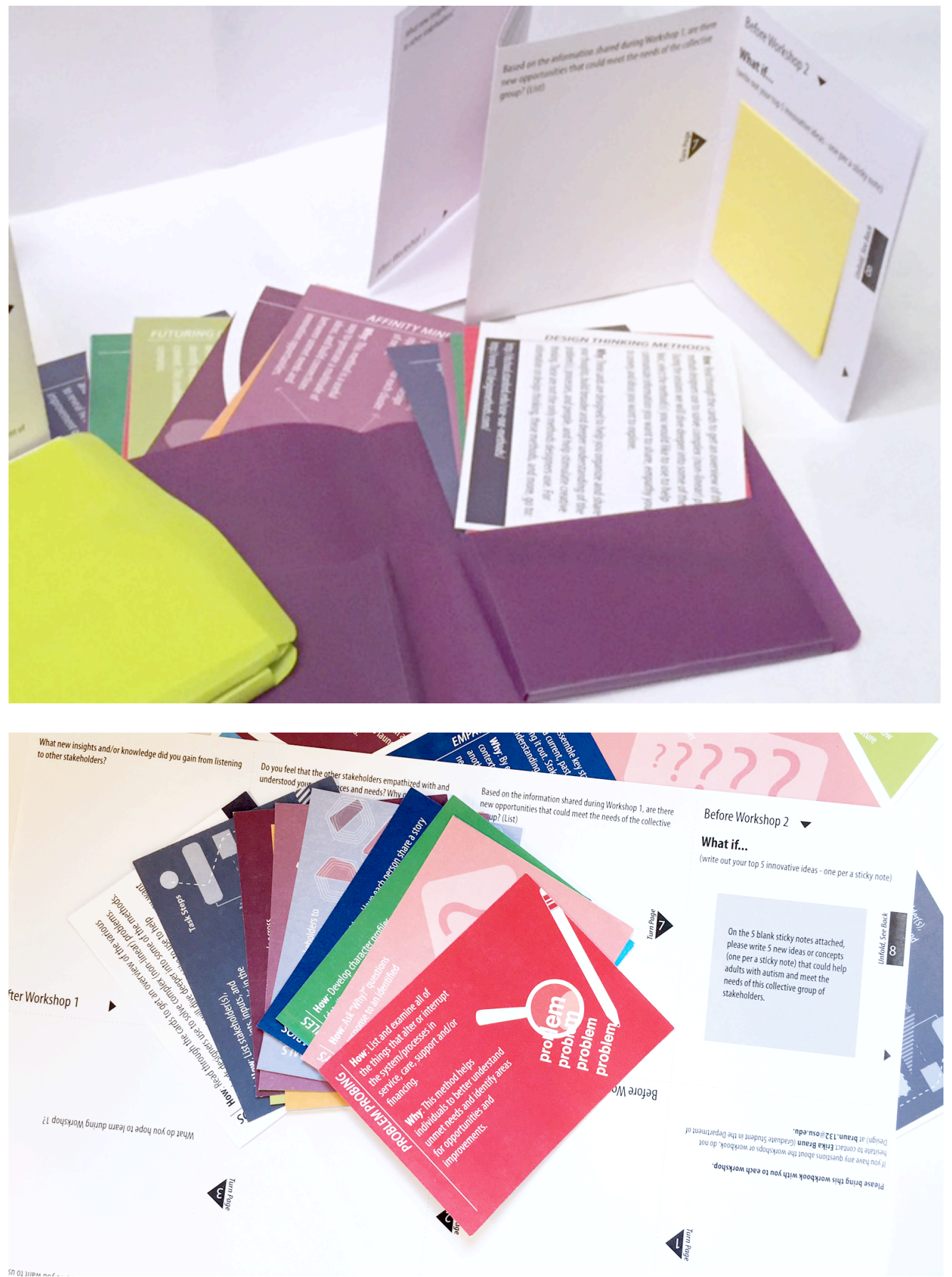


Appendix B: Phase 1 - Interviews and Focus Groups 


\section{Semi-structured Interview Guide}

\section{Semi-structured Interview:}

The aim of the interviews is to understand and visualize the complex system surrounding ASD.

\section{Estimated Total Time:}

1 Hour Individual Sessions

\section{Objective:}

Explaining the complex system and processes in healthcare, surrounding ASD by:

1 Discussing ASD healthcare processes, data, trending, etc.

2 Co-creating visualizations and maps to convey information for Workshop 1.

\section{Materials:}

Audio recorder

Paper, pen, markers for drawing diagrams and mapping processes

Sticky notes and poster board

Provotypes (concepts/maps) made in prior interviews

Spreadsheets or other materials needed to explain healthcare processes, financial allocations, etc.

Pre-Assessment Questionnaire

Reflection and preparatory Journal

Method Cards

\section{Homework:}

Write reflections and anticipations in Journal throughout various phases of the project.

\section{Agenda:}

1 Brief Introductions I 15 Min
a. Sign consent forms
b. Administer pre-assessment questionnaire
c. Research Team Introductions
d. Explanation of project objectives and goals

2 Discussion Questions I 20 Min

a. What is the current transition process for adults with autism?

b. What/Who are the main touch points in the current process?

i. What/Who are missing in the current process?

c. What are the current trends/data regarding ASD and adults with ASD?

i. What are the patterns of change in this field over the last 10 years?

ii. What are the trends/projects in this field in the next 10 years?

d. What key information do you need parents to understand from your perspective?

e. What do you hope we accomplish during this project?

3 Co-creation: Map processes and visualize information to be conveyed to others I 20 Min

a. Current environment/processes

b. Future environment/processes

4 Introduce Journal/Method Cards I 5 Min

5 Post Interviews 
a. Answer any follow up questions

b. Follow up with finalized visualizations/presentation material to ensure they accurately represent the information the participants want to share during Workshop 1 
COORDINATED CARE

inot iust for ASD? Alternative Health Gare Model?

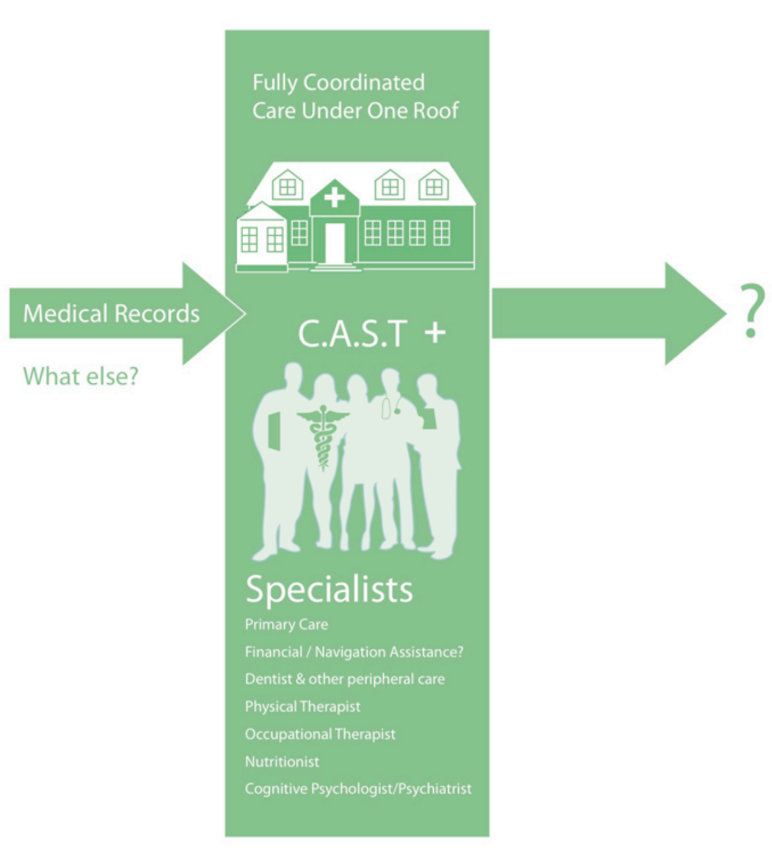

No medical care offered in clinic

TRANSITIONAL CARE

Not just for ASD? All developmental disabilities?

What about the aning population?

o they JUST need navigators to help then

chrough the system that is already in place?

(nstead of making/altering the entire system?

Does OsUMCiust need to provide/organize a

secialty service providers?

Specialists? Do they transition

to new specialist or keep the same?

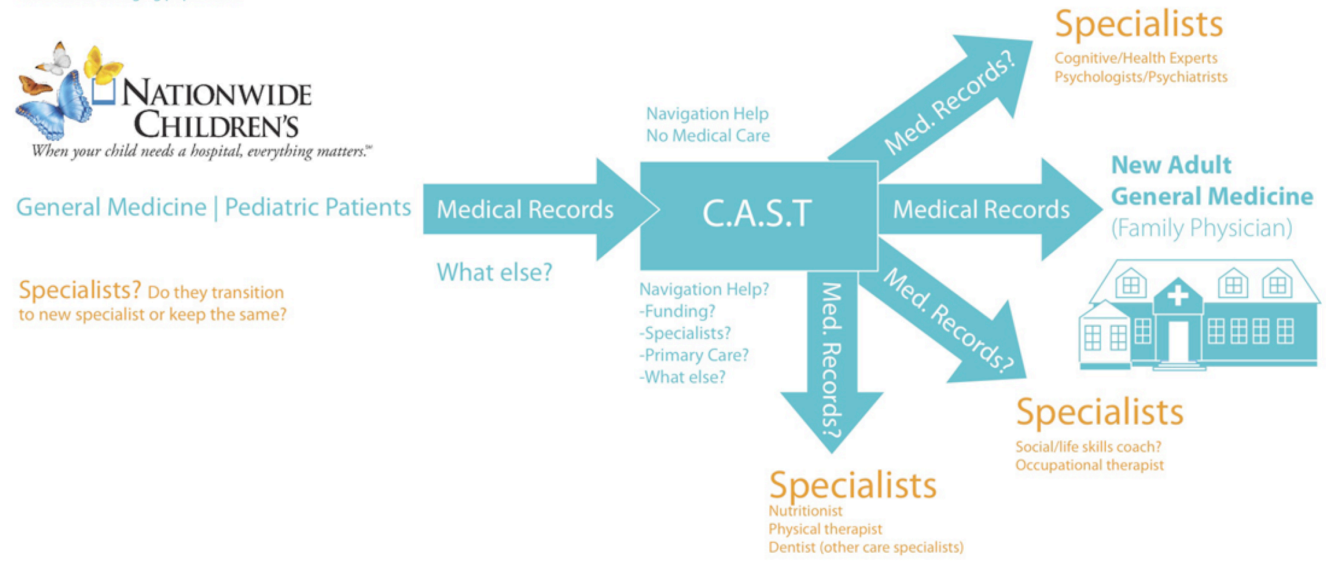


Doctor, many patients. Family Practice

GENERAL MEDICINE

Just for ASD patients? All developmental disabilities?

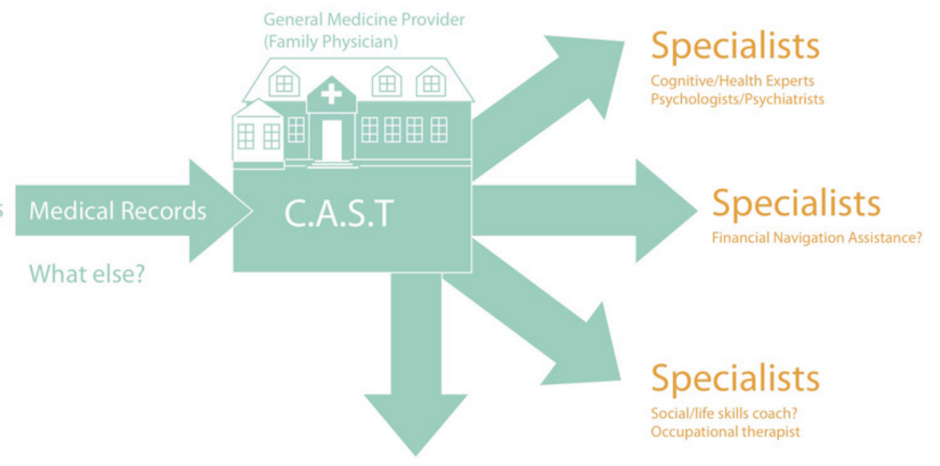

Specialists

Nutritionist

Physical therapist
Dentist (other care specialists

1 COORDINATED CARE CENTER

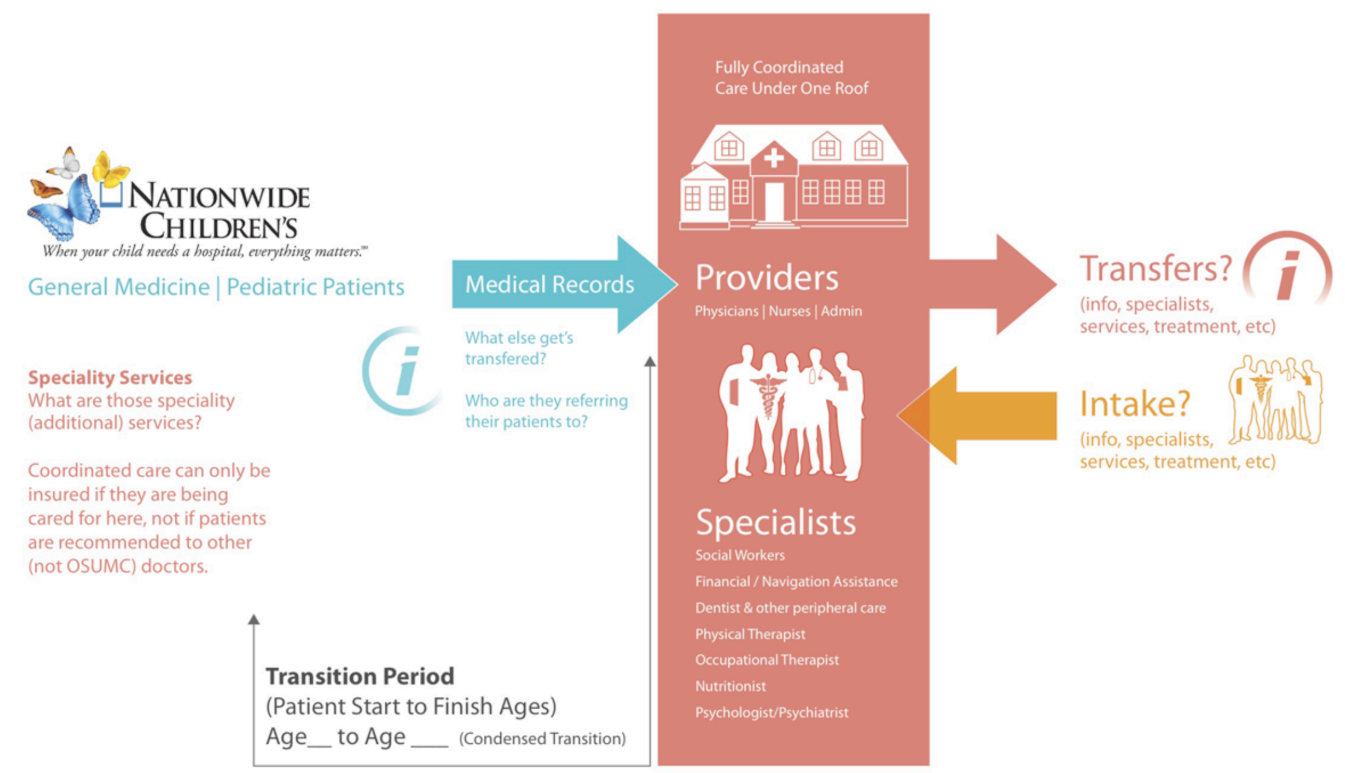


2 TRANSITIONAL SERVICE

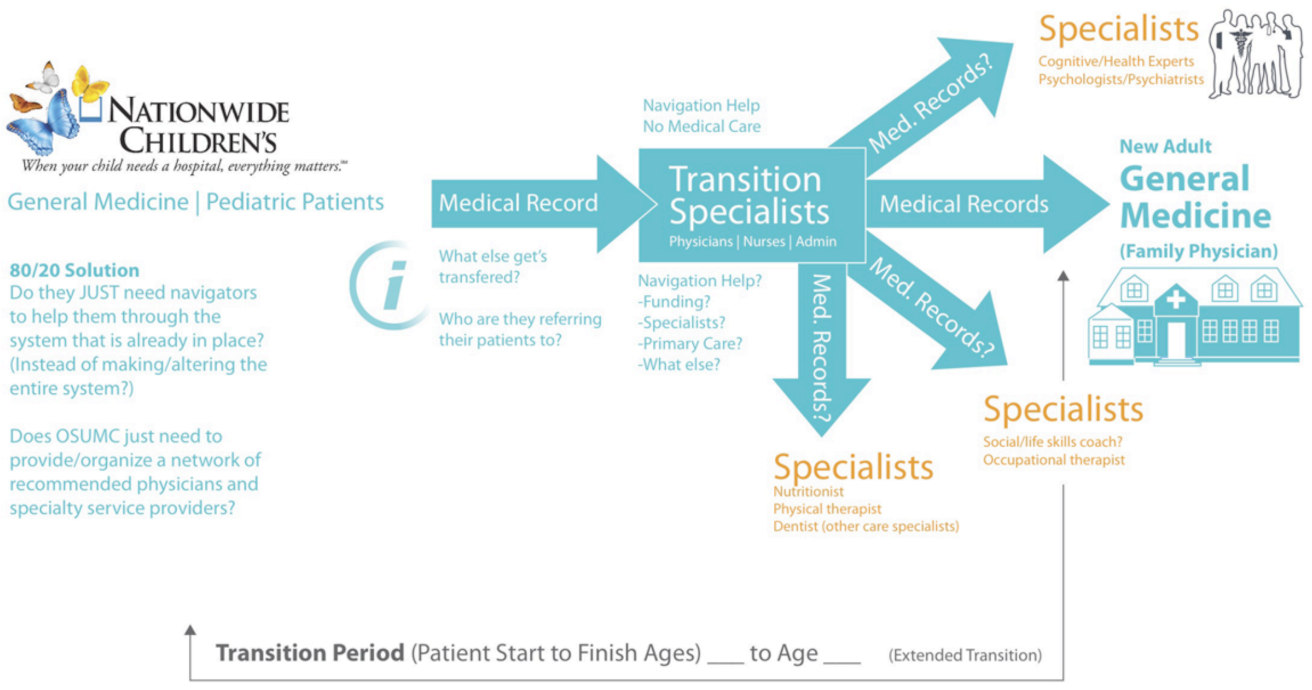

\section{CARE SERVICE TEAM}

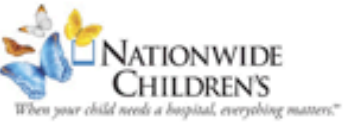

General Medicine I Pediatric Patients Medical Records

Family Practice Model

Are patients coming to clinic for

regular checkups or specific health

care issues (related to ASD)?

Is ASD being treated as a disease of

is it a social factor and youre

treating other things?

Remote Team (Not all housed in the same building.)
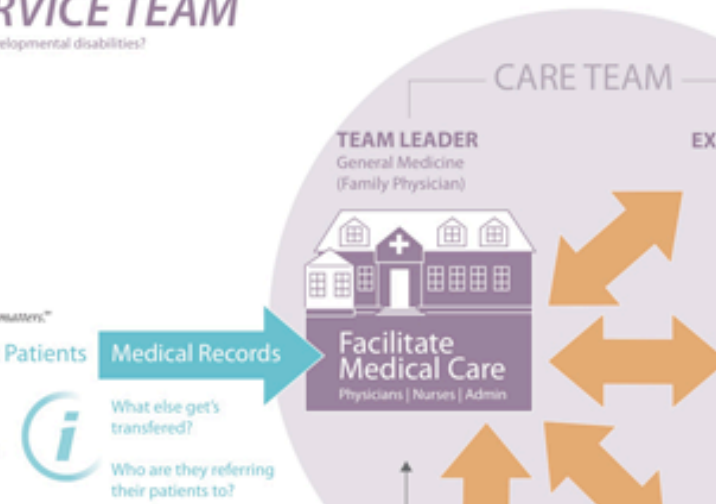

MANAGER / COORDINATOR?

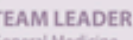
T:neral Medicine

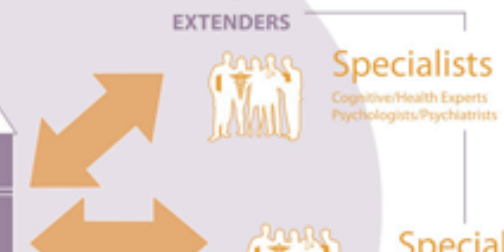
Family Physician

\section{sts}

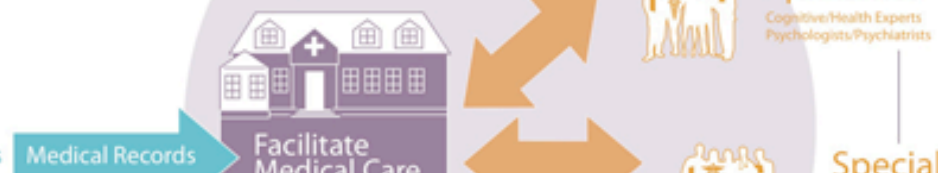

Medical Care
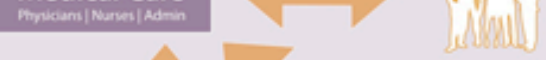

financie Numignion their patients to?

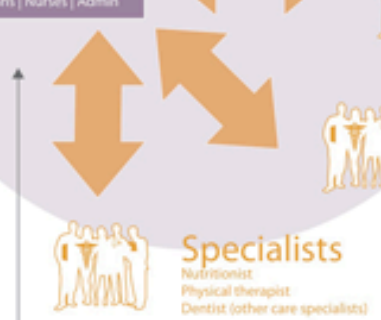

wistan

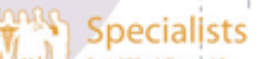

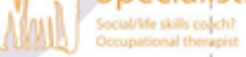

sition Period (Patient Start to Finish Ages)

Extended Transition Period (for Specialists) - to Age 


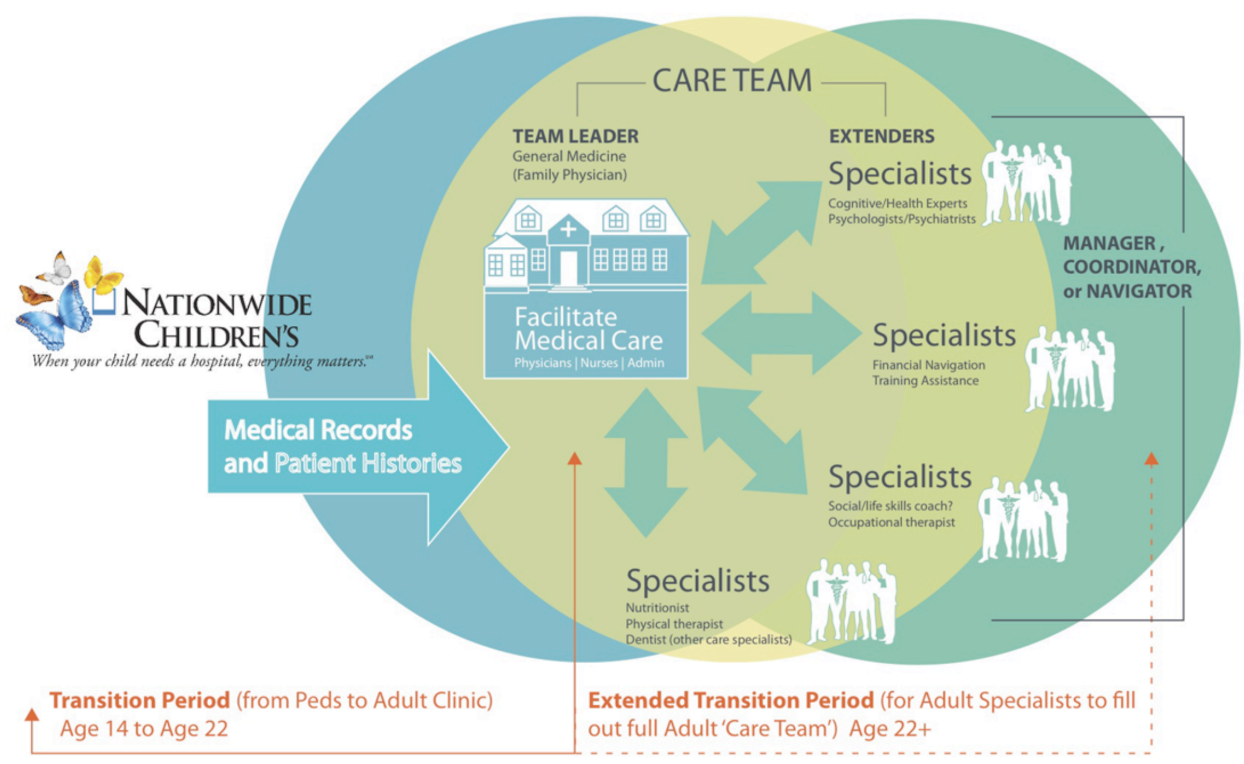




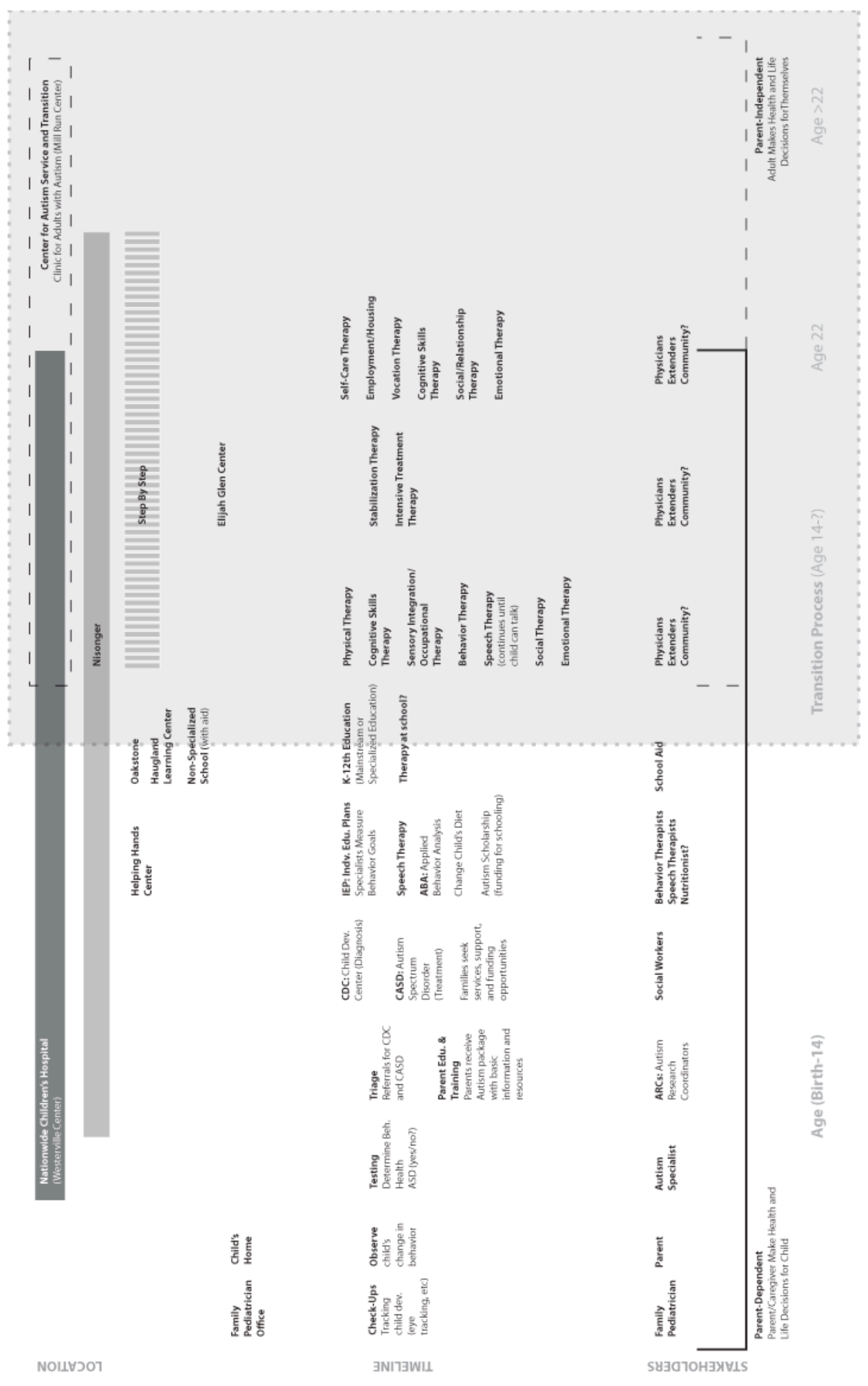


Guide for Focus Group 1 (ASD Participants at Donatos)

\section{Focus Group Discussion:}

The aim of this session will be to better understand the struggles and experiences of adults with autism by giving them an opportunity to voice their own insights, needs, and ideas for improved services and support. They will be briefly introduced to various Design Thinking methods to help them communicate their ideas and needs.

\section{Estimated Total Time:}

1-2 Hours (Depending on their attention span and comfort levels)

\section{Objective:}

Understanding Adults with Autism and helping them to express their needs and ideas by:

1 Learning about the current experiences for adults with autism.

2 Discussing unmet needs and opportunities for improvement inside and outside the clinic setting.

3 Using generative tools to help them express ideas, needs, concerns, and unique insights.

\section{Materials:}

Audio and visual recorder

Sticky notes, markers, tape/glue

2D generative tools (e.g., poster board, paper cutout shapes, images, and colored paper)

$3 \mathrm{D}$ generative tools (e.g., blocks and LEGOs)

Reflection and preparatory Journals and Method Cards

\section{Homework:}

Write reflections and anticipations in Journal throughout various phases of the project.

\section{Agenda:}

1 Welcome \& Brief Introductions I 5 Min
a. Sign consent forms
b. Administer pre-assessment questionnaire
c. Explanation of project objectives

2 Adults with autism share their current experiences and needs I 15-30 Min

3 Researchers introduce Design Thinking methods for communication and idea generation (with Method Cards) I 15-30 Min

4 Adults with autism work with researchers to construct their visions for a better clinic experience I 30Min -1 Hour

a. 2D and/or 3D generative tools can be used

$5 \quad$ Wrap-Up I 5 Min

a. What do you hope we (you) can accomplish during this project?

b. Introduce homework

6 Post Focus Group

a. Answer any follow up questions

b. Follow up with finalized visualizations/presentation material to ensure they accurately represent the information the participants want to share during Workshop 1 
Guide for Focus Group 2 (Parents and Healthcare Providers/Administrators)

\section{Focus Group Discussion:}

Helping non-designers understand what Design Thinking is and how to use various methods to establish empathy with other stakeholders, while communicating individual experiences and needs through a sticky note exercise.

\section{Estimated Total Time:}

\section{1-2 Hours}

\section{Objective:}

Understanding the various priority concerns/needs/goals of parents and healthcare

providers/administrators by:

1 Sharing various Design Thinking methods for communication/expression (telling and making).

2 Using sticky notes to write down and present unique priorities

3 Seeking out similar and conflicting priorities among the differing stakeholder groups

4 Selecting methods to use during Workshop 1 for sharing needs, concerns, and unique insights.

\section{Materials:}

Audio and visual recorder

Sticky notes, markers, tape

A large roll of paper for collective mapping (putting sticky notes up on the same surface)

Gathered research from interviews

Reflection and preparatory Journal and Method Cards

\section{Homework:}

Write reflections \& anticipations in Journal throughout various phases of the project.

\section{Agenda:}

1 Welcome \& Brief Introductions | 15 Min
a. Sign consent forms
b. Administer pre-assessment questionnaire
c. Share gathered research insights from prior interviews

2 Research Team Introduces Design Thinking and Method Cards I 15 Min.

a. Explain what Design Thinking is and how it is being used.

b. Give examples of various Design Thinking methods.

3 Write down top ten concerns, needs, and goals within stakeholder groups I 15 Min.

a. Use individual sticky notes for each concern, need, and goal

4 Present top ten concerns, needs, and goals to other stakeholder group I 15 Min.

a. Place sticky notes up on a shared wall or large roll of paper

b. Identify similarities and difference

5 Prepare a plan of action: Participants decide what content to share and how, by selecting which method(s) they would like to use during Workshop 1 I 15 Min.

6 Wrap-Up I 15 Min

a. Select Individual (or group) Design Thinking method(s). 
b. Discuss why this selection was made.

c. Reflection on focus group and Design Thinking as a whole - what do you hope we accomplish during this project?

d. Introduce homework : Journals

7 Post Focus Group

a. Answer any follow up questions

b. Follow up with finalized visualizations/presentation material to ensure they accurately represent the information the participants want to share during Workshop 1 


\section{ADULTS WITH ASD}
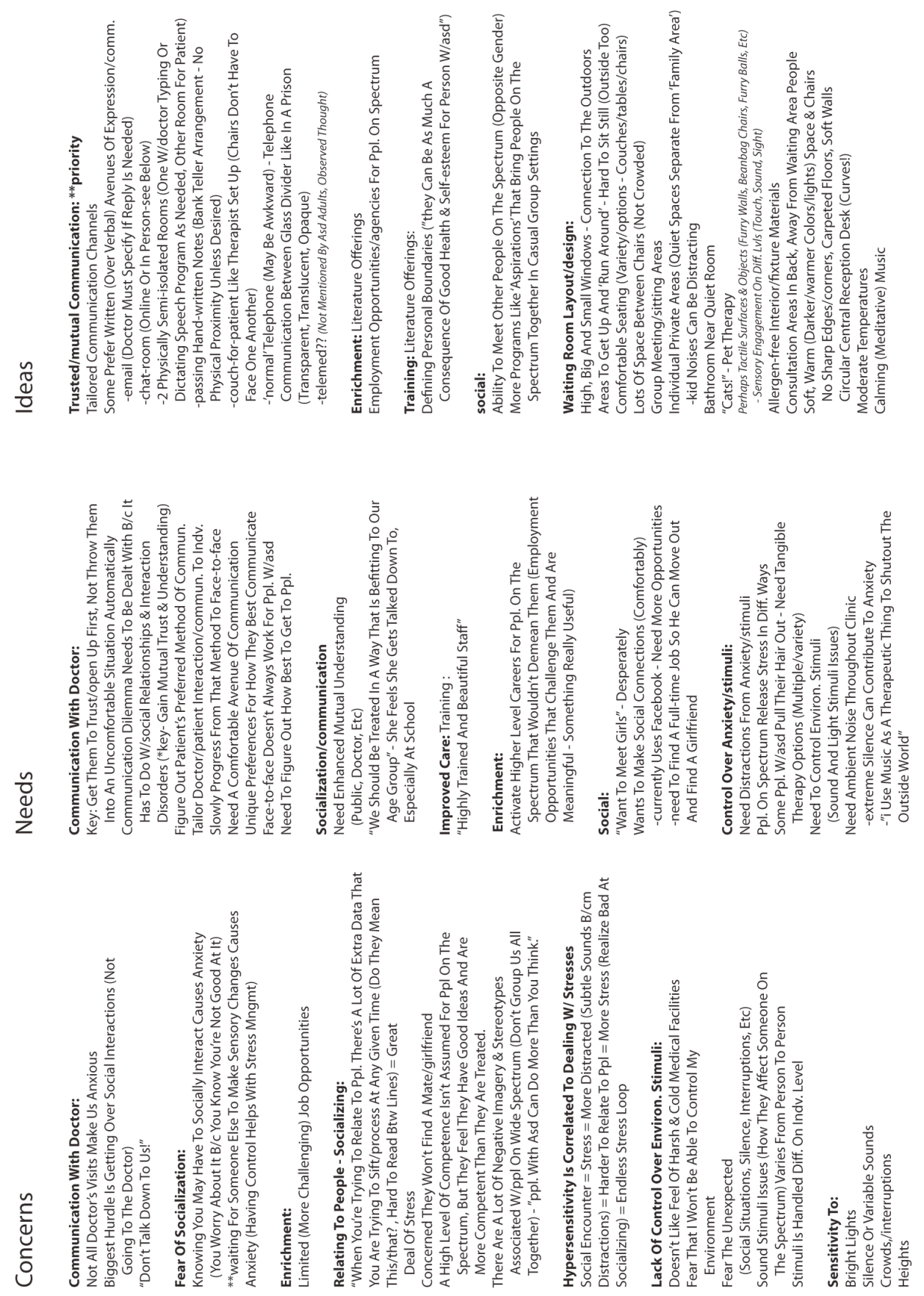


\section{ADULTS WITH ASD CONTINUED}
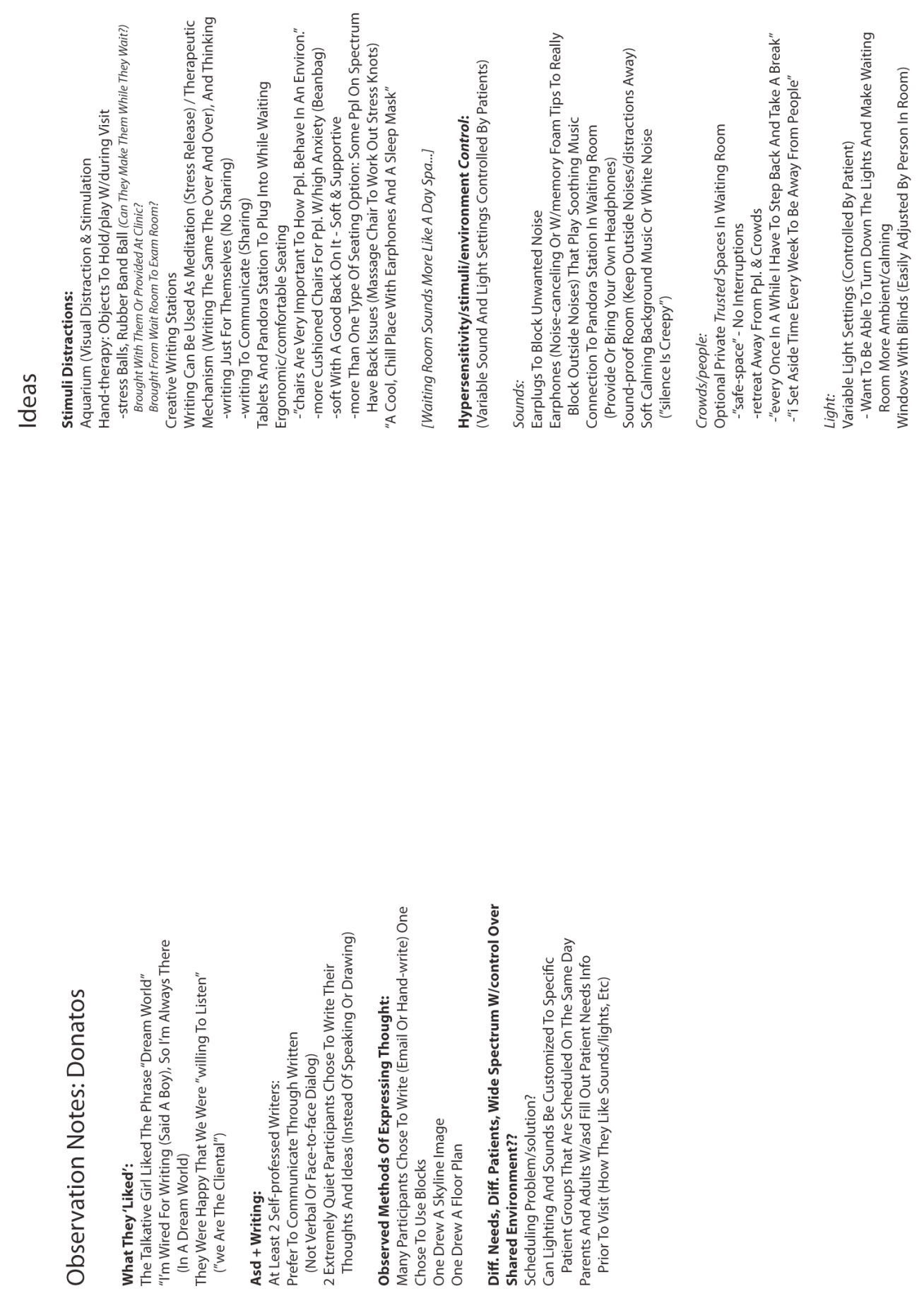


\section{PARENTS}
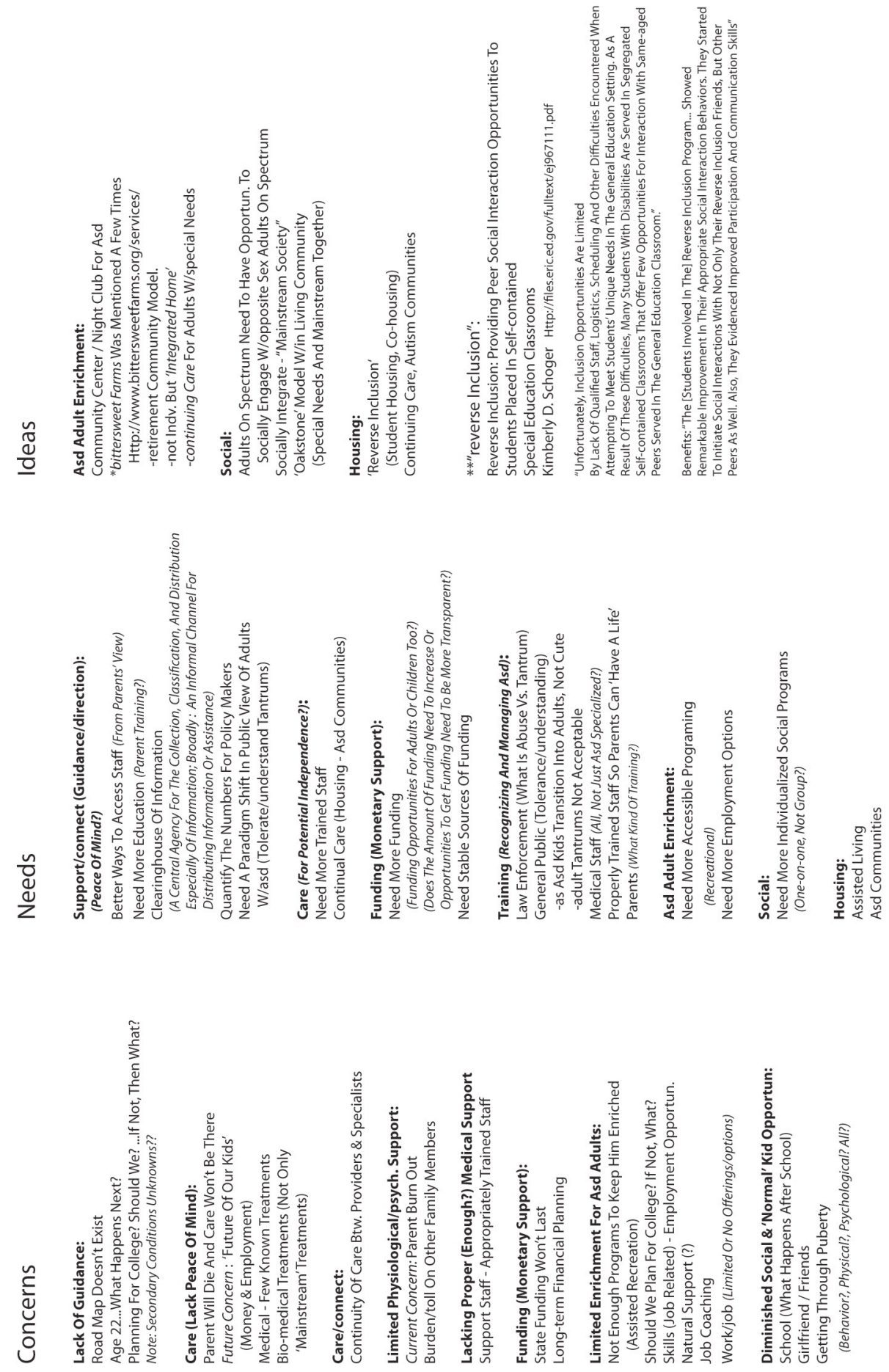


\section{PROVIDERS/ADMIN.}
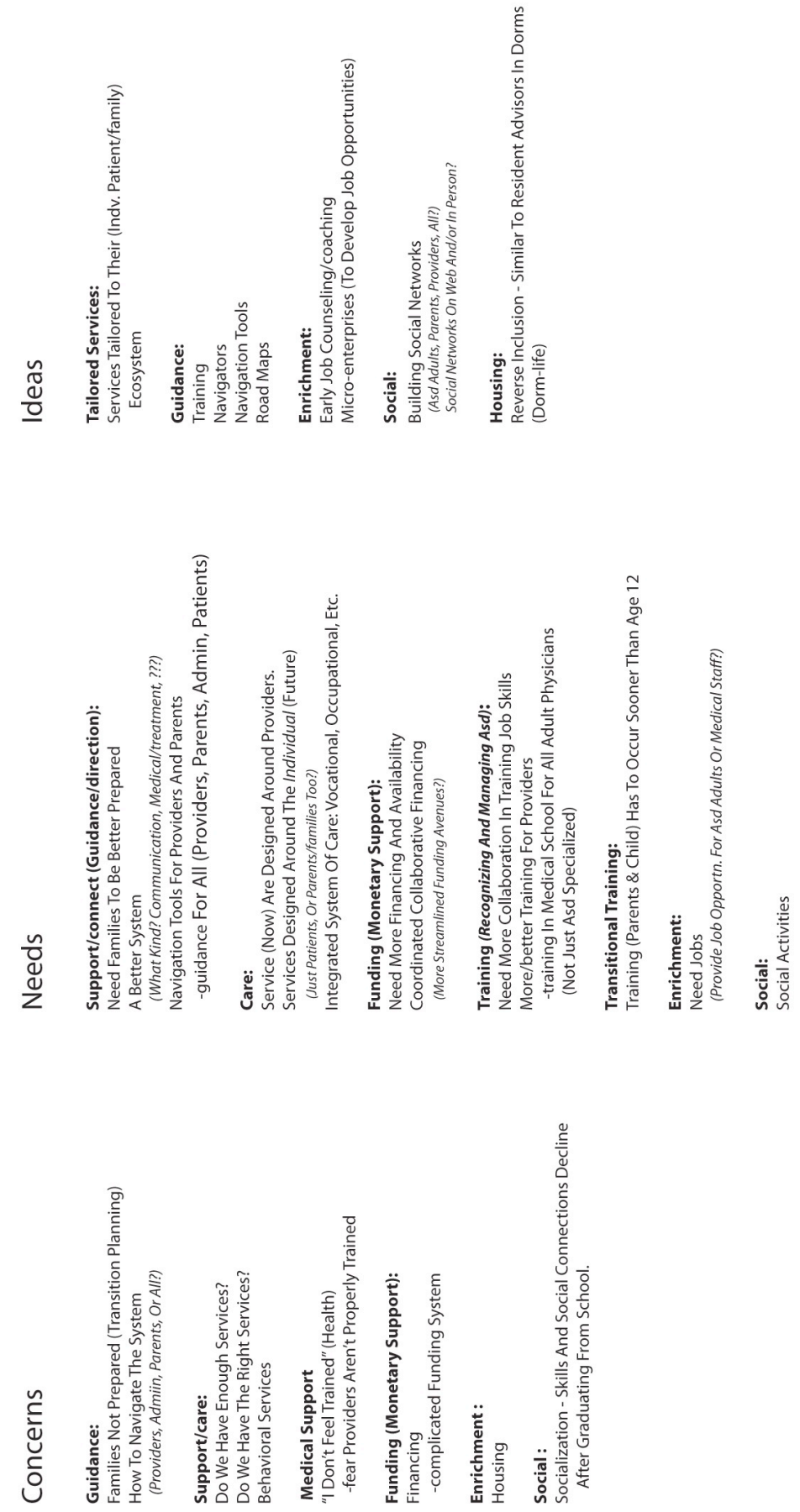


\section{PARENTS \& PROVIDERS/ADMIN.}
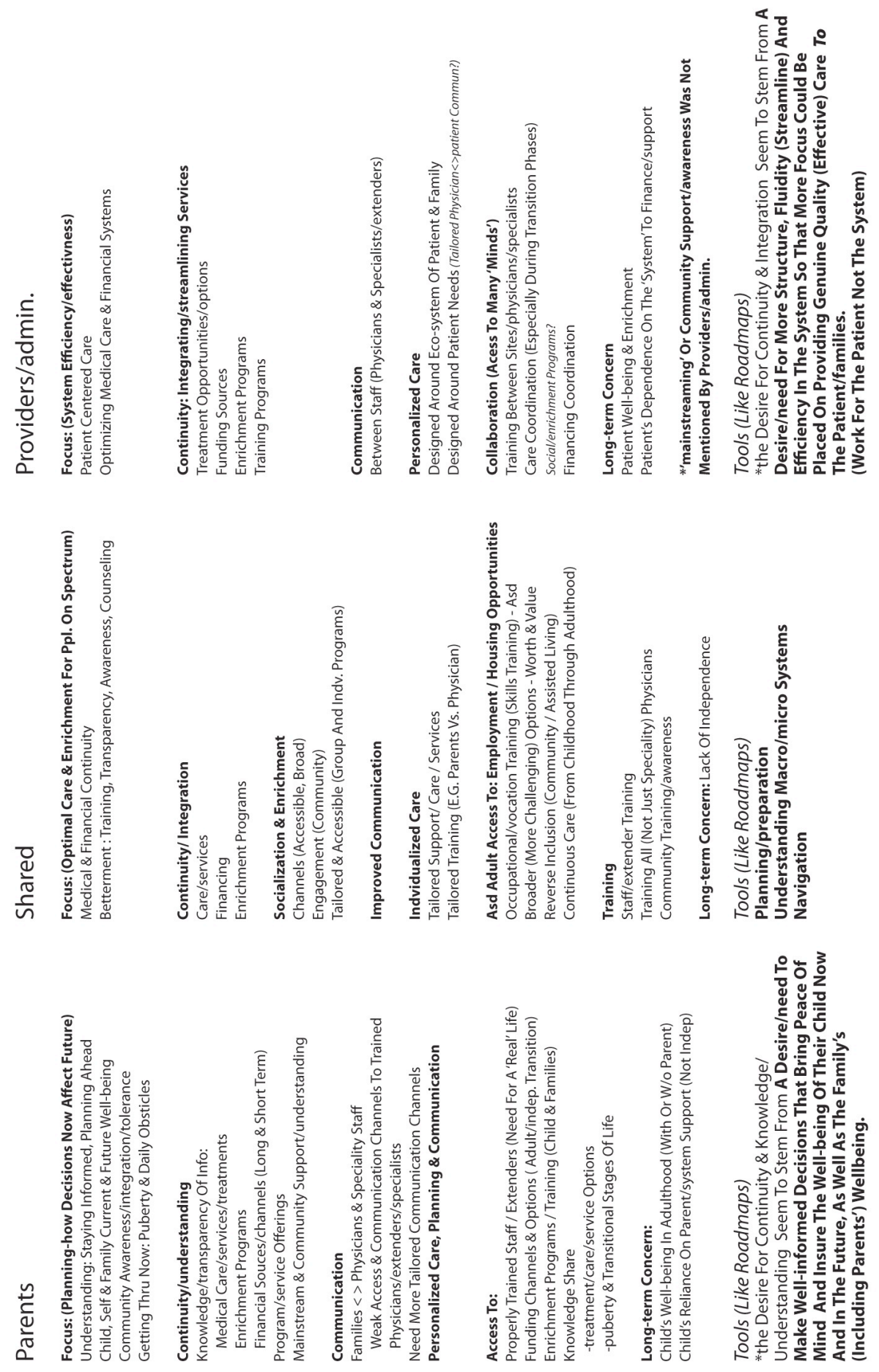


\section{OVERALL OBSERVATIONS}
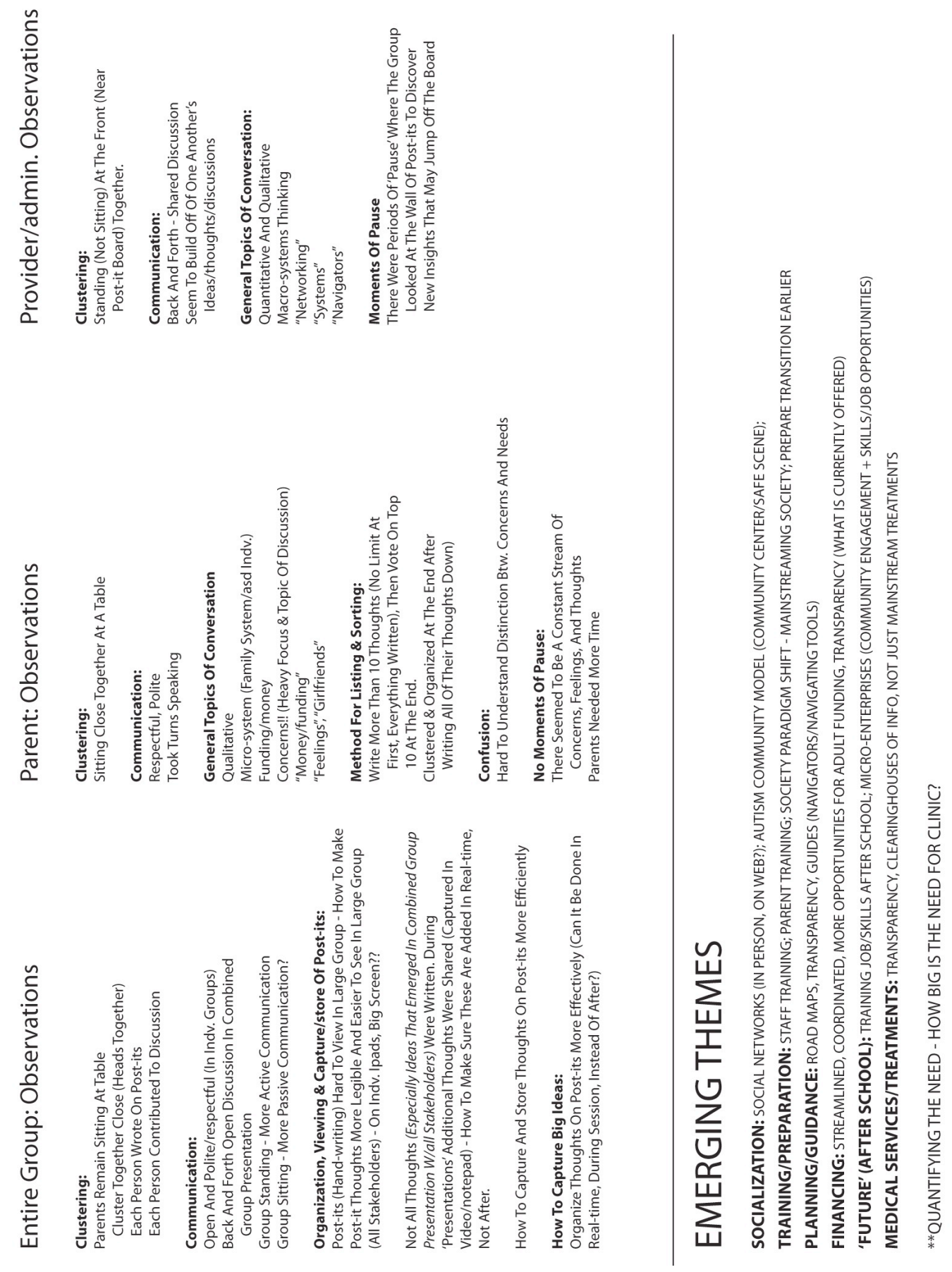

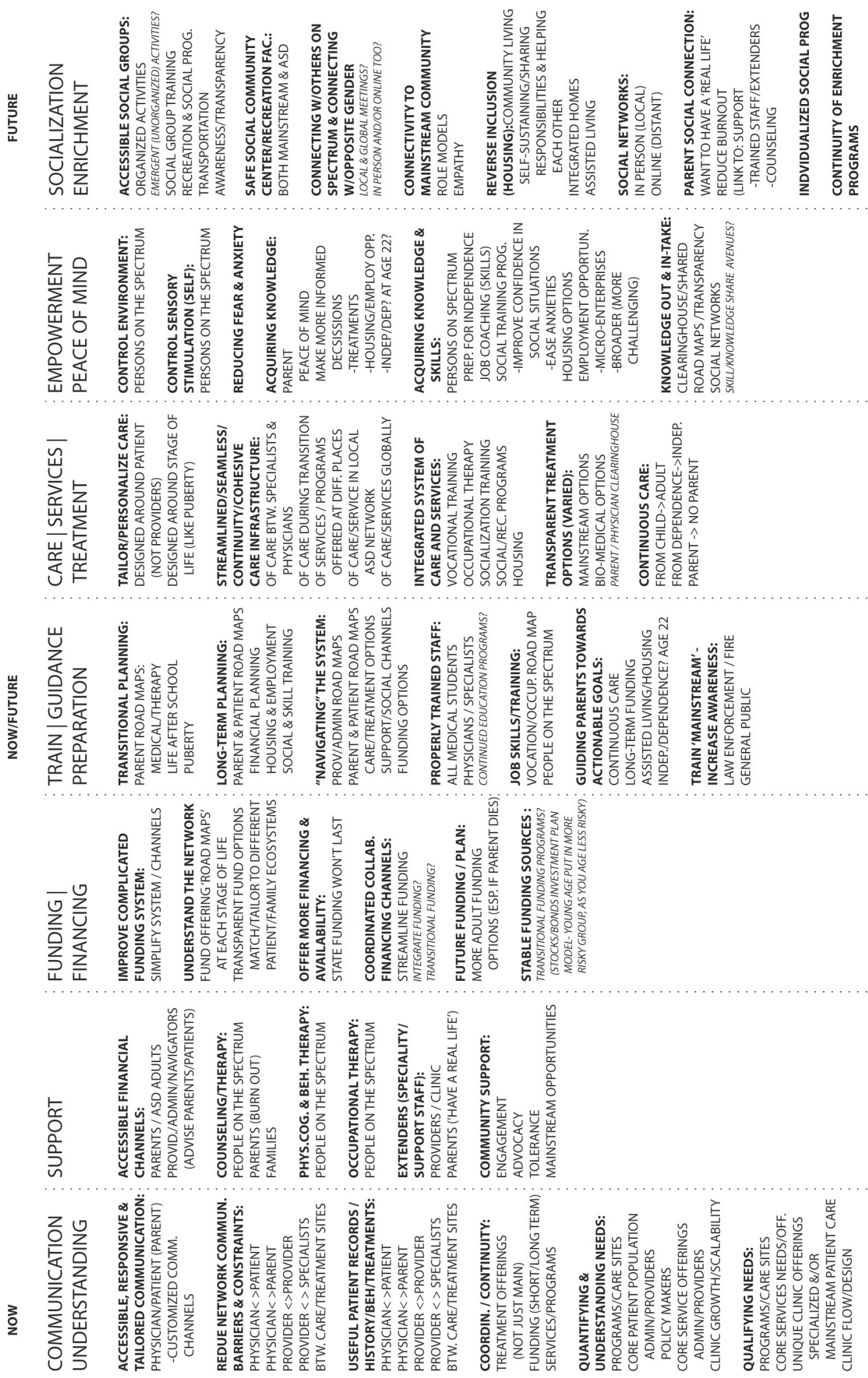

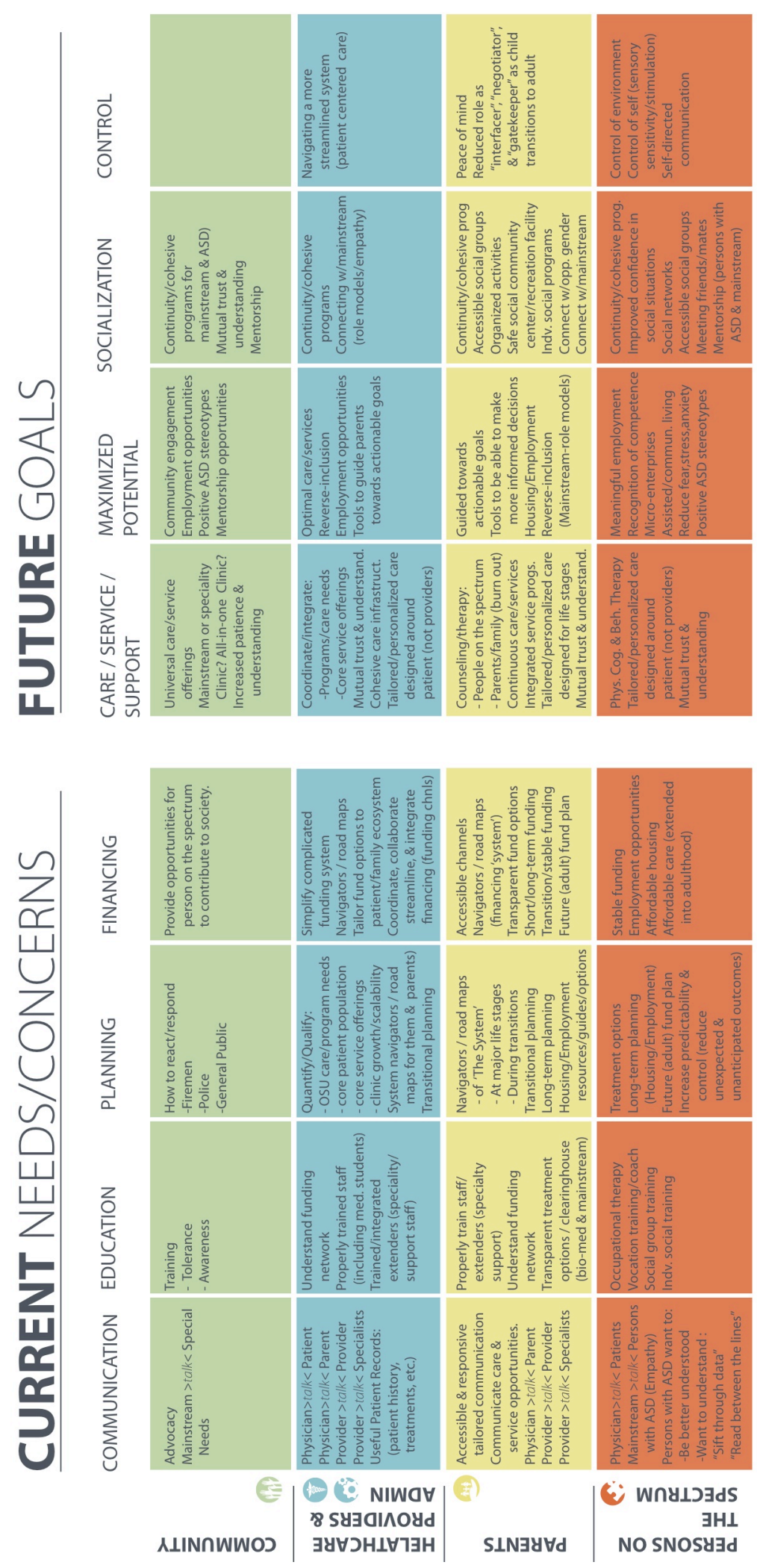


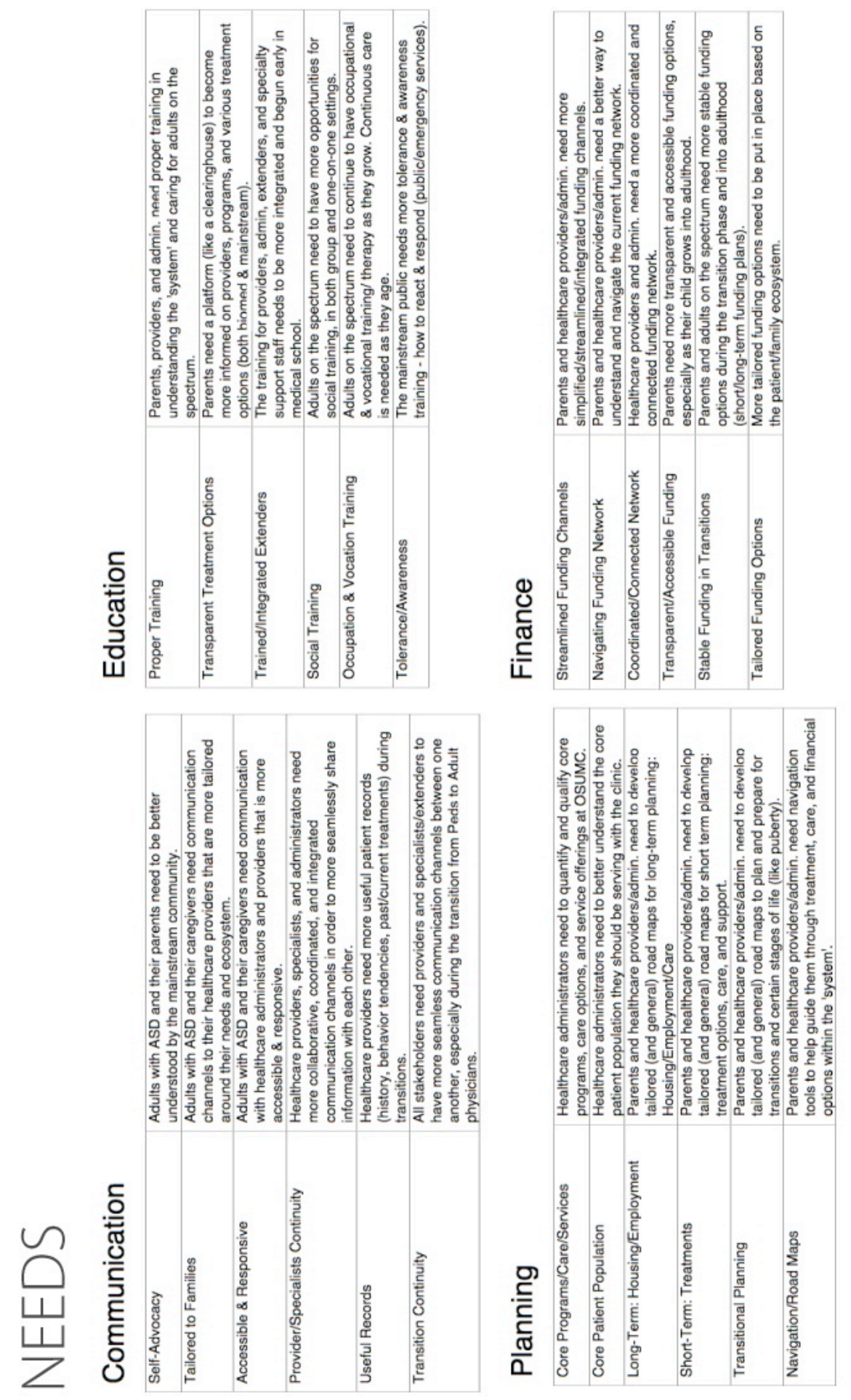



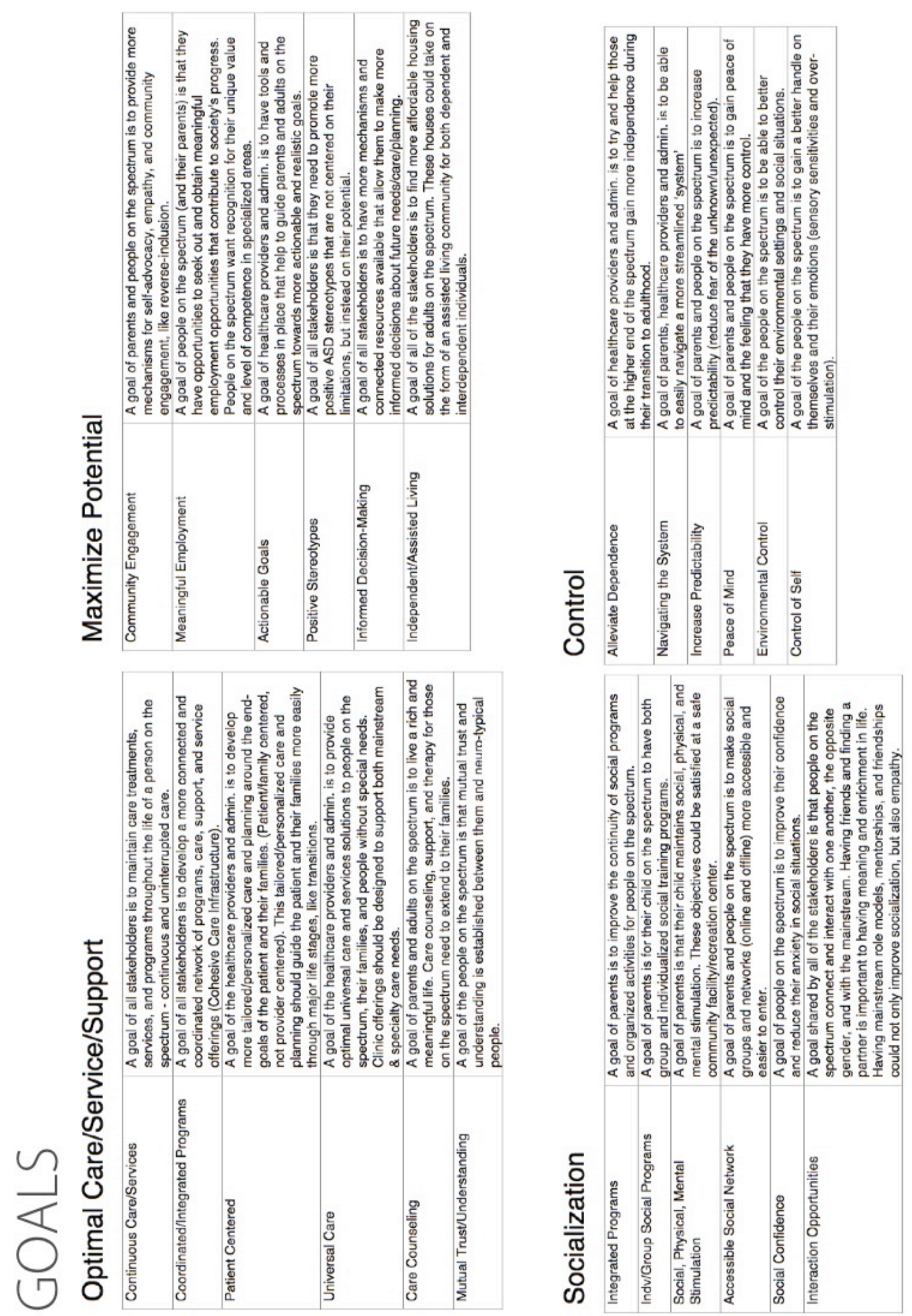
Appendix C: Phase 2-Workshop 1 


\section{Workshop 1 Discussion:}

Investigating the unmet needs of individuals with autism who are transitioning into adulthood, and the complex system surrounding ASD from the vantages of multiple stakeholders.

\section{Estimated Total Time:}

\section{Hours}

\section{Objective:}

Establish shared understanding and empathy by:

1 Sharing unique insights and perspectives into the problems and needs surrounding adults with ASD.

2 Learning more about issues surrounding ASD and the interconnected nature of this complex problem.

3 Gaining a greater awareness and appreciation for priority needs and goals, in order to make sense of the wicked problem and begin to identify root problems and innovation opportunities.

\section{Materials:}

Audio and visual recorder

Projector

Stakeholder presentations

Interactive digital tool prototype

Sticky notes and markers

Whiteboard walls

Need/goal list organized from Phase 1 findings

\section{Agenda:}

1 Welcome \& Brief Introductions | 15-30 Min

a. Warm-up exercise (empathy and/or creativity focused) to get them comfortable with speaking to the group freely and thinking creatively.

b. Introduce agenda and goals for the workshop

2 All three stakeholder groups share their experiences, needs, and perspectives I 1.5 Hour

a. Each stakeholder group is given 20 minutes to present/demonstrate/share their perspectives to the other groups

b. 10 minutes are allowed for questions/discussion/feedback after each presentation

\section{Break I 15 Min}

3 Sensemaking Activity I 1.5 Hour

a. Divide into stakeholder groups to select top ten needs/goals from the list

b. Bring the groups together to indicate priorities on a shared surface (e.g., wall, touch screen) with sticky notes or digitally

c. Have the entire interdisciplinary group re-cluster the prioritized needs/goals, draw connections among them, and determine hierarchy, in order to reframe the scope of the wicked problem

d. Capture ideas throughout the workshop

4 Wrap-Up / Reflection on Workshop 1 I 15-30 Min

a. Collective group reflects on the workshop and the new frame 
i. What new knowledge was gained?

ii. What new insights were presented?

b. Determine key needs/goals/ideas that should be addressed in the following workshop 


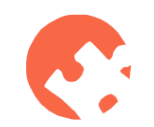

Adults on the Spectrum
Our initial session involved 10 people on the spectrum.

( 1 YO) ( 10 Y $)(0)$

Most participants had

relatively strong cognitive

and verbal abilities.
We are here to advocate for ourselves and people on the spectrum.

Kristin

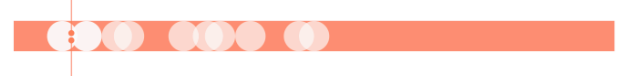

Brad

John

\section{Sensory Overload}

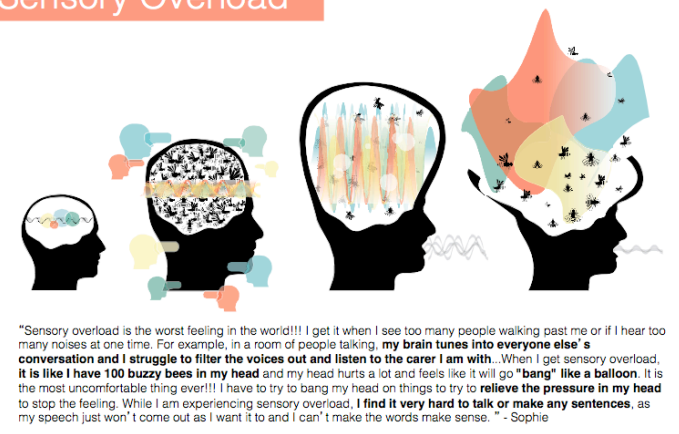

\section{Social Interaction}

Negative Feedback Loop

fear of SOCIAL INTERACTION

generates ANXIETY

causes STRESS

heightening SENSORY
SENSIIIVITY

sensitivity to SOUNDS

sensitivity to LIGHTS

sensitivity to TOUCH

causing OVERSTIMULATION

making ithard to

making it almost impossible
to SOCIALIY INTERACT

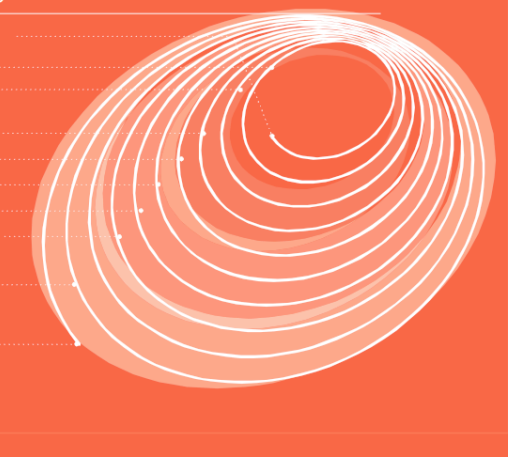

Experience Mapping

C.A.S.T. CLINIC EXPERIENCE The current olinic experience
(Shared Probiems \& I ldeas) 

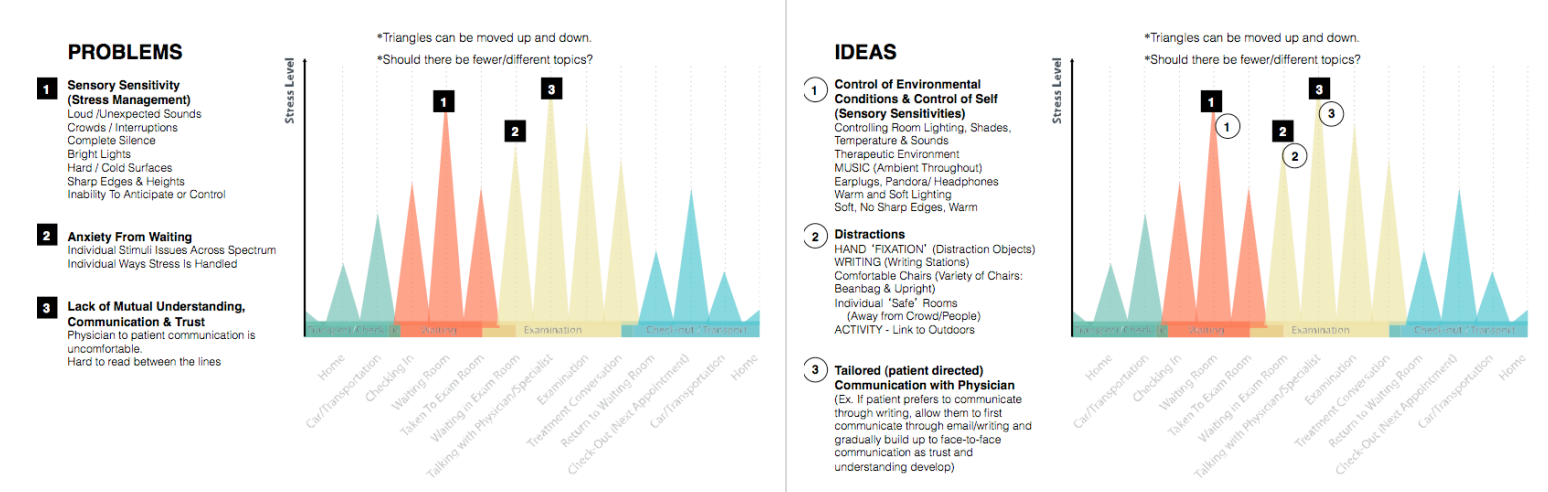

We can work on small, concrete problems, or large-scale problems.

We want to DEPENDENT

maximize

our potential
INTERDEPENDENT

INDEPENDENT

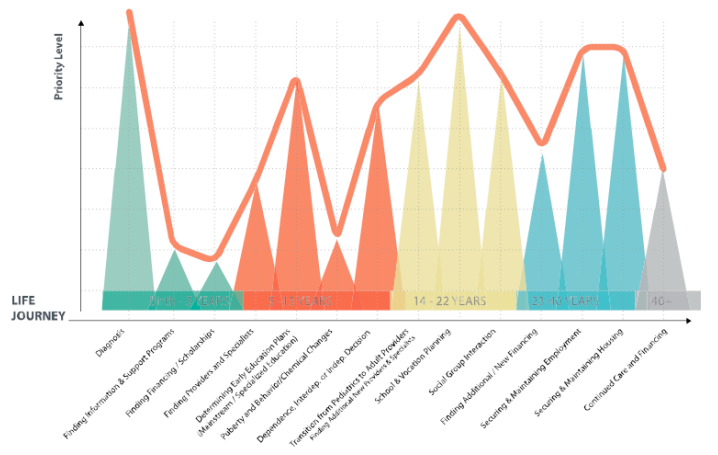

Priority Areas over our Life Journey 
public perception

\section{needs to change}

\section{'victim' $\Rightarrow$ innovator}

\section{Benefits of having ASD}

We are detail oriented, critical thinkers.

We have heightened sensitivities to sounds.

Many studies suggest that we have too much empathy.

We speculate about the future.

We look at things from a completely

different perspective.

\section{Un-tapped Employment}

Opportunities

Homeland Security

Military Intelligence

Data \& Technology Fields

Emergency Service

Search \& Rescue

Job Analyst/Consultant

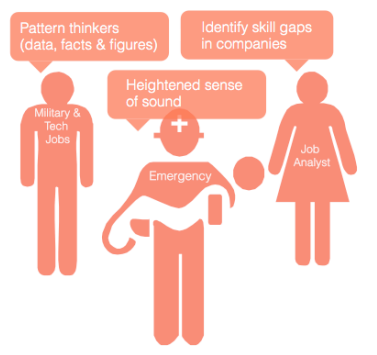

The Downside of Being Neurotypical

Being overly social can be just as much of a disability as not being social

Thinking in broad strokes can cause one to overlook the important details and the gaps.

Getting distracted more often, leads to less focus and less productivity.

\section{"We can do new things, we just have to get acclimated."}

Merit in society is measured and valued backwards

Society punishes intellect and minimizes internal gifts, while

rewarding social abilities and outward appearance. 

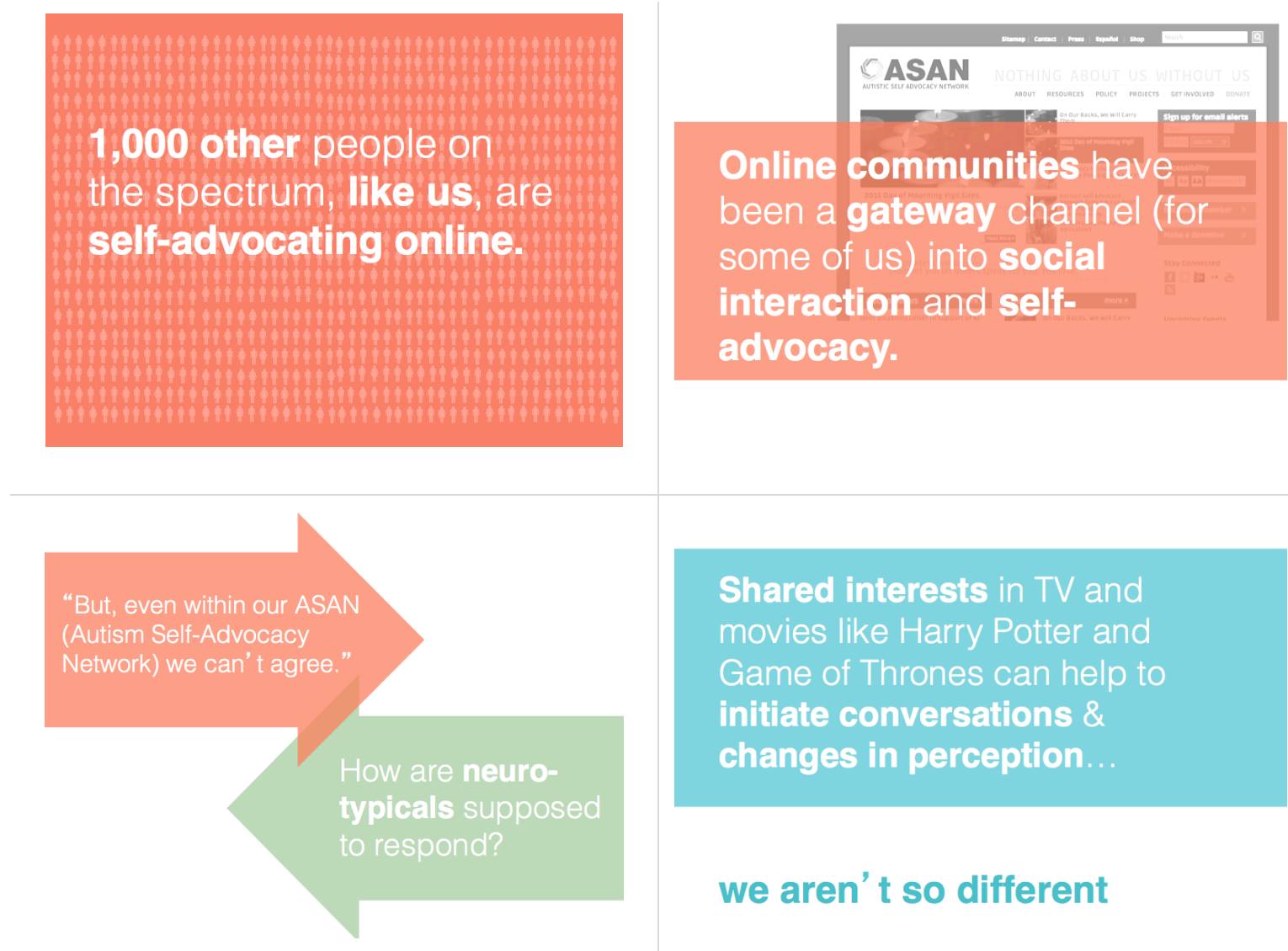

Shared interests in TV and movies like Harry Potter and Game of Thrones can help to initiate conversations \& changes in perception...

we aren' t so different

We want to lead rich and meaningful lives and have opportunities to maximize our potential.

"More inclusion and more representation is what the world needs." 
Inclusivity + Specialization

The Information Age segments us into factions. We, instead, need to find ways to connect and work together by contributing in dififerent (unique) ways.

Recognize (our specializations) Utilize (our specializations)

Our specializations can be harnessed (instead of suppressed) to contribute to innovation \& progress.

"We can help guide people through the information age... we' re a step ahead. you need us."
ACKNOWLEDGE OUR VOICF

Big or small, we want to have an impact not only on our future, but also society's future. 


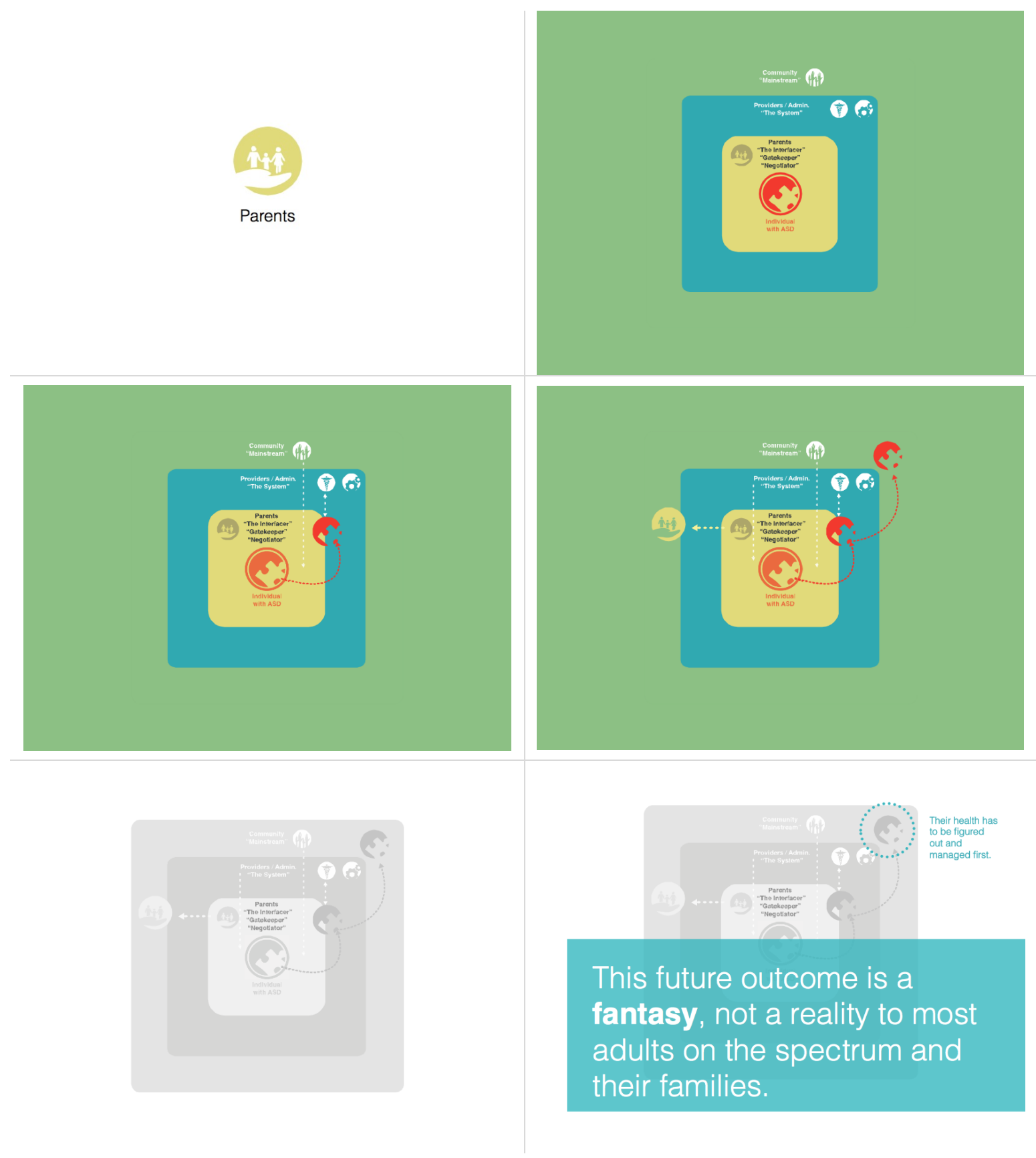


The reality is that most adults

with autism will live with their parents (or 'natural caregivers' ) for life.
Personalized care \& support should extend to the whole family.

\section{(7)} 竞

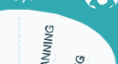

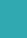

\section{Priority Areas over} the Life Journey

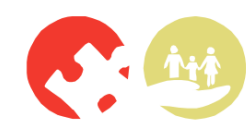

The reality is that people on the spectrum and their natural caregivers need rich and meaningful lives that are worth living and maximize their potential.

\section{top $10 \%$}

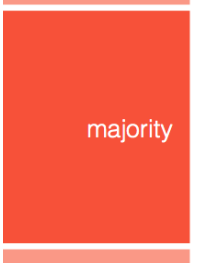

bottom $10 \%$
But, there are very few funding options for a majority of the families that fall between the top $10 \%$ and lower $10 \%$ on the spectrum. 
If we are truly going to tackle these problems, with no (or very limited) funding and resources, this 'ideal' future goal will require...

\section{DISRUPTIVE INNOVATION}

Today

\section{LACK OF}

Affordable Housing

画画 田田几田田田田
Future

More AFFORDABLE MATERIALS and

INNOVATIVE building

processes/ solutions

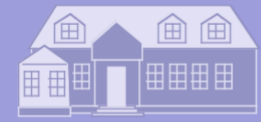

DISRUPTIVE INNOVATIONS

are needed to increase the

health support and longevity

of the natural caregiver in

order for them to better care

for their adult child.
PARENTS IN

DISAGREEMENT

WEAK NETWORK

OF SUPPORT

UNCONNECTED 


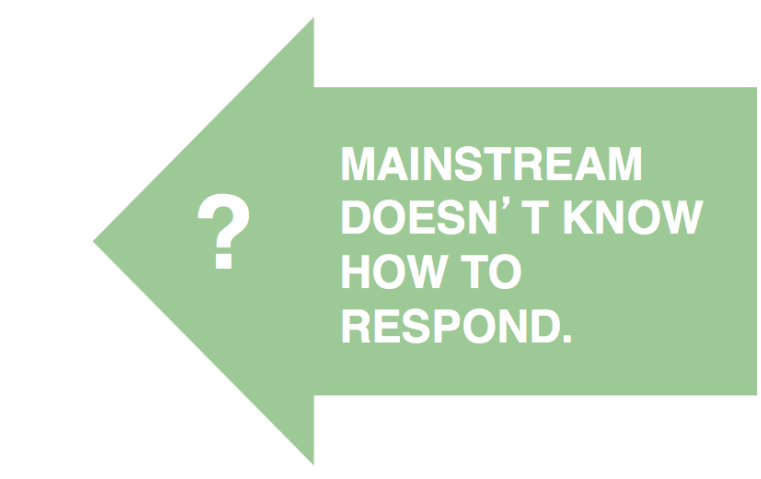

REVERSE INCLUSION

Mainstream Mentorship

CLEARINGHOUSE

sharing of treatment information and resources

TRAVELOCITY | ANGIE’ S LIST

Zip-code Based 'Navigation Maps'

Where to go first. Navigating the 'System' in your area.

Provider ratings tailored around 'type of treatments/care offered'

\section{NO-TOUCH HOME SENSORS Detect | Learn | Inform | Intervene}

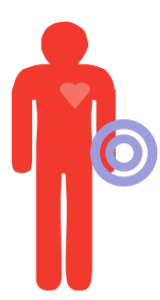

SENSORS

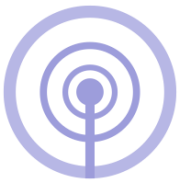

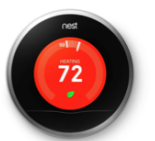

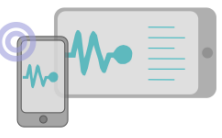

We need to generate ideas that bridge stronger connections within our 'network' and with the mainstream population.
We need to identify disruptive opportunities and technologies that simplify our lives and improve the quality of life of our children and ourselves. 


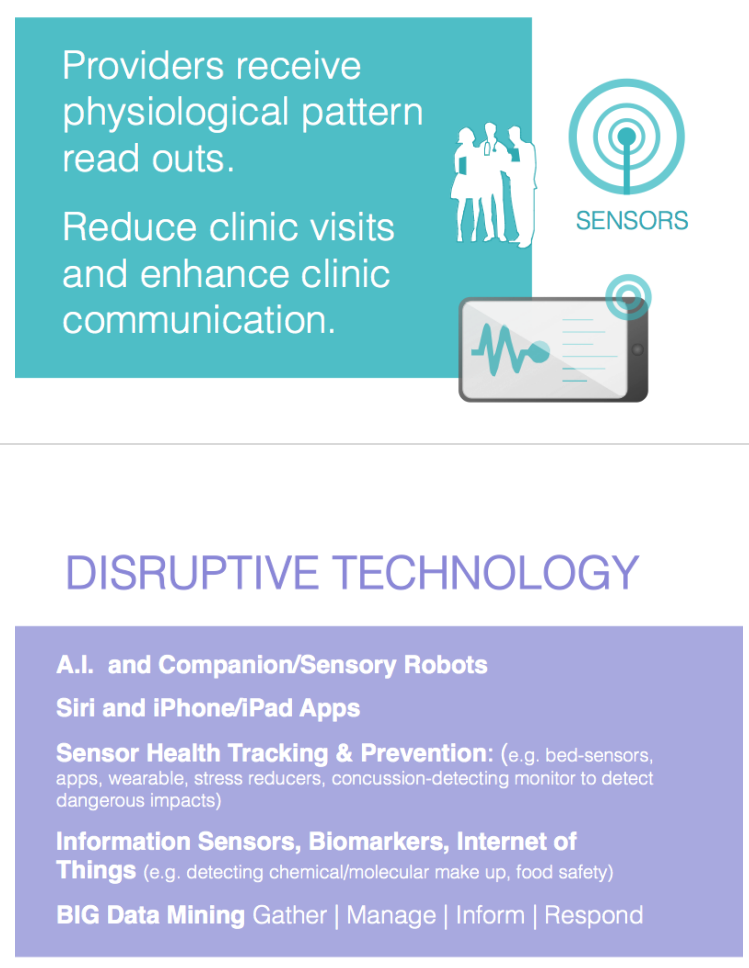

\section{OTHER DISRUPTIVE IDEAS}

Augmented Reality, Gamification

Tailored Higher Education Opportunities

(beyond the current classroom setting/model)

Self-Advocacy Mechanisms / Platforms

Creative Funding Channels

(e.g. Micro-enterprises)

Wider Caregiver Network / Mentorship
Many people on

the spectrum think

in PATTERNS.

Could they work with A.I., app

and sensor developers to work

on more meaningful projects?
In order to maximize the potential for our society, people on the spectrum, and families, we have to look at both small and large scale resolutions, untapped opportunities, and

\section{DISRUPTIVE INNOVATION}




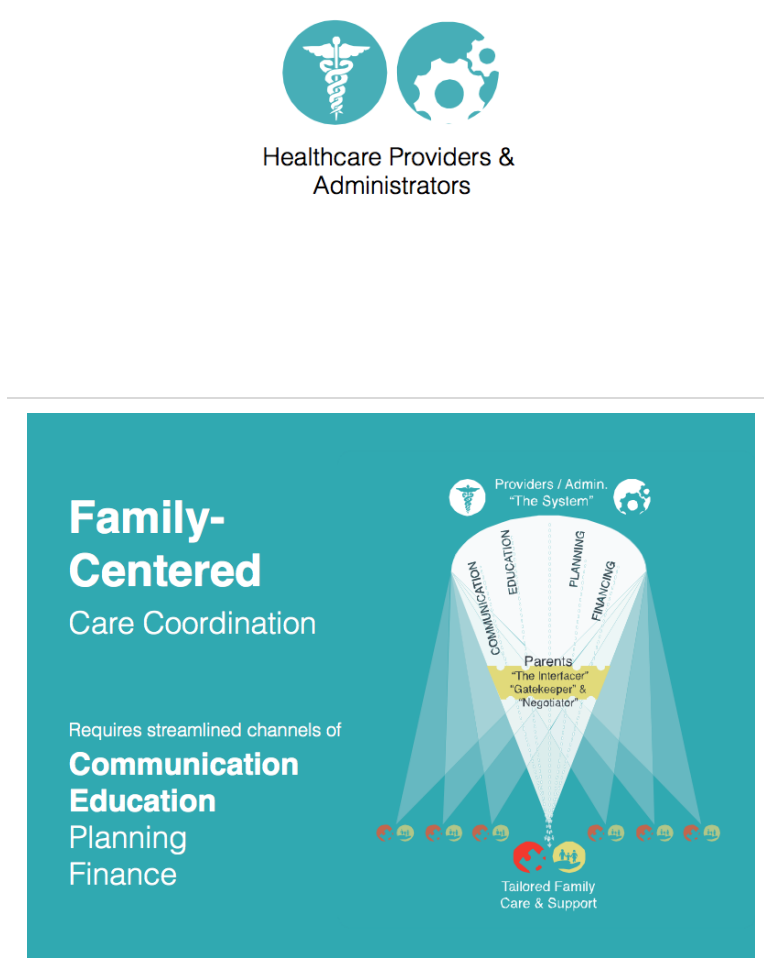

\section{EDUCATION gaps}

The biggest challenge we have is trying to apply a systemsbased approach to solving the tailored needs of patients and their families.
The success of a systemsbased approach to personalized medicine relies on overcoming two major barriers

\section{EDUCATION gaps}

COMMUNICATION gaps 


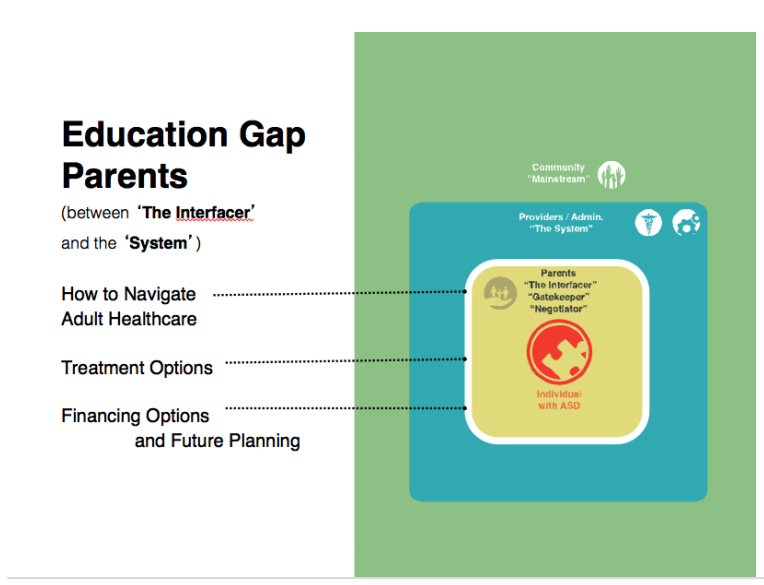

\section{Education Gap 1}

Parents need tools that make them more informed and assured.

Adults on the spectrum need tools and skill-sets to self-advocate (to step into a more 'interfacing' role).

If they can' t, a parent can step in.

\section{Education Gap}

Parents

Some young

adults on the

spectrum can

move into an

'Interfacer'

role.

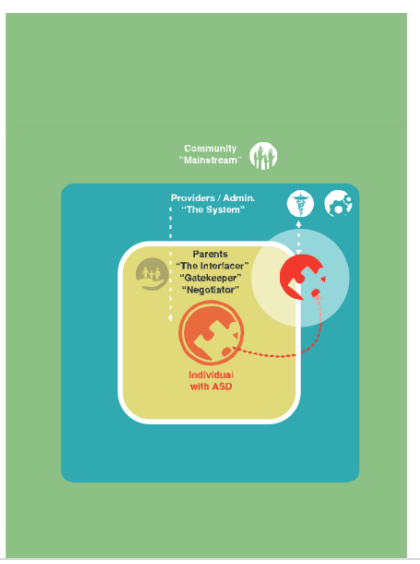

\section{Bridging Education Gap 1}

Alleviate stressors

Breakdown parent \& patient barriers

Improve mutual understanding

\section{Bridging Education Gap 1}

Bring the pharmacist into the transition process early

\section{Support a sensitive} transition process

Provide parents and young adults with tools like...

\section{A Vision Plan}

Determine end-goals \& a child's potential Deal with unrealistic goals at a young age Encourage untapped potential

Provide more personalized care, patient history, and road maps for future planning 
AASPIRE Healthcare Toolkit

Help young adults prepare for a clinic visit

Reduce stress \& responsibility on parents

Help the patient feel more empowered to ask their own questions and communicate directly with their provider

Provide helpful resources for 'outside' providers/specialists, and extenders

Who's responsible for conducting life skills training?

\section{Life Skills Training}

Person-person or group training, digital/animated simulations reinforced at home

\section{The C.A.S.T Model}

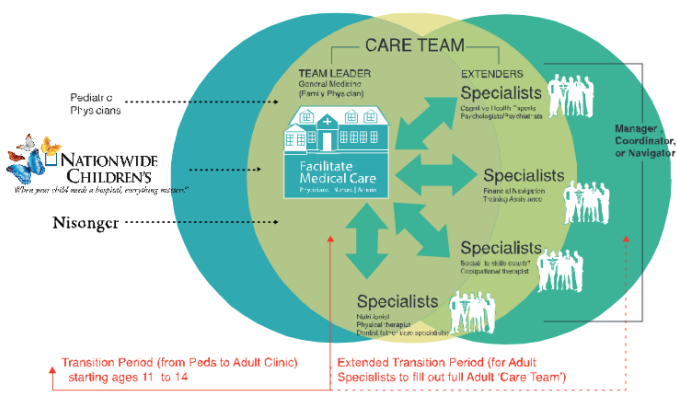

\section{Modeling \& Stories}

\section{Video Modeling \& Simulation}

Show patients and parents what to expect during their visit (like to the dentist)

Social Stories

Increase confidence and social skills in people on the spectrum
The current 'system' has LIMITED RESOURCES

to properly care for the growing ASD population

We treat 130 patients at C.A.S.T
Transition plans need to begin at age 11

Extenders and specialists (outside the ASD 'system' ) need to be better educated and trained so they feel more comfortable with people on the spectrum.

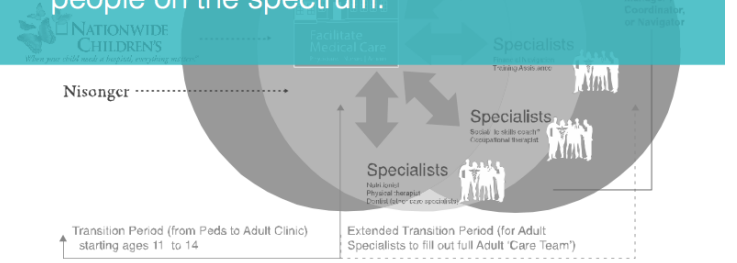


Autism 'care' is spread out.

Cancer 'Care Teams' are more connected.
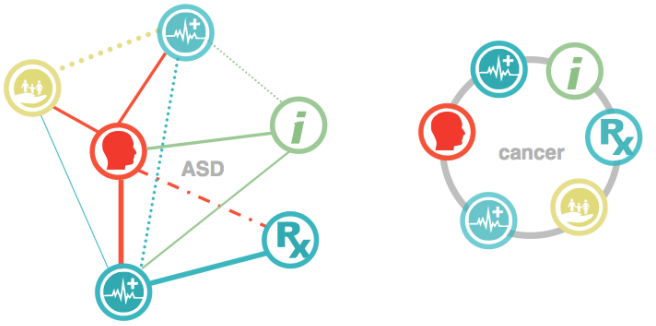

Parent's Role
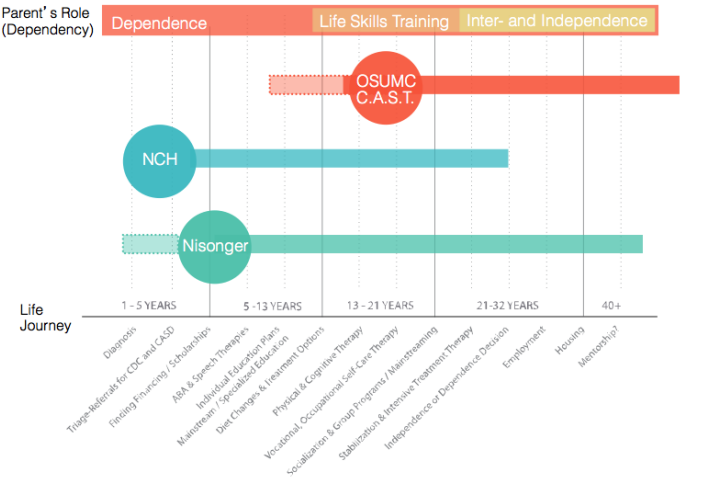

\section{Education Gap \&}

Communication Gap

between 'Mainstream' Providers

and the 'System'

Social Stigmas

Lack of Time

Lack of Comfort carmunity (At)

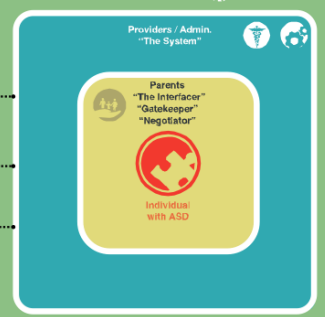

ASD Care Network over the Life Journey
How are we all connected?

How can we make the 'System'

more collaborative and streamlined?
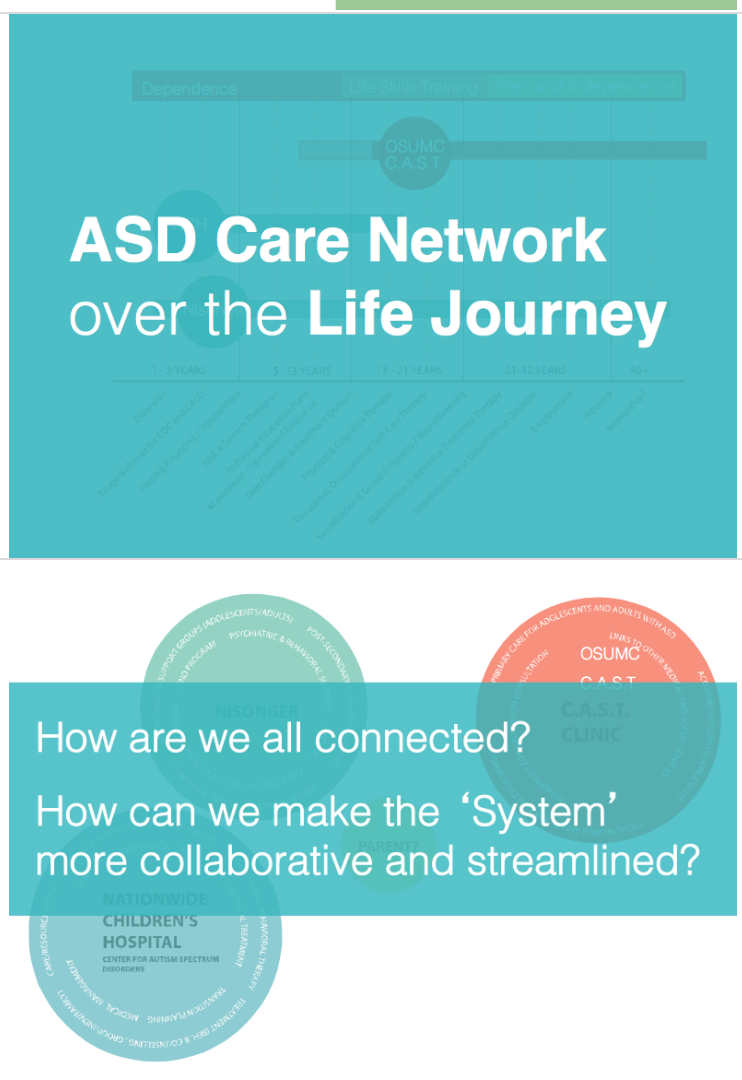

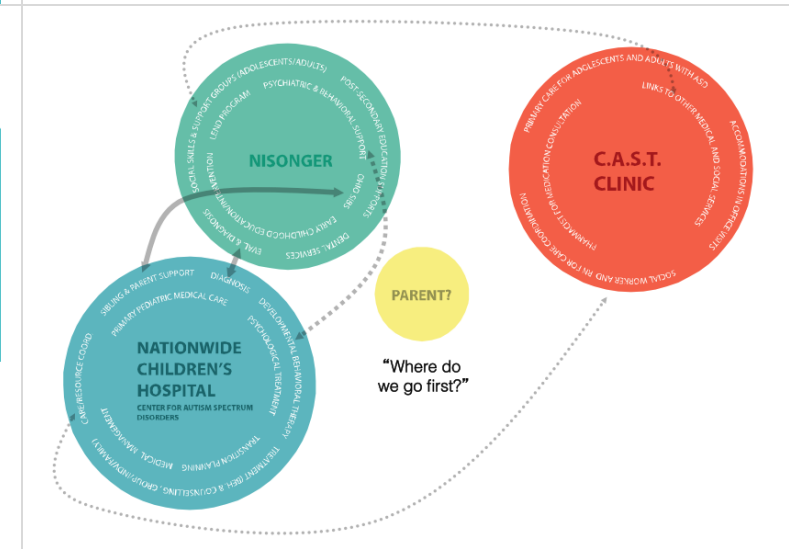




\section{Priority Areas over the Life Journey}

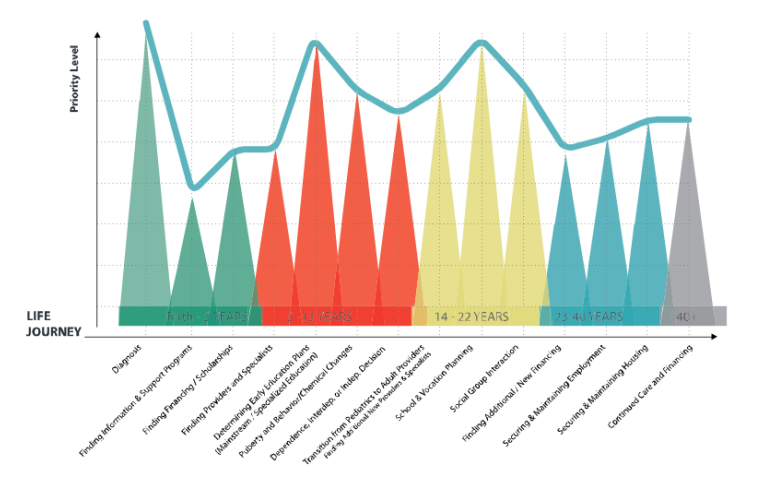

\section{Training and education needs}

to be provided to extenders and providers outside the ASD 'system,' so they feel more comfortable.

\section{Enhancing Care Coordination}

We need to improve the methods of communication between:

Pediatric $><$ adult providers

ASD 'system' providers $><$ outside providers/ extenders/specialists

Families/patients $><$ healthcare providers
Communication is HUGE in understanding where providers and specialists fit into the 'system' so that they know where and how to communicate with and care for patients and their families.

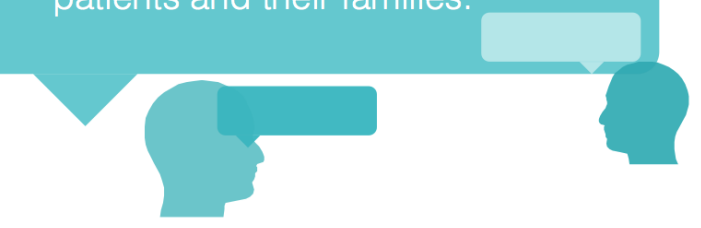

Many patients and family members would like to communicate through email but, outside/personal email is not secure and is disconnected from patient records.

MyChart allows 'email' -type functionality, but it is all linked to patient's medical records. 
"Keys to a successful visit."

More effective and readily available mechanism to view 'key' patient specific information (beyond digital medical records).

Medical app with both extended and abridged health information.

\section{Patient Passport}

Simplified patient histories/records

(1 page mostly visual icons)

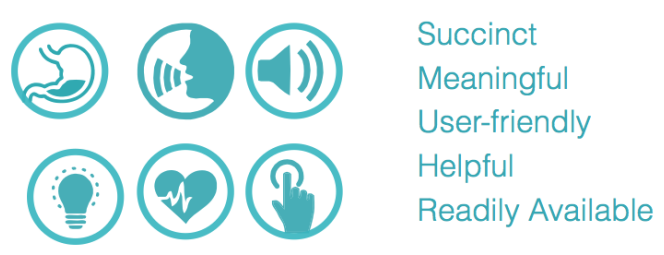

\section{App to Track Treatments}

(useful for pharmacist, physicians, parents, and specialists during visits and transitions)
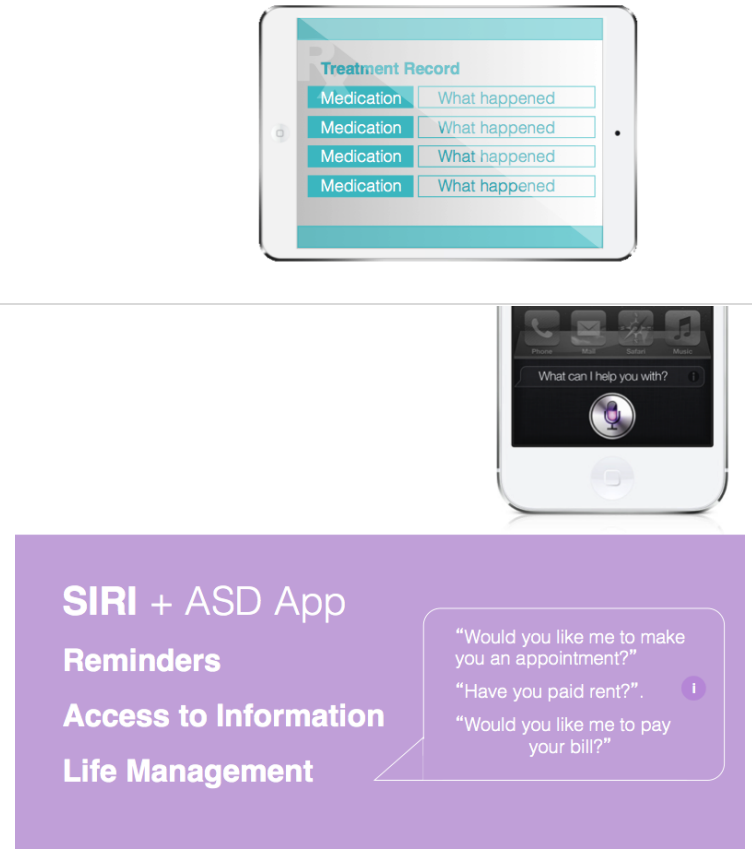

Self-Access to Medical Information/Education

WebMD-type App/Clearinghouse Physician-generated Platiorm | Parenis and patients access 247 ("If constipated try this..." remedies betiore calling/emaliling physician)

Support \& Technical Assistance for 'outside' extenders (after education and training) Similar to an IT support "help desk."

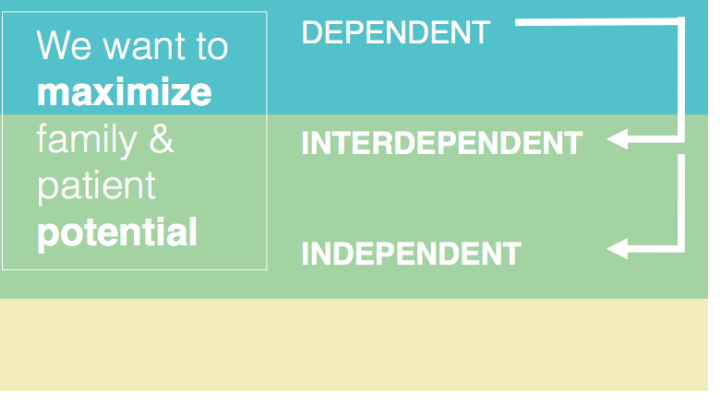




\section{Our goals at C.A.S.T are to:}

Provide primary care and support to people with intellectual and development disabilities, and their families.

Coordinate specialty/extender adult care.

Provide resources to enrich and maximize the potential of families and adults with disabilities.
Our aim as healthcare providers and administrators in the ASD system are to:

Improve the quality of life of our patient and their families.

Increase effective care and personalized medicine within system-based solutions.

Provide care/support/services to enrich and maximize patient and family potential.

Formulate sustainable supports and collaborative networks

To meet these goals we need to:

Provide tools to educate and train

Provide personalized family care

Provide means to enhance communication \& understanding

Provide means to spread resources \& responsibilities 
Priority Similarities/Dissimilarities Among the Three Stakeholder Groups

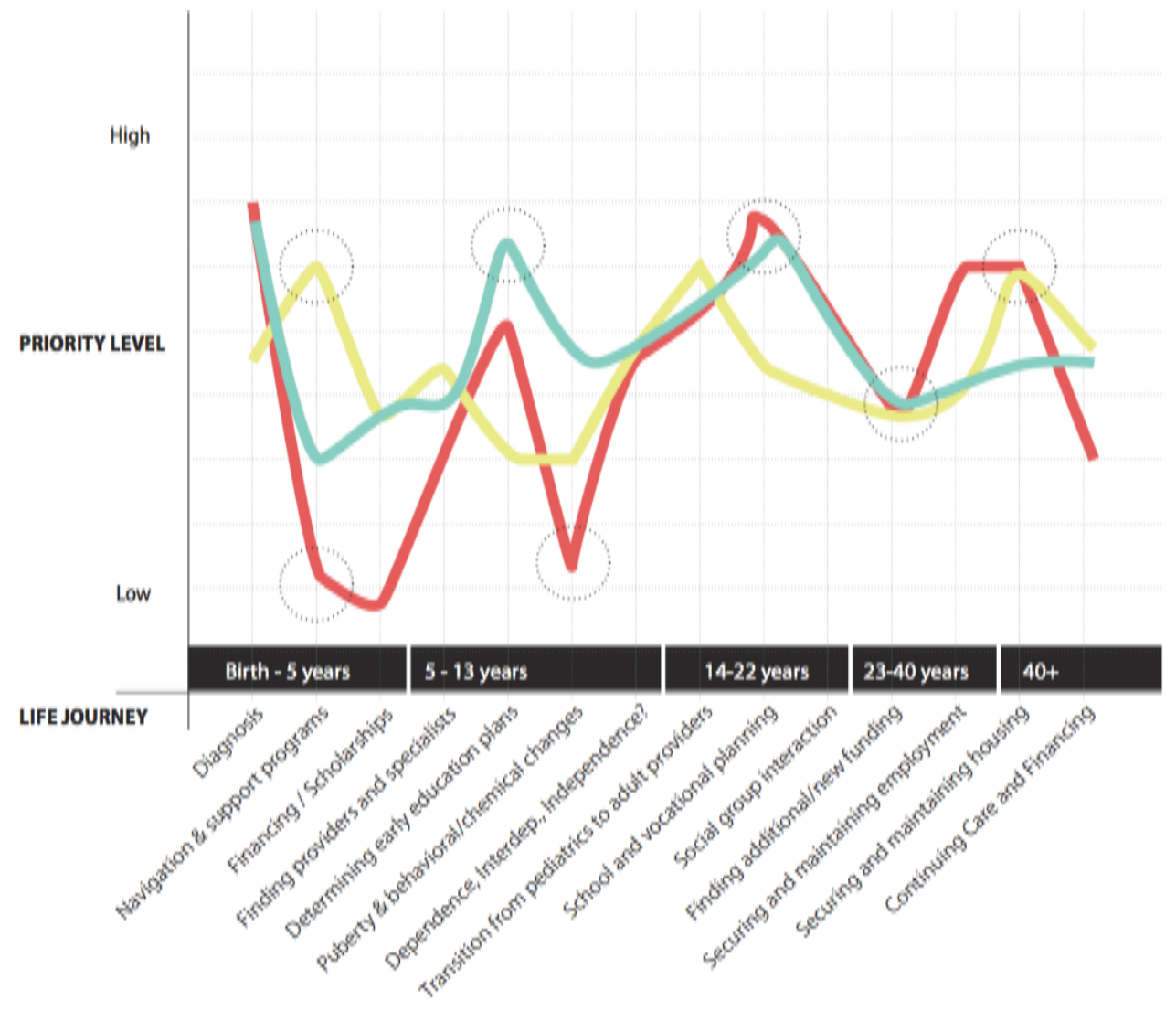



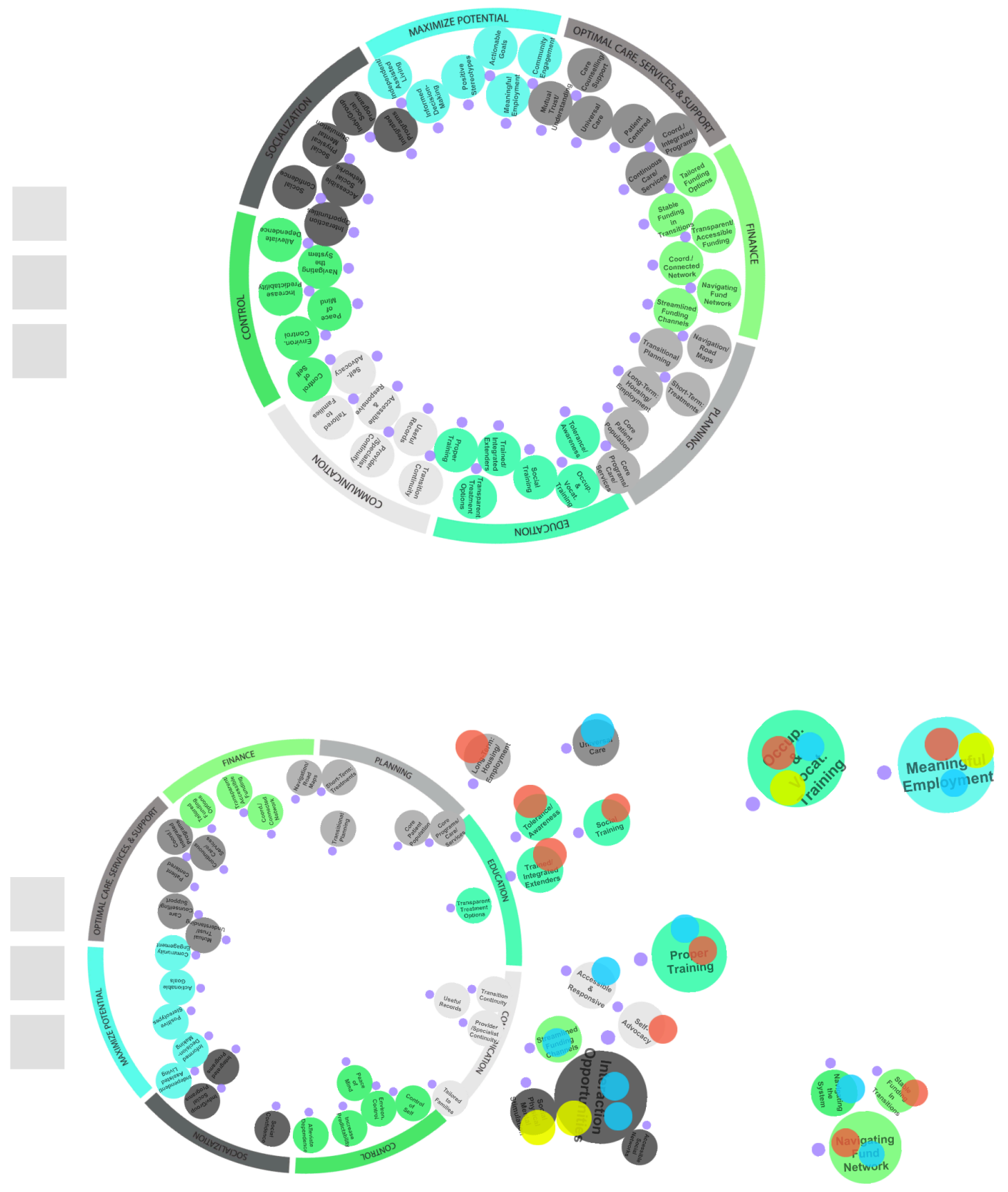


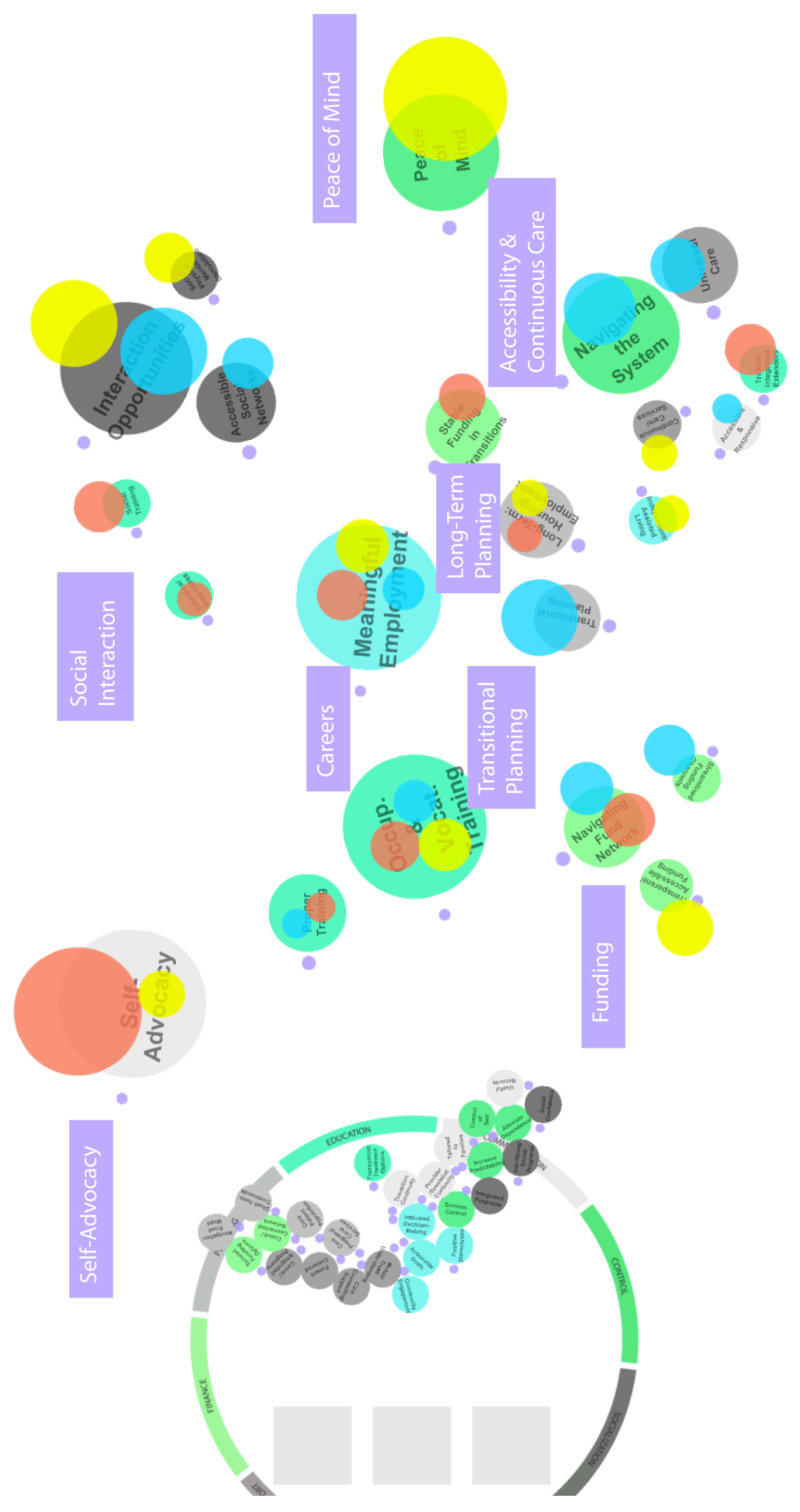




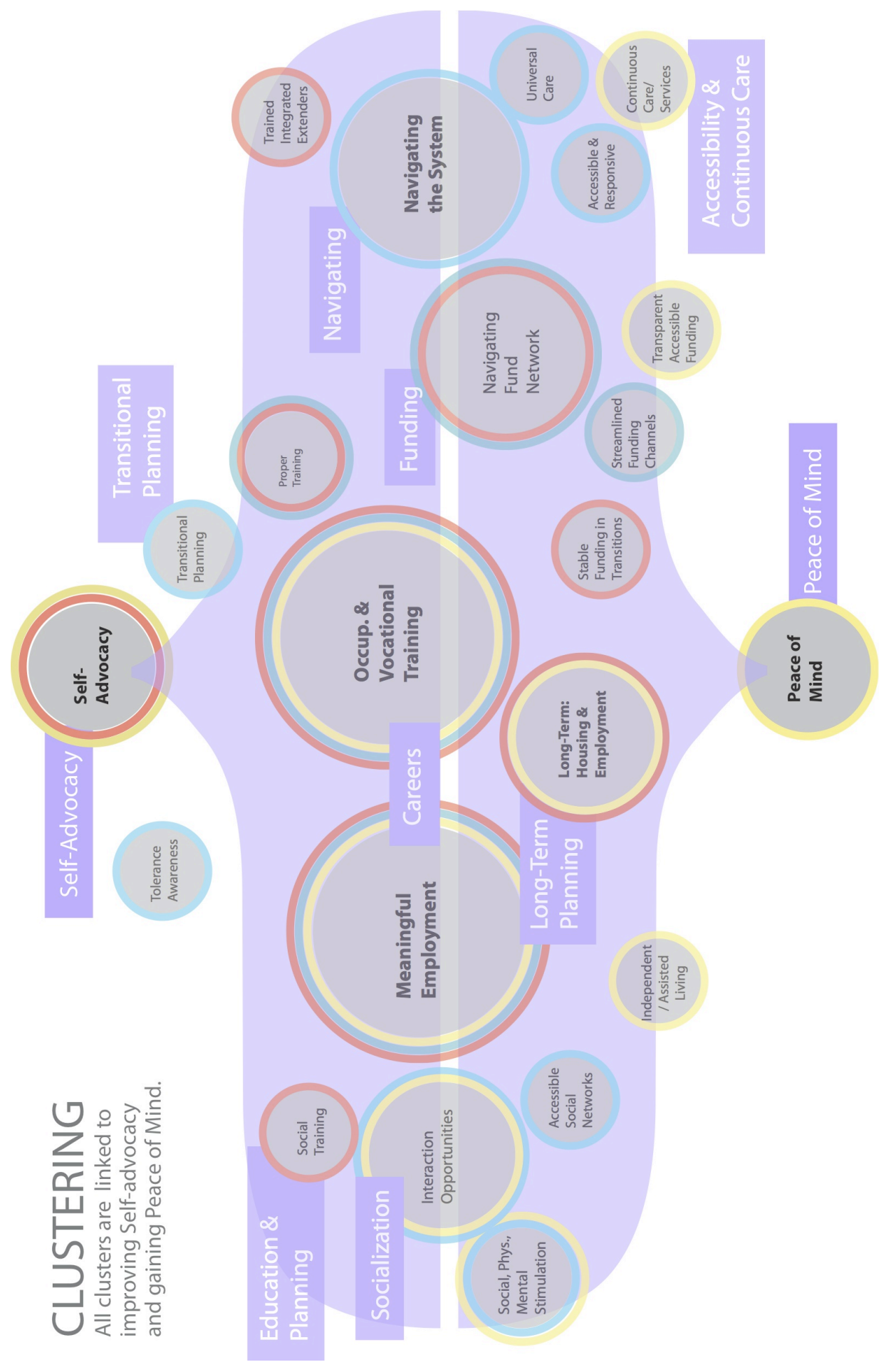




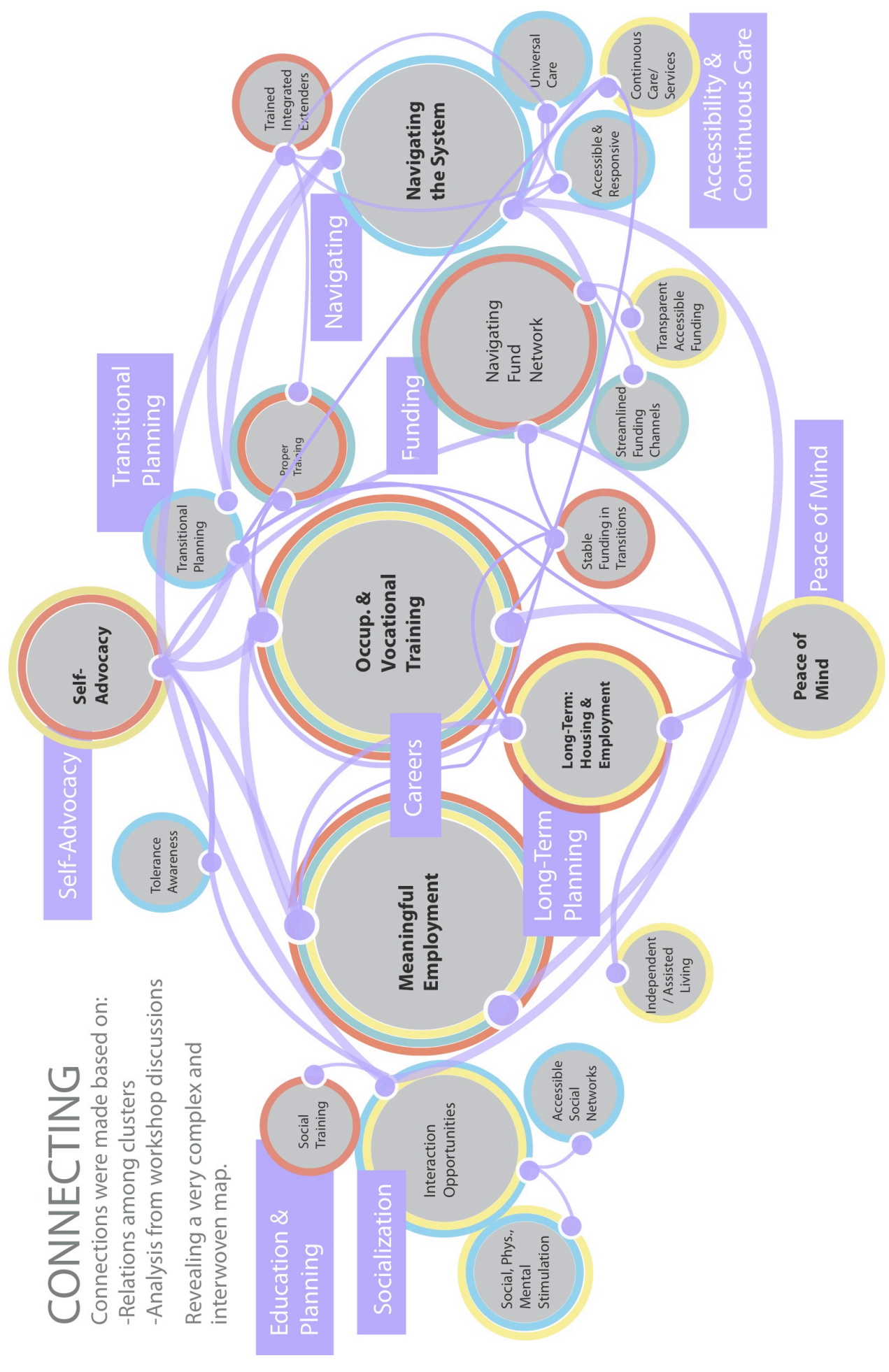




\section{CONNECTING}

The original 48 topics

and 8 categories

(including the 22

prioritized topics and

their connections from

Workshop 1) are

visualized to the

right in a different

way, using a

wheel diagram.
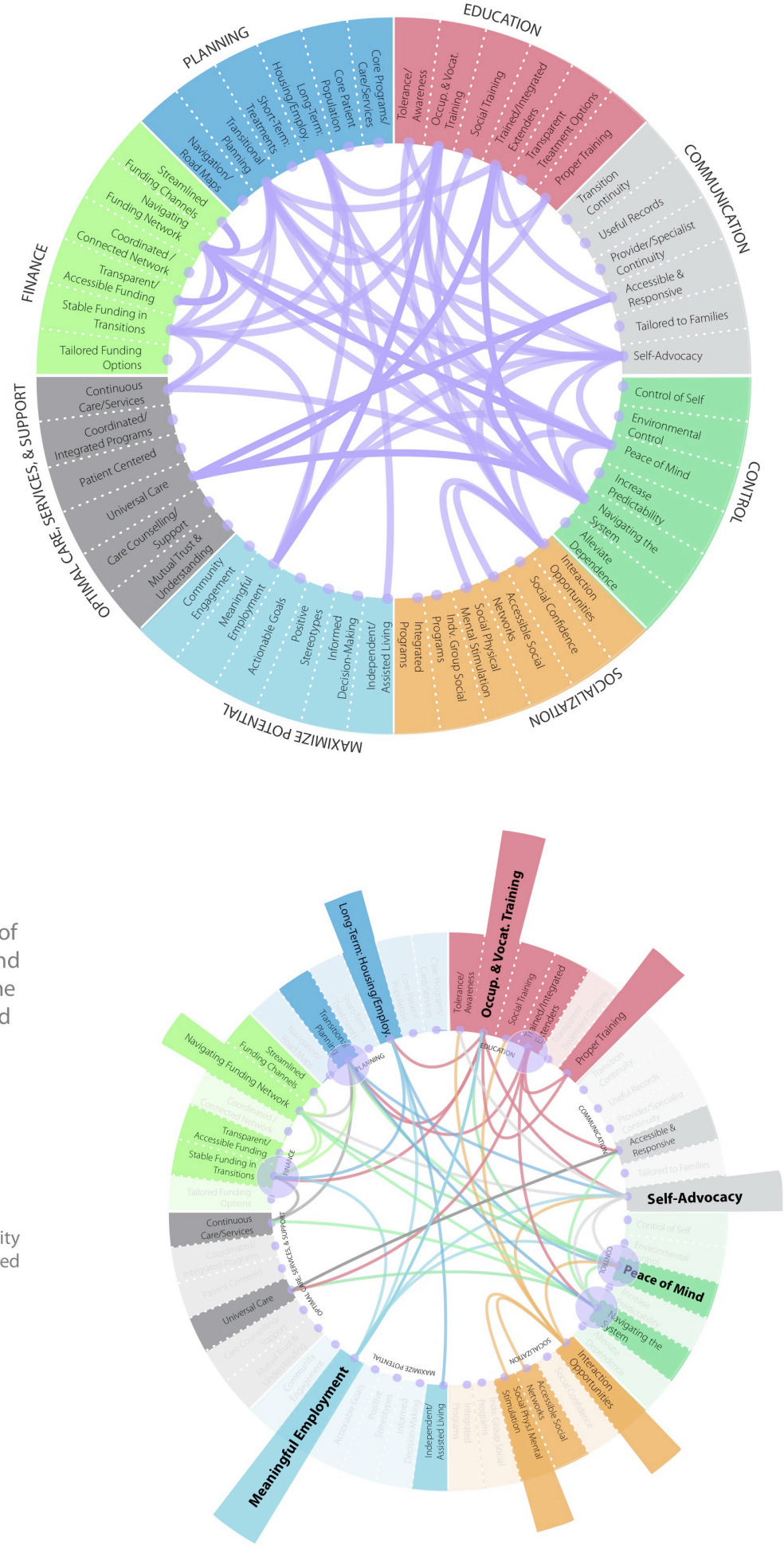

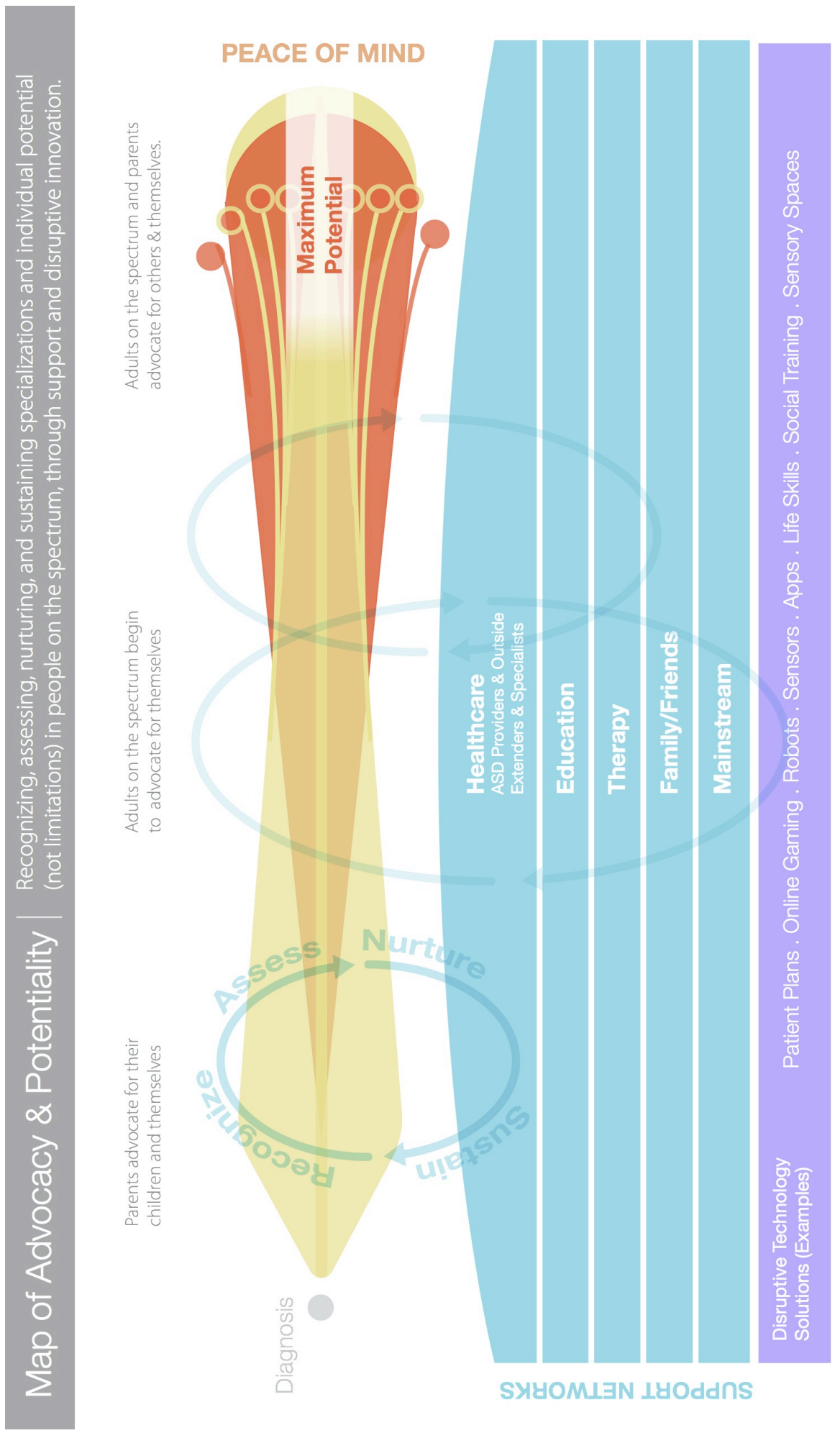


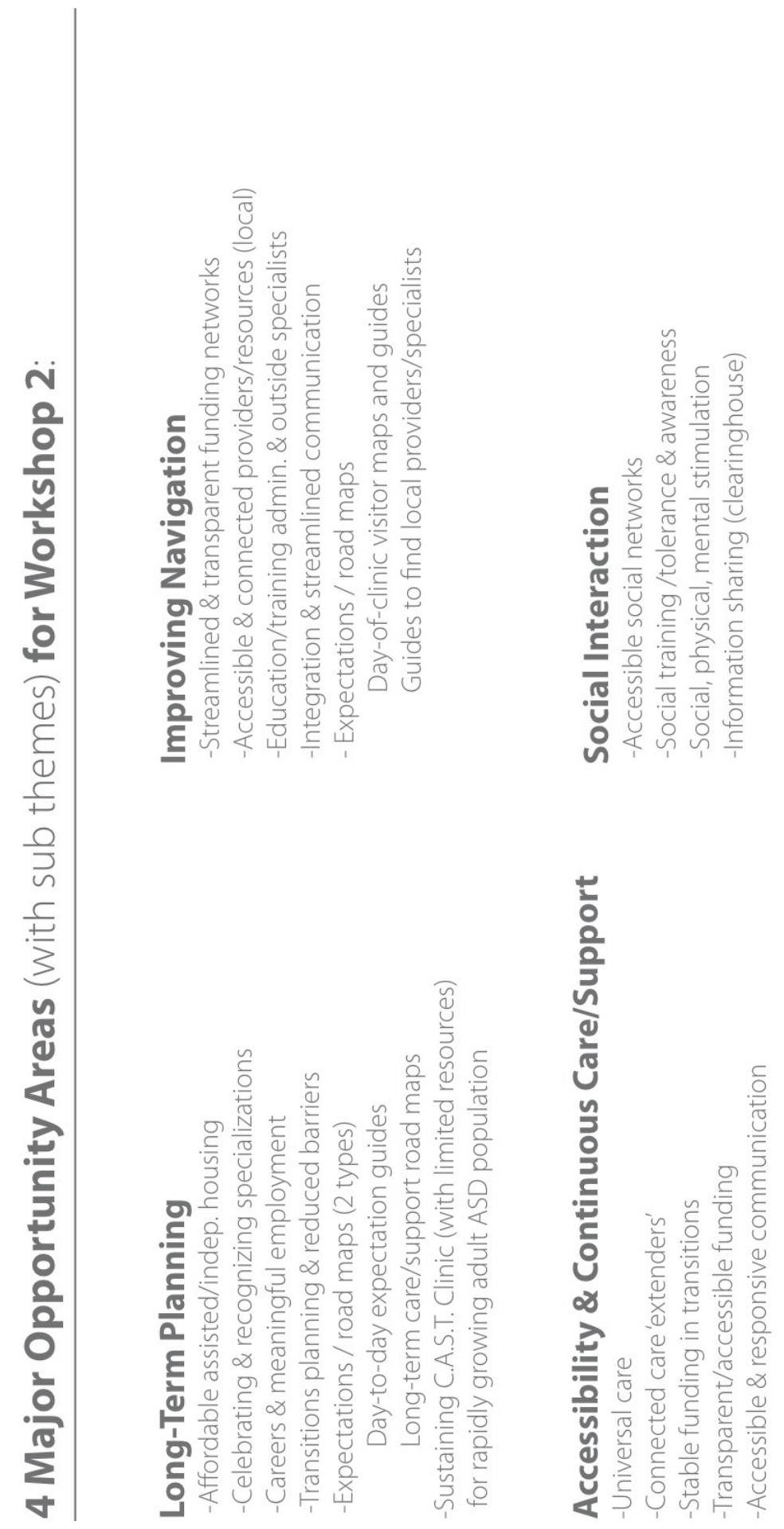


Appendix D: Phase 3 - Workshop 2 


\section{Workshop 2 Discussion:}

Identifying new opportunities within the wicked problem, collectively prototyping (building) concepts targeted at resolving these unmet needs, and determining next steps in the Design process.

\section{Estimated Total Time:}

4 Hours

\section{Objective:}

Identify new resolutions by:

1 Reviewing findings and insights shared in Workshop 1 to establish "constraints" and resolution goals.

2 Determining unmet needs and future aspirations through persona development and prospective scenario mapping.

3 Working collectively to build prototypes of resolutions (services, products, environment layouts, etc.) from identified opportunities.

\section{Materials:}

Audio and visual recorder

Visualizations: Maps, Diagrams, etc. from Workshop 1

Poster board, markers, scissors, and tape

A large roll of paper for each group

3D / 2D inspirational imagery, icons/shapes, objects, puppets, and other materials for 'making' Post-Assessment Questionnaire

\section{Agenda:}

1 Welcome I 5-10 Min

a. Warm-up exercise to get participants comfortable with each other and "what if" thinking.

b. Introduce the agenda and goals for the workshop

2 Summary of Workshop 1 Insights, Needs, Findings (from the Research Team) I 15-30 Min

a. Allow stakeholders to express what they learned and share their Journals/Vision Boards

b. Open the discussion for any additional information the participants want to add/share

c. Divide into interdisciplinary teams of equal size

3 Persona Development in Teams I 30 Min - 1 Hour

a. Build current persona and future persona

b. Bring teams together to present their personas to one another

\section{Break / 15 Min}

4 Prospective Scenario Development I 30 Min

a. Regroup into teams to map the persona's life journey, working back from their future aspirations

b. Determine gaps, barriers, and opportunities along the journey for new ideas and innovations

c. Bring teams together to present their journey maps to one another 
5 Prototype (using 3D and 2D generative tools) opportunities that have been discovered within teams I 1 Hour

a. Bring teams together to present their prototypes to one another

6 Wrap-Up / Reflection on Workshop 2 I 30 Min

a. Reflect on this workshop - what was learned

b. Select ideas to refine and determine next steps

c. Administer Post-Assessment Questionnaire

d. Reflective discussion on the overall project, methods used, and what were we able to accomplish 
Headers:

\title{
Personality
}

\section{Lifestyle}

\author{
About (Age, Location, \\ Family Situation, etc)
}

\section{Specialization}

\section{Situation Cards:}

\begin{tabular}{|c|c|c|}
\hline $\begin{array}{l}\text { Access to education and } \\
\text { fullilling careers after high } \\
\text { school (like working at } \\
\text { Microsoft) }\end{array}$ & $\begin{array}{l}\text { Living on their own / } \\
\text { Affordable housing }\end{array}$ & $\begin{array}{l}\text { Sensors to monitor and } \\
\text { communicate }\end{array}$ \\
\hline $\begin{array}{l}\text { Robots to provide support } \\
\text { and interaction }\end{array}$ & $\begin{array}{l}\text { Training and education built } \\
\text { around a specialization }\end{array}$ & $\begin{array}{l}\text { Training and ongoing } \\
\text { support for providers } \\
\text { outside ASD network }\end{array}$ \\
\hline $\begin{array}{l}\text { Planning and coordinated } \\
\text { communication through } \\
\text { transitions }\end{array}$ & Enjoying friendships & $\begin{array}{l}\text { Discovering and nurturing } \\
\text { specializations so adults on } \\
\text { the spectrum can reach } \\
\text { their full potential }\end{array}$ \\
\hline $\begin{array}{l}\text { Physical, mental and social } \\
\text { stimulation }\end{array}$ & $\begin{array}{l}\text { Self-advocacy training and } \\
\text { opportunities }\end{array}$ & $\begin{array}{l}\text { Peace of mind for parents } \\
\text { so they can live a more } \\
\text { normal life }\end{array}$ \\
\hline
\end{tabular}


Images: Faces, Homes/Places, Health \& Technology, People/Family

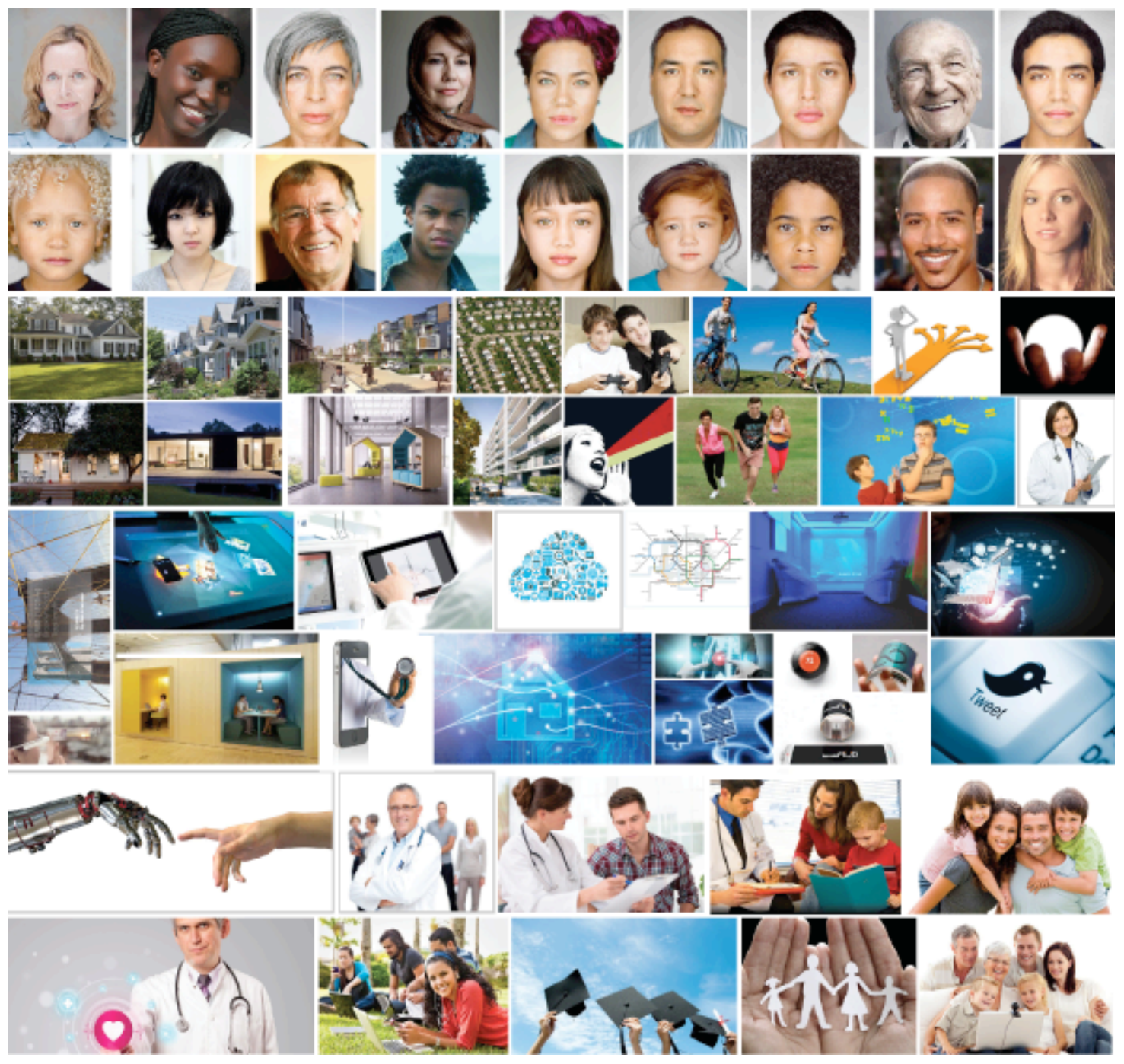


Prospective Scenario Toolkit

Headers:

\begin{tabular}{ll} 
Personality & About $\begin{array}{c}\text { Age, Location, } \\
\text { Family Stution, etc) }\end{array}$ \\
Lifestyle & Specialization \\
\hline Communication & Potential \\
\hline Support / Nurture & Transitioning \\
\hline Specialization & Training \\
\hline Planning & Optimal Care \\
\hline Finance & Peace of Mind \\
\hline Specialists & Physicians \\
\hline Career & Family \\
\hline Independence & Parents \\
\hline Guidance & Friends \\
\hline Housing & Interaction \\
\hline Education & Administrators \\
\hline Physical Stimulation & Companion \\
\hline Social Stimulation / Interaction \\
\hline Mental Stimulation & Understanding
\end{tabular}

An Assortment of 2D Paper Shapes:

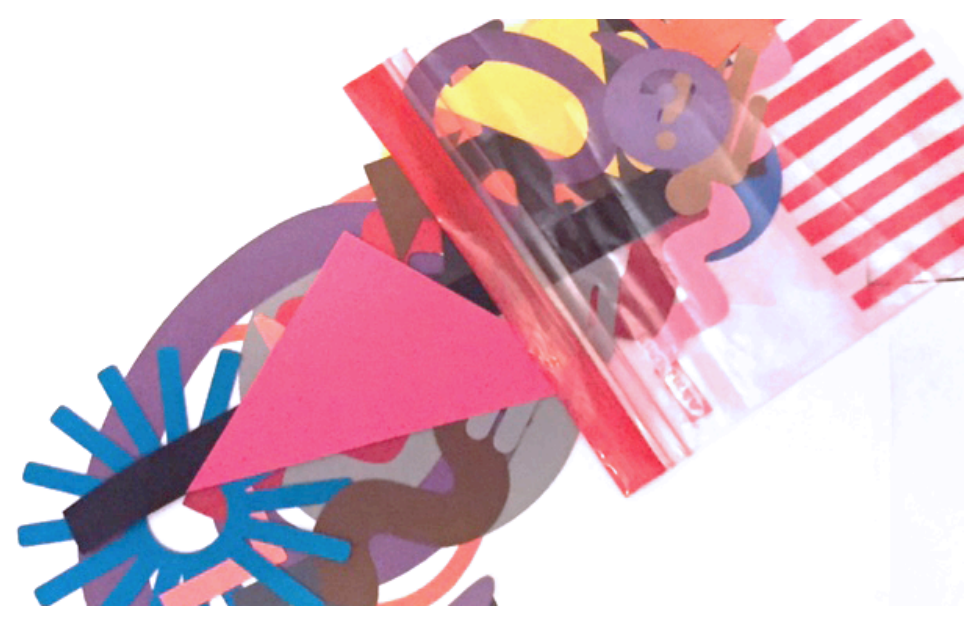


Images: Faces, Homes/Places, Health \& Technology, Lifestyle/Family

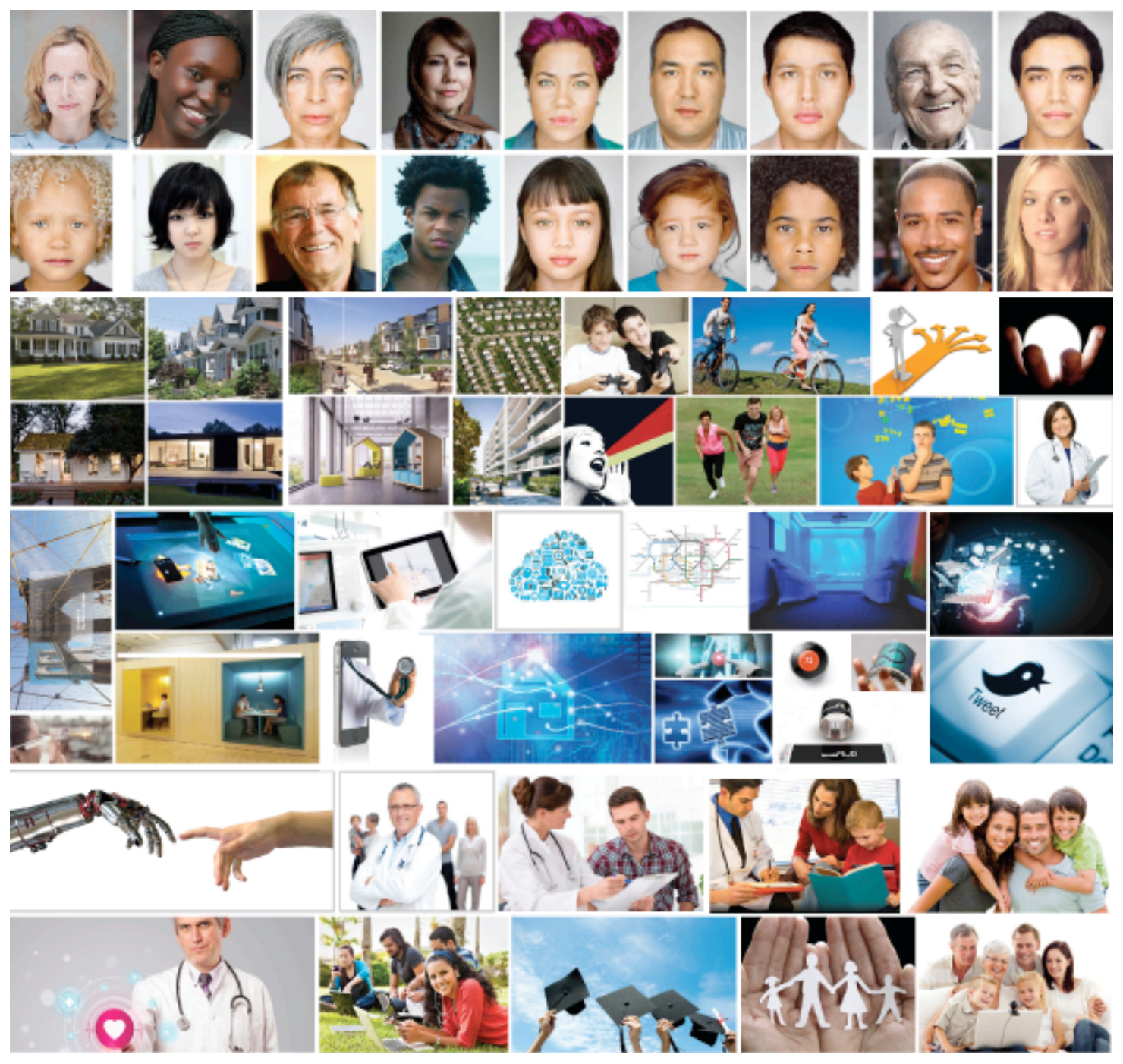




\section{Situation Cards:}

Access to education and fullfilling careers after high school (like working at Microsoft)

Robots to provide support and interaction
Living on their own /

Affordable housing
Sensors to monitor and communicate
Training and education built around a specialization
Training and ongoing support for providers outside ASD network
Planning and coordinated communication through transitions

Physical, mental and social stimulation
Self-advocacy training and opportunities
Getting through puberty

Enjoying friendships
Peace of mind for parents so they can live a more normal life 


\section{Prototyping Toolkit}

2D Shapes, Paper, Markers, Tape, and Scissors

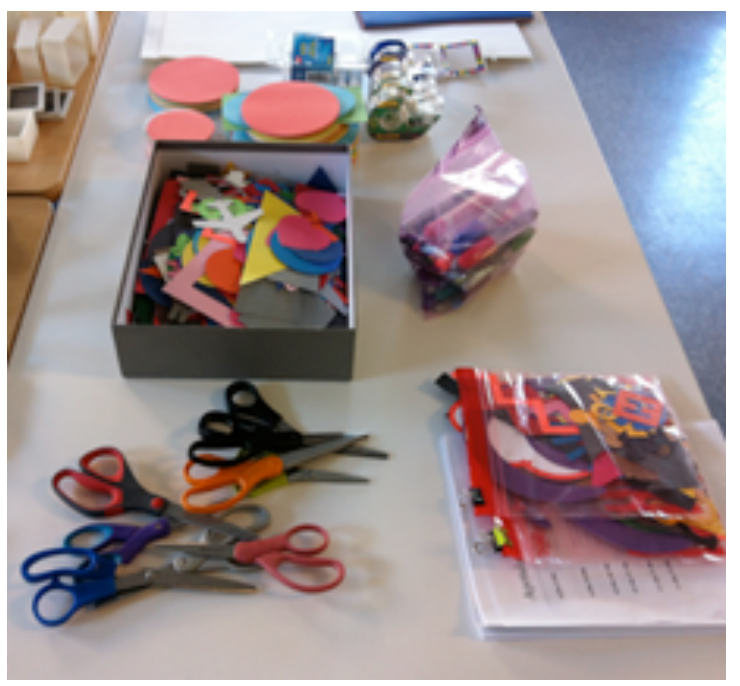

3D Velcro Shapes, White Blocks and Furniture Pieces, Random Objects, LEGOs, and Puppets
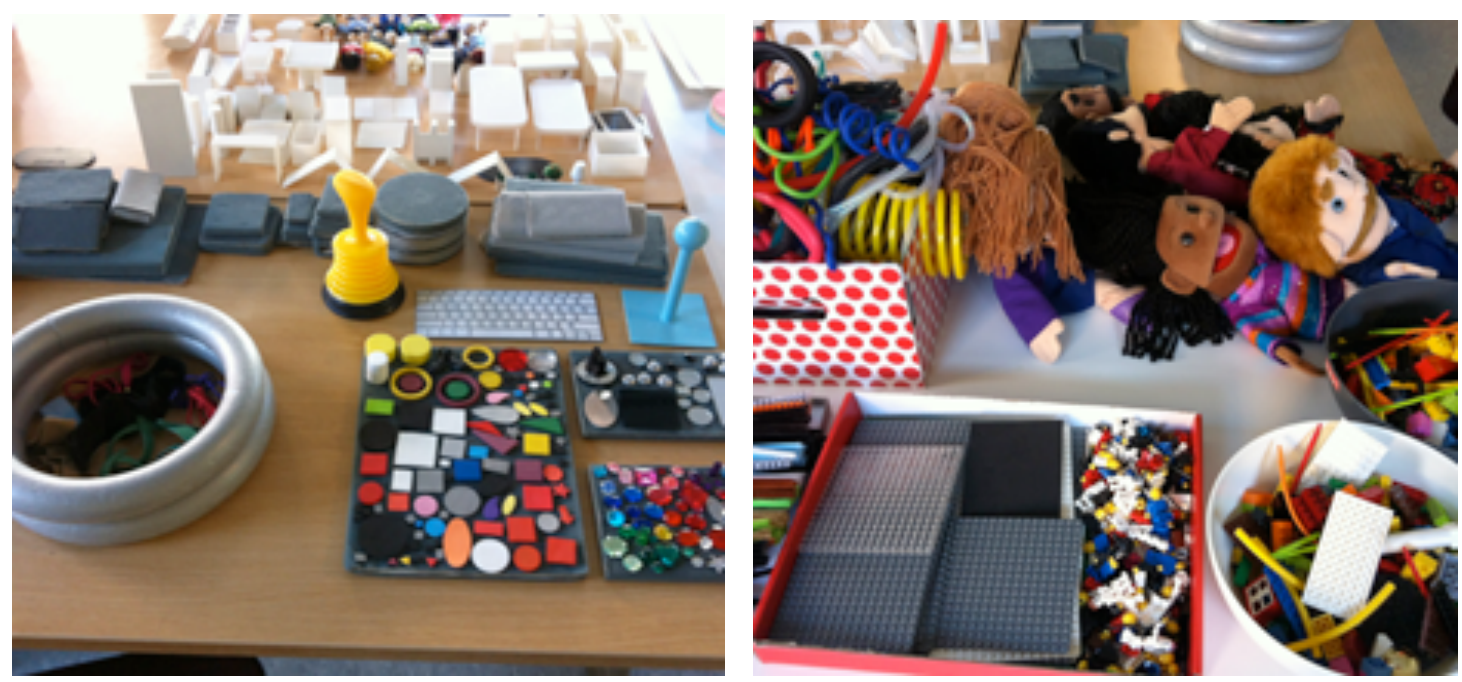
Near and Long-Term (Disruptive) Ideas Collected Over the Course of the Entire Project

\author{
Ideas: Long-Term Planning
}

\author{
NEAR-TERM \\ -Tools to help providers \& parents discover \& reinforce \\ child's specialized 'focus' \& potential \\ -The C.A.S.T. network extends to life coaches \& \\ vocation/occupation resources (job placement) \\ -Vision Planning (AASPIRE Toolkit) with IMAGES \\ -Pharmacy \& other extenders brought sooner into \\ 'sensitive' transition process \\ -Life-skills training, modeling \& social stories \\ -"Interfacer" tools for young adults on the spectrum \\ (alleviate parents' stress \& increase self-advocacy) \\ -On-going training \& support programs for outside \\ medical specialists (beyond primary care physicians) \\ -On-going healthcare and therapy support for \\ parents (at C.A.S.T.) - Family Centered Care \\ -Road maps \& clearinghouse with information for: \\ 'New' ASD parents (maps and support networks) \\ Transitions (road maps and expectations) \\ Housing/employment opportunities \& networks
}

\author{
LONG-TERM/DISRUPTIVE \\ -Tools to make school counselors more aware \\ of post-secondary opportunities for ASD persons \\ -Affordable, high efficiency homes (materials \& layouts) \\ -Life-skills \& social training + housing 'dorms' \\ -Innovative funding models (ex. micro-enterprises) \\ -Careers in technology, futuring, data intelligence, etc \\ -Post-secondary 'silo' education/training (up to PhD) for \\ specialized interests \\ -Provide means to spread resources and \\ responsibilities: between C.A.S.T. and other healthcare \\ institutions/specialists, etc. and between parents and \\ young adults (interfacer roles and responsibilities) \\ -Siri \& Apps to help people on the spectrum \\ become more interdependent/independent \\ -Maps, tools \& programs to avoid or prepare for "The Cliff" \\ after a child on the spectrum graduates from high school \\ -Programs and housing to support adults on the spectrum \\ after their parents pass away.
}

\title{
Ideas: Accessibility \& Continuous Care/Support
}

\section{NEAR-TERM}

-Calming/meditation area for parents and staff

-Open/flexible communication modes

-Infographics

-Text to speech programs on iPad tablets

-Sensory sensitivity \& awareness

'Themed' quiet focus rooms

Give them control in the space

Dark, calm music, no clashing stimuli

-Stress reducers to increase health and functional abilities of child AND parent

-Establish stronger ties with Nationwide \& other providers.

-Establish more integrated communication channels between providers/extenders and programs

\section{LONG-TERM/DISRUPTIVE}

\author{
-Ubiquitous Sensing Technology \\ -Sensors to track physiological patterns, changes, and \\ disruptions to: help caregivers better anticipate and \\ manage 'incidents', and communicate signs for changed \\ behavior to new providers, caregivers, and aides. \\ -Sensors linked to provider tablet and MyChart so \\ provider can see patterns (meaningfully detecting \\ patterns) \& know what to expect before the clinic visit \\ -Sensors and app reminders to manage a child or adult's \\ care \& give them more independence (as child ages) \\ -Stress reducers to increase health and functional \\ abilities of child AND parent \\ - Universal care solution communication ideas, \\ de-stressor environ. designs, navigation tools, \& clinic \\ 'expectation' guides (day-of and long-term) \\ -Formulate sustainable supports and collaborative \\ networks between providers and specialists
}




\section{NEAR-TERM}

-Specialist outreach program and directory

-Increased 'buy-in' from outside physicians requires: Increasing navigation fluidity for parents/patients Helping child on the spectrum know what to expect (decrease anxiety \& increase self-advocacy)

-Visual/iconic step-by-step clinic guides

-Visual time expectation cues

-Helping child know what to expect helps minimize harmful behaviors which come from confusion \& not being able to communicate.

-Portable records (provide key info - long \& short versions of medical record)

-Methods and tools to better engage w/ppl. on the spectrum to uncover indv. coping needs

-Iconic 'passports' with 'key' medical info. for a 'good visit' -Establish stronger ties w/Nationwide \& other providers

-Transparent \& accessible communication channels between healthcare institutions

\section{LONG-TERM/DISRUPTIVE}

-Integrated network trained prov., extenders, \& specialists

-More connected ASD Care Model (like Cancer Care Model)

-Care coordination mapping: understanding roles for greater impact and a smoother running system

-Care consolidation (clearinghouse) for parents and adults on the spectrum to easily access and contribute

-Effective, varied, and efficient communication channels between: Peds \& adult care, Parents/patients \& providers Virtual/online communication vs. face-to-face MyChart, TeleMed ... what else?

-Universal care-coord. mapping, navigation tools and clinic'expectation' guides (day-of and long-term)

-Apps for parents, patients, and providers/extenders to track medical histories, treatments (and outcomes), and behavior/sensory sensitivities

-Navigation app to find specialists (like Yelp): narrow search results by location, child's specific needs, etc.

\section{Ideas: Social Interaction}

\section{NEAR-TERIV}

-Open/flexible communication modes inside and outside the clinic

-Infographics

-Text to speech programs on iPad tablets

-Integrated social, physical, and mental stimulation programs (safe and accessible recreation centers)

\section{LONG-TERM/DISRUPTIVE}

-Online video game community (commun. \& education)

-Create safe spaces (digital and non-digital spaces) where ppl. feel they can be heard

-Life-skills \& social training + housing 'dorms'

-Open-source problem solving/innovative thinking community for people on the spectrum

-Innovative \& accessible commun. channels among parents, people on the spectrum, \& mainstream

-Tools and channels to help parents send out a 'cohesive message' while also recognizing that each child/adult on the spectrum is unique and has individual needs

-Mainstream + special needs higher education (similar to Oakstone model): specialized spaces and learning opportunities \& specialized services and expectations (beyond their graduates stocking shelves) 
Appendix E: Post-Phase Feedback and Reflections 


\section{Post Assessment Questionnaire}

Your participation in this questionnaire is completely voluntary. You may stop at any time.

Please consider each question carefully. Feel free to ask us to clarify any question that is not clear.

CIRCLE ONE; ADQUT,WITH AUTISM | PARENT/CAREGIVER | HEALTH CARE PRACTITIONER | ADMINISTRATOR

Ranking: $\quad 1=$ lowest $\quad 10=$ highest

1 Rate your current understanding of the daily issues facing individuals with autism and their families.

$\begin{array}{llllllllll}1 & 2 & 3 & 4 & 5 & 6 & 7 & 8 & 9 & 10\end{array}$

2 Rate your current understanding of the needs individuals with autism have as they transitions into adulthood.

$\begin{array}{llllllllll}1 & 2 & 3 & 4 & 5 & 6 & 7 & 8 & 9 & 10\end{array}$

3 Rate your current understanding of the challenges practitioners have regarding the transition of adults with autism.

$\begin{array}{llllllllll}1 & 2 & 3 & 4 & 5 & 6 & 7 & 8 & 9 & 10\end{array}$

4 Rate your knowledge of administrative processes as they relate to Autism Spectrum Disorder (Medicare, forms, billing procedures, coding, etc.).

$\begin{array}{llllllllll}1 & 2 & 3 & 4 & 5 & 6 & 7 & 8 & 9 & 10\end{array}$

5 What level of expertise do you feel you have when it comes to navigating the health care services and support offerings for individuals with autism?
Novice
Intermediate
Skillful
Experienced
Expert

6 How empowered do you feel with making changes and improvements to the current problems and system surrounding $\mathrm{ASD}$ ?

$\begin{array}{llllllllll}1 & 2 & 3 & 4 & 5 & 6 & 7 & 8 & 9 & 10\end{array}$

7 What are your current feelings towards collaboration around complex problems? (Circle all that apply.)

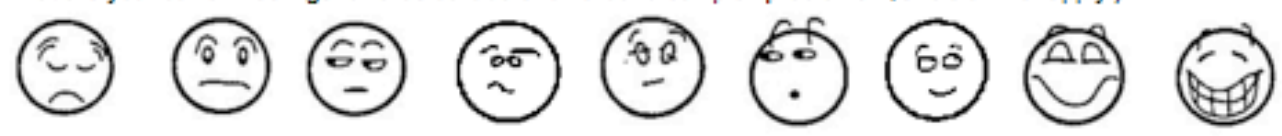

Disappointed WAnxious | Cautious | Confused | Indifferent | Curious | Interested | Confident | Ecstatic 
* Before proceeding, please RETURN to questions 1.6.

Think back to the beginning of this project. Use a square to show your position on each question before the workshops took place. So, you will have a circle and a square for each question. It is OK if you change your mind and your responses do not match the estimations you made on your pre-assessment questionnaire.

8 Will you use Design Tihinking methods in future collaborative projects to resolve complex problems?

Yes

No

9 What did you accomplish during this project?

10 What did we accomplish during this project?

11 There is value in gaining shared understanding between multiple stakeholders (connected to ASD) before discussing ideas and design opportunities.
Disagree
Somewhat Disagree
Somewhat Agree
Agree

12 There is value in prototyping and building potential resolutions to complex problems with multiple stakeholders.
Disagree
Somewhat Disagree
Somewhat Agree
Agree

13 Visualizations and diagrams help me better understand complex ideas and problems.
Disagree
Somewhat Disagree
Somewhat Agree
Agree

14 What are your current feelings towards the Center for Autism Services \& Transition (Circle all that apply.)

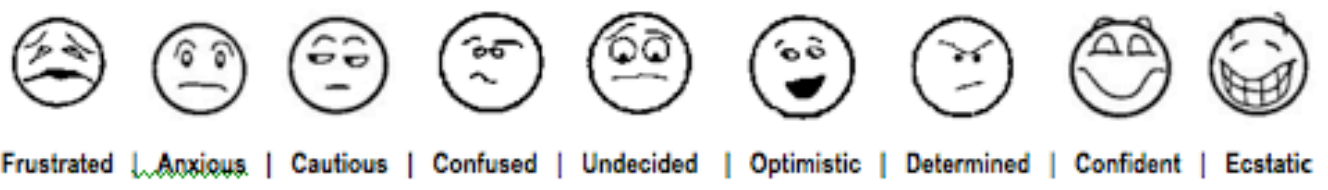




\section{Post-Assessment Questionnaire Response Summary}

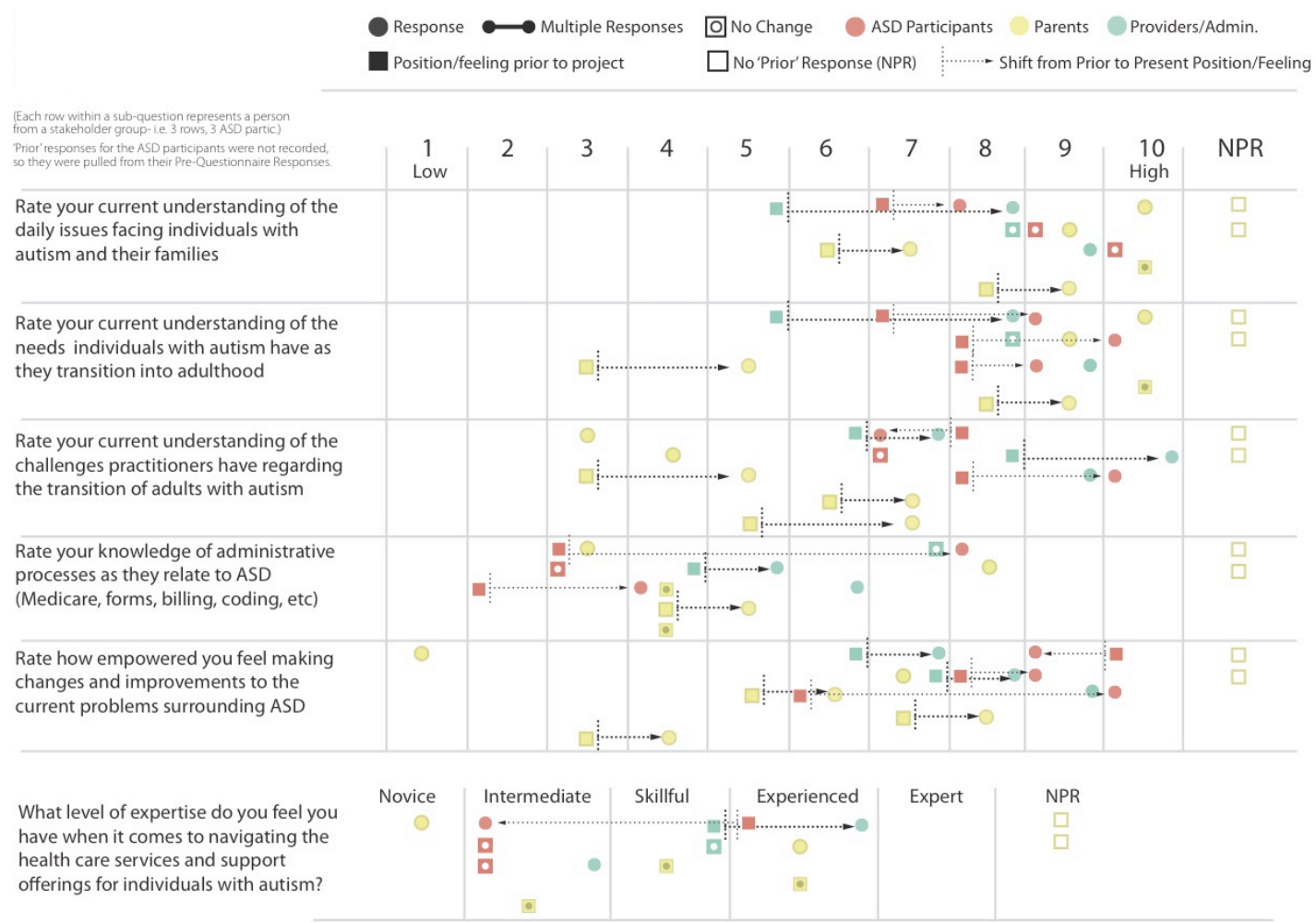

Current feelings towards collaboration Disappointed Anxious Cautious Confused Indifferent Curious Interested Confident Ecstatic NPR around complex problems (circle as many as you'd like)

(^) Note: Participant added a written response: "Militarys unwaiverable blanket ban on autism including the Air force which
pretty much NEEDS these men and women!? UNACCEPTABEI!

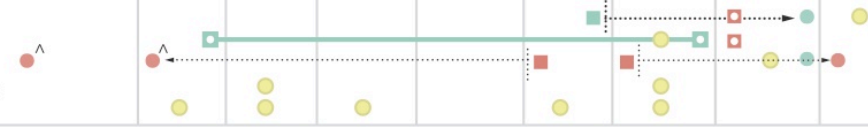

Frustrated Anxious Cautious Confused Undecided Optimistic Determined Confident Ecstatic NR

Current feelings towards the Center for Autism Services \& Transition (C.A.S.T.)

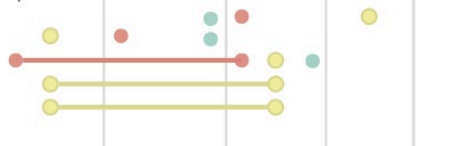

\begin{tabular}{|c|c|c|c|c|c|c|c|c|c|c|}
\hline \multirow[t]{3}{*}{$\begin{array}{l}\text { Will you use Design } \\
\text { Thinking methods in } \\
\text { future collaborative } \\
\text { projects? }\end{array}$} & \multirow{3}{*}{\multicolumn{2}{|c|}{$\begin{array}{l}\text { Yes } \\
\bullet \bigcirc \\
0\end{array}$}} & \multirow[t]{3}{*}{ No } & \multirow[t]{3}{*}{ Maybe } & \multirow[t]{3}{*}{ NR } & $\begin{array}{l}\text { There is value in gaining shared Disagree } \\
\text { understanding between multiple } \\
\text { stakeholders (connected to ASD) before } \\
\text { discussing ideas and design opportunities. }\end{array}$ & $S / D$ & S/A & $\begin{array}{c}\text { Agree } \\
0 \\
8 \\
8 \\
8\end{array}$ & NR \\
\hline & & & & & & $\begin{array}{l}\text { There is value in prototyping and building } \\
\text { potential resolutions to complex problems } \\
\text { with multiple stakeholders }\end{array}$ & & • & $\stackrel{0}{\circ}$ & \\
\hline & & & & & & $\begin{array}{l}\text { Visualizations and diagrams help me better } \\
\text { understand complex ideas and problems }\end{array}$ & & $\odot$ & $:$ & \\
\hline
\end{tabular}


Stakeholder Shifts in Understanding from Pre to Post-Assessment

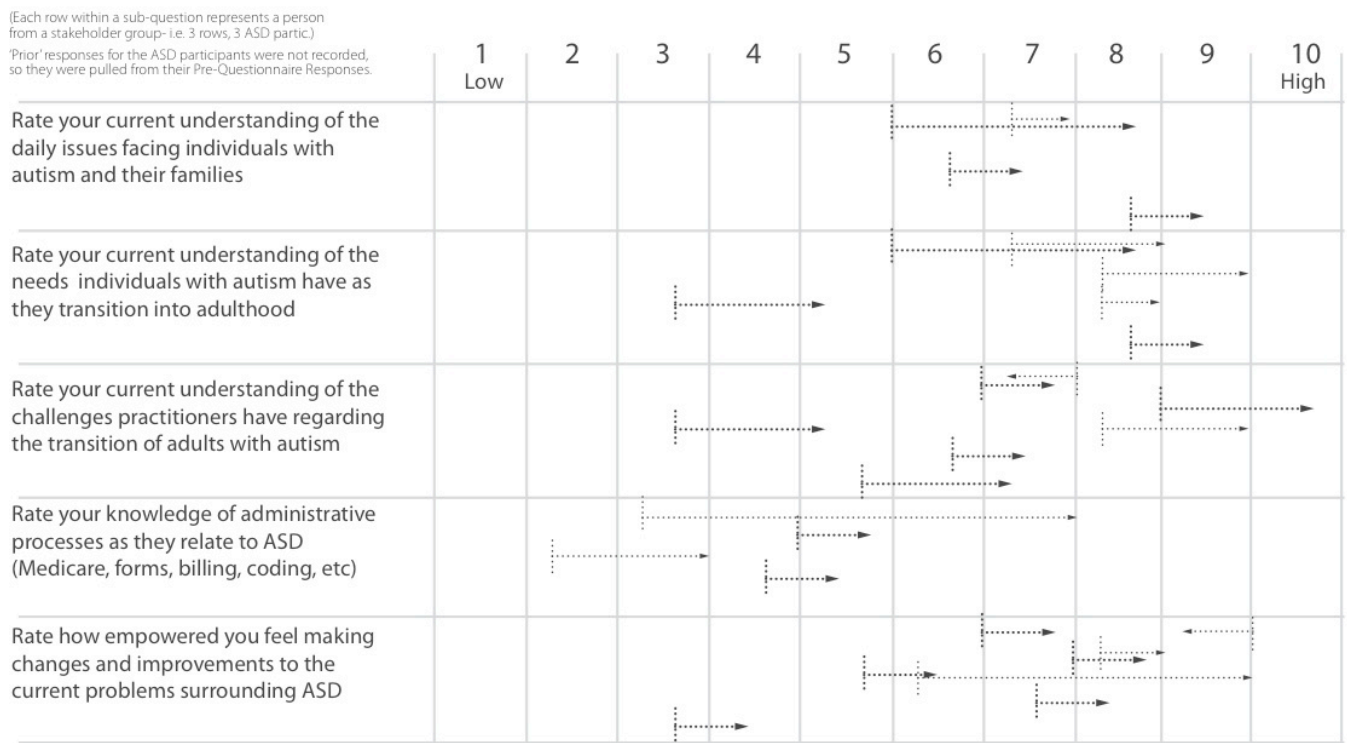


Post-Phase Feedback Questions (with responses)

\title{
1. What were your expectations prior to participating in the collaborative sessions/workshops?
}

\section{What transpired/occurred during/after the sessions (or a specific session) that was different from what you expected?}

\begin{abstract}
A Parent:
I completed the survey at the second workshop, but I wanted to answer your questions. I had no preconceived notions prior to attending the workshops. I was merely interested to be a part of a survey which included primary users, parents/family and professionals. I was very encouraged by the unanimity in the groups I was a part of regarding the needs of people on the autistic spectrum after their education is completed. As the numbers of people with autism skyrocket, and the population continues to age, it is imperative that society learns how to deal with and assist this population in as it attempts to navigate life's challenges. This workshop is a good start, but, in my opinion, this topic will become more and more relevant in the future. Clearly, more outreach and availability of post educational programs to help the autistic population deal with life are necessary to make sure these folks don't fall through society's cracks.
\end{abstract}

\begin{abstract}
A Parent:
I had assumed we would be doing a lot of "designing of space" interventions.
\end{abstract}

The conversation was much more wide-ranging than I expected. The making/designing of space was part of a much larger discussion. 


\section{A Healthcare Provider/Administrator:}

Thanks again for your excellent work on this project - I really enjoyed the process and looking forward to reading more about the outcome. I missed responding to the two questions in the initial email because I read that I didn't have to complete the survey since I was at the second workshop but realized in rereading that the two questions below were to be answered. So here are my responses.

I had no expectations. I actually had never participated in this type of workshop before. I enjoyed learning about this process and especially the use of technology to find communalities between the different stakeholder groups.

I was pleasantly surprised to see the involvement and participation of the parents and self-advocates. The self-advocates brought a lot to the table as did the parents - especially in bringing the perspective of individuals with ASD with more significant cognitive and communication deficits.

\section{A Healthcare Provider:}

I was hoping to get input and opinions from different shareholders regarding what is needed for development of CAST.

I quickly realized that CAST was not the biggest need, but just a small part of a bigger, broader need. This occurred in the session where we grouped things together with the circles on the board. 
Post-Phase Reflection Survey (with responses)

\section{Parent Participant:}

\section{Final Thoughts - Review, Reflect, and Share Your Feedback}

Thank you for your participation in my thesis research! Your participation in this final questionnaire will help me better understand specifically which methodsitools were most useful to you in the workshops and outcomes from the sessions as I wrap up my final conclusions. This questionnaire is completely voluntary. It consists of a checklist and open-ended questions. Please consider each question carefully and ask me to clarify any question that is unclear.

1. ARE YOU A(AN): (circle, bold, or underline one)

\section{ADULT WITH AUTISM | PARENT/CAREGIVER | HEALTH CARE PRACTITIONER | ADMINISTRATOR}

\section{CHECKLIST: Phases and Methods of the Collective Innovation Process}

Please rate each phase/method from $0-5$ ( 5 being most useful) Plaase just fill in the ones that you paricipated in. 'Not everyone participated in al steps. Skip or mark $(0)$ for the phases in which you did not participate.

\begin{tabular}{|c|c|c|c|}
\hline Projact Phases 8 Mathods & $\begin{array}{l}\text { (0.5) How much } \\
\text { didit it contibut to } \\
\text { your ovarall } \\
\text { undarstanding of } \\
\text { this topic? }\end{array}$ & 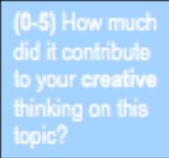 & Additional Comments: \\
\hline 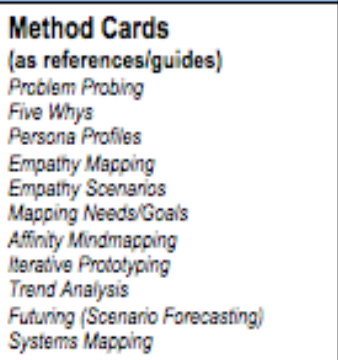 & 5 & 5 & $\begin{array}{l}\text { I use many of these techniques in my work } \\
\text { without realizing they are specific methods. } \\
\text { Having the reference cards helped me look at } \\
\text { the various autism problems/situations in new } \\
\text { and different ways. }\end{array}$ \\
\hline $\begin{array}{l}\text { Journal } \\
\text { (preparation \& reflection) }\end{array}$ & 4 & 4 & $\begin{array}{l}\text { Putting thoughts into words is my go-to problem } \\
\text { solving method and helps me organize my } \\
\text { thoughts. }\end{array}$ \\
\hline \multicolumn{4}{|l|}{ Pre-Workshop Sessions } \\
\hline \multicolumn{4}{|l|}{$\begin{array}{l}\text { One-on-One Interviews } \\
\text { (rate overall session) }\end{array}$} \\
\hline \multicolumn{4}{|l|}{$\begin{array}{l}\text { Visualizations/maps used to } \\
\text { launch into discussions } \\
\text { around needs and inital ideas }\end{array}$} \\
\hline \multicolumn{4}{|l|}{$\begin{array}{l}\text { Adults with Autism } \\
\text { Session at Donatos } \\
\text { Clinic design and needs of the } \\
\text { patient (rate overall session) }\end{array}$} \\
\hline $\begin{array}{l}\text { - Writing and discussing needs, } \\
\text { concerns \& ideas }\end{array}$ & & & \\
\hline - Bulding/constructing ideas & & & \\
\hline
\end{tabular}




\begin{tabular}{|c|c|c|c|}
\hline - Drawing ideas & & & \\
\hline $\begin{array}{l}\text { Parents/Providers \& Admin. } \\
\text { Session at McCampbell } \\
\text { (rate overall session) }\end{array}$ & 4 & 3 & $\begin{array}{l}\text { This session confirmed the common concerns } \\
\text { of ASD parents. }\end{array}$ \\
\hline $\begin{array}{l}\text { - Listing iop } 10 \text { concems, } \\
\text { needs, and goals }\end{array}$ & 4 & 3 & \\
\hline $\begin{array}{l}\text { - Mapping similarities and } \\
\text { differences between } \\
\text { stakaholder groups } \\
\end{array}$ & 5 & 3 & $\begin{array}{l}\text { Parents often feel they're on an island. I was } \\
\text { surprised by how similarly the different } \\
\text { stakeholder groups rated needs and concerns. }\end{array}$ \\
\hline \multicolumn{4}{|c|}{ Workshop 1- March 21 at Sullivant Hall (ACCAD) } \\
\hline Project Phases 8 Methods & $\begin{array}{l}\text { (0-5) Contributa to } \\
\text { Understanding }\end{array}$ & $\begin{array}{l}\text { (0.5) Contributa } \\
\text { to Creativity }\end{array}$ & Additional Comments: \\
\hline $\begin{array}{l}\text { - Presenting \& discussing: } \\
\text { sharing unique perspectives } \\
\text { with other stakeholders }\end{array}$ & 5 & 5 & $\begin{array}{l}\text { Hearing the other individual and groups' } \\
\text { perspectives was essential to reaching shared } \\
\text { understanding and prioritization. }\end{array}$ \\
\hline $\begin{array}{l}\text { Prioritization (within } \\
\text { stakeholder groups) }\end{array}$ & 5 & 4 & \\
\hline $\begin{array}{l}\text { - Mapping/clustering pricritized } \\
\text { needs/goals (all stakeholders) } \\
\text { with the Digital Tool }\end{array}$ & 3 & 3 & \\
\hline $\begin{array}{l}\text { Mapping/clustering } \\
\text { with Post-lts }\end{array}$ & 4 & 4 & \\
\hline $\begin{array}{l}\text { - Discussion around } \\
\text { connections/patterns in } \\
\text { clusters \& prionity areas }\end{array}$ & 5 & 4 & \\
\hline \multicolumn{4}{|c|}{ Workshop 1 PDF Feedback/Summary - Workshop 2 Preparation } \\
\hline Projact Phases 8 Mathods & (0.5) Contributis to & $\begin{array}{l}\text { (0-5) Contributa } \\
\text { to Creativity }\end{array}$ & Additional Comments: \\
\hline $\begin{array}{l}\text { - Constructing narratives and } \\
\text { scenarios }\end{array}$ & 4 & 4 & \\
\hline - Trend analysis/review & 4 & 4 & \\
\hline - Mindmapping & & & \\
\hline $\begin{array}{l}\text { - Reviewing needs, goals, and } \\
\text { ideas from Workshop } 1\end{array}$ & 3 & 4 & \\
\hline \multicolumn{4}{|c|}{ Workshop 2 - May 2 at Hayes Hall } \\
\hline Project Phases 8 Mothods & $\begin{array}{l}\text { (0.5) Contribute to } \\
\text { Understanding }\end{array}$ & $\begin{array}{l}\text { (0-5) Contibute } \\
\text { to Creativity }\end{array}$ & Addiftional Comments: \\
\hline $\begin{array}{l}\text { Homework (joumal questions } \\
\text { and future vision board) }\end{array}$ & 4 & 4 & \\
\hline $\begin{array}{l}\text { Exploring current needs - } \\
\text { bulding current personas }\end{array}$ & 4 & 5 & \\
\hline $\begin{array}{l}\text { - Future Forecasting - building } \\
\text { future personas and scenarios }\end{array}$ & 5 & 5 & \\
\hline $\begin{array}{l}\text { - Journey mapping (persona } \\
\text { development-current to future) }\end{array}$ & 4 & 5 & $\begin{array}{l}\text { This was an especially helpful technique as it } \\
\text { required us to think through how the persona } \\
\text { person would accomplish their goals. }\end{array}$ \\
\hline
\end{tabular}




\begin{tabular}{|l|l|l|l}
\hline $\begin{array}{l}\text { - Prototyping \& presenting } \\
\text { ideas/ooncepts derived from } \\
\text { identifed gaps \& opportunities }\end{array}$ & 5 & 5 & $\begin{array}{l}\text { Working out a prototype with stakeholders from } \\
\text { each group was the most meaningful part of the } \\
\text { process. }\end{array}$ \\
\hline
\end{tabular}

OPEN-ENDED QUESTIONS: (Feel free to provide short responses, lists of thoughts, or bullet points)

1. Based on reviewing the project summary, please share your thoughts on the outcomes of this project. The outcomes of this project are a significant example of the collaboration required to address the complex, multfaceted issues facing individuals with autism and their families. This work must be presented to the national autism organizations for further advancement!

2. Do the findings from our project reflect and address varying needs of the stakeholders? Please explain. Very much so. Voices from all stakeholder groups were heard and reflected in the findings.

3. As a participant, what do you feel was your role/value in this process? Bringing a lot of ideas, an open mind, an appreciation for what others' contributed to the process, and a willingness to share and listen.

4. Do you feel like you were heard and that the group valued your opinions/thoughts/ideas?

Yes. Due to near-constant advocacy for our ASD kidsladults, parents often feel like everyone else (providers, schools, social workers) are on the other team (e.g., in most IEP meetings there is one parent and at least 8 administrators on the other side of the table). In these workshops, it felt more like every person's input was valused and welcome.

5. Did anything interesting emerge during your participation (networking, ideas to pursue, new resources, etc)? If yes, please describe.

Many of the ideas generated can be furthered developed and implemented. The longer range ideas (Owen's List, housing, disruptive caregiving technologies, virtual and physical communities) must happen, as there are no existing solutions for the long term care and support of the ASD adult population.

6. Did we uncover new insights? If so, which insights?

The value of co-design as an approach for difficult, mult-faceted problems for which there are no easy answers and no singular solution.

7. Is there anything you would change about the sessions or the overall process? Maybe having more ASD adults participate. There was such consistency from the parents' perspective (despite the range of severely-affected to more-independent ASD children) that I don't think having additional parents would have changed the outcome.

8. Was there something you expected that did not occur? No, actually more occurred than I expected.

9. As for the thesis, this is as far as I can take it, but what wishes do you have for next steps? I plan to keep working on the caregiving technology, housing and Owen's List projects. I'd like to work with you to present this research to Autism Speaks so that others can benefit from what we've learned and the ideas generated.

10. Do you have any final comments or thoughts?

Thank you for tackling this challenging topic and advancing the strategies to develop solutions! 


\section{A Healthcare Provider:}

Final Thoughts - Review, Reflect, and Share Your Feedback

Thank you for your participation in my thesis research! Your participation in this final questionnaire will help me better understand specifically which methodsittools were most useful to you in the workshops and outcomes from the sessions as I wrap up my final conclusions. This questionnaire is completely voluntary. It consists of a checklist and open-ended questions. Please consider each question carefully and ask me to clarify any question that is unclear.

1. ARE YOU A(AN): (circle, bold, or ynderline one)

\section{ADULT WITH AUTISM | PARENT/CAREGIVER | HEALTH CARE PRACTITIONER | ADMINISTRATOR}

\section{CHECKLIST: Phases and Methods of the Collective Innovation Process}

Please rate each phase/method from $0-5$ ( 5 being most useful) Plaase just fill in the ones that you paricipated in.

"Not everyone participated in al steps. Skip or mark (0) for the phases in which you did not participate.

\begin{tabular}{|c|c|c|c|}
\hline Projact Phases 8 Methods & $\begin{array}{l}\text { (0-5) How much } \\
\text { did it contibule to } \\
\text { your overall } \\
\text { understanding of } \\
\text { this topic? }\end{array}$ & $\begin{array}{l}\text { (0-5) How much } \\
\text { did it contibute } \\
\text { to your creative } \\
\text { thinking on this } \\
\text { topic? }\end{array}$ & Additional Comments: \\
\hline 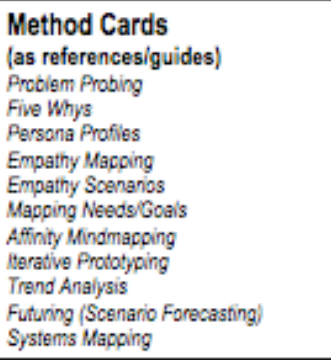 & 5 & 5 & \\
\hline $\begin{array}{l}\text { Journal } \\
\text { (preparation \& reflection) }\end{array}$ & n/a & $n / a$ & \\
\hline \multicolumn{4}{|l|}{ Pre-Workshop Sessions } \\
\hline $\begin{array}{l}\text { One-on-One Interviews } \\
\text { (rate overall session) }\end{array}$ & 5 & 5 & \\
\hline $\begin{array}{l}\text { Visualizations/maps used to } \\
\text { launch into discussions } \\
\text { around needs and inital ideas }\end{array}$ & 5 & 5 & \\
\hline $\begin{array}{l}\text { Adults with Autism } \\
\text { Session at Donatos } \\
\text { Clinic design and needs of the } \\
\text { patient (rate overall session) }\end{array}$ & $n / a$ & n/a & \\
\hline $\begin{array}{l}\text { - Writing and discussing needs, } \\
\text { concerns \& ideas }\end{array}$ & & & \\
\hline - Bulding/consiructing ideas & & & \\
\hline - Drawing ideas & & & \\
\hline
\end{tabular}




\begin{tabular}{|c|c|c|c|}
\hline $\begin{array}{l}\text { Parents/Providers \& Admin. } \\
\text { Session at McCampbell } \\
\text { (rate overall session) }\end{array}$ & n/a & n/a & \\
\hline $\begin{array}{l}\text { - Listing iop } 10 \text { concems, } \\
\text { needs, and goals }\end{array}$ & & & \\
\hline $\begin{array}{l}\text { - Mapping similarities and } \\
\text { differences between } \\
\text { stakeholder groups }\end{array}$ & & & \\
\hline \multicolumn{4}{|c|}{ Workshop 1- March 21 at Sullivant Hall (ACCAD) } \\
\hline Project Phases \& Methods & $\begin{array}{l}\text { (0.5) Contributa to } \\
\text { Understanding }\end{array}$ & $\begin{array}{l}\text { (0.5) Continbuta } \\
\text { to Creativity }\end{array}$ & Addiftional Comiments: \\
\hline $\begin{array}{l}\text { - Presenting \& discussing: } \\
\text { sharing unique perspectives } \\
\text { with other stakeholders }\end{array}$ & 5 & 5 & This was very powerful. \\
\hline $\begin{array}{l}\text { Prioritization (within } \\
\text { stakeholder groups) }\end{array}$ & 4 & 4 & \\
\hline $\begin{array}{l}\text { - Mapping/dustering pricritized } \\
\text { needs/goals (all stakeholders) } \\
\text { with the Digital Tool }\end{array}$ & 3 & 3 & \\
\hline $\begin{array}{l}\text { Mapping/clustering } \\
\text { with Post-lts }\end{array}$ & 4 & 4 & \\
\hline $\begin{array}{l}\text { - Discussion around } \\
\text { connections/patterns in } \\
\text { clusters \& prionity areas }\end{array}$ & 4 & 4 & \\
\hline \multicolumn{4}{|c|}{ Workshop 1 PDF Feedback/Summary - Workshop 2 Preparation } \\
\hline Project Phases 8 Mothods & $\begin{array}{l}\text { (0.5) Contributa io } \\
\text { Understanding }\end{array}$ & $\begin{array}{l}\text { (0.5) Contributis } \\
\text { bo Creativity }\end{array}$ & Addiftional Commenta: \\
\hline $\begin{array}{l}\text { - Constructing narratives and } \\
\text { scenarios }\end{array}$ & 3 & 3 & \\
\hline - Trend analysis/review & 3 & 3 & \\
\hline - Mindmapping & 4 & 4 & \\
\hline $\begin{array}{l}\text { - Reviewing needs, goals, and } \\
\text { ideas from Workshop } 1\end{array}$ & 3 & 3 & \\
\hline \multicolumn{4}{|c|}{ Workshop 2 - May 2 at Hayes Hall } \\
\hline Project Phases 8 Methods & $\begin{array}{l}\text { (0.5) Conkibuta to } \\
\text { Understanding }\end{array}$ & $\begin{array}{l}\text { (0.5) Conlibute } \\
\text { to Creativity }\end{array}$ & Additional Cominenta: \\
\hline $\begin{array}{l}\text { - Homework (journal questions } \\
\text { and future vision board) }\end{array}$ & $\mathrm{n} / \mathrm{a}$ & n/a & I couldn't attend \\
\hline $\begin{array}{l}\text { Exploring current needs - } \\
\text { bulding current personas }\end{array}$ & & & \\
\hline $\begin{array}{l}\text { Future Forecasting - building } \\
\text { future personas and scenarios }\end{array}$ & & & \\
\hline $\begin{array}{l}\text { - Journey mapping (persona } \\
\text { development-current to future) }\end{array}$ & & & \\
\hline $\begin{array}{l}\text { Prototyping \& presenting } \\
\text { ideas/concepts derived from } \\
\text { identifed gaps \& opportunities }\end{array}$ & & & \\
\hline
\end{tabular}

*This participant did not fill out the last page. 
Appendix F: Digital Tool 


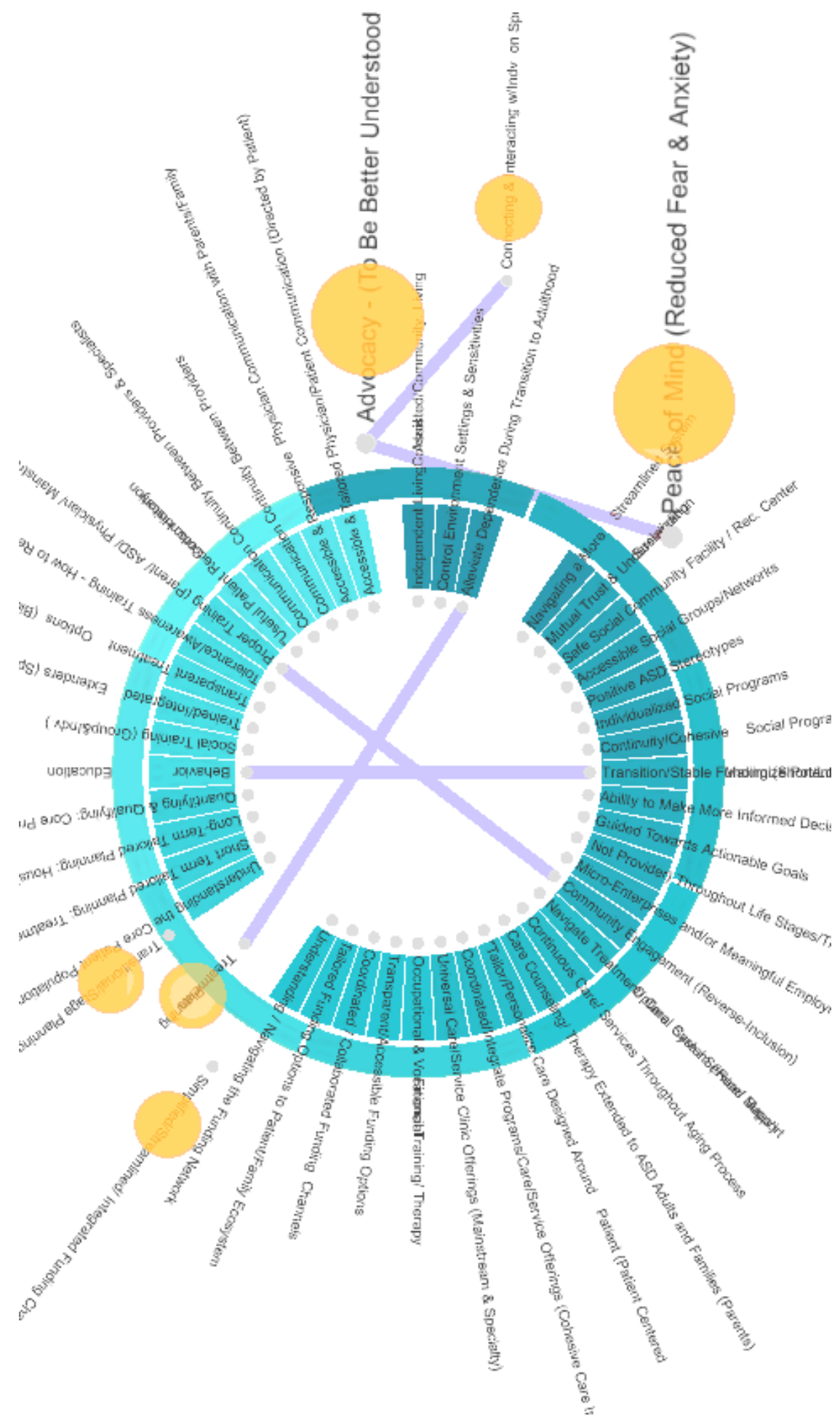



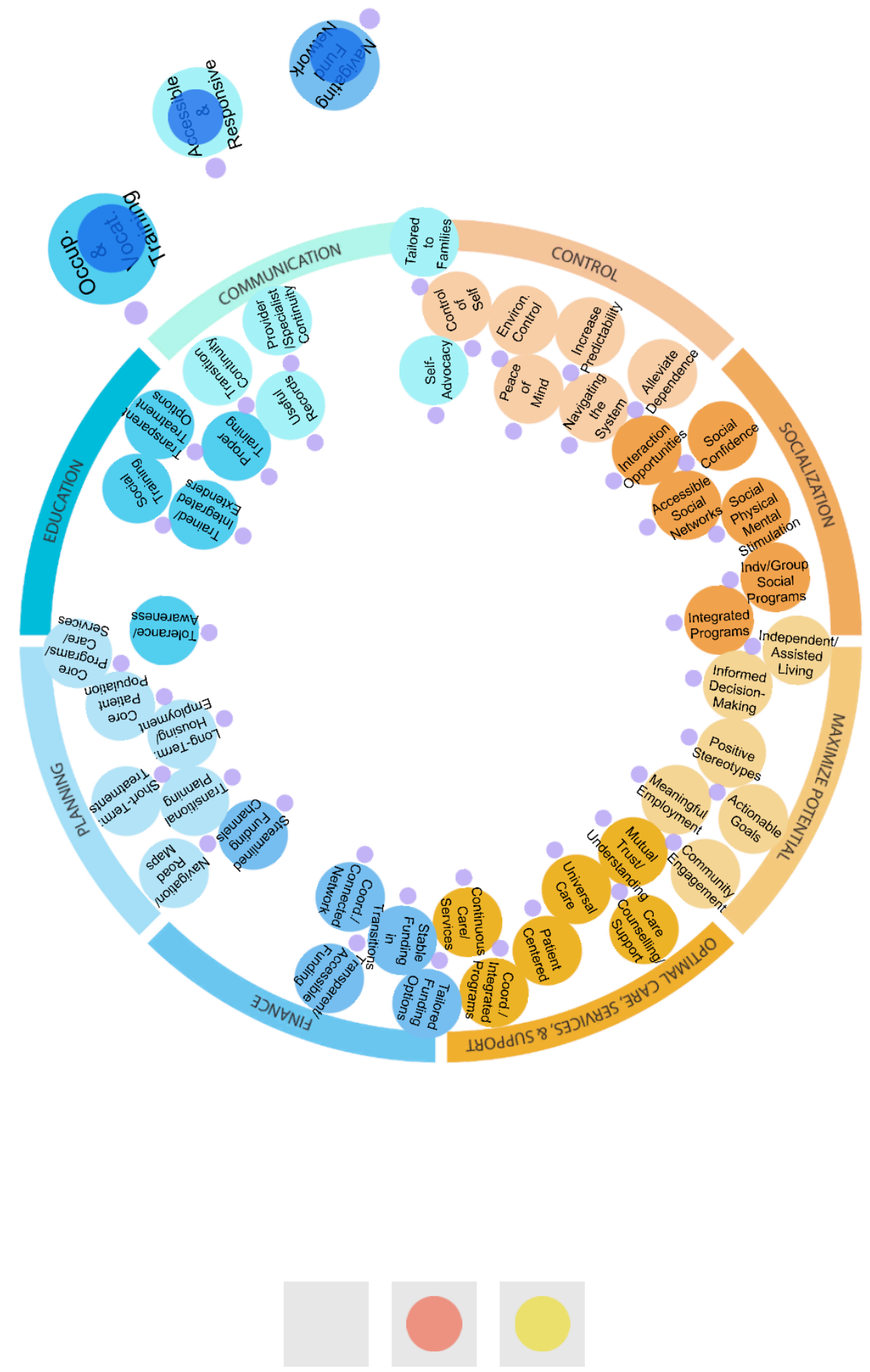

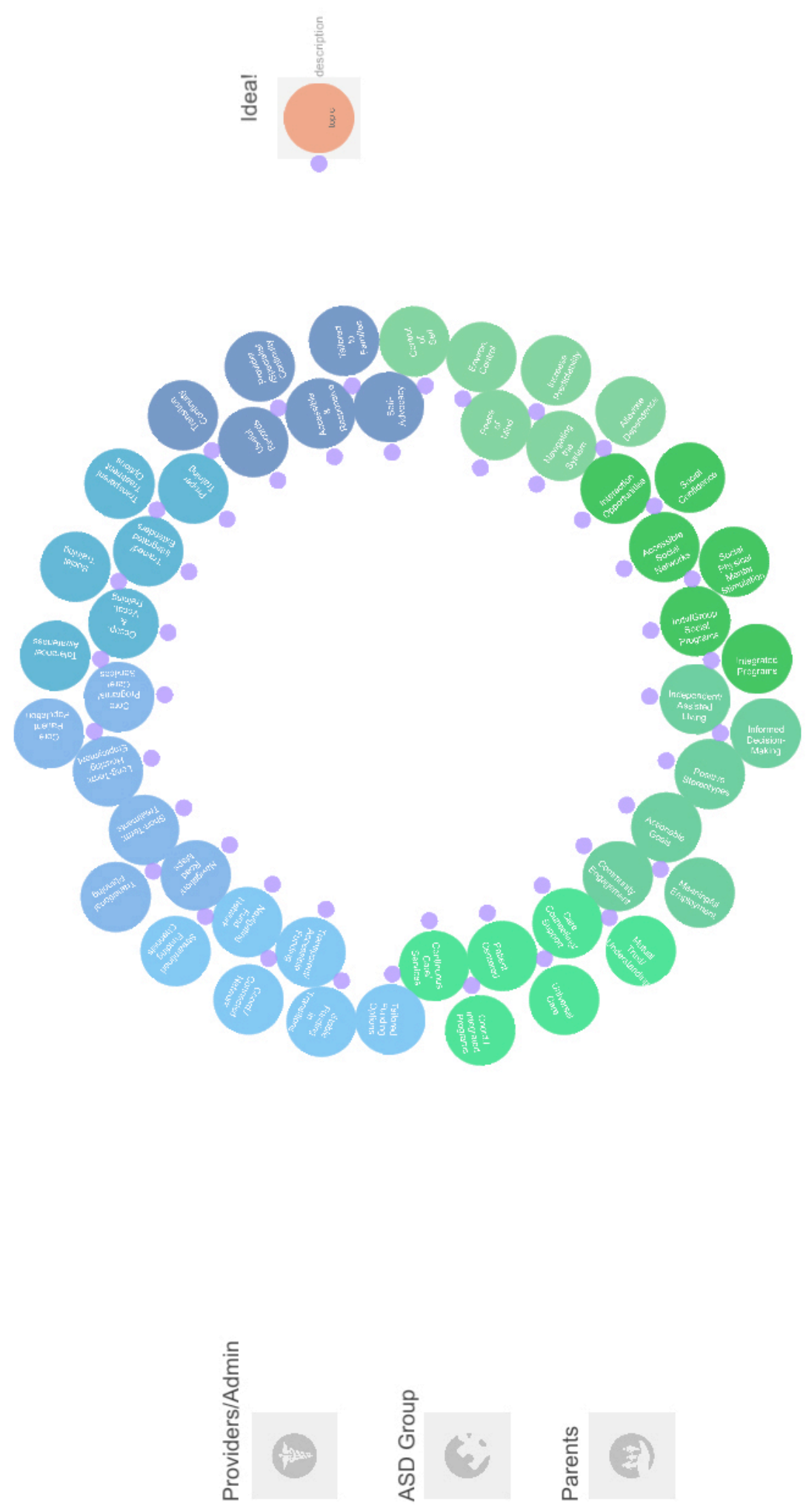

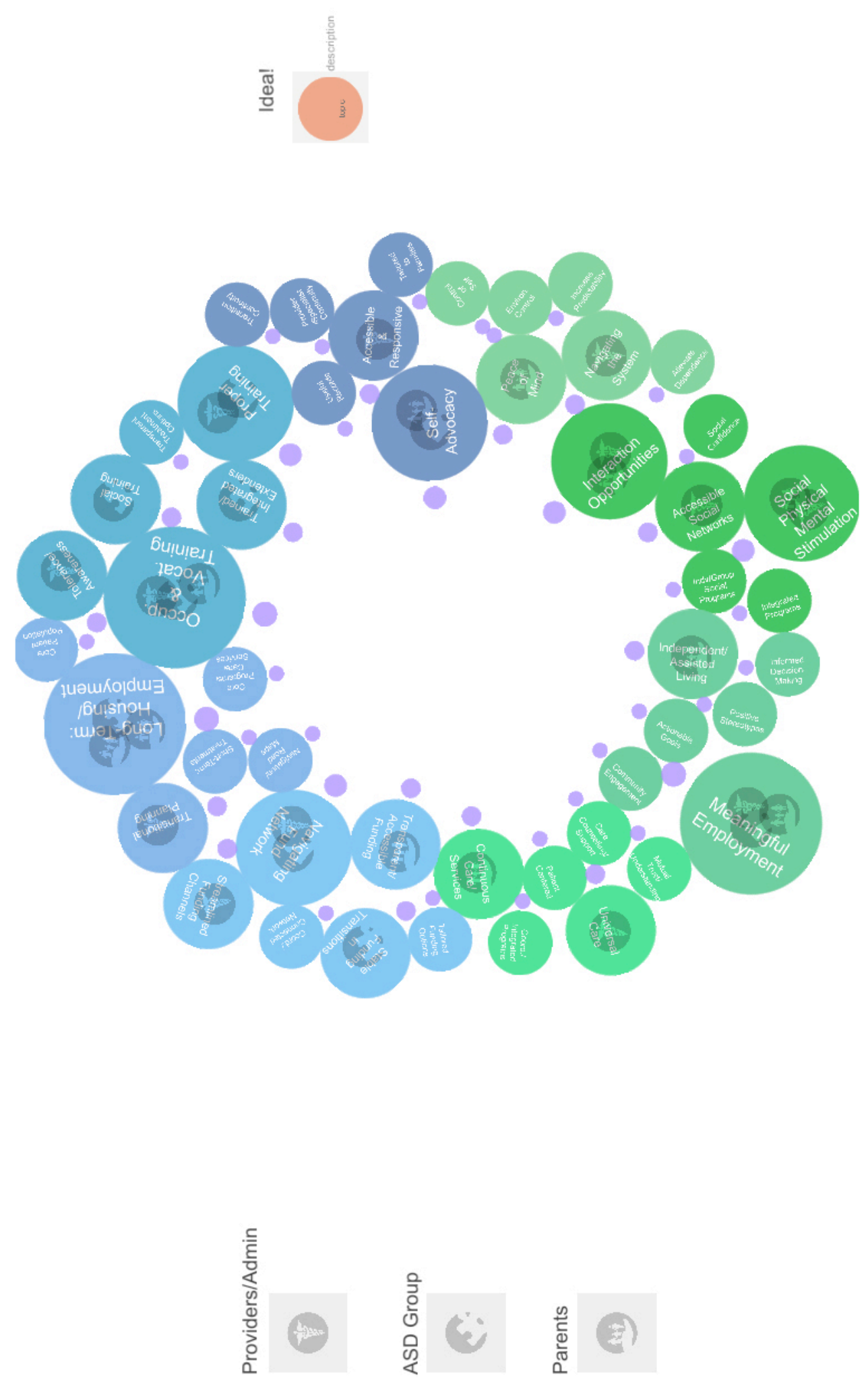


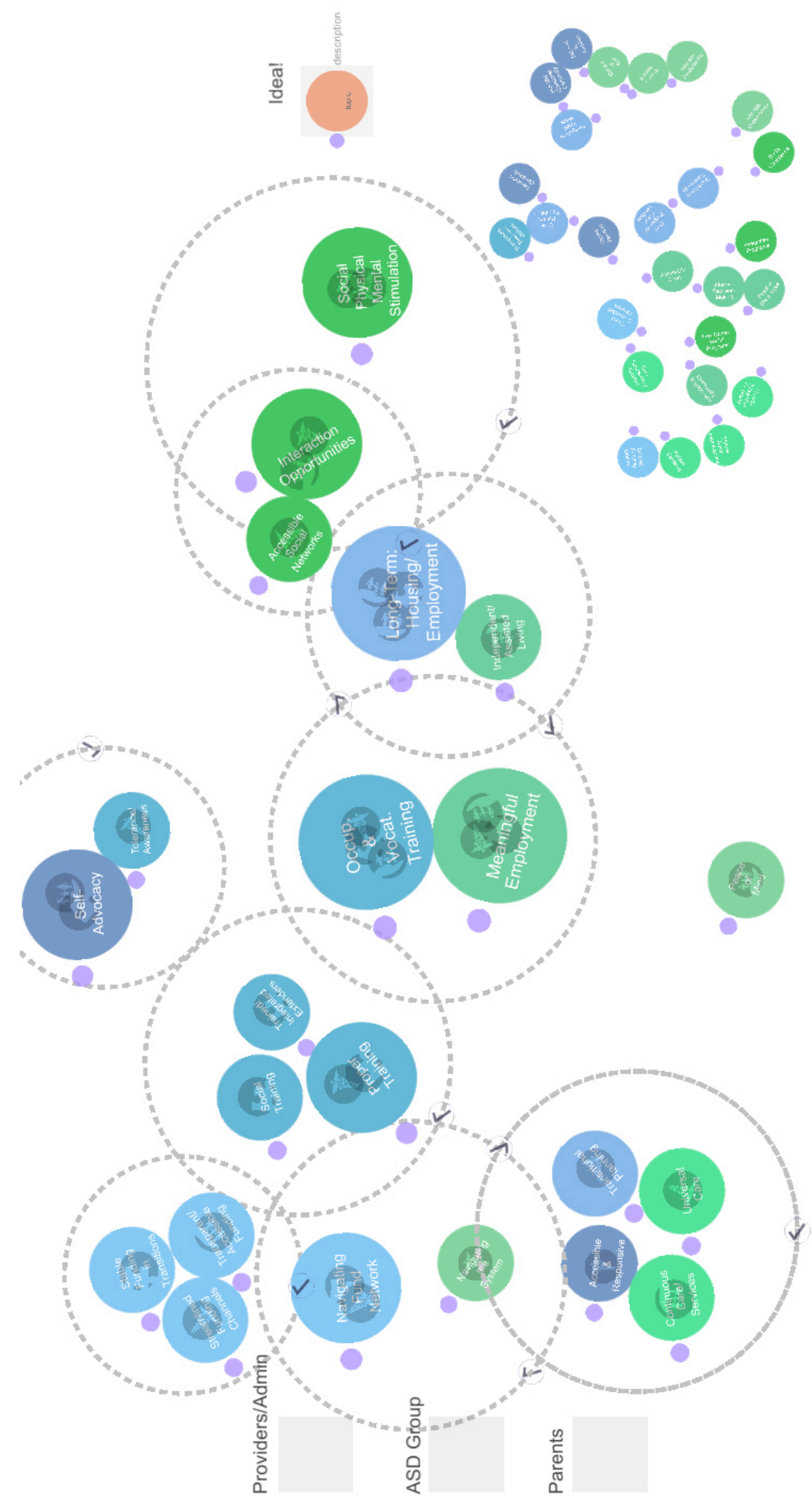




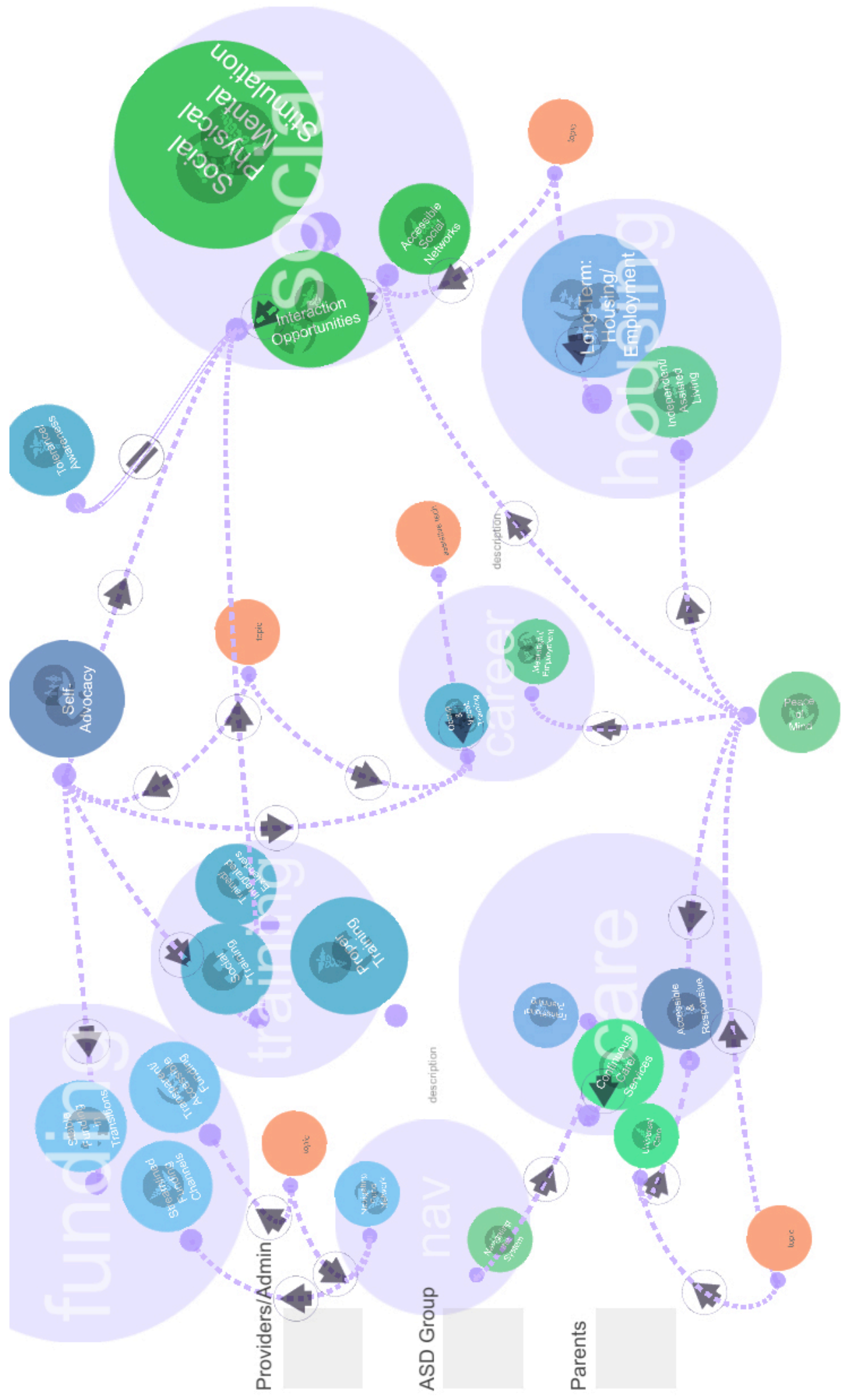

481 